\title{
Advanced Fuel Cycle Economic Analysis of Symbiotic Light-Water Reactor and Fast Burner Reactor Systems
}

D. E. Shropshire

K. A. Williams

E. A. Hoffman

J. D. Smith

D. J. Hebditch

J. J. Jacobson

J. D. Morton

A. M. Phillips

J. P. Taylor

January 2009

The INL is a U.S. Department of Energy National Laboratory operated by Battelle Energy Alliance 


\title{
Advanced Fuel Cycle Economic Analysis of Symbiotic Light-Water Reactor and Fast Burner Reactor Systems
}

\author{
D. E. Shropshire \\ K. A. Williams ${ }^{1}$ \\ E. A. Hoffman ${ }^{2}$ \\ J. D. Smith ${ }^{3}$ \\ D. J. Hebditch \\ J. J. Jacobson \\ J. D. Morton \\ A. M. Phillips \\ J. P. Taylor ${ }^{4}$ \\ ${ }^{1}$ Oak Ridge National Laboratory \\ ${ }^{2}$ Argonne National Laboratory \\ ${ }^{3}$ Sandia National Laboratory \\ ${ }^{4}$ University of Illinois - Champaign/Urbana
}

January 2009

\section{Idaho National Laboratory Idaho Falls, Idaho 83415}

http://www.inl.gov

Prepared for the

U.S. Department of Energy

Office of Nuclear Energy

Under DOE Idaho Operations Office

Contract DE-AC07-05ID14517 


\section{DISCLAIMER}

This information was prepared as an account of work sponsored by an agency of the U.S. Government. Neither the U.S. Government nor any agency thereof, nor any of their employees, makes any warranty, expressed or implied, or assumes any legal liability or responsibility for the accuracy, completeness, or usefulness, of any information, apparatus, product, or process disclosed, or represents that its use would not infringe privately owned rights. References herein to any specific commercial product, process, or service by trade name, trade mark, manufacturer, or otherwise, does not necessarily constitute or imply its endorsement, recommendation, or favoring by the U.S. Government or any agency thereof. The views and opinions of authors expressed herein do not necessarily state or reflect those of the U.S. Government or any agency thereof. 



\begin{abstract}
The Advanced Fuel Cycle Economic Analysis of Symbiotic Light-Water Reactor and Fast Burner Reactor Systems, prepared to support the U.S. Advanced Fuel Cycle Initiative (AFCI) systems analysis, provides a technologyoriented baseline system cost comparison between the open fuel cycle and closed fuel cycle systems. The intent is to understand their overall cost trends, cost sensitivities, and trade-offs. This analysis also improves the AFCI Program's understanding of the cost drivers that will determine nuclear power's cost competitiveness vis-a-vis other baseload generation systems. The common reactor-related costs consist of capital, operating, and decontamination and decommissioning costs. Fuel cycle costs include front-end (pre-irradiation) and back-end (post-iradiation) costs, as well as costs specifically associated with fuel recycling. This analysis reveals that there are large cost uncertainties associated with all the fuel cycle strategies, and that overall systems (reactor plus fuel cycle) using a closed fuel cycle are about $10 \%$ more expensive in terms of electricity generation cost than open cycle systems. The study concludes that further U.S. and joint international-based design studies are needed to reduce the cost uncertainties with respect to fast reactor, fuel separation and fabrication, and waste disposition. The results of this work can help provide insight to the costrelated factors and conditions needed to keep nuclear energy (including closed fuel cycles) economically competitive in the U.S. and worldwide. These results may be updated over time based on new cost information, revised assumptions, and feedback received from additional reviews.
\end{abstract}




\section{EXECUTIVE SUMMARY}

The Advanced Fuel Cycle Economic Analysis of Symbiotic Light-Water Reactor and Fast Burner Reactor Systems, prepared in support of the Advanced Fuel Cycle Initiative (AFCI) systems analysis, consists of system cost comparisons between closed fuel cycles and the once-through or "open" fuel cycle. This analysis combines fuel cycle costs with the reactor costs (with associated uncertainties) to produce a total cost of electricity representing the levelized cost of a system strategy consisting of hundreds of reactors and all the supporting fuel cycle services. In these scenarios, the reactors include light-water reactors using uranium oxide (UOX) and mixed-oxide (MOX) fuel that are used in combination with advanced recycling reactors (fast reactors). The common reactor-related costs consist of capital, operating, and decontamination and decommissioning costs. Fuel cycle costs include front-end and back-end costs, as well as costs associated with fuel recycling. System cost comparisons are presented in terms of cost distributions and/or ranges rather than single point values. System development costs that include reactor and fuel cycle research, development and demonstration, as well as facility start-up costs are not addressed in this report. Nor does this analysis address site-specific costs or specific business opportunities.

The objective of the analysis is to provide a technology-oriented baseline system cost comparison between the open fuel cycle and closed fuel cycle systems and to understand their overall cost trends, cost sensitivities and tradeoffs. The analysis also improves the AFCI Program's understanding of the cost drivers that will determine nuclear competitiveness vis-à-vis other baseload generation systems. The objectives support two economic goals:

- At all times, ensure that advanced fuel cycle technologies cause no significant decrease in the economic competitiveness of nuclear electricity.

- The system should remain as economical as possible.

This economic analysis builds on the AFCI Program cost data collection, economic methodology and algorithm development, and economics studies. This study was integrated with the AFCI systems analysis and modeling. All noneconomic assumptions including reactor and fuel cycle technology choices and performance, nuclear growth rates, facility timing and deployment, fast reactor conversion ratios, material balance, and waste management assumptions were based on a common set of specifications used for the Dynamic Systems Analysis Report for Nuclear Fuel Recycle (DSARR) analysis (Analysis and Integration 2008).

One of the primary resources developed by the AFCI Systems Analysis is the "2008 Advanced Fuel Cycle Cost Basis" (AFC Cost Basis) report (Shropshire 2008). Since 2004, the AFCI Program has been developing an economic cost basis, and developing capabilities to perform engineering economic comparisons of advanced fuel cycles. The initial "AFC Cost Basis" report was produced in 2004, with annual updates in 2005, 2006, and 2007. The most current "AFC Cost Basis" report that was completed in March 2008 is 614 pages (11.7 MB of PDF text) and contains approximately 400 reference citations. In Fiscal Year 2008, there has been an increased focus on the management and disposition of the waste streams resulting from used fuel separation. These functions (or modules) 
have been greatly expanded with costs for the conditioning and disposition of specific waste streams (e.g., off gases, cesium/strontium, Iodine, and Technetium). Additionally, this study includes the costs for Light-Water Reactors (LWR) and fast reactors (FR).

The "AFCI Economic Tools, Algorithms, and Methodology" report (EAWG 2009) was initially drafted in FY-07 to present the cost models and methodologies used in advanced fuel cycle economic analysis. These capabilities were developed to evaluate the economic ramifications of a range of fuel cycle scenarios, including the determination of which components have the largest associated uncertainties, and which components have the largest impact on overall cost. The primary elements of the economic methodology include the economics submodel of Verifiable Fuel Cycle Simulation (VISION.ECON) and the Generation IV Excel Calculations of Nuclear Systems (G4 ECONS) modeling and analysis, definition of front-end costs (uranium and secondary supply analysis, enrichment and fuel fabrication market structure economics), evaluation of reactor construction learning (facility deployment analysis), and insights into current projected fuel separation costs in the U.S. Other aspects of the report that focus on potential international considerations can be utilized in future international studies.

The original AFCI economic analysis of advanced fuel cycles was completed in 2006 (Shropshire et al. 2006). This analysis consisted of a comparison of a range of closed fuel cycles to the open cycle under "static" or "snapshot-in-time" equilibrium fuel cycle material balance conditions. This analysis provided an initial baseline for comparison of the broad options that can be used for subsequent analysis. The current analysis is an extension of the 2006 work with continued improvements to and details on the cost data, improved modeling algorithms, and increased definition of closed fuel cycle systems. Another significant change was the addition of reactor life cycle costs to the fuel cycle analysis. The capital cost differences, expressed in $\$ / \mathrm{KWe}$ of net capacity, between light-water reactors (LWRs) and fast reactors are extremely important in defining the cost differences between system strategies. It should be noted that cost information on the reactors was gathered nearly 18 months ago, and the values shown may appear to be low in light of the higher $(\$ 3,000 / \mathrm{KWe}$ to $\$ 6,000 / \mathrm{KWe}$ ) capital estimates now appearing in the trade press for LWRs. Two major factors have contributed to this increase: extremely high recent escalation in construction commodities and services, and cost estimates for real projects are increasingly conservative as utilities seek investment funding and favorable economic regulation from Public Service Commissions. Note, however, that all costs in the current analysis are internally consistent, and these models still provide a credible basis for comparison of technical options.

The bulk of the economic modeling was performed using G4 ECONS (Miller and Williams 2008) to provide static (snapshot-in-time) cost under equilibrium system conditions. The spreadsheet data were evaluated for their cost uncertainty using a software program called Decision Programming Language (DPL). The overall economic analysis also considers dynamic conditions such as start-up, ramp-down, end-of-life conditions, intermittent or long-term storage strategies, and fuel cycle facility and reactor deployment scenarios. VISION.ECON was used to provide the dynamic cost analysis, consistent with the cases and types of systems analysis performed by the AFCI Analysis and Integration team. The 
software was also used to perform sensitivity analysis and evaluate uncertainties in the cost due to system performance effects. For verification purposes, the static spreadsheet model was used as a check on the accuracy of the dynamic model results.

As a precursor to the economic analysis, this report identifies all the applicable general and case-specific assumptions for the fuel cycle front-end, reactor, fuel cycle back-end, and recycling requirements. The economic analysis contains a cost sensitivity analysis, including cost distributions based on multivariable analysis and the cost sensitivity due to specific variables of interest (single variable analysis). The analysis identifies key cost drivers that have the greatest impact on economic discrimination between alternatives. System uncertainties, such as process performance, which have a large influence on costs, are identified and evaluated in combination with the uncertainties that are intrinsically associated with the input cost values. The costs of transportation are also evaluated in the sensitivity analysis to bound the magnitude of these costs relative to the total cost of electricity, and describe their primary uncertainties and sensitivities.

This engineering economic analysis provides a cost baseline for discussion and subsequent fine-tuning of the assumptions driving the comparison of costs of AFCI strategies for cost, performance, and operating parameters. These results may be updated over time based on new cost information, revised assumptions, and feedback received from additional reviews. Additionally, the present analysis does not quantify the fuel cycle in terms of improvement to national energy security, reduction of carbon and other toxic emissions, or impacts on national security. However, Systems Analysis has begun to evaluate the potential tradeoffs between economics and national security. Through this investigation, Systems Analysis concluded that the most desirable future fuel cycle strategies will be both sustainably economic and beneficial for nuclear nonproliferation, and it is a worthy endeavor to develop and cultivate such solutions.

\section{System Cost Basis}

The system costs were developed from a foundation of citable cost information. In Table ES-1 the model input costs ranges are provided for the primary cost categories ${ }^{\mathrm{a}}$ that include fuel cycle front-end and back-end, recycling processes, and LWR and fast reactors. The associated low, nominal, high costs and units of measure are primarily based on the March 2008 "AFC Cost Basis" report (Shropshire 2008). Some subsequent analysis was performed to further define costs in specific areas of the analysis (e.g., waste conditioning, storage, and disposition). Updated cost information is included in the assumptions in Section 3. All cost estimates are in constant year dollars (2008 base year) and are intended to be constant dollar projection of costs $15+$ years into the future and support comparative assessments of scenarios including nuclear growth. A comparison of the costs used in this study were made to previous economic studies, including the OECD/NEA, "Advanced Nuclear Fuel Cycles and Radioactive Waste Management" report (OECD/NEA 2006) and the Massachusetts Institute of Technology (MIT) report on "The Future of Nuclear Power" (MIT 2003). Section 10 includes a comparison of approaches,

a. The cost modules belonging to the four cost categories are color coded as an aid to understanding. 
methodologies, assumptions, unit costs, total fuel cycle cost, and total costs of energy for comparable scenarios.

Table ES-1. Cost ranges for fuel cycle and reactor cost modules.

\begin{tabular}{|c|c|c|c|c|}
\hline Variable & Low & Nominal & High & Units \\
\hline A - Natural Uranium Mining and Milling & 25 & 60 & 240 & $\$ / k g \cup$ \\
\hline B - Conversion Processes & 5 & 10 & 15 & $\$ / \mathrm{kg} \mathrm{U}$ \\
\hline C1 - Enrichment & 80 & 105 & 130 & \$ISWU \\
\hline D1-1 - LWR UO2 Fuel Fab & 200 & 240 & 300 & $\$ / \mathrm{kg} \mathrm{U}$ \\
\hline D1-2 - LWR MF Fuel Fab & 1,000 & 1,950 & 4,000 & $\$ / \mathrm{kg} \mathrm{HM}$ \\
\hline K1 - Depleted Uranium Disposition & 5 & 10 & 50 & $\$ / \mathrm{kgU}$ \\
\hline E2 - Dry Storage ( $\$$ normally included with reactor costs) & 100 & 120 & 300 & $\$ / \mathrm{kg} \mathrm{HM}$ \\
\hline I - Monitored Retrievable Storage & 94 & 96 & 116 & $\$ / \mathrm{kg} \mathrm{HM}$ \\
\hline L1 - Geologic Repository (SNF) & 400 & 1,000 & 1,600 & $\$ / \mathrm{kg} \mathrm{HM}$ \\
\hline L2-1 - Geologic Repository (HLW FPs+Ln+Tc) & 2,500 & 10,000 & 12,500 & $\$ / \mathrm{kg} \mathrm{FP}$ \\
\hline L2-2 - Geologic Repository (activated hulls) & 400 & 1,000 & 1,600 & $\$ / \mathrm{kg}$ metal \\
\hline M1 GTCC Intermediate Depth Disposal (GTCC lodine+hulls) & 70,000 & 100,000 & 440,000 & $\$ / \mathrm{m} 3 \mathrm{GTCC}$ \\
\hline F1-1 UREX+1A Aqueous Separation & 500 & 1,000 & 1,500 & $\$ / \mathrm{kg} \mathrm{HM}$ \\
\hline F1+ (HYBRID) UREX+3, Product Conditioning, 15 years storage (2-Tier) & 700 & 1,320 & 2,080 & $\$ / \mathrm{kg} \mathrm{HM}$ \\
\hline F2/D2 - Reprocessing - Electrochemical \& Remote Fuel Fab & 2,500 & 5,000 & 7,500 & $\$ / \mathrm{kg} \mathrm{HM}$ \\
\hline E3-1 - Recycled U/TRU Product Storage & 7,000 & 10,000 & 13,000 & $\$ / \mathrm{kg} \mathrm{TRU}$ \\
\hline E3-2 - Recycled U/Pu Product Storage & 3,500 & 5,000 & 6,500 & $\$ / \mathrm{kg} \mathrm{Pu}$ \\
\hline G3-1 - LLW Conditioning, Storage, Packaging (solids) & 400 & 500 & 1,000 & $\$ / \mathrm{m} 3$ solids \\
\hline G3-2 - LLW Conditioning, Storage, Packaging (liquids) & 3,300 & 11,000 & 22,000 & $\$ / \mathrm{m} 3$ liquids \\
\hline G3-3 - LLW Conditioning, Storage, Packaging (resins) & 81,000 & 90,000 & 99,000 & $\$ / \mathrm{m} 3$ resins \\
\hline $\mathrm{J}$ - Near Surface Disposal & 450 & 1,250 & 2,500 & $\$ / m 3$ LLW \\
\hline G4-1A - Aqueous LLW-GTCC Offgas absorber $(\mathrm{H} 3, \mathrm{Kr}, \mathrm{Xe})$ & 8,000 & 11,200 & 15,000 & $\$ / \mathrm{m} 3$ gas \\
\hline G4-2A - Aqueous GTCC Ceramic Conditioning (Cs/Sr) & 5,700 & 7,800 & 12,000 & $\$ / \mathrm{kg} \mathrm{Cs} / \mathrm{Sr}$ \\
\hline G4-1E - EChem LLW-GTCC Offgas absorber (H3, Kr, Xe) & 8,000 & 11,200 & 15,000 & $\$ / \mathrm{m} 3$ gas \\
\hline G4-3E - Echem GTCC GBZ Conditioning (Cs/Sr+l) & 5,700 & 7,800 & 12,000 & $\$ / \mathrm{kg} \mathrm{Cs} / \mathrm{Sr}+1$ \\
\hline E4 - Managed Decay Storage (Cs/Sr) & 10,000 & 22,500 & 35,000 & $\$ / \mathrm{kg} \mathrm{Cs} / \mathrm{Sr}$ \\
\hline G4-4A - Aqueous LLW-GTCC Ag Zeolite (lodine) & 50,000 & 67,000 & 80,000 & $\$ / \mathrm{m} 3$ lodine \\
\hline G4-5A - Aqueous GTCC Metal Alloy Conditioning (ZrSS) & 200 & 540 & 1,800 & $\$ / \mathrm{kg}$ metal \\
\hline G1-1A - Aqueous HLW Conditioning, Storage, Packaging (FP+Ln) & 1,800 & 2,000 & 2,700 & $\$ / \mathrm{kg} \mathrm{FP}$ \\
\hline G1-2A - Aqueous Metal Alloy (Tc) & 18,000 & 25,000 & 30,000 & $\$ / \mathrm{kg} \mathrm{Tc}$ \\
\hline G1-2E - EChem HLW Metal Alloy Conditioning (ZrSS+Tc) & 200 & 540 & 1,800 & $\$ / \mathrm{kg}$ metal \\
\hline G2 - UOX or (UOX/MOX) Conditioning \& Packaging & 50 & 100 & 130 & $\$ / \mathrm{kg} \mathrm{HM}$ \\
\hline G5 - CH-TRU Conditioning, Storage, and Packaging & 69,000 & 70,000 & 90,000 & $\$ / m 3$ TRU \\
\hline K2 - RU Disposition from Aqueous Reprocessing & 6 & 12 & 30 & $\$ / \mathrm{kg} \mathrm{RU}$ \\
\hline K3 - RU Conditioning for Electrochemical Reprocessing & 75 & 93 & 150 & $\$ / k g R U$ \\
\hline R1 - Thermal LWR Reactor (Overnight Capital) & 1,800 & 2,300 & 3,500 & $\$ / k W(e)$ \\
\hline R2 - Advanced Recycling Reactor (Overnight Capital) & 1,800 & 2,900 & 5,000 & $\$ / k W(e)$ \\
\hline$r$ - Real Discount Rate & 5.0 & 7.5 & 10.0 & $\%$ \\
\hline c - Construction Time & 3.5 & 4.0 & 5.0 & years \\
\hline R1 - Thermal LWR Reactor (O\&M Fixed) & 55 & 64 & 75 & $\$ / k W e-y r$ \\
\hline R2 - Advanced Recycling Reactor (O\&M Fixed) & 60 & 68 & 80 & $\$ / k W e-y r$ \\
\hline R1 - Thermal LWR Reactor (O\&M Variable) & 0.8 & 1.8 & 2.5 & mills/kWh \\
\hline R2 - Advanced Recycling Reactor (O\&M Variable) & 1.0 & 2.0 & 2.7 & mills/kWh \\
\hline
\end{tabular}

\section{Total System Cost breakdown}

A breakdown of the cost composition (i.e., the breakdown of the $\$ / M W h$ total cost of electricity for the major cost categories of the open and the two closed fuel cycle systems) is provided in Figure ES-1. The majority of the costs for the open and closed systems are in the reactor capital and O\&M. These costs make up $82-85 \%$ of the total cost of electricity of the systems. The non-reactor (fuel cycle) cost for the once-through system is less expensive at $15 \%$ of the total cost of electricity (TCOE), as compared to $18 \%$ for the 1-Tier and 2-Tier systems.

In the once-through system, the largest cost components are the LWR capital and operations and maintenance (O\&M), which comprise $85 \%$ of the total costs. The front-end fuel cycle and back-end (spent fuel management and geologic disposal) fuel cycle costs cover the remaining $15 \%$, and there are no recycling costs. For the 1-Tier system, the largest cost components are reactor capital and O\&M, which encompasses $82 \%$ of the total costs. The fuel cycle front-end and 


\section{Cost Composition of Systems}

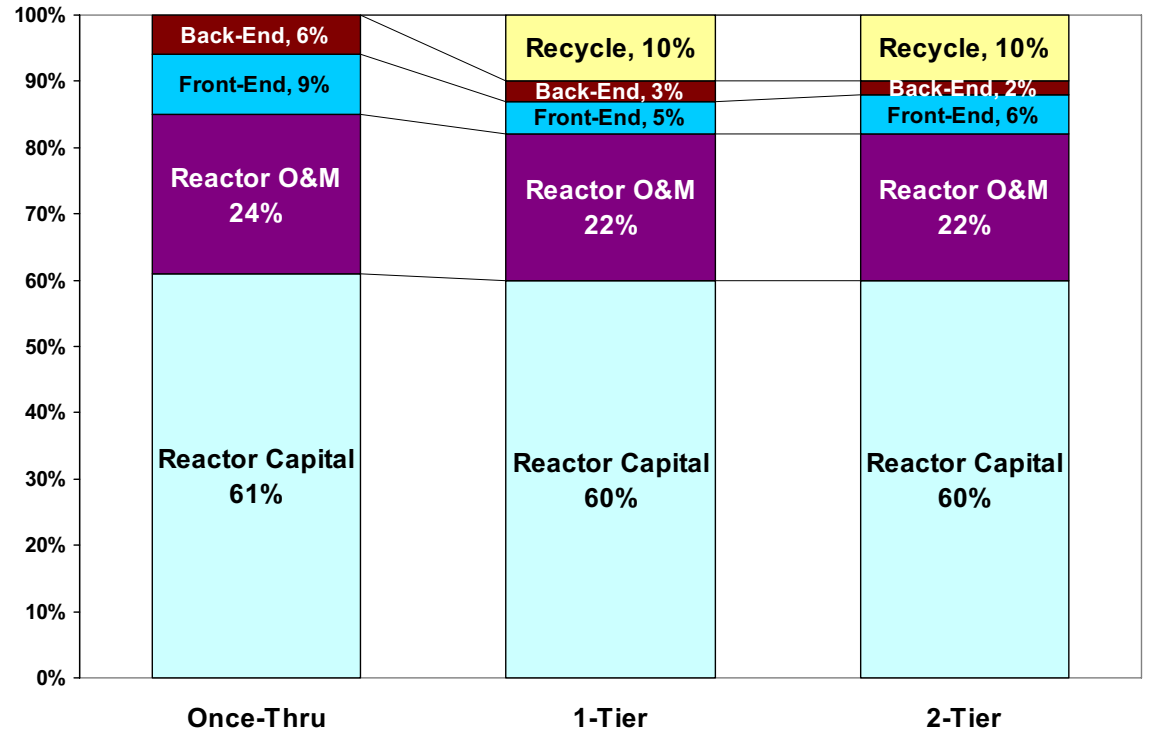

Figure ES-1. Cost breakdown of the once-through, 1-Tier, and 2-Tier systems ( $\%$ of TCOE).

back-end (geologic disposal of high-level wastes) costs are a lower percentage than for the once-through cycle, and the recycle costs (head end, separations and waste treatment/packaging) comprise $10 \%$ of the remaining costs. For the 2-Tier system, the largest cost components are reactor capital and O\&M, which consist of $82 \%$ of the total costs. The front-end and back-end costs are a lower percentage than for the once-through cycle, and the recycle costs comprise $10 \%$ of the remaining costs.

Analysis of fuel cycle system costs involve large uncertainties due to variation of input reactor capital costs, uranium ore prices, recycle facility costs, variations in waste forms, and repository costs, etc. These model input uncertainties are reflected in the resultant probability distributions for total fuel cycle costs.

The economic analysis provided in Figure ES-2 shows the large cost uncertainties associated with all the fuel cycle strategies, with equilibrium TCOE ranging from $\$ 30$ to $\$ 80 / \mathrm{MW} \cdot \mathrm{h}^{\mathrm{b}}$, with a most likely range of $\$ 40$ to $\$ 60 / \mathrm{MW} \cdot \mathrm{h}$.

While the distributions overlap, the expected values for both closed fuel cycle cases are $\$ 5-\$ 6 / \mathrm{MW} \cdot \mathrm{h}$ higher than the expected value for once-through (or about $10 \%$ higher cost). The primary contributor to this cost "gap" is due to the fast reactor capital cost premium above the LWR capital costs. Additionally, the fuel cycle cost differences can contribute up to $40 \%$ of the gap. The economics team has identified reactor cost, performance improvements, and fuel cycle cost reductions that can (when combined) effectively close the cost gap between closed and open cycles.

b. Note: " $\$ / M W \cdot h "$ is the same as "mills/kW·h". Divide above numbers by 10 to get cents/kwh. 


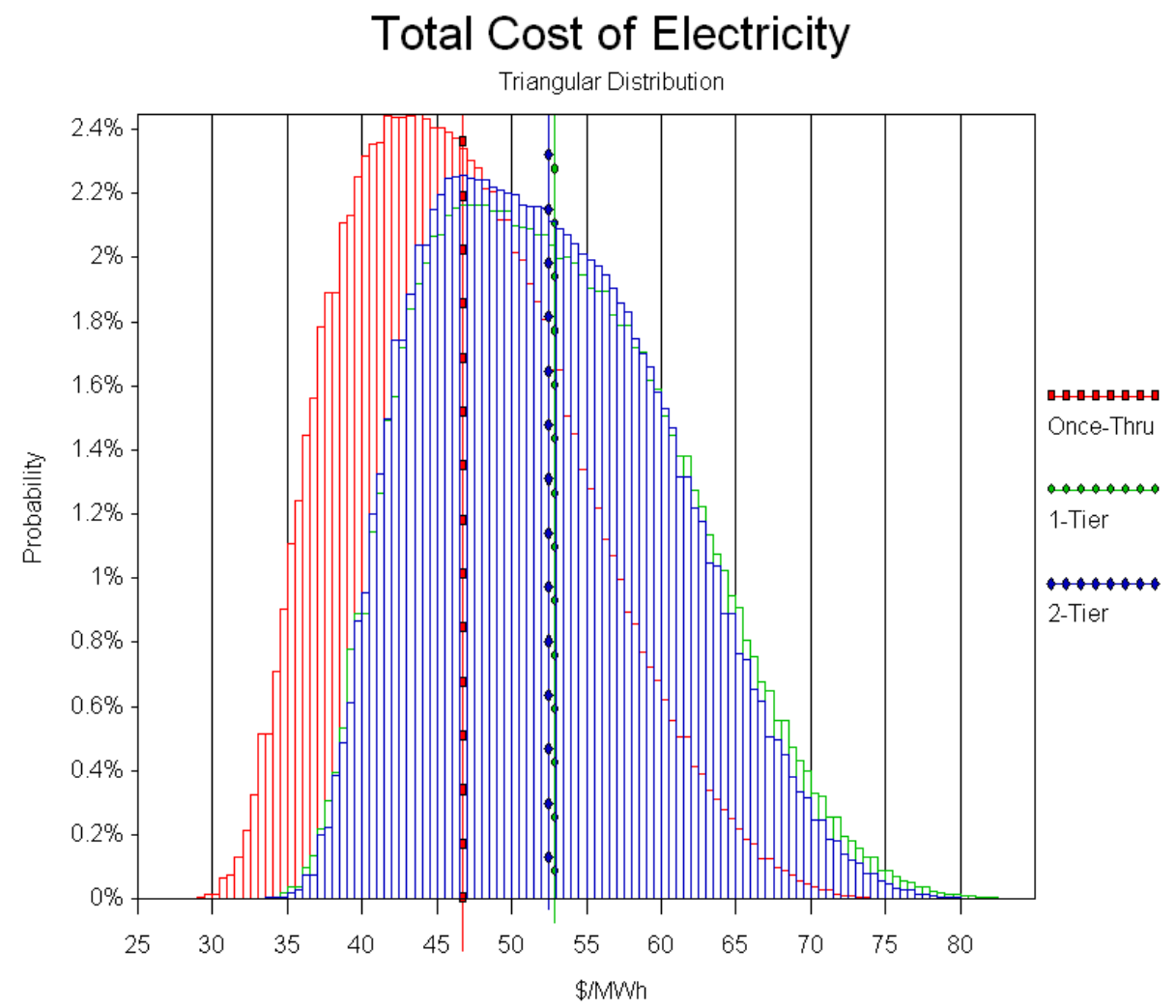

Figure ES-2. Total cost of electricity including uncertainty for the once-through, 1-Tier, and 2-Tier systems.

The reactor cost distributions and uncertainties shown in Figure ES-3 include the LWR and FR operations and maintenance, LWR overnight capital, FR overnight capital, and financing interest rate variables. For each of the three fuel cycles, color-coded horizontal lines with double-headed arrows are superimposed on the uncertainty cost distributions to show the variable cost range. The ranges are in respect to the nominal costs (from Table ES-1) for each of the distributions, which are shown as color-coded vertical dotted lines in Figure ES-3. The uncertainties include:

1. Construction Finance Interest - a large and important source of cost variability with potential to affect all nuclear reactor deployments. The interest rates, which could range from $5 \%$ to $10 \%$, are related to the perceived risk of the nuclear capital investment. The newer, less proven FRs would be considered to have higher risks than the proven LWR designs. General market conditions (e.g., conservatism in borrowing) could also drive the market toward higher (or lower) rates for LWRs and FRs. An important consideration is whether the utility or owner operates in a "regulated" (limited rate of return, lower risk to investors) or a "merchant" (no captive market, higher risk) environment.

2. Overall system FR overnight capital - a source for significantly increasing the costs of the closed fuel cycle. These costs are affected by their wide potential cost range $(\$ 1,800-\$ 5,000 / \mathrm{kWe})$ and the percentage of FRs in the system. The capital costs for the 1-Tier have a higher cost range than the 2-Tier due to the larger percentage of FRs in the fleet for the 1-Tier system. 


\section{AFCI Economic Analysis Summary Reactor Cost Uncertainties}

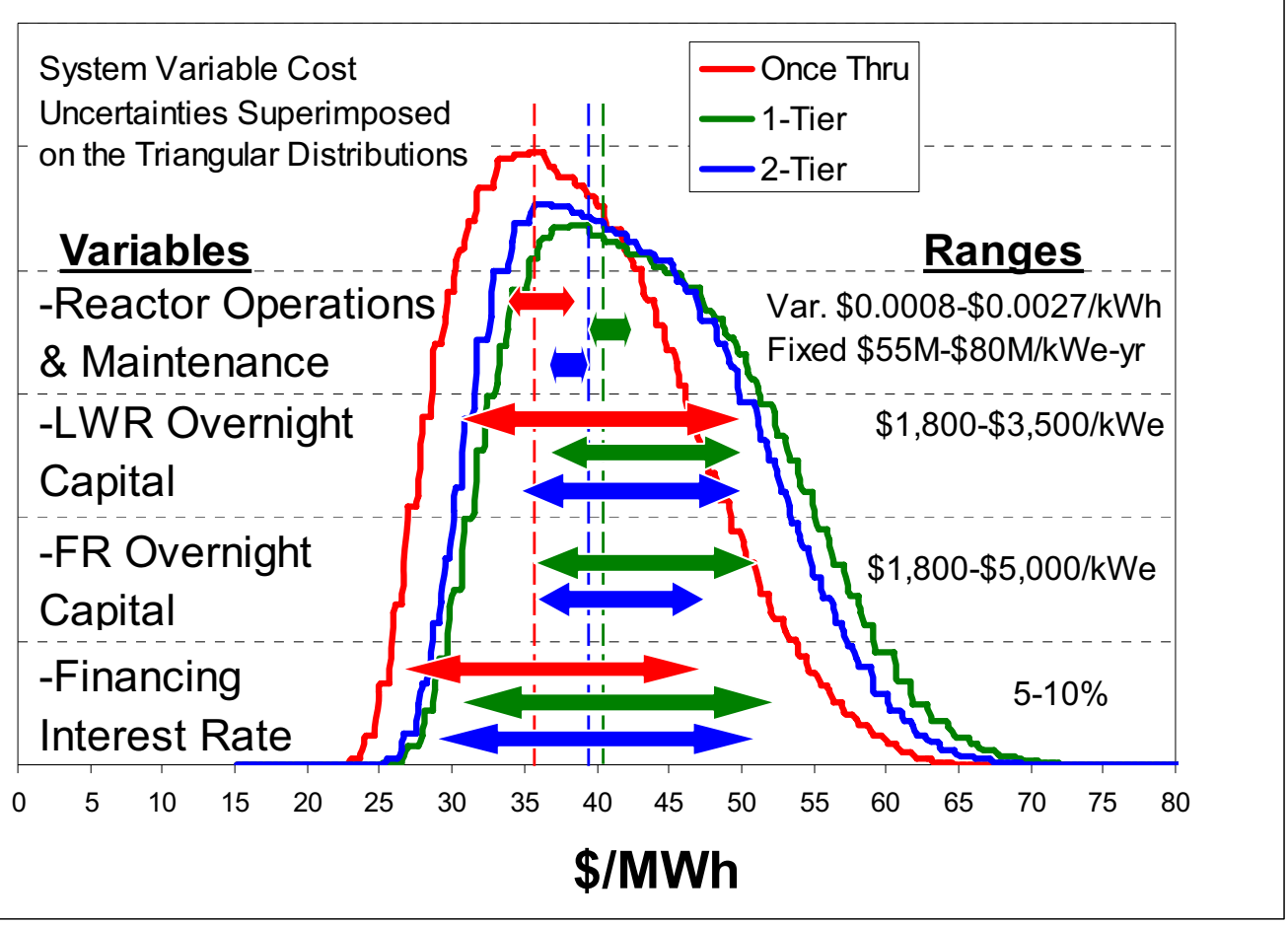

Figure ES-3. Reactor cost uncertainties.

3. LWR overnight capital - a large source of potential cost uncertainty $(\$ 1,800$ $\$ 3,500 / \mathrm{kWe}$ ) for the once-through cycle as well as closed systems that include LWRs. There are also common construction cost factors (construction materials, escalation, etc.) that could drive up the costs of all power plants (i.e., nuclear, fossil, renewable). (Overnight costs do not include financing, the model adds in the financing cost, depending on the discount rate and construction duration.)

4. Reactor O\&M- a relatively low cost uncertainty as compared to the other variables shown. It has two components: a fixed component not dependent on power generated, and variable component that varies with power generated.

\section{Learning}

There is a tremendous opportunity for operational improvements and cost reductions during the period from when the first-of-a-kind (FOAK) facilities are built, proceeding to the second through the nth-of-a-kind (NOAK) facilities. This is particularly applicable to fast reactors, since the initial learning gained from the fast reactor and the first few commercial fast reactors can drive down construction and start-up costs, lead to technology refinements, and improve the operational efficiencies that allow reactors to be operated at high capacity factors.

The "Advanced Fuel Cycle Economic Analysis of Symbiotic Light-Water Reactor and Fast Burner Reactor Systems" utilizes reactor and fuel cycle 
facilities that represent NOAK costs. Due to the relatively rapid deployment of the new systems, the costs are expected to be driven down from the FOAK to NOAK systems within the first decade of deployment. For the purposes of consistency and comparability, any further economic evaluation of the FOAK to NOAK progression is left to a future cost sensitivity analysis.

\section{Nuclear Energy Costs in Perspective}

Nuclear energy generation costs were compared to generation costs projected for other future base-load electricity producing technologies, including a review of the market competitiveness of nuclear as compared to fossil fuel derived electrical energy (particularly if carbon is taxed or carbon capture/storage is required). The overall assessment (Figure ES-4) shows that the projected busbar costs $(\$ / \mathrm{MWh})$ for the nuclear options (for Low and Nominal cost cases only) and coal (no carbon tax) are within 6-13\% of each other. In this comparison, coal is more competitive if no cost of carbon (tax, carbon capture/storage, or "cap and trade" program) is imposed. For the High cost cases, nuclear is more competitive, even when coal incurs no carbon cost. A carbon cost of only \$5-\$15/MT C will shift nuclear vis-à-vis coal to become the more competitive generation choice for all nuclear fuel cycle scenarios. Natural gas, a relatively clean energy source that until recently has been the preferred source for new baseload electricity, now suffers from high costs and continued price volatility. Again, any carbon tax greater than a few dollars per metric ton of carbon dioxide would shift the fossil energy cost advantage toward nuclear, even for closed nuclear fuel cycle systems.

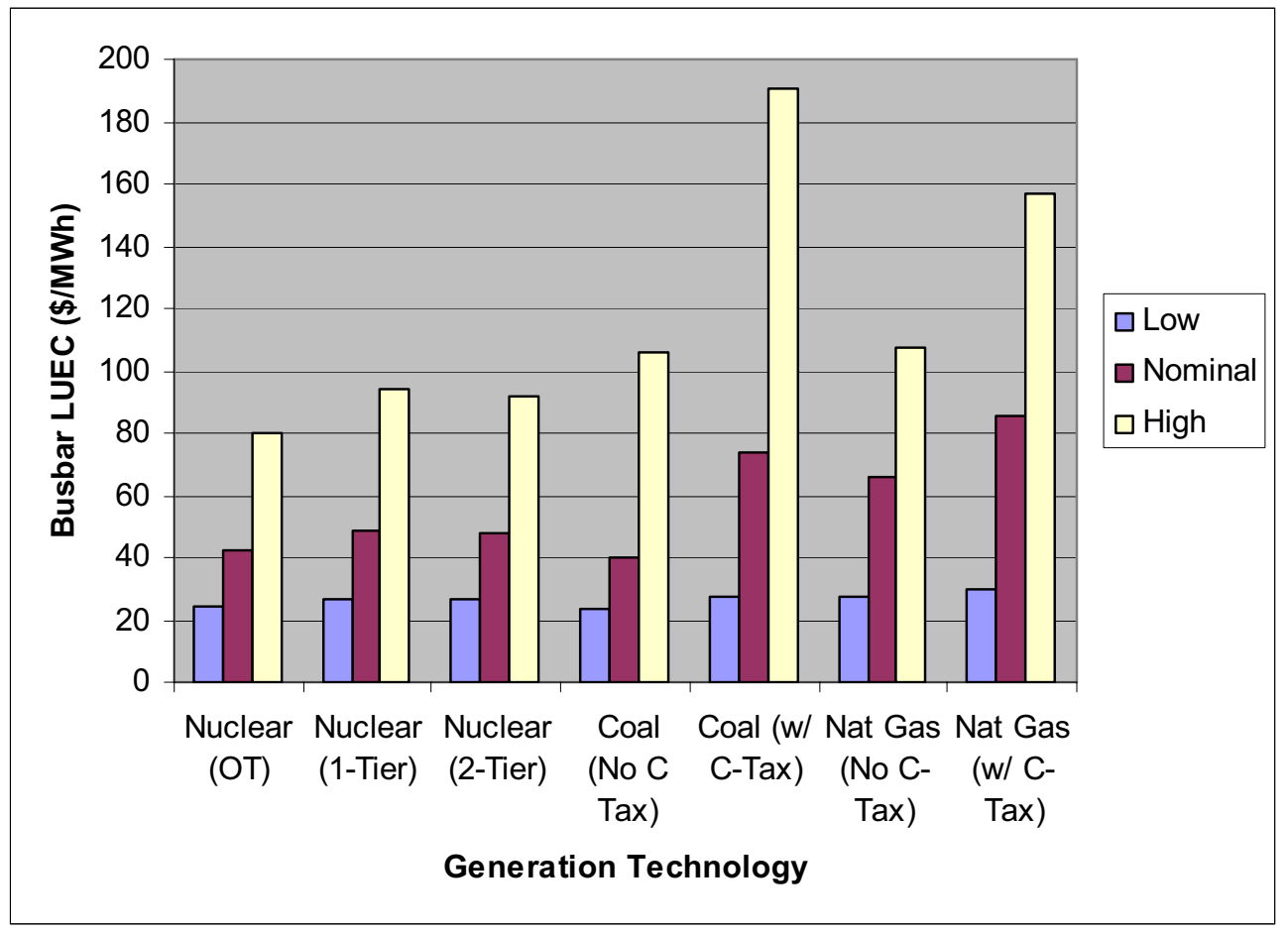

Figure ES-4. Comparisons of busbar costs of baseload energy systems. 
A principal assumption underlying recent AFCI system analysis is that for the rest of the $21^{\text {st }}$ century, nuclear power will continue to supply a significant fraction (approximately $20 \%$ or higher) of the electrical energy generation in the United States. However, in order for this to occur, nuclear energy must be economically competitive with other forms of baseload electricity generation such as coal and natural gas. In this assessment, the competiveness issue is examined for these two predominant U.S. fossil fuel choices: coal and natural gas. The figures-of-merit for competiveness are the same as those for nuclear power (i.e., the levelized busbar cost of electricity [LUEC or TCOE]) and the specific overnight and specific "all-in" (total) capital costs of the generation facility (power plant) expressed in constant $2008 \$$ per kilowatt of electrical capacity.

In Figure ES-4, costs for the fossil fuel technologies are shown both with and without the cost of carbon. These costs are shown alongside the predicted costs for the three nuclear systems with the costs of each parameter or module set at the low, nominal, or high values throughout. The uncertainty in the cost of carbon is the largest uncertainty driver for the fossil fuel technologies. For natural gas, the volatility in fuel pricing is also a highly significant contributor to the wide distribution.

The following conclusions can be drawn from these studies:

- Closing the nuclear fuel cycle would result in a much smaller cost increase to the cost of "nuclear" electricity than the addition of a "cost of carbon" (tax or CCS) to fossil fuel-based electricity.

- The potential cost of carbon is a major cost driver for the fossil technologies.

- The fossil energy costs are driven heavily by the more volatile unit costs of the fuels themselves, which tend to move with the price of crude oil. The oillinkage effect is greatest for natural gas.

- The cost of carbon is assumed to cover the social and environmental costs of greenhouse gas emission or the costs of mitigation/adaptation. Nuclear costs include all costs for safety, emissions control, regulation, and accident prevention measures. This inclusion of such "externalities" for this study gives a more realistic cost comparison of generation technologies. 


\section{Nuclear Energy Compared to Other Future Baseload Energy (Total Cost of Electricity)}

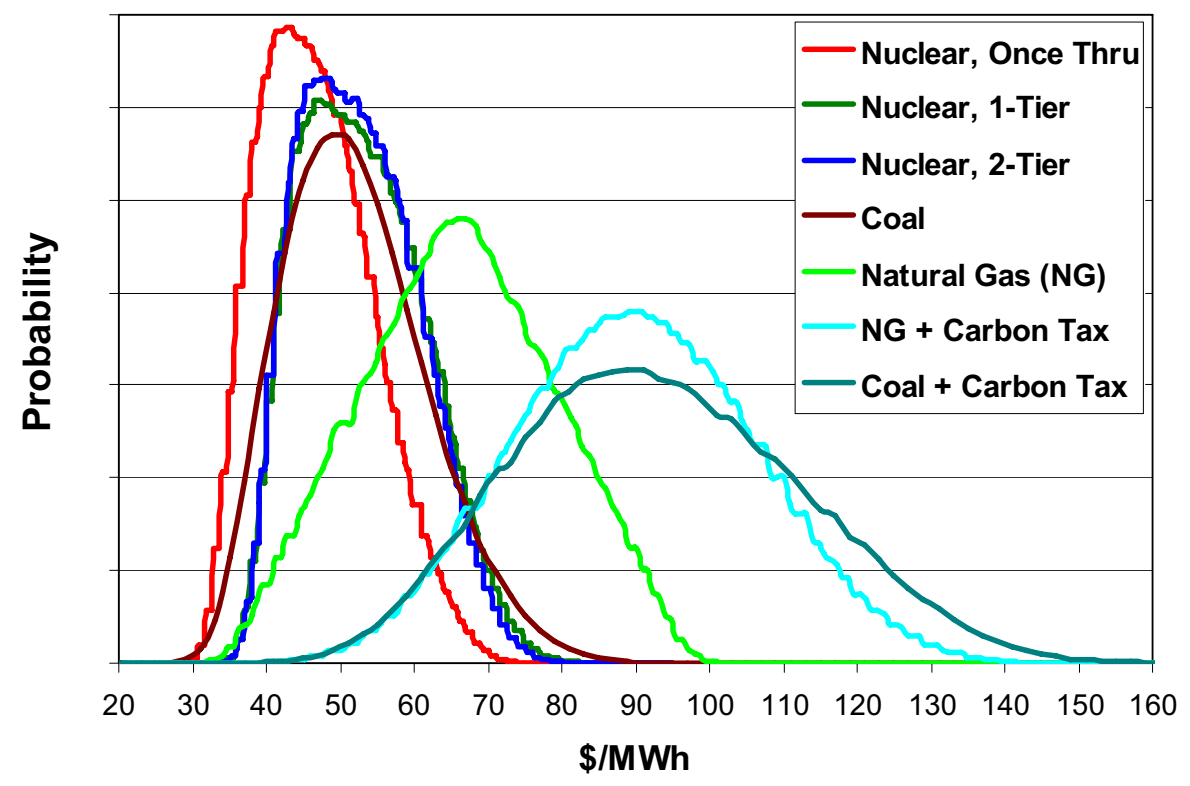

Figure ES-5. Uncertainty cost distributions for baseload energy technologies.

Figure ES-5 shows the results of an "uncertainty analyses" for the threegeneration technologies. The uncertainty in the cost of carbon is the largest uncertainty driver for all the fossil fuel technologies. Coal-based energy costs nearly double with the cost of carbon. For natural gas, fuel price volatility is a significant contributor to cost uncertainty for both the "carbon tax" and "no carbon tax" natural gas cases.

\section{Fuel Cycle Costs}

\section{Primary Fuel Cycle Cost Drivers}

According to this analysis, the main fuel cycle cost discriminator/driver for the open cycle is the unit cost for uranium mining and milling, which can vary the fuel cycle component of the generation cost by up to $\$ 5 / \mathrm{MW} \cdot \mathrm{h}$. The primary areas of cost uncertainty from the closed fuel cycle are aqueous and electrochemical separation and fuel fabrication, mixed oxide fuel fabrication, and separated waste conditioning and final disposition and their associated unit costs. Figure ES-6 shows a fuel cycle cost range from $\$ 5 / \mathrm{MW} \cdot \mathrm{h}$ to $\$ 13 / \mathrm{MW} \cdot \mathrm{h}$. The distributions represent the cost uncertainties from the LWR front-end (uranium, conversion, enrichment, depleted uranium disposition, and UOx fuel fabrication); mixed-oxide thermal fuel recycle (LWR fuel separation, MOx fabrication, U-Pu product storage, and separated waste disposition); fast reactor recycle (LWR fuel separation, TRU product storage, metal fuel fabrication, and separated waste disposition and their associated unit costs); and waste disposal of SNF and HLW. 


\section{AFCI Economic Analysis Summary Fuel Cycle Cost Uncertainties}

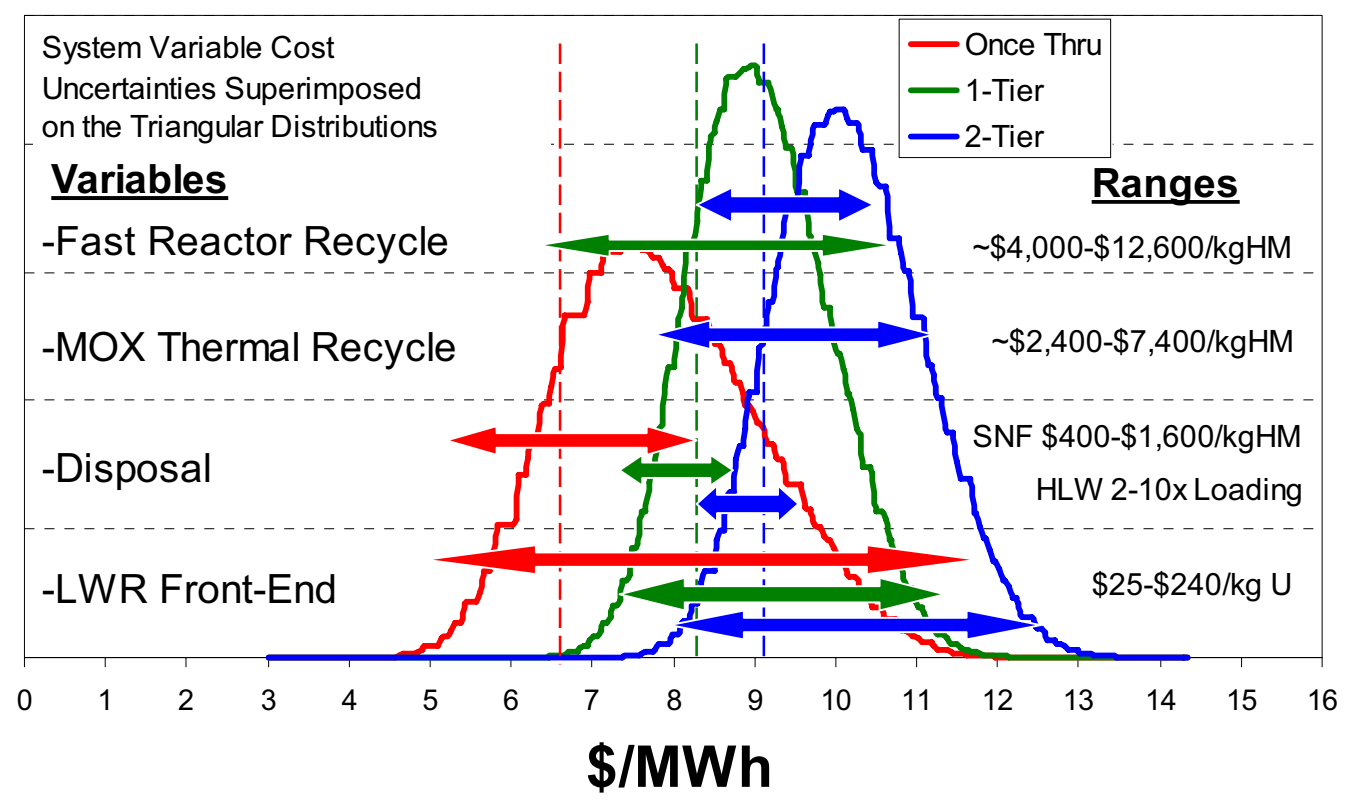

Figure ES-6. Fuel cycle cost uncertainties.

The fuel cycle cost distributions and uncertainties shown in Figure ES-6 include the fast reactor recycle, MOx thermal recycle, disposal, and LWR frontend variables. For each of the three fuel cycles, color coded horizontal lines with double headed arrows are superimposed on the uncertainty cost distributions to show the variable cost range. The ranges are in respect to the nominal costs (from Table ES-1) for each of the distributions, which are shown as color-coded vertical dotted lines in Figure ES-6. The uncertainties include:

1. LWR Front-End - a large source of uncertainty due to uranium (natural $\left.\mathrm{U}_{3} \mathrm{O}_{8}\right)$ mining and milling costs $(\$ 25-\$ 240 / \mathrm{kgU})$. The closed fuel cycles are less dependent on uranium; and therefore, show a smaller range of cost uncertainty.

2. Disposal - a large uncertainty of waste $(\$ 400-\$ 1,600 / \mathrm{kg} \mathrm{HM})$ for the oncethrough cycle since all fuel is disposed of rather than recycled. The primary cost uncertainty driver for the closed fuel cycle is the HLW loading of the waste form (ranging from 2 to $10 x$ relative to SNF) that is sent to the geologic repository.

3. MOx Thermal Recycle-used only in the 2-Tier cycle, MOx has a significant associated range of cost uncertainty $(\$ 2,400-\$ 7,400 / \mathrm{kgHM})$ due to the large potential variation in the fuel separation and fabrication unit costs. A key assumption on the 2-Tier cycle is that no actinides are disposed in the geologic repository. This assumption increases the recycling costs for the MOx thermal cycle by $30-50 \%$ over the costs of separation and fabrication of only U-Pu. 
4. Fast Reactor Recycle - a high cost uncertainty $(\$ 4,000-\$ 12,600 / \mathrm{kgIHM})$ on the 1-Tier cycle, the fuel cycle cost uncertainty is associated with the many unknowns concerning the ultimate process design and commercial costs of the less-mature EChem separation/fabrication process.

\section{Evaluation of the Cost Gap between Open and Closed Systems}

The overall electrical generation system level cost increase due to closing the fuel cycle versus direct disposal was found to be minimal ( $\sim 10 \%$ effect).

Figure ES-7 shows the impact of different cost reduction measures geared toward bringing the 2-Tier closed fuel cycle system closer in cost to the open fuel cycle system. The cost measures to close-in the cost gap are divided between reactor measures (blue arrows on the left) and fuel cycle measures (green arrows on the right). Based on the current nominal cost values, it is unlikely that a single cost measure could entirely close the gap. These cost differences are analyzed to identify measures, taken singly or in combination, that could close this gap.

The cost gap can be reduced the most by decreasing fast reactor capital costs (through improved designs, etc.). The blue arrows on the upper left show the impact of reducing FR capital costs by $10 \%$ and $20 \%$. The reduction of the FR O\&M costs to match LWR O\&M costs has an impact of about $\$ 0.8 / \mathrm{MWh}$. Improvements in FR capacity factor from 82 to $90 \%$ (through longer fuel cycles, etc.) directly translates to higher energy production, and lower electricity costs. Thermal efficiency (through higher outlet temperatures or more efficient energy conversion technologies) has a small impact $(\$ 0.2 / \mathrm{MWh})$ on overall costs.

Fuel cycle measures also have a large potential to close the gap. Reductions of the MOx thermal recycle system cost by $50 \%$ and the fast reactor recycle system cost by $35 \%$ could (together) more than make-up for the fuel cycle cost gap between the open and closed fuel cycles. Improvements on the HLW loading $(2.5 \mathrm{x}-10 \mathrm{x}$ higher than the once-through) in the repository could also close about one-third of the fuel cycle gap.

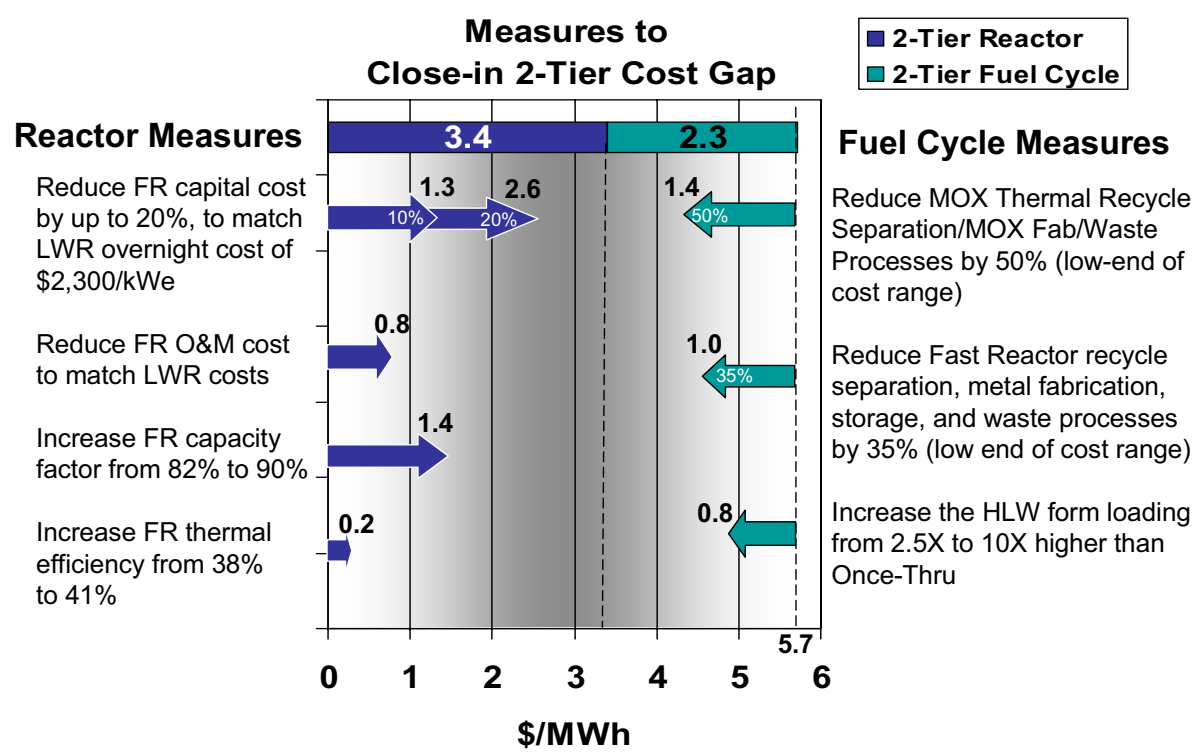

Figure ES-7. Measures to close the 2-Tier to once-through cost gap. 
Figure ES-8 shows the impact of different cost reduction measures intended to bring the 1-Tier closed fuel cycle system closer in cost to the open fuel cycle system.

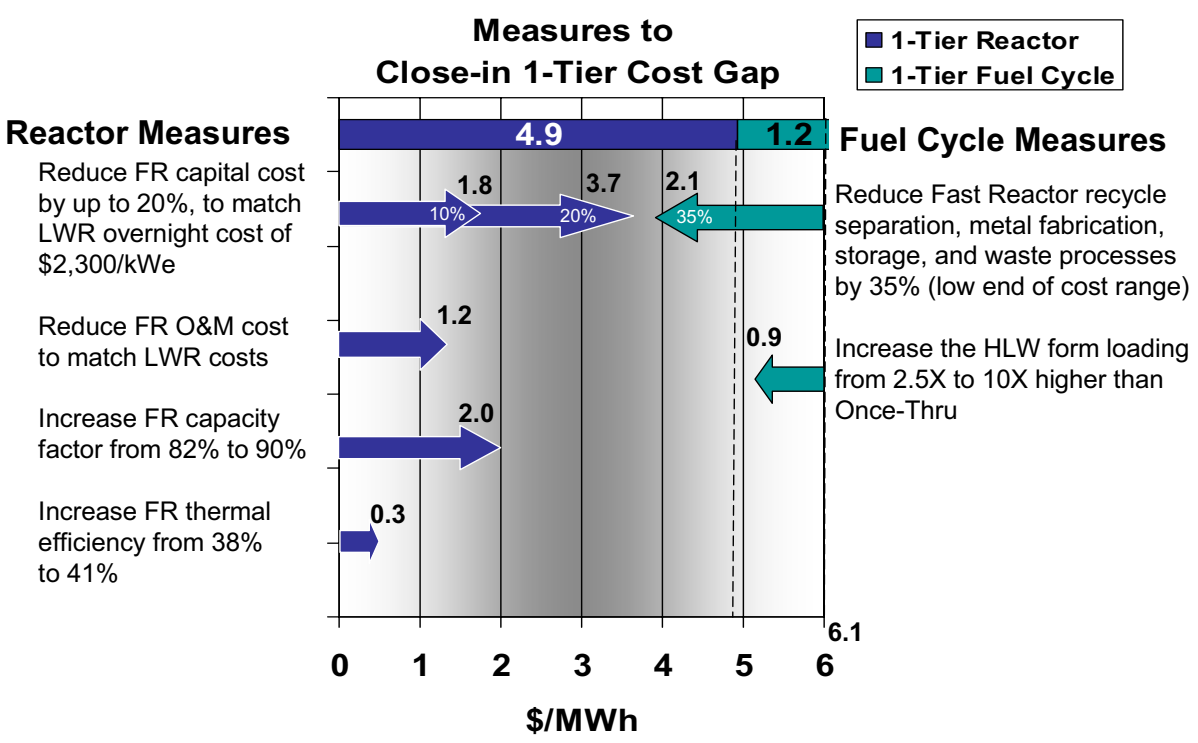

Figure ES-8. Measures to close the 1-Tier to once-through cost gap.

Due to the larger proportion of fast reactors used in the 1-Tier as opposed to the 2-Tier, the majority (or $80 \%$ ) of the cost gap is due to reactor capital costs, with the remainder due to fuel cycle costs. The blue arrows on the upper left show the impact of reducing FR capital costs. Reactor measures match the 2-Tier comparison in Figure ES-7 and consist of cost reductions to FR capital costs and improved operations resulting in higher capacity factors and improved designs resulting in higher thermal efficiency (such as through higher outlet temperatures or more efficient energy conversion technologies).

Fuel cycle improvements could also help to offset the costs of reactor capital costs. Fuel cycle improvements could be made through improved waste form loading (via research), reductions in aqueous and EChem separations costs (through a number of process-related measures), reduced cesium/strontium decay storage costs (alternate designs, etc.), and reductions to the cost of Greater-ThanClass C (GTCC) conditioning of zirconium and stainless steel resulting from aqueous processing.

\section{Transportation Costs in Perspective}

Operation of advanced fuel cycles will require movement of nuclear materials in a multitude of compositions, forms, and transportation modes. The report "Once-Through, Single and Dual Tier Fuel Cycles Transportation Cost Analyses" (Bailey and Smith 2008) provides an economic comparison of the transportation and associated infrastructure requirements for the once-through fuel cycle and the 1-Tier and 2-Tier closed fuel cycles. In the study, the cost to transport fuel cycle and waste materials through each step of the nuclear fuel cycle was assessed and the results are summarized in Figure ES-9. Most of these 


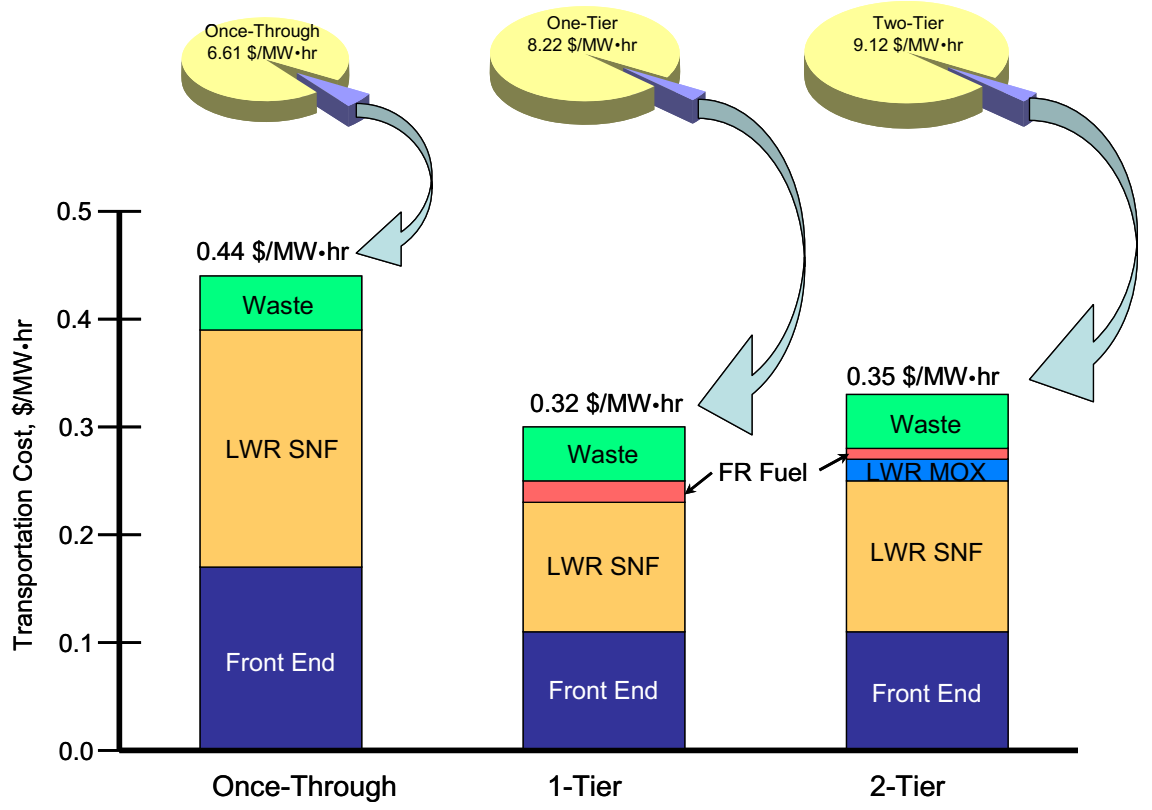

Figure ES-9. Once-through, 1-Tier, and 2-Tier nuclear fuel cycle transportation costs.

transportation costs are incorporated through the unit costs in the "Advanced Fuel Cycle Economic Analysis of Symbiotic Light-Water Reactor and Fast Burner Reactor Systems." This analysis assumes economic conditions that favor larger fuel cycle facilities where key fuel recycle operations (fuel separation, fabrication, and storage) are collocated to minimize transportation.

The estimated expenditures for transportation represent a small portion of the overall fuel cycle costs, amounting to less than $7 \%$ (6.7\% for the once-through fuel cycle and $3.7 \%$ in the 1-Tier and 2-Tier). Transportation costs in the closed fuel cycles are substantially less than the once-through fuel cycle. The largest transportation cost component is the shipment of LWR spent nuclear fuel, which constitutes about half the transportation cost in the once-through fuel cycle, and about $40 \%$ in the 1-Tier and 2-Tier cases. The closed cycles avoid about $40 \%$ of the "front-end" costs, which are partly offset by the additional transportation required to process the recycled fuel.

\section{Executive Summary Conclusion}

This analysis revealed that there are large cost uncertainties with all the fuel cycle strategies, and that systems using a closed fuel cycle are about 10\% more expensive than open cycle systems. Closed fuel cycle cost uncertainties are driven by technology uncertainties for fast reactors, used fuel separation, "hot" (remote handling) fuel fabrication, and process waste disposition. In closed systems, the resources needed to produce reactor fuels will be less sensitive to uranium resource and market pressures and will produce less waste subject to disposal in future geological repositories.

A further insight is that future nuclear energy costs appear very competitive to other base-load fossil fuel sources of energy, especially if carbon constraints 
are imposed. The market competitiveness of nuclear was compared to fossil fuelderived electrical energy (particularly if carbon is taxed or carbon storage is required). The overall assessment shows that the busbar electricity costs from nuclear and conventional pulverized coal power are similar (with a small advantage to coal for the low and nominal cases) if no cost of carbon (tax, carbon capture/storage, or "cap and trade" program) is imposed. Natural gas, a relatively clean energy source that until recently has been the preferred source new base load electricity, now suffers from high costs and continued price volatility. A carbon tax greater than a few dollars per metric ton of carbon dioxide, would shift the fossil energy cost edge toward nuclear, even for closed fuel cycle systems.

The study concludes that further U.S. and joint international-based design studies are needed to reduce the cost uncertainties with respect to fast reactor, fuel separation and fabrication, and waste disposition. The results of this work can help provide insight to the economic conditions needed to keep nuclear energy (including closed fuel cycles) economically competitive in the U.S. and globally. This cost study is integral to the complete evaluation of advanced fuel cycles, which also includes an assessment of environmental, safety, waste, and proliferation impacts. The AFCI Systems Analysis team has developed the engineering economic analysis capabilities through the development of a cost basis, static and dynamic cost models, and economic methodologies to analyze the system cost sensitivities of nuclear fuel cycles. These capabilities will continue to be improved and applied to the AFCI programs.

\section{Reference for the Executive Summary}

Analysis and Integration, 2008, "Dynamic Systems Analysis Report for Nuclear Fuel Recycle,” GNEP-SYSA-AI-SS-RT-2008-000264, December 2008.

Bailey, G.F. and J. D. Smith, 2008, "Draft Once-Through, Single and Dual Tier Fuel Cycles Transportation Cost Analyses," GNEP-SYSA-ECON-SS-RT2008-0000251, January 2008.

Economic Analysis Working Group, 2009, “AFCI Economic Tools, Algorithms, and Methodology," INL/EXT-07-13293, (Released as a draft report in 2007.)

Economic Modeling Working Group, 2006, "Cost Estimating Guidelines for Generation IV Nuclear Energy Systems," GenIV Program Economic Modeling Working Group, 2006.

MIT, 2003, The Future of Nuclear Power, Massachusetts Institute of Technology, ISBN 0-615-12420-8, 2003.

Miller, K. and K. Williams, 2008, "User's Manual for G4 ECONS Version 2.0," GenIV Program Economic Modeling Working Group, 2008.

OECD/NEA, 2006, Advanced Nuclear Fuel Cycles and Radioactive Waste Management, NEA No. 5990, OECD 2006.

Shropshire, D., et al., 2008, "2008 Advanced Fuel Cycle Cost Basis," INL/EXT-06-11536, March 2008.

Shropshire, D., K. Williams, J. D. Smith, B. Boore, 2006, “Advanced Fuel Cycle Economic Sensitivity Analysis,” INL/EXT-06-11947, December 2006. 


\section{ACKNOWLEDGEMENTS}

AFCI Systems Analysis wishes to acknowledge the technical and cost support provided by Dirk Gombert, Steve Piet, Brent Dixon, Trond Bjornard, Bob Cherry, and Gretchen Matthern (INL); Ann Reisman, John Lee, and Vatsal Bhatt (Brookhaven National Laboratory); Erich Schneider and Anthony Scopatz (University of Texas-Austin); and Geoffrey Rothwell (Stanford University). 


\section{CONTENTS}

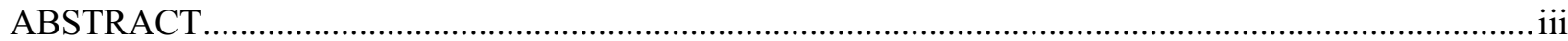

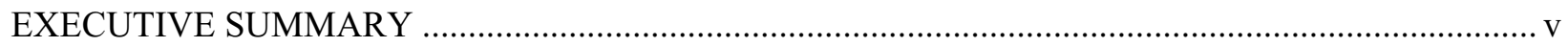

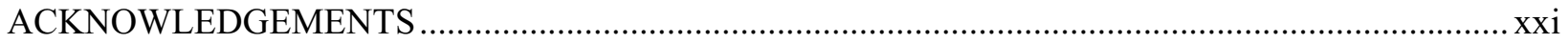

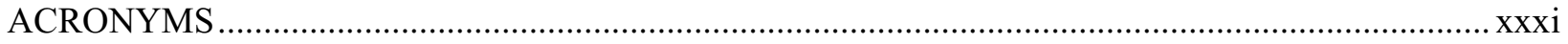

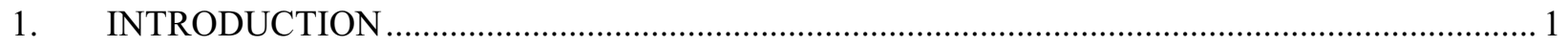

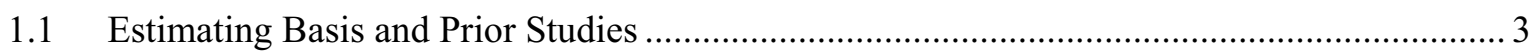

1.2 Structure of Economic Analysis for AFCI .................................................................... 4

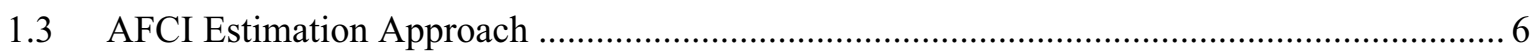

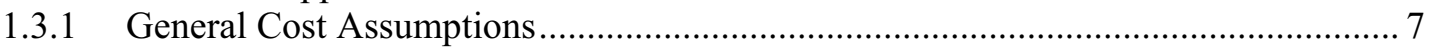

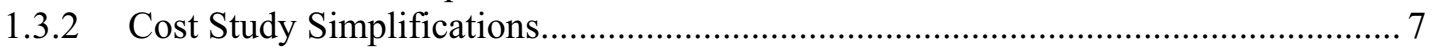

1.3.3 Impacts from Discounted Cash Flows and Present Worth Analysis........................... 8

1.3.4 Impacts from Learning Effects on Reactor and Fuel Cycle Facility Costs ................ 10

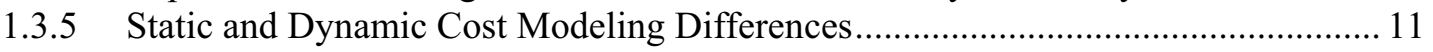

1.3.6 Impacts from Static and Dynamic Equilibrium Effects ....................................... 11

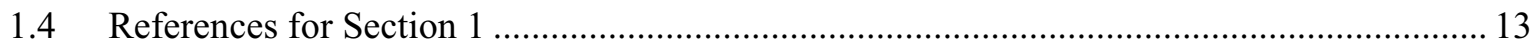

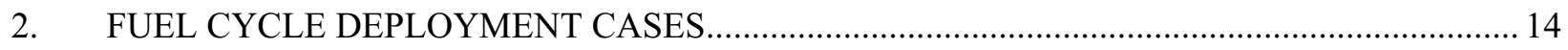

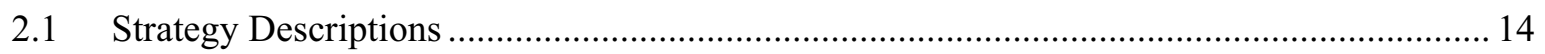

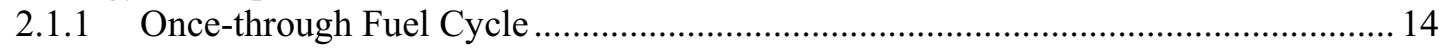

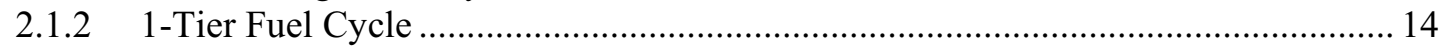

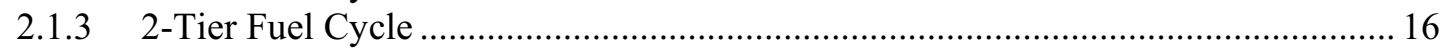

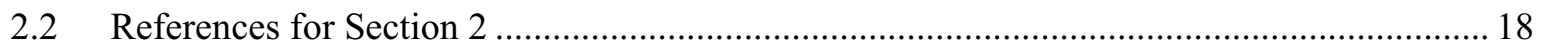

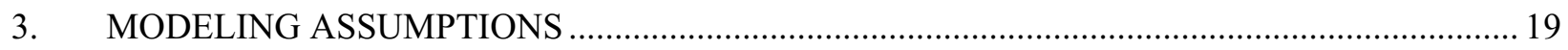

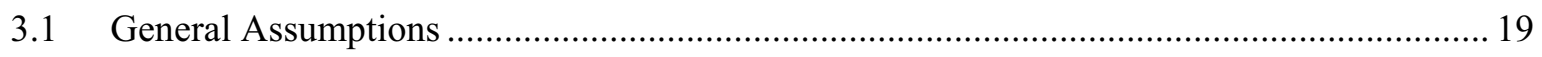

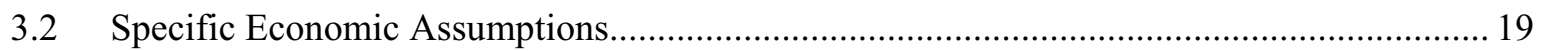

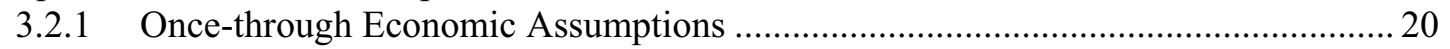

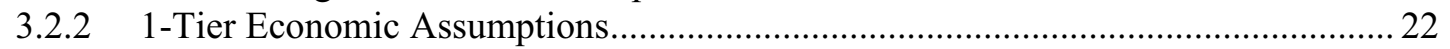

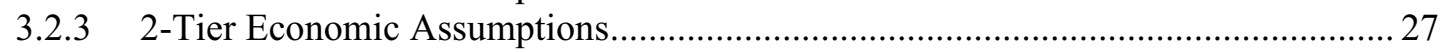

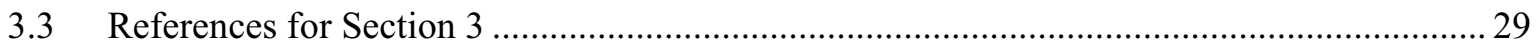

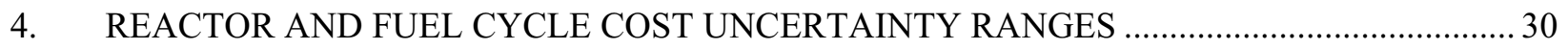

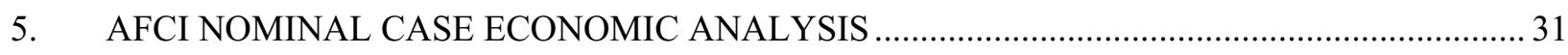

5.1 Cost Composition of the Three Systems ........................................................................ 31

5.2 System Static Cost Comparisons using G4 ECONS at Low, Nominal, and High

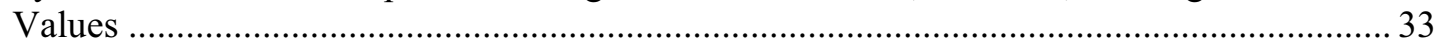

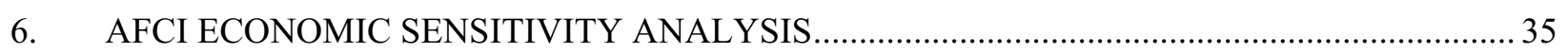

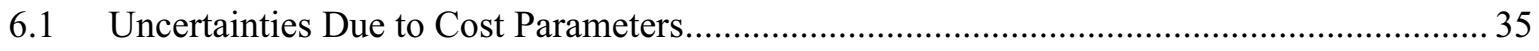

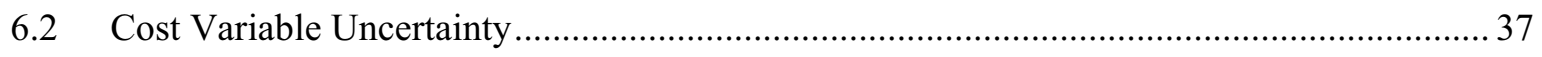

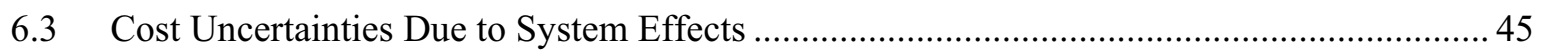


6.3.1 Once-through Cost Uncertainties Due to System Effects ....................................... 46

6.3.2 1-Tier Cost Uncertainties Due to System Effects .................................................... 53

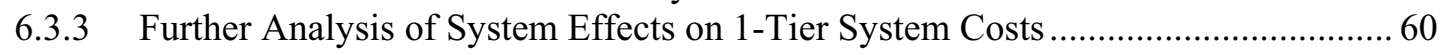

6.4 Economic Analysis of Cesium/Strontium Management .................................................. 61

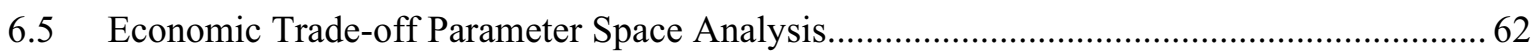

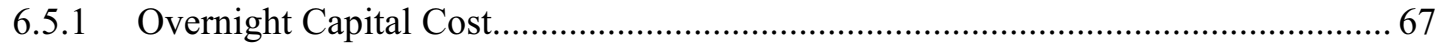

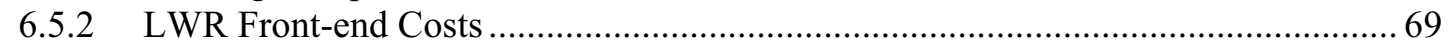

6.5.3 SNF Disposal and Once-through Recycling Costs ............................................... 71

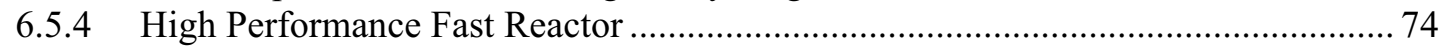

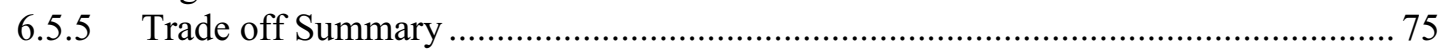

6.6 Dynamic Cost Comparisons between the AFCI Nominal Growth Cases Using

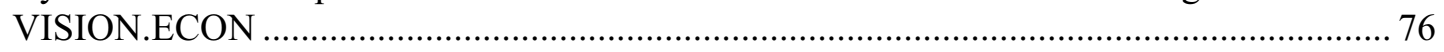

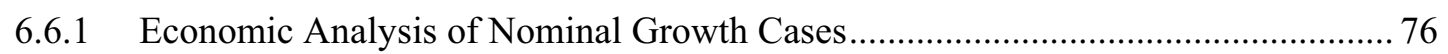

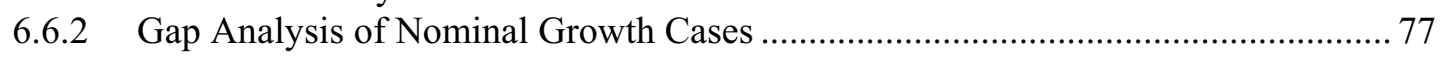

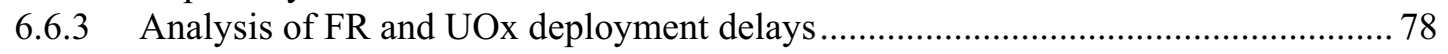

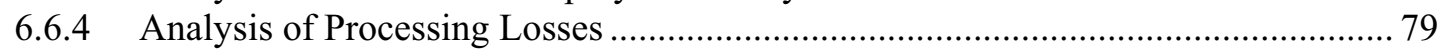

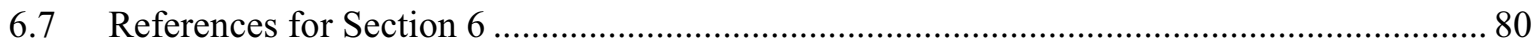

7. SUMMARY OF FUEL CYCLE TRANSPORTATION COST ANALYSIS ................................. 81

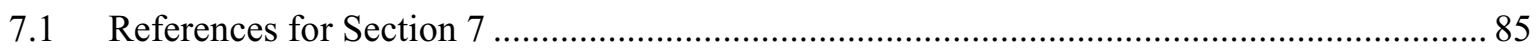

8. DISCUSSION ON THE COMPARISON OF NUCLEAR GENERATION COSTS TO

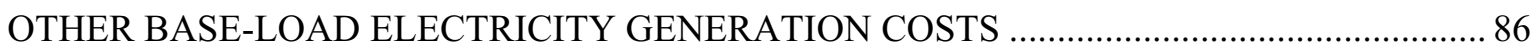

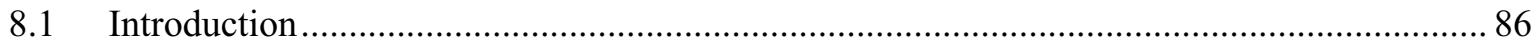

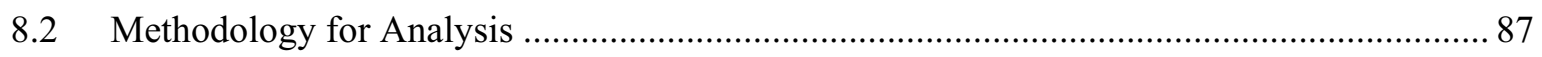

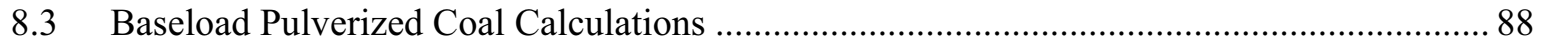

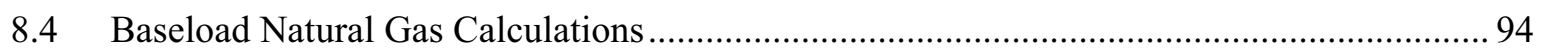

8.5 Comparison of Baseload Generation Technologies .......................................................... 99

8.6 Some Comments on the Above Economic Comparison and More Recently

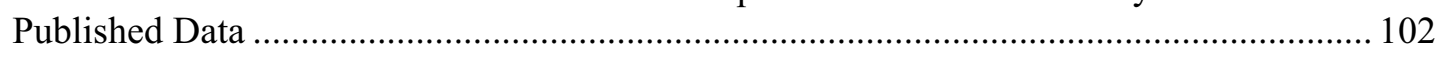

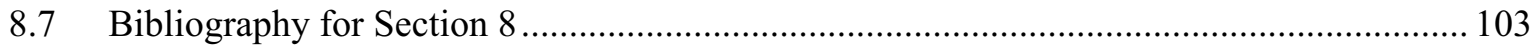

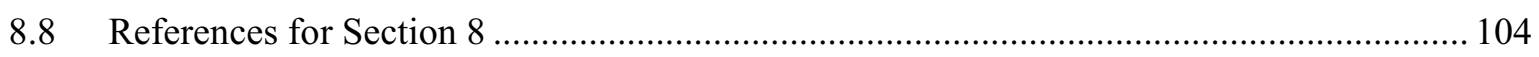

9. COMPARISON OF THE G4 ECONS AND VISION.ECON STATIC EQUILIBRIUM

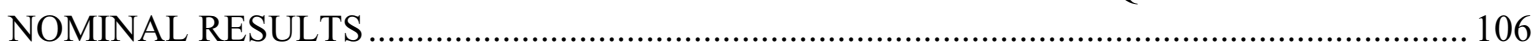

10. COMPARISON OF THE CURRENT ECONOMIC ANALYSIS TO PREVIOUS

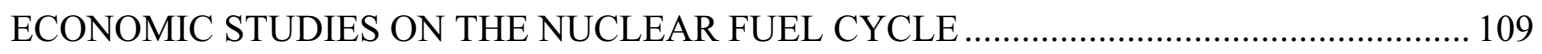

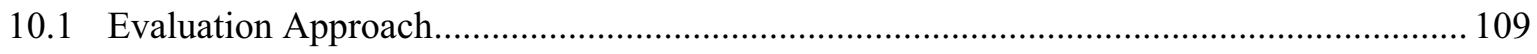

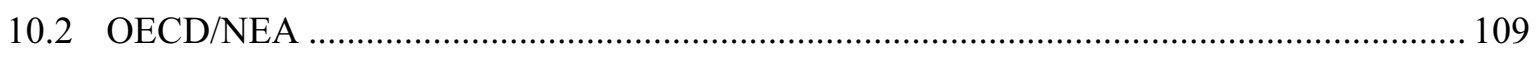

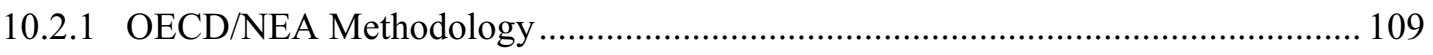

10.2.2 OECD/NEA Comparison to the "Advanced Fuel Cycle Economic Analysis of Symbiotic Light-Water Reactor and Fast Burner Reactor Systems".................. 109

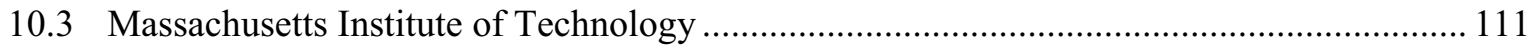

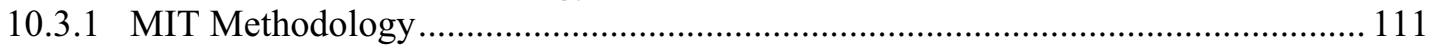

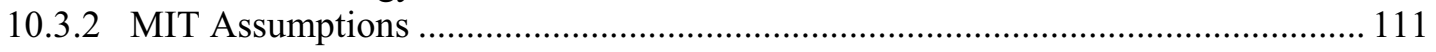

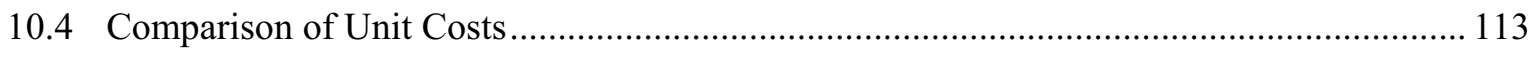


10.5 Fuel Cycle Costs

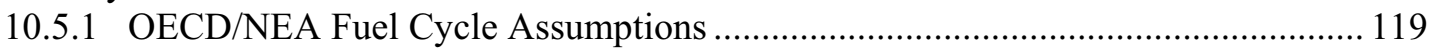

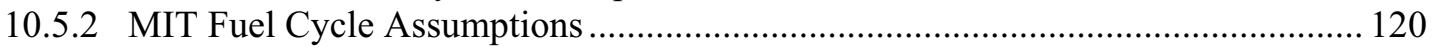

10.5.3 Comparisons between AFCI and OECD/NEA and MIT Cost Distributions .......... 120

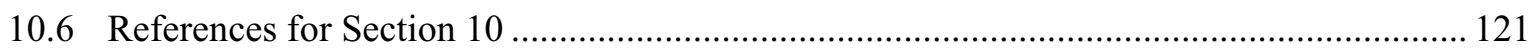

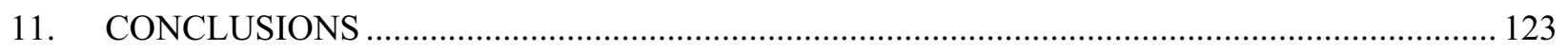

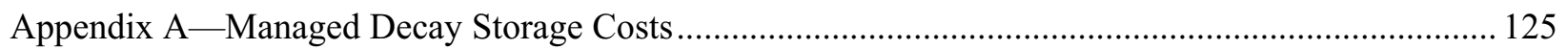

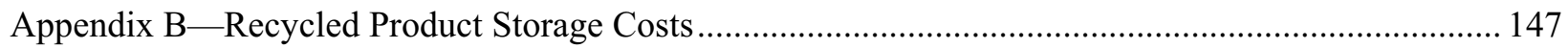

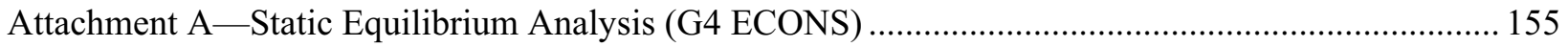

Attachment B —Dynamic Analysis of AFCI Nominal Growth Cases................................................. 179

\section{FIGURES}

Figure ES-1. Cost breakdown of the once-through, 1-Tier, and 2-Tier systems (\% of TCOE).................ix

Figure ES-2. Total cost of electricity including uncertainty for the once-through, 1-Tier, and 2-

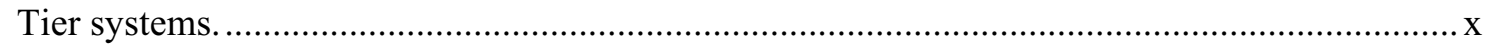

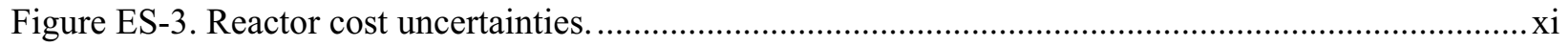

Figure ES-4. Comparisons of busbar costs of baseload energy systems. ..................................................ii

Figure ES-5. Uncertainty cost distributions for baseload energy technologies. ......................................xiv

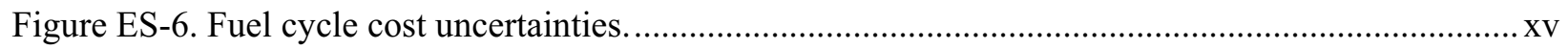

Figure ES-7. Measures to close the 2-Tier to once-through cost gap.......................................................

Figure ES-8. Measures to close the 1-Tier to once-through cost gap.......................................................ii

Figure ES-9. Once-through, 1-Tier, and 2-Tier nuclear fuel cycle transportation costs.........................xviii

Figure 1-1. Economic reports supporting the DSARR ....................................................................... 4

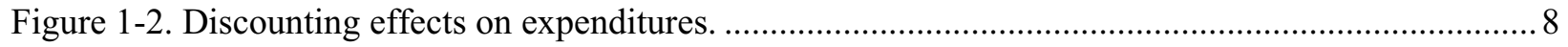

Figure 1-3. Example of the financial expenses and revenues for a Light-Water Reactor.......................... 9

Figure 1-4. Fast reactor learning rates for a range of learning curves. .................................................. 11

Figure 1-5. Comparison of fast reactor nuclear generation due to static and dynamic equilibrium

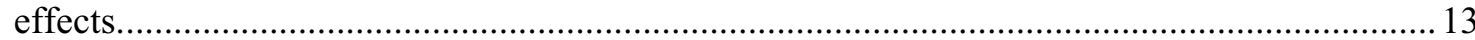

Figure 2-1. Once-through operating on an open fuel cycle. …....................................................... 15

Figure 2-2. 1-Tier fuel cycle operating on an open fuel cycle............................................................ 16

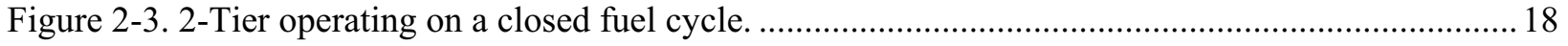

Figure 5-1. Once-through cost breakdown of the total cost of electricity (TCOE). ................................31

Figure 5-2. 1-Tier cost breakdown of the TCOE............................................................................... 32

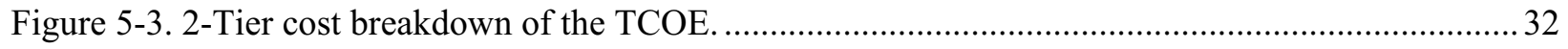

Figure 5-4. Static TCOE comparisons of strategies using low, nominal, and high values........................ 33 
Figure 5-5. Static FCC comparisons of strategies using low, nominal, and high values..... .33

Figure $6-1$. Total cost of energy using a triangular distribution. ............................................................ 36

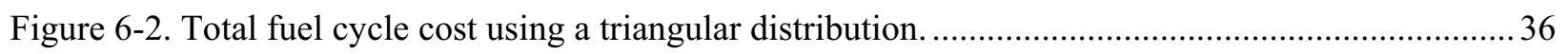

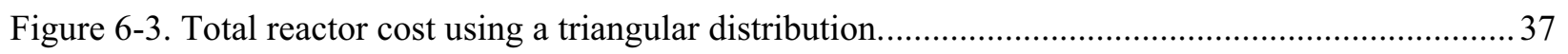

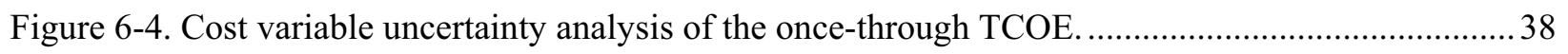

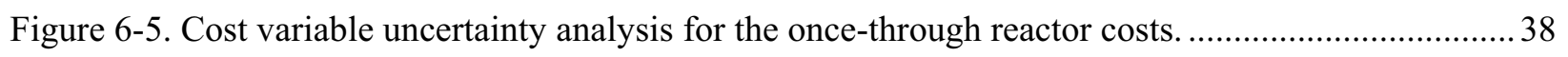

Figure 6-6. Cost variable uncertainty analysis of the once-through fuel cycle cost. ................................. 39

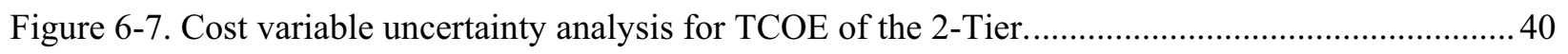

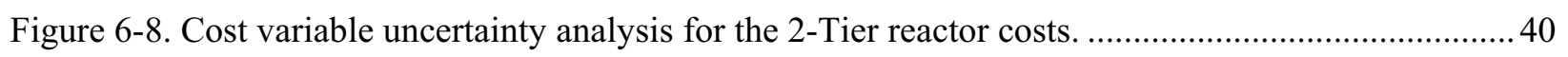

Figure 6-9. Cost variable uncertainty analysis of the 2-Tier fuel cycle cost.......................................... 41

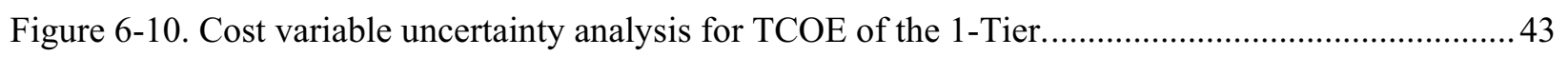

Figure 6-11. Cost variable uncertainty analysis for the 1-Tier reactor costs. ........................................... 43

Figure 6-12. Cost Variable Uncertainty Analysis of the 1-Tier Fuel Cycle Cost. ....................................4 44

Figure 6-13. Fuel cycle diagram for Base Case 1 scenario.................................................................. 47

Figure 6-14. Total fuel cycle costs annually by component at equilibrium for once-through

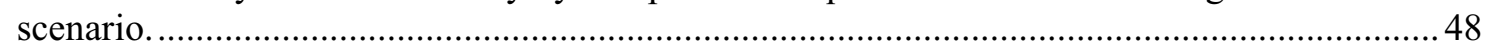

Figure 6-15. Total cost annually by component at equilibrium for once-through scenario...................... 48

Figure 6-16. Tornado diagram showing low and high values of TCOE with variations in capacity factor, reactor construction time, burnup, and thermal efficiency...........................................50

Figure 6-17. Tornado diagram showing low and high values of FCC with variations in burnup and

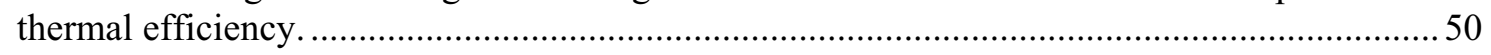

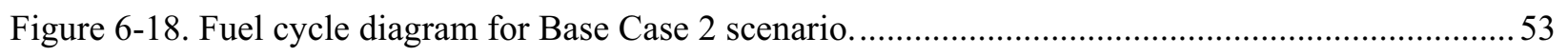

Figure 6-19. Total fuel cycle costs annually by component at equilibrium for 1-Tier scenario................54

Figure 6-20. Total costs annually by component at equilibrium for 1 -Tier scenario................................5 54

Figure 6-21. Tornado diagram showing low and high values of TCOE with variations in FR

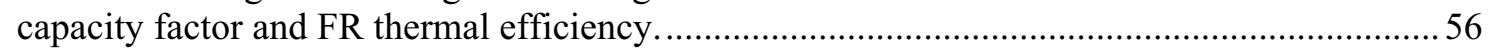

Figure 6-22. Tornado diagram showing low and high values of FCC with variations in FR

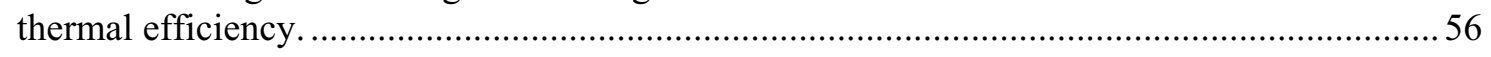

Figure 6-23. Fuel cycle costs for discrete FR conversion ratio values for 1-Tier.....................................58

Figure 6-24. Total cost of electricity for discrete FR conversion ratios values for 1-Tier..........................58

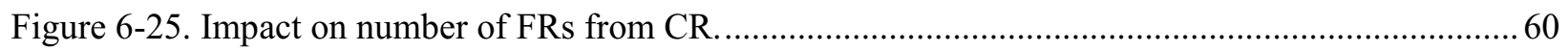

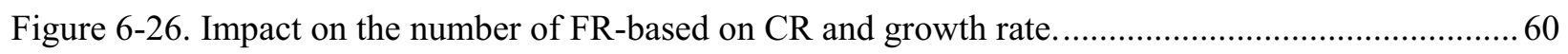

Figure 6-27. Dynamic depiction of impact on costs of CR and growth rate on system costs for 1-Tier.

Figure 6-28. Comparison of the composite fuel cycle cost for the comparison of the dynamic cases with and without $\mathrm{CsSr}$ removal. . .62

Figure 6-29. Total cost gap and 1-Tier cost gap as a function of conversion ratio. .66 
Figure 6-30. Cost sensitivity to the overnight cost for the once-through and 1-Tier fuel cycle. .68

Figure 6-31. Cost sensitivity to the overnight cost for the once-through and 2-Tier fuel cycle. ................68

Figure 6-32. Cost sensitivity to the overnight cost for the 1-Tier and 2-Tier closed fuel cycles. .69

Figure 6-33. Cost sensitivity to the LWR front-end and overnight capital cost for the oncethrough and 1-Tier fuel cycle.

Figure 6-34. Cost sensitivity to the LWR front-end and overnight capital cost for the oncethrough and 2-Tier fuel cycle.

Figure 6-35. Cost sensitivity to the LWR front-end and overnight capital cost for the 1-Tier and 2-Tier closed fuel cycles.

Figure 6-36. Cost sensitivity to LWR SNF fee and UOx recycling costs for the once-through and 1-Tier fuel cycle.

Figure 6-37. Cost sensitivity to LWR SNF fee and UOx recycling costs for the 2-Tier and 1-Tier fuel cycle.

Figure 6-38. Cost sensitivity to LWR SNF fee and UOx recycling costs for the once-through and 2-Tier fuel cycle.

Figure 6-39. Dynamic analysis of the total annual cost of electricity and fuel cycle cost........................ 76

Figure 6-40. Dynamic analysis comparison of 1-Tier and 2-Tier recycling costs...................................77

Figure 6-41. Breakdown of the cost gap between the once-through and 1-Tier (undiscounted and discounted)

Figure 6-42. Breakdown of the Cost Gap between the once-through and 2-Tier (undiscounted and discounted costs).

Figure 6-43. Chart showing the cost distributions for TCOE and FCC against delaying starting deployment of fast reactors.

Figure 6-44. Yearly profile of fuel cycle costs on an annual basis looking at different fast reactor start dates.

Figure 6-45. Dynamic analysis of the total annual cost of electricity and FCC for process losses ranging from $3.00 \%$ to $0.03 \%$.

Figure 6-46. Breakdown of the cost gap resulting from $3.00 \%$ to $0.03 \%$ process losses (undiscounted).

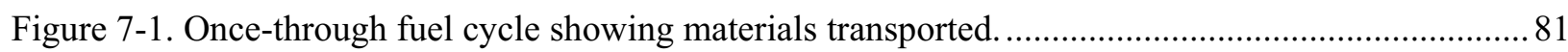

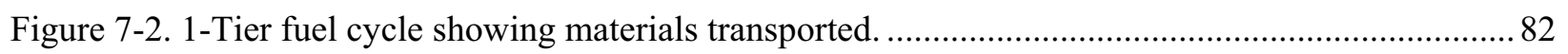

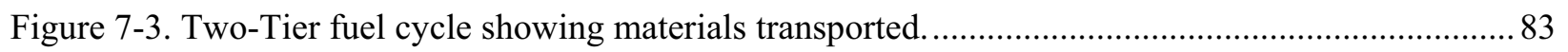

Figure 8-1. Economics of pulverized coal powerplant technology using a levelized cost model of the G4 ECONS type (spreadsheet image of nominal case: annual C emissions assumed to be $\mathrm{CO}_{2}$ only).

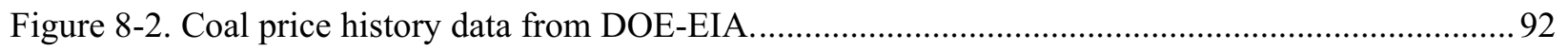

Figure 8-3. Cost variable uncertainty for coal without carbon cost..................................................... 93

Figure 8-4. Cost variable uncertainty for coal with carbon cost........................................................ 93

Figure 8-5. Cost variable uncertainty for coal both with and without carbon cost................................. 94 
Figure 8-6. Economics of natural gas turbine combined-cycle technology using levelized cost model of the G4 ECONS type (spreadsheet image of nominal case).

Figure 8-7. Variation in price projections for natural gas compared with the historical average annual wholesale wellhead price (Wiser 2004). 97

Figure 8-8. Cost variable uncertainty for natural gas without carbon cost. ............................................. 98

Figure 8-9. Cost variable uncertainty for natural gas with carbon cost. ............................................... 98

Figure 8-10. Cost variable uncertainty for coal both with and without carbon cost. ............................... 99

Figure 8-11. Graphical representation of the low, nominal, and high LUECs for baseload generation technologies. 100

Figure 8-12. Total cost of energy for all generation technologies. .................................................... 101

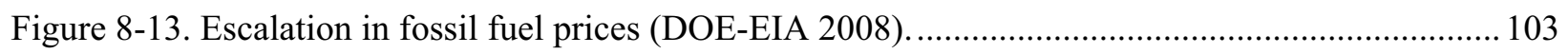

Figure 9-1. TCOE and FCC comparison of the DPL results to the VISON.ECON for the once-

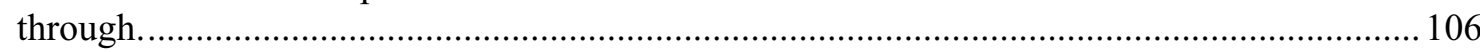

Figure 9-2. TCOE and FCC comparison of the DPL results to the VISON.ECON for the 1-Tier........... 106

Figure 9-3. TCOE and FCC comparison of the DPL results to the VISON.ECON for the 2-Tier........... 107

Figure 9-4. Comparison of TCOE modeling results for the conversion ratio......................................... 107

Figure 9-5. Comparison of TCOE modeling results for the conversion ratio....................................... 108

Figure 10-1. Unit cost comparisons: natural uranium mining and milling, and conversion (2008\$)....... 113

Figure 10-2. Unit cost comparison: enrichment and Depleted Uranium Disposition (2008\$)............... 114

Figure 10-3. Unit cost comparison: $\mathrm{LWR} \mathrm{UO}_{2}$ fuel fabrication and LWR MOx fuel fabrication

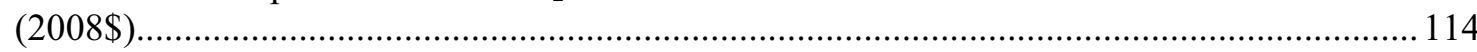

Figure 10-4. Unit cost comparison: electrochemical and remote fuel fabrication (2008\$)................... 115

Figure 10-5. Unit cost comparison: aqueous recycling (2008\$) ........................................................ 115

Figure 10-6. Unit cost comparison: RU disposition from aqueous recycling (2008\$). ......................... 116

Figure 10-7. Unit cost comparison: near surface disposal (2008\$) ..................................................... 116

Figure 10-8. Unit cost comparison: geologic repository (SNF) (2008\$) ............................................ 117

Figure 10-9. Unit cost comparison: geologic repository for high-level waste (2008\$) ........................ 117

Figure 10-10. Unit cost comparison of overnight capital: thermal LWR and fast reactor (2008\$)......... 118

Figure 10-11. Annual cost comparison: thermal LWR O\&M, based on a 1,300 MWe LWR

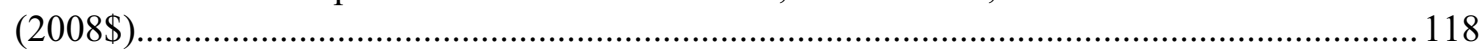

Figure 10-12. Annual cost comparison: FR O\&M, based on a 380 MWe FR (2008\$)........................ 119

Figure 10-13. Cost ranges for fuel cycles (not including reactors) ..................................................... 120

Figure 10-14. Cost ranges for the Total Cost of Electricity (including reactors).................................. 121

Figure A-1. Maximum centerline temperature difference of waste form as a function of decay time. 131

Figure A-2. WVP VPS natural convection air-cooled storage system. 136 
Figure A-3. WVP VPS charge face showing waste product flasks over storage channels (BNFL 1991).

Figure A-4. Schematic of modular vault dry storage of spent fuel at PAKS, Hungary (Ordogh et al. 2004) 140

Figure A-5. Fort St. Vrain modular vault dry store (MVDS) showing charge face. 140

Figure A-6. Paks MVDS in 2000 showing natural convection cooling exhaust outlets (Ordogh et al. 2004). 142

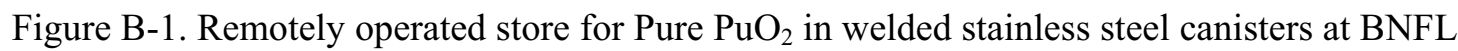
Sellafield. 150

\section{TABLES}

Table ES-1. Cost ranges for fuel cycle and reactor cost modules. viii

Table 1-1. Advanced Fuel Cycle (AFC) economic analysis functional areas and outputs. . .5

Table 3-1. AFCI economic analysis nominal assumptions. 19

Table 3-2. Nominal assumptions for the once-through scenario. 20

Table 3-3. Assumptions for 1-Tier nominal case. .22

Table 3-4. Assumptions for 2-Tier nominal case. .27

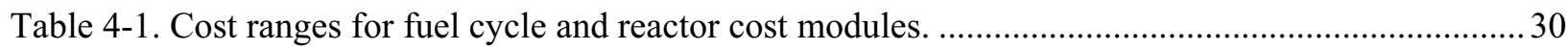

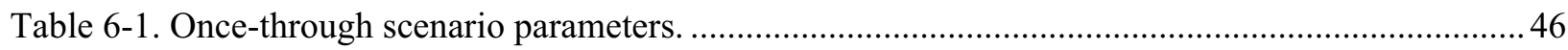

Table 6-2. TCOE and FCC with defining components at high and low ranges of burnup......................51

Table 6-3. TCOE and FCC with defining components at high and low ranges of thermal efficiency. .51

Table 6-4. TCOE and FCC with defining components at high and low ranges of capacity factors. ..........52

Table 6-5. TCOE and FCC with defining components at high and low ranges of reactor construction time. .52

Table 6-6. 1-Tier scenario parameters. .53

Table 6-7. TCOE and FCC with defining components at high and low ranges of FR thermal efficiency. .57

Table 6-8. TCOE and FCC with defining components at high and low ranges of FR capacity factors.

Table 6-9. TCOE and FCC with defining components for discrete FR conversion ratio values for 1-Tier.

Table 6-10. Defining components of back end cost for discrete FR conversion ratio values for 1-Tier. .59

Table 6-11. Defining components of recycling costs for varying FR conversion ratios for 1-Tier. .59

Table 6-12. Difference in cost from separate management of CsSr. .62

Table 6-13. Difference in LUEC between 1-Tier closed fuel cycle and once-through. .66

Table 6-14. UOx SNF recycling cost. .72 
Table 7-1. Transportation cost breakdown.

Table 8-1. Projected capital cost economics for planned coal powerplants (costs are in constant 2008 dollars unless otherwise noted; no CCS equipment is included)......

Table 8-2. Pulverized coal inputs and results from coal "Mini-Model." .................................................... 91

Table 8-3. CCGT natural gas inputs and results from "Mini-model." .................................................... 96

Table 8-4. EIA historical price for wholesale natural gas consumed by electric powerplants $\left(1 \$\right.$ thousand $\mathrm{ft}^{3}=0.988 \$$ /MMBTU; April 2008 value added by ORNL author) (DOE-EIA 2008).

Table 8-5. Tablular representation of the low, nominal, and high LUECs for baseload generation technologies. 100

Table 8-6 Escalation rates affecting powerplant costs. (Source: American Electric Power: Harding 2008)

Table 8-7. Recently published specific capital costs for selected proposed U.S. LWR projects. 102

Table 10-1. Comparison of key parameters used in the economic analyses. 112

Table 10-2. AFCI and OECD/NEA fuel cycle cases for comparative evaluation. 119

Table 10-3. Impact of MIT carrying charge factor on fuel cycle costs. 120

Table A-1. Immobilization waste form options. 129

Table A-2. Decay heat power of FPs and transuranics $(\mathrm{Pu}, \mathrm{Am}, \mathrm{Cm})$ during long-term storage, $\mathrm{W} / \mathrm{t}(\mathrm{iHM})$. 130

Table A-3. Thermal conductivity ranges for various HLW forms in temperature range 100$500^{\circ} \mathrm{C}$. 130

Table A-4. Representative CsSr-Rb-Ba waste forms 132

Table A-5. Canister and waste parameters for operating vitrified HLW stores. 138

Table A-6. Status of MVDS facilities by Foster Wheeler and ALSTEC (Roberts et al. 2003). 141

Table A-7. Estimated dry storage costs for oxide spent fuels (Fairlie 2000). 141

Table A-8. Summary cost data for managed decay storage. 143

Table B-1. Judged cost increase factor. 151

Table B-2. Capital, total, and unit charges (50 t capacity store). 152 


\section{ACRONYMS}

ADS

AFC

AFCI

ALWR

$\mathrm{AVH}$

AVM

BSG

CR

CCGT

CCS

CSF

CST

CWCCIS

D\&D

DE

DPL

DSARR

DU

DUD

DWPF

EAS

EAWG

EFR

EMWG

EPC

EV

FCC

FOAK

FOEAS

FP

FR

G4 ECONS

GE
Accelerator-Driven System

Advanced Fuel Cycle

Advanced Fuel Cycle Initiative

Advanced Light-Water Reactor

Ateliers Vitrification La Hague

Ateliers Vitrification Marcoule

borosilicate glass

conversion ratio

combined cycle gas turbine

carbon capture and storage

Consolidated Storage Facility

Crystalline Silicotitanate

Civil Works Construction Cost Index System

decontamination and decommissioning

dynamic equilibrium

Decision Programming Language

Dynamic Systems Analysis Report on Nuclear Fuel Recycle

depleted uranium

depleted uranium disposition

Defense Waste Processing Facility

Engineering Alternative Studies

Economic Analysis Working Group (AFCI)

European Fast Reactor

Economic Modeling Working Group (GenIV))

engineer, procure, construct

expected value

fuel cycle costs

first of a kind

Follow-on EAS

fission product

fast reactor

Generation IV Excel Calculations of Nuclear Systems

General Electric 


\begin{tabular}{|c|c|}
\hline GENIV & Generation IV \\
\hline GTCC & Greater-than-class-C \\
\hline HEPA & high-efficiency particulate air \\
\hline HIPing & hot isostatic pressing \\
\hline HLLW & high-level liquid waste \\
\hline HLW & high-level waste \\
\hline HM & heavy metal \\
\hline HTO & hydrous titanium oxide \\
\hline IDC & interest during construction \\
\hline IGCC & integrated (coal) gasification combined cycle \\
\hline ILW & intermediate level wastes \\
\hline INEEL & Idaho National Engineering and Environmental Laboratory \\
\hline INL & Idaho National Laboratory \\
\hline IWMS & Integrated Waste Management Strategy \\
\hline IWVF & Idaho Waste Vitrification Facility \\
\hline IX & ion exchange \\
\hline JSFR & Japanese Sodium-cooled Fast Reactor \\
\hline $\mathrm{LCC}$ & life cycle costs \\
\hline LLW & low-level waste \\
\hline LNG & liquid natural gas \\
\hline LUEC & levelized unit of electricity cost \\
\hline LWR & Light-Water Reactor \\
\hline LWRmf & Light-Water Reactor mixed fuel \\
\hline MA & minor actinide \\
\hline MMBTU & million British Thermal Unit \\
\hline $\mathrm{MOx}$ & mixed oxide \\
\hline MRS & monitored retrievable storage \\
\hline MT & metric tonnes \\
\hline MTiHM & metric tons of initial heavy metal \\
\hline MVDS & modular dry vault air-cooled stores \\
\hline NGO & non-governmental organization \\
\hline NOAK & nth of a kind \\
\hline NPP & nuclear power plant \\
\hline NRC & Nuclear Regulatory Commission \\
\hline NTS & Nevada Test Site \\
\hline
\end{tabular}




$\begin{array}{ll}\text { NWPA } & \text { Nuclear Waste Policy Act of } 1982 \\ \text { O\&M } & \text { operations and maintenance } \\ \text { ON } & \text { overnight } \\ \text { ORNL } & \text { Oak Ridge National Laboratory } \\ \text { OTC } & \text { once-through cycle } \\ \text { PNNL } & \text { Pacific Northwest National Laboratory } \\ \text { PRISM } & \text { Power Reactor Innovative Small Module } \\ \text { PUREX } & \text { Plutonium and Uranium Recovery by EXtraction } \\ \text { PWR } & \text { Pressurized Water Reactor } \\ \text { R\&D } & \text { research and development } \\ \text { ROM } & \text { rough order of magnitude } \\ \text { RU } & \text { reprocessed uranium } \\ \text { SE } & \text { static equilibrium } \\ \text { SFR } & \text { Sodium-Cooled Fast Reactor } \\ \text { SNF } & \text { spent nuclear fuel } \\ \text { SWU } & \text { specific work unit } \\ \text { TAD } & \text { Transportation, Aging, and Disposal } \\ \text { TCOE } & \text { total cost of electricity } \\ \text { TIG } & \text { Tungsten Inert Gas } \\ \text { TRU } & \text { transuranic } \\ \text { TWRS } & \text { Tank Waste Remediation System } \\ \text { UOx } & \text { uranium oxide } \\ \text { UREX } & \text { uranium extraction } \\ \text { VISION } & \text { Verifiable Fuel Cycle Simulation model } \\ \text { VISION.ECON } & \text { Economics Submodel of the Verifiable Fuel Cycle Simulation model } \\ \text { VPS } & \text { vitrified product store } \\ \text { VWISF } & \text { Vitrified Waste Interim Storage Facility } \\ \text { WVP } & \text { WMP }\end{array}$


Xxxiv 


\section{Advanced Fuel Cycle Economic Analysis of Symbiotic Light-Water Reactor and Fast Burner Reactor Systems}

\section{INTRODUCTION}

The Advanced Fuel Cycle Economic Analysis of Symbiotic Light-Water Reactor and Fast Burner Reactor Systems, prepared in support of the Advanced Fuel Cycle Initiative (AFCI) systems analysis, consists of system cost comparisons between nominal 1-Tier and 2-Tier closed fuel cycles and the oncethrough fuel cycle. This analysis combines fuel cycle costs with the reactor costs (with associated uncertainties) to produce a total cost of electricity representing the levelized costs of a system strategy consisting of hundreds of reactors and all the supporting fuel cycle services. In these scenarios, the reactors include light-water reactors (LWRs) using uranium oxide and mixed-oxide fuel that are used in combination with fast reactors. The reactor costs consist of reactor capital, operating, and decontamination and decommissioning components. Fuel cycle costs include front-end and back-end costs, as well as costs associated with fuel recycling. System cost comparisons are presented in terms of cost distributions and/or ranges rather than single point values.

There are some important exclusions in this economic analysis:

1. Does not account for total development costs, including the research, development, and demonstration facilities required for the start-up of fast reactors and new fuel cycle facilities.

2. Does not address site-specific costs, geographic placement of facilities, or specific business opportunities.

3. Does not discriminate between or identify the sources of revenue needed to start-up or operate the advanced fuel cycle system. However, the capital recovery component embedded in the unit fuel cycle costs assumes a reasonable rate of return to investors. It is also assumed that any sales revenues would cover the O\&M costs of all fuel cycle facilities and funding of an escrow account for their eventual decommissioning.

4. Does not make recommendations for specific technologies or companies.

The objective of the analysis is to provide a technology-oriented baseline system cost comparison between the open fuel cycle and closed fuel cycle systems and to understand their cost sensitivities and trade-offs. The economic analysis was performed using primarily two cost models: one static and one dynamic. Generation IV Excel Calculations of Nuclear Systems (G4 ECONS) is a spreadsheet model that provides static (snapshot-in-time) cost analysis and was used to generate the total equilibrium costs for each system. The spreadsheet data are evaluated for their cost uncertainty using a software program called Decision Programming Language. The Economics Submodel of Verifiable Fuel Cycle Simulation (VISION.ECON) is a system dynamics model that provides dynamic costs analysis, consistent with the cases and types of analysis performed by the AFCI Analysis and Integration team. The software also has a capability to perform sensitivity analysis. Through use of this model capability, Systems Analysis evaluated uncertainties in the cost due to system performance affects. For verification purposes, the static spreadsheet model based on G4 ECONS is used as a check on the dynamic results. This analysis was integrated with the AFCI System Analysis, particularly the Analysis and Integration modeling. All noneconomic assumptions including reactor and fuel cycle technology choices and performance, nuclear growth rates, facility timing and deployment, fast reactor conversion ratios, and waste management assumptions were based on a common set of specifications used for the Dynamic Systems Analysis Report on Nuclear Fuel Recycle (DSARR) (Analysis and Integration 2008). 
As a precursor to the economic analysis, all the applicable general and case-specific assumptions for the front-end, reactor, back-end, and recycling requirements are identified. The economic analysis contains cost sensitivity analysis, including cost distributions and the cost sensitivity due to specific variables of interest. The analysis identifies key cost drivers that have the greatest impact on economic discrimination between alternatives. System uncertainties, such as process performance, which have a large influence on costs, are identified and evaluated in combination with the uncertainties that are intrinsically associated with the input unit cost values. The costs of transportation are also evaluated in the sensitivity analysis to bound the magnitude of these costs relative to the total cost of electricity, and describe their primary uncertainties and sensitivities.

The economic analysis showed large cost uncertainties with all the fuel cycle strategies, with equilibrium total costs of electricity ranging from $\sim 30$ to $80 \mathrm{mills} / \mathrm{kW} \cdot \mathrm{h}^{\mathrm{c}}$ with a most likely range of 40 to $60 \mathrm{mills} / \mathrm{kW} \cdot \mathrm{h}$. The total fuel cycle costs can range from $5 \mathrm{mills} / \mathrm{kW} \cdot \mathrm{h}$ to $13 \mathrm{mills} / \mathrm{kW} \cdot \mathrm{h}$. The main cost discriminator for the once-through cycle is the LWR overnight $(\mathrm{ON})$ capital cost which contributes about $61 \%$ of the total costs, with an uncertainty range of -6 to +13 mills $/ \mathrm{kW} \cdot \mathrm{h}$. The main fuel cycle cost discriminator for the open cycle is the unit cost of uranium mining and milling. The main cost discriminator on the closed cycles is the fast reactor $\mathrm{ON}$ capital costs that contribute about $60 \%$ of the total costs, with an uncertainty range of -4 to $7 \mathrm{mills} / \mathrm{kW} \cdot \mathrm{h}$ for the $2-$ Tier and -5 to $10 \mathrm{mill} / \mathrm{s} / \mathrm{kW} \cdot \mathrm{h}$ for the 1 -Tier. The primary areas of cost uncertainty from the closed fuel cycle are the unit costs for aqueous and electrochemical separation and fuel fabrication, mixed oxide fuel fabrication, and separated waste conditioning and final disposition. Additional cost uncertainties due to system effects were also evaluated. These factors included LWR and fast reactor capacity factors and thermal efficiency, fuel burn-up, construction time, and impacts due to varying the fast reactor conversion ratio.

The insight from this analysis is that the costs for the open fuel cycle are driven by uncertainties in open-market pricing for uranium and front-end fuel services, and the policies defining the costs for used nuclear fuel disposition. Alternatively, closed fuel cycle costs are driven by technology uncertainties for fast reactors, used fuel separation, "hot" (remote handling) fuel fabrication, and process waste disposition. In the closed fuel cycle case, the resources needed to produce reactor fuels will be less sensitive to market dynamics and will produce less waste subject to disposal in future geological repositories. A further insight is that future nuclear energy costs appear very competitive to other base load source of energy, especially when greenhouse gas (mainly $\mathrm{CO}_{2}$ ) associated costs are assessed to fossil generation technologies.

Nuclear energy costs were compared to the costs projected for other future base load electricity producing technologies, including a review of the market competitiveness of nuclear as compared to fossil fuel derived electrical energy (particularly if carbon is taxed or carbon storage is required). The overall assessment shows that the busbar electricity cost from nuclear and conventional pulverized coal power are similar (with a slight advantage to coal or the "low" and "nominal" cases) if no cost of carbon (tax, carbon capture/storage, or "cap and trade" program) is imposed. Natural gas, a relatively clean energy source that until recently has been the preferred source new base load electricity, now suffers from high costs and continued volatility. A carbon tax greater than a few dollars per metric ton of carbon dioxide, would shift the fossil energy cost edge toward nuclear, even for closed fuel cycle systems.

The study concludes that further U.S. and joint international-based design studies are needed to reduce the cost uncertainties with respect to fast reactor, fuel separation and fabrication, and waste disposition. The results of this work can help provide insight to the economic conditions needed to keep nuclear energy (including closed fuel cycles) economically competitive in the U.S. and globally. These results may be updated over time based on new cost information, revised assumptions, and feedback

c. Note: "mills/kW·h" is the same as "\$/MWh." Divide above numbers by 10 to get cents/kwh. 
received from additional reviews. This systematic cost study is integral to the complete evaluation of advanced fuel cycles, which also includes an assessment of environmental and proliferation impacts.

This report is logically organized from front to back, so the reader is advised to review the first four sections as a prerequisite to the sections that follow. The first half of the report covers the estimating basis and approach, assumptions, strategy descriptions, and cost ranges (Sections 1-4), then the most basic economic analysis and comparisons are provided (Section 5), leading to the more complex analysis of cost uncertainties and dynamic sensitivity analysis (Section 6). In the second half of the report, the reader will find a summary of the fuel cycle transportation costs (Section 7), a comparison of nuclear to other base-load energy system costs (Section 8), a comparison between the static and dynamic modeling results (Section 9), and finally, a review and comparison of previous economic studies (Section 10). In the third half of the report, two appendices provide the cost basis for managed decay storage and recycled product storage. Additionally, two attachments are provided with $11 \mathrm{in.} \times 17 \mathrm{in}$. foldouts that show the cost details and results from the static spreadsheet model and the dynamic economics model.

\subsection{Estimating Basis and Prior Studies}

This economic analysis builds on the Advanced Fuel Cycle Initiative (AFCI) Program cost collection, economic methodology and algorithm development, and economics studies prepared under the AFCI Program. One of the primary resources developed by Systems Analysis is the "2008 Advanced Fuel Cycle Cost Basis" (AFC Cost Basis) report (Shropshire 2008). Since 2004, Systems Analysis has been developing an economic cost basis, and developing capabilities to perform engineering economic comparisons of advanced fuel cycles. The initial "AFC Cost Basis" report was produced in 2004, with annual updates in 2005, 2006, and 2007. The most current "AFC Cost Basis" report was completed in March 2008, is 614 pages (11.7 MB of PDF text), and contains approximately 400 reference citations. In Fiscal Year 2008 there has been an increased focus on the management and disposition of the waste streams resulting from used fuel separation. These functions (or modules) have been greatly expanded with costs for the conditioning and disposition of specific waste streams (e.g., off gases, cesium/strontium, iodine, technetium). Additionally, this study includes the costs for LWR and fast reactors (FR).

The "AFCI Economic Tools, Algorithms, and Methodology" report (EAWG 2009) developed the cost models and methodologies for use in the "Advanced Fuel Cycle Economic Analysis of Symbiotic Light-Water Reactor and Fast Burner Reactor Systems." These capabilities were developed to evaluate the economic ramifications of a range of AFCI fuel cycle scenarios, including determination of which components have the largest uncertainty, and which components have the largest impact on overall cost. The primary elements of the economic methodology used in this analysis include the VISION.ECON and G4 ECONS modeling and analysis, definition of front-end costs (uranium and secondary supply analysis, enrichment and fuel fabrication market structure economics), evaluation of reactor construction learning (AFCI facility deployment analysis), and insights to current fuel separation costs in the U.S. (AFCI Facility Economic Liaison Analysis). Other aspects of the report that focus on AFCI international deployment will be utilized in future international studies.

The initial advanced fuel cycle economic sensitivity analysis, completed in 2006 (Shropshire et al. 2006), consisted of equilibrium fuel cycle conditions that provide an initial tool for comparison of open and closed fuel cycles. Fuel cycle mass flows and costs were calculated using the fuel cycle analysis methodology in the G4 ECONS (Williams 2007), and following the Cost Estimating Guidelines for Generation IV Nuclear Energy Systems (Economic Modeling Working Group 2006). The spreadsheet analysis was performed on four fuel cycles to provide modeling insights to overall facility capacity requirements and flow rates. This analysis provides a baseline for comparison of the broad options that can be used for subsequent analysis (e.g., "Advanced Fuel Cycle Economic Analysis of Symbiotic LightWater Reactor and Fast Burner Reactor Systems"). This analysis is an extension of the earlier 2006 work 
with continued improvements and details on the cost data, improved modeling algorithms, and increased definition of the 1-Tier and 2-Tier systems. Another significant change was the addition of reactor costs to the fuel cycle analysis. The capital cost differences between LWRs and FRs are extremely important in defining the cost differences between system strategies.

The AFCI Systems Analysis has been closely integrated with the GenIV Economic Modeling Working Group (EMWG). Kent Williams (Oak Ridge National Laboratory [ORNL]) has been a U.S. representative on the group since its initiation in 2003, and David Shropshire (Idaho National Laboratory [INL]) became involved in the EMWG as the AFCI Program representative in 2004. The AFCI Program adopted the EMWG cost estimating guidelines and G4 ECONS model for use and application to advanced fuel cycles. The joint work and participation is continuing in 2008 and for the near future.

\subsection{Structure of Economic Analysis for AFCI}

The documents and flow of economic analysis supporting the AFCI systems analysis are shown in Figure 1-1. The economic documentation consists of the "2008 Advanced Fuel Cycle Cost Basis" report (Shropshire 2008), the "AFCI Economic Tools, Algorithms, and Methodologies" report (EAWG 2009), and the current "Advanced Fuel Cycle Economic Analysis of Symbiotic Light-Water Reactor and Fast Burner Reactor Systems" report, which when combined, provide the complete set of AFCI economic documentation. The results from the economic analysis are summarized in the Dynamic Systems Analysis Report on Nuclear Fuel Recycle (DSARR) (Analysis and Integration 2008).

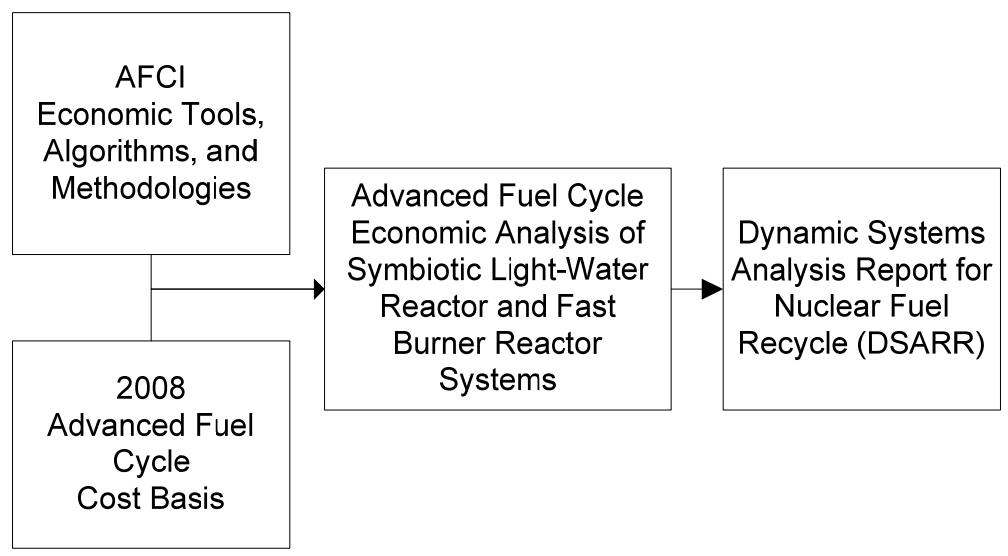

Figure 1-1. Economic reports supporting the DSARR

The "Advanced Fuel Cycle Economic Analysis of Symbiotic Light-Water Reactor and Fast Burner Reactor Systems" has supported five key functional areas during FY-07 and FY-08, as shown in Table 1-1. The studies supporting these functions are provided in the previously mentioned "AFCI Economic Tools, Algorithms, and Methodologies" report (EAWG 2009). Each of the economic functions represent a key concentration of activities that, when integrated, provide the cost basis and methods to support AFCI systems analysis. The functional areas are composed of various studies and assessments, new economic estimating methods, cost bases, and engineering cost models. Functions 1, 2, and the market structures described in Function 3, primarily focuses on economic issues associated with potential international deployment. The fast reactor economic measures in Function 3 and all of Function 4 support domestic deployment. Function 5 provides additional cost details supporting international and domestic analysis. The current analysis primarily focuses on the comparison of U.S.-based open and closed fuel cycles. However, these same processes could be extended to evaluate the economic impacts from international user/supplier fuel arrangements. The following paragraphs describe the functions in additional detail. 
Table 1-1. Advanced Fuel Cycle (AFC) economic analysis functional areas and outputs.

\begin{tabular}{|l|l|}
\hline \multicolumn{1}{|c|}{ Functions Supported } & \multicolumn{1}{|c|}{ Outputs } \\
\hline $\begin{array}{l}\text { 1. AFC technology penetration in U.S. and international } \\
\text { markets }\end{array}$ & $\begin{array}{l}\text { Annual electricity demand and technology } \\
\text { comparisons }\end{array}$ \\
\hline $\begin{array}{l}\text { 2. AFC deployment within reactor and fuel cycle facility } \\
\text { system constaints }\end{array}$ & $\begin{array}{l}\text { Module flows, facility assumptions and } \\
\text { dependencies }\end{array}$ \\
\hline $\begin{array}{l}\text { 3. Fast reactor and fuel cycle economic measures and } \\
\text { market structures }\end{array}$ & $\begin{array}{l}\text { Improved AFC cost basis modeling } \\
\text { algorithms }\end{array}$ \\
\hline $\begin{array}{l}\text { 4. AFC system level static and dynamic economic } \\
\text { analysis under conditions of uncertainty }\end{array}$ & Static and dynamic costs \\
\hline $\begin{array}{l}\text { 5. Detailed economic assessments at AFC facility and } \\
\text { technology levels }\end{array}$ & Independent studies and estimates \\
\hline
\end{tabular}

The first functional level assesses the potential for AFC technology penetration into domestic and international energy markets. The energy market assessments with domestic and international market models, which evaluate the future deployment potential for nuclear technologies based on the market competitiveness of nuclear energy compared to other energy sources. A comparison of nuclear to future new electricity generation technology alternatives further supports the market analysis.

The second functional level addresses the flows and constraints related to deployment of AFC reactors and fuel cycles facilities. A system dynamic was developed to evaluate facility deployment rates as supported by the AFC technology penetration into the domestic and international markets. From an international perspective, the nuclear capabilities, transfer of materials, and stockpile buildups are directly related to potential physical protection and nonproliferation concerns. A white paper study was developed to evaluate when economics and nonproliferation have common and conflicting objectives. A nuclear materials exchange capability was also developed to evaluate current and potential future reactor and fuel cycle capacities globally, create linkages between current/future users and suppliers, and evaluate new supplier arrangements (e.g., fuel bank, multi-lateral facilities).

In the third functional level, targeted studies were developed to improve the AFC estimating cost basis and rigor of analysis. This level includes a study of uranium ore prices and secondary supplies of uranium from re-enrichment of depleted uranium (DU) and use of reprocessed uranium (RU) recycle in LWRs. An analysis of the international market structure economics for enrichment and fuel fabrication of LWR fuel was prepared. A method was created for incorporating learning effects from first-of a kind (FOAK) to nth-of-a-kind (NOAK) facilities. A study was prepared to identify the key economic questions/issues and data requirements needed to define the costs for new AFC facilities.

Supported by the first three functional areas, the fourth functional level includes creation of static and dynamic models with uncertainty analysis to evaluate the economics of various fuel cycle and reactor scenarios. The G4 ECONS spreadsheet-based model provides a static analysis (or "snapshot in time" view) of the economics and mass flows for various reactor and fuel cycle scenarios. VISION.ECON provides dynamic analysis of fuel cycle and reactor scenarios. Both of these models use data from the "AFC Cost Basis" report, which provides unit cost ranges for fuel cycle capabilities and capital and operating cost ranges for thermal and fast reactors (Shropshire 2008).

The fifth functional level identifies the activities that are producing the most detailed economic information for advanced fuel cycles. Systems Analysis support was provided to these areas in an effort to gain access to the most current and comprehensive facility and cost data produced on the AFC technologies. 


\subsection{AFCI Estimation Approach}

This assessment provides a baseline for discussion and subsequent fine tuning of the assumptions driving the comparison of costs of AFCI strategies for cost, performance, and operating parameters.

The AFCI Systems Analysis estimating approach has evolved from equilibrium fuel cycle conditions to include dynamic conditions such as start-up, ramp-down, end-of-life conditions, intermittent or long-term storage strategies, transportation, or the reactor costs. The AFCI Economic Analysis Working Group (EAWG) calculated the fuel cycle mass flows and costs using the fuel cycle analysis methodology in the G4 ECONS spreadsheet model developed by the Gen IV EMWG (Economic Modeling Working Group). The model uses the estimating approach developed by the EMWG and was reported in the Cost Estimating Guidelines for Generation IV Nuclear Energy Systems (Economic Modeling Work Group 2006). Fuel cycle cost uncertainties were evaluated using Decision Programming Language (DPL). The EMWG performed the spreadsheet analysis on the three fuel cycles to provide modeling insights to overall facility capacity requirements and flow rates. This analysis provides a baseline for comparison of the broad options, which can be used for subsequent analysis.

The estimating approach for AFCI concentrates on evaluating the overall cost trends and differences between strategies and understanding the cost drivers that will determine nuclear competitiveness. This analysis illustrates each of these areas.

Systems Analysis has worked to establish the specific cases and system parameters to use in the economic assessments. Cost sensitivity analysis is performed in conjunction with the sensitivity analysis to evaluate the system in other areas (e.g., repository loading, etc.).

The economic analysis provides comparison and sensitivity analysis in a number of areas, such as:

- Closed fuel cycles versus the current open fuel cycle operating in the U.S.

- Single (only fast recycle) and double-tiered (thermal recycle followed by fast recycle) systems.

- Varying fuels in thermal reactors (e.g., uranium oxide [UOx], UPu-mixed oxide [MOx], and Transuranic [TRU]).

- Varying separations technologies (e.g., coex variations, uranium extraction [UREX]+, and electrochemical [EChem]) and resulting waste streams.

- Varying thermal burn-up and fast reactor conversion or breeding ratios.

- Varying waste management options (reactor storage time, recycled product storage, waste forms, decay storage, and final disposition).

In addition, timing issues were examined by changing startup dates for the various facilities and reactors types. The timing and cost effects from deploying various facility capacities are also studied.

From a global standpoint, the analysis could ultimately be used to understand the effects (timing, capacities, etc.) from supplying new fuel to user countries and associated demands on system economics arising from used fuel take back. This assessment is not part of the current study, but could be performed in the future.

Not all cost information required to compare these cases may be available. For example, the cost differentials for various UREX $+1,2,3,4$ separation schemes, fuel costs for heterogeneous fuels (e.g., target fuel) with transuranics, oxide fuels used in fast reactors, inert matrix fuels for LWRs, new waste forms for interim storage of reprocessed streams, and electrochemical costs at commercial scales are not well defined. The economic analysis presently uses broad cost ranges until design information on reactors 
and fuel cycle facilities becomes better defined through bottom-up cost analyses. Future economic models containing this information will have higher "fidelity" than those presently available.

\subsubsection{General Cost Assumptions}

The following are general cost assumptions that apply to all the nominal and sensitivity analysis cases. This analysis is:

- Intending to provide relative cost comparisons rather than specific point cost estimates. As mentioned previously, the results should be used for evaluating overall cost trends, cost differences between strategies, and cost drivers.

- Taking a general conservative approach using existing conventional practices to define the assumptions used. For example, the assumptions include waste form and operational efficiencies, based on today's understanding of process technologies. In actual practice, improved and streamlined processes and technologies would be deployed to increase efficiencies and minimize costs.

- Basing the static cost analysis on equilibrium fuel cycles with yearly material flows. Even if times between reactor refuelings were greater than 1 year, the flow data was annualized to expedite the economic analysis.

- Developing Advanced Light-Water Reactors (ALWRs) and Gen IV fast reactors to support commercial deployment. Large commercial LWRs would be deployed to realize gains from economy of scale. Costs are generally based on NOAK facilities that would be realized over multiple deployments of similar design facilities over the course of the $21^{\text {st }}$ century.

- Sizing and siting electrochemical separation and metal fabrication plants coincident to one or more fast reactor facilities.

- Using the cost distributions for a projection of constant dollar nuclear fuel cycle costs $15+$ years into the future, and are presented in current year dollars for cycle year 2008 (CY 2008). The cost ranges used in the nominal cost analysis are provided in Section 4.

- Including decontamination and decommissioning for reactor overnight capital costs and fuel cycle facility costs (expressed as unit costs) include, but generally not including site-specific costs that may consist of taxes, site infrastructure improvements, or extension of electricity transmission systems.

- Developing specific DSARR case assumptions for the systems analysis (Analysis and Integration 2008). Nuclear growth is predicated on resolution of used fuel management and risk assumptions, and that incentives (e.g., loan guarantees) are provided to industry to build the first plants. Significant growth of nuclear power could result from lower overnight capital costs and the introduction of a carbon tax.

\subsubsection{Cost Study Simplifications}

Cost study simplifications that apply to all the nominal and sensitivity analysis cases include:

- Performing a simplified transportation cost analysis (Bailey and Smith 2008). A summary of this evaluation is provided in Section 6.5. Transportation costs are not expected to be cost discriminators between the AFCI nominal cases; however, transportation costs may be a significant risk contributor in health effects and safety analysis.

- The first 63,000 metric tons of initial heavy metal (MTiHM) of used fuel from commercial reactors will have accumulated at reactor sites within the next few years. The costs for disposition will have been charged to the utilities and fees accumulated in the nuclear waste fund. This fuel and their associated accumulated fees are considered a "sunk cost" and are not included in this economic 
analysis. Furthermore, this analysis assumes that disposal beyond the first 63,000 MTiHM (metric tonnes of initial heavy metal) will not be limited to the $1 \mathrm{mill} / \mathrm{kwh}$ charge or space constraints defined by the Nuclear Waste Policy Act of 1982 (NWPA) (42 U.S.C. 10101). Expansion of the first repository and/or new repositories will be adequate to support additional demands for high-level waste (HLW) disposal from existing or newly built reactors over the remainder of the $21^{\text {st }}$ century.

\subsubsection{Impacts from Discounted Cash Flows and Present Worth Analysis}

This section describes the impacts from discounting future costs to the present. In Figure 1-2, an illustration of the discounting effects on expenditures is provided. As an example, $\$ 1,000$ in year 2000 dollars is compared to dollars discounted at interest rates from 1 to $6 \%$. The effects on future year costs are substantial, even from low interest rates. For example, a year 2000 cost of $\$ 1,000$ is worth less than half $(\$ 478)$ if the future cost is discounted at 3\% interest for 25 years. Discounting cost tends to minimize the impacts from costs late in the century (e.g., fast reactor deployments) and highlights expenditures early in the century (e.g., capital construction of separation plants).

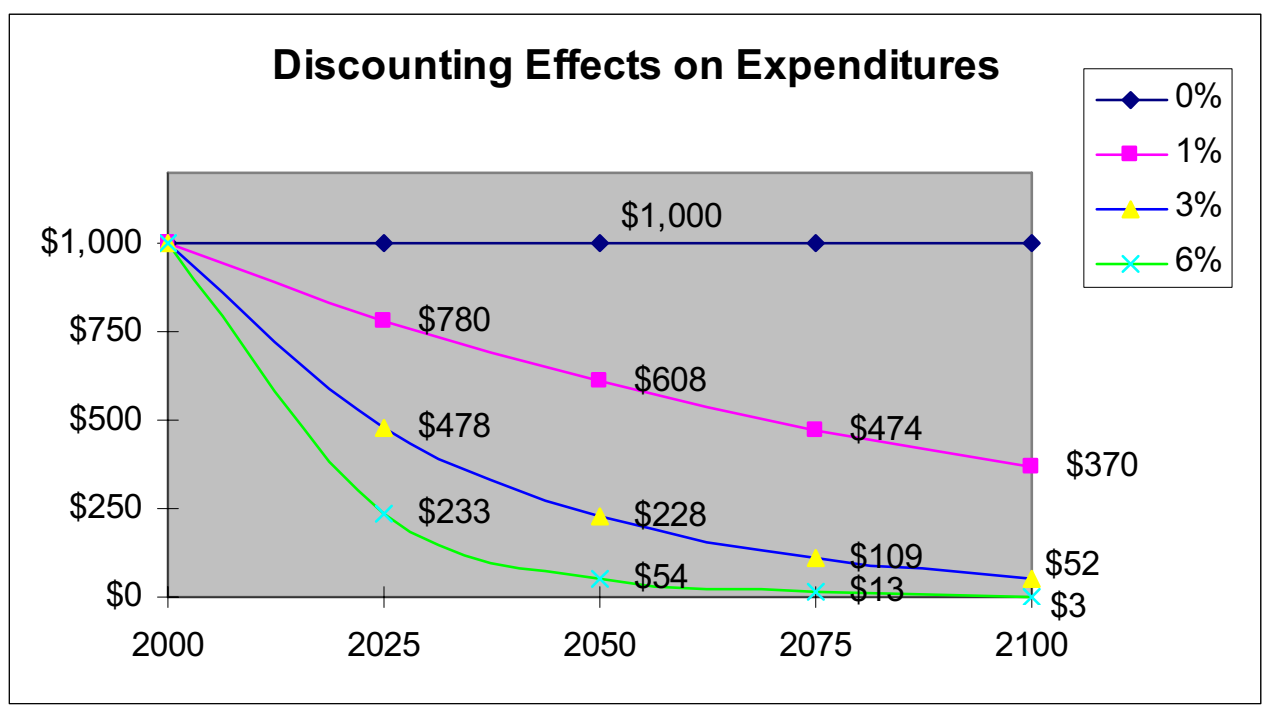

Figure 1-2. Discounting effects on expenditures.

The Systems Analysis objective is to provide a comparative analysis of options over the analysis period of 2008 to 2100 . This analysis does not provide data suitable for short-term planning where details are given on annual expenditures of capital and consequences on risk to the investors. The analysis is not the result of a "business" model that deals with financing sources and conditions and timing of revenues from sales of electricity and fuel cycle services.

Often capital projects are evaluated based on the present worth of the sum of cash flows from the expenses and revenues. These will include large capital investments on the order of tens of billions of dollars over a short time (5-10 years) near the beginning of the project.

Currently fuel cycle facility costs are amortized in the cost model, so that near-term capital expenditures are not highlighted. For example, the capital investment in the reprocessing plant is levelized via the unit cost over the life of the plant and is included in the fuel cost of the reactors that use the recycled material. If a reprocessing plant for LWR spent fuel is built, then, in order to utilize all recovered TRU, a huge investment in fast reactors is required or it requires the manufacture of MOx fuel for utilization in existing LWRs. 
Figure 1-3 provides an example of the type of financial outlay for a LWR including the expenses and revenues. This assumes that the initial capital is not financed through bonds, and therefore there are no interest payments that are part of the expenses. The fuel costs assume no lead time, which is likely a few years, and the decontamination and decommissioning (D\&D) costs are levelized as part of the operations and maintenance (O\&M) costs. This shows that for a large capital investment, such as the LWR for the example or for a reprocessing plant or fuel manufacturing plant, there will be a large expenditure of capital prior to operation and then a large reduction in expenses during the operating life and the revenue stream (levelized cost) is constant over the plant life.

This is important to understanding the cash flows because levelization of the cost has the effect of spreading the costs over time and embeds the large capital outlays that are necessary for new facilities, such as a reprocessing plant or fuel manufacturing plant. The owner sees its cost as the expenses and the consumers see their costs as the levelized revenue that includes the capital, O\&M, and return on investment. For this study, the costs are treated as the cost to the owners of the power plants, which is why the fuel cycle facilities are levelized and the power plant pays the unit cost for the product. However, if expenses and revenues are looked at for all major facilities, they will tend to look very much like Figure 1-3.

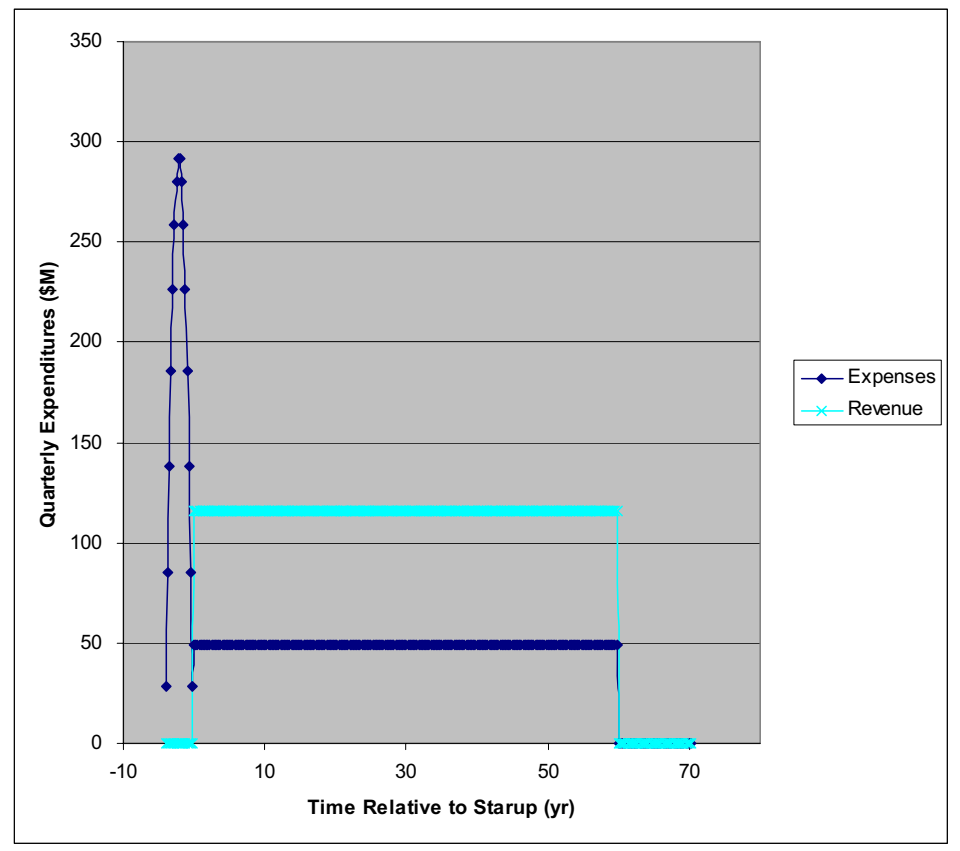

Figure 1-3. Example of the financial expenses and revenues for a Light-Water Reactor.

Both industry and the government prefer to balance the costs over the financial life of the facility. Industry prefers to reduce their capital at risk and recover fees high enough to have a relatively short economic payback period and provide a reasonable return on shareholder investment. The government prefers to avoid large capital outlays occuring over a short time that requires government subsidies. The preferred strategy is a joint government-industry approach where the investment risks are shared and the costs are deferred.

Sensitivity analysis of cash flows were examined and are included in Section 7.5.2. 


\subsubsection{Impacts from Learning Effects on Reactor and Fuel Cycle Facility Costs}

This report utilizes reactor and fuel cycle facilities that represent NOAK costs. In reality, during the initial deployment of new systems, the costs from the FOAK to NOAK systems are likely higher due to technology refinements, operational learning, etc.

In the "AFCI Economic Tools, Algorithms, and Methodologies Report" (EAWG 2009), a study was conducted on the economics of advanced nuclear power technologies with particular application to modular sodium fast reactors. The purpose of the study was to develop a general economic model to forecast the costs of a FOAK and NOAK fast reactor. The model was based on previous cost engineering and econometric studies. The study assumed that future learning rates in the U.S. nuclear construction industry would be between 3 and 10\% (U of Chicago 2004). The cost relationships between FOAK to NOAK were developed from industry cost estimates for NOAK system costs. The study identified five key assumptions for fast reactor deployment, where deployment is assumed to consist of at least $8 \mathrm{GWe}$ of capacity with a commercial fleet size of $32 \mathrm{GWe}$. These five assumptions are:

1. The deployment of advanced recycle reactors (e.g., a Power Reactor Innovative Small Module [PRISM]-like reactor) would involve more or less continuous construction of two reactors per power block with 8 power blocks (i.e., 16 to 24 modular reactors, with an equilibrium production of two reactors per year from 8 to 12 years), thus capturing "economies of series" in production.

2. Reactor modules would be built at one national (or international) location and shipped in pieces for assembly at the site, thus allowing the internalization of factory learning (because of the smaller reactor vessel, it is assumed that there are competitive manufacturers of these reactor vessels; if there are few reactor vessel manufacturers, then much of the cost savings, attributable to learning, could be captured by monopolistic equipment manufacturers). Construction crews could work nationally or internationally on modular sequential sites, thus capturing "learning by doing."

3. An internationally certified standardized design would support capturing "economies of standardization."

4. The construction industry is assumed to bid these jobs aggressively. However, to help align the incentives of all participants, equity positions in the plant could be held by a construction manager, the architect-engineer, the nuclear energy system supplier, the fuel supplier, and the plant owner and operator. This incentive structure maximizes the discovery of cost and risk reduction opportunities, thus helping to capture "economies of diversification and risk management."

5. Fast reactor deployment is well orchestrated with all regulatory bodies and most stakeholders are on board, as well as assured continuous government and corporate funding of research, development, demonstration, deployment, and commercialization.

The study used a range of learning rates (3\% to $10 \%)$ to evaluate the FOAK to the NOAK decrement in cost with each doubling of capacity to $8 \mathrm{GWe}$. At the highest learning rate of $10 \%$, the FOAK costs would be $53 \%$ higher than the NOAK unit. For more likely learning rates between $3-6 \%$ (per doubling), FOAK capital costs range $12 \%$ to $23 \%$ higher, respectively, than NOAK costs. This range of cost difference is well within the range of uncertainty of the fast reactor costs used in this study. These results are shown in Figure 1-4. The impact of learning on NOAK costs is significant and supports the value of planning for a learning period during fast reactor deployment as a way to reduce the relative cost differential between LWRs and fast reactors. (This differential is discussed in Section 6). 


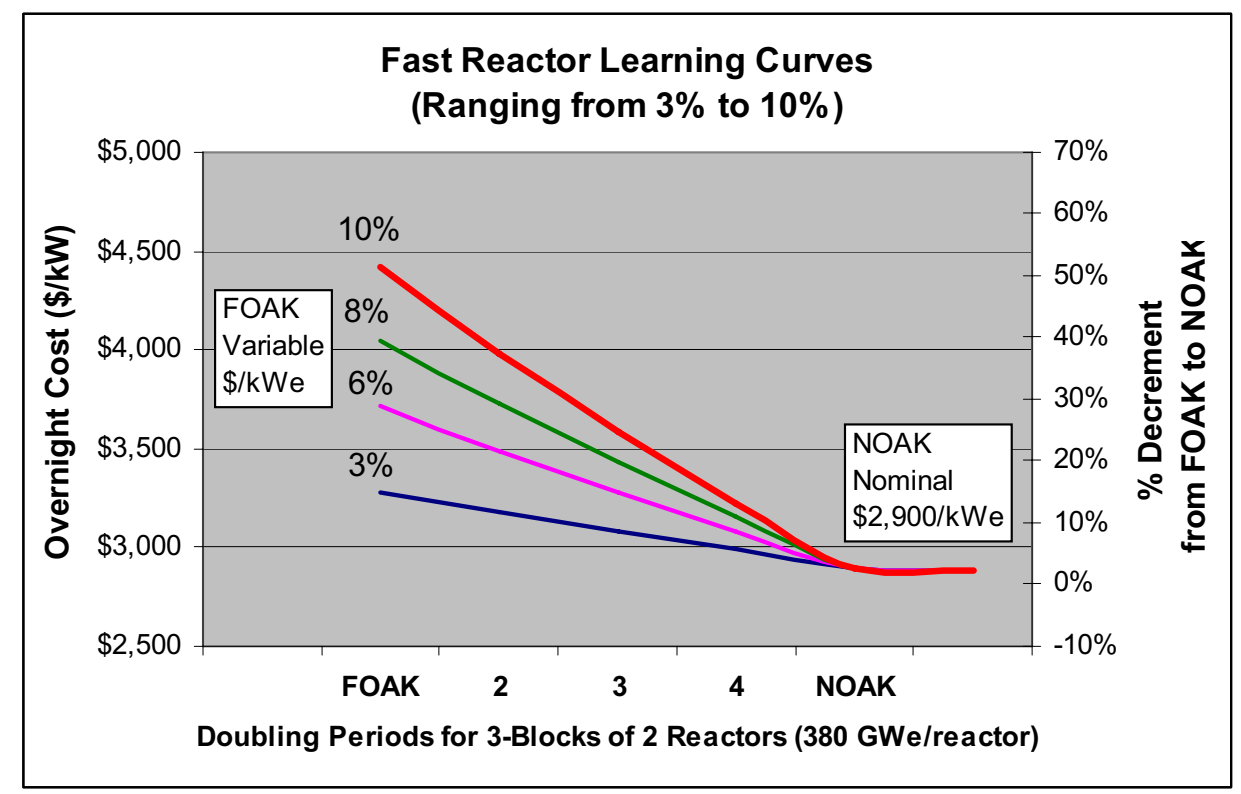

Figure 1-4. Fast reactor learning rates for a range of learning curves.

\subsubsection{Static and Dynamic Cost Modeling Differences}

As mentioned in Section 1.2, this analysis has utilized static and dynamic models. Using both types of models has been very useful as a check on results (further discussed in Section 9) and to gain understanding about the differences between static (equilibrium) and dynamic (including growth) results. The G4 ECONS spreadsheet modeling has been used to produce equilibrium costs for the AFCI nominal strategies, and the VISION.ECON model was used to produce non-equilibrium, dynamic costs over time. The static cost analysis is inclusive of all fuel cycle and reactor costs for a given strategy (no costs are missing). In the dynamic modeling the costs are incurred over time as the various fuel cycle functions are brought online (i.e., receive a mass flow) and reactors become operational (i.e., produce electricity).

Under growth conditions, multiple fuel cycle facilities and scores of reactors are brought online during the simulation run through the year 2100 . The annual costs for production of energy under the dynamic case account for all current year expenditures. The costs for electricity production are based on the annual costs divided by the energy produced in that year (in units of mills $/ \mathrm{kWh}$ ). These production costs provide a reasonable proxy for the unit energy costs, but show some variation (generally a few mills $/ \mathrm{kWh}$ ) to the static nominal costs. System variables that impact the dynamic costs include nuclear growth rates, system delays (storage), and transitions between reactor systems (thermal to fast). Cost differences are also due to modeling differences, such as counting whole number of reactors versus fractional numbers. Further treatment of this topic is provided in Section 1.3.6, where the impacts of dynamic system equilibrium are described.

\subsubsection{Impacts from Static and Dynamic Equilibrium Effects}

The steady state system will balance the transuranic (TRU) that is discharged from the LWRs with the makeup requirements of the fast reactors and startup fuel for new reactors. For the static equilibrium (SE) case, the system is assumed to have a zero growth rate in energy generation and therefore requires no new fast reactor fuel to support a growing fleet. The dynamic equilibrium (DE) case assumes an exponential growth rate in the energy generation and therefore a constant growing fleet of fast reactors that will require new fuel to startup the reactors. Balancing equations are derived to establish the static and dynamic equilibrium relationships between LWRs and fast reactors. 
Static Equilibrium : TRU $\mathrm{LWR}_{\mathrm{LWh}}^{\text {Dische }}[\mathrm{kg} / \mathrm{yr}]=\mathrm{TRU}_{\mathrm{FR}}^{\text {Makeup }}[\mathrm{kg} / \mathrm{yr}]$

LWR TRU Supply:TRU $\mathrm{LWR}^{\text {Discharge }}=\alpha_{L W R} * P_{L W R} ; \alpha_{L W R}[\mathrm{~kg} / G W e-y r] ; P_{L W R}[G W e-y r / y r]$

FR TRU Demand for Makeup Feed : TRU $U_{\mathrm{FR}}^{\text {Makeup }}=\alpha_{F R} * P_{F R} ; \alpha_{F R}[\mathrm{~kg} / G W e-y r] ; P_{F R}[G W e-y r / y r]$

Static Equilibrium LWR fraction $: \mathrm{f}_{\mathrm{LWR}}^{\mathrm{SE}}=\frac{\alpha_{F R}}{\alpha_{L W R}+\alpha_{F R}}$

The dynamic equilibrium has the same form as the static equilibrium, but the TRU supplied from the LWRs varies over time so the balance occurs with a lag time that allows for cooling of the discharged LWR fuel and the time to recycle the spent LWR fuel and utilize the TRU recovered from the LWR in the fast reactors. The recovered TRU from the spent LWR fuel is utilized as makeup feed for fast reactors operating at conversion ratios below breakeven $(\mathrm{CR} \sim 1.0)$ as is the case in static equilibrium. Additionally the TRU from the LWR is used to make the necessary fuel to startup new fast reactors that are coming online as a result of the growth in nuclear power. This means that all TRU from LWRs can be consumed with conversion ratios substantially above 1.0 assuming the growth rate is sufficient that the demand for new TRU exceeds the surplus produced by a breeder $(\mathrm{CR}>1.0)$ fast reactor.

Dynamic Equilibrium Mass Balance: $\operatorname{TRU}_{\mathrm{LWR}}^{\text {Discharge }}\left(\mathrm{t}-\Delta \mathrm{t}_{\text {lag time }}^{\mathrm{LWR} \text { Recycle }}\right)=\operatorname{TRU}_{\mathrm{FR}}^{\text {Makeup }}(\mathrm{t})+\operatorname{TRU}_{F R}^{\text {New Fuel }}(\mathrm{t})$

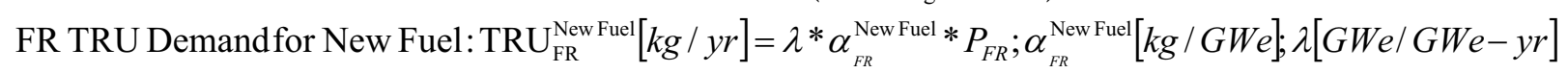
Time Dependent Power Level: $P(t)=P_{0}^{*} e^{\lambda t}$

The following equation relates the mass balance of the past generation of LWR to the current requirements of fast reactors. However, the equilibrium ratio is that of both the current LWRs and current fast reactors.

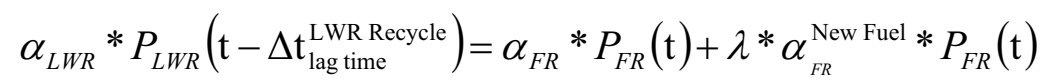

Dynamic Equilibrium LWR fraction $\mathrm{f}_{\mathrm{LWR}}^{\mathrm{DE}}=\frac{P_{L W R}(\mathrm{t})}{P_{L W R}(\mathrm{t})+P_{F R}(\mathrm{t})}$

$\alpha_{L W R} * P_{L W R}(\mathrm{t}) * e^{-\lambda \Delta t_{\text {lag time }}^{\mathrm{LWR} \text { Recle }}}=\left(\alpha_{F R} *+\lambda * \alpha_{F R}^{\text {New Fuel }}\right) * P_{F R}(\mathrm{t})$

$\mathrm{f}_{\mathrm{LWR}}^{\mathrm{DE}}=\frac{\alpha_{F R}+\lambda * \alpha_{F R}^{\text {New Fuel }}}{\alpha_{L W R} * e^{-\lambda \Delta \mathrm{t}_{\text {lag fime }}^{\mathrm{LWR} \text { Recyle }}}+\alpha_{F R}+\lambda * \alpha_{F R}^{\text {New Fuel }}}$

This following equation simplifies to the static equilibrium equation if the growth rate is set to zero $(\lambda=0)$. The calculations predict that when the growth rate (and lag time) increases, the fast reactor fraction will decline. The dynamic system results in fewer fast reactors (declining from approximately $33 \%$ to $16 \%$ ) as the nuclear power growth rate is increased from $0.3 \%$ to $3.3 \%$, while the static result is unaffected by growth (holding steady at $37 \%$ over all growth rates), as illustrated in Figure 1-5 for a 1 -Tier system operating at a 0.50 TRU conversion ratio. The analysis is performed using static and dynamic models to estimate the fast reactor requirements in nuclear growth cases, and is further evaluated in Section 6.3.3.

$$
\mathrm{f}_{\mathrm{LWR}}^{\mathrm{DE}}=\frac{\mathrm{f}_{\mathrm{LWR}}^{\mathrm{SE}}}{1+\frac{\alpha_{L W R} *\left(e^{-\lambda \Delta \Delta_{\mathrm{lag} \text { time }}^{\mathrm{LWR} \text { Recle }}}-1\right)+\lambda * \alpha_{F R}^{\text {New Fuel }}}{\alpha_{L W R}+\alpha_{F R}}}+\frac{\lambda * \alpha_{F R}^{\text {New Fuel }}}{\alpha_{L W R} * e^{-\lambda \Delta t_{\text {lag time }}^{\mathrm{LWR} R \text { Reyle }}}+\alpha_{F R}+\lambda * \alpha_{F R}^{\text {New Fuel }}}
$$




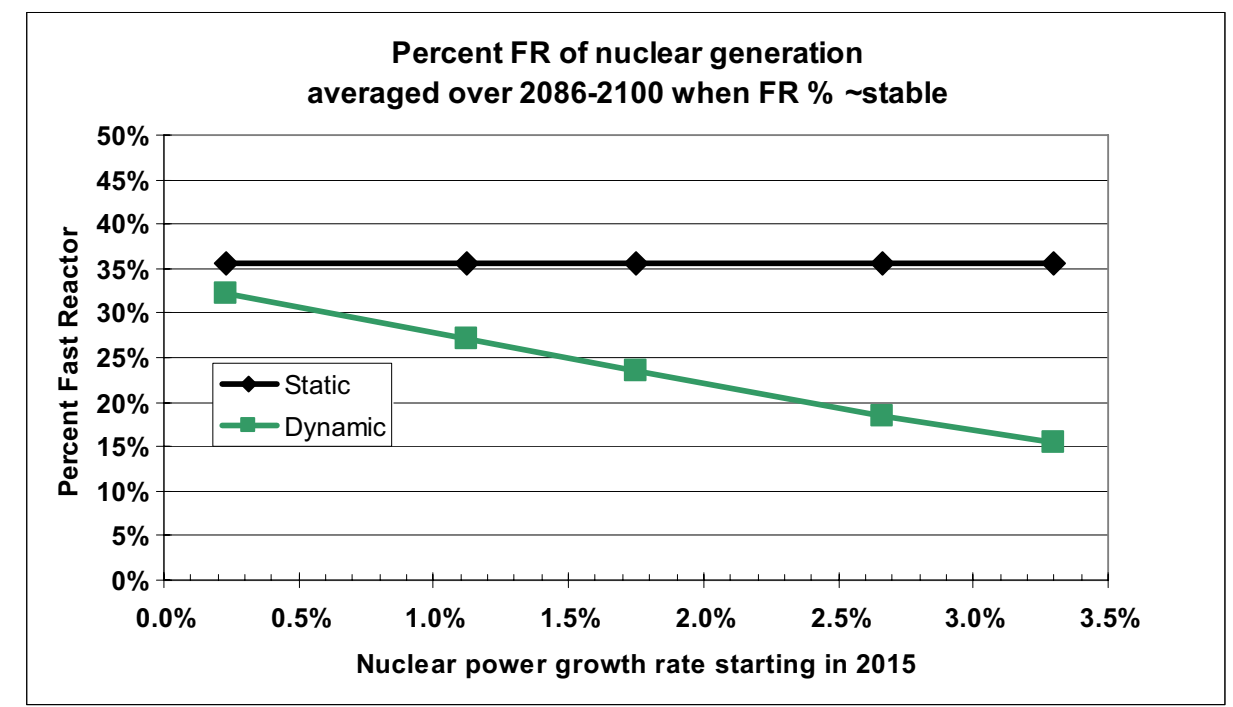

Figure 1-5. Comparison of fast reactor nuclear generation due to static and dynamic equilibrium effects.

\subsection{References for Section 1}

Bailey, G.F., J. D. Smith, 2008, "Draft Once-Through, Single and Dual Tier Fuel Cycles Transportation Cost Analyses," (GNEP-SYSA-ECON-SS-RT-2008-0000251), January 2008.

Economic Analysis Working Group, 2009, “AFCI Economic Tools, Algorithms, and Methodology," INL/EXT-07-13293, (Released as a draft report in 2007.)

Economic Modeling Work Group, 2006, "Cost Estimating Guidelines for Generation IV Nuclear Energy Systems," GenIV Program Economic Modeling Working Group, 2006.

Analysis and Integration, 2008, "Dynamic Systems Analysis Report for Nuclear Fuel Recycle," GNEPSYSA-AI-SS-RT-2008-000264, December 2008.

Shropshire, D., et al., 2008, “2008 Advanced Fuel Cycle Cost Basis,” INL/EXT-06-11536, March 2008.

Shropshire, D., K. Williams, J. D. Smith, B. Boore, 2006, “Advanced Fuel Cycle Economic Sensitivity Analysis," INL/EXT-06-11947, December 2006.

University of Chicago, 2004, The Economic Future of Nuclear Power, Argonne National Laboratory, August 2004.

Williams, K., 2007, “The G4 ECONS Economic Evaluation Tool for Generation IV Reactor Systems," 2007 ANS Summer Meeting, Boston, Massachusetts, TRANSACTIONS, pg. 131-132, (June 24-28, 2007). 


\section{FUEL CYCLE DEPLOYMENT CASES}

Systems Analysis chose the fuel cycle cases in this study to represent one option for implementing the 1-Tier and 2-Tier strategies. A strategy is a general approach to fuel management that encompasses a range of options with similar basic characteristics. The strategy identifies which materials are recycled, the type of nuclear power plant utilizing the fuel, the type of used fuel processing technology, and which materials, used fuel, or separated products go to geologic disposal or to some other disposal concept.

For each of the strategies, the cost ranges correspond to a particular case within each strategy, but the cost ranges do not represent the full range of possible ways to implement each strategy. Subsequent to this analysis, other cases may be studied that include different reactor and fuel cycle performance assumptions, optimized mass flows, and facility operational characteristics that have the potential to reduce the uncertainties of the fuel cycle cost and the total cost of energy. Further details on the strategies and their system assumptions are provided in the DSARR assumptions document (Analysis and Integration 2008).

\subsection{Strategy Descriptions}

\subsubsection{Once-through Fuel Cycle}

The once-through cycle (OTC or once-through) is the current U.S. strategy of utilizing slightly enriched uranium as uranium oxide (UOx) fuel in LWRs. All the components of spent fuel are kept intact and are ultimately directly disposed in a geologic repository. This is considered an open fuel cycle because significant quantities of actinides (partially burned fuel) will be disposed in the repository.

The process flow diagram for the once-through strategy, as shown in Figure 2-1, describes the linkage between fuel cycle modules, reactor capacity and energy output, and primary mass flows.

\subsubsection{1-Tier Fuel Cycle}

This strategy builds on the once-through cycle. However, instead of directly disposing of the intact spent LWR fuel, the TRU elements are recovered and used in fast reactors. This is referred to as a closed fuel cycle, since the actinides are repeatedly recycled, and only a small fraction of the actinides will end up in the waste stream. The fast reactors operate as waste burners for the LWR through a "sustained thermal/fast recycle." There is a symbiotic mix of thermal and fast reactors, which balance the recycled transuranic elements from the LWR with the demands of the fast reactor. The introduction of fast reactors makes this strategy sustainable from the repository standpoint. Fast reactors serving as transuranic element burners control and limit the accumulation of transuranic elements during repeated recycle.

Fission products and only characteristic TRU waste processing losses would go to geologic disposal, but transuranic elements would not. Reprocessed uranium ${ }^{\mathrm{d}}$ from used fuel and most of the enrichment "tails" in DU would be disposed of as low-level waste (LLW). This strategy requires a certain fraction of the nuclear power plants in the overall nuclear power plant (NPP) fleet to be fast reactors. Because of the significance of this process, the Generation IV Nuclear Energy Systems initiative is researching Sodium-Cooled Fast Reactor (SFR)-type nuclear power plants.

The process flow diagram for the 1-Tier strategy, as shown in Figure 2-2, describes the linkage between fuel cycle modules, reactor capacity and energy output, recycling paths and operations, and primary mass flows.

d The reprocessed uranium could be converted to UF6, enriched, and refabricated into LWR fuel. The analysis of these processing costs were not included in this economic analysis. 


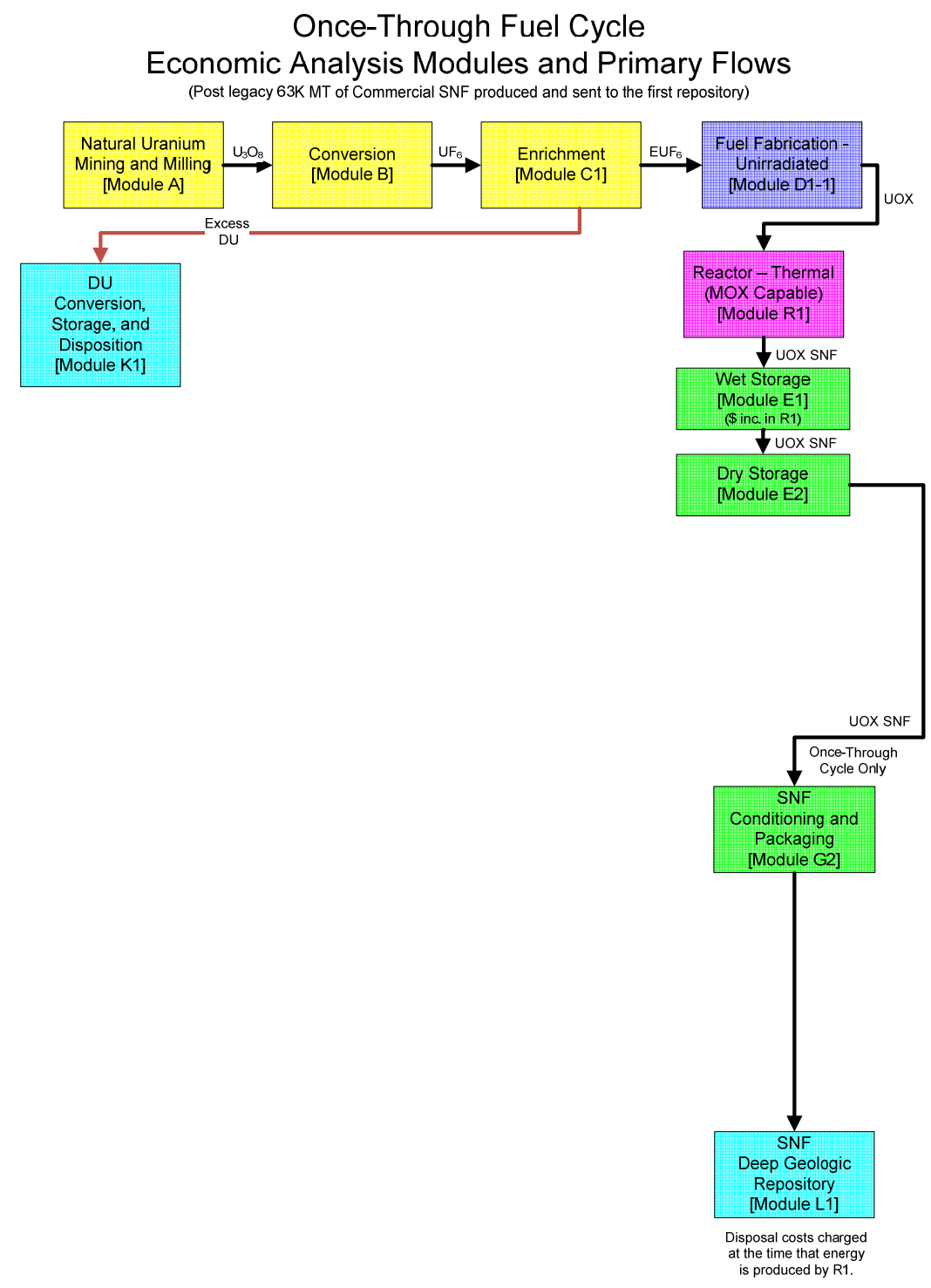

Figure 2-1. Once-through operating on an open fuel cycle.

An equilibrium system with zero growth rate would consist of a $\sim 37 \%$ mix by GWe capacity of fast reactors to $63 \%$ mix of LWRs, an equivalent thermal to fast reactor system was defined. For the nominal case, CFRs were assumed to operate at a conversion ratio $(\mathrm{CR})^{\mathrm{e}}$ of 0.50 . This means that one $1,300 \mathrm{MWe}$ LWR produces used fuel that is reprocessed, and the separated actinides provide the fissile material requirement for approximately 2.2 sodium-cooled fast reactor "burners" operating at $380 \mathrm{MWe}$. The fuel cycle cost in mills $/ \mathrm{kW} \cdot \mathrm{h}$ is calculated for both the LWRs that are operating on a partially closed cycle, and for the fast reactors, which are operating in the burner mode. Also calculated was the properly weighted "mills $/ \mathrm{kW} \cdot \mathrm{h}$ " fuel cycle cost component for the hybrid multiple reactor system as a whole. The reprocessed LWR actinides comprise the make up stream of fissile material to the fast reactor.

e. Conversion ratio is defined as the ratio of the integral number of TRU atoms produced (mostly capture in U-238) to the integral number of TRU atoms destroyed (mostly fission) that occurs during reactor operation. This is a reactor physics parameter that is consistent with previous calculations for fissile breeding calculations. It is based on atoms and not mass, does not account for decay during storage of the fuel, and neglects effects of direct fission of uranium, which are secondary effect that impact the TRU mass and energy balance for the system. 
1-Tier Fuel Cycle

Economic Analysis Modules and Primary Flows

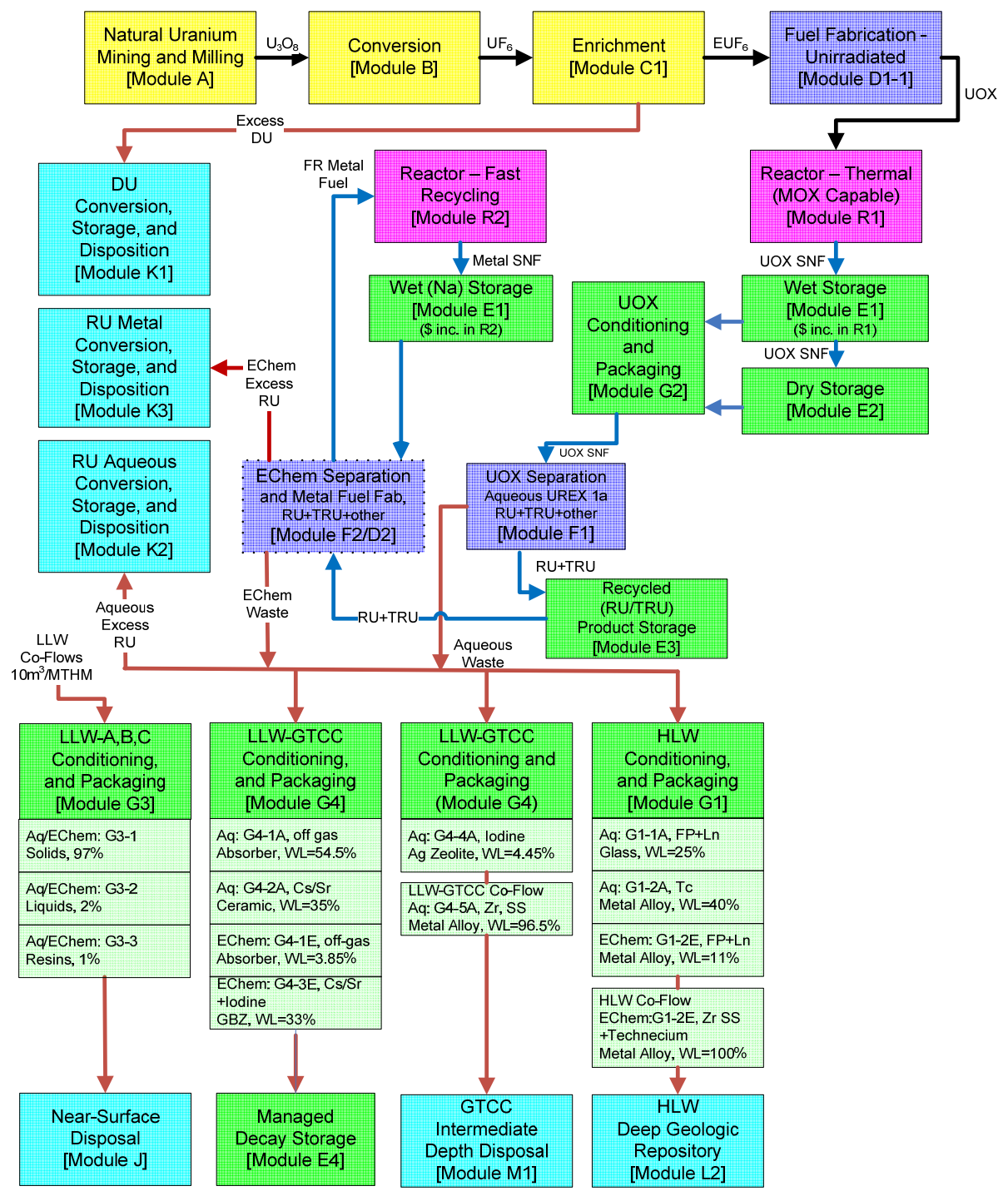

Figure 2-2. 1-Tier fuel cycle operating on an open fuel cycle.

\subsubsection{2-Tier Fuel Cycle}

The 2-Tier fuel cycle strategy builds on the traditional once-through fuel cycle. However, instead of directly disposing of the intact spent LWR UOx fuel, the separated uranium/plutonium (or uranium/plutonium and neptunium) is recovered by the separation process and then manufactured into mixed oxide (MOx) fuel for use in LWRs. The remaining transuranic elements are recovered from the spent UOx fuel and used as makeup feed in the fast reactor. After the MOx fuel is irradiated in the LWR, the spent MOx fuel is recycled and all of the transuranic elements are used as makeup feed in the fast reactor. This is referred to as a closed fuel cycle, since the actinides are repeatedly recycled and as small a fraction as practical of the actinides will end up in the waste stream. The fast reactors operate as waste burners for the LWR where through a "sustained thermal/fast recycle." There is a symbiotic mix of thermal UOx, MOx, and fast reactors, which balance the recycled transuranic elements from the LWR UOx with the demands of the MOx and fast reactor for fuel for new reactors and makeup material for 
existing reactors. The introduction of fast reactors makes this strategy sustainable from the repository standpoint. The accumulation of transuranic elements during repeated recycle passes is controlled and limited by fast reactors serving as transuranic element burners.

This strategy would use current Generation II and future Generation III ALWRs to perform the initial thermal MOx fuel recycle, and then Generation IV fast reactors would be used to further burn the remaining transuranic materials. Separated uranium would also be recycled in thermal and fast reactors, deriving additional energy. As with other recycle strategies, long-lived fission products would go to geologic disposal, and short-lived fission products would be stored and ultimately disposed of as LLW after sufficient decay.

The process flow diagram for the 2-Tier strategy, as shown in Figure 2-3, describes the linkage between fuel cycle modules, reactor capacity and energy output, recycling paths and operations, and primary mass flows.

This equilibrium system supports a $\sim 25 \%$ mix by GWe capacity of CFRs to $\sim 75 \%$ mix of LWRs, of which the LWRs are split into $67 \%$ LWRs burning UOx and $8 \%$ burning recycled MOx fuel. For the nominal case, the fast reactors were assumed to operate at a $\mathrm{CR}=0.50$. This means that approximately eight 1,300 MWe LWRs produce used fuel that is reprocessed, and the separated actinides provide the fissile material requirement for approximately one MOx burning LWR. The MOx LWR fuel is subsequently recycled in $\sim 12$ SFR "burners" operating at $380 \mathrm{MWe}$. The fuel cycle cost in mills/kW.h is calculated for LWRs burning UOx and MOx operating on a closed cycle, and for the fast reactors, which are operating in the burner mode. Also calculated was the weighted "mills $/ \mathrm{kW} \cdot \mathrm{h}$ " fuel cycle cost component for the hybrid multiple reactor system as a whole. The reprocessed LWR MOx actinides provide the make up stream of fissile material to the fast reactors.

The 2-Tier scenario requires a three-stage separation process so that all actinides are eventually recycled. The steps include:

1. Initial $\mathrm{UOx}$ aqueous separation $(\mathrm{U}+\mathrm{Pu})$ to support $\mathrm{MOx} \mathrm{UPu}$ fuel fabrication.

2. Remaining product stream is waste conditioned, then stored for 15 years awaiting subsequent separation.

3. Final aqueous separation in UREX +1 a to separate the remaining transuranics (neptunium-americiumcurium), cesium/strontium, technetium, iodine, and residual fission products (FPs). The MOx separation would be available 15 years after the $\mathrm{U}+\mathrm{Pu}$ separation and is sized at $10 \%$ of the size of the UOx front-end separation, but $100 \%$ of the size of all the remaining TRU separation processes. 


\section{Economic Analysis Modules and Primary Flows}

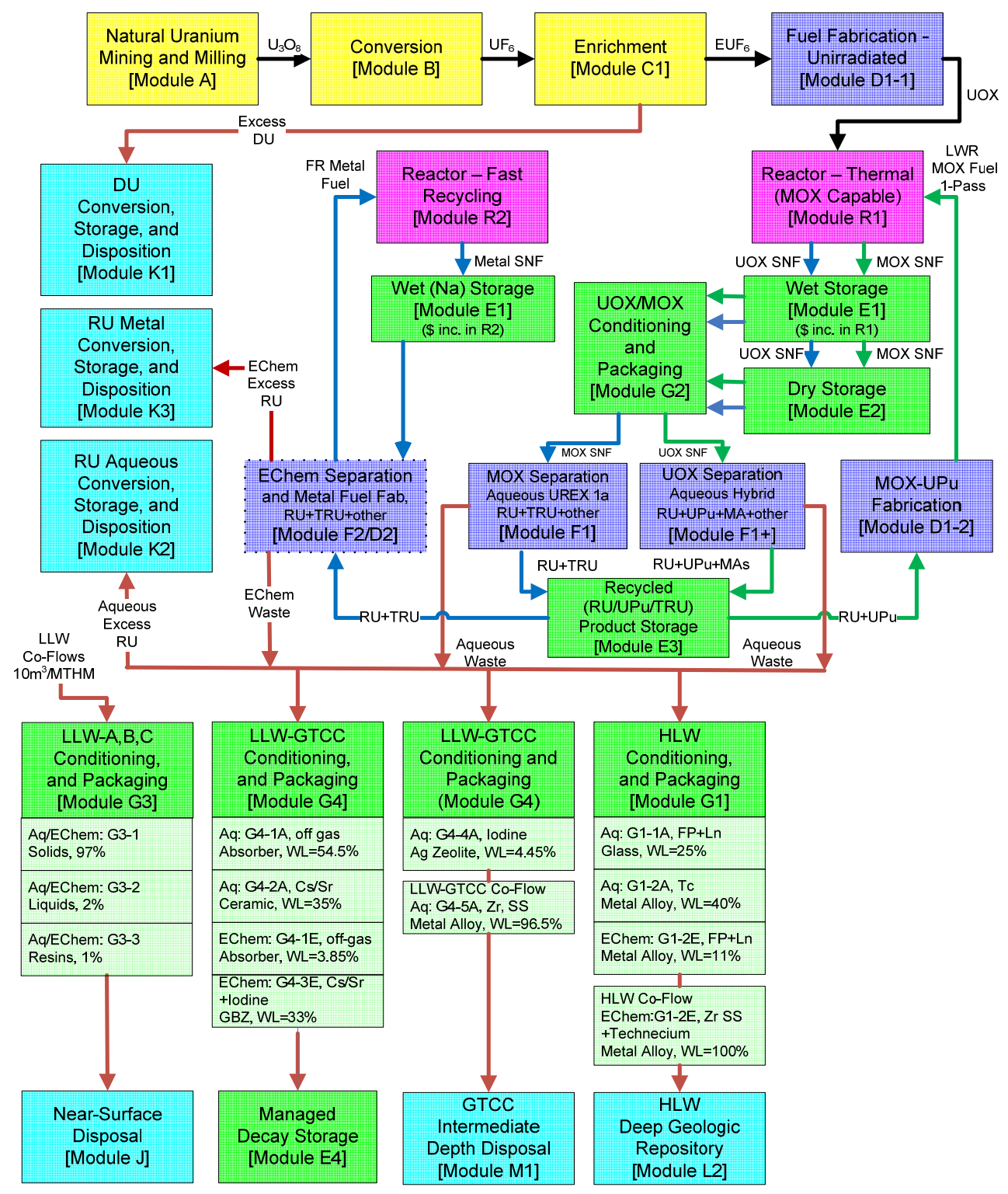

Figure 2-3. 2-Tier operating on a closed fuel cycle.

\subsection{References for Section 2}

Analysis and Integration, 2008, "Dynamic Systems Analysis Report for Nuclear Fuel Recycle," GNEPSYSA-AI-SS-RT-2008-000264, December 2008. 


\section{MODELING ASSUMPTIONS}

\subsection{General Assumptions}

The assumptions for each of the three nominal cases are described in the tables in this section. These assumptions are critical to defining the appropriate fuel cycle components, mass flows, and the application of the cost data. These assumptions can be used in conjunction with the graphical depictions of the strategies provided in Figures 1-2-1-4.

The nominal assumptions refer to the values that were initially used in the strategies. Additional sensitivity analysis can be performed with variations to these assumptions. Some assumptions are generally applicable across the various strategies, and are so noted in Table 3-1.

Table 3-1. AFCI economic analysis nominal assumptions.

\section{Nominal Assumptions}

All non-economic assumptions are based on the specifications in the DSARR (Analysis and Integration 2008).

The "AFC Cost Basis" report (Shropshire 2008) provides the module descriptions and cost distributions for the majority of costs used in this study. Some new costs were developed to support the DSARR study assumptions. Particularly, costs for waste management (Modules E and G) processes were expanded to support a more complete evaluation of the separated waste streams. Aqueous separation facility capacities and costs were scaled to reflect the nominal assumed capacities. New costs were developed for managed decay storage for cesium and strontium (Module E4) and recycled (TRU/uranium) product storage (Module E3). The estimating basis for these costs are provided in Appendix A and B, respectively. Section 3.2 notes the new and revised costs used in the "Advanced Fuel Cycle Economic Analysis of Symbiotic Light-Water Reactor and Fast Burner Reactor Systems" under the applicable cost modules.

Many of the front-end and back-end module costs include the associated transportation costs. The transportation costs differentials for centralized versus regionalized separation/fabrication facility deployment are not included in this analysis. Fuel recycling and fabrication facilities will generally be sized to gain from the improved economies of scale, which suggest that fewer more centralized facilities would be built. A summary of the fuel cycle transportation costs are included in Section 7.

No costs are applied to monitored retrievable storage (MRS) or retrievable repository in the nominal strategies.

No costs were included for the disposal of the legacy 63,000 metric tonnes (MT) spent nuclear fuel (SNF) since this will be a common cost to each of the AFCI strategies. This includes the legacy conditioning of SNF coming from the reactors (Module G2) and disposition in the repository (Module L). These costs were charged to the utilities through a $1 \mathrm{mill} / \mathrm{kwh}$ fee and are considered a sunk cost. Also, costs of the financial liability of the United States Department of Energy due to delays in opening the repository are not included in this analysis.

\subsection{Specific Economic Assumptions}

The specific economic assumptions are further defined for the three scenarios. The assumptions are described for the applicable modules as identified in Figures 2-1-2-3. The specific economic assumptions for the once-through scenario are included in Table 3-2, 1-Tier assumptions in Table 3-3, and 2-Tier assumptions in Tables 3-4. The cost ranges for the fuel cycle and reactor modules are provided in Section 4. 


\subsubsection{Once-through Economic Assumptions}

Table 3-2. Nominal assumptions for the once-through scenario.

\begin{tabular}{|c|c|}
\hline Modules & Nominal Assumptions \\
\hline $\begin{array}{l}\text { A-Natural } \\
\text { Uranium Mining } \\
\text { and Milling, } \\
\text { B-Conversion }\end{array}$ & $\begin{array}{l}\text { Front-end modules are for market-based commodities or services that are subject } \\
\text { to international market competition and pricing. The first set of these (Modules } \\
\mathrm{A} \& \mathrm{~B} \text { ) considers the steps (mining \& milling: Module A) required to produce } \\
\mathrm{U}_{3} \mathrm{O}_{8} \text { or "yellowcake" and eventually uranium hexafluoride }\left(\mathrm{UF}_{6}\right) \text { from the } \mathrm{U}_{3} \mathrm{O}_{8} \\
\text { (Conversion: Module B). A broad cost distribution was developed to consider } \\
\text { future market conditions. The analysis of the conditions affecting uranium costs } \\
\text { is provided in Module A of the "AFC Cost Basis" report (Shropshire 2008). }\end{array}$ \\
\hline C1-Enrichment & $\begin{array}{l}\text { For this assessment that looks toward future nuclear growth, enrichment is } \\
\text { assumed to be performed by the gas centrifuge enrichment process, which is less } \\
\text { energy intensive than the older gaseous diffusion process. All environmental } \\
\text { costs including LLW disposal, but not tails conversion/disposal, are covered in } \\
\text { the separative work unit (SWU) cost. } \\
\text { The unit cost of enrichment includes transportation of the enriched } \mathrm{UF}_{6} \text { product } \\
\left(\mathrm{EUF}_{6} \text { ) in } 2.5 \text { ton cylinders to the fuel fabricator (Module D1-1) and temporary }\right. \\
\text { storage of the depleted } \mathrm{UF}_{6}\left(\mathrm{DUF}_{6} \text { ) "tails" in 14-ton cylinders at the enrichment }\right. \\
\text { plant site. The tails (tta) assay is not optimized, and is set at } 0.25 \% \text { U- } 235 \text { based } \\
\text { on the nominal costs of uranium ore (Module A), conversion (Module B), and } \\
\text { enrichment (Module } \mathrm{C} 1 \text { ). }\end{array}$ \\
\hline $\begin{array}{l}\text { D1-1-Fuel } \\
\text { Fabrication- } \\
\text { Unirradiated }\end{array}$ & $\begin{array}{l}\text { The unit cost for fuel fabrication is expressed in } \$ / \mathrm{kg} \text { of heavy metal }(\mathrm{HM}) \text {, } \\
\text { which in this case is } \mathrm{U} \text { in the form of ceramic uranium dioxide }\left(\mathrm{UO}_{2}\right) \text {. The } \mathrm{HM} \\
\text { mass does not include the mass of the zirconium or zirc alloy cladding, spacers, } \\
\text { grids, etc., which make up around one-third of the mass of a typical fuel } \\
\text { assembly. The cost of these zirc components, however, is included in the unit } \\
\text { cost of fuel fabrication. } \\
\text { The fuel fabricator receives } \mathrm{EUF}_{6} \text {, defluorinates it, produces an enriched } \\
\text { uranium dioxide }\left(\mathrm{EUO}_{2}\right) \text { powder meeting a "fuel specification" morphology } \\
\text { requirement, and presses the powder into ceramic pellets, which are sintered, } \\
\text { ground, inspected, and loaded into zirconium tubes to produce "fuel rods." The } \\
\text { rods are inserted into square grids to form the complete LWR fuel assembly. } \\
\text { The unit cost includes all of the above steps plus transportation of the finished } \\
\text { fuel assemblies to the reactor. Also included are LLW disposal, hydrogen } \\
\text { fluoride disposition or sale, and other environmental compliance costs. }\end{array}$ \\
\hline $\begin{array}{l}\text { E1-Wet Storage, } \\
\text { E2-Dry Storage }\end{array}$ & $\begin{array}{l}\text { Wet and dry fuel storage is included as part of the reactor costs. Future LWRs } \\
\text { will include adequate storage for all the used fuel required over the life of the } \\
\text { facility ( } 60 \text { years). Used fuel is removed from wet storage and placed in } \\
\text { multipurpose Transportation, Aging, and Disposal (TAD) containers at the } \\
\text { reactor site. }\end{array}$ \\
\hline $\begin{array}{l}\text { G2-SNF } \\
\text { Conditioning and } \\
\text { Packaging }\end{array}$ & $\begin{array}{l}\text { SNF Conditioning and Packaging is separately estimated and is not assumed to } \\
\text { be included in the disposal costs. The NWPA may no longer apply to post } \\
63,000 \text { MT SNF disposal. }\end{array}$ \\
\hline
\end{tabular}


Table 3-2. (continued).

\begin{tabular}{|c|c|}
\hline Modules & Nominal Assumptions \\
\hline $\begin{array}{l}\text { K1-Uranium } \\
\text { Conversion, } \\
\text { storage, and } \\
\text { disposition }\end{array}$ & $\begin{array}{l}\text { Regulators are unlikely to allow permanent storage of } \mathrm{DUF}_{6} \text { at the enrichment } \\
\text { plant site. The } \mathrm{DUF}_{6} \text { is assumed to be shipped to a facility that can convert the } \\
\text { material to a more stable chemical form, in this case } \mathrm{DU}_{3} \mathrm{O}_{8} \text {. The conversion } \\
\text { facility defluorinates the } \mathrm{DUF}_{6} \text {, converts it to a crude } \mathrm{U}_{3} \mathrm{O}_{8} \text { powder, and } \\
\text { packages the powder in a container suitable for shallow burial disposal at an } \\
\text { LLW facility. } \\
\text { Transportation of } 14 \text {-ton } \mathrm{DUF}_{6} \text { cylinders from the enrichment plant site and } \\
\text { transportation of DU3O8 containers to the burial site are included in the unit } \\
\text { cost. } \\
\text { Disposition costs/benefits from hydrogen fluoride neutralization or resale } \\
\text { resulting from the defluorination step are included in the unit cost. K1 includes } \\
\text { final LLW disposal costs of the DU (Module } \mathrm{J} \text { is not additionally needed). }\end{array}$ \\
\hline $\begin{array}{l}\text { L1-Geologic } \\
\text { Repository for SNF }\end{array}$ & $\begin{array}{l}\text { For the AFCI once-through analysis, additional repositories or additional space } \\
\text { in the first repository will be required to accept any SNF produced after the first } \\
63,000 \mathrm{MT} \text {. All newly generated SNF that is sent to the repository would be in } \\
\text { excess of the } 63,000 \mathrm{MT} \text {, and not longer bound to the stipulations in the NWPA. } \\
\text { Costs would not assume the validity of a } 1-\mathrm{mill} / \mathrm{kWh} \text { fee or that the repository is } \\
\text { at Yucca Mountain. The costs in the Module } \mathrm{L} 1 \text { distribution would be assumed } \\
\text { to cover the range of repository options for a second repository, expanded YMP, } \\
\text { etc. } \\
\text { The AFCI costs range from a low of } \$ 400 / \mathrm{kg} \mathrm{HM} \text { up to } \$ 1,600 / \mathrm{kg} \mathrm{HM} \text {. A } \\
\text { nominal cost of } \$ 1,000 / \mathrm{kg} \mathrm{HM} \text { represents the costs for a hypothetical facility } \\
\text { costing } \$ 100 \mathrm{~B} \text {, supporting disposal of } 100,000 \mathrm{MTiHM} \text {. } \\
\text { SNF assemblies are transported in multi-use containers (e.g., TAD) to a (clean) } \\
\text { geologic repository where the containers are placed in tunnels. Disposal costs } \\
\text { include the casks, packaging, transportation, emplacement, and perpetual } \\
\text { monitoring. }\end{array}$ \\
\hline $\begin{array}{l}\text { R1-Reactor- } \\
\text { Thermal }\end{array}$ & $\begin{array}{l}\text { The specification for the LWR/LWRmf (mixed actinide fuel burning reactor) } \\
\text { reflect future reactors (not legacy LWRs) } \\
\text { LWR/LWRmf }=1300 \mathrm{MWe} \text { (versus } 928 \mathrm{MWe} \text { for legacy } 104 \text { reactors), b.u. = } \\
51 \mathrm{MWd} / \mathrm{KgiHM} \text {, capacity factor of } 0.90 \text {, thermal efficiency }=0.34 \text {, nominal } \\
\text { construction time }=4 \text { years, and } 60 \text { year life (operational and economic). } \\
\text { The real discount rate (range from } 5 \text { to } 10 \% \text { ) will apply to calculations of the } \\
\text { interest during construction and the fixed charge rate. Construction time was also } \\
\text { evaluated over a range of } 3.5 \text { to } 5 \text { years. } \\
\text { Assume the R } 1 \text { costs represent NOAK costs; no learning curves will be applied } \\
\text { to initial strategies. See Section } 1.3 .4 \text { for a discussion on the impacts from } \\
\text { learning. }\end{array}$ \\
\hline Other & $\begin{array}{l}\text { All fuel cycle unit costs are assumed to include an imbedded component for } \\
\text { D\&D of the fuel cycle facility at the end of the facility operational life. This is } \\
\text { normally handled by an escrow fund maintained by the facility owner/operator. }\end{array}$ \\
\hline
\end{tabular}




\subsubsection{1-Tier Economic Assumptions}

Table 3-3. Assumptions for 1-Tier nominal case.

\begin{tabular}{|c|c|}
\hline Modules & Nominal Assumptions \\
\hline $\begin{array}{l}\text { LWR cycle Modules } \\
\text { A, B, C1, D1, E1, } \\
\text { E2, K1, and R1 }\end{array}$ & $\begin{array}{l}\text { The front-end fuel cycle material balance and unit cost ranges for each } 1,300 \\
\text { MWe LWR are identical to those in the once-through strategy. } \\
\text { In the static equilibrium analysis, the assumption was that }(\sim 54) 1,300 \mathrm{MWe} \\
\text { LWRs are required to supply enough fissile material make up to fuel the }(\sim 119) \\
380 \text { MWe ARRs. This means that approximately } 1,324,367 \mathrm{kgHM} / \text { year of used } \\
\text { fuel from the LWRs is sent to the UREX+1a plant (see Module F1). }\end{array}$ \\
\hline $\begin{array}{l}\text { G2-UOx } \\
\text { Conditioning and } \\
\text { Packaging }\end{array}$ & $\begin{array}{l}\text { Assume the same costs for fuel conditioning prior to transport as for legacy } \\
\text { SNF going to the geologic repository. }\end{array}$ \\
\hline $\begin{array}{l}\text { F1-Aqueous } \\
\text { separation } \\
(\mathrm{UREX}+1 \mathrm{a})\end{array}$ & 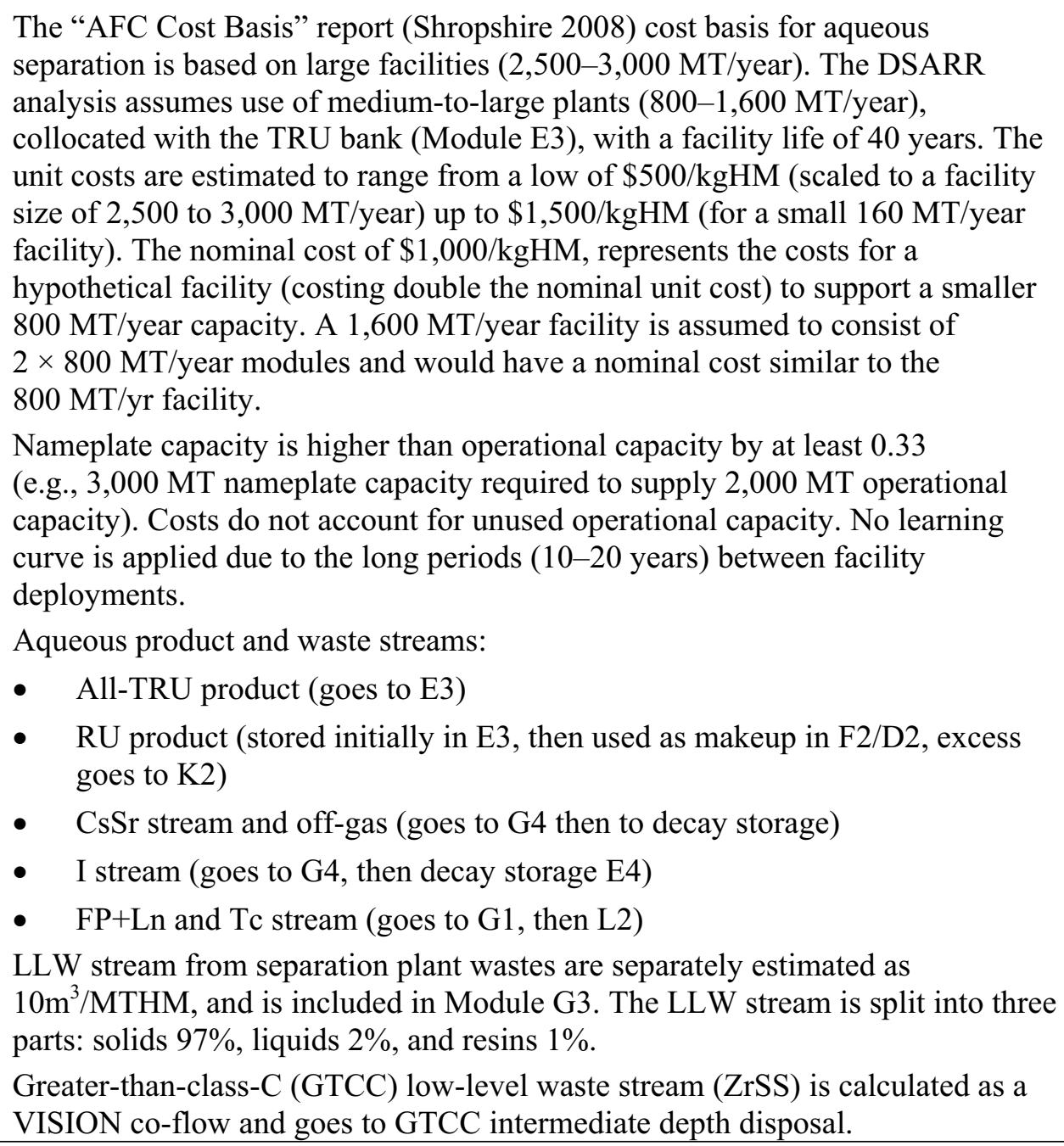 \\
\hline $\begin{array}{l}\text { E3-Recycled } \\
\text { Actinide Product } \\
\text { Storage }\end{array}$ & $\begin{array}{l}\text { This module supports the safe and secure storage of actinides as oxides from the } \\
\text { UREX+ processes. These actinides remain in safe storage until needed for MOx } \\
\text { LWR fuel (Module D1-2) or used as fast reactor TRU make-up (Module } \\
\text { F2/D2). The unit cost for storage is on a } \$ / \mathrm{kg} \text { HM actinide emplaced/removed } \\
\text { per year basis. No additional carrying charge is included for extended storage. }\end{array}$ \\
\hline
\end{tabular}


Table 3-3. (continued).

\begin{tabular}{|c|c|}
\hline Modules & Nominal Assumptions \\
\hline & $\begin{array}{l}\text { Amortization of the facility capital and annual operational costs are factored } \\
\text { into the unit cost. E3 would be collocated with the aqueous separation facilities } \\
\text { (F1). } \\
\text { The costs for TRU product storage would be greater than for UPu due to } \\
\text { increased shielding requirements due to the higher radiation and shielding } \\
\text { requirements required by the minor actinides. The recycle storage cost for TRU } \\
\text { would range from } \$ 7,000 \text { to } \$ 13,000 / \mathrm{Kg} \text { (based on the total TRU content). The } \\
\text { nominal cost of } \$ 10,000 / \mathrm{Kg} \text { TRU is a factor of } 2 \mathrm{x} \text { higher than for storage of } \\
\mathrm{RU} / \mathrm{Pu} \text {. The cost basis for recycled product storage is provided in Appendix B. } \\
\text { Facility capacity is assumed to be equivalent to } 2 \text { years of largest separations } \\
\text { facility, defined as } 3 \times 1600 \text { tonne/yr * } 0.01 \text { (Actinides portion) * } 2 \text { (years) }=96 \\
\text { tonnes (actinides). } \\
\text { Module F2/D2 includes actinide storage generated within the metal recycle } \\
\text { process, E3 is not additionally required. }\end{array}$ \\
\hline $\begin{array}{l}\text { K2-RU Disposition } \\
\text { from Aqueous } \\
\text { Reprocessing }\end{array}$ & $\begin{array}{l}\text { This module includes the costs for disposition of excess RU from aqueous and } \\
\text { electrochemical separation. } \\
\text { Separated RU from the UREX+ processes are assumed to be converted to } \mathrm{U}_{3} \mathrm{O}_{8} \text {, } \\
\text { packaged, and stored or disposed as LLW. The uranium is not re-enriched or } \\
\text { refabricated to REPUOx fuel for this scenario. } \\
\text { This aqueous derived uranium may contain small amounts of fission product } \\
\text { technetium and transmutation product } \mathrm{U}-232 \text {, which has strong gamma emitting } \\
\text { daughters. The U-236 radioisotope formed by irradiation is a neutron absorber } \\
\text { and was not present in the unirradiated (virgin) } \mathrm{UO}_{2} \text { fuel. Unless natural } \\
\text { uranium prices rise to a high level, these contaminants and undesirable products } \\
\text { make the reprocessed } \mathrm{U}_{3} \mathrm{O}_{8} \text { less attractive for re-enrichment and re-fabrication } \\
\text { into UOx fuel, thus Module } \mathrm{K} 2 \text { is invoked to handle its packaging and disposal. } \\
\text { Reprocessed } \mathrm{U}_{3} \mathrm{O}_{8} \text { and "tails" } \mathrm{U}_{3} \mathrm{O}_{8} \text { are poured into steel boxes capable of } \\
\text { holding } 1 \mathrm{metric} \text { ton of } \mathrm{U}_{3} \mathrm{O}_{8} \text {. This is the storage path followed in France for } \mathrm{U} \\
\text { from the La Hague facility and from their enrichment plants. The unit cost for } \\
\text { the } \mathrm{K} 1 \text { and } \mathrm{K} 2 \text { steps include acquisition of the boxes and transportation to the } \\
\text { warehouse area or to shallow LLW burial. }\end{array}$ \\
\hline $\begin{array}{l}\mathrm{K} 3-\mathrm{RU} \\
\text { Conditioning from } \\
\text { EChem }\end{array}$ & $\begin{array}{l}\text { This module includes the costs to convert RU from aqueous reprocessing as } \\
\text { make-up RU in metal fabrication (F2/D2). }\end{array}$ \\
\hline $\begin{array}{l}\text { F2/D2-EChem } \\
\text { Reprocessing and } \\
\text { Remote Fuel } \\
\text { Fabrication }\end{array}$ & $\begin{array}{l}\text { This very complex facility includes metal fuel separation, fabrication, and } \\
\text { actinide storage. Metal fast reactor fuel is assumed reprocessed by the EChem } \\
\text { method using molten salt chemistry. New metal fuel is cast and clad in the same } \\
\text { building, which means that the two fuel cycle steps of remote-handled FR fuel } \\
\text { reprocessing and remote-handled FR fuel fabrication are accomplished in one } \\
\text { integrated facility. Because of the complexity of this remote-handled fuel } \\
\text { fabrication operation, the unit cost will be much larger than for conventional } \\
\text { LWR UOx or U, Pu only MO } \mathrm{O}_{\mathrm{X}} \text { fuel, which can be contact handled. } \\
\text { The F2/D2 facilities will be "build-as-you-go" with fast reactor (FR) builds. } \\
\text { They would be collocated with the metal fast reactors (R2) to gain from the } \\
\text { common infrastructure, and for security and radiochemical safety reasons. }\end{array}$ \\
\hline
\end{tabular}


Table 3-3. (continued).

\begin{tabular}{|c|c|}
\hline Modules & Nominal Assumptions \\
\hline & $\begin{array}{l}\text { The metal fuel is assumed homogeneous with no separation into drivers and } \\
\text { targets/blankets, and is a blended and cast mix of DU, Pu, Np, Am, and Cm. } \\
\text { EChem product and waste streams: } \\
\text { - } \quad \text { All-TRU product (stays within F2/D2) } \\
\text { - } \quad \text { RU product (recycled within F2/D2), } 3.4 \% \text { loss to waste } \\
\text { - } \quad \text { CsSr +I and off-gas stream (goes to G4 then to decay storage E4) } \\
\text { - } \quad \text { FP+Ln stream (goes to G1). } \\
\text { LLW stream from separation plant wastes are separately estimated as } \\
10 \mathrm{~m}^{3} / \mathrm{MTHM} \text {, and is costed in Module G3. LLW stream is split into three parts: } \\
\text { solids } 97 \% \text {, liquids } 2 \% \text {, and resins } 1 \% \text {. } \\
\text { HLW co-flow for ZrSS+Tc (goes to G1 then L2). } \\
\text { The process contaminated salts are not specifically costed. }\end{array}$ \\
\hline R2-Fast Reactor & $\begin{array}{l}\text { The specification for the fast reactors at } 380 \mathrm{MWe} \text { (net), } 38 \% \text { thermal } \\
\text { efficiency, FR lifetime of } 60 \text { years, FR capacity factor } 0.82 \text {, and } 4 \text { years } \\
\text { construction time. } \\
\text { FR CR=0.50 (burn up of } 131.9 \text { ), using metal fuel with EChem separation. } \\
\text { (An oxide version is not estimated.) } \\
\text { The real discount rate (range from } 5 \text { to } 10 \% \text { ) will apply to calculations of the } \\
\text { interest during construction and the fixed charge rate. Construction time was } \\
\text { also evaluated over a range of } 3.5 \text { to } 5 \text { years. } \\
\text { Fast reactor costs are based on NOAK, as cumulative build rates exceed } 8 \text { GWe } \\
\text { early in the deployment. The reactors could be built in power blocks of two } \\
\text { reactors per one steam turbine generator. Deployment of fast reactors would be } \\
\text { initially constrained to } 1-2 \text { GWe/year (2032-2041), and then build rates will } \\
\text { increase as separations capacity is available. No learning curves were applied to } \\
\text { the initial deployment; however, the impacts from learning were considered and } \\
\text { are described in Section } 1.3 .4 \text {. }\end{array}$ \\
\hline \multirow[t]{2}{*}{$\begin{array}{l}\text { G1-HLW } \\
\text { Conditioning and } \\
\text { Packaging (Aqueous } \\
\text { and EChem) }\end{array}$} & $\begin{array}{l}\text { Costs for G1 modules were further detailed in order to support current } \\
\text { assumptions on the aqueous and EChem separated HLW streams, waste forms, } \\
\text { and waste loading. } \\
\text { The dedicated HLW conditioning and packaging facility will be located } \\
\text { immediately adjacent to the head end and separations areas of Module F1 or } \\
\text { Module F2/D2. }\end{array}$ \\
\hline & $\begin{array}{l}\text { Aqueous derived HLW: Module G1-1A includes conditioning, storage, and } \\
\text { packaging of FPs and lanthanides. Costs were adjusted from the cost basis to } \\
\text { reflect higher assumed waste loadings of } 30 \%, 25 \% \text {, and } 20 \% \text {. The associated } \\
\text { costs are } \$ 1,800 / \mathrm{kg} \text { FPs, } \$ 2,000 / \mathrm{kg} \text { FPs, and } \$ 2,700 / \mathrm{kg} \text { FPs. } \\
\text { Module G1-2A, includes aqueous derived Technetium (Tc) metal wastes. A } \\
\text { nominal cost of } \$ 25,000 / \mathrm{kg} \text { Tc was developed from a capital cost of } \$ 2.4 \mathrm{~B} \text { and } \\
\$ 9,000 / \mathrm{kg} \text { FP O\&M, for a } 150 \mathrm{t} / \mathrm{year} \text { throughput of U/Tc. Costs range from } \\
\$ 18,000 / \mathrm{kg} \text { Tc to } \$ 30,000 / \mathrm{kg} \text { Tc. The disposition costs for the fission products } \\
\text { and activated metals would include acquisition of the cans enclosing the waste. }\end{array}$ \\
\hline
\end{tabular}


Table 3-3. (continued).

\begin{tabular}{|c|c|}
\hline Modules & Nominal Assumptions \\
\hline & $\begin{array}{l}\text { EChem derived HLW: Module G1-2E includes the metal alloy conditioning, } \\
\text { storage, and packaging of zirconium and stainless steel (ZrSS) plus Tc. Costs } \\
\text { were derived from GTCC waste conditioning costs from the Cost Basis report, } \\
\text { Module G4. Costs range from a low of } \$ 200 / \mathrm{kg} \text { metal up to } \$ 1,800 / \mathrm{kg} \text { metal. A } \\
\text { nominal cost of } \$ 540 / \mathrm{kg} \text { metal. The disposition costs for the activated metals } \\
\text { would include acquisition of the cans enclosing the waste. }\end{array}$ \\
\hline $\begin{array}{l}\text { G3-LLW } \\
\text { Conditioning, } \\
\text { Storage, and } \\
\text { Packaging }\end{array}$ & $\begin{array}{l}\text { LLW stream from separation plant wastes are separately estimated as } \\
10 \mathrm{~m}^{3} / \mathrm{MTHM} \text {. LLW streams consist of three types: } 97 \% \text { LLW solids/debris } \\
(\mathrm{G} 3-1), 2 \% \text { LLW liquids (G3-2), and 1\% LLW resins (G3-3). Unit costs are } \\
\text { based on the March } 2008 \text { "AFC Cost Basis" report. }\end{array}$ \\
\hline \multirow[t]{5}{*}{$\begin{array}{l}\text { G4-LLW-GTCC } \\
\text { Waste Conditioning } \\
\text { (Aqueous and } \\
\text { EChem) }\end{array}$} & $\begin{array}{l}\text { Costs for G4 modules were further detailed in order to support current } \\
\text { assumptions on the aqueous and EChem separated HLW streams, waste forms, } \\
\text { and waste loading. These wastes consist of gases (H3, Kr, Xe), metals (CsSr, } \\
\text { ZrSS) and Iodine. Costs were developed for each type of waste. }\end{array}$ \\
\hline & $\begin{array}{l}\text { Gas wastes: Modules G4-1A (Aqueous) and G4-1E (EChem) provide waste } \\
\text { conditioning for the gaseous wastes (H3, Kr, Xe). Cost basis was derived from } \\
\text { study on Krypton encapsulation preconceptual design (Knecht } 1994) \text { ). Off-gas } \\
\text { conditioning costs range from } \$ 8,000 / \mathrm{m}^{3} \text { gas to } \$ 15,000 / \mathrm{m}^{3} \text { gas, with a nominal } \\
\text { cost of } \$ 11,200 / \mathrm{m}^{3} \text { gas. Aqueous and EChem off gases are conditioned and } \\
\text { packaged then placed in long-term managed decay storage. } \\
\text { The Knecht study was based on } 233 \mathrm{~m}^{3} / \mathrm{yr} \text { of off-gas }(99 \% \text { krypton) produced } \\
\text { from a } 2,000 \mathrm{MTHM} / \mathrm{yr} \text { reprocessing plant. This rate corresponds to } 873 \mathrm{~kg} \\
\mathrm{Kr} / \mathrm{yr} \text { for the zeolite encapsulation part of the reprocessing complex. Table } 7-7 \\
\text { from the Knecht report gives a discounted life cycle cost of } \$ 21.9 \mathrm{M} \text { in } 1994 \$ \text { or } \\
\$ 32.4 \mathrm{M} \text { if converted to today's dollars. Since unit costs }(\$ / \mathrm{unit}) \text { can be } \\
\text { calculated by dividing discounted life cycle costs by discounted annual } \\
\text { production, the discounted production of } 233 \mathrm{~m}^{3} \text { gas per year for } 30 \text { years at a } \\
7 \% \text { discount rate gives an overall discounted production of } 2891 \mathrm{~m}^{3} . \text { Dividing } \\
\$ 32.4 \mathrm{M} \text { by } 2891 \text { gives a unit cost of } \$ 11,200 / \mathrm{m}^{3} \text { of off-gas. This was designated } \\
\text { as the nominal value. The high and low values were selected to give } \\
\text { approximately a plus or minus } 30 \% \text { variation from the nominal value. }\end{array}$ \\
\hline & $\begin{array}{l}\text { Cesium and Strontium: Modules G4-2A (Aqueous) and G4-3E (EChem) } \\
\text { provide cesium/strontium conditioning. The aqueous derived wastes go into a } \\
\text { ceramic waste form, and the EChem derived wastes go into a glass bonded } \\
\text { zeolite waste form. The nominal cost of } \$ 7,800 / \mathrm{CsSr} \text { is based on an engineering } \\
\text { estimate of } \$ 3.7 \mathrm{~B} \text { capital and } \$ 40 \mathrm{M} / \mathrm{year} \mathrm{O} \& \mathrm{M} \text { for a } 28,400 \mathrm{~kg} \text { CsSr/year } \\
\text { throughput. The costs range from } \$ 5,700 / \mathrm{Kg} \text { CsSr (lower capital costs) to } \\
\$ 12,000 / \mathrm{Kg} \text { CsSr (lower throughput). Aqueous and EChem CsSr wastes are } \\
\text { conditioned and packaged then placed in long-term managed decay storage. }\end{array}$ \\
\hline & $\begin{array}{l}\text { Iodine: Module G4-4A (Aqueous derived) conditions Iodine for placement in } \\
\text { GTCC intermediate depth disposal. The nominal cost of } \$ 67,000 / \mathrm{m}^{3} \text { Iodine is } \\
\text { based on an engineering estimate of } \$ 25 \mathrm{M} \text { capital and } \$ 2 \mathrm{M} / \mathrm{year} \text { O\&M for a } \\
50 \mathrm{~m}^{3} / \text { year Iodine throughput. The costs range from } \$ 50,000 / \mathrm{m}^{3} \text { to } \$ 80,000 / \mathrm{m}^{3} \\
\text { Iodine. }\end{array}$ \\
\hline & $\begin{array}{l}\mathbf{Z r} / \mathbf{S S} \text { : Module G4-5A (Aqueous) is estimated the same as G1-2E (EChem } \\
\text { metal alloy conditioning of ZrSS). This waste is dispositioned to GTCC } \\
\text { intermediate depth disposal. }\end{array}$ \\
\hline
\end{tabular}


Table 3-3. (continued).

\begin{tabular}{|c|c|}
\hline Modules & Nominal Assumptions \\
\hline $\begin{array}{l}\text { J-Near Surface } \\
\text { Disposal }\end{array}$ & $\begin{array}{l}\text { Module } J \text { is for near surface or shallow burial of the packaged waste at private } \\
\text { licensed or government facilities such as Envirocare or Nevada Test Site (NTS). } \\
\text { The generated LLW is calculated as co-flows from Module F1 and F2/D2 and is } \\
\text { treated in Module G3. }\end{array}$ \\
\hline $\begin{array}{l}\text { M1-GTCC } \\
\text { Intermediate Depth } \\
\text { Disposal }\end{array}$ & $\begin{array}{l}\text { GTCC intermediate depth disposal is expected to cost less than geologic } \\
\text { disposal. The engineering estimates are based on experience from the Waste } \\
\text { Isolation Pilot Plant costs for waste conditioning and disposition of transuranic } \\
\text { waste provided in the "AFC Cost Basis" report Module G5. The nominal cost of } \\
\$ 100,000 / \mathrm{m}^{3} \text { GTCC, with a cost range from } \$ 70,000 / \mathrm{m}^{3} \text { to } \$ 440,000 / \mathrm{m}^{3} \text { GTCC. }\end{array}$ \\
\hline $\begin{array}{l}\text { L2-1 and L2-2- } \\
\text { Geologic Repository } \\
\text { for HLW }\end{array}$ & $\begin{array}{l}\text { A repository will be available with unconstrained input capacity after the } \\
63,000 \text { MT has been dispositioned. For the } 1 \text {-Tier and } 2 \text {-Tier strategies, all } \\
\text { HLW sent to the repository would be in excess of the } 63,000 \text { MT SNF and } \\
\text { would not assume a } 1 \text { mill/kWh fee or that the repository is in Nevada. The } \\
\text { costs in the Module L } 2 \text { distribution would be assumed to cover the range of } \\
\text { repository options for a second repository, expanded first repository, etc. } \\
\text { Module L2-1 has costs derived from Module L1, where the nominal cost for } \\
\text { SNF disposition is } \$ 1,000 / \mathrm{kg} \text { HM. The waste loading of the HLW for L2-1 is } \\
\text { estimated to be improved by a factor of } 2 x \text { to } 10 x \text {, with a nominal loading of } \\
2.5 x \text {. Based on an average fission product composition of approximately } 4 \% \text { of } \\
\text { the initial heavy metal, the related HLW disposition costs (L2-1) are estimated } \\
\text { from } \$ 2,500 / \mathrm{kg} \text { FP to } \$ 12,500 / \mathrm{kg} \text { FP. The HLW disposition charges are } \\
\text { incurred as the HLW mass is moved from the G1 Module to the deep geologic } \\
\text { repository (Module L2-1). } \\
\text { Module L2-2 is for the HLW disposition cost of the activated hulls (not the } \\
\text { fission products or used fuel). These costs are estimated similarly to Module L1, } \\
\text { with a low range cost of } \$ 400 / \mathrm{kg} \text { metal, nominal } \$ 1,000 / \mathrm{kg} \text { metal, and high } \\
\$ 1,600 / \mathrm{kg} \text { metal. The HLW disposition charges are incurred as the HLW mass } \\
\text { is moved from the G1 Module to the deep geologic repository (Module L2-2). } \\
\text { HLW assemblies are transported in multi-use containers (e.g., TAD) to a (clean) } \\
\text { geologic repository where the containers are placed in tunnels. Disposal costs } \\
\text { include the casks, packaging, transportation, emplacement, and perpetual } \\
\text { monitoring. }\end{array}$ \\
\hline Other & $\begin{array}{l}\text { All fuel cycle unit costs are assumed to include an imbedded component for } \\
\text { D\&D of the fuel cycle facility at the end of life. This is normally handled by an } \\
\text { escrow fund maintained by the facility owner/operator. }\end{array}$ \\
\hline
\end{tabular}




\subsubsection{2-Tier Economic Assumptions}

Table 3-4. Assumptions for 2-Tier nominal case.

\begin{tabular}{|c|c|}
\hline Modules & Nominal Assumptions \\
\hline $\mathrm{A}, \mathrm{B}, \mathrm{C} 1, \mathrm{~K} 1, \mathrm{D} 1$ & $\begin{array}{l}\text { The front-end fuel cycle material balance will be adjusted based on the LWRmf } \\
\text { reactor requirements. } \\
\text { In the static analysis, the assumption was that }(\sim 56) 1,300 \text { MWe LWRs are } \\
\text { required to supply the UOx SNF for }(\sim 7) 1,300 \text { MWe MOx LWRs, which in } \\
\text { turn provides enough fissile material make up to fuel }(\sim 82) 380 \text { MWe fast } \\
\text { reactors. }\end{array}$ \\
\hline $\begin{array}{l}\text { R1-MOx fueled } \\
\text { LWR }\end{array}$ & $\begin{array}{l}\text { 1-pass MOx-RU/Pu } 51 \mathrm{MW} \text {-day/kg-iHM (initial heavy metal) with aqueous } \\
\text { separation. Same capacity, lifetime, thermal efficiency as UOx-fueled reactor. } \\
\text { Gen III LWR reactors will be able to burn MOx without additional cost. }\end{array}$ \\
\hline $\begin{array}{l}\text { E1-Wet Reactor } \\
\text { Storage, }\end{array}$ & $\begin{array}{l}\text { Wet and dry storage for UOx and MOx fuel. No cost premium for MOx. Wet } \\
\text { and dry fuel storage is included in the reactor costs. }\end{array}$ \\
\hline $\begin{array}{l}\text { E2-Dry Reactor } \\
\text { Storage }\end{array}$ & $\begin{array}{l}\text { Used fuel is removed from wet storage and placed in multipurpose TAD casks } \\
\text { at the reactor site. }\end{array}$ \\
\hline $\begin{array}{l}\text { E3-1 \& E3-2- } \\
\text { Recycled Product } \\
\text { Storage }\end{array}$ & $\begin{array}{l}\text { Module E3-1 costs for TRU storage are the same as for the } 1 \text {-Tier. For the } \\
\text { 2-Tier scenario, recycled uranium/plutonium is stored prior to fabrication of } \\
\text { MOx LWR fuel. Assume same cost basis for storage as for TRU product } \\
\text { coming from UREX+1A separation. The costs are based on } \$ / \mathrm{kg} \text { RUPu, where } \\
\text { the mass of the RU is not counted. The recycle storage cost for UPu would } \\
\text { range from } \$ 3,500 \text { to } \$ 6,500 / \mathrm{Kg} \text { (based on the Pu content of the UPu). The } \\
\text { nominal cost is } \$ 5,000 / \mathrm{Kg} \mathrm{UPu} \text {. The basis for the costs of RUPu and actinide } \\
\text { storage is further discussed in Appendix B. }\end{array}$ \\
\hline $\mathrm{R} 2, \mathrm{~F} 2 / \mathrm{D} 2, \mathrm{~K} 3$ & Same assumptions as for 1 -Tier \\
\hline K2-RU Disposition & Receives excess RU from MOx and UOx aqueous separation \\
\hline $\begin{array}{l}\text { F1+ Hybrid } \\
\text { Aqueous } \\
\text { UOx separation }\end{array}$ & $\begin{array}{l}\text { This module provide the UOx separation that initially separates the UPu that } \\
\text { will subsequently fabricated into MOx fuel and burned in MOx compatible } \\
\text { LWRs. The first plant will produce } 800 \text { t/year (net), with subsequent plants } \\
\text { sized twice as large, at } 1,600 \text { t/year (or } 2 \mathrm{x} 800 \mathrm{t} / \mathrm{year} \text { lines). } \\
\text { This module includes the combined cost for aqueous separation, conditioning, } \\
\text { and storage, which is estimated at } \$ 1,320 / \mathrm{kg} \text { (nominal value). Aqueous } \\
\text { separation is based on a UREX+3 separations process, which is based on } 10 \% \\
\text { higher process complexity (hence higher costs) costs than for UREX+1 } \\
\text { (Module F1), or } \$ 1,100 / \mathrm{kg} \mathrm{HM} \text { (nominal value). The separated products are } \\
\text { conditioned (Module G2) at a cost of } \$ 100 / \mathrm{kg} \mathrm{HM} \text { (nominal value), then stored } \\
\text { for } 15 \text { years in Module E2 at } \$ 120 / \mathrm{kg} \mathrm{HM} \text { (nominal value). Low and high range } \\
\text { costs are based on the same estimating rationale as the nominal costs. } \\
\text { Initial products of separation: U+Pu+RU product goes to MOx fuel fabrication } \\
\text { (D1-2) and burned in a MOx LWR for one pass. The one-pass MOx-used fuel is } \\
\text { then recycled in the aqueous MOx separation process (Module F1). } \\
\text { Other product and waste streams: } \\
\text { NpAmCm product (goes to E3) }\end{array}$ \\
\hline
\end{tabular}


Table 3-4. (continued).

\begin{tabular}{|c|c|}
\hline Modules & Nominal Assumptions \\
\hline & $\begin{array}{l}\text { RU product (stored initially in E3, then used as makeup in F2/D2, excess goes } \\
\text { to K2) } \\
\text { CsSr stream and off-gas (goes to G4 then to decay storage) } \\
\text { I stream (goes to G4, then decay storage E4) } \\
\text { FP+Ln and Tc stream (goes to G1, then L2). } \\
\text { LLW stream from separation plant wastes are separately estimated as } \\
10 \mathrm{~m}^{3} / \mathrm{MTHM} \text {, and is costed in Module G3. LLW stream is split into three parts: } \\
\text { solids } 97 \% \text {, liquids } 2 \% \text {, and resins } 1 \% \text {. } \\
\text { GTCC stream (ZrSS) is calculated as a VISION co-flow and goes to GTCC } \\
\text { intermediate depth disposal. }\end{array}$ \\
\hline $\begin{array}{l}\mathrm{D} 1-2-\mathrm{MOx} \mathrm{RU} / \mathrm{Pu} \\
\text { fabrication }\end{array}$ & $\begin{array}{l}\text { MOx fabrication for LWRs utilizes the RU/Pu that is separated in the F1+ } \\
\text { Hybrid module. The low and nominal range costs for this module were revised } \\
\text { from the "AFC Cost Basis" to better represent the current costs for MOx fuel } \\
\text { production in the European Union. The low cost value of } \$ 1,000 / \mathrm{kg} \mathrm{HM} \\
\text { represents current fabrication costs in existing (paid off) facilities. Nominal } \\
\text { costs of } \$ 1,950 \text { reflect the projected costs for new (green field) fuel fabrication } \\
\text { facilities, based on European experience with contact-handled MOX. The high } \\
\text { cost range ( } \$ 4,000 / \mathrm{kg} \mathrm{HM)} \mathrm{is} \mathrm{based} \mathrm{on} \mathrm{a} \mathrm{U.S.} \mathrm{commercial} \mathrm{LWR} \mathrm{MOx} \mathrm{project} \\
\text { with some degree of remote handling associated with perhaps using separated } \\
\text { MOX-Pu with multiple recycled oxides of higher actinides (Am, Cm, Np, etc.), } \\
\text { and/or potentially more restrictive personnel exposure constraints in the future. }\end{array}$ \\
\hline $\begin{array}{l}\text { F1-Aqueous (MO) } \\
\text { separation }\end{array}$ & $\begin{array}{l}\text { In the 2-Tier, the aqueous MOx separation plant is assumed to be built } 15-17 \\
\text { years after the UOx separation plant (Module F1+) and would be scaled at a } \\
\text { ratio of } 1 / 9 \text { to } 1 / 10 \text { in units of heavy metal. The TRU throughput in the UOx } \\
\text { and the following MOx separation processes is estimated to be equivalent due } \\
\text { to the higher concentration of TRU in the MOx fuel. } \\
\text { The unit costs for the separation are estimated to be the same as in the } 1-\text { Tier } \\
\text { scenario. The unit costs are estimated to range from a low of } \$ 500 / \mathrm{kgHM} \text { up to } \\
\$ 1,500 / \mathrm{kgHM} \text { (based on a } 160 \mathrm{MT} / \text { year facility). The nominal cost of } \\
\$ 1,000 / \mathrm{kgHM} \text {, best represents the costs for a hypothetical facility with a smaller } \\
\text { front-end process, but similar sizing of subsequent separation processes as a } \\
\text { UOx plant operating at } 800 \mathrm{MT} / \text { year capacity. } \\
\text { Nameplate capacity is higher than operational capacity by at least } 0.33 \\
\text { (e.g., } 500 \text { MT nameplate capacity required to supply } 160 \mathrm{MT} \text { operational } \\
\text { capacity). Costs do not account for unused operational capacity. No learning } \\
\text { curve is applied due to the long periods (10-20 years) between facility } \\
\text { deployments. } \\
\text { Aqueous product and waste streams: } \\
\text { All-TRU product (goes to E3) } \\
\text { RU product (stored initially in E3, then used as makeup in F2/D2, excess goes } \\
\text { to K2) } \\
\text { CsSr stream and off-gas (goes to G4 then to decay storage) }\end{array}$ \\
\hline
\end{tabular}


Table 3-4. (continued).

\begin{tabular}{|c|c|}
\hline Modules & Nominal Assumptions \\
\hline & $\begin{array}{l}\text { I stream (goes to G4, then decay storage E4) } \\
\text { FP+Ln and Tc stream (goes to G1, then L2) } \\
\text { LLW stream from separation plant wastes are separately estimated as } \\
10 \mathrm{~m}^{3} / \mathrm{MTHM} \text {, and is included in the G3 module. The LLW stream is split into } \\
\text { three parts: solids } 97 \% \text {, liquids } 2 \% \text {, and resins } 1 \% \text {. } \\
\text { GTCC stream (ZrSS) is calculated as a VISION co-flow and goes to GTCC } \\
\text { intermediate depth disposal. }\end{array}$ \\
\hline $\begin{array}{l}\text { G1, G3, G4, J, E4, } \\
\text { M1 and L2 }\end{array}$ & All waste management and disposition modules same as in 1-Tier. \\
\hline Other & $\begin{array}{l}\text { All fuel cycle unit costs are assumed to include an imbedded component for } \\
\text { D\&D of the fuel cycle facility at the end of its life. This is normally handled by } \\
\text { an escrow fund maintained by the facility owner/operator. }\end{array}$ \\
\hline
\end{tabular}

\subsection{References for Section 3}

Analysis and Integration, 2008, "Dynamic Systems Analysis Report for Nuclear Fuel Recycle," GNEPSYSA-AI-SS-RT-2008-000264, December 2008.

Knecht, D., 1994, "Design Documentation: Krypton Encapsulation Preconceptual Design," Ralph M. Parsons Company (INEL Contract), INEL-94/0054, October 1994.

Shropshire, D., et al., 2008, “2008 Advanced Fuel Cycle Cost Basis,” INL/EXT-06-11536, March 2008. 


\section{REACTOR AND FUEL CYCLE COST UNCERTAINTY RANGES}

Table 4-1 defines the costs ranges for the fuel cycle front-end and back-end, recycling processes, and LWR and fast reactors. The associated low, nominal, high costs and units of measure are primarily based on the March 2008 "AFC Cost Basis" report (Shropshire 2008). Some subsequent analysis was performed to further define costs in specific areas of the analysis (e.g., waste conditioning, storage, and disposition). Details on the updated cost information are provided in Section 3. All cost estimates are in current year dollars (2008) and are intended to be a projection of costs 15+ years into the future and support comparative assessments of scenarios including growth. These costs do not support site-specific costs or specific business opportunities. The objective of the analysis is to provide a baseline system cost comparison between the open fuel cycle and closed fuel cycle systems and to understand their cost sensitivities and trade-offs.

Table 4-1. Cost ranges for fuel cycle and reactor cost modules.

\begin{tabular}{|c|c|c|c|c|}
\hline Variable & Low & Nominal & High & Units \\
\hline A - Natural Uranium Mining and Milling & 25 & 60 & 240 & $\$ / \mathrm{kg} \mathrm{U}$ \\
\hline B - Conversion Processes & 5 & 10 & 15 & $\$ / k g U$ \\
\hline C1 - Enrichment & 80 & 105 & 130 & \$ISWU \\
\hline D1-1 - LWR UO2 Fuel Fab & 200 & 240 & 300 & $\$ / \mathrm{kg} U$ \\
\hline D1-2 - LWR MF Fuel Fab & 1,000 & 1,950 & 4,000 & $\$ / \mathrm{kg} \mathrm{HM}$ \\
\hline K1 - Depleted Uranium Disposition & 5 & 10 & 50 & $\$ / \mathrm{kgU}$ \\
\hline E2 - Dry Storage (\$ normally included with reactor costs) & 100 & 120 & 300 & $\$ / \mathrm{kg} \mathrm{HM}$ \\
\hline I - Monitored Retrievable Storage & 94 & 96 & 116 & $\$ / \mathrm{kg} \mathrm{HM}$ \\
\hline L1 - Geologic Repository (SNF) & 400 & 1,000 & 1,600 & $\$ / \mathrm{kg} \mathrm{HM}$ \\
\hline L2-1 - Geologic Repository (HLW FPs+Ln+Tc) & 2,500 & 10,000 & 12,500 & $\$ / k g$ FP \\
\hline L2-2 - Geologic Repository (activated hulls) & 400 & 1,000 & 1,600 & $\$ / \mathrm{kg}$ metal \\
\hline M1 GTCC Intermediate Depth Disposal (GTCC lodine+hulls) & 70,000 & 100,000 & 440,000 & $\$ / \mathrm{m} 3 \mathrm{GTCC}$ \\
\hline F1-1 UREX+1A Aqueous Separation & 500 & 1,000 & 1,500 & $\$ / \mathrm{kg} \mathrm{HM}$ \\
\hline F1+ (HYBRID) UREX+3, Product Conditioning, 15 years storage (2-Tier) & 700 & 1,320 & 2,080 & $\$ / \mathrm{kg} \mathrm{HM}$ \\
\hline F2/D2 - Reprocessing - Electrochemical \& Remote Fuel Fab & 2,500 & 5,000 & 7,500 & $\$ / \mathrm{kg} \mathrm{HM}$ \\
\hline E3-1 - Recycled U/TRU Product Storage & 7,000 & 10,000 & 13,000 & $\$ / \mathrm{kg}$ TRU \\
\hline E3-2 - Recycled U/Pu Product Storage & 3,500 & 5,000 & 6,500 & $\$ / \mathrm{kg} \mathrm{Pu}$ \\
\hline G3-1 - LLW Conditioning, Storage, Packaging (solids) & 400 & 500 & 1,000 & $\$ / m 3$ solids \\
\hline G3-2 - LLW Conditioning, Storage, Packaging (liquids) & 3,300 & 11,000 & 22,000 & $\$ / m 3$ liquids \\
\hline G3-3 - LLW Conditioning, Storage, Packaging (resins) & 81,000 & 90,000 & 99,000 & $\$ / \mathrm{m} 3$ resins \\
\hline$J$ - Near Surface Disposal & 450 & 1,250 & 2,500 & $\$ / m 3$ LLW \\
\hline G4-1A - Aqueous LLW-GTCC Offgas absorber $(\mathrm{H} 3, \mathrm{Kr}, \mathrm{Xe})$ & 8,000 & 11,200 & 15,000 & $\$ / m 3$ gas \\
\hline G4-2A - Aqueous GTCC Ceramic Conditioning (Cs/Sr) & 5,700 & 7,800 & 12,000 & $\$ / \mathrm{kg} \mathrm{Cs} / \mathrm{Sr}$ \\
\hline G4-1E - EChem LLW-GTCC Offgas absorber (H3, Kr, Xe) & 8,000 & 11,200 & 15,000 & $\$ / m 3$ gas \\
\hline G4-3E - Echem GTCC GBZ Conditioning (Cs/Sr+l) & 5,700 & 7,800 & 12,000 & $\$ / \mathrm{kg} \mathrm{Cs} / \mathrm{Sr}+1$ \\
\hline E4 - Managed Decay Storage (Cs/Sr) & 10,000 & 22,500 & 35,000 & $\$ / \mathrm{kg} \mathrm{Cs} / \mathrm{Sr}$ \\
\hline G4-4A - Aqueous LLW-GTCC Ag Zeolite (lodine) & 50,000 & 67,000 & 80,000 & $\$ / m 3$ lodine \\
\hline G4-5A - Aqueous GTCC Metal Alloy Conditioning (ZrSS) & 200 & 540 & 1,800 & $\$ / \mathrm{kg}$ metal \\
\hline G1-1A - Aqueous HLW Conditioning, Storage, Packaging (FP+Ln) & 1,800 & 2,000 & 2,700 & $\$ / k g$ FP \\
\hline G1-2A - Aqueous Metal Alloy (Tc) & 18,000 & 25,000 & 30,000 & $\$ / \mathrm{kg} \mathrm{Tc}$ \\
\hline G1-2E - EChem HLW Metal Alloy Conditioning (ZrSS+Tc) & 200 & 540 & 1,800 & $\$ / \mathrm{kg}$ metal \\
\hline G2 - UOX or (UOX/MOX) Conditioning \& Packaging & 50 & 100 & 130 & $\$ / \mathrm{kg} \mathrm{HM}$ \\
\hline G5 - CH-TRU Conditioning, Storage, and Packaging & 69,000 & 70,000 & 90,000 & $\$ / m 3$ TRU \\
\hline K2 - RU Disposition from Aqueous Reprocessing & 6 & 12 & 30 & $\$ / k g R U$ \\
\hline K3 - RU Conditioning for Electrochemical Reprocessing & 75 & 93 & 150 & $\$ / \mathrm{kg} \mathrm{RU}$ \\
\hline R1 - Thermal LWR Reactor (Overnight Capital) & 1,800 & 2,300 & 3,500 & $\$ / k W(e)$ \\
\hline R2 - Advanced Recycling Reactor (Overnight Capital) & 1,800 & 2,900 & 5,000 & $\$ / k W(e)$ \\
\hline r - Real Discount Rate & 5.0 & 7.5 & 10.0 & $\%$ \\
\hline c - Construction Time & 3.5 & 4.0 & 5.0 & years \\
\hline R1 - Thermal LWR Reactor (O\&M Fixed) & 55 & 64 & 75 & $\$ / k W e-y r$ \\
\hline R2 - Advanced Recycling Reactor (O\&M Fixed) & 60 & 68 & 80 & $\$ / k W e-y r$ \\
\hline R1 - Thermal LWR Reactor (O\&M Variable) & 0.8 & 1.8 & 2.5 & mills/kWh \\
\hline R2 - Advanced Recycling Reactor (O\&M Variable) & 1.0 & 2.0 & 2.7 & mills/kWh \\
\hline
\end{tabular}

Cost comparisons between Table 4-1 and other cost studies are provided in Section 10. 


\section{AFCI NOMINAL CASE ECONOMIC ANALYSIS}

In this economic analysis, a variety of different cost comparisons were analyzed using different economic tools (static and dynamic). The initial comparisons between the scenarios are provided based on the simplest static equilibrium analysis using G4 ECONS spreadsheet. Using the cost data defined in Section 4, some basic cost comparisons were made between the systems. The first comparison shows the cost composition of the three systems and the range of total costs based on the low, nominal, and high module cost distributions. In the Section 6, more dynamic cost analysis is explored using the VISION model. Attachment A provides the static equilibrium analysis spreadsheets that show the material flows and the related costs for the once-through, 1-Tier, and 2-Tier systems.

\subsection{Cost Composition of the Three Systems}

The static costs for the systems described in Section 2 are broken into their five primary cost components (front-end, back-end, recycling, reactor capital, and reactor O\&M) as color coded from top to bottom in Table 4-1. The pie chart cost breakdown for the once-through fuel cycle is shown in Figure 5-1, 1-Tier in Figure 5-2, and 2-Tier in Figure 5-3. In the once-through system, the largest cost components are the LWR capital and operations and maintenance (O\&M), which comprise $85 \%$ of the total costs. The front-end fuel cycle and back-end (spent fuel management and geologic disposal) fuel cycle costs covers the remaining $15 \%$, and there are no recycling costs.

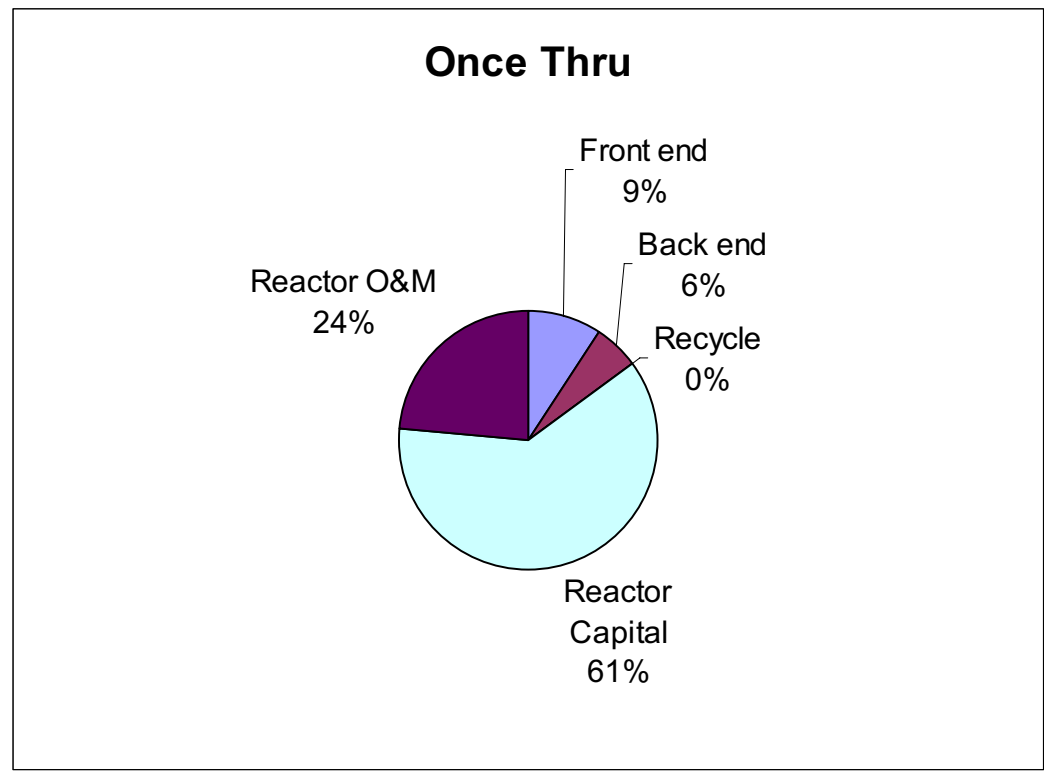

Figure 5-1. Once-through cost breakdown of the total cost of electricity (TCOE).

For the 1-Tier system, the largest cost components are reactor capital and O\&M, which encompasses $82 \%$ of the total costs. These costs include both LWRs and FRs comprising the symbiotic system. The fuel cycle front-end and back-end (geologic disposal of wastes) costs are a lower percentage than for the once through, and the recycle costs (head end, separations and waste treatment/packaging) comprise $10 \%$ of the remaining costs. 


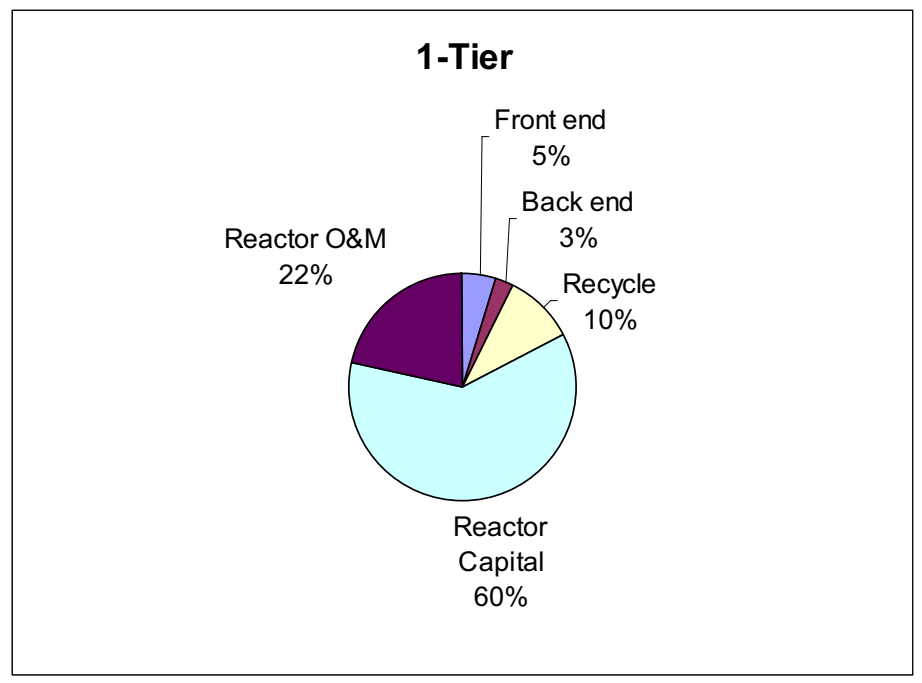

Figure 5-2. 1-Tier cost breakdown of the TCOE.

For the 2-Tier system, the largest cost components are reactor capital and O\&M, which again consist of $82 \%$ of the total costs, including LWRs and FRs. The fuel cycle front-end and back-end costs are again a lower percentage than for the once-through cycle, and the recycle costs comprise $10 \%$ of the remaining costs.

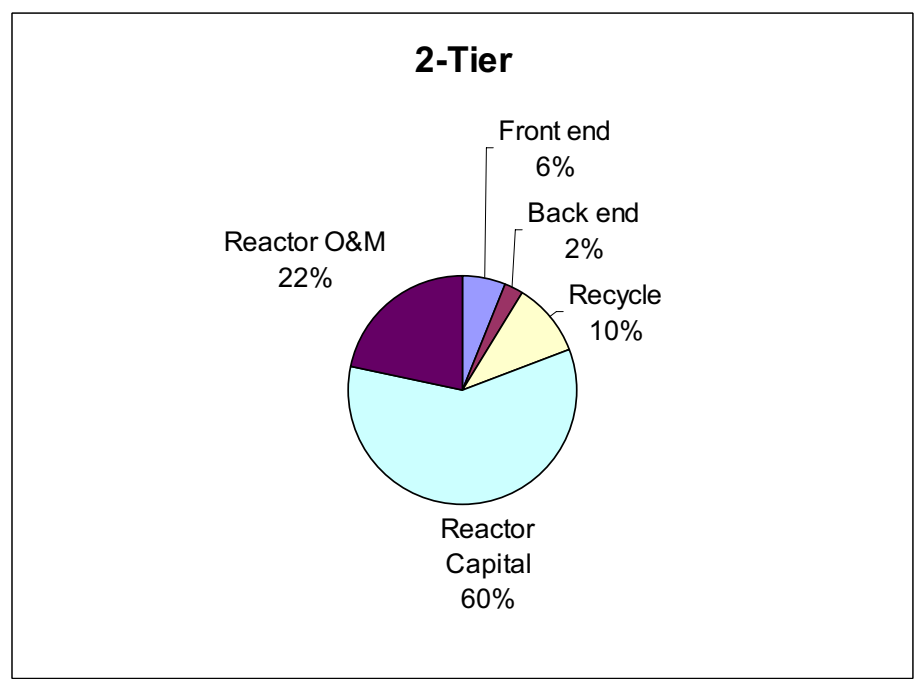

Figure 5-3. 2-Tier cost breakdown of the TCOE.

The majority of the costs for all three of these systems are in the reactor capital and O\&M. These costs make up $82-85 \%$ of the total cost of electricity of the systems. The non-reactor (fuel cycle) cost for the once-through are less expensive at $15 \%$ of the TCOE, as compared to $18 \%$ for the 1-Tier and 2-Tier systems. 


\subsection{System Static Cost Comparisons using G4 ECONS at Low, Nominal, and High Values}

The cost ranges described in Section 4 were used to determine the extreme points and the nominal costs values for each of the three scenarios. Figure 5-4 presents the cost ranges for the total cost of electricity, and Figure 5-5 shows the range of fuel cycle costs for the three systems.

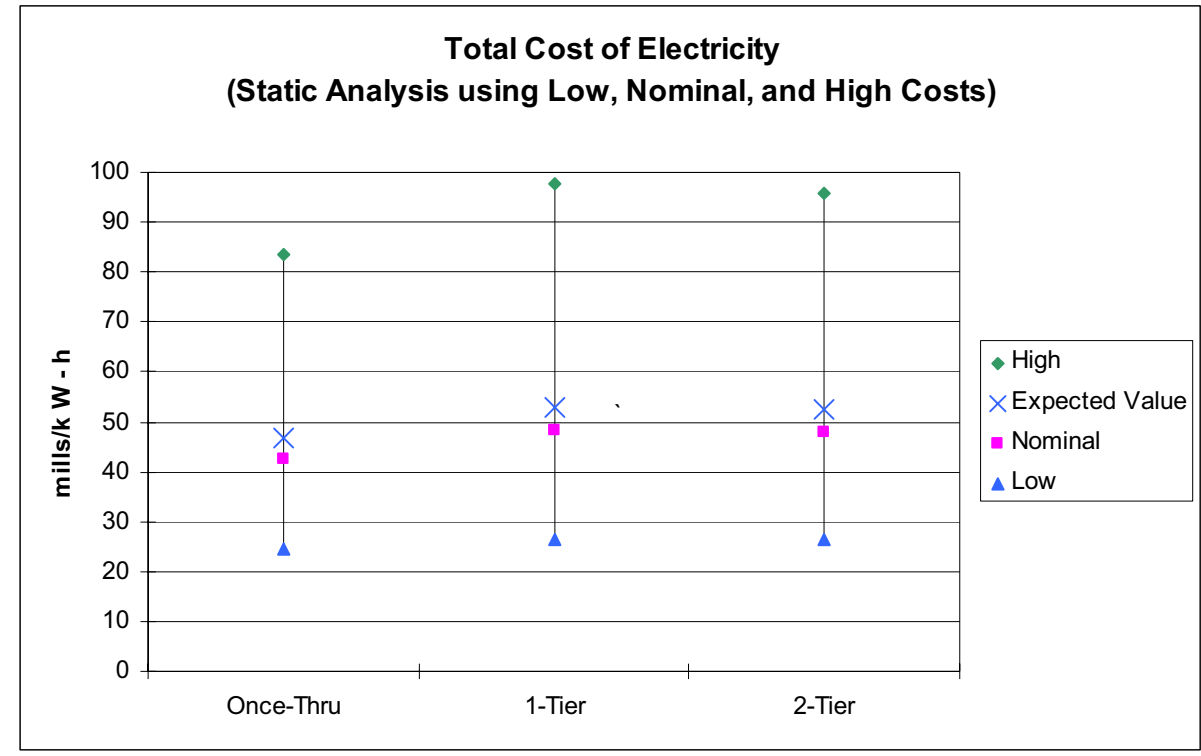

Figure 5-4. Static TCOE comparisons of strategies using low, nominal, and high values.

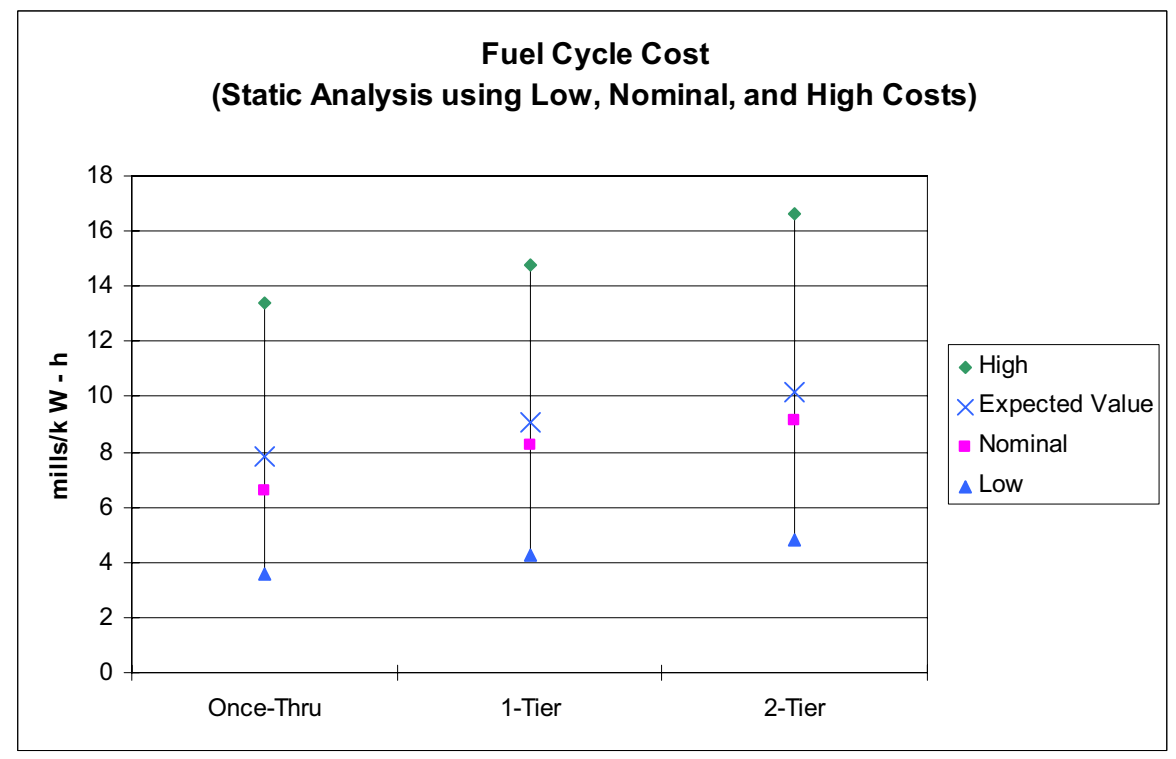

Figure 5-5. Static FCC comparisons of strategies using low, nominal, and high values. 
The ranges reflect the cost points for the three scenarios based on all cost modules values set to their low, nominal, and high values, respectively. This comparison provides the full extent of the uncertainty of each system. The nominal costs shown on each of the charts are based on the cost module values where a maximum occurs in their frequency distribution (i.e., mode of the distribution). The expected value (EV) costs (similar to a weighted statistical average), are higher than the nominal costs in all cases due to the skewing of the cost distributions toward the high end of the distribution. The cost ranges shown in these charts provide the boundary conditions for each of the systems. Section 6 shows the expected distribution of costs within these ranges. 


\section{AFCI ECONOMIC SENSITIVITY ANALYSIS}

The AFCI economic sensitivity analysis was developed using the DPL tool to evaluate cost distributions based on the assigned probability conditions. In this analysis, two types of simulations were performed:

1. Monte Carlo simulation with a triangular distribution, where specific data points were assigned for the low (minimum), high (maximum), and nominal (mode) points for each module (input variable).

2. Discrete simulations with a three-point normal distribution generated from probabilities for the low, nominal, and high costs. This type of simulation was used to prepare the cost variable uncertainty analysis in Section 6.2.

\subsection{Uncertainties Due to Cost Parameters}

Using the Monte Carlo simulation, the cost distributions were developed for the three scenarios, as shown in Figures 6-1, 6-2, and 6-3. The distributions are described as costs occurring over a range of probability. The values on the $\mathrm{Y}$-axis indicate the relative probability of the cost occurring, which is a function of the number of times the distribution is sampled by the model. The probability values are not important; however, the relative height of the distributions is a function of the overall range (breadth) of the cost uncertainty. The higher the cost distribution, the less cost uncertainty and vice versa. The vertical lines extending through each of the three systems represent their weighted statistical averages (i.e., expected values $[\mathrm{EVs}]$ ) for the distribution. ${ }^{\mathrm{f}}$ The source of the cost uncertainties are described in Section 6.2.

- Figure 6-1 shows that the equilibrium total cost of electricity (TCOE) ranges from $\sim 30$ to $80 \mathrm{mills} / \mathrm{kW} \cdot \mathrm{h}$. The distributions are very broad, with the once-through overlapped by the 1-Tier and 2-Tier distributions by greater than 90\%. The 1-Tier and 2-Tier distributions are almost entirely overlapped with very similar sizes of distributions. The cost uncertainty (or range of the distribution) for the once-through is almost as wide as the closed fuel recycling systems.

- Figure 6-2 shows the cost distributions for the fuel cycle ranging from $\sim 5$ to $13 \mathrm{mills} / \mathrm{kW} \cdot \mathrm{h}$. The oncethrough cycle has the lowest costs, then the 1-Tier cycle with costs about $1.5 \mathrm{mill} / \mathrm{kwh}$ higher than the once-through, and the 2-Tier cycle with the highest fuel cycle costs. The closed cycle costs are higher than the once-through due to the additional costs for recycling the fuel. The recycling costs account for about $10 \%$ of the TCOE, as shown on the pie charts in Section 5.1. Recycling results in lower front-end and back-end costs than the once-through cycle due to lower uranium and enrichment requirements and less waste requiring a geologic repository. The 2-Tier cycle shows higher fuel cycle costs than the 1-Tier due primarily to the three stage separation process so that all the actinides are eventually recycled, as described in Section 2.1.3.

- Figure 6-3 shows the cost distribution for the total reactor costs ranging from $\sim 23$ to $70 \mathrm{mills} / \mathrm{kW} \cdot \mathrm{h}$. The distributions are broad and overlapping, as described for the TCOE, due to large variable cost uncertainties further described in Section 6.2.

As mentioned previously, a conservative approach was used to define key parameters in this study. In actual practice, with additional design, development, and operational experience, streamlined processes and technologies would be deployed to increase efficiencies and minimize costs. Alternative strategies incorporating new technologies (e.g., thermal recycle using inert matrix fuels) could reduce costs and the cost uncertainties associated with closed fuel cycles.

f. Expected values are different from the nominal values used in the cost variable uncertainty analysis in Section 6.2. The expected values are determined through statistical analysis using DPL or VISION.ECON and are based on the distributions for each of the cost modules. Typically, the EVs represent higher costs than the nominal values due to the skewedness in the module triangular distributions toward higher costs. 


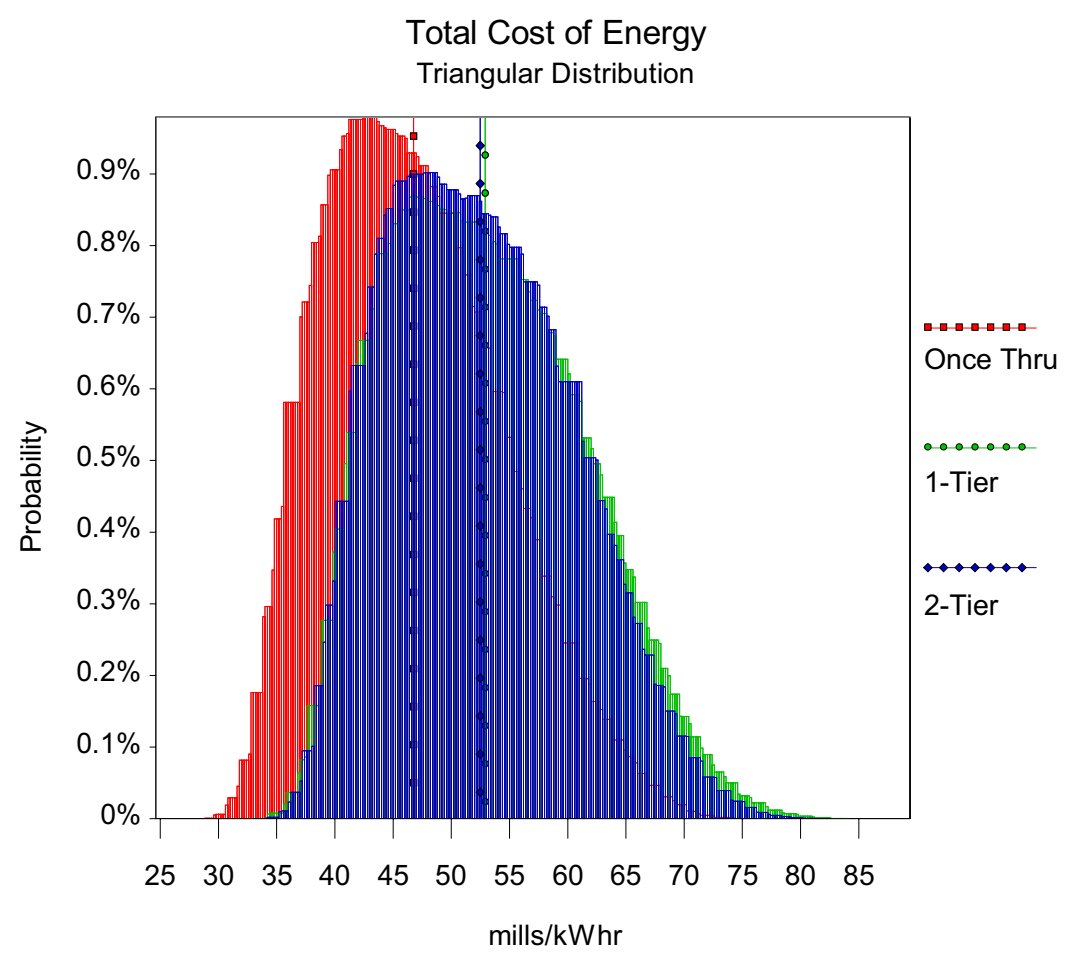

Figure 6-1. Total cost of energy using a triangular distribution.

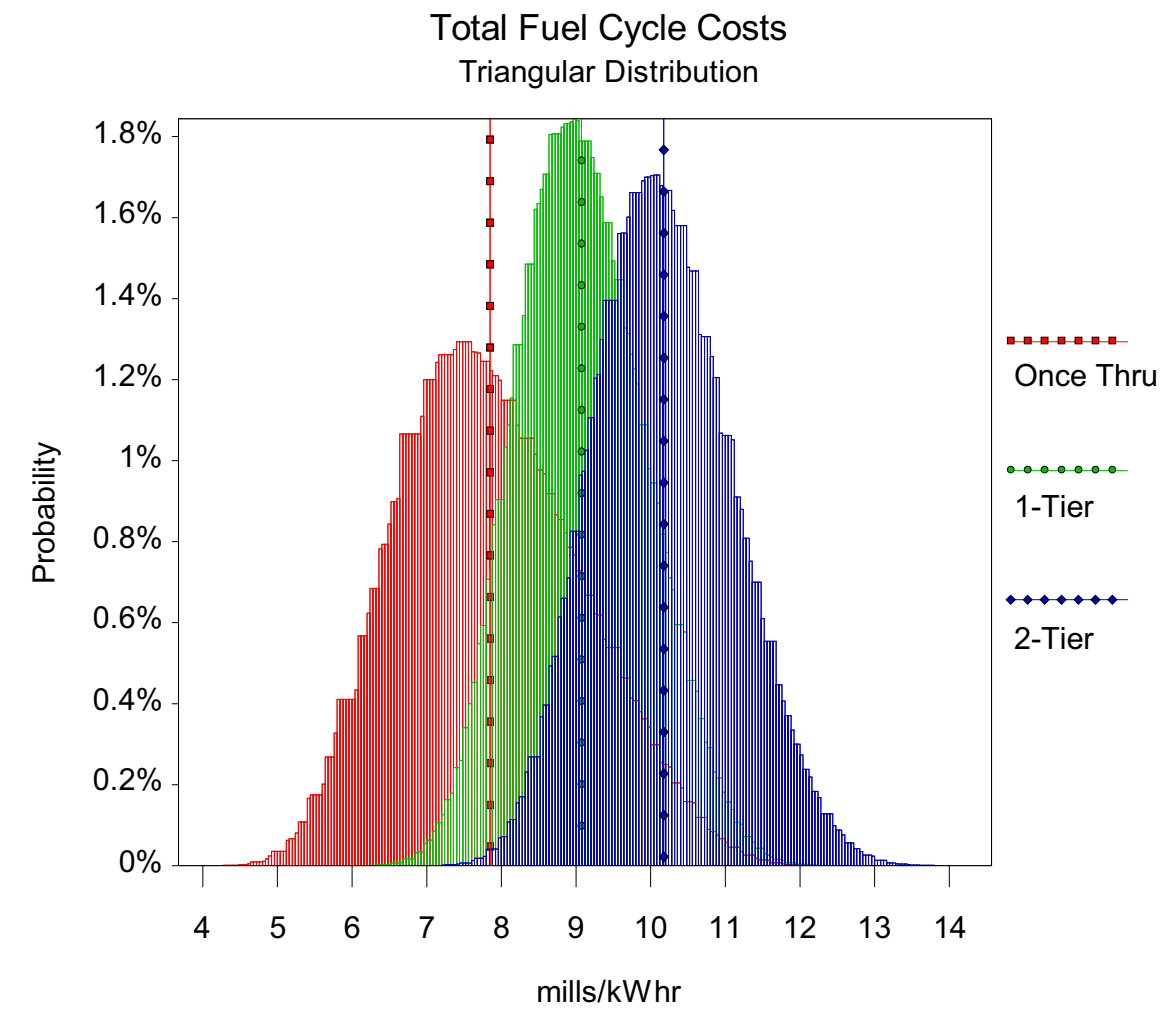

Figure 6-2. Total fuel cycle cost using a triangular distribution. 


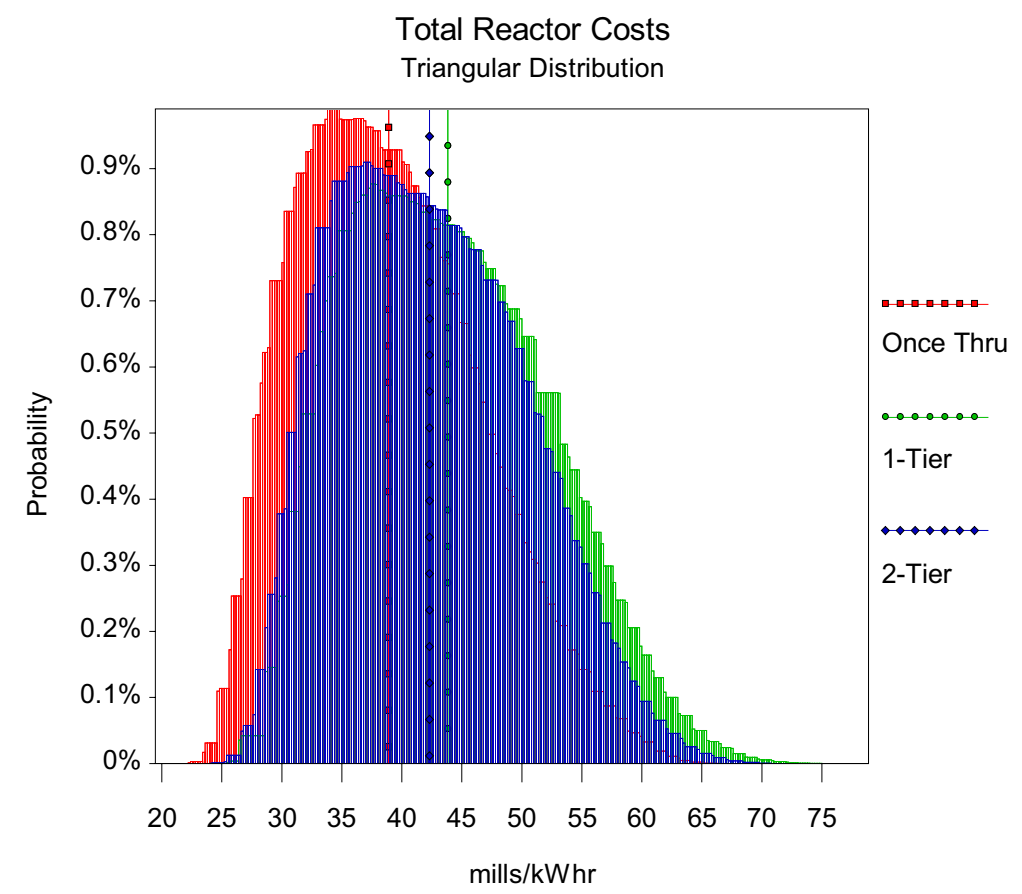

Figure 6-3. Total reactor cost using a triangular distribution.

\subsection{Cost Variable Uncertainty}

This section describes the costs sensitivity due to specific variables. The Value Tornado Diagrams produced by the DPL software are used to display the change in costs as each variable is changed from the low to high-value setting while all other variables are held at the nominal settings. The specific low, nominal, and high costs for each variable and the resulting impact on the cost is shown below each of the uncertainty bars. The bars are sorted so the variables with the greatest potential impact for increasing or decreasing the cost are located at the top of the diagram.

The DPL diagram in Figure 6-4 shows the key variables for the once-through strategy that are most likely to increase or decrease the cost. The relative cost influence is shown by the width of the bar on the chart. The value shown above the center line is the cost based on all modules set to their nominal cost. The ends of each bar show the low or high value of the variable and their impact on the expected costs. For example, on the top bar labeled "R1 ALWR ON Capital" a value of 1800/36.802 is at the left (low end) and 3500/55.954 is at the right (high cost) end. This means that the R1 ALWR capital cost ranges from $\$ 1,800-\$ 3,500 / \mathrm{kWe}$, and the impact on the TCOE at those cost points range from 36.802-55.954-mills/kWhr, respectively.

In Figures 6-5, 6-7, 6-8, 6-9, 6-10, and 6-11 the Tornado diagram variable uncertainties (displayed as vertical bar charts) show more than one color (i.e., green plus magenta or green plus blue). The DPL software generates these two-tone bars whenever the variable has a high or low cost value where there is the potential for another strategy to become the optimal lowest-cost policy. ${ }^{\mathrm{g}}$

g. The preferred policy is the strategy (i.e., once-through, 1-Tier, 2-Tier) with the least cost. DPL acts as an optimizer to seek out new strategies that minimize the cost (TCOE, fuel cycle, or reactor). When a new strategy (e.g., 2-Tier) is close in cost to the current preferred policy (e.g., once-through), then DPL identifies the variables that have the potential to switch the optimal policy to the new strategy. 
The specific variable cost value where the optimal policy changes cannot be determined by the location of the color bar. However, DPL does have the capability to determine these cost points. In Section 6.4, we further examine these types of trade-offs, in the economic trade-off parameter space analysis.

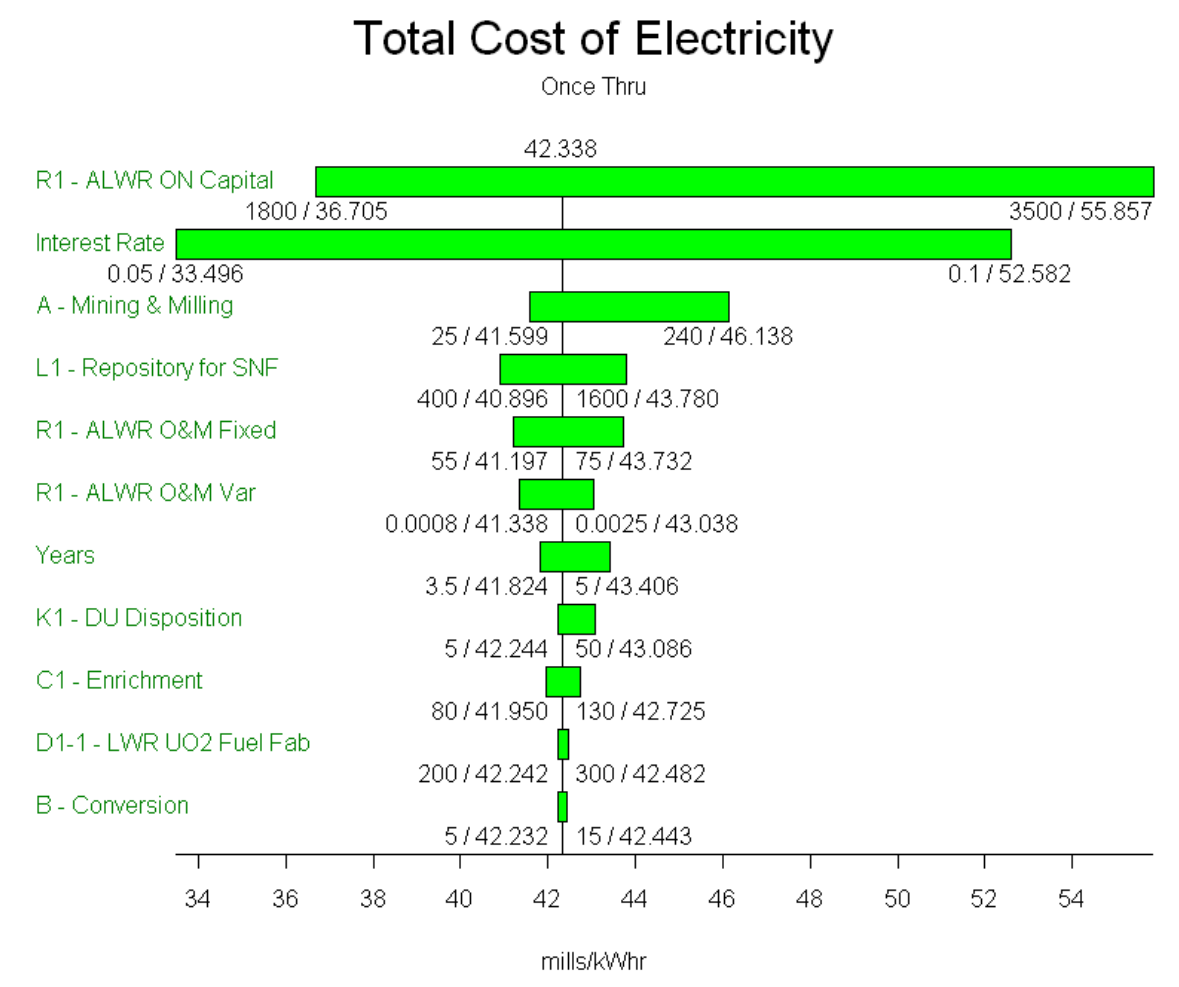

Figure 6-4. Cost variable uncertainty analysis of the once-through TCOE.

In Figure 6-4, the DPL tornado diagram shows the relative influence from each of the variables on the TCOE for the once-through cycle. The specific impacts are further described in Figures 6-5 and 6-6.

\section{Reactor Cost Uncertainties}

Once Thru

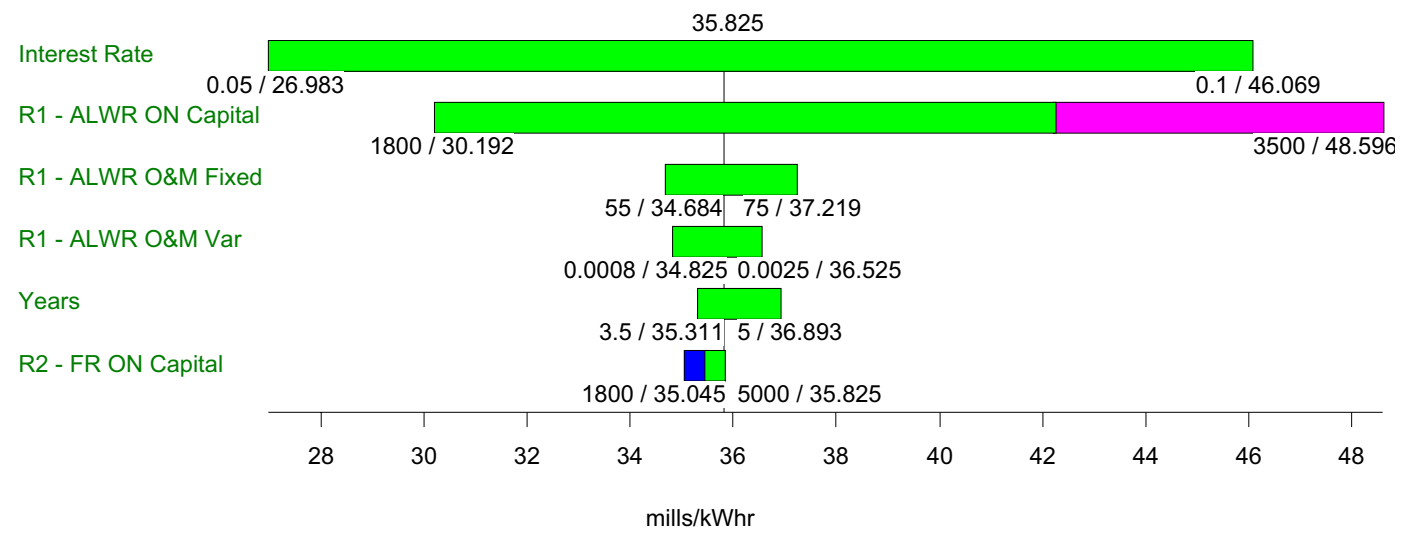

Figure 6-5. Cost variable uncertainty analysis for the once-through reactor costs. 
The DPL tornado diagram in Figure 6-5 shows only the reactor variables for the once-through strategy, where the largest cost uncertainties are in the R1-ALWR Capital and the Interest Rate variables. An assessment of the variables that have the most influence on the costs are described as follows:

- The cost range of Module R1 has the potential to swing the costs -6 mill $/ \mathrm{kW} \cdot \mathrm{h}$ to $13 \mathrm{mill} / \mathrm{kW} \cdot \mathrm{h}$

- The interest rate also has the potential to swing the costs by $-9 \mathrm{mill} / \mathrm{kW} \cdot \mathrm{h}$ to $10 \mathrm{mill} / \mathrm{kW} \cdot \mathrm{h}$

- The reactor O\&M has fixed and variable components with a range of costs of $+/-2 \mathrm{mills} / \mathrm{kW} \cdot \mathrm{h}$

- The reactor construction duration has a potential range of impact of -0.5 to $1.0 \mathrm{mill} / \mathrm{kW} \cdot \mathrm{h}$ over the range of 3.5 to 5 years, respectively.

This diagram also identifies the variables (i.e., R1 ALWR ON Capital, R2- FR ON Capital) that have the potential to switch the lowest-cost strategy (currently once-through) to another strategy as the lowest-cost policy. The diagram shows that at some cost above the nominal cost value of the ALWR reactor, or alternatively at some cost below the nominal FR capital cost, the policy would switch from the once-through to one of the closed fuel strategies. The chart does not specifically define the cost point where the optimal cycle changes. In Section 6.4, we further examine these types of trade-offs, in the economic trade-off parameter space analysis.

\section{Fuel Cycle Cost Uncertainties}

Once Thru

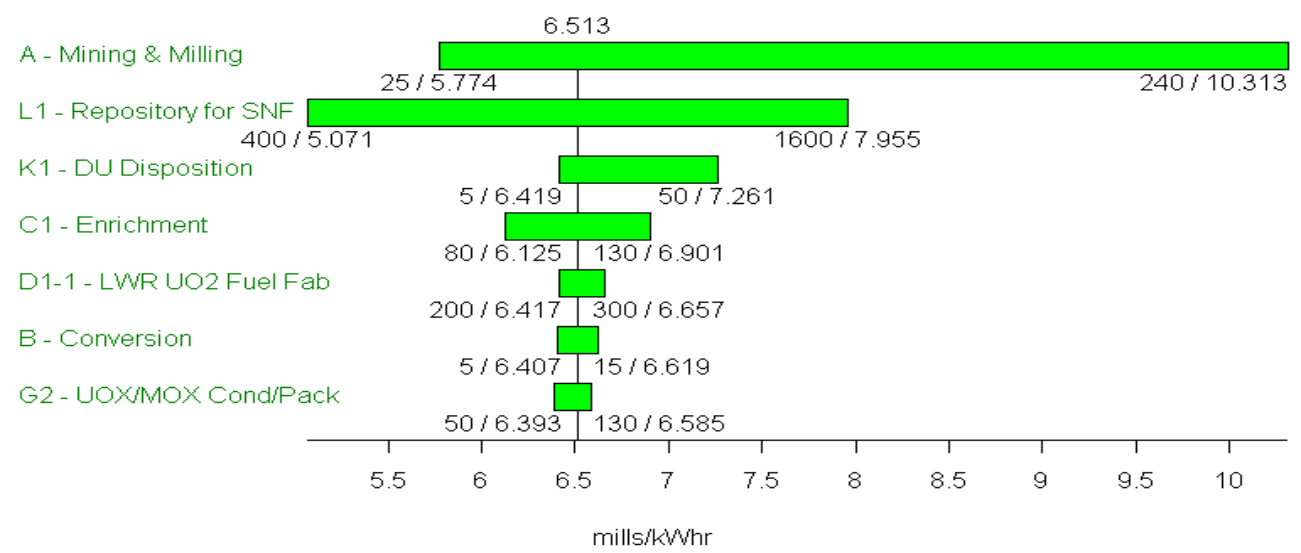

Figure 6-6. Cost variable uncertainty analysis of the once-through fuel cycle cost.

The Value Tornado Diagram in Figure 6-6 shows the fuel cycle variables for the once-through strategy, where the largest cost uncertainties are in the A-Mining and Milling (uranium) and the L1-Repository for SNF variables. Assessments of the variables that have the most influence on the costs are described as follows:

- The cost of uranium mining and milling has the potential to increase costs by 4 mills $/ \mathrm{kW} \cdot \mathrm{h}$ over the nominal costs. There is also a $0.8 \mathrm{mill} / \mathrm{kW} \cdot \mathrm{h}$ cost reduction if uranium costs returns to historical cost levels around $\$ 25 / \mathrm{kg} \mathrm{U}$.

- The repository costs for SNF could fall from the nominal cost by $-1.5 \mathrm{mills} / \mathrm{kW} \cdot \mathrm{h}$ if the future costs are held to the current fees defined in the NWPA. More likely however, is the likelihood that costs would increase over the current levels based on the escalation in the geologic repository life cycle cost estimates. The high value costs equivalents would increase the nominal costs by $1.5 \mathrm{mills} / \mathrm{kW} \cdot \mathrm{h}$.

- Depleted uranium disposition has the potential to increase costs by $0.8 \mathrm{mills} / \mathrm{kW} \cdot \mathrm{h}$. 
- Enrichment and conversion often move in price as a function of the costs for uranium. If future uranium costs increase, then together the enrichment and conversion costs could rise $0.5 \mathrm{mills} / \mathrm{kW} \cdot \mathrm{h}$.

- Cost uncertainties from $\mathrm{LWR} \mathrm{UO}_{2}$ fuel fab and UOx conditioning/packaging are expected to have a small impact on the fuel cycle costs.

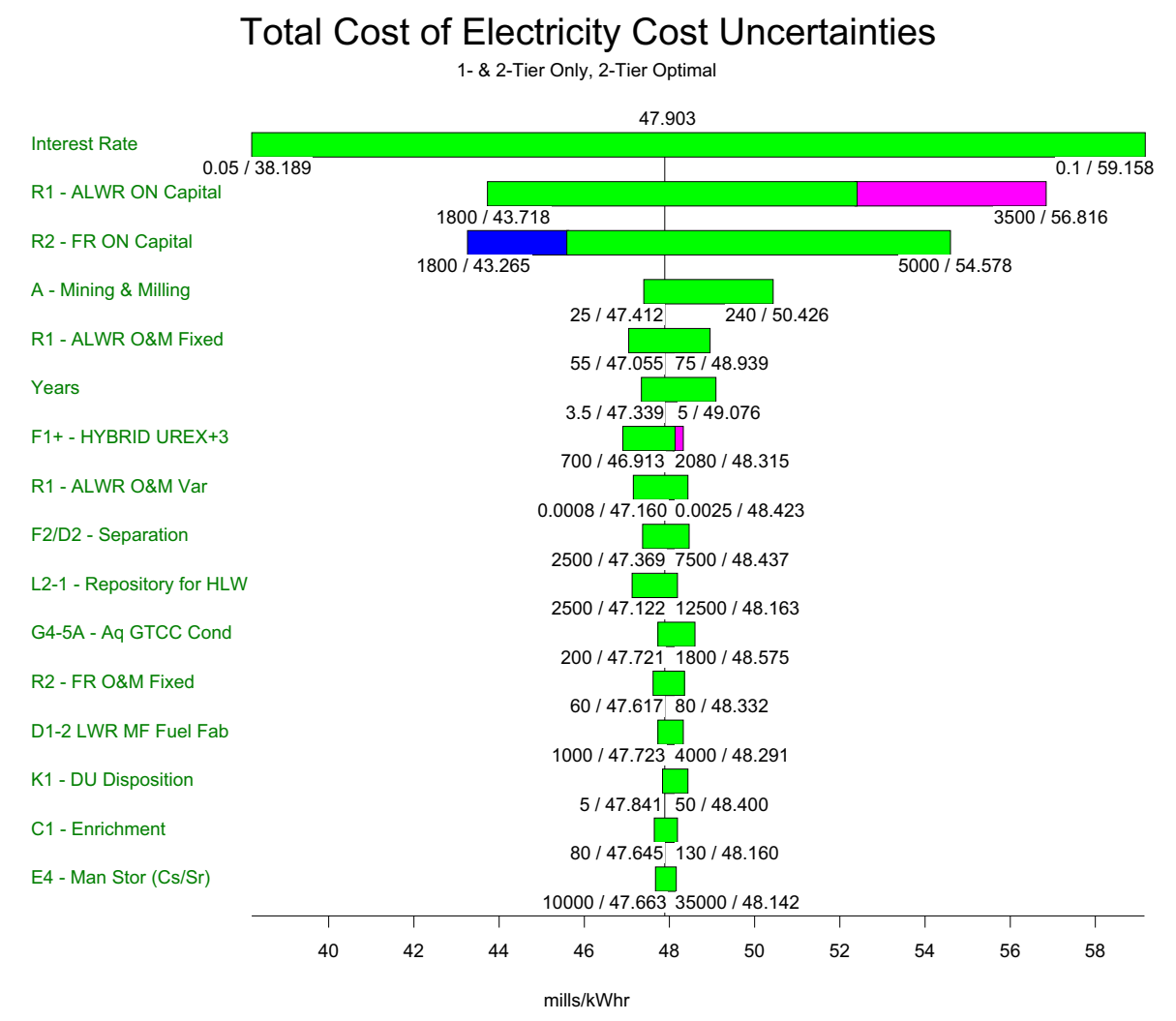

Figure 6-7. Cost variable uncertainty analysis for TCOE of the 2-Tier.

The DPL Tornado Diagram in Figure 6-7 shows the key variables for the 2-Tier strategy that are most likely to increase or decrease the costs. The specific impacts are described in Figures 6-8 and 6-9.

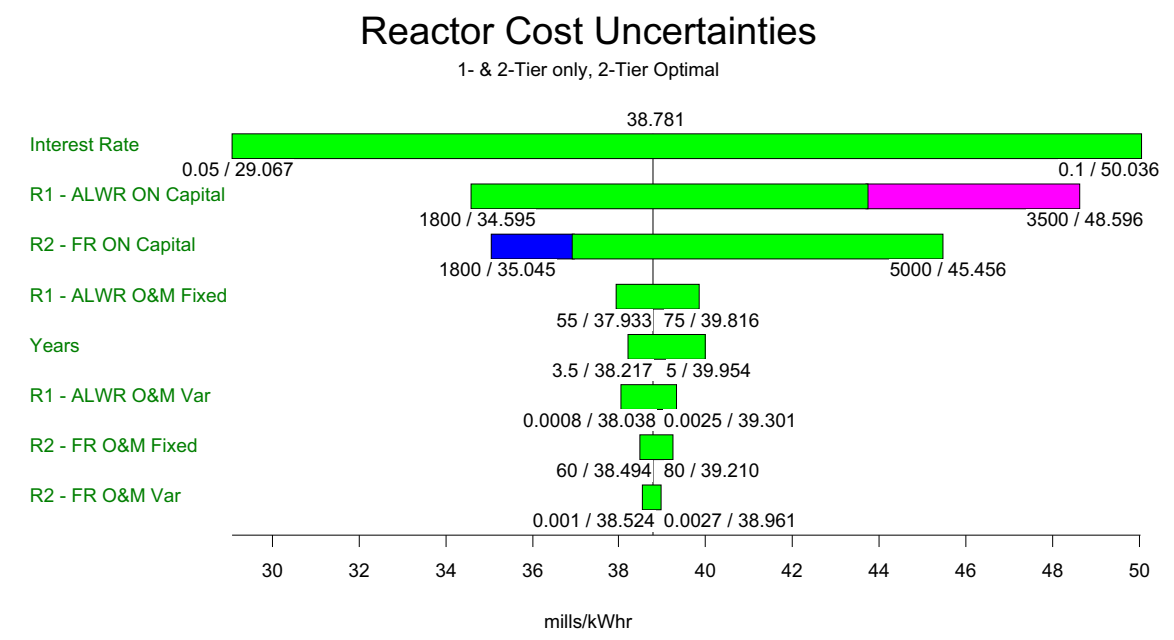

Figure 6-8. Cost variable uncertainty analysis for the 2-Tier reactor costs. 
The DPL tornado diagram in Figure 6-8 shows the only the reactor cost variables for the 2-Tier strategy, where the largest cost uncertainties are in the Interest Rates, R1-ALWR overnight capital, and R2-FR overnight capital variables. Assessments of the variables that have the most influence on the costs are described as follows:

- The interest rate also has the potential to swing the costs by $-10 \mathrm{mill} / \mathrm{kW} \cdot \mathrm{h}$ to $11 \mathrm{mill} / \mathrm{kW} \cdot \mathrm{h}$.

- The ALWR capital has the potential to swing the costs -4 mills $/ \mathrm{kW} \cdot \mathrm{h}$ to $10 \mathrm{mills} / \mathrm{kW} \cdot \mathrm{h}$. The ALWR O\&M can also affect the costs $+/-1 \mathrm{mill} / \mathrm{kW} \cdot \mathrm{h}$.

- The R2 fast reactor capital has the potential to swing the costs -4 mill $/ \mathrm{kW} \cdot \mathrm{h}$ to $7 \mathrm{mill} / \mathrm{kW} \cdot \mathrm{h}$. The FR O\&M can also affect the costs $+/-0.5 \mathrm{mills} / \mathrm{kW} \cdot \mathrm{h}$.

- The reactor construction duration has a potential range of impact of -0.5 to $1.2 \mathrm{mill} / \mathrm{kW} \cdot \mathrm{h}$ over the range of 3.5 to 5 years, respectively.

- The FR O\&M has fixed and variable components with a range of costs of $+/-0.5 \mathrm{mill} / \mathrm{kW} \cdot \mathrm{h}$.

The ALWR capital and FR capital variables indicate a shift in color from green to magenta (or green to blue) when moving from the nominal cost (center line) toward the extremes low or high cost values. This means that the variables have the potential at some unknown value to make the 1-Tier or 2-Tier TCOE less than the once-through (i.e., the optimal policy). The chart does not specifically define the cost point where the optimal cycle changes. In Section 6.4, we further examine these types of trade-offs, in the economic trade-off parameter space analysis.

\section{Fuel Cycle Cost Uncertainties}

1- \& 2-Tier Only, 2-Tier Optimal (Forced)

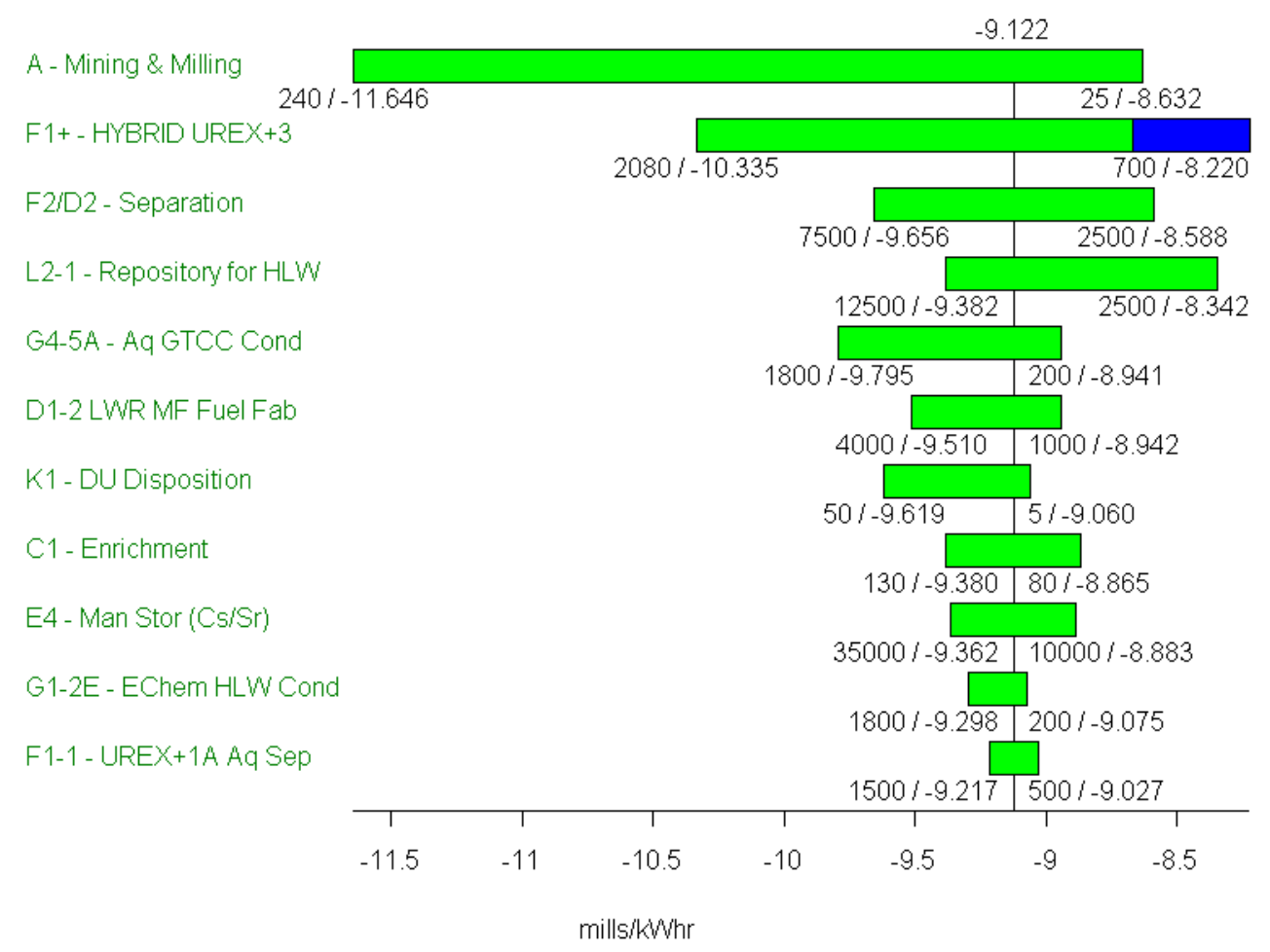

Figure 6-9. Cost variable uncertainty analysis of the 2-Tier fuel cycle cost. 
The Tornado diagram in Figure 6-9 shows the fuel cycle cost variables for the 2-Tier strategy, where the largest cost uncertainties are A-Mining and Milling (uranium), the F1+ Hybrid aqueous separation, and the F2/D2 EChem separation. (Notice that the costs are negative on this diagram, this was done in order to be able to display the variable uncertainty. The strategy must be forced to be the optimal policy in order to display the tornado diagram). Assessments of the variables that have the most influence on the costs are described as follows:

- The cost of uranium mining and milling has the potential to increase costs by $2.5 \mathrm{mills} / \mathrm{kW} \cdot \mathrm{h}$ over the nominal costs. There is also a $0.5 \mathrm{mill} / \mathrm{kW} \cdot \mathrm{h}$ cost reduction if uranium costs returns to historical cost levels around $\$ 25 / \mathrm{kg} \mathrm{U}$.

- The UOx aqueous separation, as described in Section 2.1.3, includes an initial separation of UPu, conditioning of the remaining materials, storage for 15 years, and a final separation of the minor actinides. If these separation functions could be completed for $\$ 700 / \mathrm{kg} \mathrm{HM}$, a fuel cycle saving of $0.9 \mathrm{mill} / \mathrm{kW} \cdot \mathrm{h}$ would result. A high cost for this function would result in an increase of $1.2 \mathrm{mills} / \mathrm{kW} \cdot \mathrm{h}$.

- EChem separation ranges $+/-0.5 \mathrm{mills} / \mathrm{kW} \cdot \mathrm{h}$ from nominal costs.

- The HLW repository costs could decrease costs by $0.5 \mathrm{mill} / \mathrm{kW} \cdot \mathrm{h}$ at the low range, and would increase costs by $0.2 \mathrm{mill} / \mathrm{kW} \cdot \mathrm{h}$ at the high end of the cost range. This cost is a function of the repository cost and the HLW loading.

- Aqueous GTCC conditioning could decrease costs by $0.2 \mathrm{mill} / \mathrm{kW} \cdot \mathrm{h}$ at the low range, and would increase costs by $0.7 \mathrm{mill} / \mathrm{kW} \cdot \mathrm{h}$ at the high end of the cost range.

- MOx fuel fabrication could decrease costs by $0.2 \mathrm{mill} / \mathrm{kW} \cdot \mathrm{h}$ at the low range, and would increase costs by $0.4 \mathrm{mill} / \mathrm{kW} \cdot \mathrm{h}$ at the high end of the cost range.

- Cesium/Strontium decay storage has a cost uncertainty of $+/-0.2 \mathrm{mills} / \mathrm{kW} \cdot \mathrm{h}$. In a managed decay scenario these costs in addition to the cost differentials for aqueous separations, waste conditioning, and waste disposal would need to be considered. See Section 6.4 for further analysis.

- A number of additional variables (e.g., DU disposition, enrichment) have minimal cost uncertainty (i.e., total of less than $0.5 \mathrm{mills} / \mathrm{kW} \cdot \mathrm{h}$ ).

The F1+ aqueous separation (Hybrid) variable indicates a shift in color from green to blue when moving from the nominal cost (center line) toward the high cost values. This means that the variable has the potential at some value to make the 2-Tier cost less than the 1-Tier (i.e., the optimal policy). The chart does not specifically define the cost point where the optimal cycle changes. In Section 6.4, we further examine these types of trade-offs, in the economic trade-off parameter space analysis. 


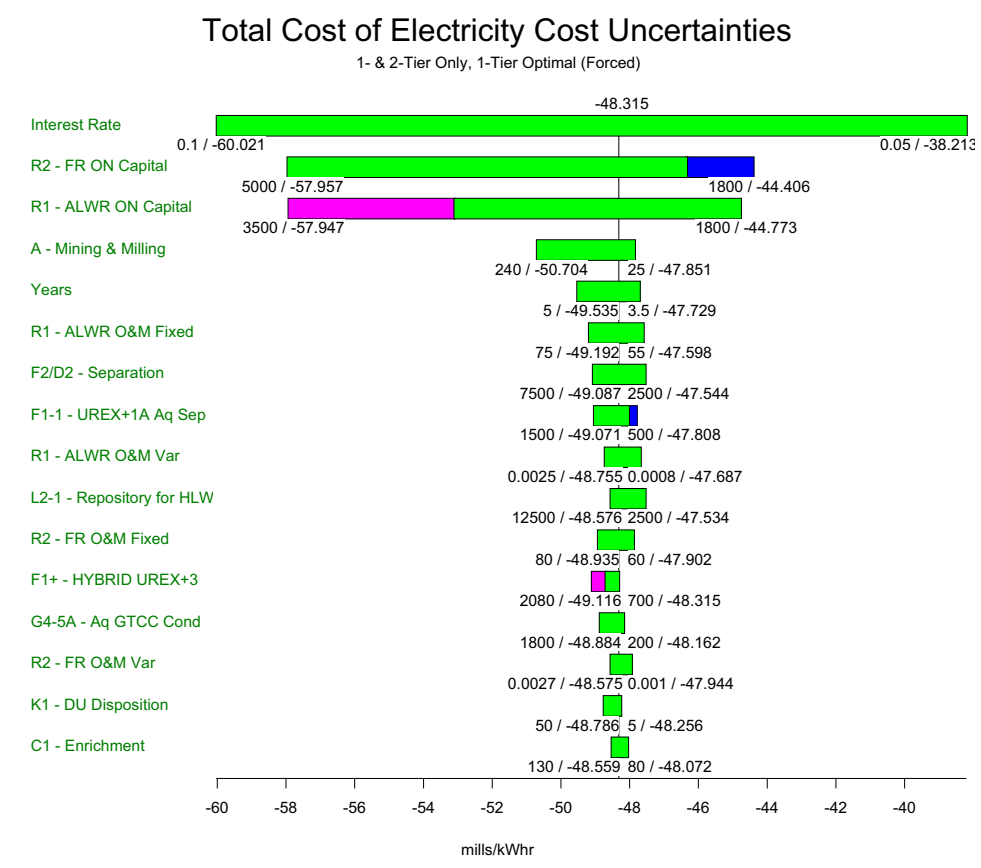

Figure 6-10. Cost variable uncertainty analysis for TCOE of the 1-Tier.

The DPL Tornado Diagram in Figure 6-10 shows the key variables for the 1-Tier strategy that are most likely to increase or decrease the costs. (Notice that the costs are negative on this diagram, this was done in order to be able to display the variable uncertainty. The strategy must be forced to be the optimal option in order to display the tornado diagram). The specific impacts are described in Figures 6-11 and 6-12.

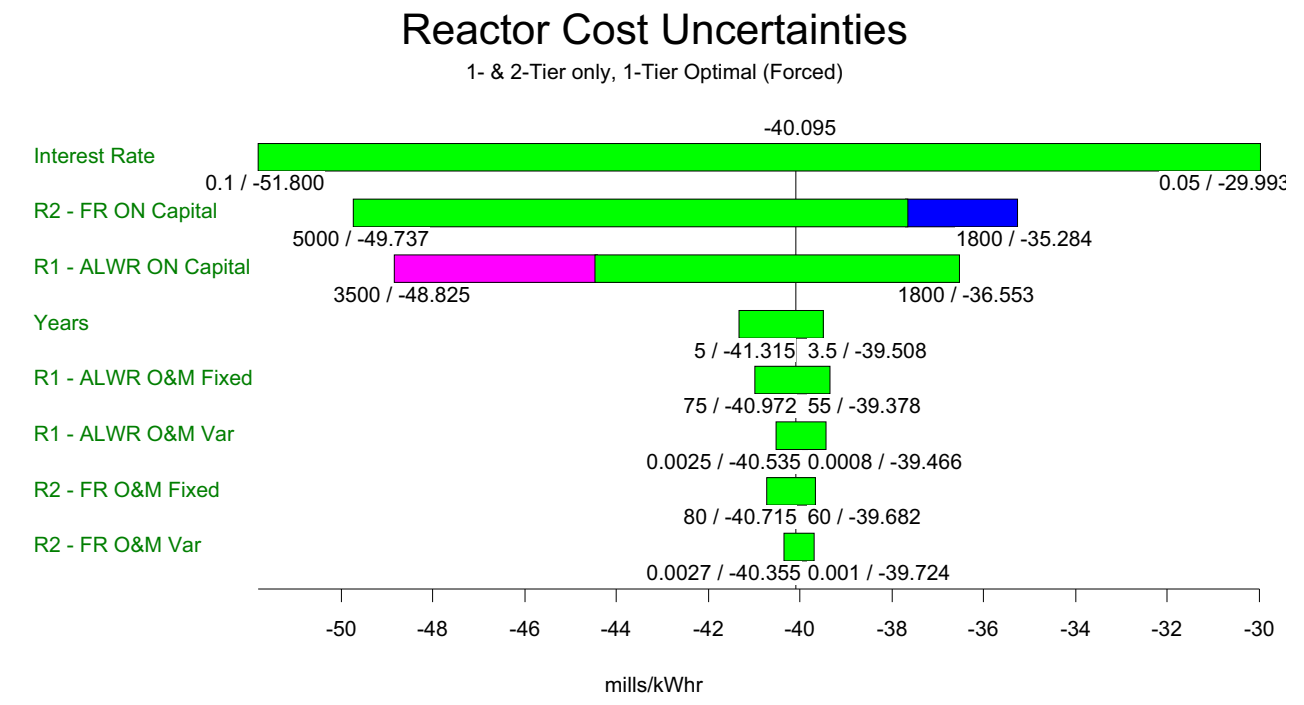

Figure 6-11. Cost variable uncertainty analysis for the 1-Tier reactor costs.

The DPL tornado diagram in Figure 6-11 shows the only the reactor cost variables for the 1-Tier strategy, where the largest cost uncertainties are the in the Interest Rate, R2-FR overnight capital, and the R1-ALWR overnight capital variables. (Notice that the costs are negative on this diagram, this was done 
in order to be able to display the variable uncertainty. The strategy must be forced to be the optimal policy in order to display the tornado diagram). Assessments of the variables that have the most influence on the costs are described as follows:

- The interest rate has the potential to swing the costs by $-10 \mathrm{mill} / \mathrm{kW} \cdot \mathrm{h}$ to $12 \mathrm{mill} / \mathrm{kW} \cdot \mathrm{h}$.

- The cost range for the FR capital has the potential to swing the costs $-5 \mathrm{mill} / \mathrm{kW} \cdot \mathrm{h}$ to $+9 \mathrm{mill} / \mathrm{kW} \cdot \mathrm{h}$. The FR O\&M has fixed and variable components with a range of costs of $+/-1.0 \mathrm{mill} / \mathrm{kW} \cdot \mathrm{h}$.

- The ALWR overnight capital has the potential to swing the costs -4 mills $/ \mathrm{kW} \cdot \mathrm{h}$ to 8 mills $/ \mathrm{kW} \cdot \mathrm{h}$. The ALWR O\&M can also affect the costs $+/-1.4 \mathrm{mill} / \mathrm{kW} \cdot \mathrm{h}$.

- The reactor construction duration has a potential range of impact of -0.5 to $1.2 \mathrm{mill} / \mathrm{kW} \cdot \mathrm{h}$ over the range of 3.5 to 5 years, respectively.

The ALWR capital and Fast Reactor capital variables indicate a shift in color from green to magenta (or green to blue) when moving from the nominal cost (center line) toward the extremes low or high cost values. This means that the variables have the potential at some unknown value to make the 1-Tier or 2Tier TCOE less than the once-through (i.e., the optimal policy). The chart does not specifically define the cost point where the optimal cycle changes. In Section 6.4, we further examine these types of trade-offs, in the economic trade-off parameter space analysis.

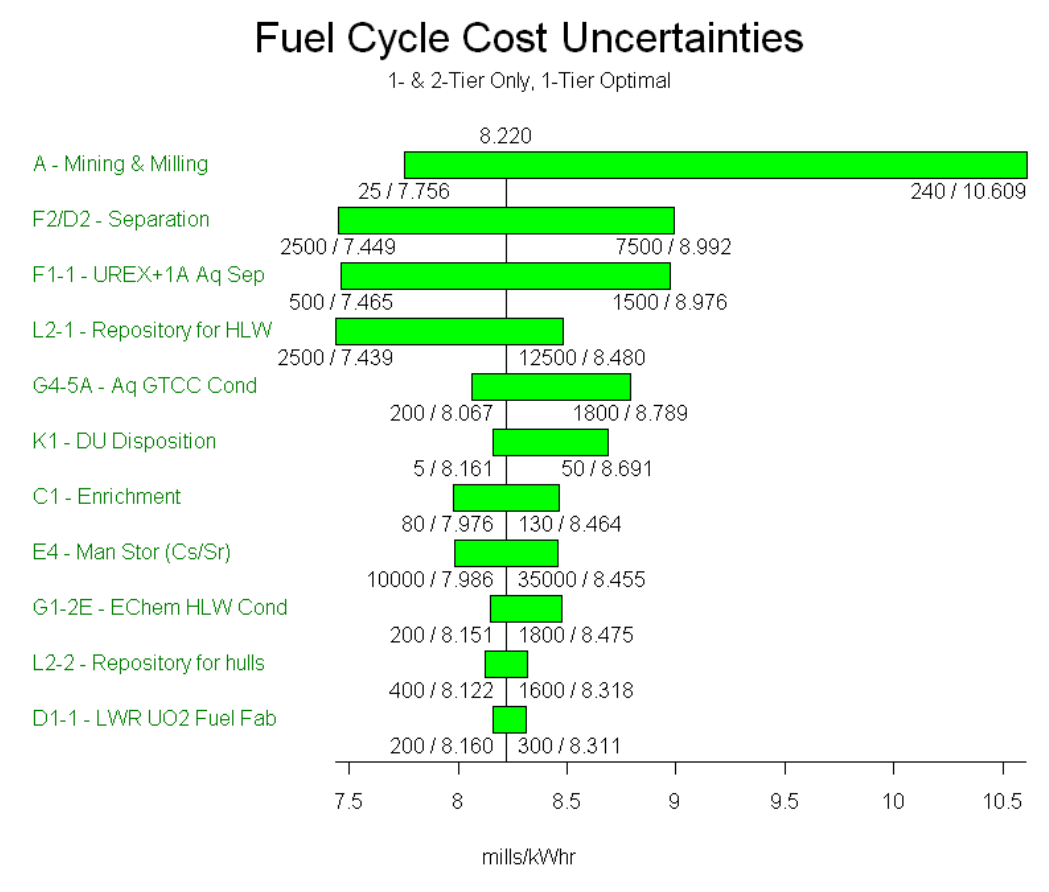

Figure 6-12. Cost Variable Uncertainty Analysis of the 1-Tier Fuel Cycle Cost.

The Value Tornado Diagram in Figure 6-12 shows the fuel cycle variables for the 1-Tier strategy, where the largest cost uncertainties are the A-Mining and Milling (uranium), F2/D2 Separation (EChem), F1 UREX+1a separation, and the L2-Repository for HLW variables. Assessments of the variables that have the most influence on the costs are described as follows:

- The cost of uranium mining and milling has the potential to increase costs by $2.5 \mathrm{mill} / \mathrm{s} / \mathrm{kW} \cdot \mathrm{h}$ over the nominal costs. There is also a $0.5 \mathrm{mill} / \mathrm{kW} \cdot \mathrm{h}$ cost reduction if uranium costs returns to historical cost levels around $\$ 25 / \mathrm{kg} \mathrm{U}$. 
- The EChem separation at the low range of costs would result in a fuel cycle saving of $0.7 \mathrm{mill} / \mathrm{kW} \cdot \mathrm{h}$ would result. A high cost for this function would result in an increase of $0.8 \mathrm{mills} / \mathrm{kW} \cdot \mathrm{h}$.

- The UOx aqueous separation at the low range of costs would result in a fuel cycle saving of $0.5 \mathrm{mill} / \mathrm{kW} \cdot \mathrm{h}$ would result. A high cost for this function would result in an increase of $0.8 \mathrm{mills} / \mathrm{kW} \cdot \mathrm{h}$.

- The HLW repository costs could decrease costs by $0.7 \mathrm{mill} / \mathrm{kW} \cdot \mathrm{h}$ at the low range, and would increase costs by $0.2 \mathrm{mill} / \mathrm{kW} \cdot \mathrm{h}$ at the high end of the cost range. This cost is a function of the repository cost and the HLW loading.

- Aqueous GTCC conditioning could decrease costs by $0.2 \mathrm{mill} / \mathrm{kW} \cdot \mathrm{h}$ at the low range, and would increase costs by $0.5 \mathrm{mill} / \mathrm{kW} \cdot \mathrm{h}$ at the high end of the cost range.

- DU disposition and enrichment, together could decrease costs by $0.2 \mathrm{mill} / \mathrm{kW} \cdot \mathrm{h}$ at the low range, and would increase costs by $0.6 \mathrm{mill} / \mathrm{kW} \cdot \mathrm{h}$ at the high end of the cost range.

- Cesium/Strontium decay storage has a cost uncertainty of $+/-0.2 \mathrm{mill} / \mathrm{s} / \mathrm{kW} \cdot \mathrm{h}$. In a managed decay scenario these costs in addition to the cost differentials for aqueous separations, waste conditioning, and waste disposal would need to be considered. See Section 6.4 for further analysis.

- EChem HLW conditioning could decrease costs by $0.1 \mathrm{mill} / \mathrm{kW} \cdot \mathrm{h}$ at the low range, and would increase costs by $0.2 \mathrm{mill} / \mathrm{kW} \cdot \mathrm{h}$ at the high end of the cost range.

- A number of additional variables (e.g., DU disposition, enrichment) have minimal cost uncertainty (i.e., total of less than $0.5 \mathrm{mills} / \mathrm{kW} \cdot \mathrm{h}$ ).

The F1+ aqueous separation (hybrid) variable has a shift in color from green to blue when moving from the nominal cost (center line) toward the extremes low value. This means that the variable has the potential at some unknown value to make the 1-Tier less than the 2-Tier fuel cycle cost (i.e., the optimal policy). The chart does not specifically define the cost point where the optimal cycle changes. In Section 6.4, we further examine these types of trade-offs, in the economic trade-off parameter space analysis.

\subsection{Cost Uncertainties Due to System Effects}

This section covers cost uncertainties due to system effects for the once-through and the 1-Tier. The scenarios allow for opportunities to analyze different aspects of cost uncertainties due to system effects based on the complexity of each. The once-through cost uncertainties were analyzed with respect to LWR system variables (Taylor 2008). The 1-Tier cost uncertainties are analyzed looking at effects due to FR system variables and changes in conversion ratio. The 2-Tier cost uncertainties were not analyzed due to a lack of model recipes for all the 2-Tier conversion ratios; however, the results are expected to be similar to the 1-Tier.

The Powersim platform on which VISION is built contains a tool for sensitivity analysis, which is denoted as the "Risk Assessment" analysis capability (Powersim 2007). This risk analysis capability varies selected parameters and measures the effect on selected results. The risk analysis has four variable types: assumptions, decisions, objectives, and effects. Assumptions are parameters set by the user and are sampled from a user-selected distribution, which includes normal, triangular, and uniform distributions. Decisions are parameters that the user changes to a static value that differs from the nominal value in the model. Objectives are computed parameters that are a target for optimization and are not utilized in this analysis. The effects are parameters that are analyzed due to changes in the assumption and decision variables. Multiple assumption, decision, and effects variables can be chosen for a single-risk analysis. 
Simulation settings, such as start year, end year, and time step, must be defined specifically for the risk analysis. The user must also define the sampling method from the choices of Latin Hypercube or Monte Carlo. The Latin Hypercube is the recommended sampling method. It partitions the data space according to its probability distribution and then randomly samples each partitioned data set. The Monte Carlo method randomly samples the entire data space. The drawback to the Monte Carlo method is longer run times due to more samples being needed to ensure that the entire data space is sampled for accurate results.

Other settings for the risk assessment include the run count, seed type and number, and the history of effects. The run count specifies the number of simulation runs for the analysis and decides how many samples to create. The seed type is a variation between random and fixed seed values. The seed value is the start value for the random function. The random option creates a random seed number from a generator for each run and the fixed option is user input so that the user may repeat the results. The risk analysis samples the assumption variable using the user-defined distribution and method. The modeling algorithm analyzes the effect variable for each given sample and returns the results in percentile values selected by the user. The user may also select high or low values, average, and the standard deviation of the samples.

\subsubsection{Once-through Cost Uncertainties Due to System Effects}

The scenario examined is a once-through fuel cycle with a single LWR using UOx fuel. Parameters for this scenario are listed in Table 6-1. The authors acknowledge that this scenario is not truly representative of the existing U.S. commercial reactor fleet. This scenario is designed simplistically to analyze system parameters that significantly affect costs. For this scenario, the model was set to begin on January 1, 2000 and continues for 100 years with a time step of 3 months. Figure 6-13 diagrams the specific fuel cycle processes for Base Case 1.

Table 6-1. Once-through scenario parameters.

\begin{tabular}{|lr|}
\hline Mining Time & $1 \mathrm{yr}$ \\
Conversion Time & $0.25 \mathrm{yr}$ \\
Enrichment Time & $1 \mathrm{yr}$ \\
Fabrication Time & $1 \mathrm{yr}$ \\
Estimated Conventional Resources & $12000 \mathrm{kt} \mathrm{U}$ \\
Tails Enrichment & $0.25 \%$ \\
\hline Number of Batches & 5 \\
Cycle Length & $1 \mathrm{yr}$ \\
Reactor Power & $1.3 \mathrm{GWe}$ \\
Capacity Factor & 0.9 \\
Thermal Efficiency & 0.34 \\
\hline Wet Storage Time & $5 \mathrm{yrs}$ \\
Dry Storage Time & $5 \mathrm{yrs}$ \\
\hline Interest Rate & $10 \%$ \\
Scenario Start Date & $1 / 1 / 2000$ \\
Scenario End Date & $1 / 1 / 2100$ \\
Timestep & $0.25 \mathrm{yr}$ \\
\hline
\end{tabular}




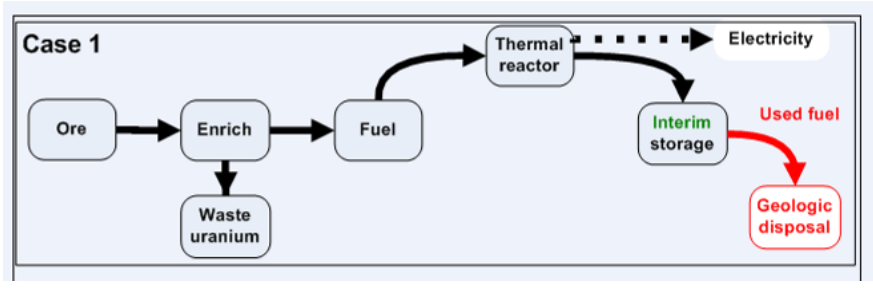

Figure 6-13. Fuel cycle diagram for Base Case 1 scenario.

The front end of the fuel cycle consists of mining, conversion, enrichment, and fuel fabrication. Uranium mining is contingent upon world supply markets and demand from the reactor. Conversion, enrichment, and fuel fabrication are limited by the amount of material from the previous step and process time. In this scenario, fuel fabrication facilities are assumed to be commercial entities possessing excess capacity, and are not expected to be a limiting factor. The reactor lifetime of the LWR is set to span the simulation lifetime, meaning no additional reactors are constructed or will begin operating. At the simulation start point, the LWR is constructed and operated with reserve fuel. There are five batches of fuel in the reactor with a batch cycle length of 1 year.

The back end of the fuel cycle in this scenario consists of spent nuclear fuel repository disposal costs. Subsequent more complex scenarios will also feature recycling in the back end of the fuel cycle. Fuel cycle batches are removed from the reactor and sent to wet storage for a period of 5 years. The spent fuel is then sent to dry storage for the remainder of the simulation. Wet and dry storage is located at the reactor site and is included in reactor costs. Materials in product storage are altered by decay during their tenure by VISION.

When examining this scenario, a run of the VISION code is analyzed to examine the largest contributing factors to the main economic measures, TCOE, and fuel cycle costs (FCC). Figure 6-14 shows components that constitute the total fuel cycle cost annually at the scenarios' equilibrium. The fuel cycle cost each year defines the fuel cycle cost when divided by electricity production. The approximate percentage of the component that comprise the fuel cycle cost each year is $19 \%$ for ore mining, $3 \%$ for ore conversion, $25 \%$ for enrichment, $9 \%$ for fuel fabrication, $3 \%$ for depleted uranium disposition (DUD), $4 \%$ for conditioning, and 38\% for repository disposal.[5] The unit costs used in this analysis are based on nominal near term (next 10-15 years) cost expectations, and represent an average cost over a wide range of costs that have considered recent increases in uranium ore, enrichment services, and power plant construction costs. Figure 6-15 shows components that constitute the total cost annually at the scenarios' equilibrium. TCOE is defined as the total cost each year divided by the electricity produced. The approximate percentage of each component that comprises the total cost each year is $9 \%$ for the front end, up to $6 \%$ for the back end, $61 \%$ for reactor capital costs, and $24 \%$ for reactor operations costs. 


\section{Annual Fuel Cycle Costs $\$ 67.6 \mathrm{M}$}

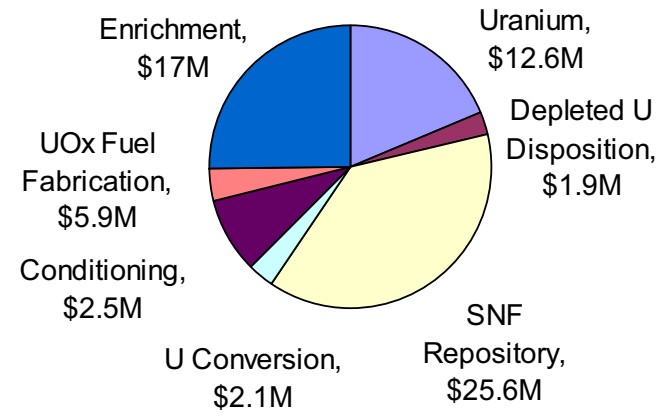

Figure 6-14. Total fuel cycle costs annually by component at equilibrium for once-through scenario.

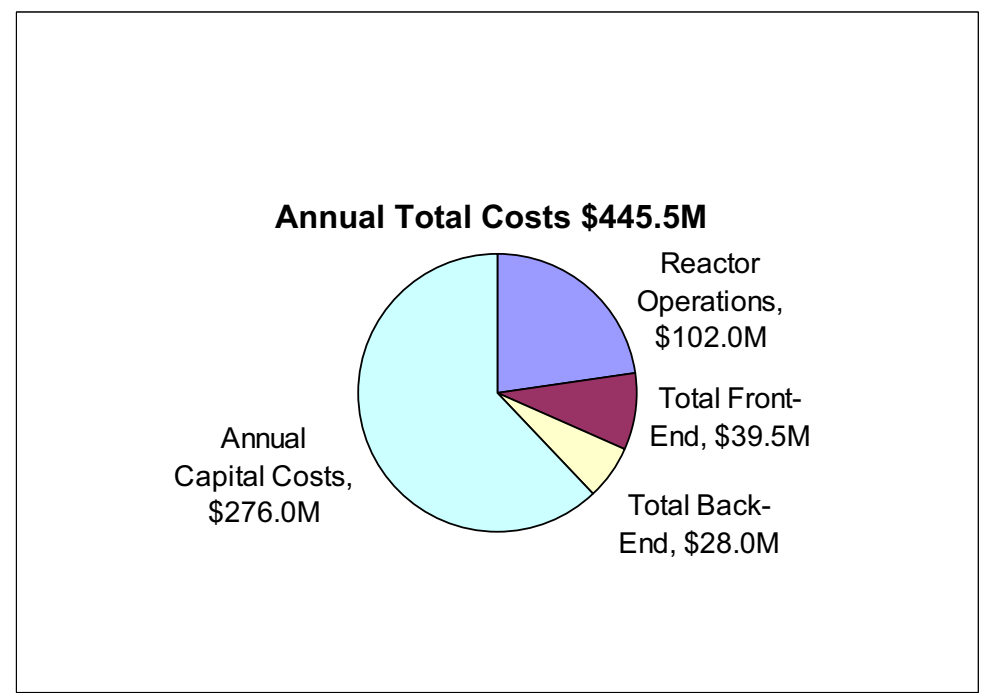

Figure 6-15. Total cost annually by component at equilibrium for once-through scenario.

Systems Analysis chose the assumption parameters based on component contribution to costs and electricity production. The largest contributing component to total fuel cycle costs is repository disposal costs followed by uranium enrichment costs. The user-input system variable that greatly influences these costs is burnup. Higher burnups will require higher initial enrichment, but less mass of uranium. However, higher burnups lead to greater electricity production, which offsets the enrichment cost increase when analyzing fuel cycle cost. Due to these interesting tradeoffs, burnup was chose to be an assumption variable. Thermal efficiency was also chosen as an assumption variable because it is a user-defined variable that is directly input. Thermal efficiency also has implications in the two largest components to total fuel cycle costs.

The largest contributor to total costs each year is the reactor annual capital costs. Reactor capital costs are based on reactor size, overnight capital costs, and the interest during construction (IDC). The reactor capital costs are annualized by amortizing the costs over the economic lifetime of the reactor using a 
capital recovery factor. An assumption parameter that influences the reactor capital is the interest costs. Varying the interest rate would influence the total reactor capital cost. However, this sensitivity analysis is interested in the effects due to system parameters, not economic parameters; therefore, reactor construction time was chosen as a variable because it determines the amount of time that the interest is charged. The reactor capacity factor was also chosen as an assumption variable due to its direct affect on the amount of electricity annually produced.

Reactor annual capital costs are directly dependent on the reactor size, and it would seem that reactor size would be a choice as an assumption variable. However, reactor size is also directly involved with the calculation of electricity produced yearly. The main economic measures TCOE and fuel cycle cost are ratios of costs-to-electricity production. A change in costs due to reactor size would scale proportionately with changes in electricity production causing no change in the economic measures. For this reason, reactor size was not chosen as an assumption parameter.

System variables directly input by the user were candidates as assumption parameters. Systems Analysis evaluated all modeling times for the various fuel cycle process steps (i.e., mining time, conversion time, etc.) and facility construction times. However, the simplicity of the scenario used for risk analysis made the some of the system parameters irrelevant as assumption variables. Mining time was found to be irrelevant due to the fact that the scenario is to begin with sufficient fuel for reactor operation, which negates delays in mining; the same is true for conversion time. Other system variables not directly input were also considered. However, these variables were not selected because they were auxiliary calculations, values calculated from other variables, and were inappropriate candidates for use in risk analysis. Reactor power is an example of one such system variable. Reactor power is defined as the product of reactor size and a capacity factor representing availability. Capacity factor and reactor size are both user input variables. Therefore, reactor power was not chosen as an assumption parameter to avoid redundancy and aid simplicity of analysis. Finally, some system parameters were eliminated as assumption parameter choices because of their embedded definitions. Fuel enrichment is defined in VISION based on fuel recipes, which are user input variables. Fuel recipes, represented in an isotopic array, would need to be modified in order to model enrichment changes. Further research on the embedded definitions will be undertaken, but are not included in this analysis.

Using capacity factor, reactor construction time, burnup, and thermal efficiency as assumption parameters, and TCOE and fuel cycle cost as effect parameters, the risk analysis produces results as illustrated in the tornado diagrams in Figures 6-16 and 6-17. Each assumption variable was sampled while the other variables were set to their nominal values. Therefore, each bar of the tornado diagram denotes a different risk analysis. The risk analysis gives the value of $43.41 \mathrm{mills} / \mathrm{kWh}$ for the TCOE and $6.59 \mathrm{mills} / \mathrm{kWh}$ for the fuel cycle cost with nominal values set for every assumption variable. The capacity factor is shown to be uniformly sampled between 0.80 and 0.95 with a nominal value of 0.90 . Reactor construction time is uniformly sampled from 4 to 6 years with a nominal value of 5 years. Burnup is uniformly sampled from 50 to $80 \mathrm{GWth} * \mathrm{~d} / \mathrm{MT}$ with a nominal value of $51 \mathrm{GWth}$ d/MT. Thermal efficiency is uniformly sampled from 0.30 to 0.35 with a nominal value of 0.34 . 


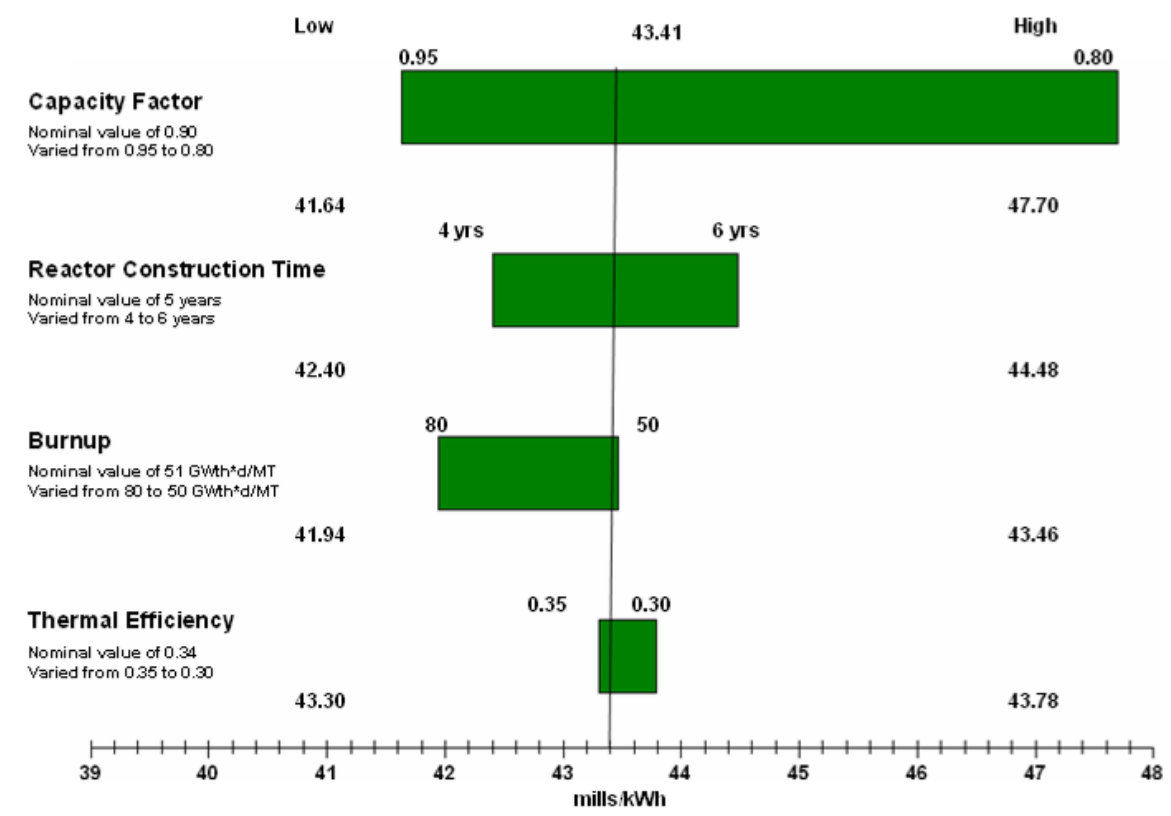

Figure 6-16. Tornado diagram showing low and high values of TCOE with variations in capacity factor, reactor construction time, burnup, and thermal efficiency.

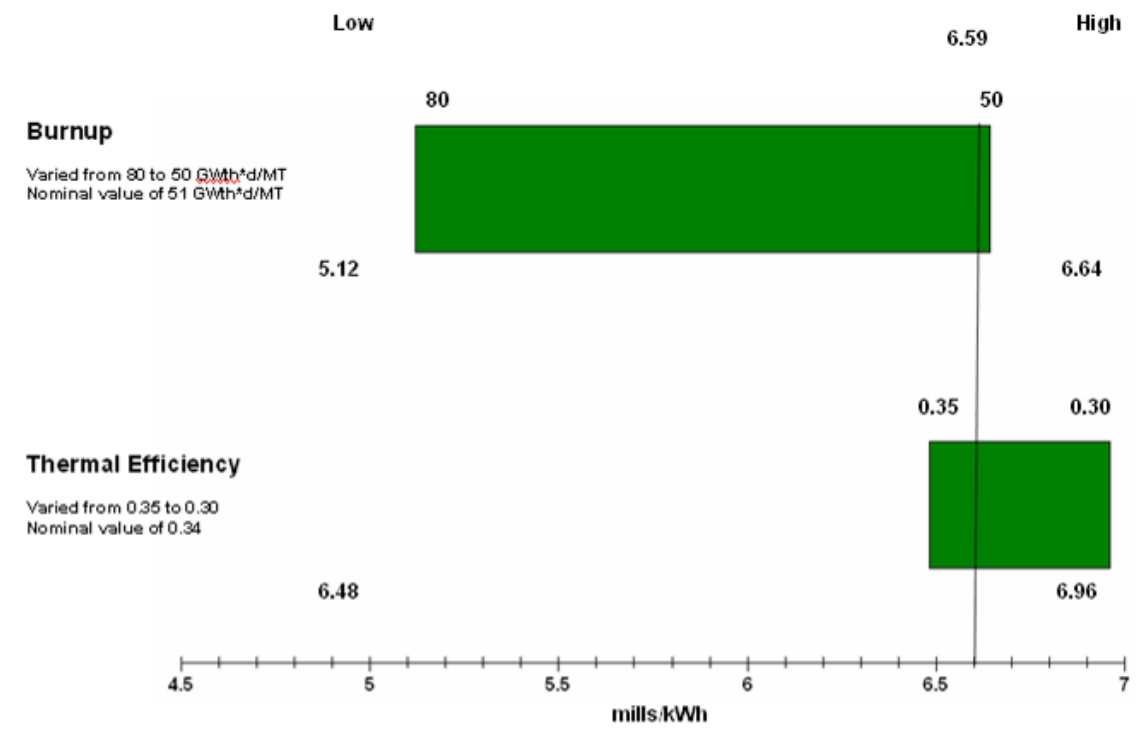

Figure 6-17. Tornado diagram showing low and high values of FCC with variations in burnup and thermal efficiency.

As shown in Figure 6-16, an increase in TCOE results from reductions in the reactor capacity factor and thermal efficiency, and from lower fuel burnup. Longer construction times also cause an increase in the TCOE. In Figure 6-17, increases in fuel cycle cost can result from lower reactor burnup and lower thermal efficiency. Changes in the reactor capacity factor and construction time have no affect on the fuel cycle cost.

The VISION model coding and output were analyzed to understand the system implications from these results. Analysis of the results begins with single runs of the main VISION model using the high and low values of the assumption variables to discern their affects on TCOE and the fuel cycle cost. 
Burnup is a user input variable given in GWth*d/MT. VISION uses burnup to calculate the fuel consumption rate, which is defined as the thermal power divided by the burnup. The fuel consumption rate dictates how much fuel is ordered, the front-end process rates, such as mining rate and conversion rate, and the back-end storage rates. Changes in burnup do not influence the electricity produced. Lower burnup rates require more mass at a lower enrichment to meet the specified power demand. Costs are calculated by multiplying process flow rates by a unit cost basis. As shown in Table 6-2, the lower burnup value increases the fuel cycle costs due to the increase in fuel mass flows in the front and back end of the fuel cycle. A decrease in burnup increases front-end costs by increasing the demand for uranium ore mining, conversion, fabrication, and depleted uranium disposition. However, fuel enrichment requirements (energy needed to produce fissile LWR fuel) for low burnup fuels are less on a per unit basis than for high burnup fuels. Back-end costs increase due to more reactor total fuel mass for disposition. Reactor costs do not vary with change in burnup. Table 6-2 illustrates the differences in cost due to varying burnup.

Table 6-2. TCOE and FCC with defining components at high and low ranges of burnup.

\begin{tabular}{|r|rrl|}
\hline Burnup Value & $50 \mathrm{GWth}{ }^{*} \mathrm{~d} / \mathrm{MT}$ & $80 \mathrm{GWth}{ }^{*} \mathrm{~d} / \mathrm{MT}$ & Units \\
\hline Electricity Produced Yearly & $1.03 \mathrm{E}+10$ & $1.03 \mathrm{E}+10$ & $\mathrm{kWh}$ \\
\hline Total Front End & $4.03 \mathrm{E}+07$ & $2.52 \mathrm{E}+07$ & $\$$ \\
U Ore Mining & $1.29 \mathrm{E}+07$ & $8.05 \mathrm{E}+06$ & $\$$ \\
U Ore Conversion & $2.15 \mathrm{E}+06$ & $1.34 \mathrm{E}+06$ & $\$$ \\
U Enrichment & $1.74 \mathrm{E}+07$ & $1.08 \mathrm{E}+07$ & $\$$ \\
Fuel Fabrication & $6.03 \mathrm{E}+06$ & $3.77 \mathrm{E}+06$ & $\$$ \\
DUD & $1.90 \mathrm{E}+06$ & $1.18 \mathrm{E}+06$ & $\$$ \\
\hline Total Back End repository Costs & $2.81 \mathrm{E}+07$ & $2.72 \mathrm{E}+07$ & $\$$ \\
Conditioning & $2.56 \mathrm{E}+07$ & $2.56 \mathrm{E}+07$ & $\$$ \\
& $2.50 \mathrm{E}+06$ & $1.56 \mathrm{E}+06$ & $\$$ \\
\hline Reactor Costs & $3.78 \mathrm{E}+08$ & $3.78 \mathrm{E}+08$ & $\$$ \\
\hline Fuel Cycle Costs & & & \\
\hline Total Cost of Electricity & 6.67 & 5.11 & $\mathrm{mills} / \mathrm{kWh}$ \\
& 43.50 & 41.93 & $\mathrm{mills} / \mathrm{kWh}$ \\
\hline
\end{tabular}

Thermal efficiency is a user input variable. VISION uses thermal efficiency to determine how much thermal energy generated is converted to electric energy in the reactor. Increasing thermal efficiency decreases front and back-end costs. The increase in thermal efficiency decreases the amount of source material needed in the front-end processes and mass of material needing to be disposed. Reactor costs and electricity production are not influenced by thermal efficiency and are constant. Table 6-3 illustrates the effect of thermal efficiency on TCOE and the fuel cycle cost.

Table 6-3. TCOE and FCC with defining components at high and low ranges of thermal efficiency.

\begin{tabular}{|r|rrl|}
\hline Thermal Efficiency Value & 0.3 & 0.35 & Units \\
\hline Electricity Produced Yearly & $1.03 \mathrm{E}+10$ & $1.03 \mathrm{E}+10$ & kWh \\
\hline Total Front End & $4.48 \mathrm{E}+07$ & $3.84 \mathrm{E}+07$ & $\$$ \\
U Ore Mining & $1.43 \mathrm{E}+07$ & $1.23 \mathrm{E}+07$ & $\$$ \\
U Ore Conversion & $2.39 \mathrm{E}+06$ & $2.04 \mathrm{E}+06$ & $\$$ \\
U Enrichment & $1.93 \mathrm{E}+07$ & $1.65 \mathrm{E}+07$ & $\$$ \\
Fuel Fabrication & $6.70 \mathrm{E}+06$ & $5.74 \mathrm{E}+06$ & $\$$ \\
DUD & $2.11 \mathrm{E}+06$ & $1.81 \mathrm{E}+06$ & $\$$ \\
\hline Total Back End & $2.84 \mathrm{E}+07$ & $2.80 \mathrm{E}+07$ & $\$$ \\
Repository Disposal & $2.56 \mathrm{E}+07$ & $2.56 \mathrm{E}+07$ & $\$$ \\
Conditioning & $2.78 \mathrm{E}+06$ & $2.38 \mathrm{E}+06$ & $\$$ \\
\hline Reactor Costs & $3.78 \mathrm{E}+08$ & $3.78 \mathrm{E}+08$ & $\$$ \\
\hline Fuel Cycle Costs & & & \\
\hline Total Cost of Electricity & 7.14 & 6.48 & $\mathrm{mills} / \mathrm{kWh}$ \\
& 43.96 & 43.30 & $\mathrm{mills} / \mathrm{kWh}$ \\
\hline
\end{tabular}

Capacity factor is a user input variable, which defines the availability of the reactor. Reactor power is then defined as a product of capacity factor and reactor size. An increase in capacity factor corresponds to an increase in front and back-end costs, as well as increased annual electricity production. Total fuel cycle cost increases are offset by the increase in electricity production leaving the fuel cycle cost unchanged 
with varying capacity factor. Due to the reactor costs, this is not the case with TCOE. Reactor costs have a strong dependence on reactor size. Reactor size does not vary with the capacity factor. Therefore, reactor costs have only a slight change with the change in capacity factor. TCOE is the ratio of total annual costs, reactor and fuel cycle, to annual electricity production. Therefore, with a higher capacity factor, the total costs are outpaced by the increase in electricity, hence lowering the TCOE. Table 6-4 illustrates the changes in TCOE and fuel cycle cost with variations in capacity factor.

Table 6-4. TCOE and FCC with defining components at high and low ranges of capacity factors.

\begin{tabular}{|r|lll|}
\hline Capacity Factor Value & 0.8 & 0.95 & Units \\
\hline Electricity Produced Yearly & $9.12 \mathrm{E}+09$ & $1.08 \mathrm{E}+10$ & kWh \\
\hline Total Front End & $3.51 \mathrm{E}+07$ & $4.17 \mathrm{E}+07$ & $\$$ \\
U Ore Mining & $1.12 \mathrm{E}+07$ & $1.33 \mathrm{E}+07$ & $\$$ \\
U Ore Conversion & $1.87 \mathrm{E}+06$ & $2.22 \mathrm{E}+06$ & $\$$ \\
U Enrichment & $1.51 \mathrm{E}+07$ & $1.80 \mathrm{E}+07$ & $\$$ \\
Fuel Fabrication & $5.25 \mathrm{E}+06$ & $6.24 \mathrm{E}+06$ & $\$$ \\
DUD & $1.65 \mathrm{E}+06$ & $1.96 \mathrm{E}+06$ & $\$$ \\
\hline Total Back End & $2.50 \mathrm{E}+07$ & $2.97 \mathrm{E}+07$ & $\$$ \\
Repository Disposal & $2.28 \mathrm{E}+07$ & $2.71 \mathrm{E}+07$ & $\$$ \\
Conditioning & $2.18 \mathrm{E}+06$ & $2.59 \mathrm{E}+06$ & $\$$ \\
\hline Reactor Costs & $3.76 \mathrm{E}+08$ & $3.79 \mathrm{E}+08$ & $\$$ \\
\hline Fuel Cycle Costs & & & \\
\hline Total Cost of Electricity & 6.59 & 6.59 & $\mathrm{mills} / \mathrm{kWh}$ \\
\hline
\end{tabular}

Construction time is used to evaluate the accrued IDC. The interest is amortized over the reactor lifetime and contributes to annual capital cost. A change in reactor construction time does not affect any fuel cycle or reactor operations cost. However, this change does have a significant impact on the reactor annual capital costs, which comprise three-fourths of the total cost of electricity. This leads to a significant increase in TCOE. Table 6-5 illustrates the changes in the TCOE with variation in reactor construction time.

Table 6-5. TCOE and FCC with defining components at high and low ranges of reactor construction time.

\begin{tabular}{|r|ccl|}
\hline Construction Time Value & 4 yrs & 6 yrs & Units \\
\hline Electricity Produced Yearly & $1.03 \mathrm{E}+10$ & $1.03 \mathrm{E}+10$ & $\mathrm{kWh}$ \\
\hline Total Front End & $3.95 \mathrm{E}+07$ & $3.95 \mathrm{E}+07$ & $\$$ \\
U Ore Mining & $1.26 \mathrm{E}+07$ & $1.26 \mathrm{E}+07$ & $\$$ \\
U Ore Conversion & $2.10 \mathrm{E}+06$ & $2.10 \mathrm{E}+06$ & $\$$ \\
U Enrichment & $1.70 \mathrm{E}+07$ & $1.70 \mathrm{E}+07$ & $\$$ \\
Fuel Fabrication & $5.91 \mathrm{E}+06$ & $5.91 \mathrm{E}+06$ & $\$$ \\
DUD & $1.86 \mathrm{E}+06$ & $1.86 \mathrm{E}+06$ & $\$$ \\
\hline Total Back End & $2.81 \mathrm{E}+07$ & $2.81 \mathrm{E}+07$ & $\$$ \\
Repository Disposal & $2.56 \mathrm{E}+07$ & $2.56 \mathrm{E}+07$ & $\$$ \\
Conditioning & $2.45 \mathrm{E}+06$ & $2.45 \mathrm{E}+06$ & $\$$ \\
\hline Reactor Costs & $3.67 \mathrm{E}+08$ & $3.89 \mathrm{E}+08$ & $\$$ \\
Reactor Operations & $1.02 \mathrm{E}+08$ & $1.02 \mathrm{E}+08$ & $\$$ \\
Annual Capital & $2.65 \mathrm{E}+08$ & $2.87 \mathrm{E}+08$ & $\$$ \\
\hline Fuel Cycle Costs & & & \\
\hline Total Cost of Electricity & 6.59 & 6.59 & $\mathrm{mills} / \mathrm{kWh}$ \\
& 42.37 & 44.50 & $\mathrm{mills} / \mathrm{kWh}$ \\
\hline
\end{tabular}

The once-through scenario is a starting point for sensitivity analysis of VISION. Additional sensitivity analysis work includes extending the analysis to the 1-Tier and 2-Tier scenarios with increasing levels of complexity. An analysis of the tiered fuel cycle scenarios begins with the assumption parameters selected for the once-through scenario. The introduction of fast reactors into the scenario includes fast reactor assumption parameters, such as fast reactor construction time, reactor power, and capacity factor. The introduction of recycling in these latter scenarios will allow selection of assumption variables, such as conversion ratio. These more complex system studies include variations in queuing times between processes. Analyses will progress from the simplistic once-through scenario, testing appropriate variables, and adding complexity as modeling confidence and user knowledge grows. 


\subsubsection{1-Tier Cost Uncertainties Due to System Effects}

The scenario examined is a 1-Tier fuel cycle with LWR using UOx fuel, and FR. Parameters for this scenario are listed in Table 6-6. This scenario is designed simplistically to analyze system parameters pertaining to fast reactors that significantly affect costs. This scenario also analyzes changes in the main economic measures of FCC and TCOE due to variations in conversion ratio. For this scenario, the model began on January 1, 2000, and continues for 100 years with a time step of 3 months. Figure 6-18 diagrams the specific fuel cycle processes.

Table 6-6. 1-Tier scenario parameters.

\begin{tabular}{|lr|}
\hline Mining Time & $1 \mathrm{yr}$ \\
Conversion Time & $0.25 \mathrm{yr}$ \\
Enrichment Time & $1 \mathrm{yr}$ \\
Fabrication Time & $1 \mathrm{yr}$ \\
Estimated Conventional Resources & $12000 \mathrm{kt} \mathrm{U}$ \\
Tails Enrichment & $0.25 \%$ \\
\hline Reactor Power (LWR) & $1.3 \mathrm{GWe}$ \\
Reactor Power (FR) & $0.38 \mathrm{Gwe}$ \\
Capacity Factor (LWR) & 0.9 \\
Capacity Factor (FR) & 0.82 \\
Thermal Efficiency & 0.34 \\
\hline Wet Storage Time & $5 \mathrm{yrs}$ \\
Dry Storage Time & $5 \mathrm{yrs}$ \\
\hline Interest Rate & $10 \%$ \\
Scenario Start Date & $1 / 1 / 2000$ \\
Scenario End Date & $1 / 1 / 2100$ \\
Timestep & $0.25 \mathrm{yr}$ \\
\hline
\end{tabular}

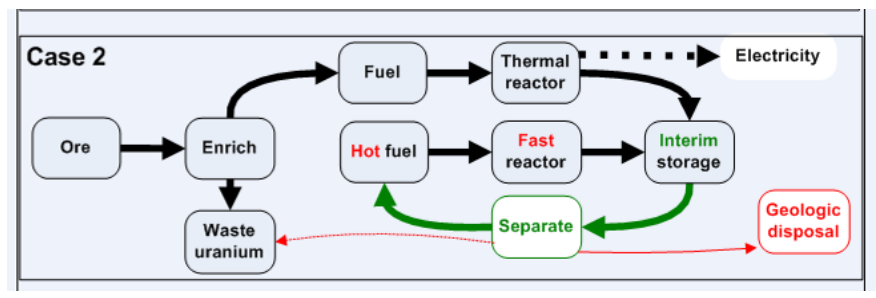

Figure 6-18. Fuel cycle diagram for Base Case 2 scenario.

The front end of the fuel cycle consists of mining, conversion, enrichment, and fuel fabrication. Uranium mining is contingent upon world supply markets and demand from the reactor. Conversion, enrichment, and fuel fabrication are limited by the amount of material from the previous step and process time. In this scenario, fuel fabrication facilities are assumed to be commercial entities possessing excess capacity, and are not expected to be a limiting factor. At the simulation start point there are no operating reactors. LWRs are constructed beginning in 2005, and FRs are constructed beginning in 2010 to meet nuclear energy market share.

The back end of the fuel cycle in this scenario consists of intermediate disposal and HLW repository disposal costs. Recycling costs consists of aqueous separations, electrochemical separations, conditioning and storage costs as well as co-flow processes. Fuel is removed from the LWRs and sent to wet storage and then dry storage. The LWR fuel is then available for recycling and use in the fast reactors. Wet and dry storage is located at the reactor site and is included in reactor costs. Materials in product storage are altered by decay during their tenure by VISION. 
When examining this scenario, a run of the VISION code is analyzed to examine the largest contributing factors to the main economic measures, TCOE, and fuel cycle cost. Figure 6-19 shows components that constitute the total fuel cycle cost annually at the scenarios' equilibrium. The fuel cycle cost each year defines the fuel cycle cost when divided by electricity production. The approximate percentage of the component that comprise the fuel cycle cost each year is $30 \%$ for front-end costs, $15 \%$ for back end costs, and 55\% for recycling costs . The unit costs used in this analysis are based on nominal near-term (next 10-15 years) cost expectations, and represent an average cost over a wide range of costs that have considered recent increases in uranium ore, enrichment services, and power plant construction costs. Figure 6-20 shows components that constitute the total cost annually at the scenarios' equilibrium. TCOE is defined as the total cost each year divided by the electricity produced. The approximate percentage of each component that comprises the total cost each year is $5 \%$ for the front end, $2 \%$ for the back end, $10 \%$ for recycling costs, $60 \%$ for reactor capital costs, and $22 \%$ for reactor operations costs.

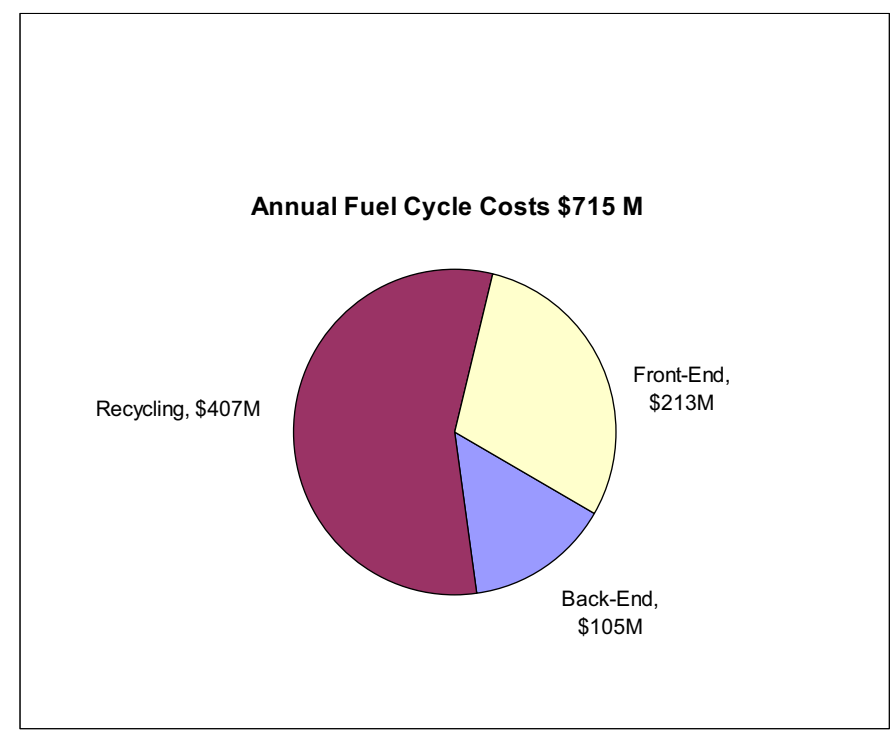

Figure 6-19. Total fuel cycle costs annually by component at equilibrium for 1-Tier scenario.

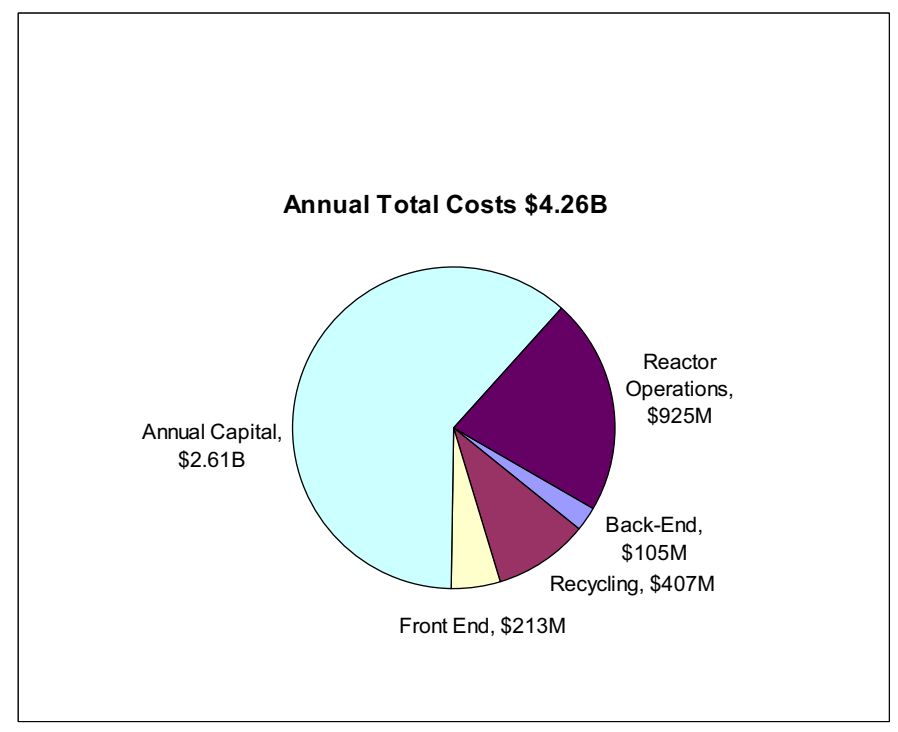

Figure 6-20. Total costs annually by component at equilibrium for 1-Tier scenario. 
Assumption parameters are chosen based on component contribution to costs due to fast reactors and electricity production. The largest contributing component to total fuel cycle costs is recycling costs. Recycling costs are dedicated by the amount of material recycled and the composition of the material.

The user-input system variable that greatly influences these costs for fast reactors is conversion ratio. ${ }^{\mathrm{h}}$ Additional sensitivity analyses were done using fuel conversion ratios, but due to the complexity of the changes necessary to perform the analyses, the risk analysis tool embedded in Powersim was not capable of performing the analyses. Conversion ratio in fast reactors determines how much fissile material is burned (consumed) in the reactor - the lower the conversion ratio, the higher the burnup of fissile material. Conversion ratio is a very important parameter in reactor performance, and as such is a good candidate for sensitivity analysis. In order to test different conversion ratios in VISION it is necessary to adjust a number of system parameters such as the fuel recipes, number of batches, cycle time, and burnup. The sensitivity analysis was performed manually by running common scenarios and varying the conversion ratio from 0.25 to 0.50 to 0.75 and finally 1.0 .

The conversion ratio is imbedded in fast reactor fuel recipes input by the user before VISION is run. Conversion ratio is varied manually by the input parameters and the effects are noted below risk analysis variations. Fast reactor thermal efficiency was chosen as an assumption parameter because it is a userdefined variable that is directly input and intuitively affects costs. The fast reactor capacity factor was also chosen as an assumption parameter due to its direct affect on the amount of electricity annually produced.

Using fast reactor capacity factor, and thermal efficiency as assumption parameters and TCOE and fuel cycle cost as effect parameters, the risk analysis produces results as illustrated in the tornado diagrams in Figures 6-21 and 6-22. Each assumption variable was sampled while the other variables were set to their nominal values. Therefore, each bar of the tornado diagram denotes a different risk analysis. The risk analysis gives the value of $48.28 \mathrm{mills} / \mathrm{kWh}$ for the TCOE and $8.23 \mathrm{mills} / \mathrm{kWh}$ for fuel cycle cost with nominal values set for every assumption variable. The capacity factor is uniformly sampled between 0.80 and 0.90 with a nominal value of 0.82 , the fast reactor thermal efficiency is uniformly sampled from 0.37 to 0.41 with a nominal value of 0.38 , and the sampling effect on TCOE is shown in Figure 6-21. Figure 6-22 shows the effect of sampling thermal efficiency on fuel cycle cost.

h. Conversion ratio is defined as the ratio of the integral number of TRU atoms produced (mostly capture in U-238) to the integral number of TRU atoms destroyed (mostly fission) that occurs during reactor operation. This is a reactor physics parameter that is consistent with previous calculations for fissile breeding calculations. It is based on atoms and not mass, it does not account for decay during storage of the fuel, and it neglects effects of direct fission of uranium, which are secondary effects that impact the TRU mass and energy balance for the system. 


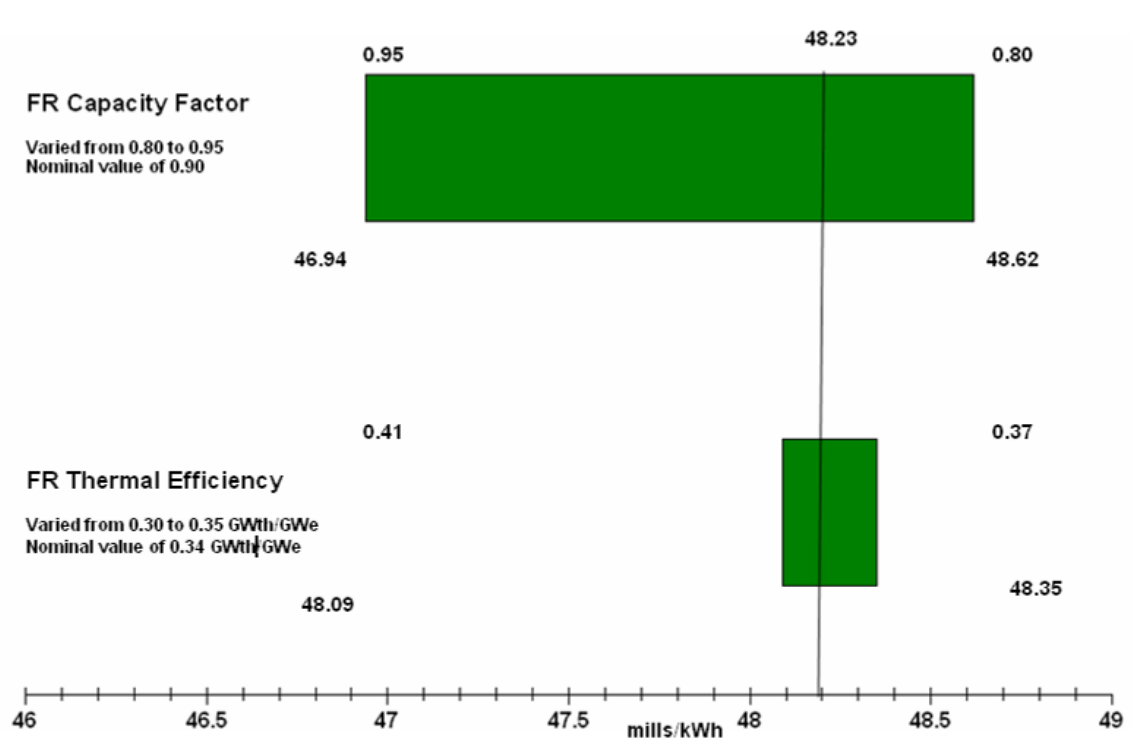

Figure 6-21. Tornado diagram showing low and high values of TCOE with variations in FR capacity factor and FR thermal efficiency.

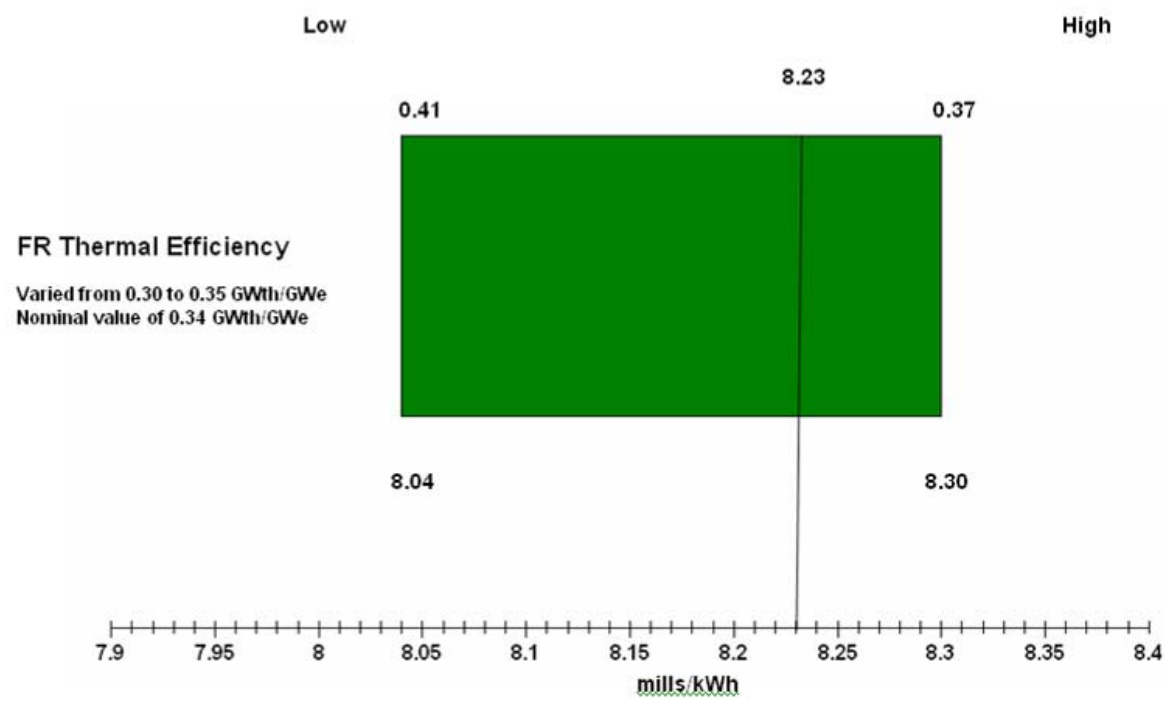

Figure 6-22. Tornado diagram showing low and high values of FCC with variations in FR thermal efficiency.

As shown in Figure 6-21, an increase in TCOE results from reductions in the reactor capacity factor and thermal efficiency. In Figure 6-22, increases in fuel cycle cost result from lower thermal efficiency. A change in the reactor capacity factor has no affect on the fuel cycle cost. The VISION model coding and output were analyzed to understand the system implications from these results. Analysis of the results begins with single runs of the main VISION model using the high and low values of the assumption variables to discern their affects on TCOE and fuel cycle cost. 
Fast reactor thermal efficiency is a user input variable. VISION uses FR thermal efficiency to determine how much thermal energy generated is converted to electric energy in fast reactors. Increasing fast reactor thermal efficiency does not affect front-end costs and increases back-end costs and decreases recycling costs. The increase in LWR thermal efficiency decreases the amount of source material needed in the front-end processes and mass of material needing to be disposed. However, fast reactor thermal efficiency has no front-end cost effect because fast reactor source material is derived from LWR spent fuel. Lower FR thermal efficiency increases the mass of material needed to fulfill fast reactor energy demands. This increase in material is shown as an increase in recycling costs of LWR spent fuel. An increase in back-end costs due to increase in FR thermal efficiency is due to increased mass flow to the HLW repository. Reactor costs and electricity production are not influenced by thermal efficiency and are constant. Table 6-7 illustrates the effect of thermal efficiency on TCOE and FCC.

Table 6-7. TCOE and FCC with defining components at high and low ranges of FR thermal efficiency.

\begin{tabular}{|l|rrl|}
\hline FR Thermal Efficiency Value & 0.37 & 0.41 & Units \\
\hline Electricity Produced Yearly & $8.82 \mathrm{E}+11$ & $8.82 \mathrm{E}+11$ & $\mathrm{kWh}$ \\
\hline Total Front End & $2.13 \mathrm{E}+03$ & $2.13 \mathrm{E}+03$ & $\$ \mathrm{M}$ \\
\hline Total Back End & $1.02 \mathrm{E}+03$ & $1.06 \mathrm{E}+03$ & $\$ \mathrm{M}$ \\
Total Recycling Costs & $4.13 \mathrm{E}+03$ & $3.94 \mathrm{E}+03$ & $\$ \mathrm{M}$ \\
& $1.46 \mathrm{E}+01$ & $1.46 \mathrm{E}+01$ & $\$ \mathrm{M}$ \\
REPU & $7.49 \mathrm{E}+02$ & $7.35 \mathrm{E}+02$ & $\$ \mathrm{M}$ \\
\multicolumn{1}{|c|}{ Conditioning } & $1.09 \mathrm{E}+02$ & $1.13 \mathrm{E}+02$ & $\$ \mathrm{M}$ \\
Storage & $3.25 \mathrm{E}+03$ & $3.07 \mathrm{E}+03$ & $\$ \mathrm{M}$ \\
\hline Co Flow/FP & $3.53 \mathrm{E}+04$ & $3.53 \mathrm{E}+04$ & $\$ \mathrm{M}$ \\
Reactor Costs & 8.31 & 8.04 & $\mathrm{mills} / \mathrm{kWh}$ \\
Fuel Cycle Costs & 48.35 & 48.08 & mills/kWh \\
\hline
\end{tabular}

Capacity factor is a user input variable, which defines the availability of the reactor. Reactor power is then defined as a product of capacity factor and reactor size. An increase in capacity factor corresponds to an increase in back-end costs, an increase annual electricity production, and a decrease in recycling costs. The change in back-end cost is due to an increase in material mass to the HLW repository associated with increased energy production. Total fuel cycle cost increases are offset by the increase in electricity production leaving the fuel cycle cost unchanged with varying FR capacity factor. Table 6-8 illustrates the effect of thermal efficiency on TCOE and fuel cycle cost.

Table 6-8. TCOE and FCC with defining components at high and low ranges of FR capacity factors.

\begin{tabular}{|c|c|c|c|}
\hline FR Capacity Factor Value & 0.8 & 0.9 & Units \\
\hline Electricity Produced Yearly & $8.74 \mathrm{E}+11$ & $9.14 \mathrm{E}+11$ & $\mathrm{kWh}$ \\
\hline Total Front End & $2.13 E+03$ & $2.13 E+03$ & $\$ M$ \\
\hline Total Back End & $1.04 \mathrm{E}+03$ & $1.10 \mathrm{E}+03$ & $\$ M$ \\
\hline Total Recycling Costs & $4.26 \mathrm{E}+03$ & $4.03 E+03$ & $\$ M$ \\
\hline REPU & $1.46 \mathrm{E}+01$ & $1.45 E+01$ & $\$ M$ \\
\hline Conditioning & $7.42 E+02$ & $7.59 E+02$ & $\$ M$ \\
\hline Storage & $1.09 \mathrm{E}+02$ & $1.13 \mathrm{E}+02$ & $\$ M$ \\
\hline Co Flow/FP & $3.39 E+03$ & $3.14 \mathrm{E}+03$ & $\$ M$ \\
\hline Reactor Costs & $3.53 E+04$ & $3.53 E+04$ & $\$ M$ \\
\hline Total Cost of Electricity & 48.64 & 46.91 & mills/kWh \\
\hline
\end{tabular}

The fuel cycle cost and TCOE for the 1-Tier are illustrated for discrete values of FR conversion ratio in Figures 6-23 and 6-24, respectively. Both economic measures increase with the rising FR conversion ratio. The fuel cycle cost is at its lowest value of $7.52 \mathrm{mills} / \mathrm{kWh}$ for a FR conversion ratio of 0.25 and at its highest value of $10.54 \mathrm{mills} / \mathrm{kWh}$ for a FR conversion ratio of 1.00. TCOE is at is lowest value of $47.57 \mathrm{mills} / \mathrm{kWh}$ for a FR conversion ratio of 0.25 and at its highest value of $50.29 \mathrm{mill} / \mathrm{s} / \mathrm{kWh}$ for a FR conversion ratio of 1.00. The TCOE and fuel cycle costs given for all discrete FR conversion ratio values 
and their defining costs are shown in Table 6-9. A change in fast reactor conversion ratio corresponds to changes in recycling and back-end costs. Back-end costs consist of only HLW repository costs and are shown in Table 6-10 for the discrete conversion ratio values. As the conversion ratio increases, more fissionable isotopes are left in the FR waste causing more mass to be classified as HLW, which would increase the HLW repository rate and costs. Recycling cost has many components, and the components and their costs for the discrete conversion ratio values for the 1-Tier are shown in Table 6-11.

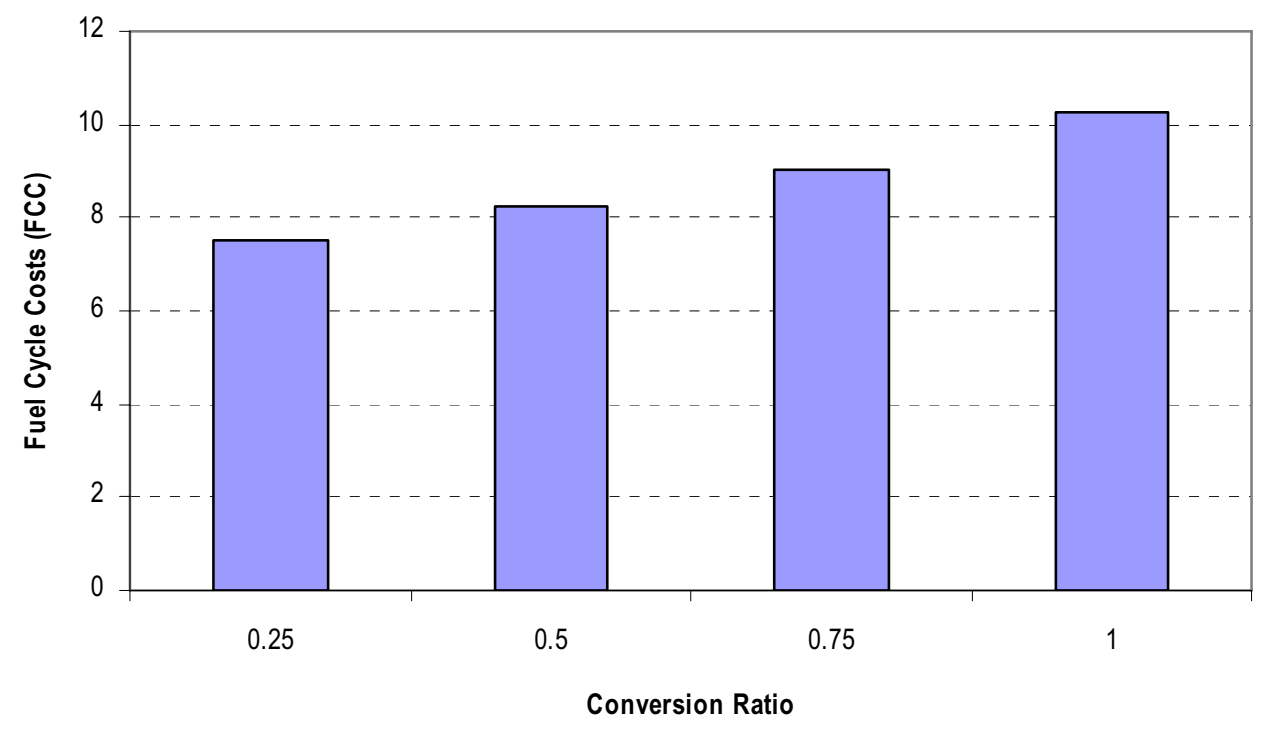

Figure 6-23. Fuel cycle costs for discrete FR conversion ratio values for 1-Tier.

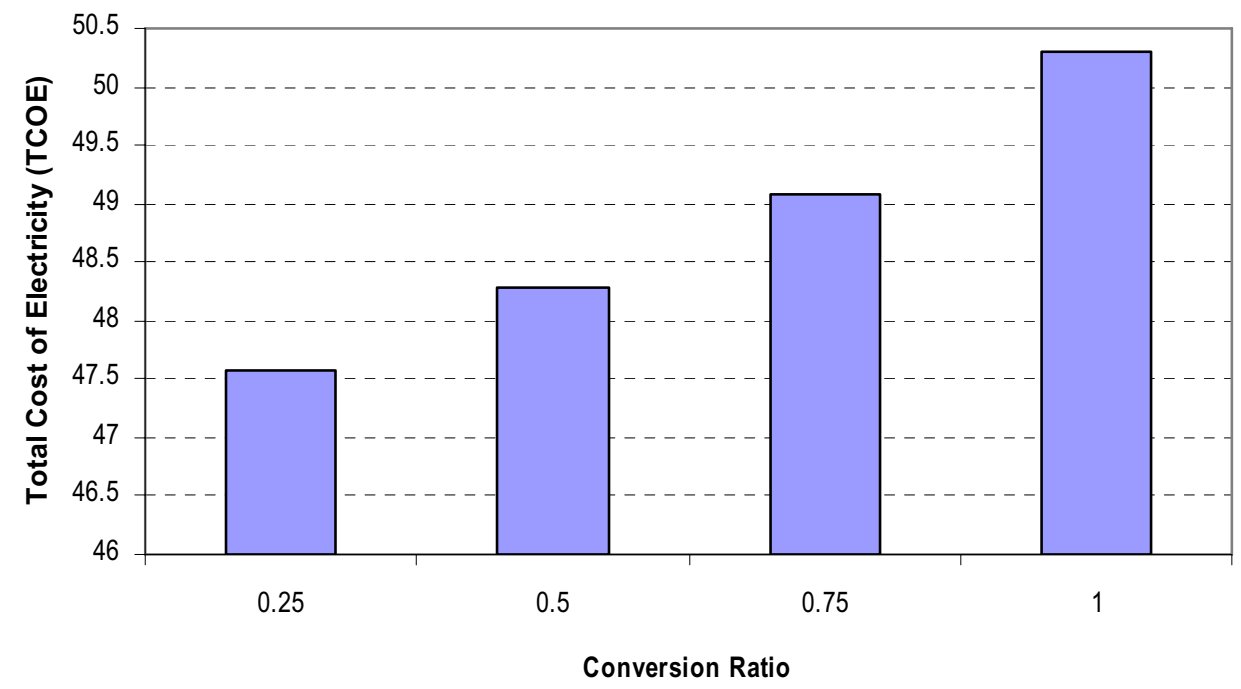

Figure 6-24. Total cost of electricity for discrete FR conversion ratios values for 1-Tier. 
Table 6-9. TCOE and FCC with defining components for discrete FR conversion ratio values for 1-Tier.

\begin{tabular}{|c|c|c|c|c|c|c|c|c|c|}
\hline & $\begin{array}{l}\text { Total Front } \\
\text { End Costs } \\
\text { each year }\end{array}$ & $\begin{array}{l}\text { Total Back } \\
\text { End Costs } \\
\text { Each Year }\end{array}$ & $\begin{array}{c}\text { Total } \\
\text { Recycling } \\
\text { Cost each } \\
\text { Year }\end{array}$ & $\begin{array}{c}\text { Fuel Cycle Cost } \\
\text { each Year }\end{array}$ & $\begin{array}{c}\text { Total } \\
\text { Reactor } \\
\text { Cost each } \\
\text { Year }\end{array}$ & $\begin{array}{l}\text { Total Cost } \\
\text { each Year }\end{array}$ & $\begin{array}{l}\text { Total Electricity } \\
\text { Produced } \\
\text { Yearly }\end{array}$ & $\begin{array}{l}\text { Fuel Cycle Costs } \\
\text { (FCC) }\end{array}$ & $\begin{array}{c}\text { Total Cost of } \\
\text { Electricity (TCOE) }\end{array}$ \\
\hline & $\$ M^{\prime} / y r$ & $\$ \mathrm{M}^{\prime} / \mathrm{yr}$ & $\$ M^{\prime} / y r$ & $\$ M^{\prime} / y r$ & $\$ \mathrm{M}^{\prime} / \mathrm{yr}$ & $\$ \mathrm{M}^{\prime} / \mathrm{yr}$ & $\mathrm{KW}(\mathrm{e})^{1 \star} \mathrm{hr} / \mathrm{yr}$ & 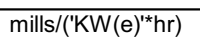 & mills/('KW(e)'t*hr) \\
\hline CR 0.25 & $2.13 \mathrm{E}+03$ & $1.02 \mathrm{E}+03$ & $3.48 \mathrm{E}+03$ & $6.63 \mathrm{E}+03$ & $3.53 \mathrm{E}+04$ & $4.19 E+04$ & $8.82 \mathrm{E}+11$ & 7.52 & 47.57 \\
\hline CR 0.50 & $2.13 \mathrm{E}+03$ & $1.05 \mathrm{E}+03$ & $4.07 \mathrm{E}+03$ & $7.26 \mathrm{E}+03$ & $3.53 E+04$ & $4.26 \mathrm{E}+04$ & $8.82 \mathrm{E}+11$ & 8.23 & 48.28 \\
\hline CR 0.75 & $2.13 \mathrm{E}+03$ & $1.10 \mathrm{E}+03$ & $4.74 \mathrm{E}+03$ & $7.97 \mathrm{E}+03$ & $3.53 E+04$ & $4.33 E+04$ & $8.82 \mathrm{E}+11$ & 9.04 & 49.09 \\
\hline CR 1.00 & $2.13 \mathrm{E}+03$ & $1.16 \mathrm{E}+03$ & $5.74 \mathrm{E}+03$ & $9.03 E+03$ & $3.53 E+04$ & $4.43 E+04$ & $8.82 \mathrm{E}+11$ & 10.24 & 50.29 \\
\hline
\end{tabular}

Table 6-10. Defining components of back end cost for discrete FR conversion ratio values for 1-Tier.

\begin{tabular}{|c|c|c|c|c|c|}
\hline & \multicolumn{3}{|c|}{$\begin{array}{c}\text { Cost of HLW in Repository by } \\
\text { Year }\end{array}$} & \multirow{2}{*}{$\begin{array}{c}\text { Total Cost of } \\
\text { HLW in } \\
\text { Repository by } \\
\text { Year }\end{array}$} & \multirow[t]{2}{*}{$\begin{array}{l}\text { Total Back } \\
\text { End Costs } \\
\text { Each Year }\end{array}$} \\
\hline & therm & Rtherm & Rfast & & \\
\hline CR 0.25 & $5.79 \mathrm{E}+02$ & $0.00 \mathrm{E}+00$ & $4.42 \mathrm{E}+02$ & $1.02 \mathrm{E}+03$ & $1.02 E+03$ \\
\hline CR 0.50 & $5.79 \mathrm{E}+02$ & $0.00 \mathrm{E}+00$ & $4.73 E+02$ & $1.05 E+03$ & $1.05 E+03$ \\
\hline CR 0,75 & $5.79 \mathrm{E}+02$ & $0.00 \mathrm{E}+00$ & $5.19 E+02$ & $1.10 \mathrm{E}+03$ & $1.10 \mathrm{E}+03$ \\
\hline CR 1.00 & $5.79 \mathrm{E}+02$ & $0.00 \mathrm{E}+00$ & $5.83 E+02$ & $1.16 \mathrm{E}+03$ & $1.16 \mathrm{E}+03$ \\
\hline
\end{tabular}

Table 6-11. Defining components of recycling costs for varying FR conversion ratios for 1-Tier.

\begin{tabular}{|c|c|c|c|c|c|c|c|c|c|}
\hline & $\begin{array}{c}\text { Aqueous UOX } \\
\text { Separations Cost }\end{array}$ & $\begin{array}{c}\text { Echem } \\
\text { Separations } \\
\text { Cost }\end{array}$ & $\begin{array}{l}\text { Total Cost of } \\
\text { Separations }\end{array}$ & $\begin{array}{l}\text { Total Cost } \\
\text { Recycled } \\
\text { Product } \\
\text { Storage }\end{array}$ & & $\begin{array}{l}\text { Cost of LLW } \\
\text { Conditioning }\end{array}$ & & $\begin{array}{l}\text { Total Cost of } \\
\text { LLW } \\
\text { Conditioning }\end{array}$ & $\begin{array}{c}\text { Cost of Near } \\
\text { Surface } \\
\text { Disposal }\end{array}$ \\
\hline & & & & & Solids & Liquids & Resins & & \\
\hline CR 0.25 & $1.32 \mathrm{E}+03$ & $9.68 \mathrm{E}+02$ & $2.29 \mathrm{E}+03$ & $1.65 E+02$ & $7.36 \mathrm{E}+00$ & $3.34 \mathrm{E}+00$ & 1.37E+01 & $2.44 \mathrm{E}+01$ & $1.90 \mathrm{E}+01$ \\
\hline CR 0.50 & $1.32 \mathrm{E}+03$ & $1.36 \mathrm{E}+03$ & $2.69 \mathrm{E}+03$ & $1.65 \mathrm{E}+02$ & $7.75 \mathrm{E}+00$ & $3.51 \mathrm{E}+00$ & $1.44 \mathrm{E}+01$ & $2.56 \mathrm{E}+01$ & $2.00 \mathrm{E}+01$ \\
\hline CR 0.75 & $1.32 \mathrm{E}+03$ & $1.80 \mathrm{E}+03$ & $3.13 E+03$ & $1.65 E+02$ & $8.17 \mathrm{E}+00$ & $3.71 \mathrm{E}+00$ & $1.52 \mathrm{E}+01$ & $2.71 \mathrm{E}+01$ & $2.11 \mathrm{E}+01$ \\
\hline CR 1.00 & $1.32 E+03$ & $2.46 \mathrm{E}+03$ & $3.79 E+03$ & $1.65 \mathrm{E}+02$ & $8.81 \mathrm{E}+00$ & $4.00 \mathrm{E}+00$ & $1.64 \mathrm{E}+01$ & $2.92 \mathrm{E}+01$ & $2.27 \mathrm{E}+01$ \\
\hline
\end{tabular}

(a)

\begin{tabular}{|c|c|c|c|c|c|c|c|c|}
\hline & $\begin{array}{c}\text { Cost of Aq Off } \\
\text { Gas }\end{array}$ & $\begin{array}{c}\text { Cost of Aq Cs } \\
\mathrm{Sr}\end{array}$ & $\begin{array}{c}\text { Cost of Echem } \\
\text { Off Gas }\end{array}$ & $\begin{array}{l}\text { Cost of Echem } \\
\text { Cs Sr }\end{array}$ & $\begin{array}{c}\text { Cost of } \\
\text { Managed Decay } \\
\text { Storage }\end{array}$ & Cost of lodine & $\begin{array}{c}\text { Cost of Coflow } \\
\text { Zr SS }\end{array}$ & $\begin{array}{l}\text { Cost of Aq } \\
\text { Glass FP LN }\end{array}$ \\
\hline \begin{tabular}{|l|} 
CR 0.25 \\
\end{tabular} & $2.30 \mathrm{E}+00$ & $5.60 \mathrm{E}+01$ & $7.54 \mathrm{E}-01$ & $3.69 \mathrm{E}+01$ & $7.51 \mathrm{E}+01$ & $5.53 \mathrm{E}+00$ & $2.15 \mathrm{E}+02$ & $1.11 \mathrm{E}+02$ \\
\hline CR 0.50 & $2.30 \mathrm{E}+00$ & $5.60 \mathrm{E}+01$ & 7.53E-01 & $3.69 \mathrm{E}+01$ & $7.52 \mathrm{E}+01$ & $5.53 E+00$ & $2.15 \mathrm{E}+02$ & $1.11 \mathrm{E}+02$ \\
\hline CR 0.75 & $2.30 \mathrm{E}+00$ & $5.60 \mathrm{E}+01$ & 7.70E-01 & $3.83 E+01$ & $7.62 \mathrm{E}+01$ & $5.53 E+00$ & $2.15 \mathrm{E}+02$ & $1.11 \mathrm{E}+02$ \\
\hline CR 1.00 & $2.30 \mathrm{E}+00$ & $5.60 \mathrm{E}+01$ & 7.76E-01 & $3.98 \mathrm{E}+01$ & $7.74 \mathrm{E}+01$ & $5.53 E+00$ & $2.15 \mathrm{E}+02$ & $1.11 \mathrm{E}+02$ \\
\hline
\end{tabular}

(b)

\begin{tabular}{|c|c|c|c|c|c|c|c|c|}
\hline & Cost of Aq Tc & $\begin{array}{l}\text { Cost of Echem } \\
\text { FP Ln }\end{array}$ & $\begin{array}{l}\text { Cost of Echem } \\
\text { Coflow Zr SS }\end{array}$ & $\begin{array}{l}\text { Cost of UOX } \\
\text { MOX } \\
\text { Conditioning }\end{array}$ & $\begin{array}{l}\text { Total Cost } \\
\text { REPU Rate }\end{array}$ & $\begin{array}{l}\text { Total Cost of } \\
\text { Conditioning }\end{array}$ & $\begin{array}{l}\text { Total Cost } \\
\text { Storage }\end{array}$ & $\begin{array}{c}\text { Total } \\
\text { Recycling } \\
\text { Cost each } \\
\text { Year }\end{array}$ \\
\hline CR 0.25 & $6.42 \mathrm{E}+01$ & $1.83 \mathrm{E}+01$ & $5.58 \mathrm{E}+01$ & $1.32 \mathrm{E}+02$ & $1.47 \mathrm{E}+01$ & $7.22 \mathrm{E}+02$ & $1.09 \mathrm{E}+02$ & $3.48 \mathrm{E}+03$ \\
\hline CR 0.50 & $6.42 E+01$ & 1.77E+01 & $7.84 \mathrm{E}+01$ & $1.32 E+02$ & 1.46E+01 & $7.45 \mathrm{E}+02$ & 1.10E+02 & 4.07E+03 \\
\hline CR 0.75 & $6.42 \mathrm{E}+01$ & $1.77 \mathrm{E}+01$ & $1.04 \mathrm{E}+02$ & $1.32 E+02$ & $1.44 E+01$ & $7.73 E+02$ & $1.12 \mathrm{E}+02$ & $4.74 \mathrm{E}+03$ \\
\hline CR 1.00 & $6.42 \mathrm{E}+01$ & $1.73 E+01$ & $1.41 \mathrm{E}+02$ & $1.32 E+02$ & $1.43 E+01$ & $8.14 \mathrm{E}+02$ & $1.14 \mathrm{E}+02$ & $5.74 \mathrm{E}+03$ \\
\hline
\end{tabular}

(c) 


\subsubsection{Further Analysis of System Effects on 1-Tier System Costs}

The next analysis analyzes the effect on a 1-Tier system when increasing the conversion ratio while simultaneously increasing the nuclear growth.

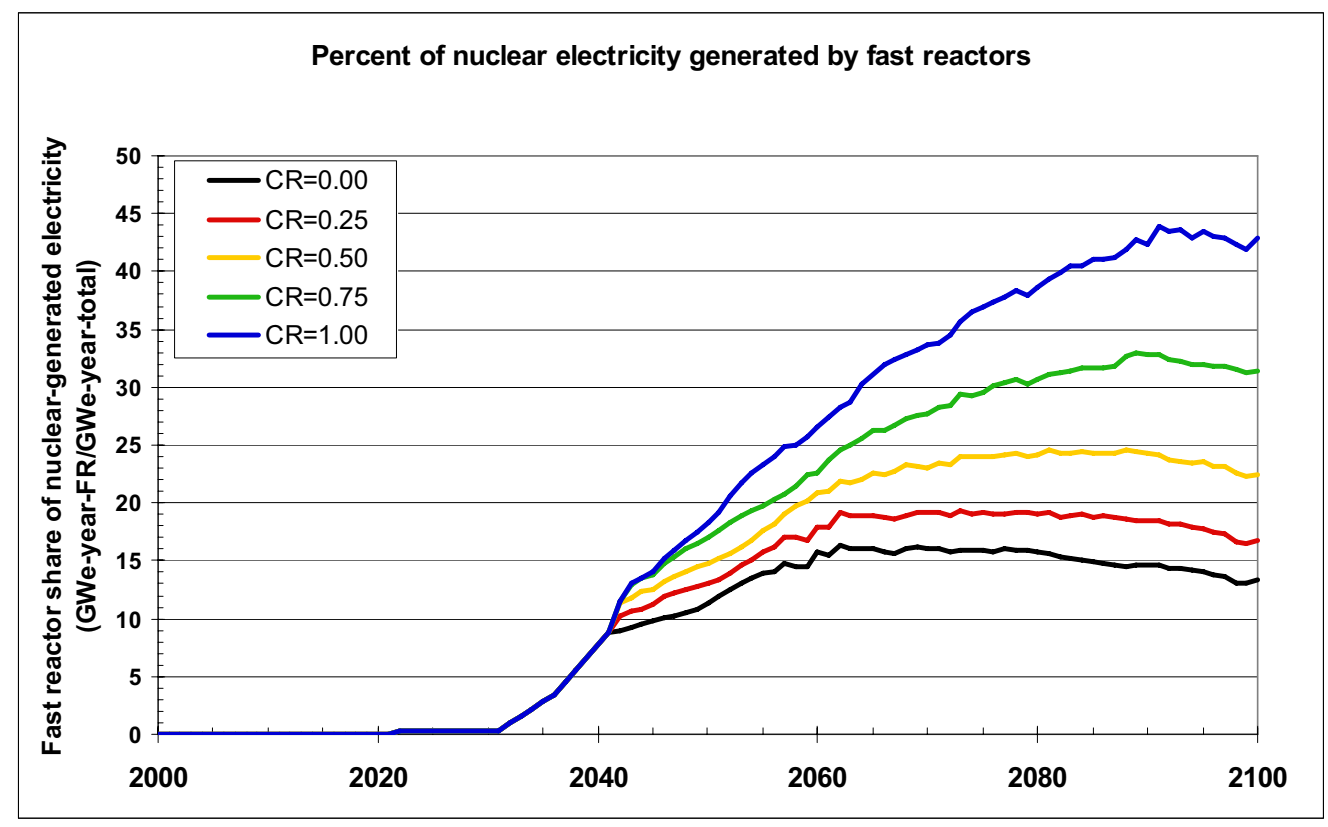

Figure 6-25. Impact on number of FRs from CR.

Figure 6-25 shows the relationships between conversion ratios and the percent of fast reactors. As the CR increases toward 1.0, the required share of fast reactors increases. For low CRs ( 0 to 0.25$)$ the share of fast reactors is in the range of $15 \%$ of the reactor fleet, nominal CRs $(0.50)$ require $20-25 \%$ of the fleet. For high CRs (0.75-1.0) the share of fast reactors would rise from over $30 \%$ to nearly $45 \%$ of the reactor fleet. Due to the premium on the capital costs for fast reactors, as the CR approaches 1.0, higher TCOE costs would result from the larger number of fast reactors.

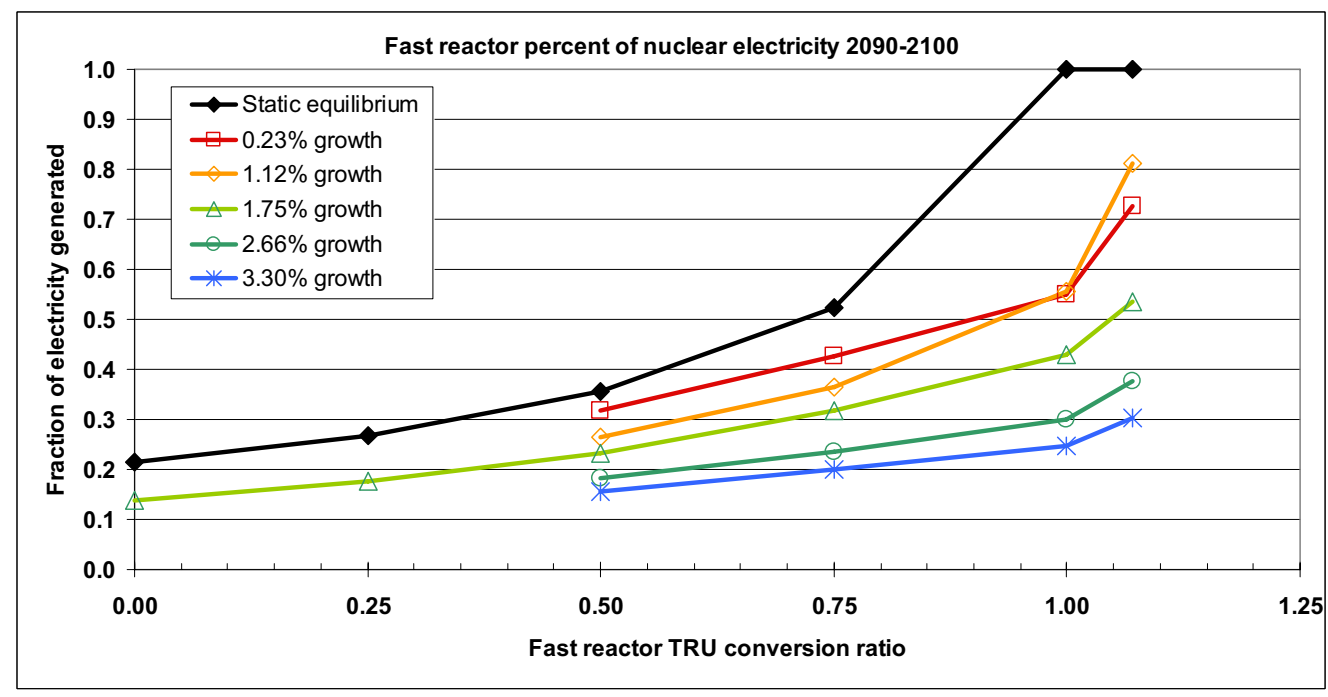

Figure 6-26. Impact on the number of FR-based on CR and growth rate. 
Figure 6-26 shows an interesting dynamic relationship between the conversion ratio, nuclear growth, and the percent of fast reactors. In a static equilibrium system, $37 \%$ of the fleet would need to be FRs if they are being run at a $\mathrm{CR}=0.50$. With growth, the actual number of FRs in the fleet would be in the range of $16 \%$ to $37 \%$ (based on growth rates from $3.3 \%$ to $0 \%$, respectively). There is a balancing effect from increasing the $\mathrm{CR}$ while also increasing the FR growth percentage. The number of fast reactors required due to high CR is increased; however, the resources (e.g., separation, fabrication) needed to support the FR growth rate and the time delays in the system tend to constrain the number of fast reactors. A consequence of these constraints is that if FRs are not available to support the required nuclear growth, then LWR reactors are used instead, hence decreasing the percentage of fast reactors. The cost impact is that the premium costs for fast reactors are not accentuated in higher nuclear growth cases due to the constraints on building additional fast reactors.
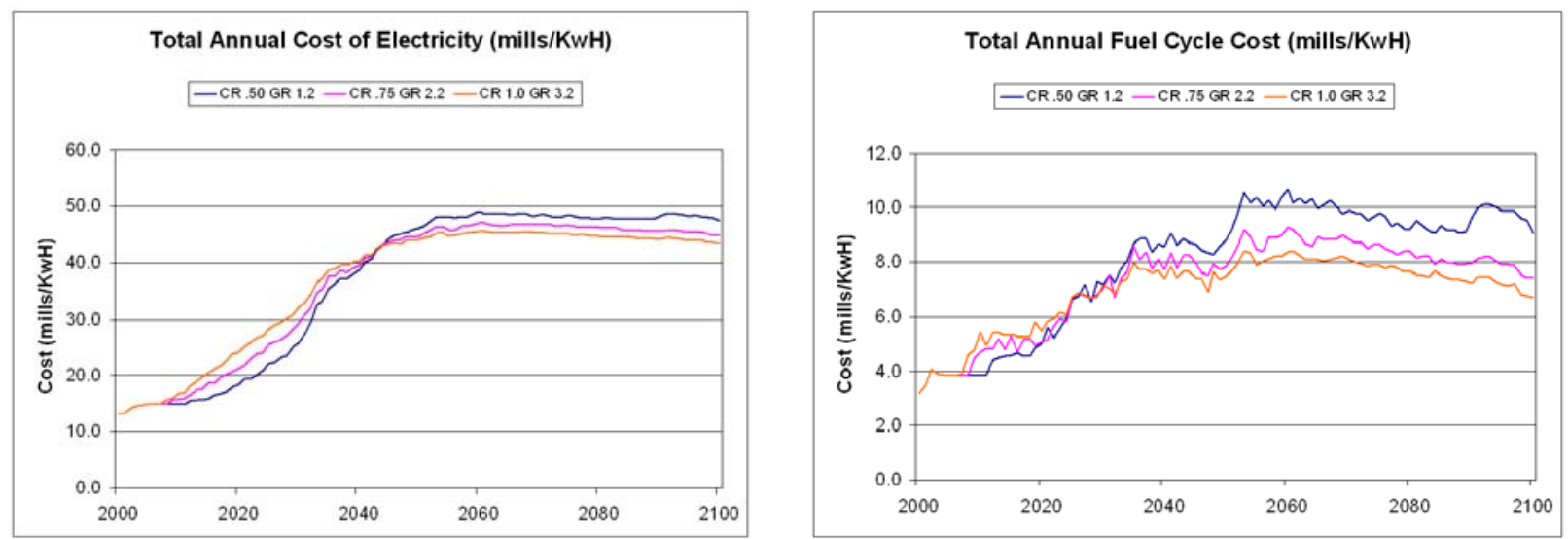

Figure 6-27. Dynamic depiction of impact on costs of CR and growth rate on system costs for 1-Tier.

Figure 6-27 shows a dynamic analysis using VISION and VISION.ECON to examine the dual affect of increasing the conversion ratio and the nuclear growth rate. Overall, the annual costs are relatively constant because the higher conversion ratios are balanced by the increased growth rates. For a high conversion ratio $(\mathrm{CR}=1.0)$ and high growth rate $(3.2 \%)$ there is actually a slight decrease in annual costs toward the end of the simulation. If there were no system constraints, then independently increasing the conversion ratio (see Figure 6-25) or the nuclear growth rate would result in higher costs due to the increased number of fast reactors.

\subsection{Economic Analysis of Cesium/Strontium Management}

An analysis was performed to assess the incremental costs for the separation, waste conditioning, managed decay storage, and final disposition of Cesium and Strontium $(\mathrm{CsSr})$. The incremental changes in the nominal costs were derived from the 1-Tier system. The related cost modules and the changes to the costs are provided in Table 6-12. Figure 6-28 shows the results from a dynamic comparison using VISION.ECON to evaluate the nominal fuel cycle cost difference between managing CsSr separately versus leaving the $\mathrm{CsSr}$ in the main waste stream. 
Table 6-12. Difference in cost from separate management of CsSr.

\begin{tabular}{|l|c|l|}
\hline \multicolumn{1}{|c|}{ Cost Module and Description } & $\begin{array}{c}\text { Delta Costs } \\
\text { (mills/kW·h) }\end{array}$ & \multicolumn{1}{c|}{ Rationale } \\
\hline E4, Managed Decay Storage & -0.422 & No decay storage required \\
\hline F1-1, UREX+1a Separation & -0.151 & Separation costs reduced by 10\%, to \$900/kgHM \\
\hline $\begin{array}{l}\text { G4-2A, Aqueous CsSr } \\
\text { Conditioning }\end{array}$ & -0.098 & No conditioning required \\
\hline G4-3E, EChem CsSr Conditioning & -0.040 & No conditioning required \\
\hline $\begin{array}{l}\text { G1-1a, Aqueous HLW } \\
\text { Conditioning }\end{array}$ & +0.025 & CsSr added to FP+Ln \\
\hline G1-2E, EChem HLW Conditioning & +0.003 & CsSr added to HLW metal alloy (ZrSS+Tc) \\
\hline L2-1 Geologic Repository HLW & +0.177 & Cs/Sr wastes disposed as HLW \\
\hline Total Delta \$ & $\mathbf{- 0 . 5 0 6}$ & \\
\hline
\end{tabular}

The static cost comparison suggests a potential nominal cost reduction of $0.5 \mathrm{mills} / \mathrm{kW} \cdot \mathrm{h}$ from the 1 -Tier costs if CsSr is not separately managed. The likely range of savings is $0.25-0.75 \mathrm{mill} / \mathrm{s} / \mathrm{kW} \cdot \mathrm{h}$, and appears reasonable based on the results from the dynamic analysis shown in Figure 6-28. If managed storage costs were to be significantly higher at $\$ 50 \mathrm{~K} / \mathrm{kg} \mathrm{CsSr}$, then the cost difference would increase to $1 \mathrm{mill} / \mathrm{kW} \cdot \mathrm{h}$. The cost basis for the 300-year storage is provided in Appendix A.

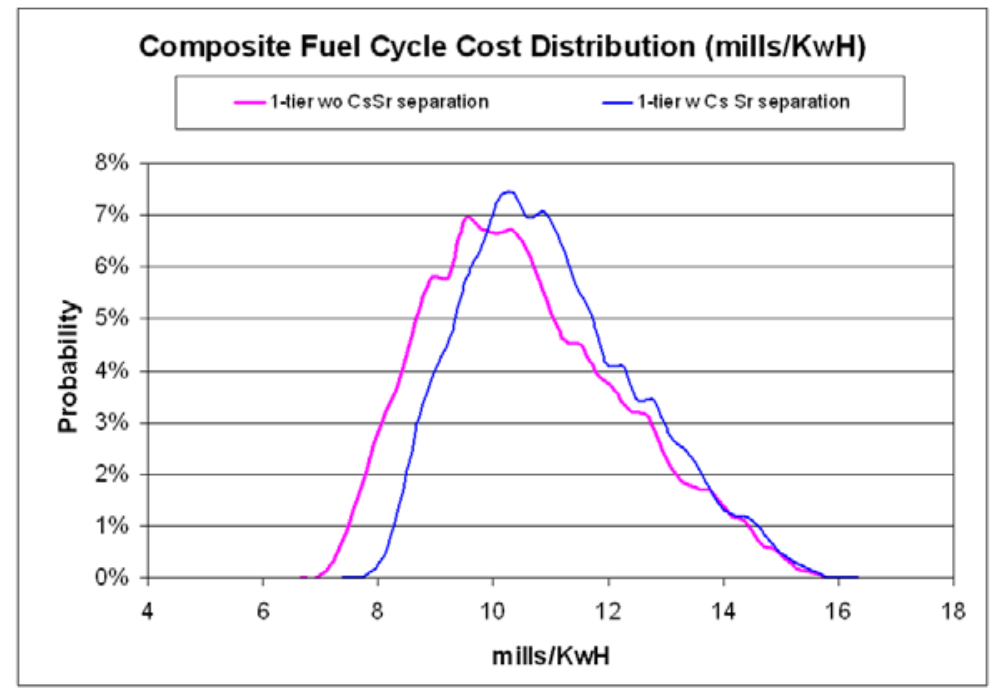

Figure 6-28. Comparison of the composite fuel cycle cost for the comparison of the dynamic cases with and without $\mathrm{CsSr}$ removal.

\subsection{Economic Trade-off Parameter Space Analysis}

The G4 ECONS (Williams 2007) spreadsheet models were developed for once-through, 1-Tier, and 2-Tier systems as described in Section 2 and shown in detail in Attachment A. The cost variable uncertainty was evaluated in Section 6.2, where the DPL software was used to identify the cost measures that have the potential to make a strategy the optimal (lowest levelized unit of electricity cost [LUEC]) policy. These models were utilized to determine under what conditions the different fuel cycles would be the economically preferred option. This section looks at what it would take to close or at least reduce this 
gap over the very wide parameter space. In preparation of these trade-off analysis, there were some minor modifications to the nominal cases in the 2 -Tier. ${ }^{\mathrm{i}}$

There are a wide range of cost measures that can affect the total costs of electricity. The module cost ranges for this analysis are provided in Section 4. This section looks at different hypothetical scenarios that could affect the relative cost of the different potential fuel cycles. A parameter or set of parameters are varied and the relative cost of the different fuel cycles is calculated. Based on the current nominal values, it is unlikely that a single parameter (e.g., uranium prices) will result in a change in the economically preferred option. However, a combination of factors could do this. For example, high performance fast reactors (low capital, high capacity, and high efficiency) along with rising uranium price and expensive repository costs for SNF disposal could result in a competitive market for closed fuel cycles. Also, cost improvements in the MOx thermal recycling processes and FR recycle processes (e.g., commercial EChem facilities) can help to close the gap between the closed fuel cycle and the once-through. Many of the important costs for the fast reactor are highly uncertain because they have limited or no commercial experience. Currently, no intact SNF has been disposed in a geologic repository anywhere in the world. There is limited experience in building and operating fast reactors, especially on a commercial basis. What will future fast reactors cost, how will they compare to ALWR costs, and to other baseload electricity generation sources? These questions could substantially change the assumption about the relative costs for the different fuel cycles.

For the trade-off analysis, simplified cost equations were derived for a single LWR using the once-through cycle and a fast reactor in a closed fuel cycle. Additionally, equations were developed for a 1-Tier system that includes a symbiotic ratio of LWRs to FRs based on the conversion ratio. These system levels costs are described in terms of the total cost of electricity (TCOE). ${ }^{j}$ The variables defined for this analysis are:

$$
\begin{aligned}
& C_{\text {Overnight }}^{L W R}=\text { Overnight capital cost of the LWR }(\$ / \mathrm{kWe})-\text { Module R1 } \\
& C_{\text {Overnight }}^{F R}=\text { Overnight capital cost of the fast reactor }(\$ / \mathrm{kWe})-\text { Module R2 } \\
& C_{N U}=\text { Cost of natural uranium }(\$ / \mathrm{kg} \mathrm{U})-\text { Module } \mathrm{A} \\
& C_{S N F}=\text { Cost of in-tact SNF disposal }(\$ / \mathrm{kg} \mathrm{HM})-\text { Module L1 } \\
& C R=\text { Conversion ratio of fast reactor (values } 0.0 \text { to } 1.0 \text { ) } \\
& C_{r e p}^{U O X}=\text { Cost of recycling spent UOx fuel }(\$ / \mathrm{kg} \mathrm{HM})-\text { Module F1, K2, E3, G3, G4, E4, G1, M1, } \\
& \text { L2 } \\
& C_{\text {rep,manu }}^{F R}=\text { Cost of recycling and remanufacturing fast reactor fuel (\$/kg HM) - Module F2/D2 }
\end{aligned}
$$

i. There were two minor modifications to the nominal cases in the 2-Tier. The first was the average discharge burnup of the MOx fuel was $50 \mathrm{MWd} / \mathrm{kg}$ instead of the $51 \mathrm{MWd} / \mathrm{kg}$ for the UOx fuel. The second was to use the mass flow data for the 1Tier in the 2-Tier cases. There was only data available for the conversion ratio of 0.5 for the 2-Tier fast reactor, so this assumption was necessary to study conversion ratio impacts. The net effect was to change the nominal Total Cost of Electricity (TCOE) for the 2-Tier from $47.86 \mathrm{mills} / \mathrm{kW} \cdot \mathrm{h}$ to $48.01 \mathrm{mills} / \mathrm{kW} \cdot \mathrm{h}$. Utilizing all of the nominal values in G4 ECONS leads to a cost disadvantage of $5.87 \mathrm{mills} / \mathrm{kW} \cdot \mathrm{h}(6.1 \mathrm{mills} / \mathrm{kW} \cdot \mathrm{h}$ including uncertainty) for the 1-Tier relative to the once-through and a cost disadvantage of $5.62 \mathrm{mills} / \mathrm{kW} \cdot \mathrm{h}(5.7 \mathrm{mills} / \mathrm{kW} \cdot \mathrm{h}$ including uncertainty $)$ for the 2 -Tier relative to the once-through.

j. Some data results in fitting reactor performance parameters (e.g., average discharge burnup, TRU makeup). These parameters were set to exactly reproduce the reference values, which are based on the $\mathrm{CR}=0.5$. 


$$
\begin{gathered}
B U_{F R}=\text { Conversion ratio dependent average discharge burnup of fast reactor fuel } \\
(\mathrm{MWd} / \mathrm{MTIHM}) .
\end{gathered}
$$

Equation 6.8-1 provides the levelized unit of electricity cost (LUEC) of a single LWR reactor using the once-through fuel cycle. ${ }^{\mathrm{k}}$

$$
L U E C_{\text {OTC }}=0.01125 * C_{\text {Overnight }}^{L W R}+9.91+2.8435+0.02111 * C_{N U}+C_{S N F}
$$

Equation 6.8-2 provides the LUEC of a single FR using a closed fuel cycle. ${ }^{1}$

$$
L U E C_{F R}=0.01234 * C_{\text {Overmight }^{F R}}^{F}+11.46+0.00814 *(1-C R)^{*} C_{r e p}^{U O X}-\frac{f_{L W R}}{1-f_{L W R}} C_{S N F}+\frac{109.65}{B U_{F R}}\left(C_{r e, \text { manu }}^{F R}+530+0.016359 * B U_{F R}\right) \quad \text { Eq. 6.8-2 }
$$

Equation 6.8-3 provides the total system TCOE (including LWRs and FRs) in a 1-Tier fuel cycle.

$$
T C O E_{1-T i e r}=f_{L W R} * L U E C_{\text {OTC }}+\left(1-f_{L W R}\right) * L U E C_{F R}
$$

Equation 6.8-4 provides the fraction of the energy generated in the 1-Tier fuel cycle that is produced by the LWRs. Similar equations could be developed for the 2-Tier fuel cycle. ${ }^{\mathrm{m}}$ Using this equation, it shows that a $\mathrm{CR}=1.0$ will result in a need for zero LWRs, versus a $\mathrm{CR}=0.0$ results in a ratio of $79 \%$ LWRs in the fleet of reactors.

$$
f_{L W R}=\frac{3.3866 *(1-\mathrm{CR})}{(3.3866 *(1-\mathrm{CR})+1)}
$$

G4 ECONS was used to perform the calculations and the results were compared to this parametric analysis. The TCOE for the 1-Tier does not deviate by more than $2 \%$ from the value calculated by G4 ECONS for the nominal parameters or the conversion ratios when varying the ratio from 0.0 to 1.0. Each of the constants can be broken down further to include the variables from the cost database or performance parameters.

For the system costs (based on the TCOE) of the 1-Tier to equal the system costs using LWRs in a once-through cycle, the 1-Tier fuel cycle (based on the LUEC) must also equal the open cycle. By setting Equation 6.8-1 equal to Equation 6.8-2, the parameter space where they would be equal can be determined. Even in these simplified equations, there are eight independent variables. Each constant embodies several more independent variable (e.g., the constant 2.8435 in Equation 6.8-1 includes conversion, enrichment, and manufacturing costs) that are assumed constant for this analysis.

The sources of difference can be lumped into different categories. Equation 6.8-5 breaks down the cost difference between the once-through and the FR. Equation 6.8-6 provides the TCOE difference between the 1-Tier and once-through. As can be seen, the sole source of the difference is a result of differences between the LUEC of the FR with the once-through because it is an energy-weighted average of this difference. The results are summarized in Table 6-13.

k. The value "9.91" mills/kWh is the O\&M (Module R1). The value " 2.8435 " mills/kWh is the other front-end costs (Modules $\mathrm{B}, \mathrm{C}, \mathrm{D} 1-1$, and K1).

1. The value " 11.46 " mills/kWh is the O\&M cost (Module R2). The value of " 0.00814 " mills/kWh includes the LWR recycling costs (Modules F1, K2, E3, G3, J, G4-2A, G4-1A, G4-4A, G4-5A, E4, G1, M1, L2-1). The "530" is the normalized cost of hulls disposal (Module L2-2). The 0.016359 is the HLW disposal (Module L2-1). The 109.65 is the fitting parameter to determine the quantity of FR fuel that is required each year. The units are normalized to give the appropriate units for cost (mills/kWh).

$\mathrm{m}$. The 3.3866 is the fitting parameter that balance the mass of TRU per unit energy produced by the LWRs with the amount of makeup TRU required per unit energy for the FRs. 
The results show that the difference between the FR and the once-through actually decreases with conversion ratio. However, the model assumes reactor costs and performance are independent of CR, while it is likely that their will be some decline in capacity factor and increase in capital costs as the conversion ratio is decreased. Most cost estimates are based on designs developed for CR from 0.8 to 1.2. For the fast reactor to be directly competitive with an LWR, the conversion ratio will likely be a selfsufficient design (CR slightly $>1.0$ ) because of the cost of utilizing spent LWR fuel as makeup feed as can be seen by the decreasing cost difference for utilizing UOx spent fuel.

For the 1-Tier fuel cycle, the steady state mixture of LWRs and fast reactors will determine the system-wide average cost. The fraction of the energy generated by LWRs decrease with increasing conversion ratio and is zero at conversion ratio of unity. Because of the large cost advantage of the LWRs over the fast reactors, the cost of the 1-Tier system will be a minimum when the LWR fraction is a maximum, which occurs at a conversion ratio of zero. However, design and technology necessary to operate fast reactors at low conversion ratios has not been demonstrated. This makes this conclusion very sensitive to any potential cost penalties that may result as fast reactors are developed for low conversion ratios. For the nominal case analysis for the 1 -Tier, the fast reactor conversion ratio is 0.50 , which relates to a cost difference around $6 \mathrm{mills} / \mathrm{kW} \cdot \mathrm{h}$.

$$
\begin{aligned}
& \Delta_{F R-O T C}=L U E C_{F R}-L U E C_{\text {OTC }}=\Delta_{\text {cap }}+\Delta_{O \& M}+\Delta_{U O X S N F}+\Delta_{\text {fuel }} \\
& \Delta_{\text {cap }}=0.01234 * C_{\text {Overmight }}^{F R}-0.01125 * C_{\text {Overnight }}^{L W R} \\
& \Delta_{\text {O\&M }}=11.46-9.91 \\
& \Delta_{U O X S N F}=0.00814 *(1-C R) * C_{r e p}^{U O X}-\frac{f_{L W R}}{1-f_{L W R}} C_{S N F}-C_{S N F} \\
& \Delta_{\text {fuel }}=\frac{109.65}{B U_{F R}}\left(C_{r e p, \text { manu }}^{F R}+530+0.016359 * B U_{F R}\right)-2.8435-0.2111 * C_{N U} \\
& \Delta_{1-\text { Tier-OTC }}=L U E C_{1-\text { Tier }}-L U E C_{\text {OTC }} \\
& \Delta_{1-T i e r-O T C}=f_{L W R} * L U E C_{\text {OTC }}+\left(1-f_{L W R}\right) * L U E C_{F R}-L U E C_{\text {OTC }} \\
& \Delta_{1-\text { Tier-OTC }}=L U E C_{F R}-L U E C_{\text {OTC }}-f_{L W R} *\left(L U E C_{F R}-L U E C_{\text {OTC }}\right) \\
& \Delta_{1-\text { Tier-OTC }}=\left(1-f_{L W R}\right) * \Delta_{F R-O T C}
\end{aligned}
$$

Table 6-13 shows the cost differences between the open and closed fuel cycle over the range of conversion ratios. The cost components are broken down into the differences in reactor capital, reactor O\&M, back-end costs for disposal, and front-end costs for UOx or fast reactor fuel. Note that the reactor cost differences are unchanged throughout the range of the conversion ratios. However, the reactor cost differences account for $61 \%-75 \%$ of the total gap. The fuel cycle cost differences make up the remaining $25 \%-39 \%$ of the gap. 
Table 6-13. Difference in LUEC between 1-Tier closed fuel cycle and once-through.

\begin{tabular}{|c|c|c|c|c|c|c|c|}
\hline $\mathrm{CR}$ & $\Delta_{\text {cap }}$ & $\Delta_{\text {O\&M }}$ & $\Delta_{\text {UOXSNF }}$ & $\Delta_{\text {fuel }}$ & $\Delta_{\text {FR }- \text { OTC }}$ & $f_{\text {LWR }}$ & $\Delta_{1-\text { Tier }- \text { OTC }}$ \\
\hline 0.0 & 9.93 & 1.55 & 7.77 & -0.40 & 18.85 & 0.79 & 3.91 \\
\hline 0.1 & 9.93 & 1.55 & 6.98 & -0.01 & 18.45 & 0.78 & 4.08 \\
\hline 0.2 & 9.93 & 1.55 & 5.86 & 0.54 & 17.88 & 0.76 & 4.35 \\
\hline 0.3 & 9.93 & 1.55 & 4.64 & 1.11 & 17.24 & 0.73 & 4.72 \\
\hline 0.4 & 9.93 & 1.55 & 3.53 & 1.62 & 16.63 & 0.69 & 5.13 \\
\hline $\mathbf{0 . 5}$ & $\mathbf{9 . 9 3}$ & $\mathbf{1 . 5 5}$ & $\mathbf{2 . 0 6}$ & $\mathbf{2 . 2 8}$ & $\mathbf{1 5 . 8 1}$ & $\mathbf{0 . 6 3}$ & $\underline{\mathbf{5 . 8 7}}$ \\
\hline 0.6 & 9.93 & 1.55 & 1.16 & 2.81 & 15.46 & 0.58 & 6.54 \\
\hline 0.7 & 9.93 & 1.55 & 0.05 & 3.47 & 15.01 & 0.49 & 7.70 \\
\hline 0.8 & 9.93 & 1.55 & -0.94 & 4.23 & 14.77 & 0.37 & 9.35 \\
\hline 0.9 & 9.93 & 1.55 & -1.73 & 5.09 & 14.84 & 0.22 & 11.55 \\
\hline 1.0 & 9.93 & 1.55 & -2.50 & 6.21 & 15.18 & 0.00 & 15.18 \\
\hline a. Reference values. & \multicolumn{7}{l}{} \\
\hline
\end{tabular}

Figure 6-29 shows the results plotted from Table 6-13 based on the simplifying equations in this section. The results show that these equations provide a quick method to evaluate relative differences and necessary values to achieve an economical 1-Tier closed fuel cycle. The results show that the cost gap for the 1-Tier is the smallest at low conversion ratios (e.g., $\mathrm{CR}=0.0$ results in a cost gap of $3.9 \mathrm{mills} / \mathrm{kWh}$ ). As the CR is increased toward 1.0, the number of fast reactors is increased, which in turn increases the cost gap between the closed cycle and the open cycle. The most likely conversion ratios that FRs would operate are around $\mathrm{CR}=0.50$, which centers the cost gap at $\sim 6 \mathrm{mills} / \mathrm{kWh}$.

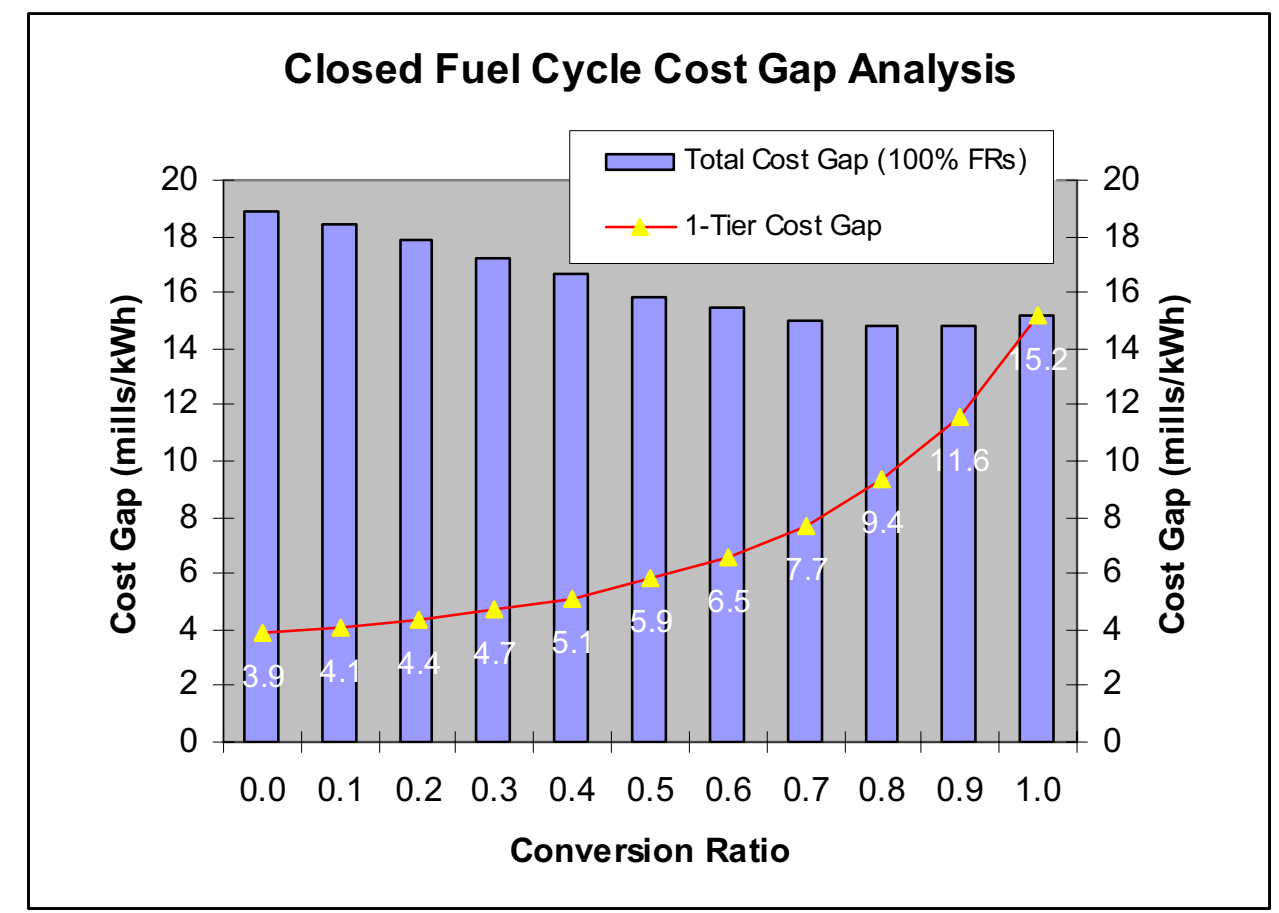

Figure 6-29. Total cost gap and 1-Tier cost gap as a function of conversion ratio. 
The important question is how these cost differences are overcome while still closing the fuel cycle and is it more effective to use a single pass of MOx (2-Tier) or recycle directly in to the fast reactor (1-Tier). There are many other variations, but these are the primary options with other options generally being deviations or modifications of these options.

\subsubsection{Overnight Capital Cost}

The dominant factor is the overnight fast reactor capital cost. The FR capital cost was determined to be $60 \%$ of the TCOE in Section 5. The "AFC Cost Basis" report indicates that the nominal cost of a fast reactor is $26 \%$ higher than the LWR (i.e., $\$ 2,900 / \mathrm{kWe}$ versus $\$ 2,300 / \mathrm{kWe}$ ). This difference in reactor costs accounts for $60-80 \%$ of the cost gap between the once-through and the closed fuel cycles. There are a number of other fast reactor handicaps in the nominal analysis including an assumed higher O\&M cost and lower capacity factors. If the cost gap is closed, the cost difference between reactors would be reduced by $60-80 \%$. The validity of these assumptions will be critical to evaluating the costs, but with limited operational experience of the fast reactors these will not be resolved any time soon. The objectives of the fast reactor design teams are to make the fast reactor as economical as possible and there are paper designs that purport to reduce the fast reactor costs to levels below that of the LWRs.

In order for the gap to be closed based solely on the overnight capital cost, the FR must have a cost advantage of $-5.87 \mathrm{mills} / \mathrm{kW} \cdot \mathrm{h}$. Equation $6.8-7$ shows the relative cost required for the once-through and 1-Tier fuel cycle to have the same cost using nominal values for all cost modules, except the overnight capital cost. Based on the nominal overnight cost of the FR $(\$ 2,900 / \mathrm{kWe})$, the overnight cost of the LWR would have risen from $\$ 2,300 / \mathrm{kWe}$ to approximately $\$ 3,700$ per $\mathrm{kWe}$, which is above the high end of the cost range for the LWR overnight cost. Additionally, whatever factors (construction materials, large forgings, etc.) causing the LWR reactor costs to rise would likely have an associated affect on the FR. It is very unlikely that the costs for the LWR would rise independent of the costs of the FR.

$$
\Delta_{\text {cap }}=-5.88=0.01234 * C_{\text {Overnight }}^{F R}-0.01125 * C_{\text {Overnight }}^{L W R}
$$

Figure 6-30 shows the breakeven overnight cost for once-through compared with the 1-Tier fuel cycle. The values show a nominal cost difference of $5.87 \mathrm{mills} / \mathrm{kW} \cdot \mathrm{h}$. When using all nominal values, except for the overnight cost, the overnight cost of the fast reactor would have to be approximately $75 \%$ of that of the LWR.

Figure 6-31 shows the breakeven overnight cost for once-through compared with the 2-Tier fuel cycle. The values are closer together with a nominal difference of $5.62 \mathrm{mills} / \mathrm{kW} \cdot \mathrm{h}$. However, since the there is only a small fraction of the energy generated $(27 \%$ versus $37 \%)$ by fast reactors in the 2-Tier fuel cycle relative to the 1-Tier fuel cycle, the cost of the 2-Tier is less sensitive to the fast reactor capital cost.

Figure 6-32 shows the breakeven overnight cost for 2-Tier compared with the 1-Tier fuel cycle. The values are much closer together with a nominal difference of $0.25 \mathrm{mills} / \mathrm{kW} \cdot \mathrm{h}$. This difference is within the cost uncertainties and shows that even if the fast reactor has an inherently higher overnight cost, the 1-Tier cycle will be competitive with the 2-Tier fuel cycle. 


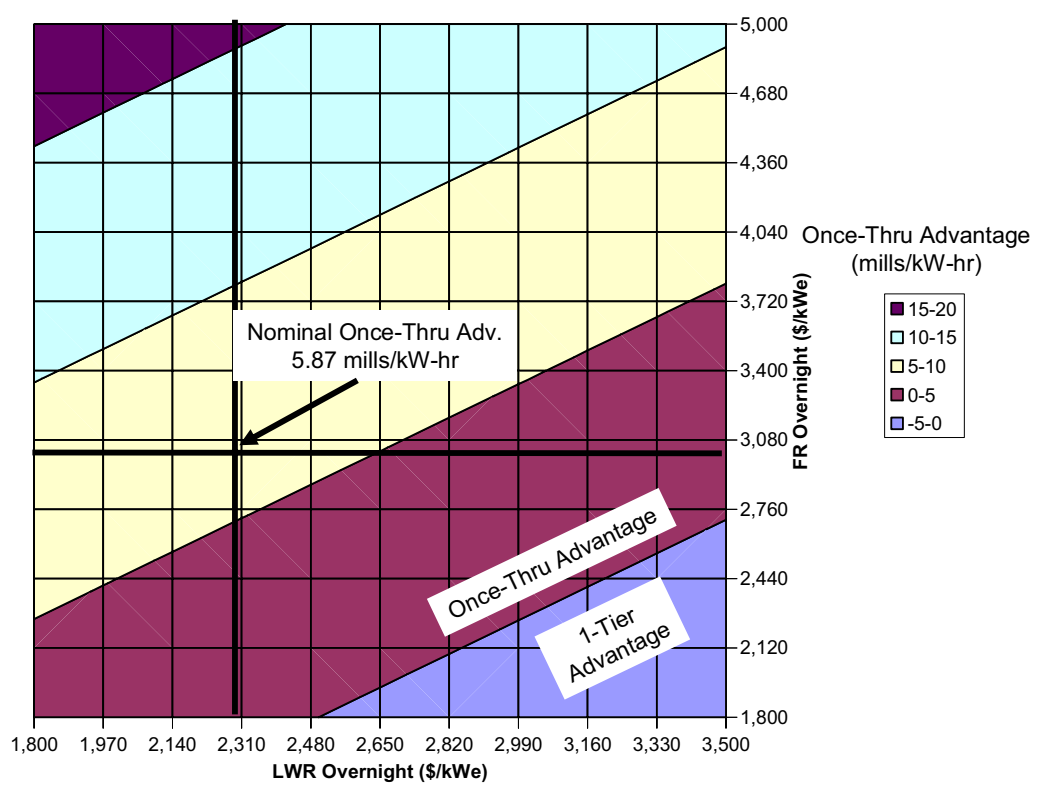

Figure 6-30. Cost sensitivity to the overnight cost for the once-through and 1-Tier fuel cycle.

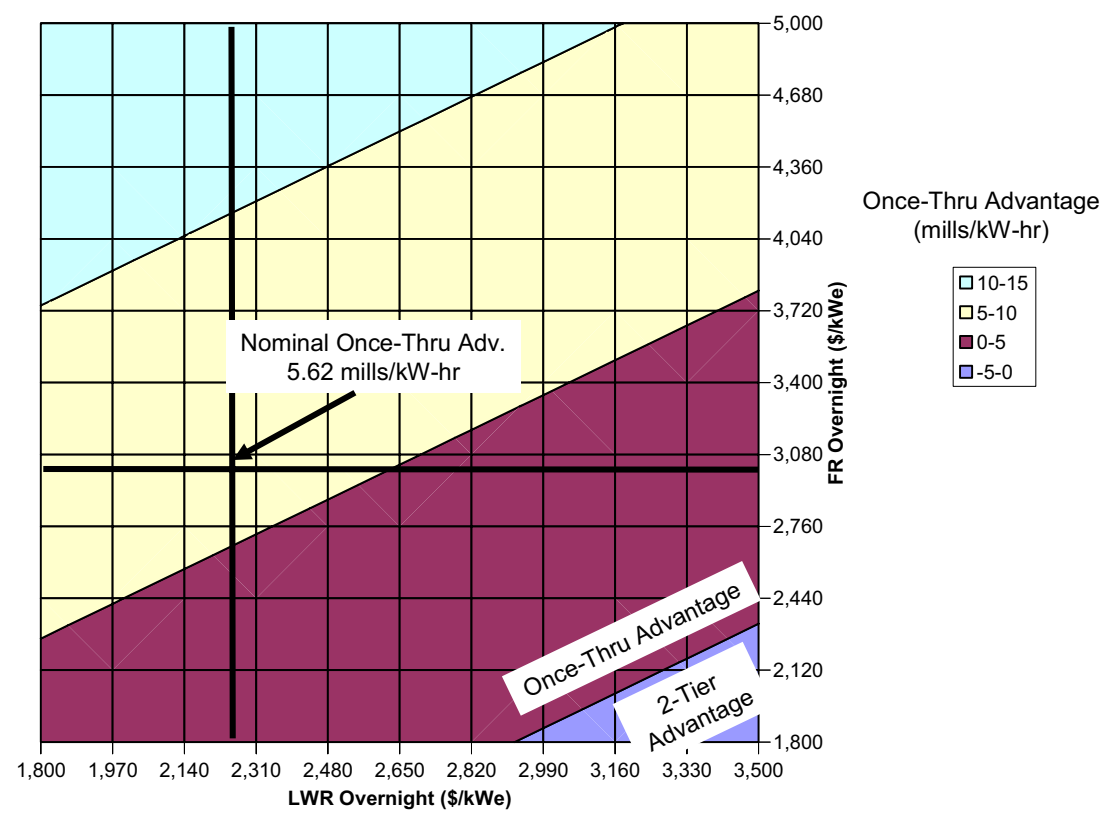

Figure 6-31. Cost sensitivity to the overnight cost for the once-through and 2-Tier fuel cycle. 


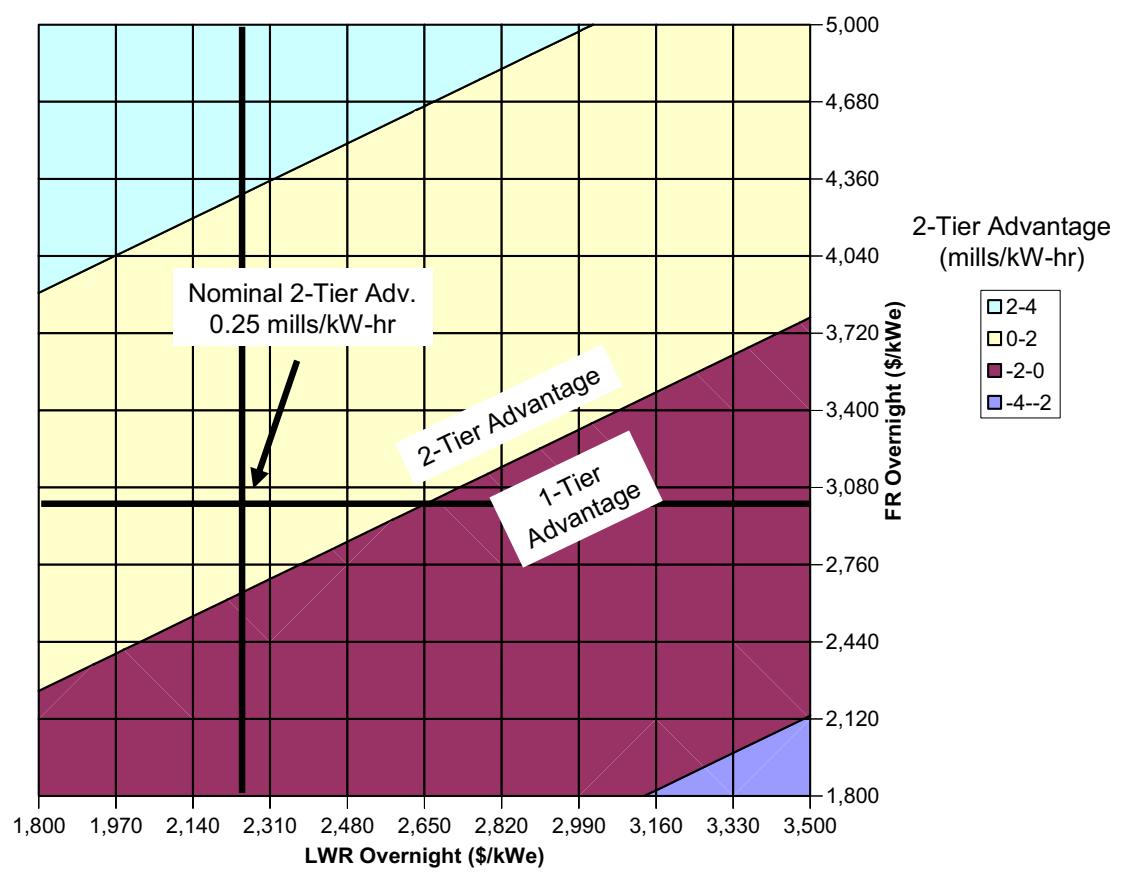

Figure 6-32. Cost sensitivity to the overnight cost for the 1-Tier and 2-Tier closed fuel cycles.

\subsubsection{LWR Front-end Costs}

A typical parameter evaluated is the cost of natural uranium that would be required to change the economics in favor of fast reactors. Natural uranium mining and milling is about $19 \%$ of the fuel cycle costs and is only a small fraction $(\sim 3 \%)$ of the TCOE for the once-through fuel cycle. Uranium costs alone would have to rise to over $\$ 800$ per $\mathrm{kgU}$ if all other costs were left at their nominal values. The entire front-end costs for the LWR is approximately 3 times the natural uranium costs. The front-end costs vary from their minimum value $(2.4 \mathrm{mills} / \mathrm{kW} \cdot \mathrm{h})$, and nominal value $(3.9 \mathrm{mills} / \mathrm{kW} \cdot \mathrm{h})$, to maximum value of $9.1 \mathrm{mills} / \mathrm{kW} \cdot \mathrm{h}$. The TCOE difference between a closed fuel cycle with $100 \%$ FRs and the oncethrough cycle is $15.8 \mathrm{mills} / \mathrm{kW} \cdot \mathrm{h}$. If the once-through front-end costs were to increase to their maximum values, this would reduce the gap by a maximum of $5.2 \mathrm{mill} / \mathrm{s} / \mathrm{kW} \cdot \mathrm{h}$. A substantial rise in all the front-end costs (to the high cost range) would reduce the cost differences between the once-through and the 1-Tier by about $30 \%$.

Figure 6-33 shows the sensitivity to the LWR front-end costs (Modules A, B, C, D1-1, and K1) and overnight capital cost for once-through compared with the 1-Tier fuel cycle. The overnight capital cost is provided as a ratio of the FR overnight cost to the LWR overnight cost and was evaluated at the nominal value for the LWR. The results show that the relative TCOE is not very sensitive to the LWR front-end cost, even when varying the LWR front-end cost to the maximum value for all modules.

Figure 6-34 shows the sensitivity to the LWR front-end costs (Modules A, B, C, D1-1, and K1) and overnight capital cost for once-through compared with the 2-Tier fuel cycle. The difference is more sensitive to the LWR front-end costs because of the slightly higher fraction of the energy derived from UOx LWRs.

Figure 6-35 shows the sensitivity to the LWR front-end costs (Modules A, B, C, D1-1, and K1) in combination with the overnight capital cost for 1-Tier and 2-Tier closed fuel cycle. The difference is fairly insensitive, with a diminished cost advantage for the 2-Tier over the 1-Tier when the front-end costs are high, due to the 2-Tier greater dependence on UOx-fueled LWRs. 


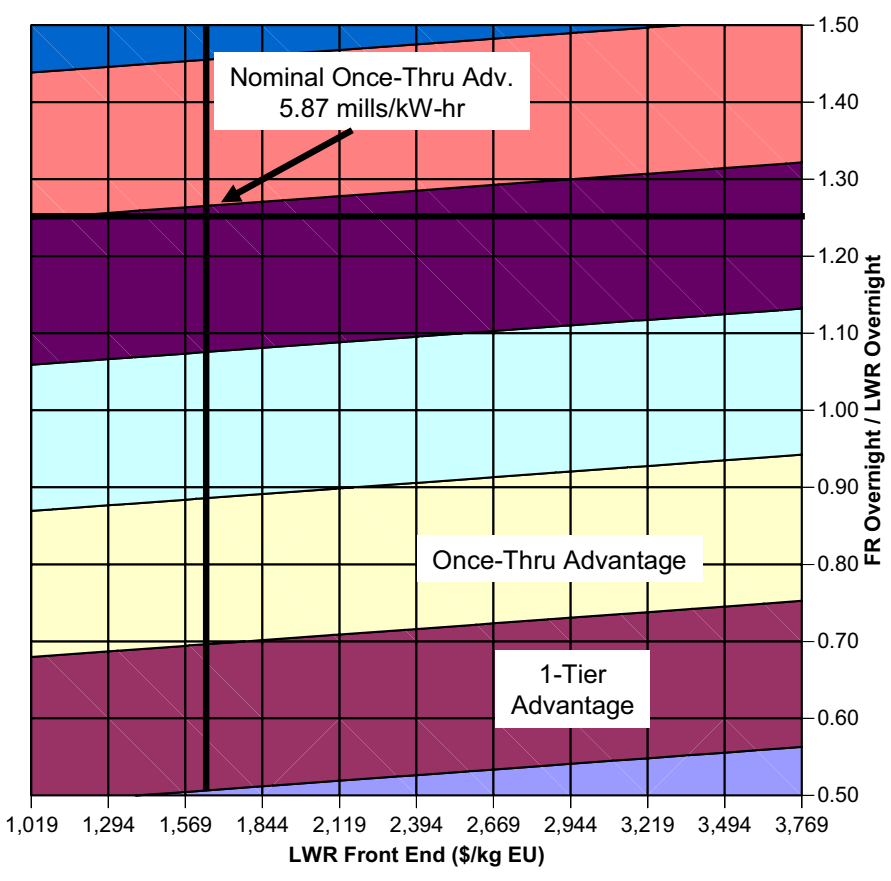

Once-Thru Advantage (mills/kW-hr)

\begin{tabular}{|l|}
\hline$\square 8-10$ \\
$\square 6-8$ \\
$\square 4-6$ \\
$\square 2-4$ \\
$\square 0-2$ \\
$\square-2-0$ \\
$\square-4--2$ \\
\hline
\end{tabular}

Figure 6-33. Cost sensitivity to the LWR front-end and overnight capital cost for the once-through and 1-Tier fuel cycle.

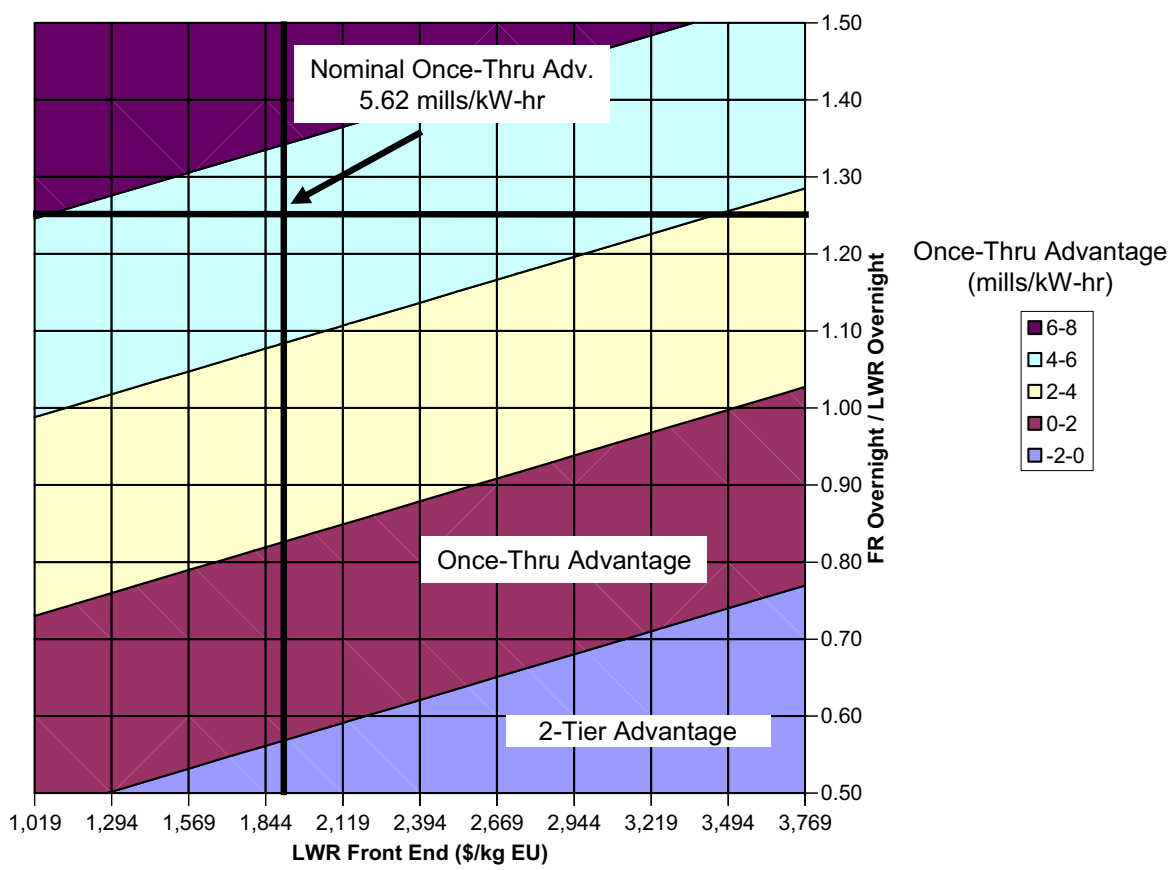

Figure 6-34. Cost sensitivity to the LWR front-end and overnight capital cost for the once-through and 2-Tier fuel cycle. 


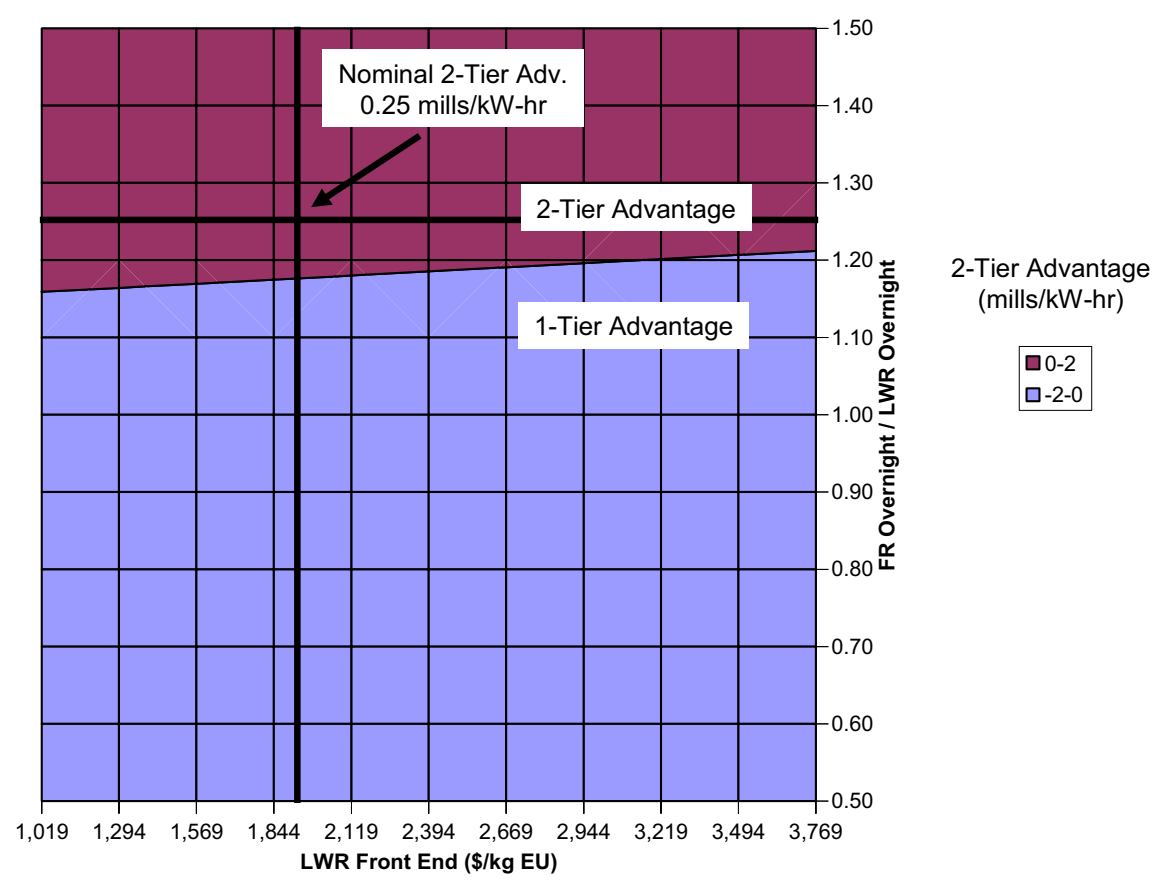

Figure 6-35. Cost sensitivity to the LWR front-end and overnight capital cost for the 1-Tier and 2-Tier closed fuel cycles.

\subsubsection{SNF Disposal and Once-through Recycling Costs}

In the once-through fuel cycle, utilities currently pay $1 \mathrm{mill} / \mathrm{kW} \cdot \mathrm{h}$ to dispose of the SNF. However, since no fuel has been actually disposed at this cost and future repositories are unlikely to cost less than the first, the nominal SNF disposal cost is estimated at $\$ 1,000 / \mathrm{kg} \mathrm{HM}$. In a closed fuel cycle, the intact SNF will not be disposed and all of the cost of disposing of the intact SNF will be a credit to the fast reactor, which will be responsible for the cost of disposing all resulting waste streams from the recycle of the SNF. Therefore, an escalating cost of SNF disposal will be an economic advantage for the closed fuel cycles.

In this economic trade-off analysis the nominal cost to recycle the UOx SNF is calculated to be $\$ 2,160 / \mathrm{kg} \mathrm{HM}$ as the cost breakdown indicates in Table 6-14. The nominal cost of conditioning and disposing the LWR SNF is $\$ 1,140 / \mathrm{kg}$ HM. This shows that it is $\$ 1,020 / \mathrm{kg}$ HM less expensive to dispose of the UOx SNF relative to the cost of recycling it. This difference is a substantial part of the cost penalty of going from the once-through cycle to a closed fuel cycle. Based on these figures, the SNF waste disposal costs would have to rise to about $\$ 2,000 / \mathrm{kg} \mathrm{HM}$, or if the waste cost is fixed then the recycling costs would have to drop to the $\$ 1,140 / \mathrm{kg}$ HM level in order to break-even between fuel cycles. 
Table 6-14. UOx SNF recycling cost.

\begin{tabular}{|l|l|c|c|}
\hline \multicolumn{1}{|c|}{ Module } & \multicolumn{1}{|c|}{ Description } & Recycling Cost $(\$ / \mathrm{kg} \mathrm{HM})$ & Fraction \\
\hline F1 & Aq Repr HdEnd\&Sep & 1,000 & $46.3 \%$ \\
\hline K2 & RepUranium Prod Dis & 11 & $0.5 \%$ \\
\hline E3 & TRU in HA storage & 130 & $6.0 \%$ \\
\hline G3 & LLW cond/pkg : F1 & 16 & $0.7 \%$ \\
\hline J & LLW disposal: F1 & 13 & $0.6 \%$ \\
\hline G4-2A & Cs/Sr/Rb/Ba C\&P:F1 & 65 & $3.0 \%$ \\
\hline G4-1A & Vol “offgas” C\&P: F1 & 2 & $0.1 \%$ \\
\hline G4-4A & Iodine C\&P: F1 & 161 & $0.2 \%$ \\
\hline G4-5A & Hulls, etc C\&Pkg:F1 & 199 & $7.5 \%$ \\
\hline E4 & Mng'd Prod Dy Stg:F1 & 85 & $9.2 \%$ \\
\hline G1 & Other FPs C\&P: F1 & 29 & $3.9 \%$ \\
\hline G1 & Tc C\&P: F1 & 12 & $1.3 \%$ \\
\hline M1 & GTCC Int Dep Disp:F1 & 434 & $0.5 \%$ \\
\hline L2 & HLW Deep Geo Disp & 2,160 & $20.1 \%$ \\
\hline & Total & & $100 \%$ \\
\hline
\end{tabular}

Figure 6-36 shows the cost advantage for the once-through cycle relative to the 1-Tier fuel cycle. The results show that over the range of costs for the LWR SNF fee and the levelized cost to the FR of recycling the LWR SNF, the entire cost gap will not be closed. The results show that combined, these actions could help to reduce the cost gap by up to $50 \%$. Note that some cost parameters (i.e., SNF disposal fee) have an associated affect on the open and closed fuel cycles. An increase in the costs for geologic disposal would directly increase the costs for the once-through cycle, but could also marginally increase the closed fuel cycle costs for HLW disposal.

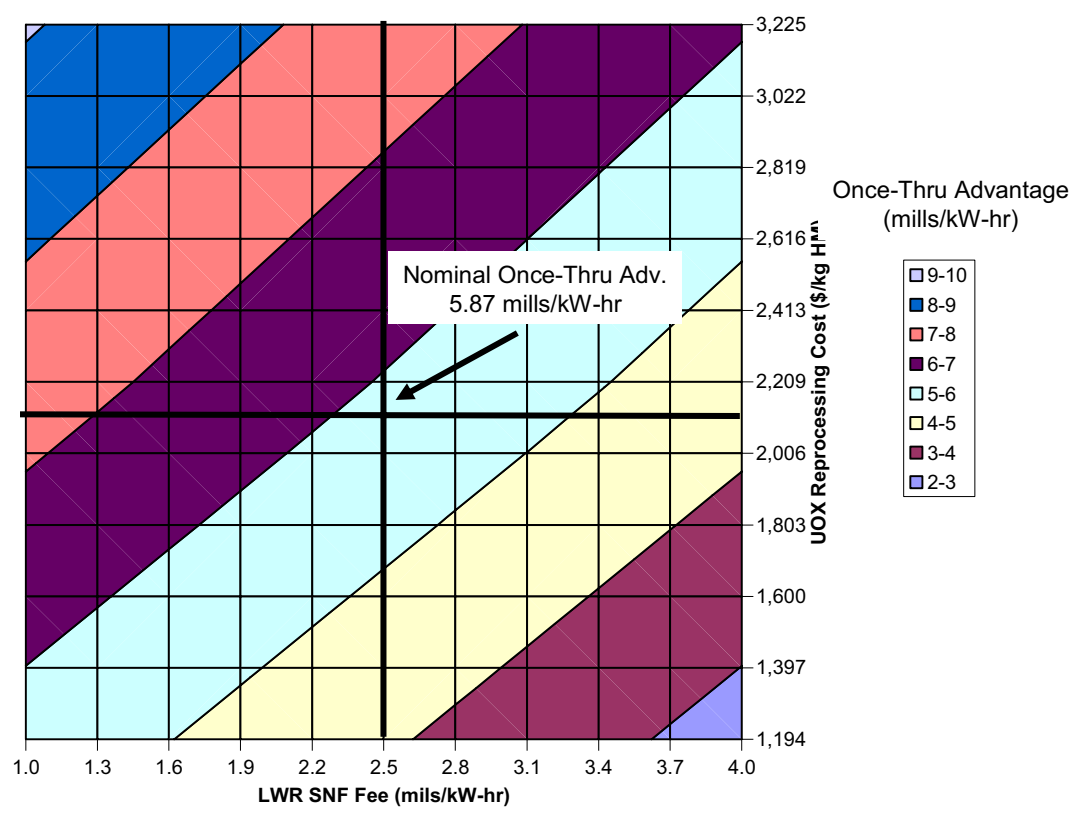

Figure 6-36. Cost sensitivity to LWR SNF fee and UOx recycling costs for the once-through and 1-Tier fuel cycle. 
Figure 6-37 shows the cost advantage for the 2-Tier relative to the 1-Tier. The relative cost is independent of the SNF waste fee since both are closed fuel cycles and no intact SNF is disposed. The results also show that rising recycling costs will result in a cost advantage for the 1-Tier relative to the 2-Tier fuel cycle because of the larger quantity of recycled LWR spent fuel. The LWR SNF that is recycled is proportional to the energy generated by the LWRs, which is $63 \%$ for the 1 -Tier and $73 \%$ for the 2-Tier. This increases the total LWR spent fuel that is recycled by $16 \%$ for the 2-Tier relative to the 1-Tier. The MOx spent fuel is assumed to cost the same as the UOx spent fuel to recycle and recovering the minor actinides separately from the UOx spent fuel was not assumed to add any cost in this analysis.

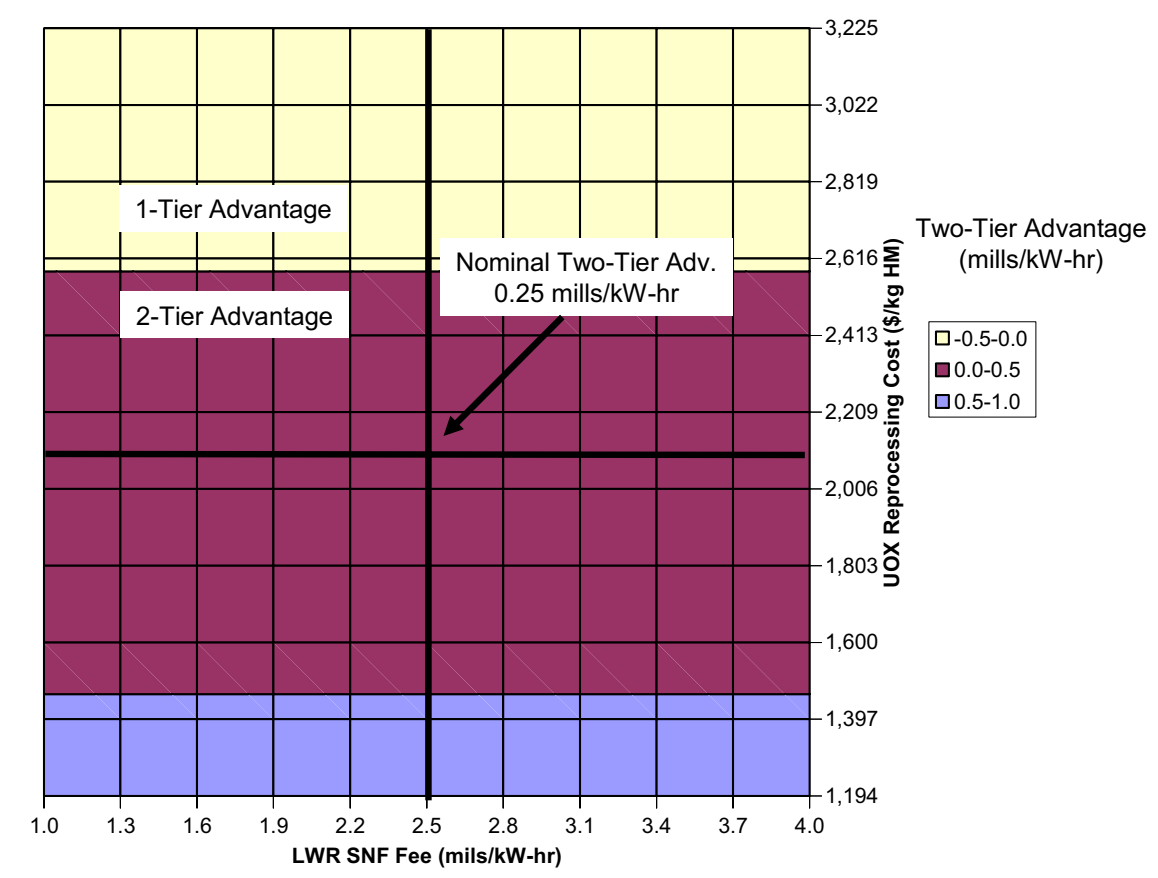

Figure 6-37. Cost sensitivity to LWR SNF fee and UOx recycling costs for the 2-Tier and 1-Tier fuel cycle.

Figure 6-38 shows the cost advantage for the once-through relative to the 2-Tier fuel cycle. The results show that over the range of costs for the LWR SNF fee and the levelized cost to the FR of recycling the LWR SNF, the entire cost gap will not be closed. The results show that combined these actions could reduce the cost gap from $5.62 \mathrm{mills} / \mathrm{kW} \cdot \mathrm{h}$ by a maximum of approximately $50 \%$. 


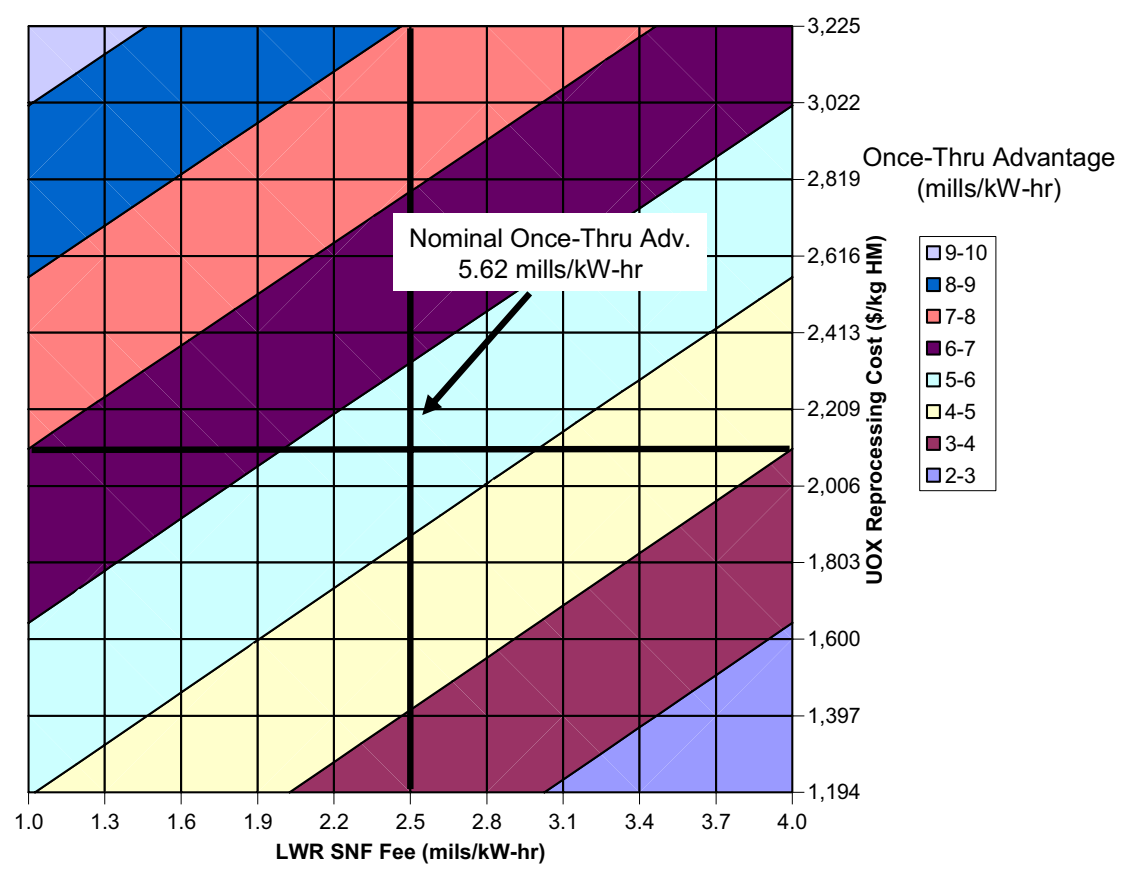

Figure 6-38. Cost sensitivity to LWR SNF fee and UOx recycling costs for the once-through and 2-Tier fuel cycle.

\subsubsection{High Performance Fast Reactor}

The assumptions about the cost and performance of the FR are based on limited experience and a few designs that have not necessarily been optimized for commercial operations. The Japanese Sodium-cooled Fast Reactor (JSFR) concept in Japan is a highly innovative concept that suggests that it is possible to greatly reduce the capital cost and significantly improve the performance (e.g., thermal efficiency). Some studies suggest the overnight cost could be under $\$ 2,000 / \mathrm{kWe}$ and thermal efficiencies about $42 \%$. Assuming these optimistic parameters, along with a capacity factor of $90 \%$, the cost difference between the once-through and the 1-Tier fuel cycle is reduced to approximately $0.25 \mathrm{mills} / \mathrm{kW} \cdot \mathrm{h}$. This is much smaller than the uncertainties presented in these calculations. The 1-Tier would have a cost advantage of $1.20 \mathrm{mills} / \mathrm{kW} \cdot \mathrm{h}$ over the 2-Tier fuel cycle. There is a large potential for closing the cost gap if highly innovative concepts like JSFR yield large improvements in cost and efficiency. Reduced differences in capital and performance will then make the other parameters such as uranium price and the SNF fee relatively more important.

In addition to the optimism expressed for the JSFR to reduce costs, there is a growing belief by current reactor vendors that low-cost fast reactors are possible and can be competitive. The AFCI estimates for fast reactors are based on projections from FOAK systems, research fast reactors, and limited cost studies. The LWR cost is based on a mature technology, does not require FOAK or research reactors, and is more driven by the uncertainties due to construction materials and critical components. This uneven playing field tends to skew the perspective for FRs toward higher costs, but this need not be so. There is no inherent reason why fast reactors should cost more than LWRs. The potential for new technologies to reduce the costs of these systems has never been higher.

The levelized capital cost is a function of five variables: overnight capital cost (Module R2), capacity factor, real discount rate, construction time, and economic lifetime. As can be seen from Equation 6.8.1.4-1, there is no benefit from improved thermal efficiency in the model. There is likely some additional economic benefit (maximum 10\% reduction) from increased thermal efficiency. Going 
from $82 \%$ capacity factor to $90 \%$ reduces the levelized capital cost by $9 \%$, and reducing the overnight cost to $\$ 2,000 / \mathrm{kWe}$ reduces the capital costs by $31 \%$. Improved unit cost along with improved capacity factor could potentially erase any substantial difference in cost between the open and closed fuel cycles.

$$
L U E C_{\text {Capital }}=f\left(r, t_{\text {cons }}, t_{\text {econ }}\right) * \frac{\mathrm{C}_{\text {overnight }}}{\mathrm{CF}}=0.010123 * \frac{\mathrm{C}_{\text {overnight }}}{\mathrm{CF}} \quad \text { Eq. 6.8.1.4-1 }
$$

\subsubsection{Trade off Summary}

The analysis in this sections shows that the dominant factor in determining the economically favored option is the overnight capital cost. Fuel cycle cost components may (in combination) significantly reduce the cost gap (up to 40\%), but without significant reductions in the FR capital cost, the economics are unlikely to favor the closed fuel cycle over the once-through cycle.

Information from the development of the JSFR concept and the General Electric (GE) S-PRISM concept suggest that optimization of the FR design that is likely to occur as design and operational experience are gained from the deployment and operation of successive generations of FRs may significantly reduce the cost gap that currently exists between the once-through and closed-fuel cycles based on the nominal costs. Small reductions $(\$ 100 / \mathrm{kWe})$ in the fast reactor overnight cost relative to the nominal cost would eliminate the cost benefit of the 2-Tier closed fuel cycle and all current NOAK cost projections for active fast reactor concepts would exceed this reduction. Even the most optimistic cost projections for the fast reactor would not completely eliminate the cost advantage of the once-through fuel cycle if all other costs remained near their nominal estimates. The ultimate cost of the NOAK FR is the dominant uncertainty in determining the relative cost of closing the fuel cycle with direct disposal of the intact SNF.

The cost of disposing of the intact SNF would have to grow well beyond current expectation (2.5-4.0 $\mathrm{mils} / \mathrm{kW} \cdot \mathrm{h})$ to change the conclusions, but if the costs go even higher, the relative cost difference between the open and closed fuel cycles will close. Higher costs of recycling LWR SNF actually favor the 1-Tier over the 2-Tier fuel cycle. This is because the 2-Tier must recycle all UOx SNF and then recycle all MOx SNF, which increases the demands on aqueous processing. Natural uranium is such a small fraction of the once-through costs that the cost would have to rise well beyond envisioned values to have a decisive impact, but rising costs will reduce the estimated cost gap between the open and closed fuel cycles.

The simple model for the cost as a function of conversion ratio implies that going to ever-lower conversion ratios will lead to lower costs of closing the fuel cycle. This model neglects any penalties likely from reduced conversion ratio (e.g., more control rods and/or shorter cycle lengths) or benefits from going to higher conversion ratios (e.g., simpler design and higher capacity factors). There may be a practical minimum to the conversion ratio, but this has not been determined. The current model treats most variables as independent variables, when in reality they are correlated. The independence of most variables (as modeled) does not truly capture many of the tradeoffs that will occur when going from one fuel cycle to another or modifying different parameters such as conversion ratio, burnup, or the relative cost of HLW and intact SNF fuel.

All combined, there is enough uncertainty that the economically favored option cannot be determined. Without significant deviation from the nominal values, the open cycle will maintain a cost advantage. If NOAK fast reactors can come online in the low range of the overnight cost $(\$ 2,000$ per $\mathrm{kWe}$ relative to $\$ 2,300$ for the ALWR) and perform above the assumed nominal values ( $>82 \%$ capacity factor), the cost of closing the fuel cycle will be greatly reduced and may even be the economically favored option. If recycling costs can be controlled and disposal of HLW is substantially cheaper than disposal of the intact 
SNF, this will improve the economics to the point that the closed fuel cycle would be the economically favored option if fast reactors can achieve the objectives of the JSFR and S-PRISM.

\subsection{Dynamic Cost Comparisons between the AFCI Nominal Growth Cases Using VISION.ECON}

This section examines the dynamic flows of materials over time and their associated costs. Selected outputs are provided in this section to illustrate the dynamic cost modeling capabilities of VISION.ECON. A complete set of model-generated outputs for the nominal growth case is provided in Attachment B. The dynamic analysis provides a different perspective from the static equilibrium analysis. The initial start-up flows generated by the VISION model are generally in non-equilibrium. Under these conditions, much fewer fast reactors are required than for the equilibrium condition. Therefore, the total costs during the start-up period (first 50 years) for the 1-Tier and 2-Tier systems will be lower than the static costs. Also, some fuel cycle disposition costs will be incurred after the end of the model run in 2100. These "orphan" costs increase for high nuclear growth. The static equilibrium costs described in Sections 5 and 6 provide a complete accounting of all the reactor and disposition costs, and should be consulted when analyzing the dynamic results.

\subsubsection{Economic Analysis of Nominal Growth Cases}

The economic analysis in this section examines the nominal growth cases for the once-through, 1 -Tier, and 2-Tier cycles, with a constant growth rate of $1.2 \%$ per year. In this analysis, the annual costs are evaluated over time to examine the overall driving cost factors resulting from nuclear growth. In the growth cases, the initial period of expanded growth (e.g., deployment of separation facilities, fast reactors) generates increases in fuel cycle costs and the total cost of electricity. After this initial expansion period, the systems enter into a dynamic equilibrium period where relative costs become stable. Dynamic equilibrium occurs when new FRs are brought online and the ratio between the FRs and LWR reactors becomes stabilized. Once stabilized, the relative annual cost of electricity and fuel cycle costs flatten (around 2050), as demonstrated in Figure 6-39.
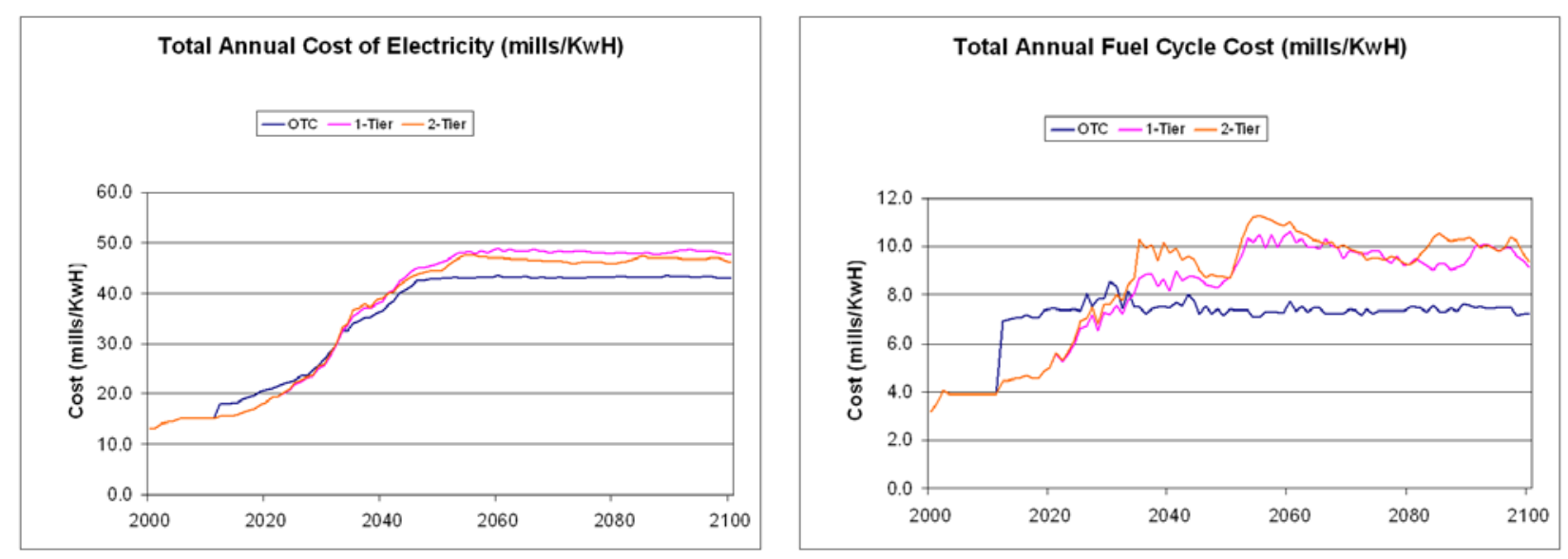

Figure 6-39. Dynamic analysis of the total annual cost of electricity and fuel cycle cost.

In this analysis, consistent with the G4 ECONS spreadsheet analysis, the fuel cycle costs are highest for the 2-Tier and the total annual cost of electricity are the highest for the 1-Tier after the system has stabilized, as shown in Figure 6-39. The once-through scenario, as in previous cases, has the lowest annual costs. 

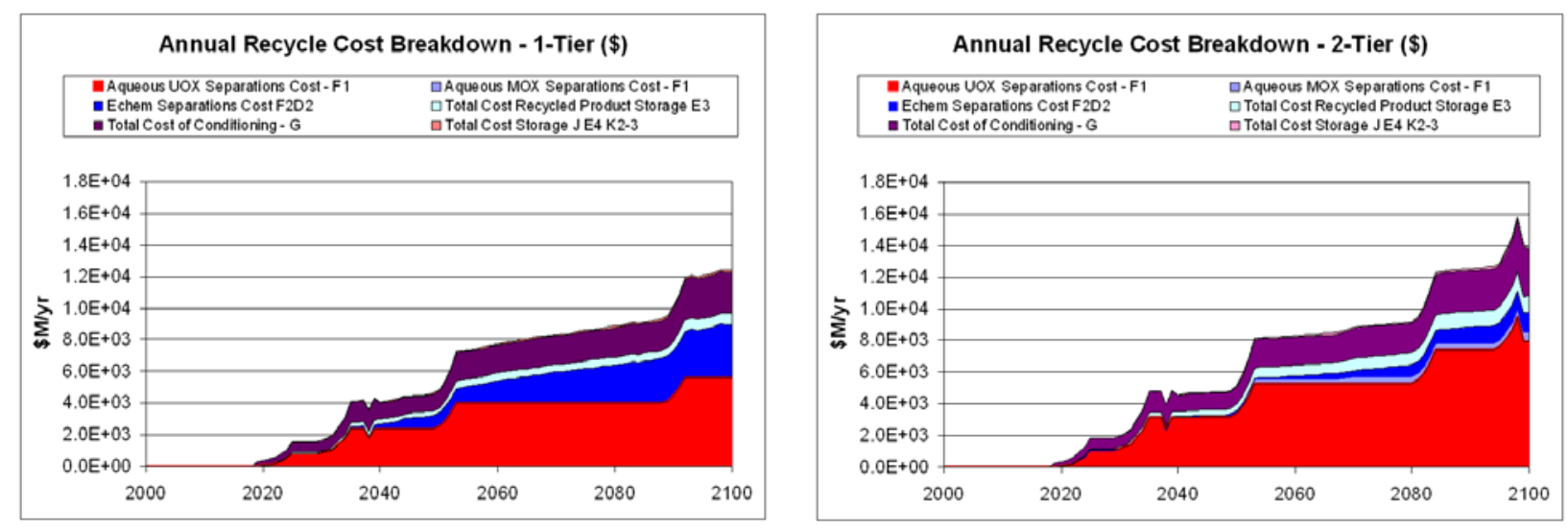

Figure 6-40. Dynamic analysis comparison of 1-Tier and 2-Tier recycling costs.

In Figure 6-40, the annual recycle cost breakdown is shown for the 1-Tier and 2-Tier. The 2-Tier has higher recycle costs than the 1-Tier due to the additional UOx separations required (red stacked bar) to support separation flows for the higher number of LWR and LWRmf reactors. However, the 1-Tier has significantly more EChem separations/metal fabrication costs than the 2-Tier (blue stacked bar).

\subsubsection{Gap Analysis of Nominal Growth Cases}

The gap analysis looks at a comparison between the once-through cycle versus the 1-Tier and the 2-Tier. The comparison looks at the difference between the fuel cycle cost and the reactor costs on a year-to-year difference. ${ }^{\mathrm{n}}$
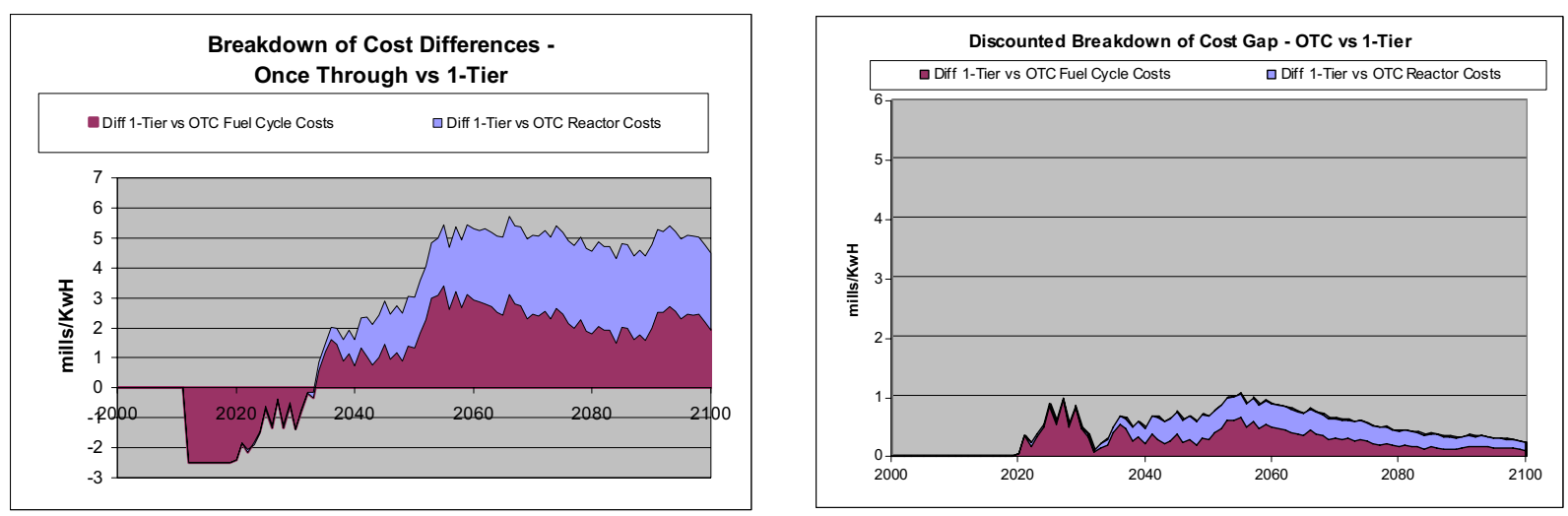

Figure 6-41. Breakdown of the cost gap between the once-through and 1-Tier (undiscounted and discounted).

Figure 6-41 shows the cost gap between the once-through and the 1-Tier. After 2030, the cost gap is evenly divided between fuel cycle costs and reactor costs. The chart on the left shows the gap based on undiscounted costs and the chart on the right shows the costs discounted at $3 \% /$ year. When discounted costs are used, the gap between cases reduces to less than $1 \mathrm{mill} / \mathrm{kW} \cdot \mathrm{h}$. The composition of the cost gap is approximately equal between fuel cycle costs and fast reactors.

n. For these scenarios, the model is set to begin on January 1, 2000 and continues for 100 years with a time step of 3 months. 

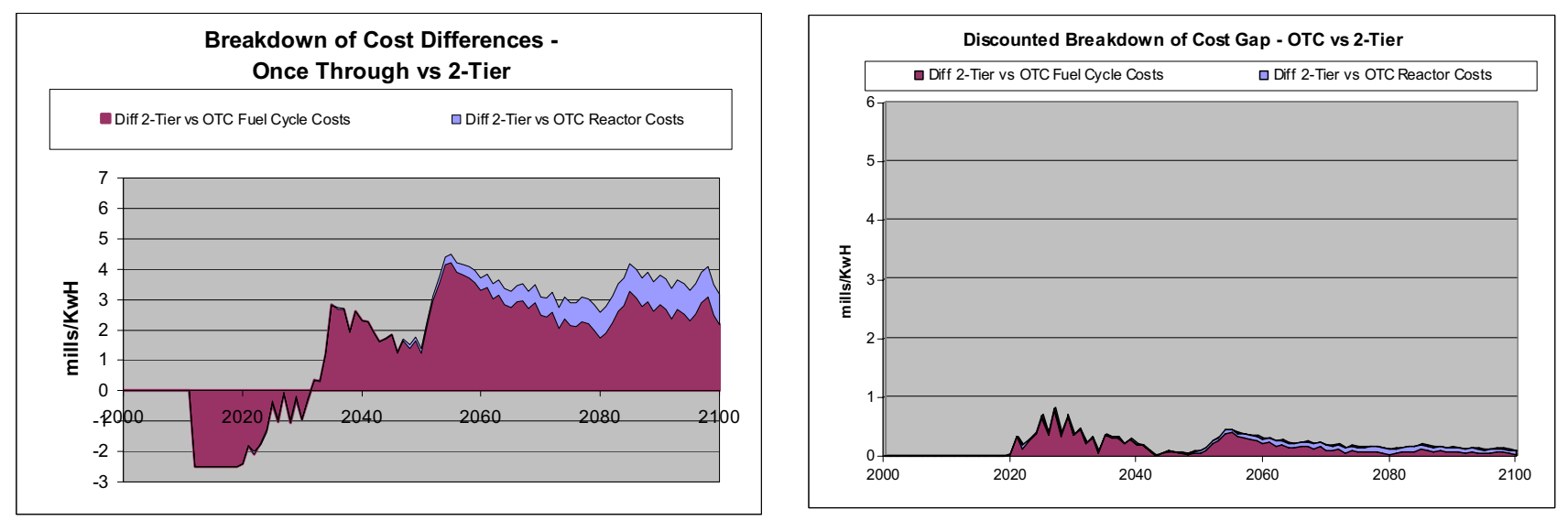

Figure 6-42. Breakdown of the Cost Gap between the once-through and 2-Tier (undiscounted and discounted costs).

Figure 6-42 shows the cost gap between the once-through and the 2-Tier. After 2050, only a small share of the cost differences are due to reactor costs, most of the gap is made up of fuel cycle cost differences. The chart on the left shows the gap based on undiscounted costs and the chart on the right shows the costs discounted at 3\%/year. When discounted costs are considered, the gap between cases reduces to less than $0.5 \mathrm{mill} / \mathrm{kW} \cdot \mathrm{h}$. The cost gap is primarily composed of fuel cycle costs, due to the relative few fast reactors (less than $10 \%$ of fleet).

In the period prior to about 2030, the once-through fuel cycle costs are higher than the 1-Tier or 2 -Tier cases (indicating negative costs). The negative costs result from low early-on costs for the closed cycles and the relatively higher costs incurred by the once-through for the waste disposal. After 2030, the 1-Tier and 2-Tier costs for separation and fabrication begin to exceed the fuel cycle costs from the once-through scenario.

\subsubsection{Analysis of FR and UOx deployment delays}

The analysis in this section examines the economic affect of delaying the deployment of fast reactors. The initial scenario starts with LWRs using UOx fuel and then transitions to FRs starting in 2020. The next four cases delay the deployment of fast reactors by an incremental 5 years/case up to 2040 . Figure 6-43 shows that there is no appreciable cost difference in either the composite cost of electricity or the fuel cycle cost. This is mainly due to the requirement that all legacy-spent fuel (fuel generated prior to starting separations) needs to be recycled by the end of the 21 st century. To meet this requirement, separations capacity has to be ramped up faster than in the baseline case of starting deployment of fast reactors in 2020. Therefore, by the end of the 100 years the same amount of spent UOx fuel has been through separations. 

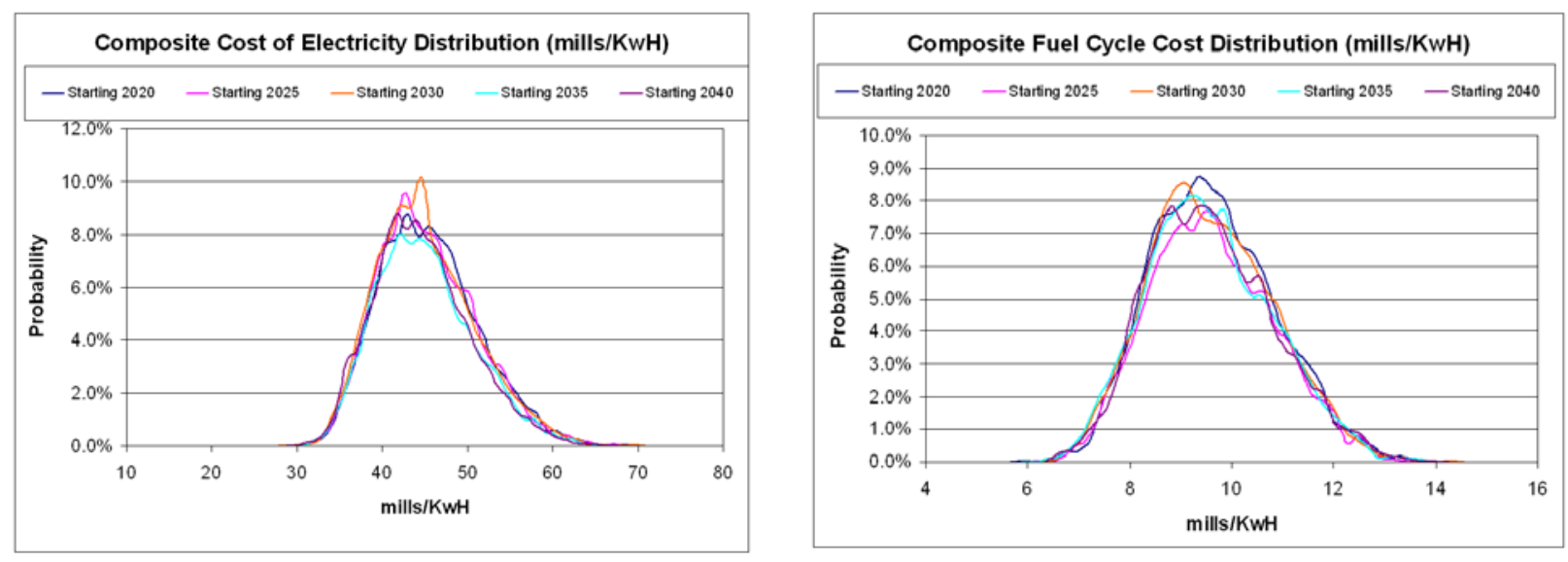

Figure 6-43. Chart showing the cost distributions for TCOE and FCC against delaying starting deployment of fast reactors.

The economic difference between early and late deployment of fast reactors is in the cost profile over time (see Figure 6-44). All the cases reach nearly the same annual fuel cycle cost by the end of the century, but the longer the delay in deploying FRs, the steeper the ramp of the cost curve. So, if there was a need to push costs out into the future, then delaying fast reactors, but ramping up separations capacity to offset that delay will still meet the spent fuel work-off requirement and not increase the overall fuel cycle cost by the end of the century.

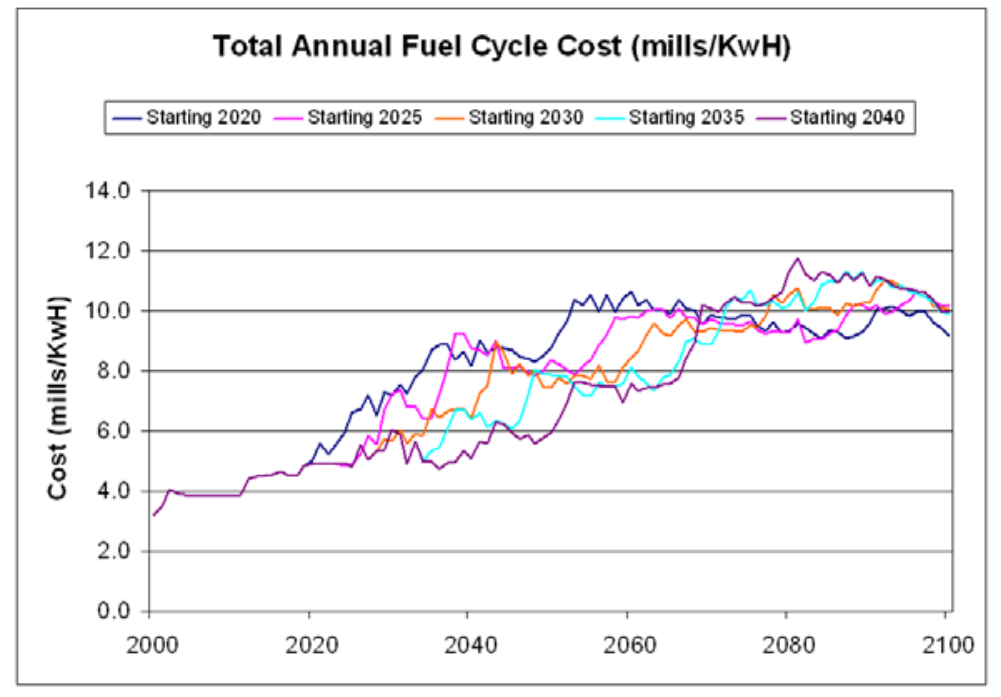

Figure 6-44. Yearly profile of fuel cycle costs on an annual basis looking at different fast reactor start dates.

\subsubsection{Analysis of Processing Losses}

In this section, the cost impacts from reducing the separation process losses for the 1-Tier by a factor of $100 \mathrm{x}$ (from $3.00 \%$ to $0.03 \%$ ) were evaluated. The nominal loss rate is based at $0.1 \%$. Figure $6-45$ shows that there is no appreciable cost difference due to reducing the processing losses. These costs only reflect the differences due to the additional waste conditioning and disposition, the costs do not include facility costs to upgrade systems or increased operations times to meet more stringent loss rates. 

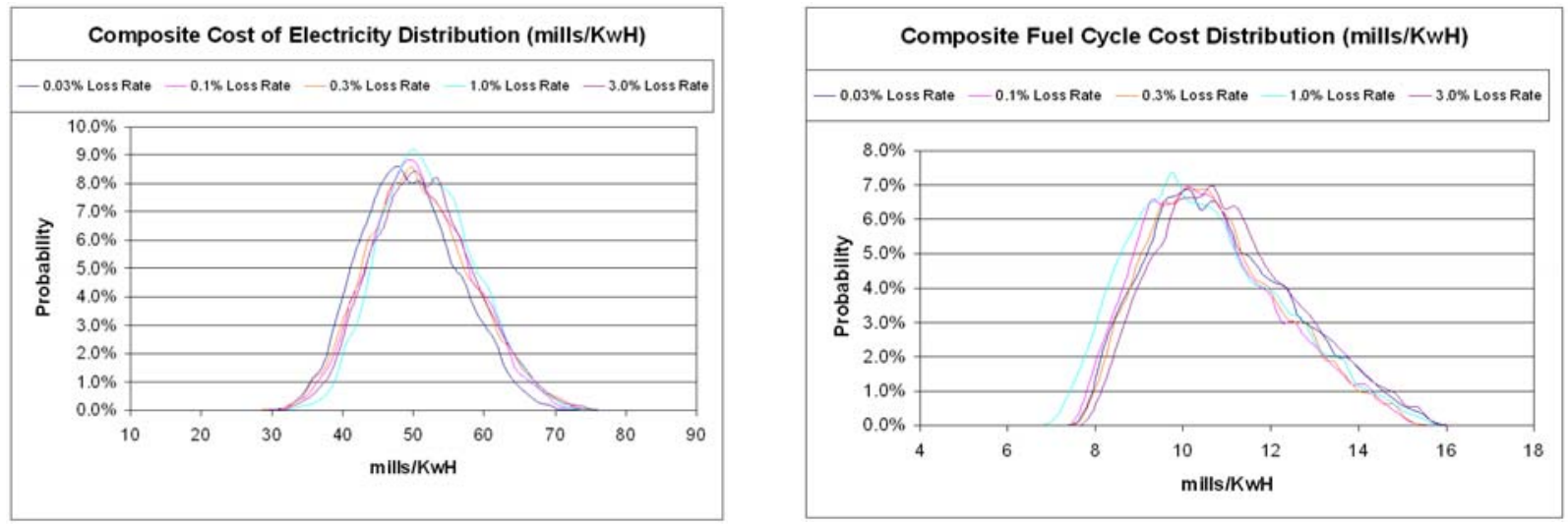

Figure 6-45. Dynamic analysis of the total annual cost of electricity and FCC for process losses ranging from $3.00 \%$ to $0.03 \%$.

Figure 6-45 shows that there is no appreciable total cost or fuel cycle cost difference due to reducing the processing losses from $3 \%$ to $0.03 \%$. Essentially all the cost differences are due to the fuel cycle.

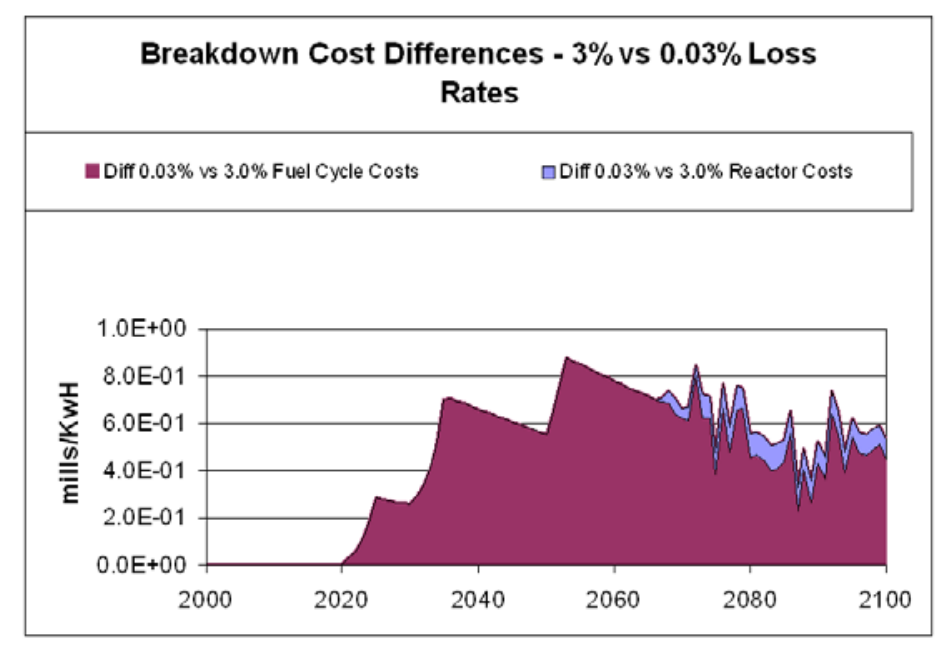

Figure 6-46. Breakdown of the cost gap resulting from $3.00 \%$ to $0.03 \%$ process losses (undiscounted).

The comparison of the cost difference between the $3.00 \%$ and the $0.03 \%$ process loss rates result in a cost difference less than $1 \mathrm{mills} / \mathrm{Kw} \cdot \mathrm{h}$ (Figure 6-46).

\subsection{References for Section 6}

Taylor, J., D. Shropshire, J. D., Jacobson, J., 2008, “A VISION of Advanced Nuclear System Cost

Uncertainty," International Conference on Nuclear Engineering, Orlando Florida, ICONE-16-48428, May 12-15 2008. 


\section{SUMMARY OF FUEL CYCLE TRANSPORTATION COST ANALYSIS}

As mentioned in the general economic assumptions in Section 3, many of the front-end and back-end module costs include the associated the transportation costs. The transportation costs for the complete fuel cycle were evaluated to determine their relative costs as compared to the open and closed fuel cycle total costs of electricity.

The final report, Once-Through, Single and Dual Tier Fuel Cycles Transportation Cost Analyses (Bailey and Smith 2008), provides an economic comparison of the transportation and associated infrastructure requirements for the current once-through fuel cycle, in which the TRU materials produced are disposed as waste, and the 1-Tier and 2-Tier cycles in which the TRU materials produced are recycled in nuclear reactors. In the study, the cost to transport fuel cycle and waste materials through each step of the nuclear fuel cycle was assessed. In practice, however, many of these costs, particularly in the "front end" of the fuel cycle (from transport of ore and mill concentrates to transport of finished fuel to reactors) are included in the unit cost of the commodity or service being procured.

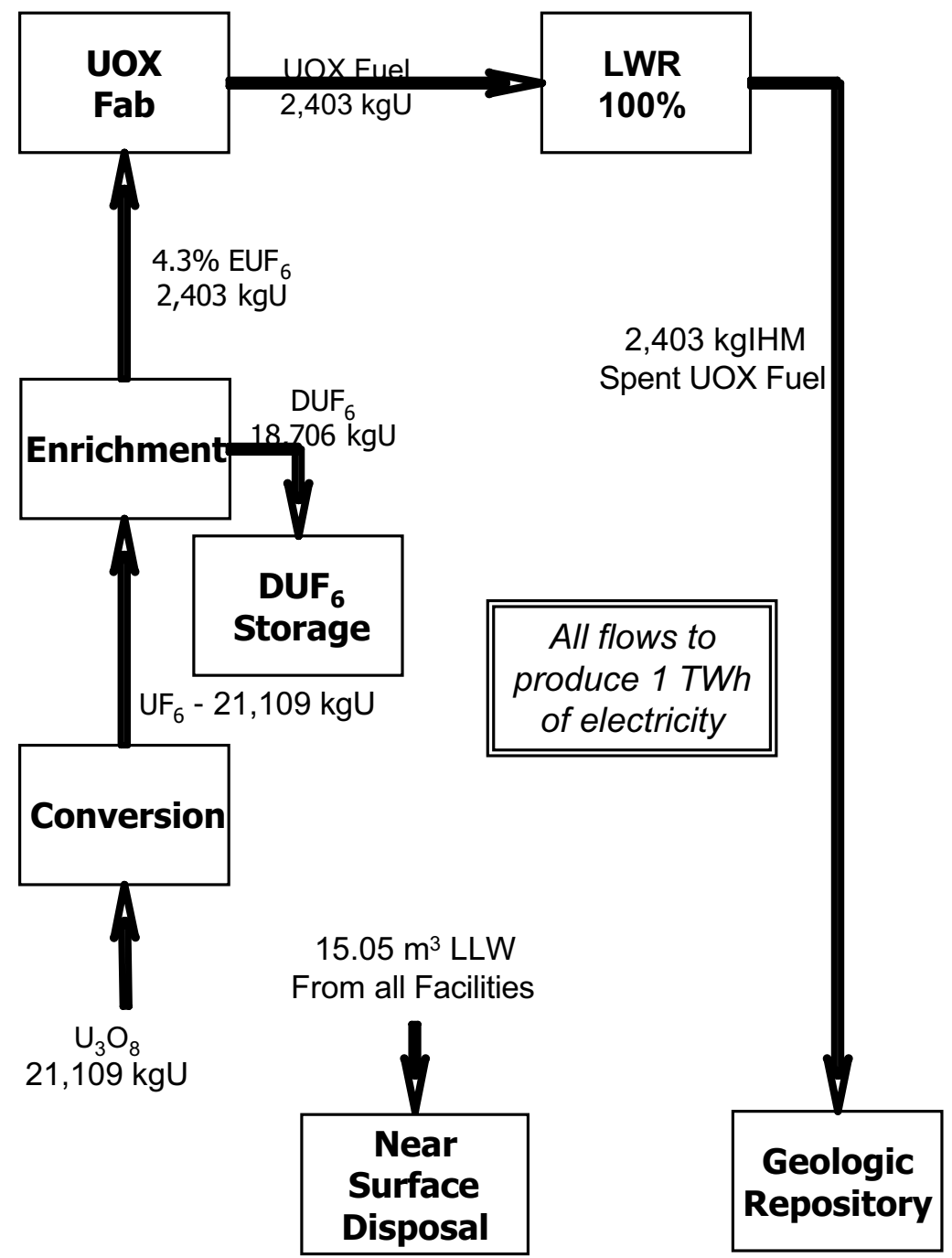

Figure 7-1. Once-through fuel cycle showing materials transported. 
The once-through scenario or "Direct Disposal" fuel cycle is the fuel cycle currently in use in the United States. As illustrated in Figure 7-1, uranium ore is mined and milled to produce a uranium oxide product (commonly called "yellowcake" or "uranium concentrates" treated as $\mathrm{U}_{3} \mathrm{O}_{8}$ ), which is then converted to uranium hexafluoride $\left(\mathrm{UF}_{6}\right)$ in preparation for enrichment. In the enrichment plant, the concentration of fissile ${ }^{235} \mathrm{U}$ is increased from the naturally occurring concentration of $0.711 \%$ to approximately $3-5 \%$, resulting in a waste/by-product stream of depleted uranium (DU) containing, typically, near $0.25 \%{ }^{235} \mathrm{U}$. The enriched $\mathrm{UF}_{6}$ is converted to a high-purity dioxide and fabricated into fuel assemblies (UOx fuel) for LWRs. The fuel assemblies are irradiated, typically to 45-60 GWd/MTU, cooled to reduce the content of short-lived fission products, and finally sent to a repository for disposal. The irradiated fuel is called SNF. In the once-through fuel cycle of Figure 7-1, and for the following 1Tier and 2-Tier cases, respectively depicted in Figures 7-2 and 7-3, the arrows with associated materials and quantities between processes denote mass flows, and accordingly, represent transportation segments between operations, which are not collocated within a single facility.

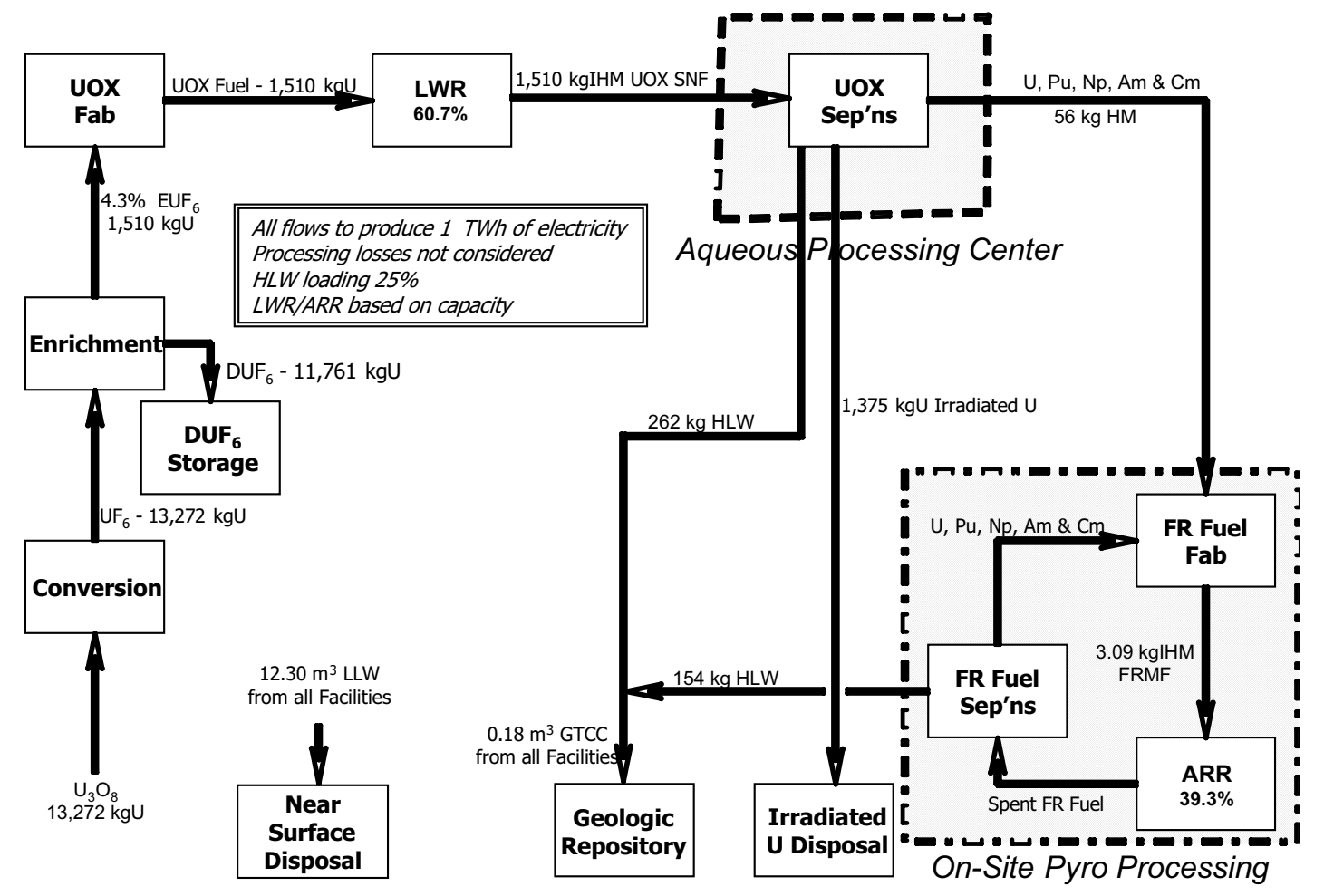

Figure 7-2. 1-Tier fuel cycle showing materials transported.

As observable in Figure 7-2 of the 1-Tier fuel cycle, the SNF from the LWRs is not sent to the repository, but is instead sent to a "separations plant" where recyclable materials (uranium and TRUtransuranic elements) are separated from the fission products, which are mostly considered wastes. The TRU materials are combined with a portion of the previously irradiated $U$ (the rest being disposed as low-level waste) to produce a fuel that can be used in fast reactors. The SNF from the fast reactors is recycled indefinitely, using the $U$ and TRU materials from the LWRs as makeup to compensate for the destruction of these materials during irradiation in the fast reactor. The fission products and a small inseparable portion of the $U$ and TRU materials (loss assumed to be $0.1 \%$ ) are mixed with a glass-like matrix to form vitrified HLW, which is sent to the repository for disposal. In the 1-Tier system, about $37 \%$ of the electricity generating capacity is associated with fast reactors. 
In the 2-Tier fuel cycle summarized in Figure 7-3, the plutonium and a portion of the irradiated $U$ from the separations plant are made into a recycle fuel for LWRs commonly called MOx fuel, of which the $\mathrm{Pu}$ concentration is typically around $10 \%$. After irradiation, the fuel is sent to a separations plant and the recyclable materials forwarded to a fabrication plant for fast reactor fuels. Under the 2-Tier system, approximately $27.5 \%$ of the electricity generating capacity is associated with fast reactors and $8 \%$ with MOx fuel in LWRs.

Radioactive materials are transported in robust containers to dissipate the heat generated and shield personnel from emanating radiation. Assuming commercial transportation by an appropriately licensed shipper, the total cost to transport depends on the cost of the container and associated capacity, the time and infrastructure required to load and unload the package (container with contents), and the shipping distance. Since the various facilities involved are located around the country, a stochastic approach to transportation cost was developed for the "AFC Cost Basis" report (Shropshire 2008). Distributions were developed for container cost, container life, loading time, unloading time, travel distance considering a variety of beginning and ending points for the trip, travel speed, labor costs, etc. Then a "Monte Carlo" statistical calculation, a computational algorithm that relies on repeated random sampling, was performed for 10,000 sets of these parameters to develop median, high (exceeded only $10 \%$ of the time), and low (exceeded $90 \%$ of the time) costs for each transportation leg.

The cost of the container was based on actual, NRC-certified packages. In reality, few of the packages used as simulants for shipping TRU-containing materials are certified to do so, and at least one has a certificate that cannot be renewed. Thus, some package certificates will require amendment for different contents and some packages will have to be redesigned and certified. Nevertheless, it is assumed the cost for any newer packages will be similar to the costs for the existing packages. This is considered to be a reasonable assumption with the possible exception of shipping TRU materials, which can have a high-heat generation rate.

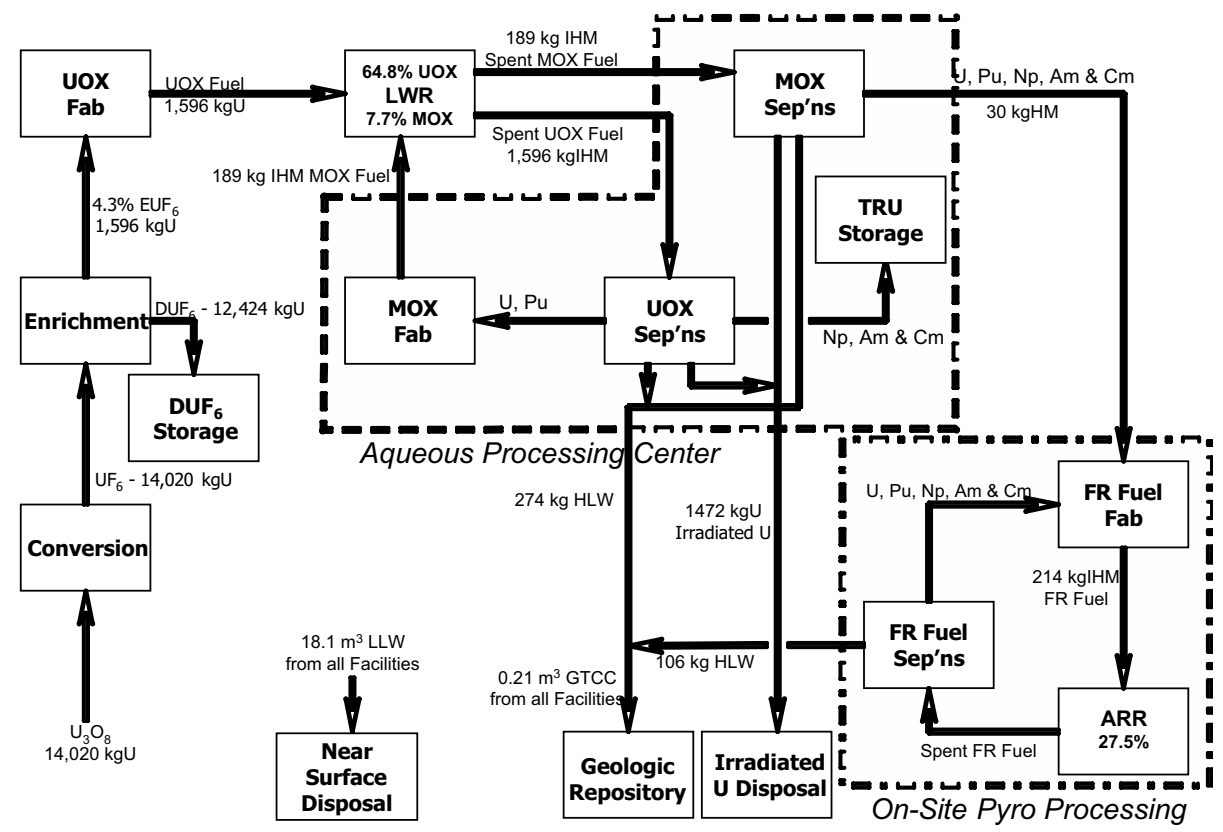

Figure 7-3. Two-Tier fuel cycle showing materials transported.

Table 7-1 presents the information for transportation costs using the median costs from the Monte Carlo calculations. The costs are given in thousands of $2007 \$$ per terawatt-hour. A 1,000 MW electric generating station would produce about 7.9 TWhr in a year. 
Table 7-1. Transportation cost breakdown.

\begin{tabular}{|c|c|c|c|c|c|c|c|}
\hline Transportation Step & \multicolumn{3}{|c|}{ Amount Transported, kg/TWh } & \multirow{2}{*}{$\begin{array}{c}\text { Cost } \\
\$ / \mathrm{kg} \mathrm{HM}\end{array}$} & \multicolumn{3}{|c|}{ Cost, $\$$ thousand/TWh } \\
\hline Fuel Cycle Type & Once-Thru & 2-Tier & 1-Tier & & $\begin{array}{c}\text { Once- } \\
\text { Through }\end{array}$ & 2-Tier & 1-Tier \\
\hline $\mathrm{U}_{3} \mathrm{O}_{8}$ to Conversion & 21,109 & 14,020 & 13,272 & 2.01 & $\$ 42.43$ & $\$ 28.18$ & $\$ 26.68$ \\
\hline $\mathrm{UF}_{6}$ to Enrich & 21,109 & 14,020 & 13,272 & 1.94 & $\$ 40.95$ & $\$ 27.20$ & $\$ 25.75$ \\
\hline EUF $_{6}$ to UOx Fabrication & 2,403 & 1,596 & 1,510 & 2.24 & $\$ 5.38$ & $\$ 3.57$ & $\$ 3.38$ \\
\hline DUF $_{6}$ to Waste & 18,706 & 12,424 & 11,761 & 2.16 & $\$ 40.41$ & $\$ 26.84$ & $\$ 25.40$ \\
\hline UOx Fuel to LWR & 2,403 & 1,596 & 1,510 & 34.08 & $\$ 81.89$ & $\$ 53.39$ & $\$ 51.47$ \\
\hline UOx SNF to MGR & 2,403 & $\mathrm{~N} / \mathrm{A}$ & & 91.46 & $\$ 219.77$ & & \\
\hline UOx SNF to UOx Seps & $\mathrm{N} / \mathrm{A}$ & 1,596 & 1,510 & 83.41 & & $\$ 132.36$ & $\$ 125.98$ \\
\hline $\mathrm{U}, \mathrm{Pu}, \mathrm{Np}$ to $\mathrm{MOx} \mathrm{Fab}$ & $\mathrm{N} / \mathrm{A}$ & 189 & & & & & \\
\hline MOx Fuel to LWR & N/A & 189 & & 83.41 & & $\$ 15.80$ & \\
\hline MOx SNF to MOx Seps & N/A & 189 & & 83.41 & & $\$ 15.80$ & \\
\hline $\mathrm{U} / \mathrm{MOx} \mathrm{HLW}$ to $\mathrm{MGR}^{\mathrm{a}}$ & $\mathrm{N} / \mathrm{A}$ & 277 & 262 & 21.21 & & $\$ 5.88$ & $\$ 5.57$ \\
\hline U to Waste & $\mathrm{N} / \mathrm{A}$ & 1,472 & 1,375 & 2.16 & & $\$ 3.18$ & $\$ 2.97$ \\
\hline U+TRU to FRMF Fab & N/A & 30 & 56 & 232.48 & & $\$ 6.97$ & $\$ 13.01$ \\
\hline FRMF to AAR & N/A & 214 & 309 & & & & \\
\hline FRM SNF to FRMF Seps & N/A & 214 & 309 & & & & \\
\hline $\mathrm{U}+\mathrm{TRU}$ to FRMF Fab & N/A & 184 & 265 & & & & \\
\hline FRMF HLW to $\mathrm{MGR}^{\mathrm{a}}$ & $\mathrm{N} / \mathrm{A}$ & 106 & 154 & 24.92 & & $\$ 2.65$ & $\$ 3.83$ \\
\hline $\mathrm{LLW}^{\mathrm{b}}$ & 28.9 & 18.1 & 18.2 & 765 & $\$ 21.57$ & $\$ 13.84$ & $\$ 13.92$ \\
\hline GTCC Waste $^{b}$ & 0 & 0.21 & 0.18 & 28,570 & & $\$ 5.93$ & $\$ 5.02$ \\
\hline Total Cost & & & & & $\$ 452.40$ & $\$ 342.59$ & $\$ 302.98$ \\
\hline Fuel Cycle Cost ${ }^{\mathrm{c}}$ & & & & & 0.45 & 0.34 & 0.30 \\
\hline \multicolumn{8}{|c|}{$\begin{array}{l}\text { a. The amount of HLW transported is the actual amount of HLW, rather than the HM content or the HM equivalent. Cost is in } \\
\$ / \mathrm{kg} \text {. } \\
\text { b. The amount of LLW or GTCC waste is given in } \mathrm{m}^{3} / \mathrm{TWh} \text {. Cost is given in } \$ / \mathrm{m}^{3} \text {. } \\
\text { c. The fuel cycle cost is given in mills } / \mathrm{kW} \cdot \mathrm{h} \text {. }\end{array}$} \\
\hline
\end{tabular}

Converting to units typically used to express the cost of electricity, $\$ 1,000$ per terawatt-hour is equivalent to $10^{-3} \mathrm{mills} / \mathrm{kW} \cdot \mathrm{h}$, yielding a transportation component cost of $0.45 \mathrm{mills} / \mathrm{kW} \cdot \mathrm{h}$ for the once-through fuel cycle. The nominal once-through fuel cycle cost is estimated at $6.61 \mathrm{mills} / \mathrm{kWh}$, so transportation contributes $6.7 \%$ of the cost. For the recycle cases, the transportation cost is $0.30-0.34$ mills $/ \mathrm{kW} \cdot \mathrm{h}$ out of the nominal $8.22-9.13 \mathrm{mill} / \mathrm{s} / \mathrm{kW} \cdot \mathrm{h}$ fuel cycle cost, or about $3.7 \%$. This analysis leads to the conclusion that transportation costs represent only a small contribution to the total fuel cycle cost.

The results presented in Table 7-1 allow some additional observations to be made regarding transportation costs:

- Irrespective of the fuel cycle, the major contributor to transportation cost is associated with shipping LWR SNF. In recycle fuel cycles, about $40 \%$ of this and all front-end costs are avoided. This savings is only partly offset $(20-40 \%)$ by the further transportation steps needed for recycle fuel.

- In the recycle cases, the transportation costs associated with the LWRs (including dealing with LWR SNF) make up about $85-90 \%$ of the transportation cost.

- To a certain extent, the amount of energy generation provided by TRU-containing fuel (either MOx or FR fuel) is limited by the production of TRU in LWRs, and as a result, the mix of LWRs and FRs does not depend strongly on the details of the fuel cycle. However, the amount of materials 
transported depends strongly on the fuel burn-up and consequently, the primary avenue to transportation cost reductions for TRU-consuming systems is by increasing the fuel burnup. If processing losses were considered, the fuel mix would shift slightly to increase the fraction of LWRs. This would not cause a significant change in transportation costs; however, consideration of processing losses would increase the amount of HLW to some extent. Nevertheless, the overall effect would be to increase transportation costs by only a very few percent.

- Only about $11-13 \%$ of the transportation cost in the recycle fuel cycles is due to the FR component. In a system consisting only of FRs, the transportation cost would be about $0.2 \mathrm{mills} / \mathrm{kW} \cdot \mathrm{h}$. Note that in such a system there would be about 2.5 times as many fast reactors.

Since the calculations of transportation cost allow consideration of variations from the median cost, it is instructive to consider the precision of the median cost. One technique, based upon the "propagation of error" approach used in many scientific calculations, yields a variability of $0.002 \mathrm{mills} / \mathrm{kW} \cdot \mathrm{h}$ for all three considered fuel cycles. Note this implies a rather precise answer for which it must be realized the precision accrues to the totality of all shipments, and not to the cost for any individual component.

\subsection{References for Section 7}

Bailey, G.F. and J. D. Smith, 2008, "Draft Once-Through, Single and Dual Tier Fuel Cycles

Transportation Cost Analyses," GNEP-SYSA-ECON-SS-RT-2008-0000251, January 2008.

Shropshire, D., et al., 2008, “2008 Advanced Fuel Cycle Cost Basis,” INL/EXT-06-11536, March 2008. 


\section{DISCUSSION ON THE COMPARISON OF NUCLEAR GENERATION COSTS TO OTHER BASE-LOAD ELECTRICITY GENERATION COSTS}

\subsection{Introduction}

A principal assumption underlying AFCI systems analysis is that for the rest of the $21^{\text {st }}$ century nuclear power will continue to supply a significant fraction (approximately $20 \%$ or higher) of the electrical energy generation in the United States. However, in order for this to occur, nuclear energy must be economically competitive with other forms of baseload electricity generation such as coal and natural gas. In this section, the competiveness issue is examined for these two predominant U.S. fossil fuel choices: coal and natural gas. The figures-of-merit for competiveness are the same as those for nuclear power (i.e., the levelized busbar cost of electricity (LUEC or TCOE) and the specific overnight and specific "all-in" (total) capital costs of the generation facility (powerplant) expressed in constant $2008 \$$ per kilowatt of electrical capacity). Note that the term "LUEC" is applied most often to a single plant, whereas the term "TCOE" applies to a system comprised of multiple reactor (LWR and FR) systems.

The economic competitiveness issue was also discussed in Attachment B of the September 2007 "AFCI Economic Tools, Algorithms, and Methodologies Report" (Economic Analysis Working Group 2009) prepared by the AFCI Systems Analysis. Most of the data for that report was gathered in the last half of FY-07. However, in FY-08, many economic factors work to affect the projected competitiveness of nuclear vis-à-vis fossil baseload generation. Many of these are driven by the rising price of crude oil and the strong demand for fuel and construction commodities from China and India. Among the resultant cost effects are:

- Prices for fossil fuels, such as natural gas and coal, were rising along with the price of crude oil, but with a significant time lag and somewhat smaller escalation rate. These price increases seen over the last 7 months normally would occur over the span of several years if only general inflation was occurring.

- Nuclear energy projected costs for new plants were rising because of the increasing costs of concrete, steel, and specialty metals. This trend is also affecting coal to a lesser extent.

- For all types of generation, some utilities are now required to supply their regulators (such as public service commissions) with more detailed cost estimates, which include the projected costs for likely project risk factors such as regulatory delays and materials or labor shortages.

- With several climate change-related bills now coming up for consideration by Congress, a better understanding of the likely cost ranges for and economic effects of a carbon tax or "cap and trade" regime for the mitigation of greenhouse gases is now available. The dollars per metric tonne of carbon (\$/MT C) or dollars per metric tonne of carbon dioxide $\left(\$ / \mathrm{MT} \mathrm{CO}_{2}\right)$ figure-of-merit is often called the "cost of carbon."

- Projected costs for carbon $\left(\mathrm{CO}_{2}\right)$ capture and storage (CCS) and advanced coal-fired generation, such as integrated (coal) gasification combined cycle (IGCC) are becoming increasingly available and more realistic.

- "All-in" capital cost estimates $(\$ / \mathrm{kWe})$ and proposed generation capacities $(\mathrm{MWe})$ for many power generation projects are now routinely available on industry Web sites and in the trade press.

This section is not intended to replace Appendix B of the "AFCI Economic Tools, Algorithms, and Methodologies Report" (Economic Analysis Working Group 2009), but rather to augment it with new data. Appendix B also briefly discusses electricity generation cost-related issues for renewable electrical generation sources such as wind and solar. In this report, however, it has been decided to deal only with the major baseload electricity generation candidates - coal, natural gas, and nuclear. (A later Subsection 
8.6 will present some new data on nuclear costs that has been gathered since the late FY-07 research period already documented in Appendix R-1 of the "AFC Cost Basis" report [Shropshire et al. 2008])

The main reason renewable sources such as solar, wind, biomass, and hydro are not included in the analysis is that the two most-often mentioned (and suggested) generation technologies (wind and solar) are not baseload ${ }^{\circ}$ electricity generators today. Hydroelectric power is capable of baseload production; however, there are so few new dam sites available in the US for new hydropower, that its potential contribution to future energy supply will be very small. Biomass (wood, agricultural residues, and municipal refuse) could also be burned in baseload facilities much in the same way as coal; however, there is insufficient operating experience and historical cost information to judge the competitiveness of large ( $>500 \mathrm{MWe}$ ) biomass plants against typically large coal, nuclear, and natural gas generation facilities.

Given the choice of these three major technologies (coal, nuclear, and natural gas), the economic assessment methodology for the fossil technologies is discussed in Section 8.2.

\subsection{Methodology for Analysis}

The first step was to construct simple economic models for calculation of the LUEC for new coal and natural gas baseload powerplants. This required modifying the G4 ECONS reactor cost model (EMWG 2008 ) in the following ways:

- Removing the fuel cycle module applicable only to nuclear power.

- Simplifying the capital cost input such that an "overnight" $\$ /$ kwe cost could be entered instead of a series of two-digit code-of-accounts lump sum cost values. Essentially a "rolled-up" capital cost value is entered. Such "rolled-up" values are also more easily found in the literature, since more detailed "broken-down" estimates (by code-of-accounts) are generally proprietary.

- Simplifying the O\&M input such that only the non-fuel "fixed" and "variable" components of the O\&M cost need be entered. Essentially this "rolls-up" the more detailed two-digit O\&M codes in the original G4 ECONS.

- Keeping the levelization (capital recovery and D\&D sinking fund) and IDC calculational methodologies exactly the same as those in the G4 ECONS reactor model.

- Adding fuel/plant performance modules appropriate to each technology that contain the fuel description, its heat value (BTUs per mass or volume), the percent carbon in the fuel, the projected future constant dollar unit cost for the fuel, the plant thermodynamic efficiency, and the projected plant average plant capacity factor.

- Adding an algorithm to calculate the additional LUEC attributable to the imposition of a carbon tax, cap and trade provisions, or carbon, capture, and storage. This variable is expressed and entered in dollars per metric ton of carbon. The corresponding $\$ / \mathrm{MT} \mathrm{CO}$ value is also calculated. The additional LUEC is usually referred to as the "cost of carbon." (In the algorithm all C emissions are assumed to be $\mathrm{CO}_{2}$. In reality there are small amounts of $\mathrm{CO}, \mathrm{CH}_{4}$, solid carbon char, and carbonate salts produced.)

o. Baseload means that a power plant is available continuously except for occasional planned and unplanned outages. In order to provide baseload power, solar and wind would require expensive energy storage technology to cover the times when the sun does not shine and when the wind is absent or insufficient. Presently, reliable numbers on the cost and performance of such storage technologies are not available. Grid stability requires that a large fraction of generation come from baseload sources. Utilities typically build additional back-up baseload power whenever renewable enegy sources are added to the energy portfolio. 
- Selecting a combustion technology within each generic "coal" and "natural gas" category. For the coal plant state-of-the-art pulverized coal technology with capture of SOx, NOx, particulates, and mercury was chosen. For natural gas, state-of-the-art high-efficiency combustion gas-turbine combined cycle technology (CCGT) was selected.

Fortunately, energy conversion algorithms and some plant data were available from a previous ORNL report comparing projected fusion energy generation costs to other fossil and nuclear technologies (Delene, et al. 1999).

The second step was to review the most recent literature (see Section 8.7), including the Web and trade publications, for recent estimates on fuel costs and plant life cycle costs for coal and natural gas facilities. Similar sources also provided some information on what various experts expect to be the value for a carbon tax or the cost of CCS (carbon capture and storage). Data on the current, projected, and historical unit prices for fossil fuels is available from the Energy Information Administration and from publicly available utility projections made for regulators.

The third step was to select low, nominal, and high-projected values for the important inputs based on the literature search and some experience-based cost analysis. Distributions were then assigned to span the range for each variable. The DPL software was used to generate total cost of energy distributions. In this manner, bell-shaped "output" \$/MWh generation cost distributions were generated for both fossil fuel technologies. Value Tornado Diagrams were prepared to represent single value, one-variable-at-a-time sensitivity studies. These can then be compared to the similarly formatted results for nuclear systems shown in Section 6.

\subsection{Baseload Pulverized Coal Calculations}

Most planned coal fired plants in the U.S. are of the conventional "pulverized coal" variety where ground-up coal is injected into a large furnace/boiler where high-pressure steam is produced to drive turbines. Nearly all newer units will include equipment for limiting emissions of SOx, NOx, particulates, and mercury. Currently, none of these plants have carbon CCS equipment. It is most likely that the $\mathrm{CO}_{2}$ "greenhouse gas" mitigation or adaptation externality will be handled initially by means of a "cap and trade" mechanism or by a carbon tax. (CCS retrofits may follow later.) The reference plant considered for the case below is assumed to burn Eastern (Appalachian in this case) coal which has a high BTU content per unit mass (i.e., high heat value), but also contains low to moderate amounts of sulfur, and therefore requires SOx scrubbing. Figure 8-1, a spreadsheet image from the economic model, shows the inputs, internally calculated variables, and final figures-of-merit for the nominal (most likely) set of inputs selected for pulverized coal technology. 


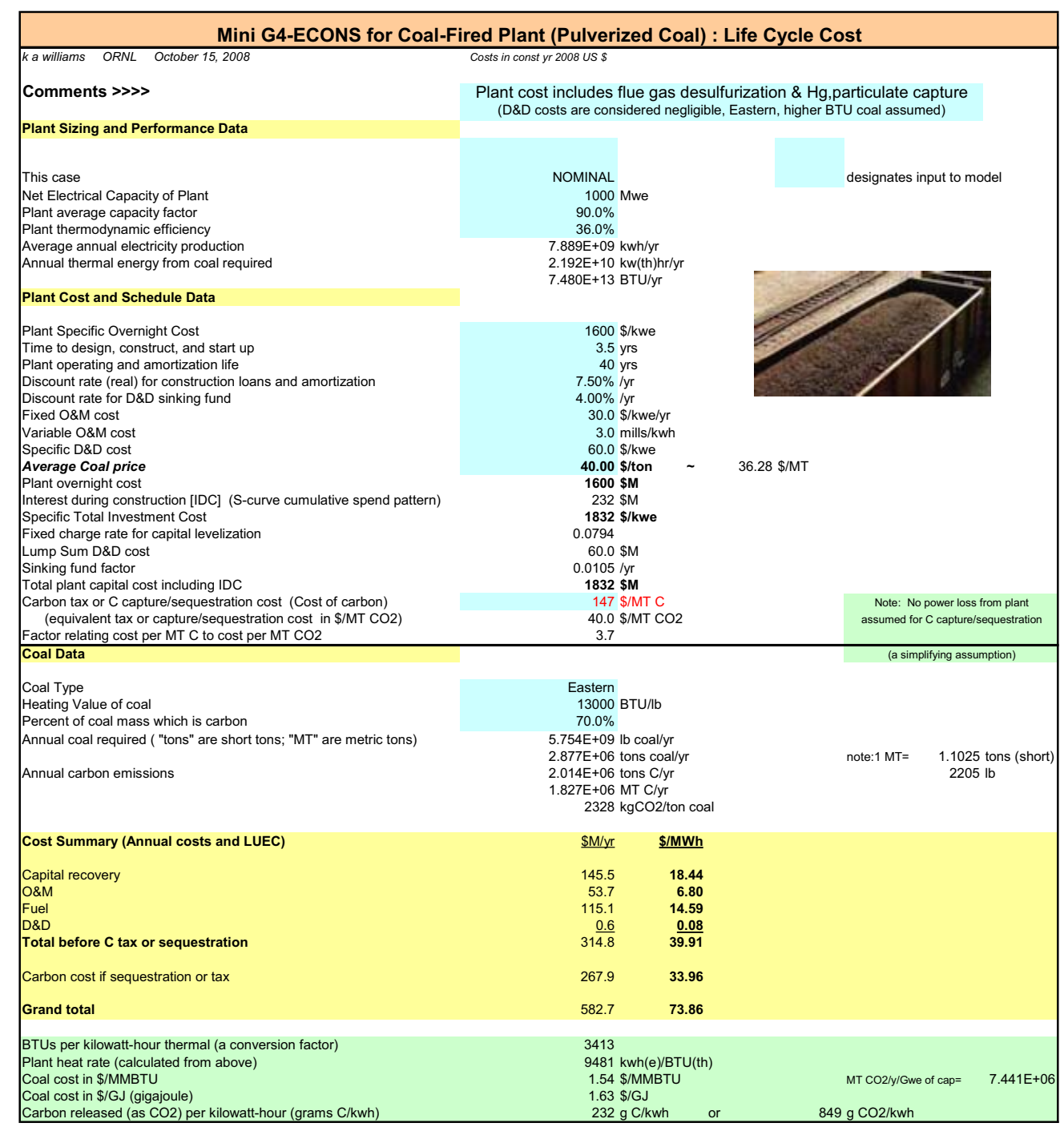

Figure 8-1. Economics of pulverized coal powerplant technology using a levelized cost model of the G4 ECONS type (spreadsheet image of nominal case: annual $\mathrm{C}$ emissions assumed to be $\mathrm{CO}_{2}$ only).

The basis upon which the specific overnight capital cost was chosen is of interest, since this variable (outside of the carbon tax/cost) is one of the main drivers of the LUEC. The following Table 8-1 shows some cost and capacity information gleaned from trade Web sites and trade press information collected over the last few years. In most cases the only capital cost data point available in the reference was the "all-in" or total capital cost. The specific all-in cost $(\$ / \mathrm{kWe})$ was calculated by dividing the all-in cost in dollars by the plant net capacity in kilowatts (electric). Most coal plants are multi-unit (boiler) plants; the specific cost calculated represents all of the proposed new units on the given site. 
Table 8-1. Projected capital cost economics for planned coal powerplants (costs are in constant 2008 dollars unless otherwise noted; no CCS equipment is included).

\begin{tabular}{|c|c|c|c|c|c|}
\hline $\begin{array}{l}\text { Project Name, } \\
\text { Owner, and } \\
\text { Location } \\
\end{array}$ & $\begin{array}{c}\text { Electrical } \\
\text { Generation } \\
\text { Capacity } \\
\end{array}$ & $\begin{array}{l}\text { Estimated } \\
\text { Capital } \\
\text { Cost } \\
\end{array}$ & $\begin{array}{l}\text { Calculated } \\
\text { Specific } \\
\text { Cost } \\
(\$ / \mathbf{k W e}) \\
\end{array}$ & Assumptions & Data Source \\
\hline $\begin{array}{l}\text { Sunflower Electric; } \\
\text { Holcomb Kansas }\end{array}$ & $\begin{array}{l}1400 \mathrm{MWe} \\
\text { total (two } \\
\text { units) }\end{array}$ & $\$ 3.6 \mathrm{~B}$ & 2570 & $\begin{array}{l}\text { Capital cost } \\
\text { is likely to be } \\
\text { "all-in" cost }\end{array}$ & $\begin{array}{l}\text { Washingtonpost.com; } \\
\text { October 19, 2007 } \\
\text { (Mufson 2007) }\end{array}$ \\
\hline $\begin{array}{l}\text { Kingsnorth, United } \\
\text { Kingdom }\end{array}$ & $\begin{array}{l}1600 \mathrm{MWe} \\
\text { (two units) }\end{array}$ & $\begin{array}{l}1.97 \mathrm{~GB} \\
\text { Pounds } \\
(\$ 3.94 \mathrm{~B}) \\
\end{array}$ & 2460 & "all-in" cost & $\begin{array}{l}\text { Platts Coal News; } \\
\text { January 7, } 2008 \\
\text { (Platt 2008) }\end{array}$ \\
\hline $\begin{array}{l}\text { Marshalltown, } \\
\text { Iowa; Alliant } \\
\text { Energy }\end{array}$ & $600 \mathrm{MWe}$ & $\$ 1.5 \mathrm{~B}$ & 2500 & "all-in" cost & $\begin{array}{l}\text { Energy Central } \\
\text { Network Web site; } \\
\text { January } 11,2008 \\
\text { (Energy Central } \\
\text { News 2008a) }\end{array}$ \\
\hline $\begin{array}{l}\text { Florence Co, South } \\
\text { Carolina; Santee- } \\
\text { Cooper Power }\end{array}$ & $600 \mathrm{MWe}$ & $\$ 1.0 \mathrm{~B}$ & 1667 & "all-in" cost & $\begin{array}{l}\text { Charleston, SC } \\
\text { Regional Business } \\
\text { Journal Web site; } \\
\text { December 10, 2007 } \\
\text { (Regional Business } \\
\text { Journal 2007) }\end{array}$ \\
\hline $\begin{array}{l}\text { Hempstead Co, } \\
\text { Alaska; SWEPCO }\end{array}$ & $600 \mathrm{MWe}$ & $\$ 1.34$ & 2233 & "all-in" cost & $\begin{array}{l}\text { Energy Central News } \\
\text { Web site; March 28, } \\
2008 \text { (Energy Central } \\
\text { News 2008b) }\end{array}$ \\
\hline $\begin{array}{l}\text { Cliffside, North } \\
\text { Carolina; Duke } \\
\text { Power }\end{array}$ & $\begin{array}{l}1600 \mathrm{MWe} \\
\text { (two units) }\end{array}$ & $\$ 3 B$ & 1875 & “all-in" cost & $\begin{array}{l}\text { Atomic Insights } \\
\text { Blog; Nov 19, } 2006 \\
\text { (Atomic Insights } \\
\text { 2006) }\end{array}$ \\
\hline $\begin{array}{l}\text { Oak Creek, } \\
\text { Wisconsin; } \\
\text { Wisconsin Power }\end{array}$ & $\begin{array}{l}1270 \mathrm{MWe} \\
\text { (two units) }\end{array}$ & $\$ 2.65 \mathrm{~B}$ & 2083 & “all-in" cost & $\begin{array}{l}\text { Amer. Council for } \\
\text { Energy Efficient } \\
\text { Economy } \\
\text { presentation (Elliot } \\
2006 \text { ) }\end{array}$ \\
\hline
\end{tabular}

All of the capital cost information in the press was in the form of "total project" or "all-in" cost, which presumably includes financing costs and perhaps escalation. The "overnight" costs that correspond with the above values would probably be $10-20 \%$ lower, depending on the interest rate and construction duration. The huge escalation in concrete, steel, and copper costs has affected coal plants as well as nuclear projects, but not to the same degree (less concrete and steel per kWe of capacity for coal). Most of the coal project cost estimates from earlier literature searches (2002-2005) showed projected specific costs considerably less than $\$ 1,900 / \mathrm{kwe}$. (Some of these are summarized in the annotated references.) There are a few projects "on the drawing board" involving the cleaner and technologically more sophisticated IGCC technology. First-of-a-kind projects of this type are likely to experience specific project costs upwards of $\$ 3,500 / \mathrm{kwe}$. NOAK IGCC coal plants are expected to be at least $20 \%$ higher in specific cost than NOAK pulverized coal plants (USA Today 2007). The addition of CCS equipment to 
pulverized coal plants could add upwards of 30\% to the project capital cost and impose a power penalty on the net generation capacity of the plant. The cases run in this study do not include such a power penalty, which would have to be made up by other generation sources. If these are fossil sources, the carbon "footprint" problem is compounded; however, for make-up nuclear capacity it is not.

Table 8-2 shows the input values selected for the three coal cases (low, nominal, and high) and the LUEC results for each case. Note that the carbon tax selected for these cases also has a low, nominal, and high value (at $\$ 5, \$ 40$, and $\$ 100 / \mathrm{MT} \mathrm{CO}_{2}$, respectively). Total costs are provided with and without the carbon tax. The carbon tax is entered in $\$ / \mathrm{MT} \mathrm{CO}_{2}$, but that the equivalent $\$ / \mathrm{MT} \mathrm{C}$ value is also provided.

Table 8-2. Pulverized coal inputs and results from coal "Mini-Model."

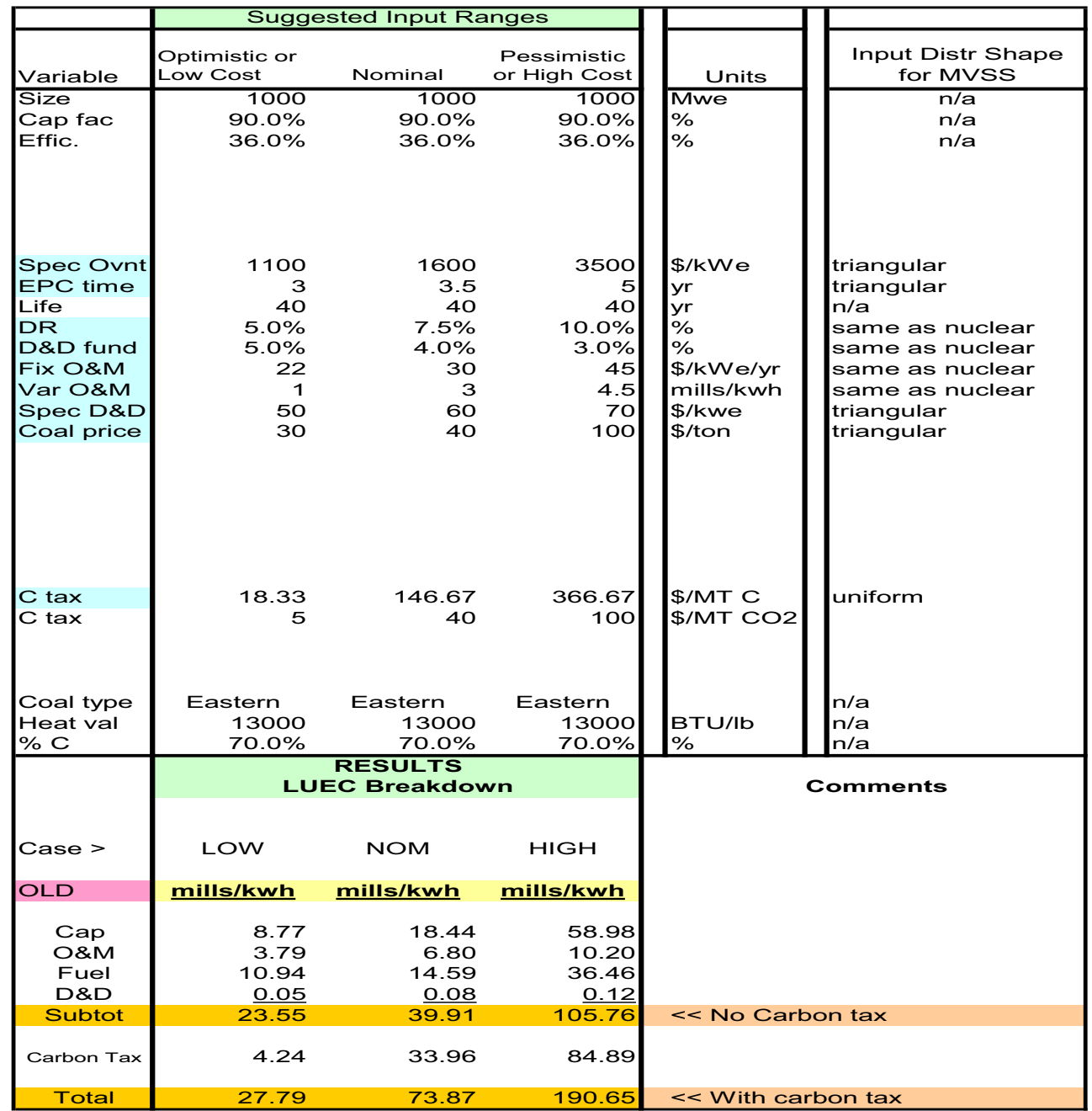

Note: "mills/kW·h" is the same as "\$/MWh." Divide above numbers by 10 to get cents $/ \mathrm{kwh}$.

Next to capital cost, the next largest cost is for coal, which makes up about $35 \%$ of the LUEC. As shown in Figure 8-2 below, the price depends on the location from which the coal is mined, and is higher for coals with higher heat value (BTUs/lb), such as Appalachian coals. If the price of coal were expressed in dollars per million BTUs, the prices would be closer together. Coal has recently experienced a run-up in price along with oil, steel, and other commodities with high international demand. The U.S. costs below are significantly less than for coal purchased for use in Japan, where large long-distance transportation costs must be added. U.S. coal is also experiencing additional costs due to the higher cost of fuel (diesel oil) required to transport coal over highways or by rail within the U.S. 


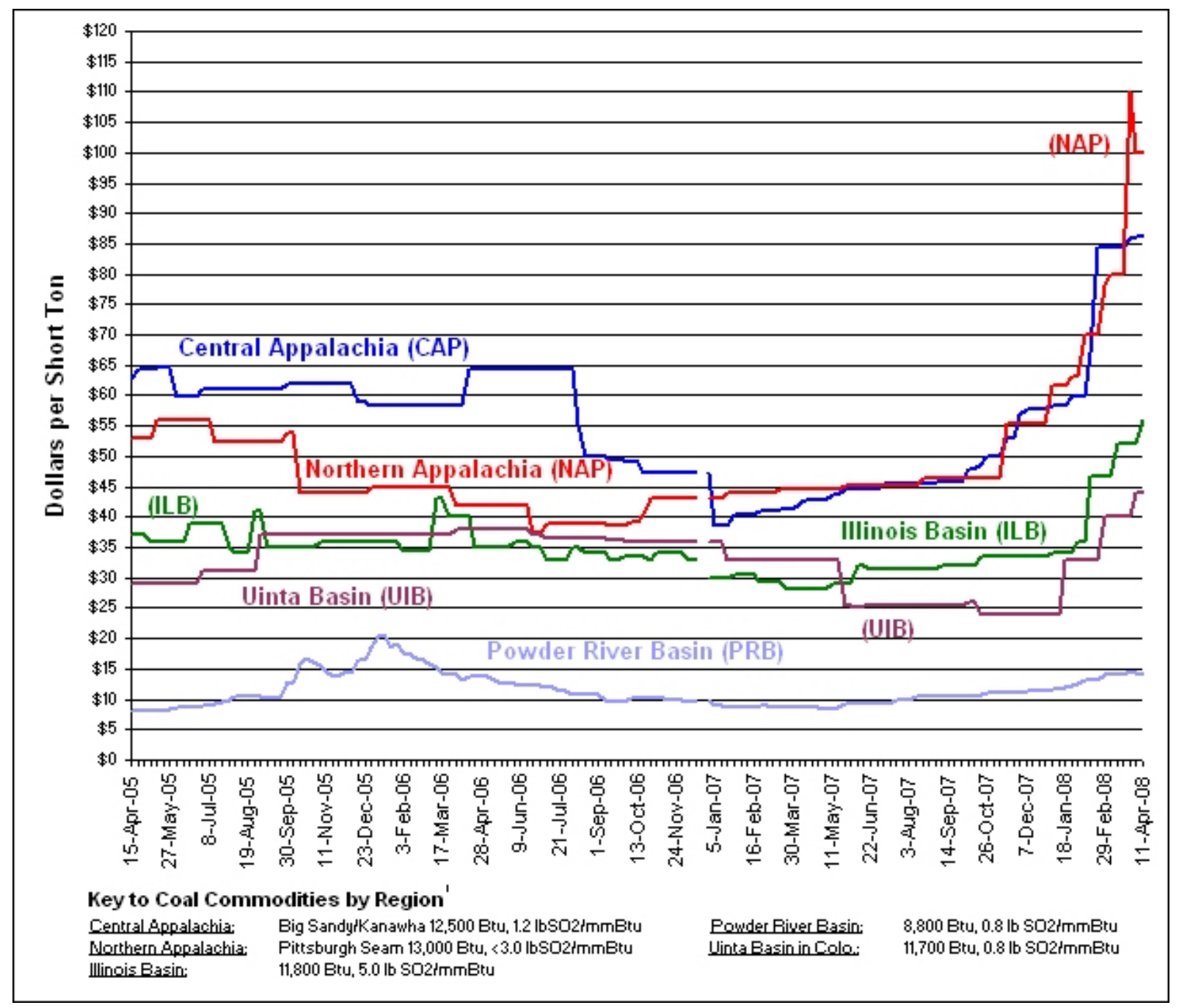

Figure 8-2. Coal price history data from DOE-EIA.

Selecting appropriate carbon costs $\left(\$ / \mathrm{MT} \mathrm{C}\right.$ or $\left.\$ / \mathrm{MT} \mathrm{CO}_{2}\right)$ was also difficult because of the uncertainties involved and wide range of suggested values. There are many bills before Congress on greenhouse gas control, and only recently have reports begun to appear that gauge the true "costs" associated with the various legislative proposals. Multiple studies were used to guide the selection of the range for the cost of carbon (Lombor 2008; McKinsey \& Co. 2007; Engineering News Record 2007; MIT 2007; Bauer 2006; and Pew Center 2008). Note that for the "nominal cost" and "high cost" cases, the carbon tax nearly doubles the LUEC compared to the cost without the tax. The following tornado diagrams summarize the cost variable uncertainty for coal without a carbon tax (Figures 8-3) and with a carbon tax (Figure 8.4). At the end of each bar are the low and high values of the input variable and the output LUEC value that corresponds to each. Note the carbon cost range in the uncertainty analysis are in units of \$/MT C, which correspond to a low range of $\$ 5 / \mathrm{MT} \mathrm{CO}_{2}(\$ 18.33 / \mathrm{MT} \mathrm{C})$ and a high range of $\$ 100 / \mathrm{MT} \mathrm{CO}_{2}(\$ 366.67 / \mathrm{MT} \mathrm{C})$. 


\section{Coal Cost Uncertainties}

without carbon tax

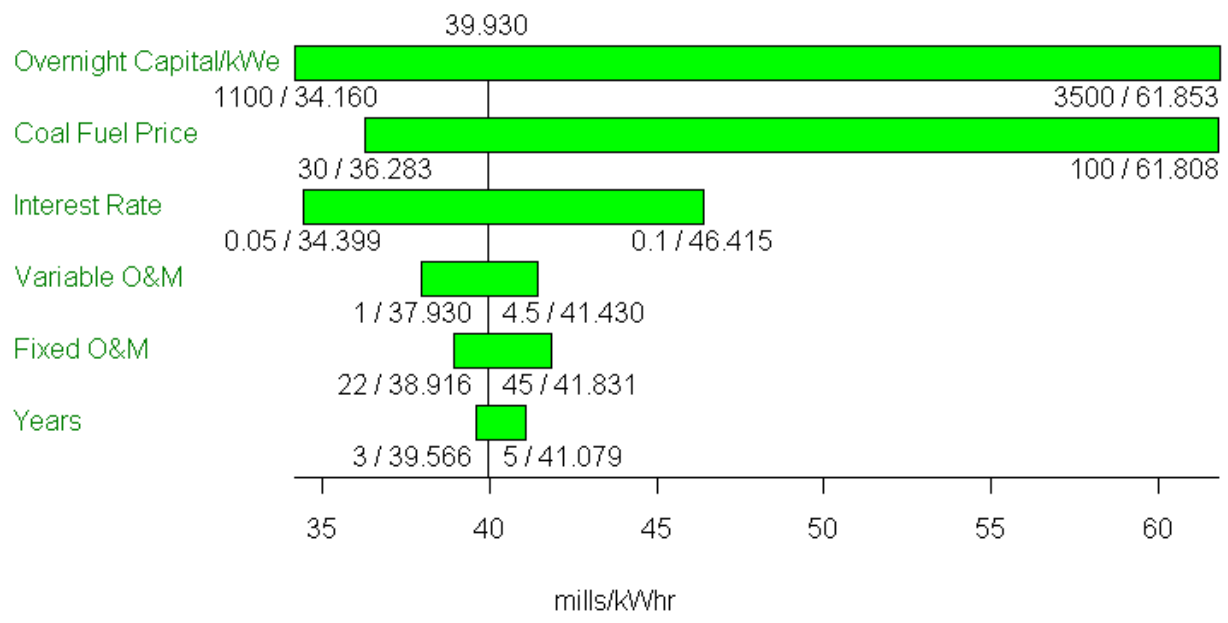

Figure 8-3. Cost variable uncertainty for coal without carbon cost.

\section{Coal Cost Uncertainties}

with carbon tax

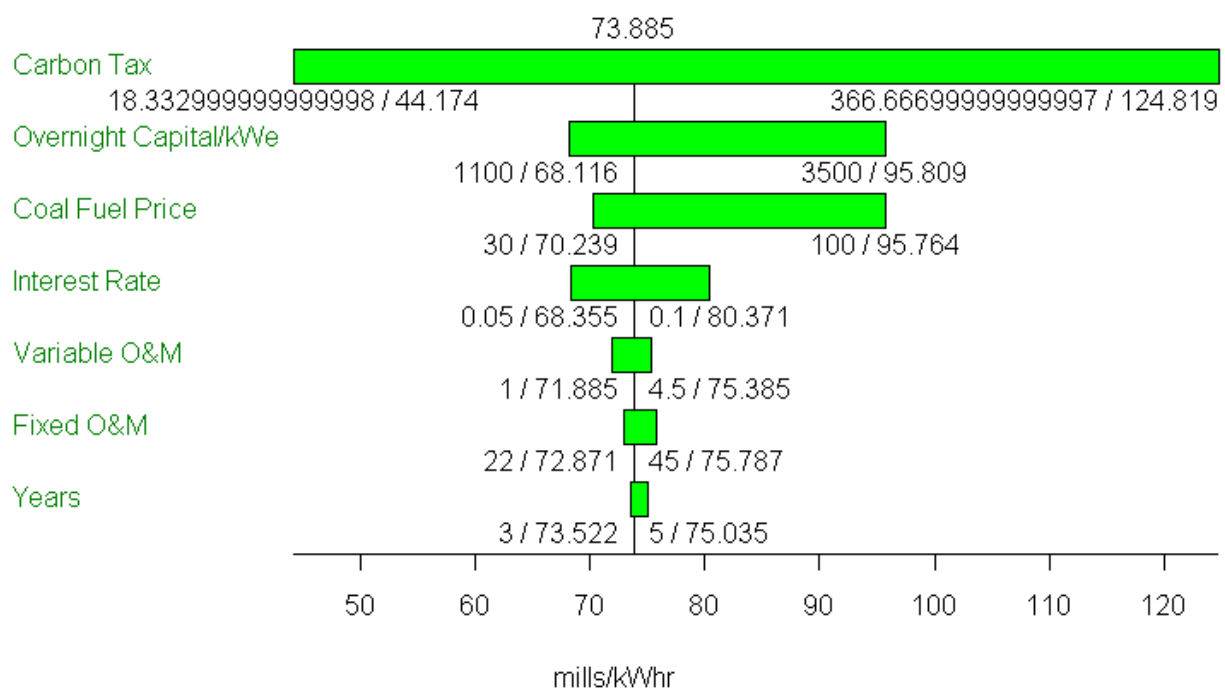

Figure 8-4. Cost variable uncertainty for coal with carbon cost.

The following relative probability chart in Figure 8-5 shows the results of a cost distribution for coal where all input variables are simultaneously considered and drawn from their assigned input distributions. The Decision Programming Language model was used to produce the cost distributions. Carbon tax (cost) has a huge effect on coal's busbar LUEC, and that the uncertainty in the long-range average $\$ / \mathrm{MT} \mathrm{CO}_{2}$ value widens the uncertainty band for the LUEC. (The carbon cost ranges from $\$ 5 / \mathrm{MT} \mathrm{CO}_{2}$ to $\$ 100 / \mathrm{MT}$ $\mathrm{CO}_{2}$, a range of $\$ 95 / \mathrm{MT} \mathrm{CO}_{2}$. The uncertainly in the carbon cost is larger than the nominal value of $\$ 40 / \mathrm{MT} \mathrm{CO}_{2}$ selected; hence, the carbon cost uncertainty is itself the major driver.) The vertical lines within each distribution on the chart represent the "expected value" or statistical mean for each curve. 


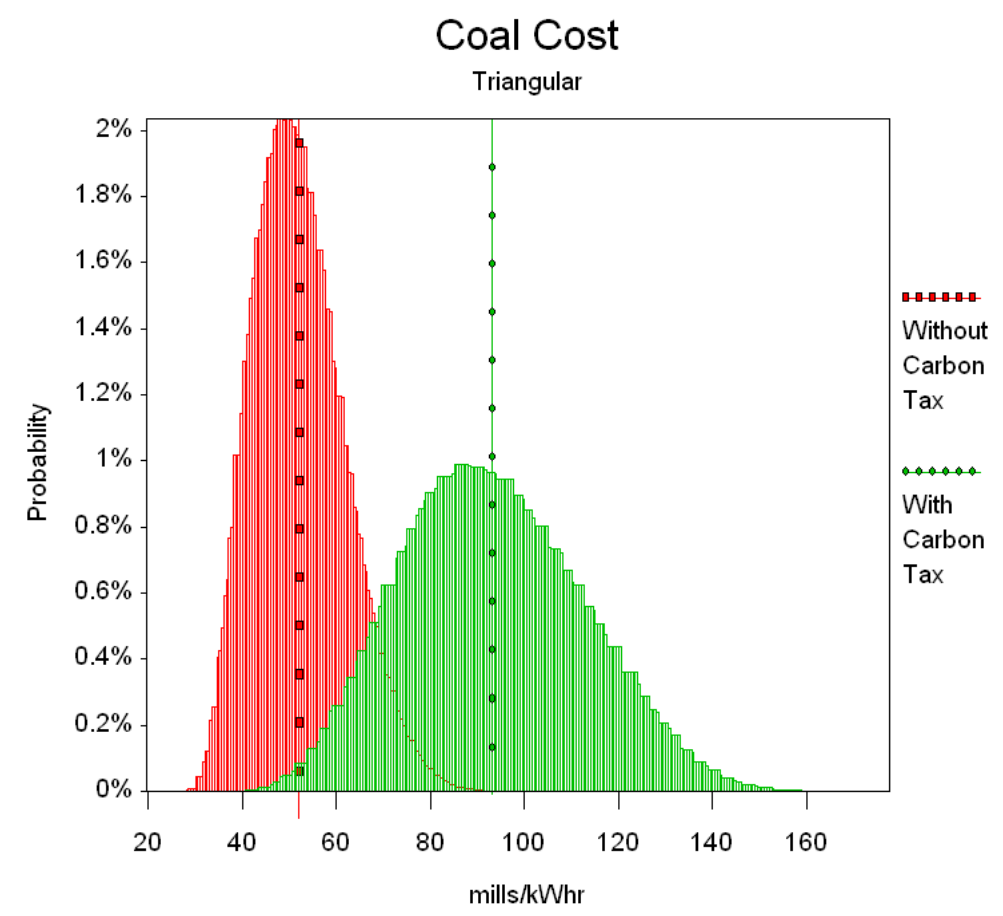

Figure 8-5. Cost variable uncertainty for coal both with and without carbon cost.

\subsection{Baseload Natural Gas Calculations}

Natural gas combustion became the technology of choice for new U.S. baseload electrical capacity in the early and mid-1990s for the following reasons:

- The specific capital investment cost ( $\$ / \mathrm{kWe}$ of capacity) for the plant is very low relative to other technologies (overnight cost $<\$ 1,000 / \mathrm{kWe}$ ).

- The plants can be constructed in half the time or less than nuclear or coal facilities (typically $1.5-3$ years).

- The plants occupy much less space (land area and volume) than coal or nuclear plants.

- If combustion turbines are used, the thermodynamic efficiency can be much higher than for coal or water reactors.

- Less $\mathrm{SOx}$ and $\mathrm{CO}_{2}$ are emitted per kilowatt-hour than for coal.

- Low particulates are emitted.

- The feedstock (natural gas) can be piped directly to the plant and minimal onsite fuel storage is required.

- Gas units can be quickly brought in or out of service, and can also be used to supply peaking power.

- In the early and mid-1990s, plentiful natural gas was available at a price of \$2-\$5 per million British Thermal Units (MMBTUs).

- Most U.S. natural gas is delivered by pipeline from "friendly" North American well sources. As new receiving terminals are constructed along the coasts, an increasing amount of liquid natural gas (LNG) will be imported into the U.S.

For this technology, the fuel cost is the predominant ( $>60 \%)$ component of the LUEC, even if the fuel cost is low. In the late 1990s, the unit cost of natural gas began to rise precipitously, mainly driven by 
demand for this versatile fuel within the electric power and petrochemical industry, where it is both an energy source and a feedstock. Irregularities due to industry deregulation, such as the ENRON crisis, also caused the price to spike. The natural gas price nearly tripled and then stabilized over the early part of this decade to around \$7/MMBTUs, which is over twice the cost seen in the early 1990s. The gas price is on the rise again (now $>\$ 10 /$ MMBTU), due to rising crude oil prices which tend to drive the prices of all fuel commodities after some time lags.

The price rise in natural gas has now made it much less competitive when compared with "no-carbon cost" coal and existing nuclear. The rapidity with which gas can be brought online (18 months), capability to meet stringent air quality regulations, and the ability to pass along fuel cost increases to the customer make gas a baseload contender in some markets. Gas generation will also be affected by the carbon costs, whether they are taxes, cap, and trade programs, or CCS. Because a significant portion of the energy from natural gas (mostly $\mathrm{CH}_{4}$ ) is derived from hydrogen (and its $\mathrm{H}_{2} \mathrm{O}$ combustion product) rather than carbon (and its $\mathrm{CO}_{2}$ combustion product) the effect of carbon costs will be significantly smaller (i.e., about half as much as coal).

The "mini G4 ECONS" type model for natural gas was run for low, nominal, and high cost cases to gauge the effects of gas cost, specific capital cost, discount rate, and carbon costs on the LUEC.

Figure 8-6, an image from the model spreadsheet, shows the model inputs and results for the nominal case.

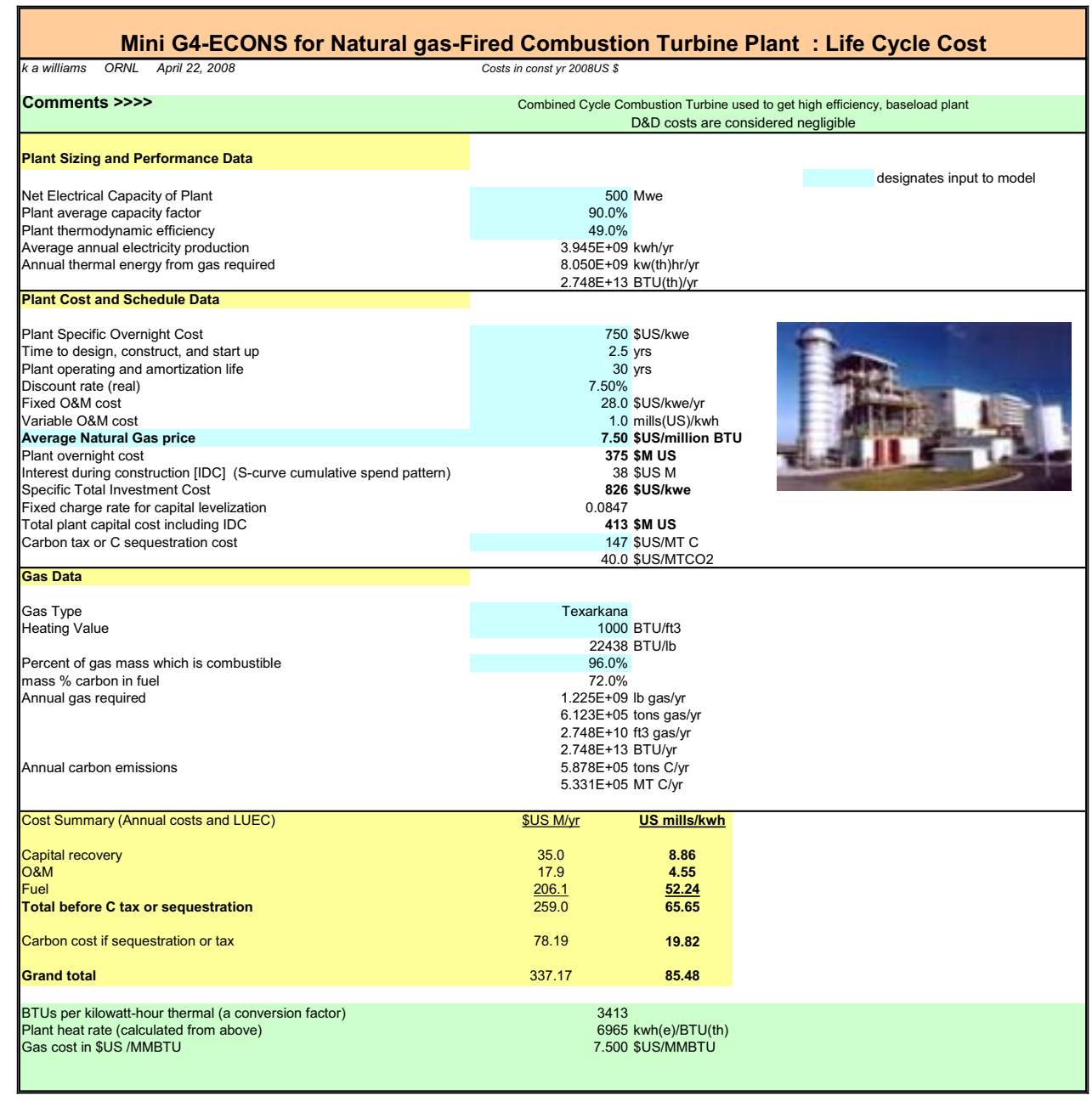

Figure 8-6. Economics of natural gas turbine combined-cycle technology using levelized cost model of the G4 ECONS type (spreadsheet image of nominal case). 
Table 8-3 shows the inputs and results for all three cases: low cost (optimistic), nominal (most likely) cost, and high (pessimistic) cost. The LUEC both with and without a carbon tax are shown. The specific capital cost of natural gas plants (combined cycle gas turbine [CCGT] assumed in this case) have also risen as a result of the recent 2004-2008 "run-up" in commodity prices (i.e., concrete, steel, copper, and specialty metals). This commodity "run-up" or real escalation above normal inflation will be discussed in a later subsection.

Table 8-3. CCGT natural gas inputs and results from "Mini-model."

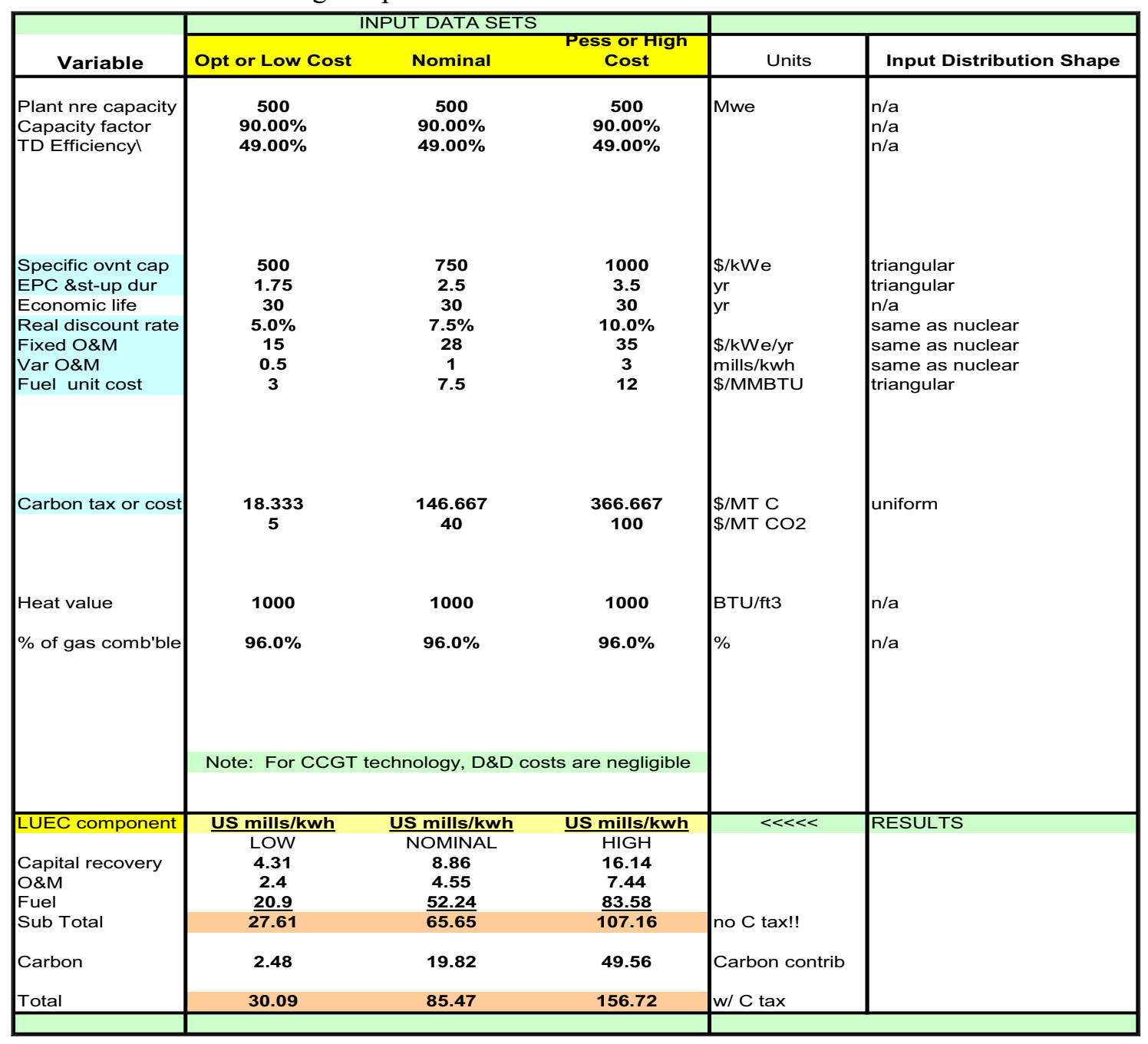

The natural gas combustion technology of choice is the high efficiency combustion turbine. If a combined cycle is used, where the hot turbine offgas is used to produce steam for a "topping" cycle, the plant thermodynamic efficiency can be much higher (40-50\% efficiency) than for a coal plant or an LWR (typically 32-38\% efficiency). This technology's efficiency helps to drive down the capital component of the LUEC for natural gas generation. As stated earlier, however, the cost of natural gas fuel is the main driver of the LUEC, and its price volatility is a serious problem for this technology. Table 8-4 shows how the gas price has varied considerably over the last 6 years. The prices shown are wholesale, national, and monthly average prices, which are less volatile than the "spot" market prices. A single natural gas powerplant plant could see greater "spikes" over its life cycle. 
Table 8-4. EIA historical price for wholesale natural gas consumed by electric powerplants $\left(1 \$ /\right.$ thousand $\mathrm{ft}^{3}=0.988$ \$MMBTU; April 2008 value added by ORNL author) (DOE-EIA 2008).

\begin{tabular}{|r|rrrrrrrrrrrrrr|r|}
\hline \multicolumn{8}{|c|}{ U.S. Natural Gas Electric Power Price (Dollars per Thousand Cubic Feet) } \\
\hline Year & Jan & Feb & Mar & Apr & May & Jun & Jul & Aug & Sep & Oct & Nov & Dec \\
$\mathbf{2 0 0 2}$ & 3.10 & 2.86 & 3.37 & 3.80 & 3.78 & 3.61 & 3.49 & 3.42 & 3.71 & 4.19 & 4.35 & 4.72 \\
$\mathbf{2 0 0 3}$ & 5.33 & 6.47 & 7.05 & 5.38 & 5.70 & 6.08 & 5.45 & 5.23 & 5.12 & 4.98 & 4.85 & 5.69 \\
$\mathbf{2 0 0 4}$ & 6.37 & 5.76 & 5.50 & 5.74 & 6.30 & 6.52 & 6.24 & 5.97 & 5.39 & 6.05 & 6.71 & 6.88 \\
& & & & & & & & & & & & \\
$\mathbf{2 0 0 5}$ & 6.72 & 6.42 & 6.84 & 7.27 & 6.83 & 7.08 & 7.57 & 8.67 & 10.99 & 11.84 & 9.87 & 11.26 \\
$\mathbf{2 0 0 6}$ & 9.15 & 8.00 & 7.36 & 7.32 & 6.89 & 6.69 & 6.69 & 7.56 & 6.27 & 5.76 & 7.48 & 7.57 \\
$\mathbf{2 0 0 7}$ & 7.05 & 8.16 & 7.64 & 7.76 & 7.96 & 7.80 & 7.01 & 6.80 & 6.35 & 7.04 & 7.27 & NA \\
$\mathbf{2 0 0 8}$ & NA & NA & NA & $>11.0$ & & & & & & & &
\end{tabular}

Figure 8-7 from Wiser's 2004 conference presentation shows the difficulty in predicting the price of natural gas. The two-digit legend labels for each curve show the year that a projection was made. From 1985 through 1996, each year's price projections trended lower than the previous year forecast (downward arrow) suggesting a period of stable and slowly escalating prices. From 1997 onward, the opposite effect has occurred (upward arrow), and the current actual wellhead price (around \$7/MMBTU) is well above any of the 1997-2004 projections (DOE-EIA 2008).

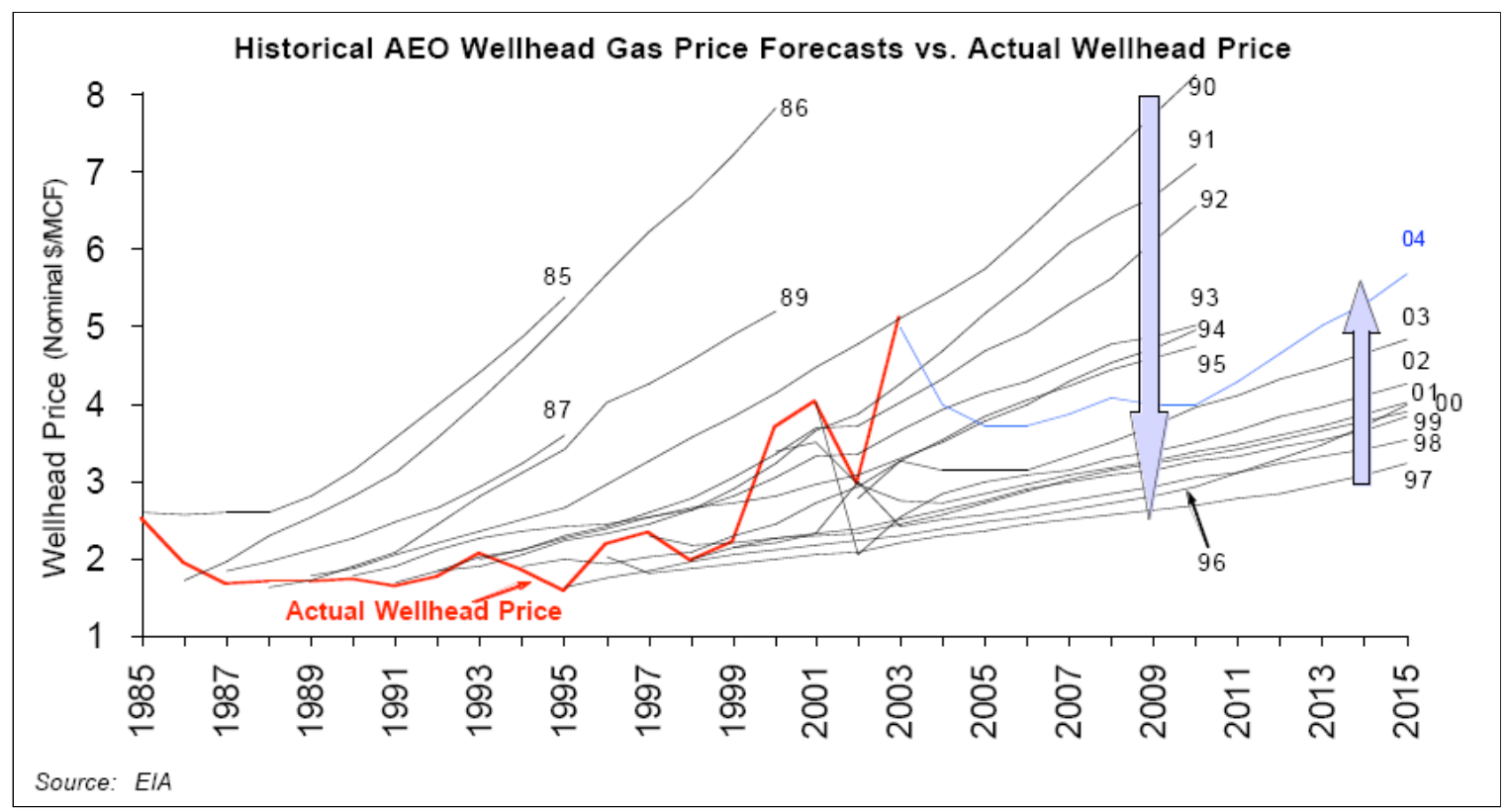

Figure 8-7. Variation in price projections for natural gas compared with the historical average annual wholesale wellhead price (Wiser 2004).

If or when a carbon tax is imposed, a likely response would be to build additional natural gas power plants due to their lower emissions of carbon and consequently lower carbon penalty (compared to coal). However, this action could cause a higher dependency on natural gas and serve to reduce supplies of natural gas more quickly than would otherwise occur. ${ }^{\mathrm{p}}$

p. Natural gas is important to preserve for non-energy uses such as plastics, petrochemicals, etc. Ammonia-based fertilizers also require natural gas as a feedstock. 
Increasing the LNG imports may have the effect of stabilizing the long-term natural gas price (Fielden 2003). Presently, the price of LNG is heavily coupled to the price of crude oil, but as a larger market develops, a decoupling is likely to occur (Gulf Times Newspaper 2007). The LNG production and transportation cost (not price) is estimated to be around \$4/MMBTU. Many existing non-U.S. contracts for LNG are now being priced over \$10/MMBTU.

The tornado diagrams in Figures 8-8 and 8-9 summarize the cost uncertainty for natural gas with and without the carbon tax. At the end of each bar are the low and high values of the input variable and the output LUEC value that corresponds to each. Note the carbon cost range in the uncertainty analysis are in units of $\$ / \mathrm{MT} \mathrm{C}$, which correspond to a low range of $\$ 5 / \mathrm{MT} \mathrm{CO}_{2}(\$ 18.33 / \mathrm{MT} \mathrm{C})$ and a high range of $\$ 100 / \mathrm{MT} \mathrm{CO}_{2}(\$ 366.67 / \mathrm{MT} \mathrm{C})$.

\section{Natural Gas Cost Uncertainties}

without carbon tax

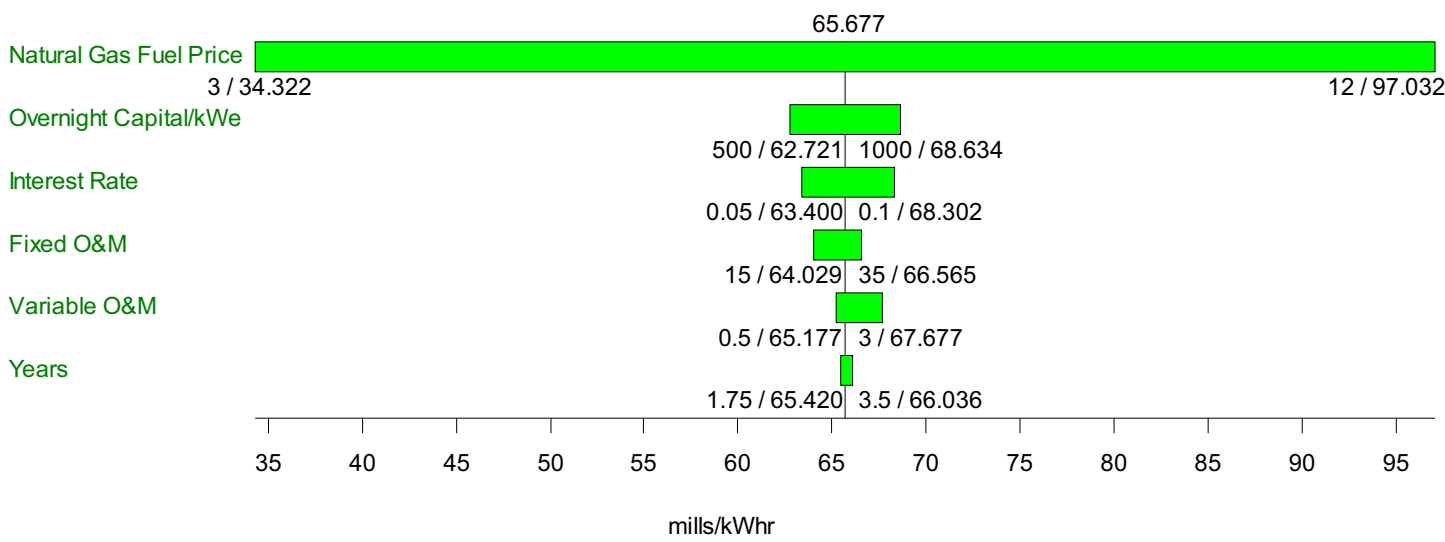

Figure 8-8. Cost variable uncertainty for natural gas without carbon cost.

\section{Natural Gas Cost Uncertainties}

with carbon tax

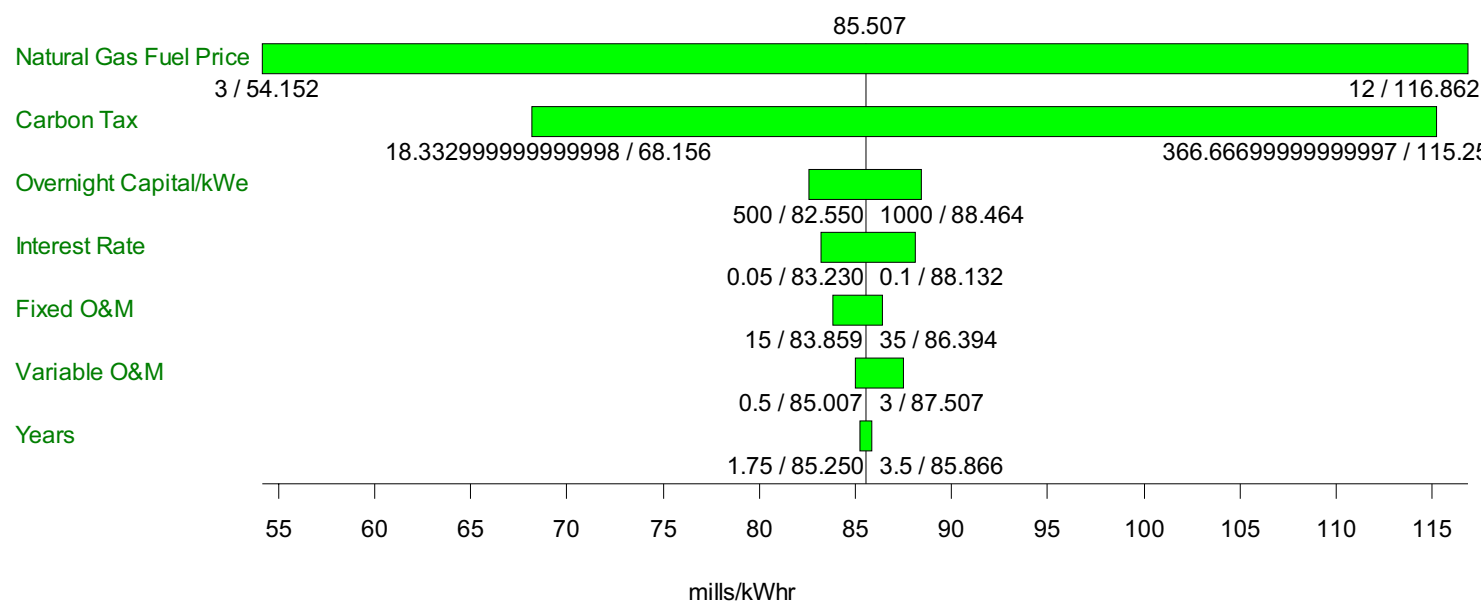

Figure 8-9. Cost variable uncertainty for natural gas with carbon cost. 
The following relative probability chart (Figure 8-10) shows the uncertainty distribution for natural gas where all input variables are simultaneously considered and drawn from their assigned input distributions. The carbon tax (cost) has a significant effect on natural gas's busbar LUEC, and the uncertainty in the long-range average $\$ / \mathrm{MT} \mathrm{CO}_{2}$ value widens the uncertainty band somewhat for the LUEC. The effects on natural gas, however, are much smaller than the carbon cost effects on coal. The vertical lines within each distribution on the Figure 8-10 chart represent the "expected value" or statistical mean for each curve.

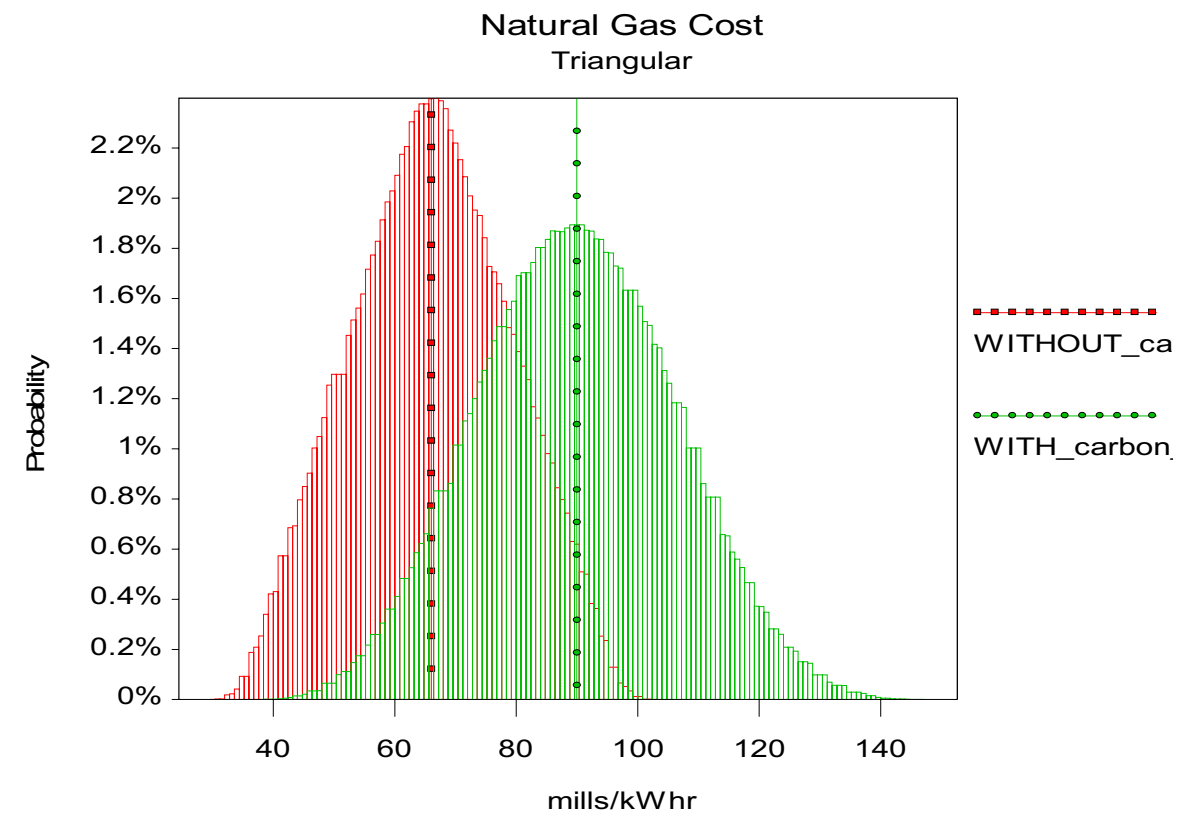

Figure 8-10. Cost variable uncertainty for coal both with and without carbon cost.

\subsection{Comparison of Baseload Generation Technologies}

A cost comparison of the three nuclear cases (once-through, 1-Tier, and 2-Tier) to the projected LUECs for coal ${ }^{\mathrm{q}}$ and natural gas is provided in Table 8-5. ${ }^{\mathrm{r}}$ Untaxed coal (no carbon costs) and "oncethrough" nuclear costs are very close, with a slight (4-6\%) cost advantage to coal for the "low" and "nominal" cases, respectively. This result is consistent with other studies discussed in Sections 8.6 and 8.7. For the "high" case comparing untaxed coal with all nuclear scenarios, coal generation is more expensive due to the high fuel costs having a much greater influence on coal than on nuclear generation. Nuclear, coal, and natural gas costs (with and without carbon tax) are plotted in Figure 8-11, along with their associated low, nominal, and high values. The notable findings are:

- A small carbon cost of only $\$ 15 / \mathrm{MT} C$ will drive the coal generation cost above even the most expensive (2-Tier fuel cycle) nuclear generation cost (for the "low" and "nominal" scenarios). No carbon cost is needed for the "high" case comparison of coal and nuclear.

- For the nominal coal case with carbon tax $\left(\$ 40 / \mathrm{MT} \mathrm{CO}_{2}\right)$ the coal LUEC increases by $75 \%$.

- The percentage increase between no-tax and tax for coal and natural gas is much less for the low C-tax $\left(\$ 5 / \mathrm{MT} \mathrm{CO}_{2}\right)$ than for the nominal $\left(\$ 40 / \mathrm{MT} \mathrm{CO}_{2}\right)$ and high $\left(\$ 100 / \mathrm{MT} \mathrm{CO}_{2}\right)$ coal cases.

q. The reference coal plant represents the latest pulverized coal technology with capture of all pollutants except $\mathrm{CO}_{2}$.

r. Note that the LUEC value is the same as the busbar TCOE or "total cost of electricity"; the value is the same whether expressed in "mills/kwh" or "\$/MWh. 
- With FY-07 natural gas prices (\$7.5/MMBTU for the nominal case), the nominal natural gas costs without the $\mathrm{C}$-tax is $30+\%$ above that for coal (without the $\mathrm{C}$-tax) and the nuclear cases.

- Addition of the C-tax to natural gas has a much smaller effect on this fuel source than it does for coal (and coal's much higher carbon content).

Table 8-5 also shows the fuel cycle component of the LUEC for all three technologies. Even with the addition of closed cycle options for nuclear, the overall fuel-related costs for nuclear are a fraction of those for the fossil technologies. This, of course, stems from nuclear fuel producing 1.E5 to 1.E6 (nearly a million times) as much energy per unit mass as compared to fossil fuels. In Table 8-5 the cost of carbon (tax or CCS) is not included in the fuel cost component of the LUEC for the fossil fuel technologies, it is called out separately in Tables 8-2 and 8-3.

Table 8-5. Tablular representation of the low, nominal, and high LUECs for baseload generation technologies.

\begin{tabular}{|c|c|c|c|c|c|c|c|}
\hline Assumptions & TECHNOLOGY & $\begin{array}{c}\text { Overall LUEC (LOW) } \\
\text { S/MWh }\end{array}$ & $\begin{array}{c}\text { Overall LUEC (NOM) } \\
\text { SIMWh }\end{array}$ & $\begin{array}{c}\text { Overall LUEC (HIGH) } \\
\text { \$IMWh }\end{array}$ & $\begin{array}{c}\text { Fuel Comp of LUEC } \\
\text { (LOW) \$/MWh }\end{array}$ & $\begin{array}{l}\text { Fuel Comp of LUEC } \\
\text { (NOM) \$/MWh }\end{array}$ & $\begin{array}{c}\text { Fuel Comp of LUEC } \\
\text { (HIGH) \$IMWh }\end{array}$ \\
\hline \multirow{7}{*}{$\begin{array}{l}\text { All LWR generation } \\
37.1 \% \text { of Gen by FRs } \\
25.7 \% \text { of Gen by FRs } \\
\text { Pulverized Coal Tech } \\
\text { Pulverized Coal Tech } \\
\text { CCGT Technology } \\
\text { CCGT Technology }\end{array}$} & \multirow{7}{*}{$\begin{array}{l}\text { Nuclear (OT) } \\
\text { Nuclear (1-Tier) } \\
\text { Nuclear (2-Tier) } \\
\text { Coal (No C Tax) } \\
\text { Coal (w/ C-Tax) } \\
\text { Nat Gas (No C-Tax) } \\
\text { Nat Gas (w/ C-Tax) }\end{array}$} & 24.65 & 4229 & 80.07 & 353 & 6.51 & 13.21 \\
\hline & & 26.43 & 48.26 & 93.84 & 4.24 & 8.22 & 14.73 \\
\hline & & 26.67 & 47.86 & 91.93 & 4.81 & 9.13 & 16.59 \\
\hline & & 23.55 & 39.91 & 105.76 & 10.94 & 14.59 & 36.46 \\
\hline & & 27.79 & 73.87 & 190.65 & & & \\
\hline & & 27.61 & 65.65 & 107.16 & 20.9 & 52.24 & 83.58 \\
\hline & & 30.09 & 85.47 & 156.72 & & & \\
\hline
\end{tabular}

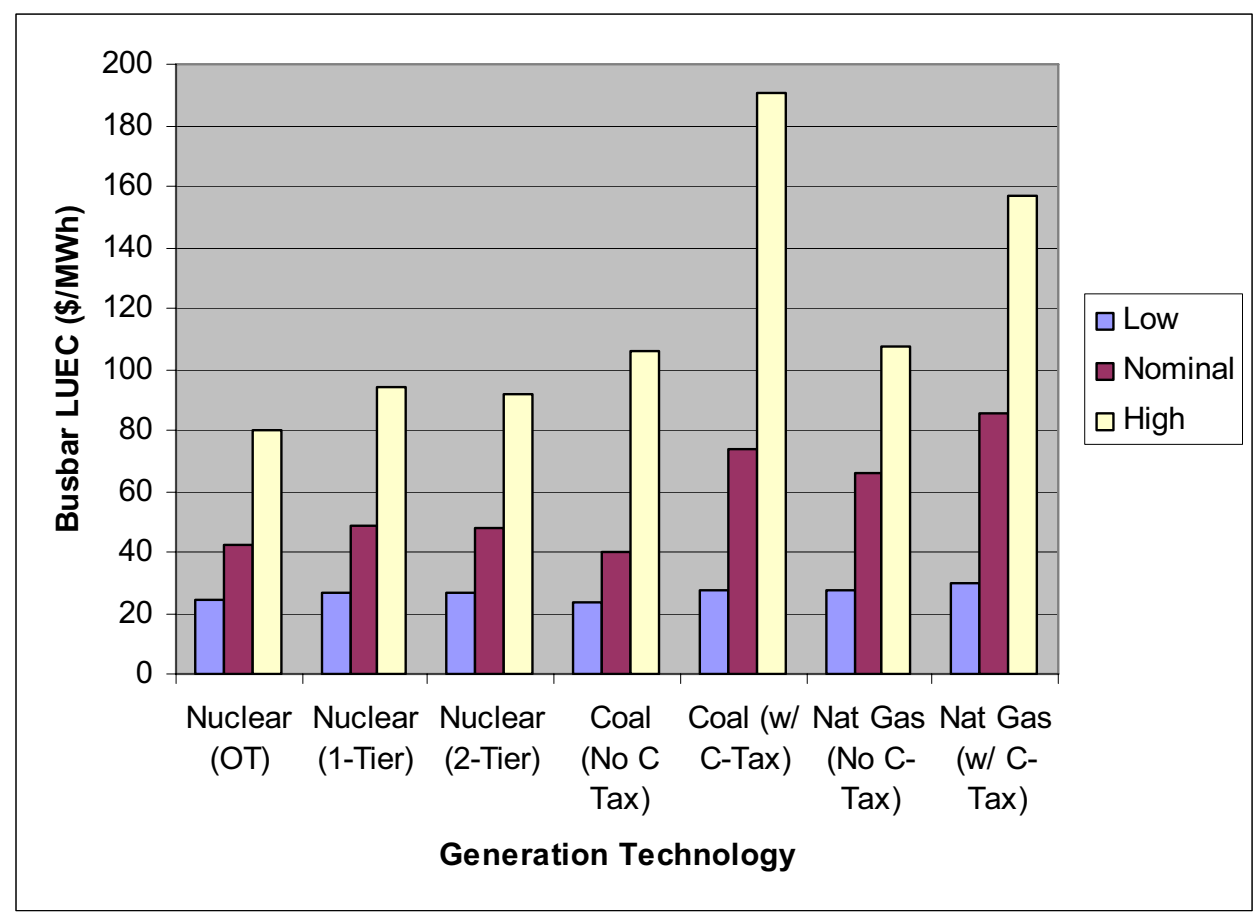

Figure 8-11. Graphical representation of the low, nominal, and high LUECs for baseload generation technologies. 
The following conclusions can be drawn from these studies:

- The likely effect of closing the fuel cycle will have a much smaller percentage-wise effect on nuclear's LUEC than the addition of a "cost of carbon" (tax or CCS) will have on the LUECs for the fossil technologies.

- The cost of carbon is a major LUEC driver for the fossil technologies.

- The fossil fuel costs are also driven by the cost of the fuels themselves, which tend to move with the cost of crude oil. The oil-linkage effect is greatest for natural gas.

- The cost of carbon is assumed to cover the social and environmental costs of greenhouse gas emission or the costs of mitigation/adaptation. Nuclear costs include all costs for safety, emissions control, regulation, and accident prevention measures. This addition of "externalities" to the fossil energy costs helps to provide a more realistic and fair comparison of generation technologies.

- A caveat: This multivariable uncertainty analysis implicitly assumes that the various input cost components vary independently of each other. This is unlikely to be exactly true. A correlation may exist between some of the input costs (e.g., coal plant construction costs and O\&M costs are probably somewhat linked). Correlations across technologies may also exist (e.g., high-cost coal plants will likely be competing with high-cost nuclear plants). There was not sufficient time nor funding to examine and mathematically represent these possible interactions.

The DPL uncertainty analysis was applied to the fossil fuel input ranges and distributions in the same manner as it was for the nuclear cases in Section 6. Figure 8-12 shows the uncertainty cost distributions for all three technologies. The fossil fuel technologies are shown both with and without the cost of carbon. The uncertainty in the cost of carbon is the largest uncertainty driver for the fossil fuel technologies. For natural gas, the volatility in pricing is also a highly significant contributor to the wide distribution.

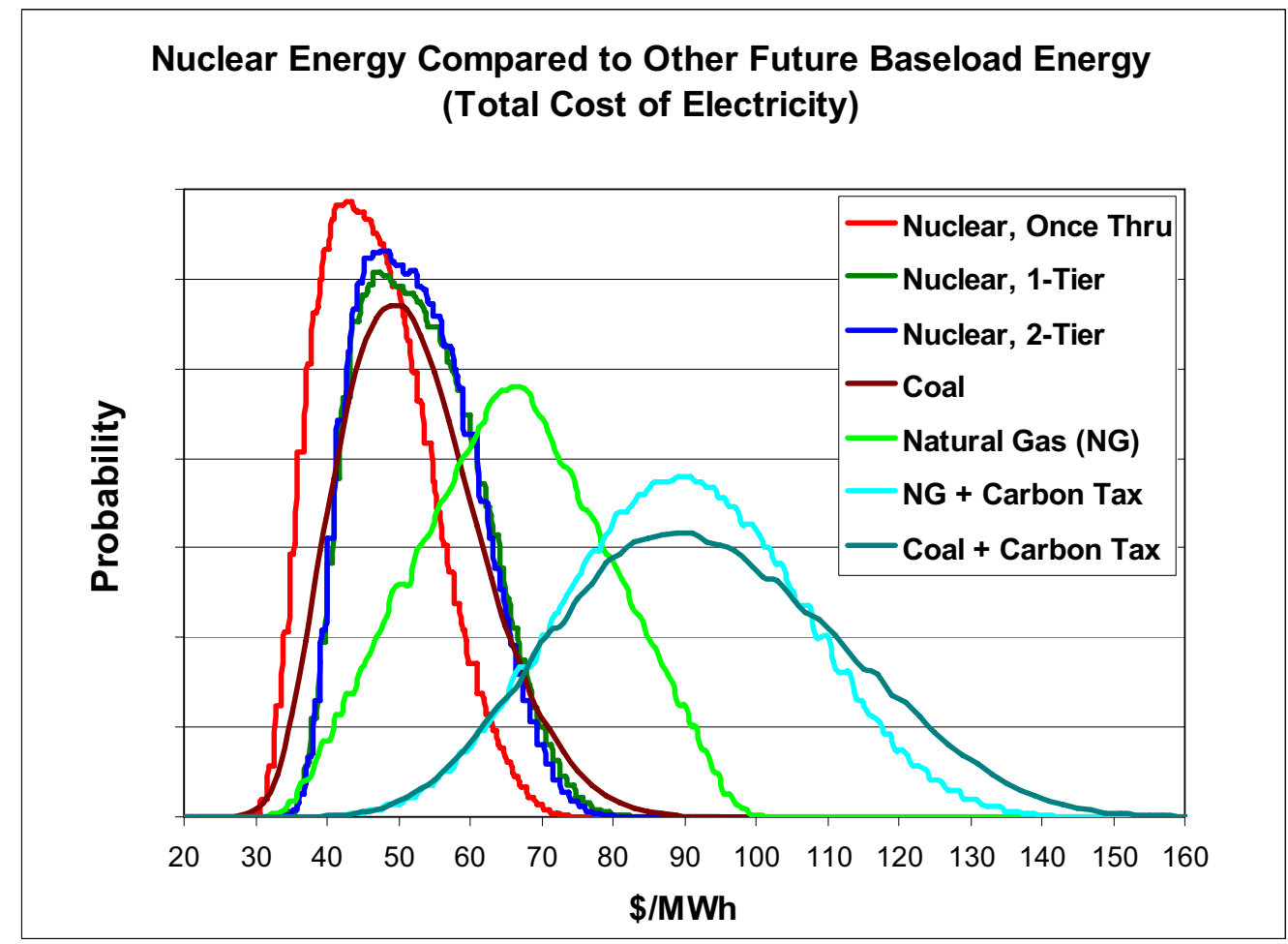

Figure 8-12. Total cost of energy for all generation technologies. 


\subsection{Some Comments on the Above Economic Comparison and More Recently Published Data}

Most of the data in the above analyses is based on 2003-2007 cost information gathered in FY-07 and reported in Shropshire et al.'s 2007 and 2008 reports. In the last several months considerable new data, much of it produced in January through October 2008, on coal and nuclear plant capital costs has become available as utilities begin obtaining pre-construction regulatory approvals from Public Service Commissions and begin seeking financing for their projects. The new data points are much less optimistic than earlier (2002-2007) data and reflect continuing escalation in prices for basic construction commodities, skilled labor, and specialized "N-stamp" engineering/fabrication services. The new data also take into account longer engineer, procure, and construct (EPC) schedules due to perceived regulatory schedule slip and the difficulties in scheduling certain long lead procurements such as large forgings (for nuclear). Table 8-6 shows the historical escalation for several items that is well above the general inflation rate as measured by normal U.S. Government price deflators. Continuing high demand for commodities and engineering/construction services both in the U.S. and abroad (mostly China and India) is likely to extend the escalation trends shown over at least the next few years.

Table 8-6 Escalation rates affecting powerplant costs. (Source: American Electric Power: Harding 2008)

\begin{tabular}{|l|c|c|}
\hline \multicolumn{1}{|c|}{ COMMODITY } & $1986-2003 \mathrm{ESC}$ & $2003-2007 \mathrm{ESC}$ \\
\hline Nickel & $3.8 \% / \mathrm{yr}$ & $60.3 \% / \mathrm{yr}$ \\
\hline Copper & $3.3 \% / \mathrm{yr}$ & $69.2 \% / \mathrm{yr}$ \\
\hline Cement & $2.7 \% / \mathrm{yr}$ & $11.6 \% / \mathrm{yr}$ \\
\hline Iron/Steel & $1.2 \% / \mathrm{yr}$ & $19.6 \% / \mathrm{yr}$ \\
\hline Heavy Construction & $\mathbf{2 . 2 \%} / \mathbf{y r}$ & $\mathbf{1 0 . 5 \% / y r}$ \\
\hline
\end{tabular}

Table 8-7 shows some overnight and "all-in" specific costs for three U.S. reactor projects for which new data has recently become available.

Table 8-7. Recently published specific capital costs for selected proposed U.S. LWR projects.

\begin{tabular}{|l|l|l|l|}
\hline \multicolumn{1}{|c|}{$\begin{array}{c}\text { Project Name and } \\
\text { Owner }\end{array}$} & \multicolumn{1}{|c|}{ Plant Capacity } & \multicolumn{1}{c|}{ Specific cost $(\$ / \mathrm{kWe})$} & \multicolumn{1}{c|}{ Source } \\
\hline $\begin{array}{l}\text { Turkey Point; Florida } \\
\text { Power \& Light }\end{array}$ & 2200 MWe (2-unit) & $\begin{array}{l}\text { Overnight Range: } \\
3108-4540 ; \\
\text { All-in Range: } 5492- \\
8071\end{array}$ & $\begin{array}{l}\text { Nuclear Information } \\
\text { Resource Service Web } \\
\text { site (NIRS 2008) }\end{array}$ \\
\hline $\begin{array}{l}\text { Levy County I \& II; } \\
\begin{array}{l}\text { Florida; Progress } \\
\text { Energy }\end{array}\end{array}$ & $\begin{array}{l}\text { 2200 MWe (2 AP- } \\
1000 \mathrm{~s})\end{array}$ & $\begin{array}{l}\text { Overnight: 4200 } \\
\text { All-in: } 6350 \text { incl esc }\end{array}$ & Progress Energy 2008 \\
\hline $\begin{array}{l}\text { Williams S. Lee III; } \\
\begin{array}{l}\text { Cherokee Co South } \\
\text { Carolina; Duke Energy }\end{array}\end{array}$ & 2234 MWe (2-unit) & All-in: 3580 & $\begin{array}{l}\text { GoUpstate.com Web } \\
\text { site (April 22, 2008) } \\
\text { (Go Upstate 2008) }\end{array}$ \\
\hline
\end{tabular}

Note that the specific overnight costs reported above for what will be essentially FOAK plants are in the high range or above the high $\$ / \mathrm{kWe}$ value $(\$ 3,500 / \mathrm{kWe}$ for a NOAK LWR) reported in the March 2008 "AFC Cost Basis" report (prepared in FY-07). (The reactor cost data in the "AFC Cost Basis" report are the basis for the reactor analyses in Table 8-5 above.) The significant specific cost increase from the "AFC Cost Basis" report data is likely more than just the "FOAK versus NOAK" cost effect, but also the 
fact that fiscal realities due to commodity and labor escalation, schedule stretch, and rising financing costs are manifesting themselves for "real" projects. This fact, however, does not invalidate the above comparative analysis, since the same factors are affecting all planned powerplant projects, not just nuclear. This is most true for coal, where regulatory and legal delays due to public opposition are becoming as common as they were during the peak of nuclear construction in the 1970s and 1980s. For natural gas plants, the fuel price and equipment costs also have changed markedly in the nearly 1 year since the data for the comparative analysis were first gathered. (The annotations to the references in the next section call out some of these new costs.) For fossils fuels the trend shown in Figure 8-13 below continues unabated, and is likely to continue if crude oil prices continue to climb.

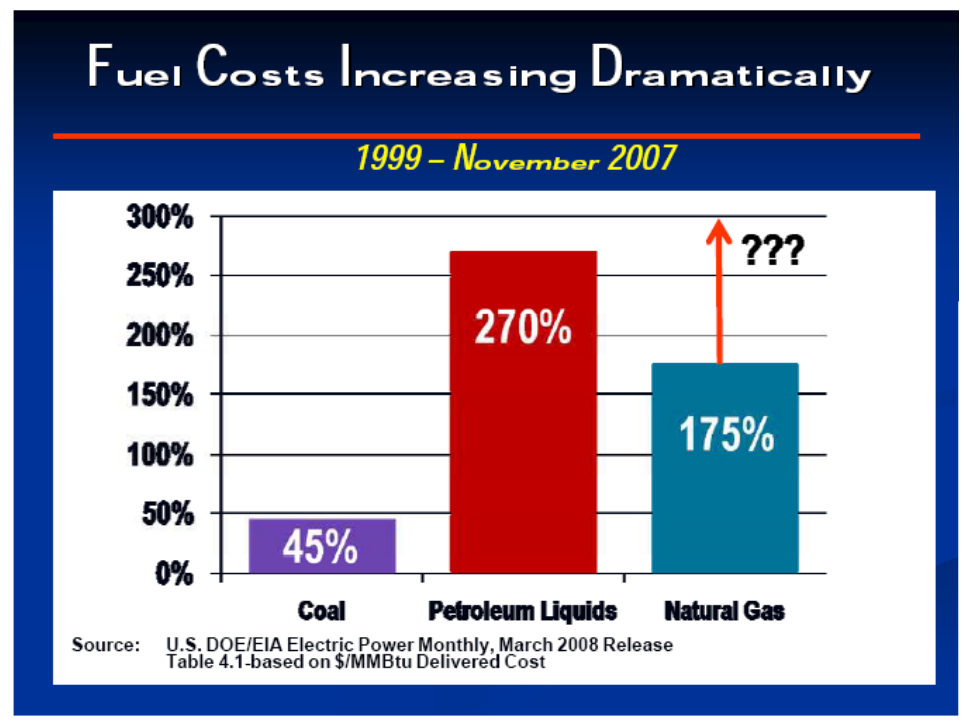

Figure 8-13. Escalation in fossil fuel prices (DOE-EIA 2008).

Note also that some form of cost for carbon is imminent in the U.S. and will certainly affect the relative competitiveness of nuclear vis-à-vis fossil sources in a manner that will favor nuclear. Nearly every month a new report dealing with the competitiveness issue and carbon cost effects is issued by some industry group, non-governmental organization (NGO), or government agency (such as the recent Congressional Budget Office study: CBO 2008). Some of the most informative of these are provided in the following bibliography.

\subsection{Bibliography for Section 8}

Bhatt, V., J. Lee, A. Reisman, and M. Todosow, 2008, A Methodology to Evaluate the Impact of Financial Mechanisms on the Economic Competitiveness of GNEP Technologies in the U.S. Energy Market, Brookhaven National Laboratory, BNL-AFCI-2008-001R0, February 29, 2008.

Felder, F. E. and A. Hajos, 2006, Using Restructured Electricity Markets in the Hydrogen Transition: The PJM Case, Proceedings of the IEEE, Vol 94, No.10, page 1864, October 2006.

Grecheck, E. S., 2008, (Dominion Energy), Building New Nuclear Plants: The Utility Decision, Viewgraph presentation from EIA 2008 Energy Conference, Washington DC, April 8, 2008.

Heller, Jamie, 2008, HellerWorx (Consulting Firm), New Baseload Coal Generation: Warts and All, Viewgraph presentation at EIA 2008 Energy Conference, Washington DC, April 8, 2008.

Hirschberg, Stefan, et al, 2004, Sustainability of Electricity Supply Technologies under German Conditions: A Comparative Evaluation, Paul Scherrer Institute, PSI Report (Bericht) No 04-15, December 2004. 
The Keystone Center, 2007, Nuclear Power Joint Fact-Finding, The Keystone Center, June 2007.

MIT, 2003, The Future of Nuclear Power: Massachusetts Institute of Technology, 2003.

Moniz, E., 2008, Future of Coal and Nuclear Power, Viewgraph presentation to National Academy of Science, March 2008.

Moody's Corporate Finance, 2007, New Nuclear Generation in the United States, Moody's Corporate Finance, October 2007.

University of Chicago, 2004, The Economic Future of Nuclear Power: A Study Conducted at The University of Chicago, 2004.

\subsection{References for Section 8}

Atomic Insights, 2006, Rising Coal and Coal Plant Capital Costs May Alter Duke Energy Plans, Atomic Insights Blog, http://atomicinsights.blogspot.com/2006/11/rising-coal-and-coal-plant-capital.html; November 19, 2006.

Bauer, C.O., 2006, National Energy Technology Laboratory, $\mathrm{CO}_{2}$ Capture Technology: Options and Experience, Viewgraph presentation, August 6, 2006.

Congressional Budget Office, Nuclear Power's Role in Generating Electricity, Publication 2986, May 2008.

Delene, J. G., J. Sheffield, K. A. Williams, et al. 2000, “An Assessment of the Economics of Future Electric Power Generation Options and the Implications for Fusion," Rev 1, ORNL/TM1999/243/R1, January 2000.

DOE-Energy Information Administration, 2008, http://www.eia.doe.gov/oil_gas/natural_gas/info_glance/natural gas.html, Web page visited on May 23, 2008.

DOE-EIA, 2008, DOE/EIA Electric Power Monthly: March 2008 Release.

Economic Analysis Working Group, 2009, “AFCI Economic Tools, Algorithms, and Methodologies Report,” Economic Analysis Working Group, Idaho National Laboratory, INL/EXT-07-13293, (Released as a draft in 2007).

Elliott, R. N., 2006, Energy Efficiency: the First Fuel in the Race for Clean Energy; Viewgraph presentation for the American Council for an Energy Efficient Economy (ACEEE), 2006.

Energy Central News, 2008a, Coal Plant Would Be "Waste of Money," www.energycentral.com/centers/news/daily/article.cfm?aid=9520943, January 11, 2008.

Energy Central News, 2008b, AEP SWEPCO Receives Louisiana Commission Approval to build 600-MW Coal-fueled Plant, www.energycentral.com/centers/news/daily/printer.cfm?aid=9991280, March 28, 2008.

Engineering News Record, 2007, "With Carbon Constraints Expected, Generation Plants Must Clean-up or Pack-up," Engineering News Record, http://enr.ecnext.com/coms2/article februar070212p, February 12, 2007.

Fielden, Sandy, 2003, "LNG Could Rescue the U.S.," EPRM Market Focus, www.eprm.com, September 2003.

Generation IV Economic Modeling Working Group (EMWG), 2008, User's Manual for G4 ECONS Version 2.0, March 2008, Printed by OECD Nuclear Energy Agency, Paris, France, March 2008. 
Gulf Times Newspaper, 2007, LNG Price for Future Supply Hits Record High on Tight Supplies; Vol 12 Issue \#6; March 28, 2007, Alexander's Gas \& Oil Connections Web site, www.gasandoil.com/goc/features/fex71379.htm.

Go Upstate, 2008, Nuclear Plant Set to Go Online in 2018, GoUpstate.com, www.goupstate.com/apps/pbcs.dll/article?Date=20080422\&Category=NEWS\&Art, April 23, 2008.

Harding, James, 2008, Reactor Economics 2008, viewgraph presentation given to National Academy of Sciences/National Research Council Panel, Washington DC, January 22, 2008.

Lomborg, Bjorn, 2008, “Let's Keep Our Cool About Global Warming,” Skeptical Inquirer, Vol 32, Issue 2, March/April 2008.

McKinsey \& Co., 2007, Reducing U.S Greenhouse Gas Emissions: How Much at What Cost?, McKinsey \& Co (Consulting firm), December 2007.

MIT, 2007, The Future of Coal: Options for a Carbon-Constrained World, Massachusetts Institute of Technology, 2007.

Mufson, Steven, 2007, Power Plant Rejected over Carbon Dioxide for First Time, October 19, 2007, www.washingtonpost.com.

NIRS, 2008, Project Total Cost Estimate Range, Nuclear Information and Research Service (NIRS), www.nirs.org/images/fplturkeypointcostchart.jpg, April 18, 2008.

Pew Center, 2008, Climate Change 101: Cap and Trade, Pew Center on Global Climate Change.

Platt, 2008, EON-UK Gets Council OK for New UK 1.6 GW Coal Plan; Platt's News Release for Risk Managers, www.platts.com/Coal/News/8447113.xml, January 7, 2008.

Progress Energy, 2008, Appendix: Need Determination Study in Support of Progress Energy Florida, Inc's Petition for Determination of Need for Levy County Units 1 and 2 Nuclear Power Plants (Docket No. 080148-EI), Progress Energy, March 11, 2008.

Regional Business Journal, 2007, Santee Cooper's Coal Plans Fire Debate, Charleston (SC), www.charlestonbusiness.com/pub/13322/news/10677-1.html, December 10, 2007.

Shropshire, D. E., Williams, K.A., et al., 2008, “Advanced Fuel Cycle Cost Basis,” Idaho National Laboratory, INL/EXT-07-12107 Rev 1, March 2008.

USAToday, 2007, "Plans for at Least 8 Clean Coal Plants Hit Snags," USAToday, www.usatoday.com/money/industries/energy/environment/2007-12-26-coalplants.html, December 27, 2007.

Wiser, Ryan H., 2004, Lawrence Berkeley National Laboratory, Managing Natural Gas Price Volatility and Escalation, Viewgraph presentation given at NEMS/AEO 2004 Conference, Washington DC, March 23, 2004. 


\section{COMPARISON OF THE G4 ECONS AND VISION.ECON STATIC EQUILIBRIUM NOMINAL RESULTS}

This section shows the comparison of modeling results using the G4 ECONS-based results, shown in Section 6.1, and comparing them to the composite results simulated in VISION.ECON. This comparison was performed in order to calibrate and verify results between the models, and to better understand why dynamic analysis may provide somewhat different answers. The first set of analysis compares the overall cost distributions for the three cases for the FCC and the TCOE. The second set of analysis compares the conversion ratio results from the two models. Figure 9-1 shows two charts for the once-through fuel cycle, the composite cost of electricity, and the composite fuel cycle costs. Very good agreement was found between the DPL distributions based on the static spreadsheet analysis and the VISION.ECON results.
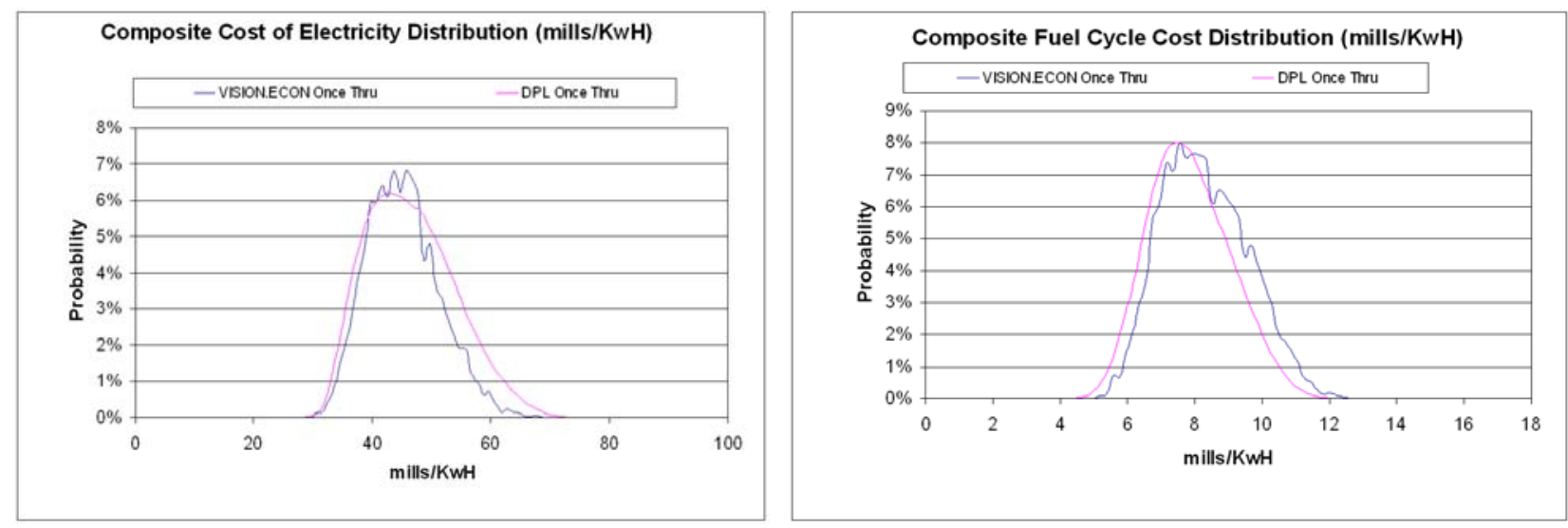

Figure 9-1. TCOE and FCC comparison of the DPL results to the VISON.ECON for the once-through.

Figure 9-2 shows two charts for the 1-Tier, the composite cost of electricity and the composite fuel cycle costs. Good agreement was found between the DPL distributions based on the static spreadsheet analysis and the VISION.ECON results.
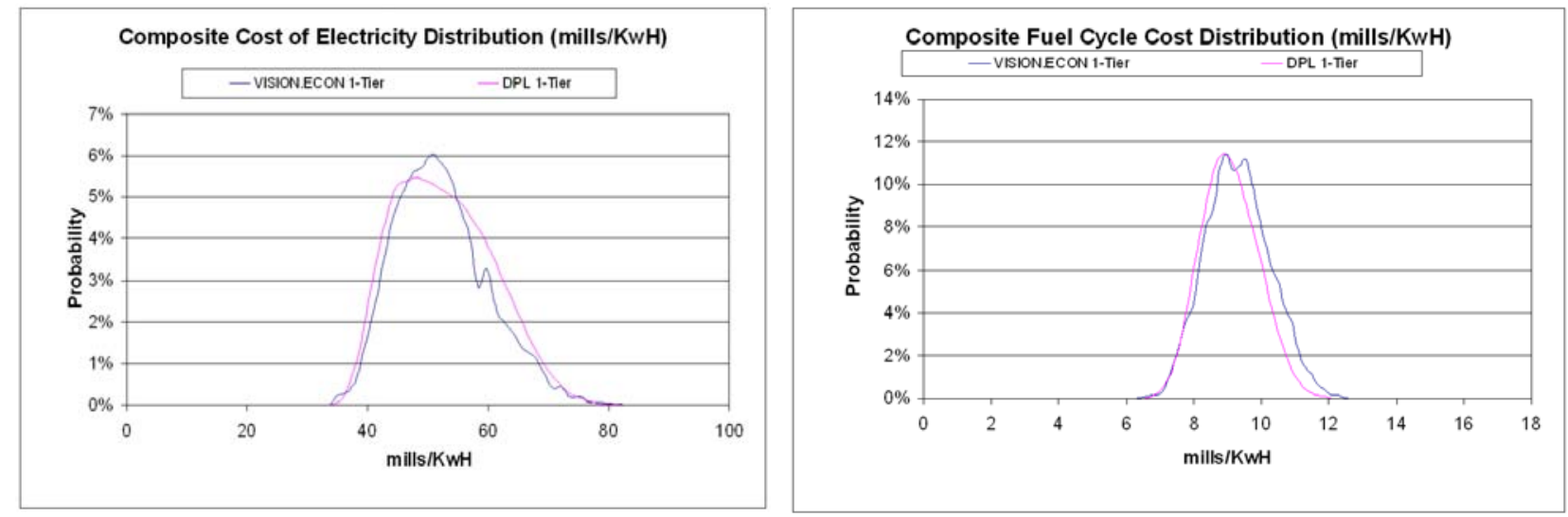

Figure 9-2. TCOE and FCC comparison of the DPL results to the VISON.ECON for the 1-Tier.

Figure 9-3 shows two charts for the 2-Tier, the composite cost of electricity, and the composite fuel cycle costs. Very good agreement was found between the DPL distributions based on the static spreadsheet analysis and the VISION.ECON results. 

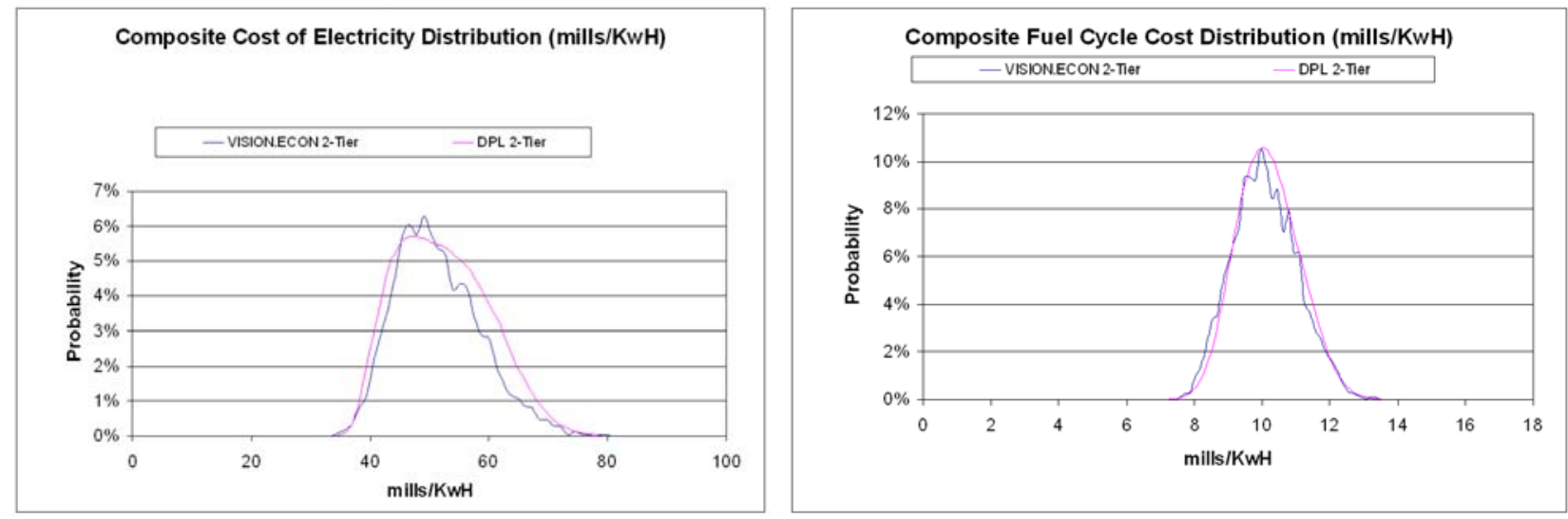

Figure 9-3. TCOE and FCC comparison of the DPL results to the VISON.ECON for the 2-Tier.

Because of the complexity of the calculations when changing conversion ratios it was deemed important to check the calculations in VISION against the G4 ECONS spreadsheet calculations. Figures 9-4 and 9-5 show how the TCOE and FCC from VISION compare to the results generated with G4 ECONS. As can be seen the results are in very good agreement. Differences between the results are due to calculations for the number of reactors (whole versus partial numbers) and the additional waste streams that are tracked in VISION, not accounted for in the spreadsheet model.

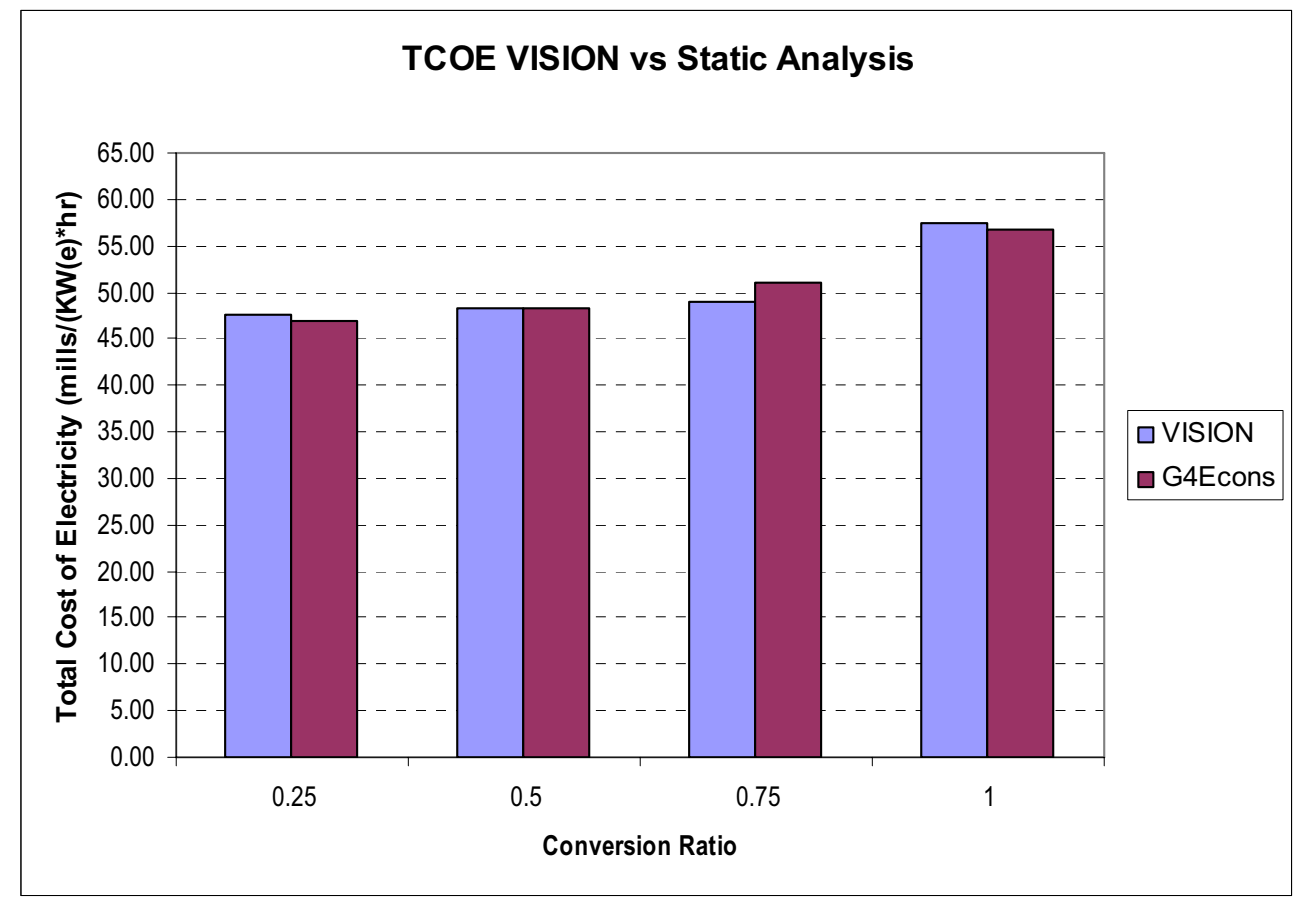

Figure 9-4. Comparison of TCOE modeling results for the conversion ratio. 


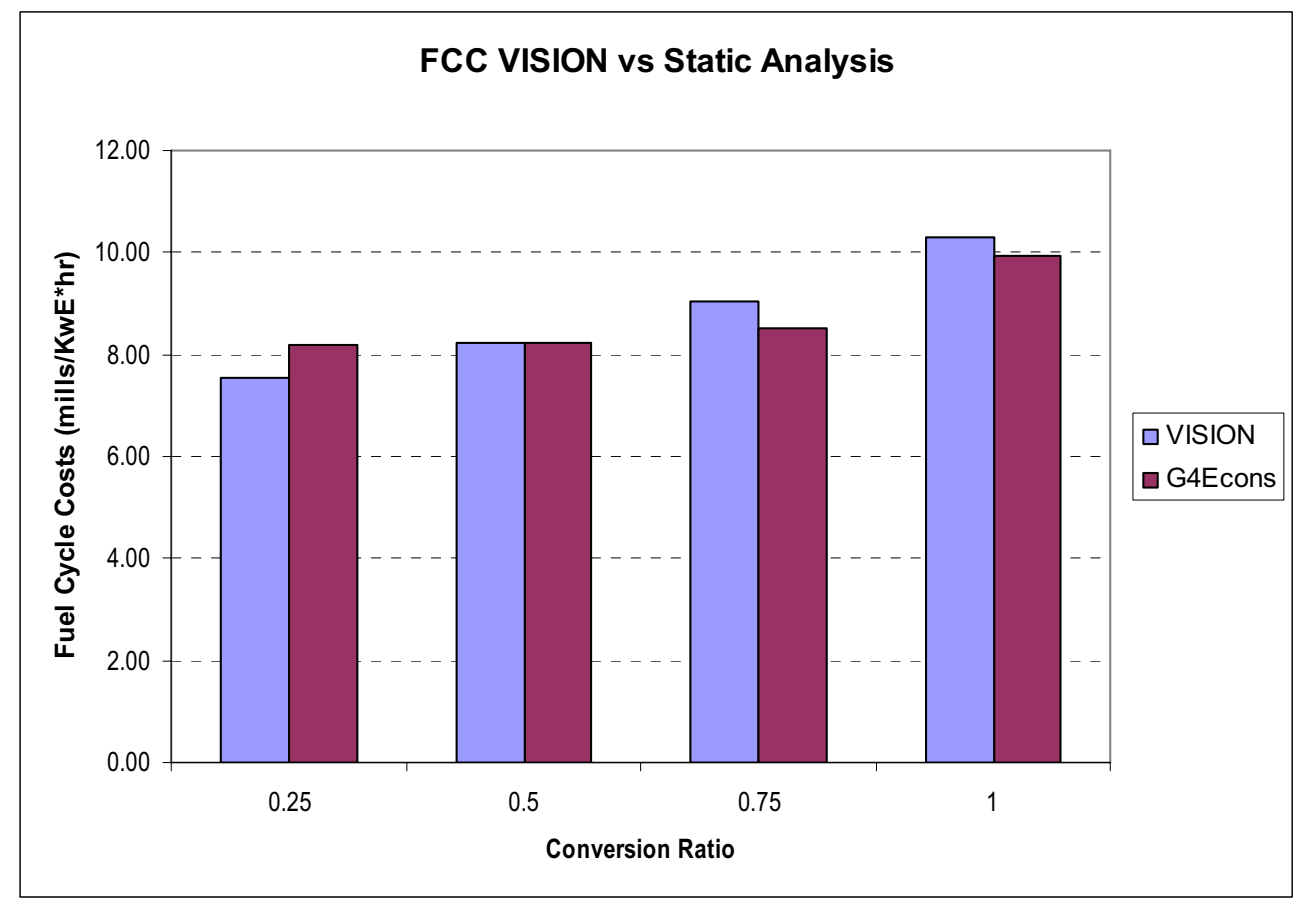

Figure 9-5. Comparison of TCOE modeling results for the conversion ratio. 


\section{COMPARISON OF THE CURRENT ECONOMIC ANALYSIS TO PREVIOUS ECONOMIC STUDIES ON THE NUCLEAR FUEL CYCLE}

The purpose of this analysis is to compare the current "Advanced Fuel Cycle Economic Analysis of Symbiotic Light-Water Reactor and Fast Burner Reactor Systems" and the associated "AFC Cost Basis" report (Shropshire et al. 2008) to previous economic studies prepared by the OECD/NEA "Advanced Nuclear Fuel Cycles and Radioactive Waste Management" (OECD/NEA 2006) and by MIT "The Future of Nuclear Power" (MIT 2003). The comparison considers the approach, assumptions, unit costs, fuel cycle costs. These studies were selected due to their relevance and relative recency in publication.

\subsection{Evaluation Approach}

The costs data and static equilibrium system results from this economic analysis were used as the basis for comparison to the OECD and MIT studies. An earlier study performed by Systems Analysis in 2006, called the "AFCI Sensitivity Analysis" (Shropshire et al. 2006) used the same basic methodology as this study, but used different fuel cycle system assumptions and earlier (2006) cost data. The "AFC Cost Basis" report was the reference source for the cost data for the AFCI and 2006 studies, but the data has been significantly changed over the past two years as more recent unit cost data became available and additional analysis was performed to refine the estimating cost basis. The most current cost data from the March 2008 Cost Basis report was used to compare to the OECD and MIT studies. The unit costs from the OECD and MIT studies were escalated from (2000 and 2002 dollars, respectively) to 2008 dollars based on the Civil Works Construction Cost Index (U.S. Army Corps of Engineers 2007). The comparison of the unit costs is provided in Section 10.4.

In the current study, the G4 ECONS spreadsheet was used to develop the cost distributions for the open and closed fuel cycle systems as presented in Section 6. The results from the static spreadsheet analysis were used for the comparisons in Section 10.5. The OECD and MIT reports were reviewed for content and the underlying structure and assumptions used to generate the content. The reported cost data was compared and matched as accurately as possible to the cost ranges in the "AFC Cost Basis" report and systems defined in this analysis.

In the following two sections, the OECD and MIT methodologies and assumptions are described along with the areas of commonality with the AFCI costs and the areas of differences. In Table 10-1, a comparison of some of the key parameters used in the studies is provided.

\subsection{OECD/NEA}

\subsubsection{OECD/NEA Methodology}

The OECD/NEA report considers costs and additional parameters relative to the fuel cycle, but only the economics portion was considered in this comparison. The general approach used in the OECD/NEA and AFCI reports is very similar. The studies were prepared from different perspectives due to OECD country fuel cycle policies related to reprocessing (PUREX separation of $\mathrm{Pu}$ ), waste regulations (highlevel and intermediate-level waste classifications), and disposition plans for used fuel (long-term storage).

\subsubsection{OECD/NEA Comparison to the "Advanced Fuel Cycle Economic Analysis of Symbiotic Light-Water Reactor and Fast Burner Reactor Systems"}

Although the OECD/NEA fuel cycles do not match the AFCI fuel cycles exactly, they are similar enough for comparison. Notable differences are that OECD's 2-Tier case (OECD Scheme 3b) includes an Accelerator-Driven System (ADS) step that is not included in the AFCI 2-Tier case and uses a larger FR. 
The OECD/NEA report studied more fuel cycle scenarios than the "Advanced Fuel Cycle Economic Analysis of Symbiotic Light-Water Reactor and Fast Burner Reactor Systems," so the three that align the most closely with the AFCI fuel cycles were considered. The fuel cycle steps vary the most for waste disposal, due to different categories of waste (e.g., intermediate level wastes) and differences in the processed used for recycling or disposing of SNF.

Although both reports studied transportation costs, these costs are not being compared at this time because of the large variation in assumptions (such as packaging and geographic location) that affect cost. In addition, transportation costs are a small factor compared to the overall costs (Section 7).

The OECD/NEA and AFCI reports have many similar assumptions. Both of the reports:

- Provide costs with uncertainty ranges for fuel cycle costs (without reactors) and total costs (including reactors). Use wider uncertainty ranges for immature technologies, and have similar data sources for the width of the uncertainty ranges.

- Examine the influence of module costs and uncertainties on the fuel cycle.

- Perform sensitivity analysis to identify the key drivers in fuel cycle cost, but use different approaches to the sensitivity analysis. OECD/NEA normalizes costs for other fuel cycles to the costs of an open fuel cycle, and uses comparisons of dependency of relative costs. AFCI uses Tornado diagrams to show the relative cost uncertainty effects and to identify variables with the potential to have the largest impact on nuclear fuel cycle costs. Have same basic fuel cycle steps.

- Divide the fuel cycle into the same basic steps (modules) with unit costs and uncertainty ranges.

- Based on equilibrium conditions using an Excel-type of spreadsheet to track mass flows and calculate cost ranges for each module and for the overall reactor and fuel cycle system.

- Use Monte Carlo simulations for uncertainty analysis.

- Normalize the cost per unit of electricity produced.

- Indicate that relative cost differences, not absolute costs values are the aim of the report.

- Include wet storage with the reactor cost.

Some differences between the OECD/NEA and AFCI reports include:

- Use of different categories of waste, with the OECD/NEA report using the IAEA categories of shortlived waste (LILW-SL), long-lived waste (LILW-LL), and HLW, while AFCI uses the waste categories of LLW and HLW that are used in the United States.

- Less details for conditioning, storage, and packaging of various waste streams. The AFCI report breaks the fuel cycle down into more modules.

- Only perform economic assessments with static spreadsheet models. The AFCI study includes the VISION model for dynamic analysis of nonequilibrium and growth scenarios.

- Using a different method (as a one time and recurring annual cost) for calculation of dry storage costs. AFCI calculates the dry storage costs as a one-time cost.

- Using separate unit costs each for long-term storage of Am and Cm. AFCI has unit costs for storage of U/TRU and $\mathrm{U} / \mathrm{Pu}$, so these costs cannot be compared directly.

- Combining product treatment/conditioning in the recycling cost and using separate unit costs for dry storage and packaging. AFCI uses a separate unit cost for recycling, and accounts for conditioning, storage, and packaging in various combinations, based on the specific waste stream.

A summary of some of the key parameters used in the OECD/NEA studies are shown in Table 10-1. 


\subsection{Massachusetts Institute of Technology}

\subsubsection{MIT Methodology}

The overall objective of the MIT report is to evaluate what would be required to maintain or increase nuclear power in the U.S. Although the MIT report considers cost, safety, proliferation, and waste, only the economics section is considered in this comparison. The report also evaluates the cost competitiveness of nuclear compared to coal and combined cycle gas turbine from the investor's perspective by estimating the specific costs for the various fuel cycles. Comparison of the MIT estimated costs for non-nuclear energy sources to those in Section 8 are also not considered in this analysis.

\subsubsection{MIT Assumptions}

The MIT study evaluated the thermal once-through uranium fuel cycle and a thermal fuel cycle with single-pass plutonium recycling. The MIT study did not include a complete analysis for the costs of advanced fuel cycles. They did examine in some detail the cost of the closed fuel cycle with a single pass PUREX/MOx relative to the open cycle. Only the once-through cycle was directly comparable to the AFCI analysis.

The MIT fuel cycle cost model showed that for the closed fuel cycle option, the fuel cycle costs are 4.5 times higher than for the once-through UOx cycle under U.S. conditions. ${ }^{\mathrm{s}}$ Further, the closed cycle could only be shown to be competitive with the once-through option if the price of uranium is high and if optimistic assumptions are made regarding the cost of reprocessing, MOx fabrication, and high-level waste disposal. In contrast, the OECD/NEA study (OECD/NEA 1994) concluded that the unit fuel cycle cost including reprocessing and recycling is 1.1 times that of the once-through cycle.

The MIT study states “...It should be noted that the cost increment associated with reprocessing and thermal recycle is small relative to the total cost of nuclear electricity generation. In addition, the uncertainty in any estimate of fuel cycle costs is extremely large." The Japanese tend to agree with this view (Japan Atomic Energy Commission 2005), by indicating that the cost increment over direct disposal for reprocessing and thermal recycle is small (about 10\%) or even negative if the cost for early shut down of nuclear power plants due to shortage of spent fuel storage capacity is considered. The "Advanced Fuel Cycle Economic Analysis of Symbiotic Light-Water Reactor and Fast Burner Reactor Systems" also concludes that cost uncertainties are large and that the fleet cost (\$/MWh for whole generation system) of using a closed fuel cycle is about $10 \%$ (on the average) more expensive than open cycle systems.

The MIT report uses 2002 costs, while the "Advanced Fuel Cycle Economic Analysis of Symbiotic Light-Water Reactor and Fast Burner Reactor Systems" uses 2008 costs. For comparison, all of the MIT costs presented in this report have been escalated to 2008 dollars. The escalation was based on the Civil Works Construction Cost Index (U.S. Army Corps of Engineers 2007).

Some similarities are that the MIT and AFCI report both:

- Divide the fuel cycle into steps (modules) and use a spreadsheet to estimate module and fuel cycle costs based on unit costs and mass flows. Both reports evaluate a steady state equilibrium condition.

- Use a similar estimating approach, have the same basic fuel cycle steps, and normalize the cost per unit of electricity produced.

Some differences include:

- $\quad$ MIT uses a less-detailed breakdown for conditioning, storage, and packaging of various waste streams. The AFCI study includes additional details for various fuel cycle steps such as packaging, storage, disposal, depleted uranium disposition, etc.

s. The factor of " 4.5 times" more expensive has been disputed since the blended increase in the cost of electricity reported by MIT is actually $50 \%$, an increase from $5.15 \mathrm{mills} / \mathrm{kWh}$ (once through) to $7.91 \mathrm{mills} / \mathrm{kWh}$ (MOx recycle). The $50 \%$ factor of increase is much more in line with the results from the "Advanced Fuel Cycle Economic Analysis of Symbiotic Light-Water Reactor and Fast Burner Reactor Systems." 
- $\quad$ MIT estimates specific cost points versus the AFCI study provides estimates of the relative differences in cost for various fuel cycles.

- MIT acknowledges that there are large uncertainties associated with fuel cycle cost estimates, but does not include uncertainty analysis. The AFCI report includes uncertainty analysis, and a study of which components of the fuel cycle have the largest impact on fuel cycle cost, based on the uncertainty ranges.

- MIT includes a credit for UOx SF in the thermal recycle scenario, while the mass flows in the AFCI analysis are used to accomplish this.

- $\quad$ MIT assumes an unregulated environment in their determination of financing rates, whereas the AFCI assumes that new reactors will be in a regulated environment.

- $\quad$ MIT uses a discounted cash flow analysis to estimate the real levelized cost of electricity. This approach considers economic factors such as interest rate, depreciation, tax rate, inflation rate, expected return to equity investor, incremental capital costs, etc. AFCI considers the effects from discounting, but has chosen to use undiscounted system costs for comparison purposes.

- The MIT model includes a carrying charge time-dependent factor to account for the cost of the delay between the investment and the return on investment. AFCI does not include a carrying charge in the calculations due to the uncertainty associated with the timing of system deployments and to simplify the calculations and comparisons between systems. The cost comparisons in Sections 10.4 and 10.5 do not include the MIT carrying charges.

A summary of some of the key parameters used in the studies are shown in Table 10-1.

Table 10-1. Comparison of key parameters used in the economic analyses.

\begin{tabular}{|c|c|c|c|c|c|c|c|}
\hline & \multicolumn{3}{|c|}{ Once-through } & \multicolumn{2}{|c|}{ 1-Tier } & \multicolumn{2}{|c|}{ 2-Tier } \\
\hline & $\mathrm{AFCI}$ & OECD & MIT & AFCI & OECD & $\mathrm{AFCI}$ & OECD \\
\hline $\begin{array}{l}\text { Burn-up (GWd/tHM) } \\
\text { Thermal Reactor } \\
\text { FR }\end{array}$ & 51 & 60 & 50 & $\begin{array}{c}51 \\
131.9\end{array}$ & $\begin{array}{c}50 \\
140\end{array}$ & $\begin{array}{c}51 \\
131.9\end{array}$ & $\begin{array}{c}50 \\
185\end{array}$ \\
\hline $\begin{array}{l}\text { Enrichment - LWR } \\
(\% \text { \%235) }\end{array}$ & 4.28 & 4.9 & 4.51 & 4.28 & 4.2 & 4.28 & 4.2 \\
\hline $\begin{array}{l}\text { Capacity Factor (\%) } \\
\text { Thermal Reactor } \\
\text { FR }\end{array}$ & 90 & 90 & 85 & $\begin{array}{l}90 \\
82\end{array}$ & $\begin{array}{l}90 \\
85\end{array}$ & $\begin{array}{l}90 \\
82\end{array}$ & $\begin{array}{l}90 \\
85\end{array}$ \\
\hline $\begin{array}{l}\text { Reactor Size (MWe) } \\
\text { Thermal Reactor } \\
\text { FR }\end{array}$ & 1300 & 1450 & 1500 & $\begin{array}{c}1300 \\
380\end{array}$ & $\begin{array}{c}1450 \\
600\end{array}$ & $\begin{array}{c}1300 \\
380\end{array}$ & $\begin{array}{l}1450 \\
1450\end{array}$ \\
\hline $\begin{array}{l}\text { Type Recycling } \\
\text { Thermal Reactor } \\
\text { FR } \\
\text { Thermal Reactor (MOx) }\end{array}$ & N/A & N/A & N/A & $\begin{array}{l}\text { UREX+1a } \\
\text { Echem }\end{array}$ & $\begin{array}{l}\text { UREX+ } \\
\text { Echem }\end{array}$ & $\begin{array}{l}\text { UREX Hybrid } \\
\text { Echem } \\
\text { UREX+1a }\end{array}$ & $\begin{array}{l}\text { Adv PUREX } \\
\text { Adv PUREX } \\
\text { Adv PUREX }\end{array}$ \\
\hline $\begin{array}{l}\text { Type Fuel } \\
\text { Thermal Reactor } \\
\text { FR } \\
\text { Thermal Reactor (MOx) }\end{array}$ & UOx & UOx & UOx & $\begin{array}{l}\text { UOx } \\
\text { Metal }\end{array}$ & $\begin{array}{l}\text { UOx } \\
\text { Metal }\end{array}$ & $\begin{array}{c}\text { UOx } \\
\text { Metal } \\
\text { MOx-UPu }\end{array}$ & $\begin{array}{l}\text { UOx } \\
\text { MOx } \\
\text { MOx }\end{array}$ \\
\hline Ratio \%FR/LWR & $\mathrm{N} / \mathrm{A}$ & N/A & $\mathrm{N} / \mathrm{A}$ & $\begin{array}{c}63 \% \text { LWR } \\
37 \% \text { FR }\end{array}$ & $\begin{array}{c}63.2 \% \text { LWR } \\
36.8 \% \text { FR }\end{array}$ & $\begin{array}{l}67 \% \text { LWR } \\
8 \% \text { LWR } \\
25 \% \text { FR }\end{array}$ & $\begin{array}{l}65 \% \text { PWR } \\
9.8 \% \text { PWR } \\
19.9 \% \text { FR } \\
5.2 \% \text { ADS }\end{array}$ \\
\hline $\begin{array}{l}\text { Type Reactor } \\
\text { Thermal Reactor } \\
\text { FR } \\
\text { Thermal Reactor }\end{array}$ & LWR & EPR & LWR & $\begin{array}{l}\text { LWR } \\
\text { SFR }\end{array}$ & $\begin{array}{c}\text { EPR } \\
\text { ALMR Sodium }\end{array}$ & $\begin{array}{c}\text { LWR } \\
\text { SFR } \\
\text { LWR MOx }\end{array}$ & $\begin{array}{l}\text { PWR N4 } \\
\text { EFR } \\
\text { PWR N4 }\end{array}$ \\
\hline $\begin{array}{l}\text { Reactor Life (yr) } \\
\text { Thermal Reactor } \\
\text { FR }\end{array}$ & 60 & 40 & 40 & $\begin{array}{l}60 \\
60\end{array}$ & $\begin{array}{l}40 \\
40\end{array}$ & $\begin{array}{l}60 \\
60\end{array}$ & $\begin{array}{l}40 \\
40\end{array}$ \\
\hline $\begin{array}{l}\text { Thermal Efficiency (\%) } \\
\text { Thermal Reactor } \\
\text { FR }\end{array}$ & 34 & 34 & 33 & $\begin{array}{l}34 \\
38\end{array}$ & $\begin{array}{l}34 \\
38\end{array}$ & $\begin{array}{l}34 \\
38\end{array}$ & $\begin{array}{l}34 \\
40\end{array}$ \\
\hline
\end{tabular}




\subsection{Comparison of Unit Costs}

Graphical comparisons of the nominal unit costs from OECD/NEA, MIT, and the "AFC Cost Basis" are shown in Figures 10-1-10-12. The vertical bar charts in these figures show the range of costs with the low cost at the bottom of the red bar, the nominal as the dividing line between red and yellow bars, and the high cost at the top of the yellow bar. The MIT study did not include a cost uncertainty range, so their costs are depicted as a solid line. Some of the costs used in the studies are not shown because they were not readily comparable due to different functional assumptions, cost breakdowns, or units.

The OECD/NEA unit costs for the front-end fuel cycle processes are comparable to the "AFC Cost Basis." AFC costs range higher for uranium, conversion, depleted uranium disposition, and MOx fuel fabrication. OECD/NEA costs range higher than the AFC costs for enrichment, RU disposition LWR fuel fabrication, electrochemical and aqueous reprocessing, and for near surface disposal. Geologic repository costs were not compared due to differences in waste categorization and disposition policies. Reactor capital costs are comparable, with the AFC high range costs exceeding the OECD/NEA estimates.

MIT's unit costs for LWR capital and O\&M exceed the nominal costs for OECD/NEA and the "AFC Cost Basis." The MIT costs do not reflect recent uranium prices and the SNF disposal costs reflect a continuation of the nuclear waste fee paid to the government (1 mill/kWh).
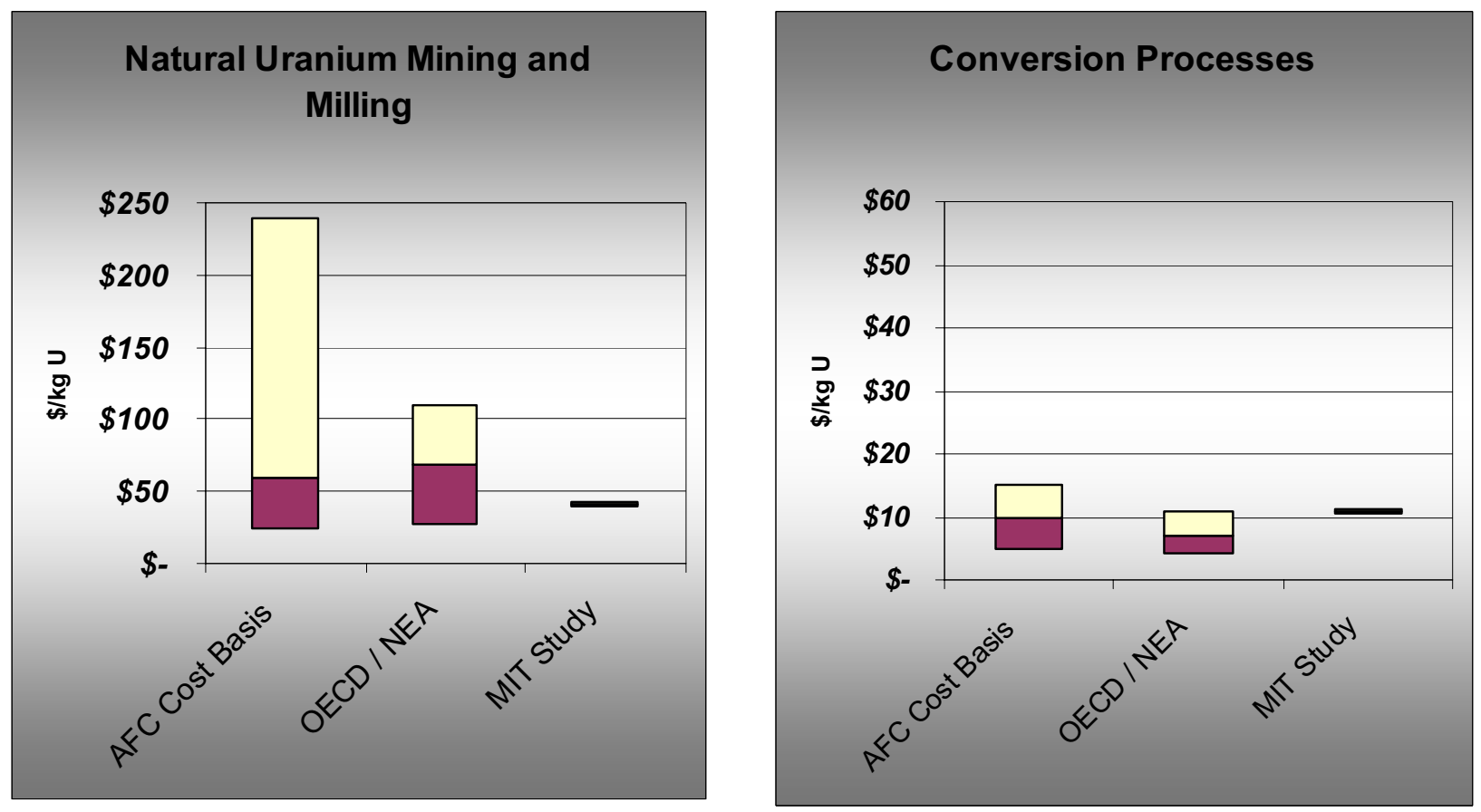

Figure 10-1. Unit cost comparisons: natural uranium mining and milling, and conversion (2008\$). 

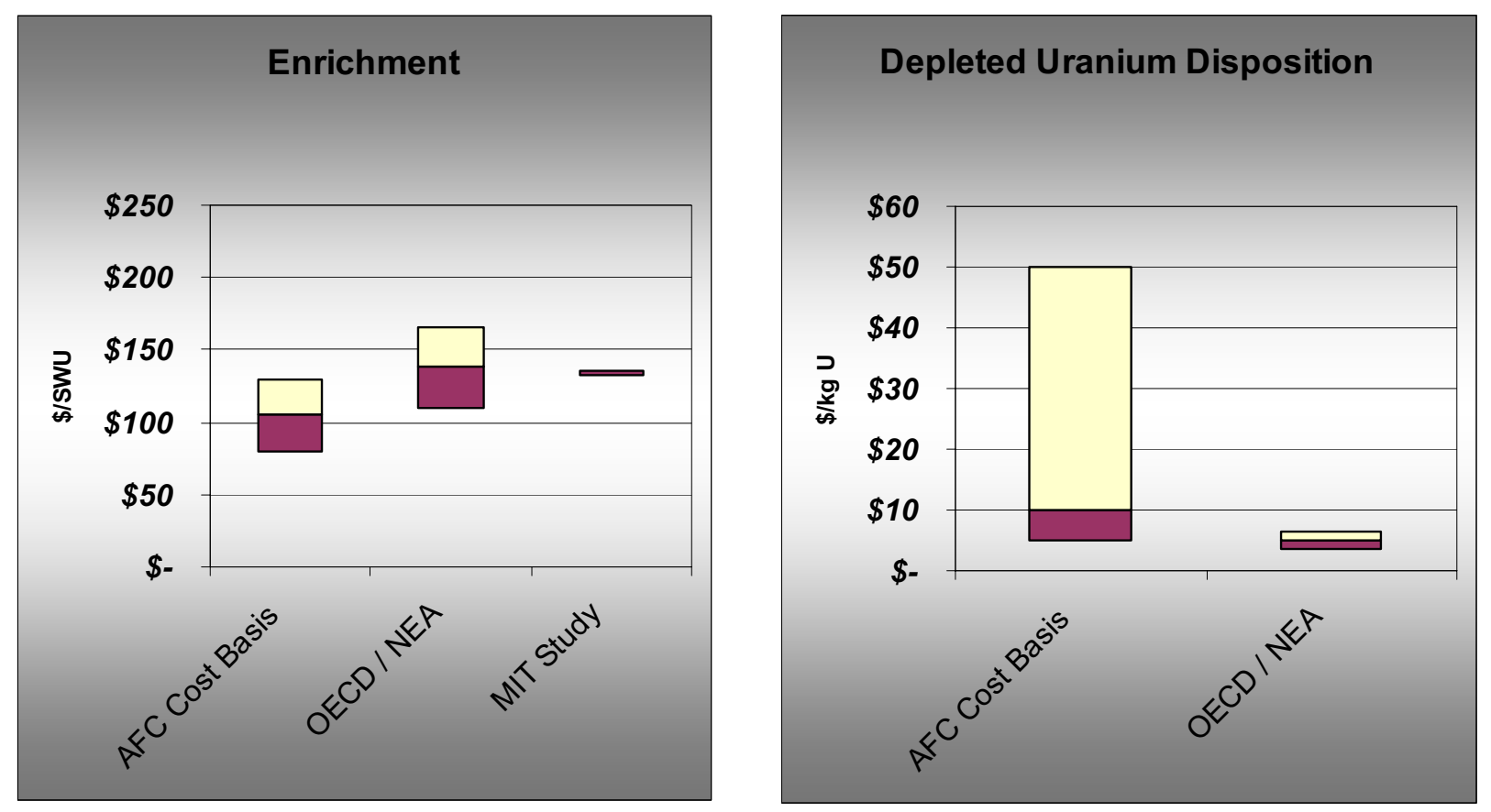

Figure 10-2. Unit cost comparison: enrichment and Depleted Uranium Disposition (2008\$).
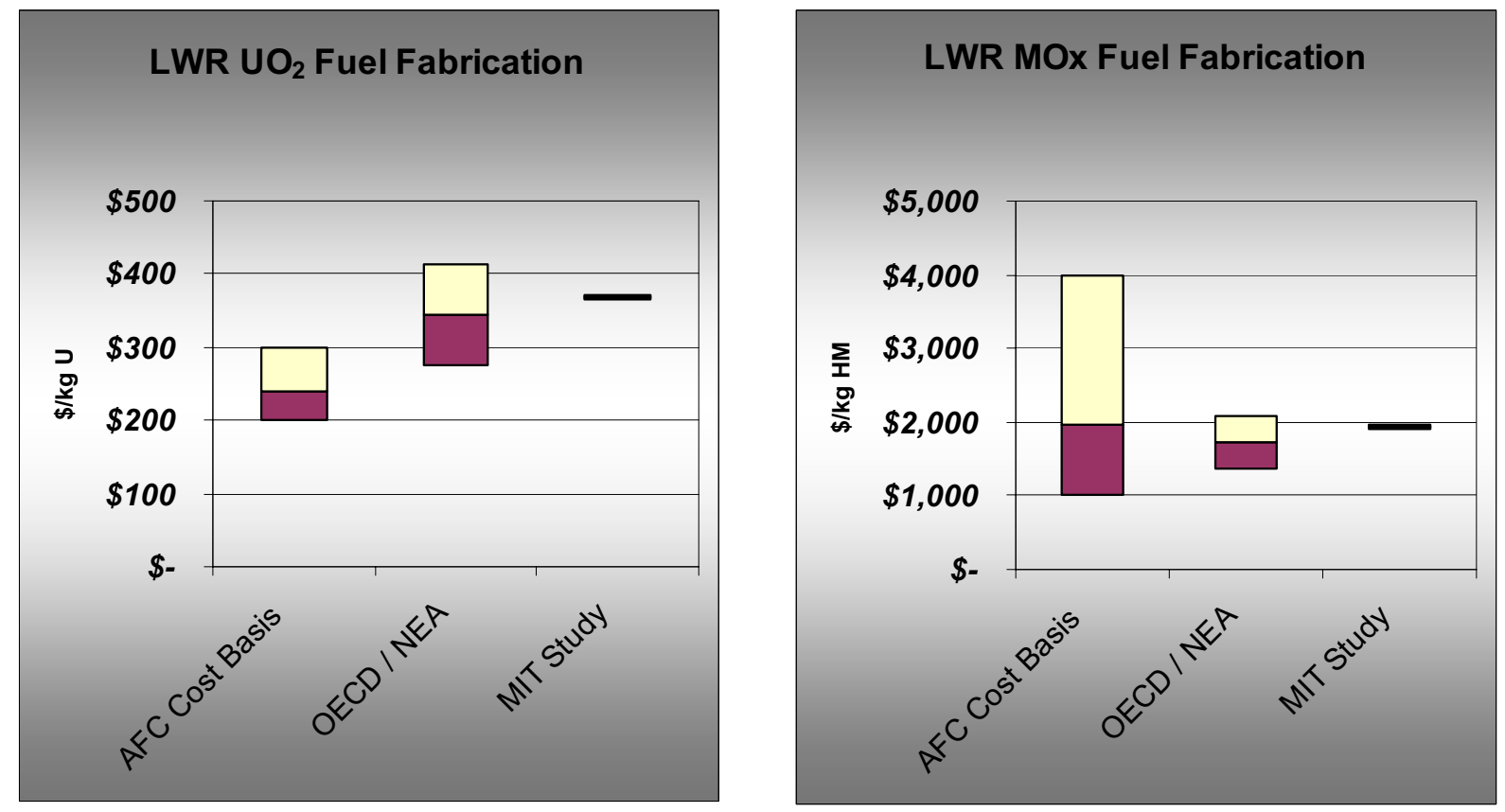

Figure 10-3. Unit cost comparison: $\mathrm{LWR} \mathrm{UO}_{2}$ fuel fabrication and LWR MOx fuel fabrication (2008\$). 


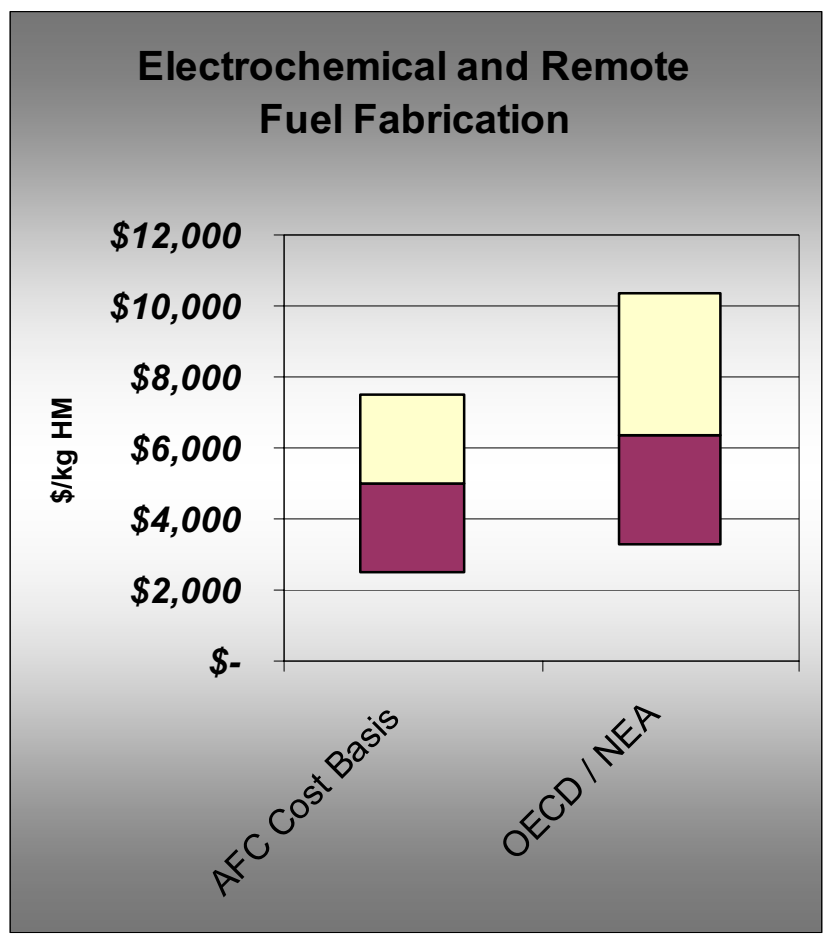

\begin{tabular}{|l|l|}
\hline AFC Cost Basis & $\begin{array}{l}\text { Combined electrochemical } \\
\text { recycling and metal fuel } \\
\text { fabrication into one unit } \\
\text { cost as likely to be in a } \\
\text { collocated facility }\end{array}$ \\
\hline OECD/NEA & $\begin{array}{l}\text { OECD/NEA's separate } \\
\text { unit costs for } \\
\text { electrochemical recycling } \\
\text { and metal fuel fabrication } \\
\text { were combined for } \\
\text { comparison }\end{array}$ \\
\hline
\end{tabular}

Figure 10-4. Unit cost comparison: electrochemical and remote fuel fabrication (2008\$).

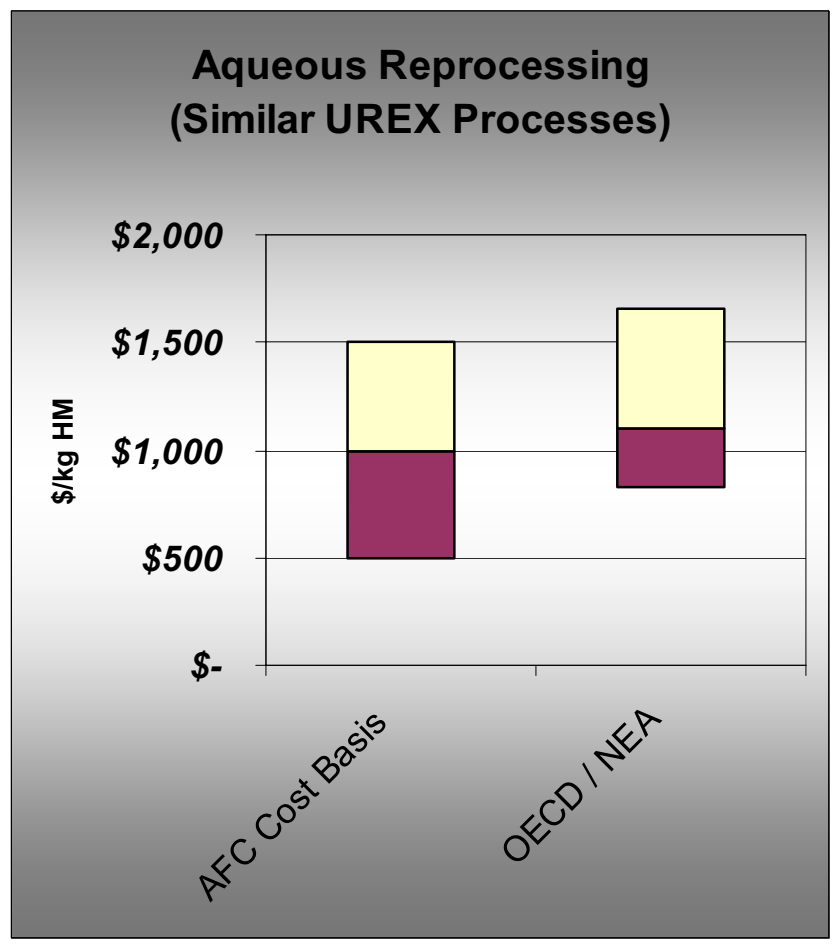

\begin{tabular}{|l|l|}
\hline AFC Cost Basis & UREX+1a; recycling alone \\
\hline OECD/NEA & $\begin{array}{l}\text { UREX; includes recycling } \\
\text { and conditioning }\end{array}$ \\
& \\
\hline
\end{tabular}

Figure 10-5. Unit cost comparison: aqueous recycling (2008\$). 

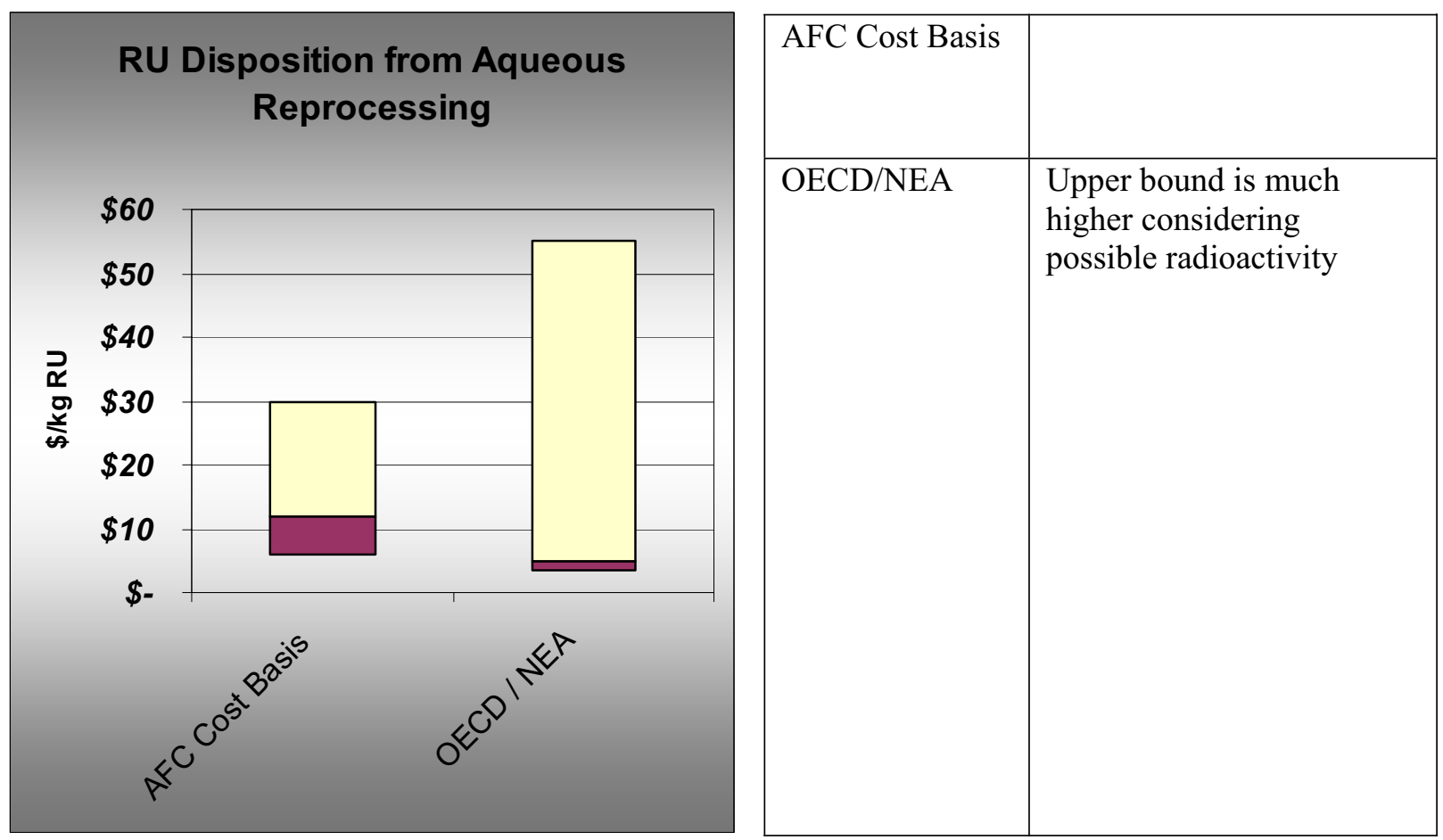

Figure 10-6. Unit cost comparison: RU disposition from aqueous recycling (2008\$).

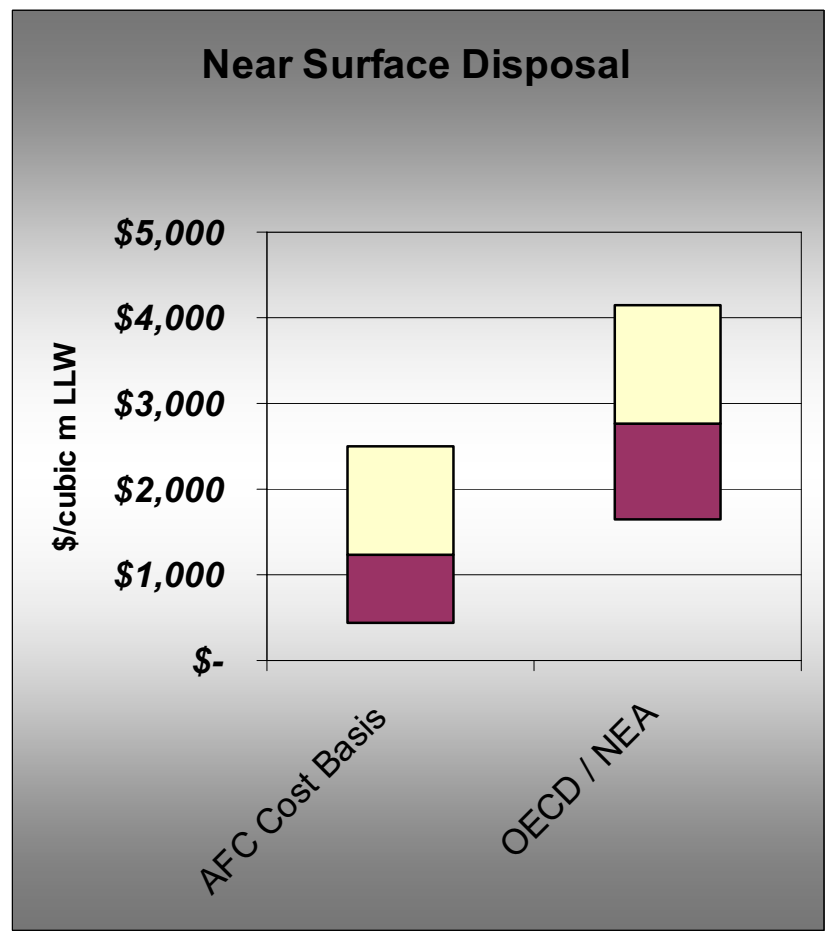

\begin{tabular}{|l|l|}
\hline AFC Cost Basis & $\begin{array}{l}\text { Near Surface Disposal of } \\
\text { debris }\end{array}$ \\
\hline OECD/NEA & $\begin{array}{l}\text { LILW (short-lived) near } \\
\text { surface disposal }\end{array}$ \\
& \\
\hline
\end{tabular}

Figure 10-7. Unit cost comparison: near surface disposal (2008\$). 


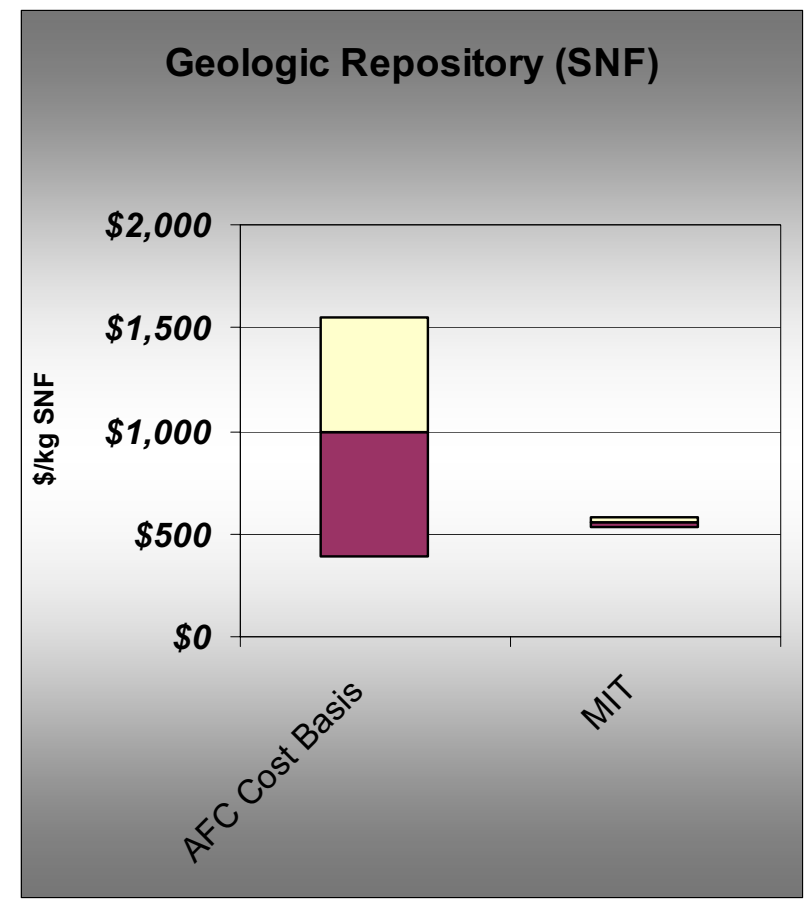

\begin{tabular}{|l|l|}
\hline AFC Cost Basis & Disposal alone \\
\hline OECD/NEA & $\begin{array}{l}\text { N/A-Assumes SNF will } \\
\text { be stored and recycled not } \\
\text { put in geologic repository }\end{array}$ \\
\hline MIT Study & $\begin{array}{l}\text { Includes storage and } \\
\text { disposal }\end{array}$ \\
\hline
\end{tabular}

Figure 10-8. Unit cost comparison: geologic repository (SNF) (2008\$).

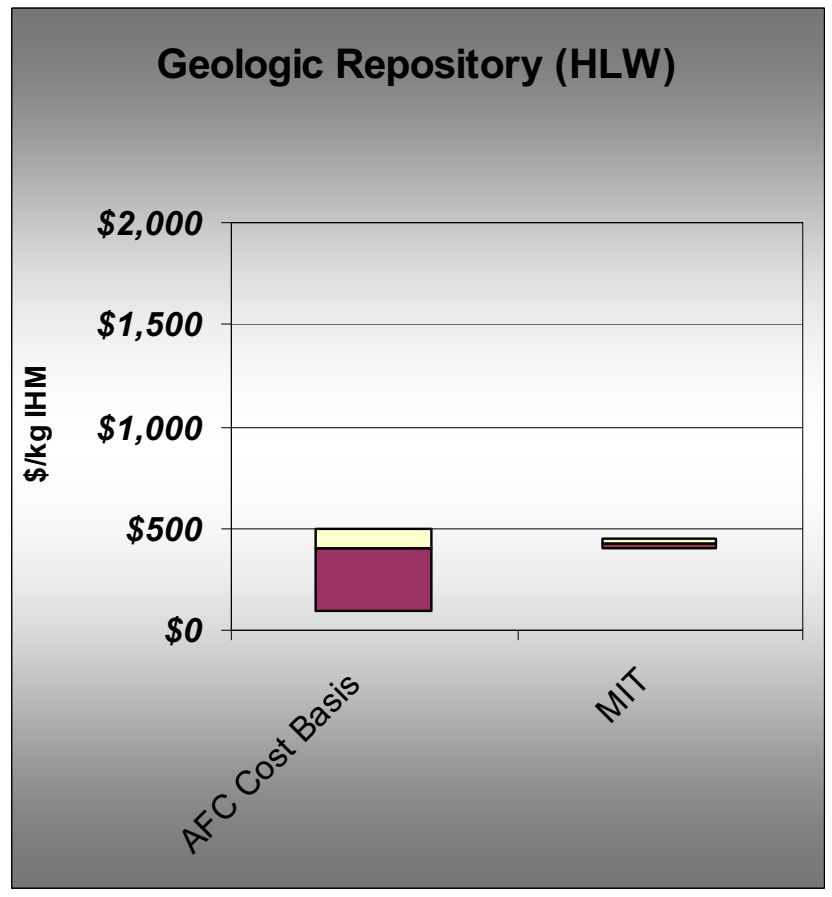

\begin{tabular}{|l|l|}
\hline AFC Cost Basis & $\begin{array}{l}\text { U.S. categories of waste } \\
\text { (LLW, HLW). Disposal } \\
\text { alone. }\end{array}$ \\
\hline OECD/NEA & $\begin{array}{l}\text { Not comparable- } \\
\text { Different categories of } \\
\text { waste (LILW-SL, } \\
\text { LILW-LL, and HLW). } \\
\text { Also different units } \\
\text { (\$/m3). }\end{array}$ \\
\hline MIT Study & $\begin{array}{l}\text { Includes storage and } \\
\text { disposal. Estimate basis: } \\
25 \% \text { of SNF disposal unit } \\
\text { cost. }\end{array}$ \\
\hline
\end{tabular}

Figure 10-9. Unit cost comparison: geologic repository for high-level waste (2008\$). 

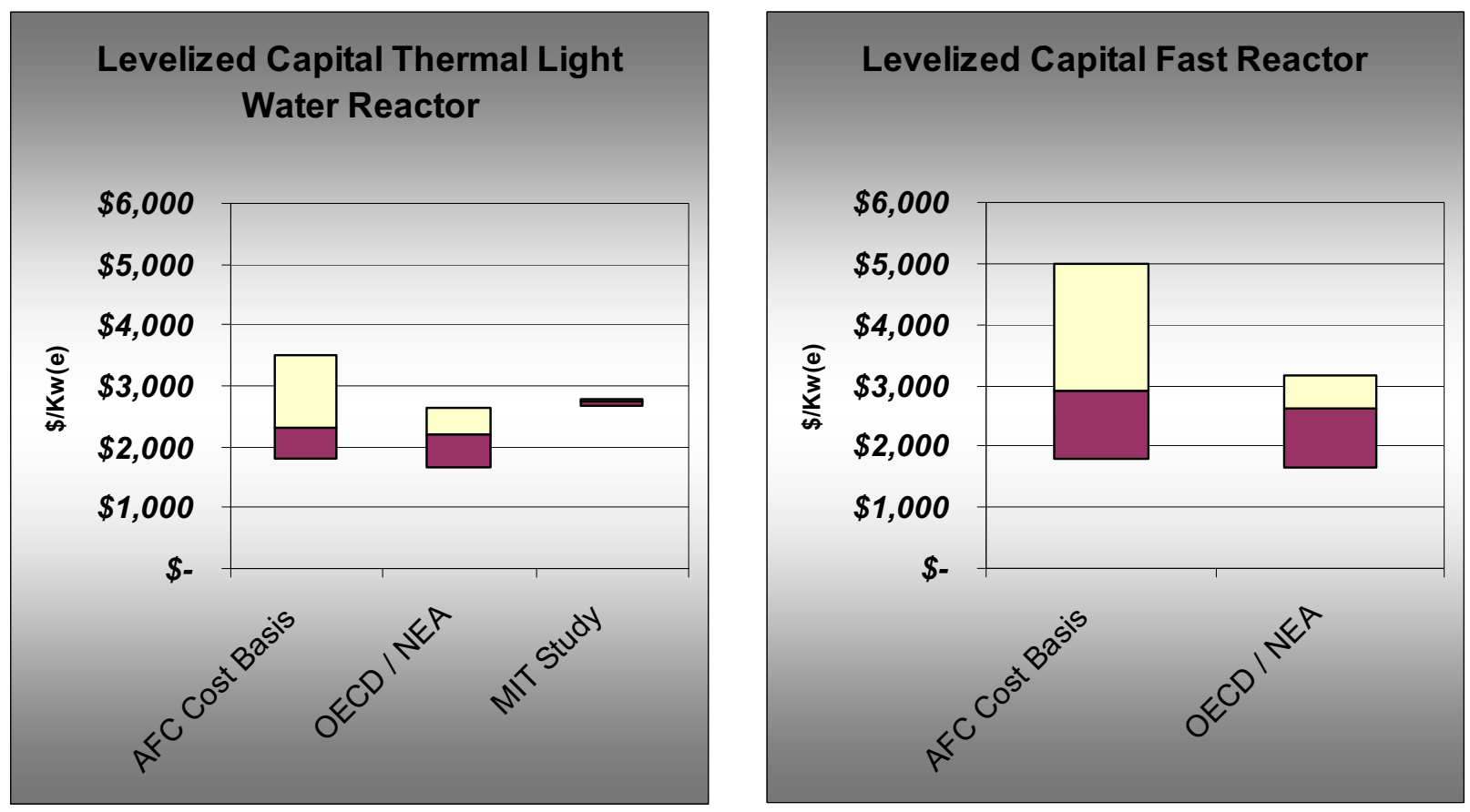

Figure 10-10. Unit cost comparison of overnight capital: thermal LWR and fast reactor (2008\$).

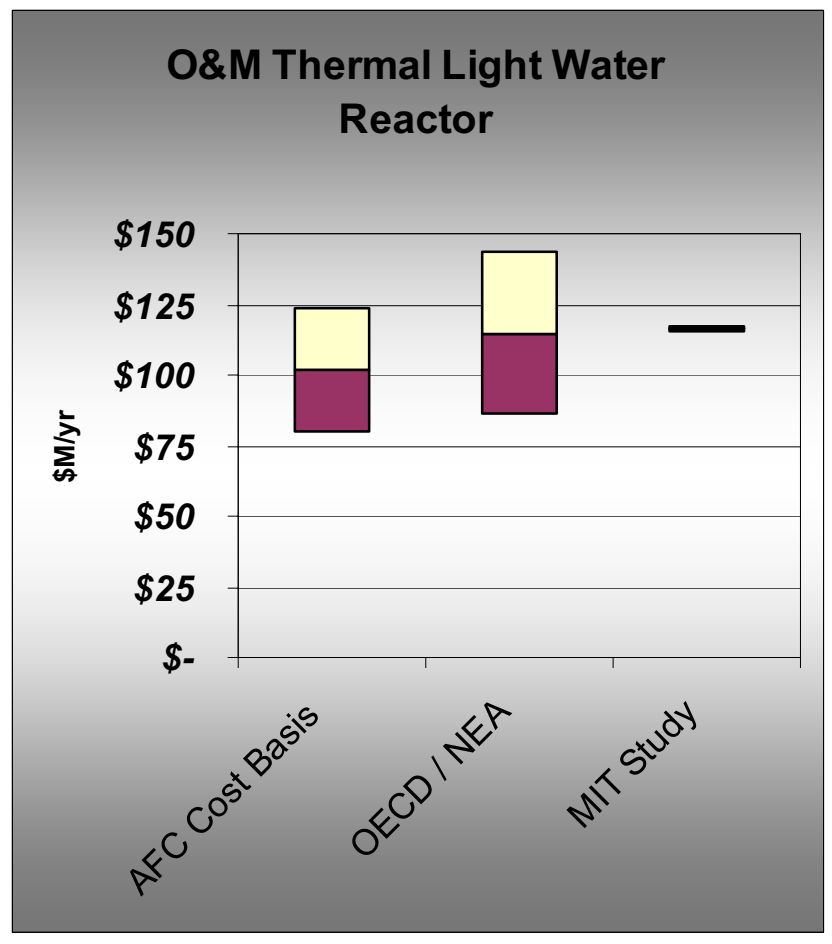

\begin{tabular}{|l|l|}
\hline $\begin{array}{l}\text { AFC Cost } \\
\text { Basis }\end{array}$ & $\begin{array}{l}\text { Fixed (\$/Kwe-yr) and } \\
\text { variable (mills/Kwh) O\&M } \\
\text { costs were combined to show } \\
\text { an annual total O\&M cost. } \\
\text { Includes capital cost for } \\
\text { replacement and D\&D. }\end{array}$ \\
\hline OECD/NEA & $\begin{array}{l}\text { Does not include D\&D. A } \\
\text { separate D\&D rate }(\% / y r) \text { is } \\
\text { used. OECD/NEA's fixed } \\
\text { O\&M rate }(\% / y r) \text { was put on } \\
\text { a similar basis to AFCI and } \\
\text { scaled to reactor size } \\
{[1,300 \text { MW(e)] for }} \\
\text { comparison. }\end{array}$ \\
\hline MIT Study & $\begin{array}{l}\text { Fixed (\$/Kwe-yr) and } \\
\text { variable (mills/Kwh) O\&M } \\
\text { costs were combined to show } \\
\text { an annual total O\&M cost. } \\
\text { Capital costs for replacement } \\
\text { are in a separate incremental } \\
\text { capital cost and are not } \\
\text { included in this unit cost }\end{array}$ \\
\hline
\end{tabular}

Figure 10-11. Annual cost comparison: thermal LWR O\&M, based on a 1,300 MWe LWR (2008\$). 


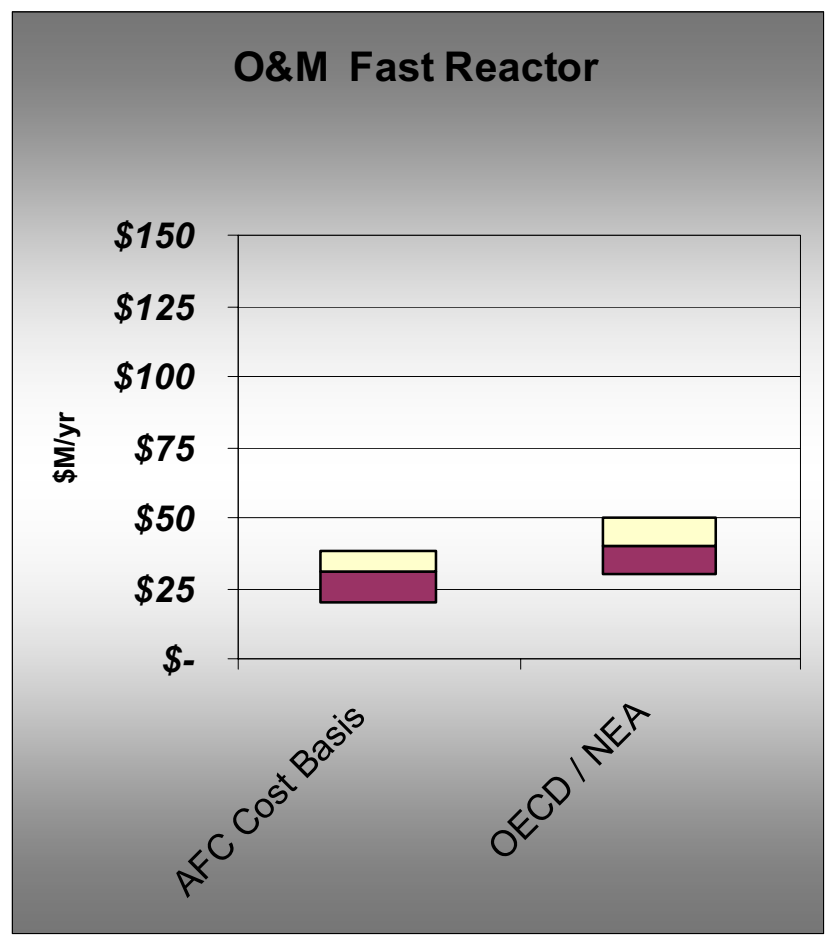

\begin{tabular}{|l|l|}
\hline AFC Cost Basis & $\begin{array}{l}\text { Fixed (\$/Kwe-yr) and } \\
\text { variable (mills/Kwh) } \\
\text { O\&M costs were } \\
\text { combined to show an } \\
\text { annual total O\&M cost. } \\
\text { Includes capital cost for } \\
\text { replacement and D\&D. }\end{array}$ \\
\hline OECD/NEA & $\begin{array}{l}\text { OECD/NEA's fixed O\&M } \\
\text { rate (\%/yr) was put on a } \\
\text { similar basis to AFCI and } \\
\text { scaled to reactor size } \\
{[380 \text { MW(e) for }} \\
\text { comparison. Does not } \\
\text { include D\&D. A separate } \\
\text { D\&D rate (\%/yr) is used. }\end{array}$ \\
\hline MIT Study & Not available \\
& \\
\hline
\end{tabular}

Figure 10-12. Annual cost comparison: FR O\&M, based on a 380 MWe FR (2008\$).

\subsection{Fuel Cycle Costs}

\subsubsection{OECD/NEA Fuel Cycle Assumptions}

The AFCI total fuel cycle costs and total costs of electricity for the open and closed systems were compared to similar systems in the OECD/NEA study. The specific cases selected for comparison are shown in Table 10-2. (Attachment A shows the actual AFCI spreadsheets.)

Table 10-2. AFCI and OECD/NEA fuel cycle cases for comparative evaluation.

\begin{tabular}{|l|l|l|l|}
\hline \multicolumn{1}{|c|}{ Study } & \multicolumn{1}{c|}{ Open Cycle } & \multicolumn{1}{c|}{ Fast Recycle } & \multicolumn{1}{c|}{ Thermal/Fast Recycle } \\
\hline AFCI & Once-through & 1-Tier & 2-Tier \\
\hline OECD/NEA & Scheme 1a & Scheme 3a & Scheme 3b \\
\hline
\end{tabular}

1. The open cycle is based on a LWR using standard UOx fuel. The primary difference is that in the AFCI once-through case the used fuel goes directly from a reactor to geologic repository versus in the OECD/NEA Scheme 1a, used fuel goes into indefinite long-term storage.

2. The fast recycle is based on thermal reactors providing make-up fuel for fast reactors. The AFCI 1-Tier and the OECD/NEA Scheme 3a used very similar ratios of LWRs to FRs, with AFCI using $62 \%$ PWRs and 38\% FRs, and OECD/NEA using 63\% PWRs and 37\% FRs. Both studies used similar uranium extraction (UREX) aqueous separations. OECD/NEA used UREX and AFCI used variations of UREX for different scenarios. They both also used metal fast reactor fuel with electrochemical processing.

3. The thermal/fast recycle is based on a one-pass thermal MOx recycle followed by fast reactor recycling. The OECD/NEA Scheme $3 \mathrm{~b}$ assumes a 1,450 MWe fast reactor using oxide MOx fuel, 
while AFCI 2-Tier assumes a modular $380 \mathrm{MWe}$ sodium-cooled fast reactor using metal fuel.

$\mathrm{OECD} / \mathrm{NEA}$ uses plutonium separation (PUREX) and AFCI uses more advanced UREX+ processes.

\subsubsection{MIT Fuel Cycle Assumptions}

The MIT study once-through fuel cycle was also compared to the OECD/NEA and AFCI oncethrough (open) fuel cycle. As seen in Table 10-3, MIT's carrying charge factor increases the once-through fuel cycle cost by $19 \%$. No breakdown of the total costs with and without carrying charge was provided in the study.

Table 10-3. Impact of MIT carrying charge factor on fuel cycle costs.

\begin{tabular}{|l|c|c|}
\hline \multicolumn{1}{|c|}{ Once-through Fuel Cycle } & MIT $(\mathrm{mills} / \mathrm{kWh})$ & AFCI (mills/kWh) \\
\hline 2008 Cost with carrying charge factor & 6.78 & N/A \\
\hline 2008 Direct cost & 5.67 & 6.72 \\
\hline
\end{tabular}

\subsubsection{Comparisons between AFCI and OECD/NEA and MIT Cost Distributions}

The fuel cycle cost distributions for the AFCI and OECD/NEA, and point-cost values for MIT are compared in Figure 10-13. The distributions are consistent in regards to the order and the relative fuel cycle costs. Both studies show that the order of cost (from least to the most) is the open cycle fuel, the fast recycle, then the thermal/fast recycle. The range of AFCI fuel cycle costs are about $2.5-4 \mathrm{mills} / \mathrm{kWh}$ higher than the OECD/NEA costs. The primary difference is due to the higher AFCI cost uncertainty for uranium, as shown in Figure 10-1, and the large differences in SNF and HLW waste disposal strategies, described in Figures 10-8 and 10-9. The MIT once-through fuel cycle cost of $5.67 \mathrm{mills} / \mathrm{kWh}$ (no carrying charge) is shown as a red vertical line Figure 10-13. The MIT costs are low relative to the AFCI costs due to relatively low uranium and SNF disposition cost estimates.

Fuel Cycle Cost Distribution

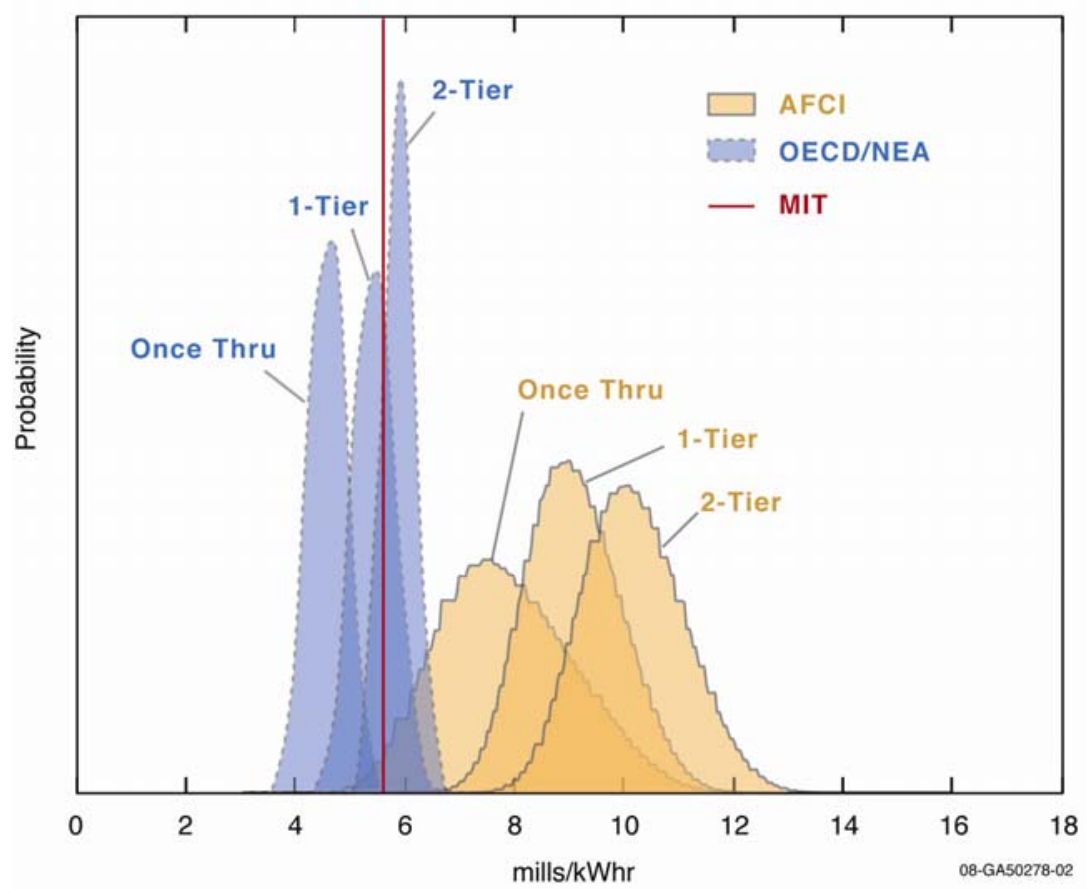

Figure 10-13. Cost ranges for fuel cycles (not including reactors). 
The total cost of electricity distributions for the AFCI and OECD/NEA, and point-cost value for MIT (including carrying charge factor) are compared in Figure 10-14. The cost distributions from the AFCI and OECD/NEA studies are quite consistent. The large cost uncertainties used in the AFCI analysis lead to wider distributions and skew toward higher costs. The higher fast reactor capital costs used in the AFCI analysis leads to a slight shift in the TCOE ranking, such that the 2-Tier costs less than the 1-Tier. In the OECD/NEA study, the 2-Tier maintains the same ranking in TCOE costs, as was seen in the costs for the fuel cycle without reactors. MIT's much higher costs of $89 \mathrm{mills} / \mathrm{kW} \cdot \mathrm{h}^{\mathrm{t}}$ are attributed to the $10 \% /$ year carrying charge, low reactor capacity factor (85\%), high finance rates applicable to unregulated markets, and high assumed costs for LWR levelized capital and O\&M.

\section{Total Cost of Electricity Distribution}

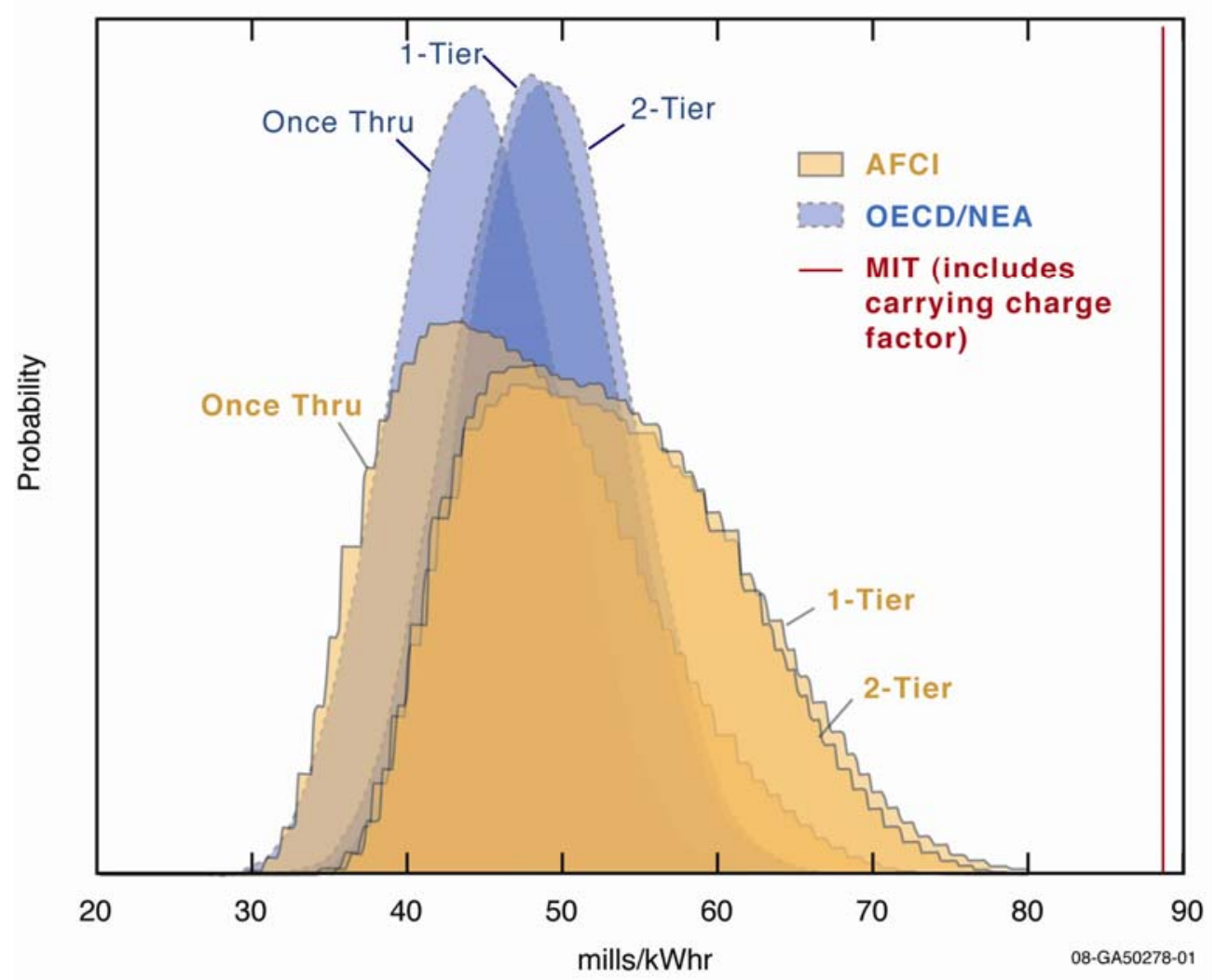

Figure 10-14. Cost ranges for the Total Cost of Electricity (including reactors).

\subsection{References for Section 10}

Japan Atomic Energy Commission (JAEC), 2005, "Framework for Nuclear Energy Policy," October 2005.

Massachusetts Institute of Technology, 2003, The Future of Nuclear Power, ISBN 0-615-12420-8, 2003.

OECD/NEA, 1994, The Economics of the Nuclear Fuel Cycle, 1994.

OECD/NEA, 2006, Advanced Nuclear Fuel Cycles and Radioactive Waste Management, NEA No. 5990, OECD 2006.

t. MIT's nominal costs were based on real levelized costs of $67 \mathrm{mills} / \mathrm{kW} \cdot \mathrm{h}$ (including the carrying charge) that were escalated to 2008 dollars to arrive at a cost of $\sim 89 \mathrm{mills} / \mathrm{kW} \cdot \mathrm{h}$. 
Shropshire, et al., 2008, “Advanced Fuel Cycle Cost Basis,” Idaho National Laboratory, INL INL/EXT-10-12107 March 2008.

Shropshire, et al., 2006, “Advanced Fuel Cycle Economic Sensitivity Analysis,” Idaho National Laboratory, INL/EXT-06-111947, December 2006.

Todreas, N., 2006, "Perspectives on the Economics of Nuclear Power from the MIT Study," Northeastern ANS Symposium on Economics in the Nuclear Industry, Troy, New York, March 30, 2006.

U.S. Army Corps of Engineers, 2007, Engineering and Design Civil Works Construction Cost Index, EM 1110-2-1304, September 30, 2007. 


\section{CONCLUSIONS}

This AFCI study has revealed that there are large cost uncertainties with all the fuel cycle strategies, and that systems using a closed fuel cycle are about $10 \%$ more expensive than open cycle systems. Closed fuel cycle cost uncertainties are driven by technology uncertainties for fast reactors, used fuel separation, "hot" (remote handling) fuel fabrication, and process waste disposition. In closed systems, the resources needed to produce reactor fuels will be less sensitive to uranium resource and market pressures and will produce less waste subject to disposal in future geological repositories.

A further insight is that future nuclear energy costs appear very competitive to other base-load fossil fuel sources of energy, especially if carbon constraints are imposed. The market competitiveness of nuclear was compared to fossil fuel-derived electrical energy (particularly if carbon is taxed or carbon storage is required). The overall assessment shows that the busbar electricity costs from nuclear and conventional pulverized coal power are similar if no cost of carbon (tax, carbon capture/storage, or "cap and trade" program) is imposed. Natural gas, a relatively clean energy source that until recently has been the preferred source new base load electricity, now suffers from high costs and continued price volatility. A carbon tax greater than a few dollars per metric ton of carbon dioxide, would shift the fossil energy cost edge toward nuclear, even for closed fuel cycle systems.

The study concludes that further U.S. and joint international-based design studies are needed to reduce the cost uncertainties with respect to fast reactor, fuel separation and fabrication, and waste disposition. The results of this work can help provide insight to the economic conditions needed to keep nuclear energy (including closed fuel cycles) economically competitive in the U.S. and globally. This cost study is integral to the complete evaluation of advanced fuel cycles, which also includes an assessment of environmental, safety, waste, and proliferation impacts. The AFCI Systems Analysis group has developed the engineering economic analysis capabilities through the development of a cost basis, static and dynamic cost models, and economic methodologies to analyze the system cost sensitivities of nuclear fuel cycles. These capabilities will continue to be improved and applied to the AFCI programs. 
Appendix A

\section{Managed Decay Storage Costs}




\title{
Appendix A
}

\section{Managed Decay Storage Costs}

\author{
Cost of Decay Storage of Immobilized CsSr Waste Form arising from Advanced Fuel Cycles.
}

\section{PURPOSE}

The purpose of this paper is to determine a cost range for decay storage of immobilized, heatgenerating, mixed cesium-strontium $(\mathrm{CsSr}-\mathrm{BaRb})$ waste as generated by the Advanced Fuel Cycle Initiative (AFCI) UREX+ based fuel cycle. The period of storage has a nominal value of 300 years and may allow subsequent shallow disposal as low-level waste (LLW).

The approach adopted is to outline immobilization processes and corresponding waste form properties for storage of the separated $\mathrm{CsSr}$ fission product waste fraction derived from advanced fuel separation processes for treatment of LWR spent nuclear fuel (SNF). Waste form properties determine acceptable canister dimensions, features, numbers, and store design, which enable prediction of capital and operational costs. Various storage designs (e.g., ponds, standalone casks, housing arrays, dry vault storage [passively or forced convection cooled], and modification of existing facilities) to provide dry storage are available for storage of solid heat generating wastes such as spent fuel and vitrified high-level waste (HLW). Cost data from existing heat-generating waste storage studies is used to determine unit costs for 300-year CsSr storage. Three centuries provide 10 half-lives of decay and is the nominal value for the CsSr waste to comply with Class C waste criteria for proposed shallow disposal as LLW. Data is taken from a variety of sources including U.S., UK, French, and Japanese vitrified HLW, SNF, and AFCI studies. These provide various costs and waste forms (e.g., aluminosilicate, bentonite, borosilicate glass, synroc, and SNF) for a variety of specific decay powers (W/L) and store designs, so that it is important to reconcile scope and consistency of data.

\section{INTRODUCTION}

Under the AFCI Programs, the U.S. is developing advanced reactor, fuel cycle, and energy products (Bresee et al. 2007). UREX+ processes are under development for the partitioning of spent fuels to enable recycling of fissile and selected fissionable materials and enhanced disposal pathways for various separated waste streams. The removal of both long and medium-term major heat emitters (e.g., Pu-Am-Cm, and CsSr), from SNF or HLWs enables a substantial (1-2 orders of magnitude) increase in repository drift loading compared to direct disposal of SNF (Wigeland et al. 2006) and may avoid the need for a second geological repository in the U.S. in the $21^{\text {st }}$ century for SNF and vitrified HLW. Generally, a holistic or lifecycle analysis should be undertaken to encompass waste immobilization, transport, storage, and disposal. The DOE waste management strategy addresses these issues (Gombert et al. 2007). The roles of storage in the management of long-lived radioactive waste have been examined (OECD-NEA 2006).

There is the wider question of justifying CsSr separation in advanced fuel cycle processing and its impact on overall waste management costs. If $\mathrm{CsSr}$ is not removed from high-level liquid wastes (HLLW), as presently occurs with the commercial LWR PUREX fuel cycle, then vitrified HLW is normally decay stored, often in a modular dry vault air-cooled store (MVDS). The planned periods of decay storage generally range between 50-100 years. LWR commercial vitrified waste in canisters often has mass of $0.5 \mathrm{t}$ and a decay power of around $3 \mathrm{~kW}$ at fabrication after 4 years ex-reactor for a maximum (centerline) temperature of $\sim 500^{\circ} \mathrm{C}$ for glass formed at $\sim 1,150^{\circ} \mathrm{C}$. Such waste can be transported (e.g., French and UK HLW returns to Japan) though this is more economical after several decades. After 
100 years of storage, 3 half-lives for $\mathrm{CsSr}$, the decay heat reduces to several hundred watts per canister and actinide decay power starts to predominate.

The principal AFCI advanced fuel processing flowsheets employ UREX+ for separation of a near pure aqueous CsSr-Rb-Ba nitrate or carboxylate (salt-free) stream and electroreduction /refining using molten chloride salts to extract CsSr halides using zeolite ion exchange/occlusion. The often lower selectivity of molten salt processing may decrease CsSr loadings, decrease specific decay power, and increase waste quantities as well as decay periods for compliance with Class $\mathrm{C}$ waste criteria, and affect subsequent waste management. These AFCI CsSr heat generating streams may be immobilized using a wide variety of waste forms and processes including vitrification and ceramic sintering. Depending on design, many repositories have good capacities for vitrified HLW at this stage though further decay, increasingly effective removal of actinides and $\mathrm{CsSr}$ and/or disposal in heat dissipating salt repositories may significantly increase repository capacity. However, this study is restricted to performing sensitivity analysis for technology and cost of decay storage of immobilized CsSr waste form arising from advanced fuel cycles. Unit costs of $\mathrm{CsSr}$ waste storage are estimated based on storage technology, waste form properties, and canister size.

\section{WASTE FORMS, CANISTER SIZES, AND STORAGE OF HEAT GENERATING WASTES}

Solidification of HLW, the main existing heat generating waste, serves the two main purposes of immobilization of waste for storage, transport, and emplacement in the repository and long-term fixation of radionuclides after repository closure and permanent disposal. Interim storage is normally required to allow further decay of the major heat emitting nuclides, and therefore reduce the early thermal loading of the repository. Thermal, mechanical and chemical stability of the waste form is required including effects of irradiation and leaching. For separated $\mathrm{CsSr}$ wastes, the main objective is that of immobilization while a secondary one is longer-term stability. For example, to prevent the leaching of long-lived isotopes such as Cs-135 and stable toxic species such as Ba (Alvarez 2007).

Various waste forms and corresponding waste immobilization processes are known for HLW. Calcines are products or intermediates obtained by partial or complete removal of volatile components of the waste, such as water and nitrates, at temperatures of $400-900^{\circ} \mathrm{C}$. This creates a mixture of oxides in particulate form and with a specific surface of $0.1-5 \mathrm{~m}^{2} / \mathrm{g}$. Calcine powders may not be very stable because of the chemical properties of some constituents, large surface area, low thermal conductivity, and friable nature of the solids creating fine dust. Depending on calcination temperature, calcines may have residual water and nitrate content. Calcine powder may be pressed or solidified within cements or concentrated solutions grouted. If waste temperatures, radiation, or canister corrosion effects are sufficient to release water, NOx or hydrogen, then canister venting, inspection, and off-gas treatment may be needed (Streatfield et al. 2006).

For passive long-term decay storage, higher process temperature, refractory, near-inert waste forms, such as glasses and ceramics are preferred. For heat generating wastes, waste form dimensions may need optimization to limit center line temperatures to acceptable values. The more important immobilization alternatives for HLW are calcine, ceramics, glass, glass ceramics, and cement (Benedict et al. 1981), see Table A-1. 
Table A-1. Immobilization waste form options.

\begin{tabular}{|l|l|l|l|}
\hline \multicolumn{1}{|c|}{ Alternative } & \multicolumn{1}{|c|}{ Calcine } & \multicolumn{1}{c|}{ Glass } & \multicolumn{1}{c|}{ Ceramic } \\
\hline Basic & $\begin{array}{l}\text { Fluidized bed } \\
\text { (particulates) } \\
\text { Pot (cake) }\end{array}$ & $\begin{array}{l}\text { Borosilicate (cylinder) } \\
\text { Phosphate (cylinder) }\end{array}$ & $\begin{array}{l}\text { Aluminosilicate } \\
\text { Bentonite }\end{array}$ \\
\hline Advanced & $\begin{array}{l}\text { Supercalcine (additives, } \\
\text { high T) }\end{array}$ & $\begin{array}{l}\text { Borosilicate glass ceramic } \\
\text { (cylinder) }\end{array}$ & $\begin{array}{l}\text { Synroc (multi-phase } \\
\text { ceramic) - Hi T, HIP }\end{array}$ \\
\hline Composite & $\begin{array}{l}\text { Multibarrier (e.g., pyroC, } \\
\text { SiC in metal matrix) }\end{array}$ & $\begin{array}{l}\text { Vitromet (glass/ glass } \\
\text { ceramic in metal matrix) }\end{array}$ & $\begin{array}{l}\text { Glass ceramic (e.g., puck } \\
\text { crystallized glasses and } \\
\text { sodalite in glass matrix) }\end{array}$ \\
\hline $\begin{array}{l}\text { Cement- } \\
\text { vented } \\
\text { Canister }\end{array}$ & $\begin{array}{l}\text { Low T encapsulation of } \\
\text { concentrated solution or } \\
\text { calcined particles. }\end{array}$ & & $\begin{array}{l}\text { Higher temperature } \\
\text { specialized cements } \\
\text { (e.g., supercalcines). }\end{array}$ \\
\hline
\end{tabular}

There is generally an increased processing cost for refractory, inert waste forms. This may take place through need for high-temperature operation, corrosive conditions limiting equipment lifetimes, volatilization of selected fission products requiring complex off-gas systems and waste recycle. Synrocs often require small batch operations using hot isostatic pressing (HIPing) at high temperature and with relatively long process cycles.

Waste forms may incorporate differing waste concentrations to meet waste performance and economic goals. Waste packaging and transportation costs are significant so that reduction of package number is desirable. However, thermal limitations apply to transportation, disposal and the waste form itself, and excessive fission product (FP) concentrations reduce the chemical performance of waste and may cause excessive internal temperatures. Composition limitations are typical for glasses where either phase separation or lack of glass forming occurs. For borosilicate glasses, for example, the FP oxide limit is normally considered to be around $20-25 \%$ by weight (plus process additives) using existing hot-wall melters. Higher concentration may create a distinct yellow crystalline phase formed of alkaline and alkaline earth molybdates. This readily soluble phase contains Cs-137 and Sr-90. Glasses can be formulated to incorporate most fission product and actinide oxides with good stability. Devitrification occurs above the glass transition temperature, for example at elevated temperatures of $\sim 500^{\circ} \mathrm{C}$ for phosphate and $\sim 600^{\circ} \mathrm{C}$ for present borosilicate glasses. Some processes employ controlled crystallization to glass ceramics to create known waste form properties. Other waste form composition/temperature limitations may arise from a wide variety of limits such as melting, volatization, and recrystallization into new phases, chemical reaction, canister pressurization, etc. Composition is not a direct limitation for calcines, which are amorphous, but high-heat load may cause further chemical decomposition, canister corrosion, and pressurization. Grouts are normally used to immobilize mineral ion exchange (IX) materials used for treatment of LLW and (in Europe) for intermediate level wastes (ILW) liquid wastes, but have also been used for encapsulation of low specific decay power raffinates from specialized recycling operations.

Industrial practice for HLW vitrification tends to use large canisters for low specific decay heat defense wastes (e.g., 0.61m o.d., 3m height and 2.1t filled mass) for Savannah River Defense Waste Processing Facility (DWPF), and small canisters for high-specific decay power LWR wastes (e.g., 0.43m o.d., $1.3 \mathrm{~m}$ height and 0.5t filled mass) for Ateliers Vitrification La Hague AVH (IAEA 1992). Even smaller diameter canisters, o.d. 0.3m, have also been used at PAMELA (Germany) and WIP (India). In general terms, canisters with diameters less than $0.2-0.15 \mathrm{~m}$ diameter are not favored industrially due to difficulties of filling with molten glass due to bridging, potentially more thimble tubes due to retention of moderate $1 / \mathrm{d}$ ratios for canister cooling, increased pressure drops with high-air velocities, and possible 
limited cost reduction of storage with decreasing thimble tube diameter. KfK Germany developed a process where HLW phosphate glass beads were cast and then embedded in molten metal in a canister. Such an approach or other internal features for heat conduction may be especially useful for $\mathrm{CsSr}$ vitrification of short-cooled, high-loaded fission products.

For LWR fuel of typical burn-up of $40 \mathrm{GW}(\mathrm{t}) \cdot \mathrm{d} / \mathrm{t}(\mathrm{iHM})$, the decay powers are given in the Table A-2, (Bergelson et al. 2005).

Table A-2. Decay heat power of FPs and transuranics $(\mathrm{Pu}, \mathrm{Am}, \mathrm{Cm})$ during long-term storage, W/t(iHM).

\begin{tabular}{rcccc}
\hline$t$ y) & Beta & Gamma & Total FP & Actinides + FP \\
\hline 0 & 2300 & 615 & 2910 & 3180 \\
1 & 1470 & 530 & 2000 & 2260 \\
3 & 892 & 451 & 1340 & 1600 \\
10 & 617 & 351 & 968 & 1230 \\
30 & 376 & 206 & 582 & 845 \\
100 & 71.0 & 39.5 & 111 & 330 \\
300 & 0.648 & 0.396 & 1.04 & 149 \\
1000 & 0.0136 & 0.00752 & 0.0211 & 63.8 \\
3000 & 0.0135 & 0.00742 & 0.0209 & 24.9 \\
10,000 & 0.0131 & 0.00707 & 0.0202 & 14.7 \\
30,000 & 0.0122 & 0.00615 & 0.0183 & 5.60 \\
100,000 & 0.00936 & 0.00380 & 0.0132 & 0.859 \\
300,000 & 0.00478 & 0.00097 & 0.00573 & 0.239 \\
\hline
\end{tabular}

Data in Table A-2 shows both total FP and total FP with transuranic ( $\mathrm{Pu}, \mathrm{Am}, \mathrm{Cm}$ ) contributions to decay power. Often the Pu contribution is omitted as vitrified HLW includes minor actinides, but not Pu. The total FP decay power reduces by $40 \%$ in the period 10 -year cooled to 30 -year cooled, which indicates the major contributions of Cs-137 $\left(\mathrm{t}_{1 / 2}=30 \mathrm{y}\right)$ and Sr-90 $\left(\mathrm{t}_{1 / 2}=28 \mathrm{y}\right)$ to decay power in this period.

Heat generation in immobilized HLW and CsSr waste causes the waste form to be at elevated temperature for more than 100 years. With some simplifications, the maximum temperature difference between the centerline and surface of a long cylindrical waste form is given by:

$\Delta \mathrm{T}_{\max }=\mathrm{qr}^{2} / 4 \kappa$

Where $\mathrm{q}$ is the power density, $\mathrm{W} / \mathrm{m}^{3}, \mathrm{r}$ is the radius of the cylinder, and $\kappa$ is the waste form thermal conductivity, $\mathrm{W} /\left(\mathrm{m} .{ }^{\circ} \mathrm{C}\right)$. The surface temperature is given by the storage conditions including canister wall and waste surface/canister interfacial properties. This enables scaling of canister radius against heat loadings from existing commercial practice (IAEA 1992). Representative values for conductivity of waste forms are given in Table A-3 (Benedict et al. 1981).

Table A-3. Thermal conductivity ranges for various HLW forms in temperature range $100-500^{\circ} \mathrm{C}$.

\begin{tabular}{|l|c|}
\hline \multicolumn{1}{|c|}{ Waste Form } & $\begin{array}{c}\text { Thermal Conductivity, } \kappa \\
\mathrm{W} /\left(\mathrm{m} .{ }^{\circ} \mathrm{C}\right)\end{array}$ \\
\hline Particulate calcine & $0.2-0.3$ \\
\hline Phosphate glass & $0.8-1.2$ \\
\hline Borosilicate glass & $0.9-1.3$ \\
\hline Borosilicate glass ceramic & $1.5-2.0$ \\
\hline Particulate calcine or glass beads in metal matrix (e.g., vitromet) & $\sim 10$ \\
\hline
\end{tabular}


Waste form conductivity clearly has a major influence on centerline (peak) temperature and corresponding canister dimension (radius), see Figure A-1, (Benedict et al. 1981).

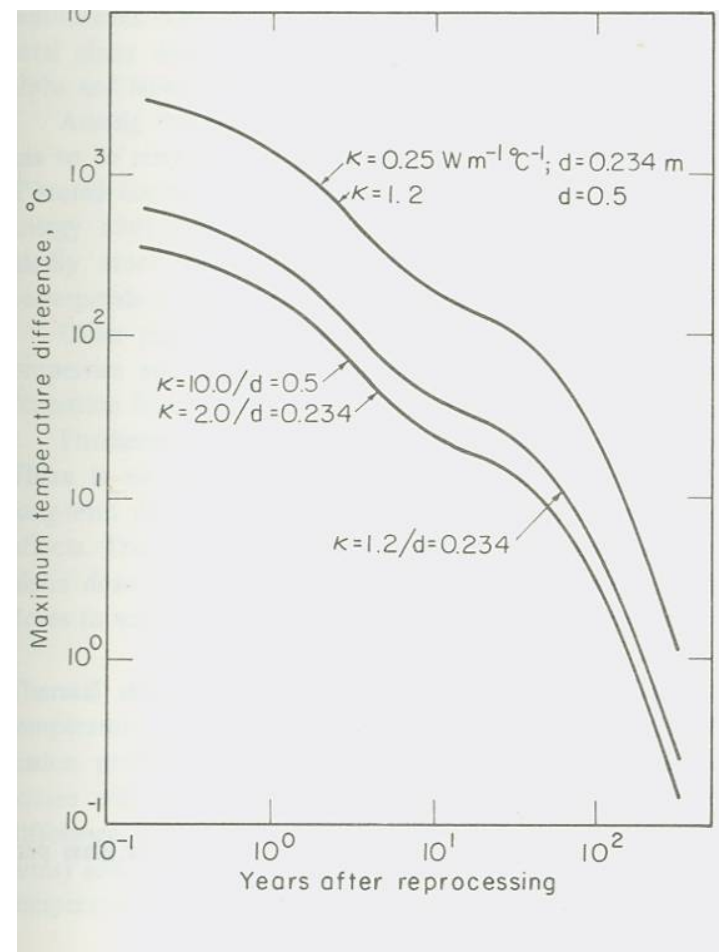

Figure A-1. Calculated maximum temperature difference in a cylinder of solidified waste for different diameters and thermal conductivities as a function of time (years) after recycling.

The heat generation rate is based on fission products and minor actinides incorporated into a waste form specific volume of $70 \mathrm{~L} / \mathrm{t}(\mathrm{iHM})$. The originating SNF burnup is $30 \mathrm{GW}(\mathrm{t}) \cdot \mathrm{d} / \mathrm{t}(\mathrm{iHM})$ and recycling taking place at 150 days SNF decay.

Figure A-1. Maximum centerline temperature difference of waste form as a function of decay time.

At the assumed waste loading, Figure A-1 indicates maximum temperature differences for glass ceramic waste with canister diameter of about $0.5 \mathrm{~m}$ of between the waste center line and surface of $>1,000^{\circ} \mathrm{C}(\equiv 100 \mathrm{~W} / \mathrm{L})$ and $>100^{\circ} \mathrm{C}(\equiv 10 \mathrm{~W} / \mathrm{L})$ for 1 year and 10 years decay after recycling respectively.

In the present study, borosilicate glass is considered the reference HLW form. This is conservative since it is somewhat more restrictive thermally than some synrocs and other ceramics. For civil design, it is generally preferable to restrict natural convection cooling air discharge temperatures to around $150-200^{\circ} \mathrm{C}$ as concrete structural components are damaged by long-term contact with air at temperatures approaching $100^{\circ} \mathrm{C}$. However, higher values can be engineered. Air cooling in forced convection stores would be less limiting, but for a long-term decay store, there is likely to be conservatism concerning highly rated systems and effects of cooling failure. This suggests that a maximum temperature difference, $\Delta \mathrm{T}$, between waste centerline and surface of around $300^{\circ} \mathrm{C}$ may be appropriate assuming a centerline maximum design temperature of $\sim 500^{\circ} \mathrm{C}$. For a canister with diameter $0.5 \mathrm{~m}$ containing borosilicate glass HLW, a maximum specific thermal power in the range 10-30 W/L appears suitable. Raising the glass transition temperature by $\sim 200^{\circ} \mathrm{C}$ increases the maximum thermal power by about $60 \%$.

\section{POTENTIAL WASTE FORMS FOR IMMOBILIZATION OF CESIUM AND STRONTIUM}

The most troublesome Cs and $\mathrm{Sr}$ isotopes are Cs-137 ( $\mathrm{t}_{1 / 2}=30.07 \mathrm{y}: 0.66 \mathrm{MeV} \gamma$ and $0.514 \mathrm{MeVmax}$ $\beta$-) and $\mathrm{Sr}-90\left(\mathrm{t}_{1 / 2}=28.78 \mathrm{y}: 0.546 \mathrm{MeVmax} \beta\right.$-), so their activities remain a concern for $\sim 300$ years (i.e., $\sim 10$ half-lives). These two isotopes generate a major portion of the decay heat in spent nuclear fuel over the first 100 years of storage, but then are essentially stable. Removing $\mathrm{Cs}$ and $\mathrm{Sr}$ for decay storage will reduce the short-term heat load on a repository waste form. 
Fission product oxide mass, excluding noble gases, is $\sim 1 \mathrm{~kg}(\mathrm{FPOx}) / \mathrm{GW}(\mathrm{t}) \mathrm{d}$, and so for a metric ton, $\mathrm{t}$, of SNF at $40 \mathrm{GW}(\mathrm{t}) . \mathrm{d} / \mathrm{t}(\mathrm{iHM})$, the mass of FP oxides is about $40 \mathrm{~kg}$. Cs and $\mathrm{Sr}$ form about 10 atom \% of the FPs, of which around half are the major heat emitting isotopes-Cs-137 and Sr-90. Total CsSr also form around $10 \%$ by weight of the FP oxides, that is $4 \mathrm{~kg} / \mathrm{t}(\mathrm{iHM})$ of spent fuel. Total $\mathrm{CsSr}-\mathrm{Rb}-\mathrm{Ba}$ form around $15 \%$ by weight of the FP oxides, that is $6 \mathrm{~kg} / \mathrm{t}(\mathrm{iHM})$ of spent fuel. From Table A-2, where Cs-137 and $\mathrm{Sr}-90$ are the only major FP isotopes with half lives between 10-50 years, the decay power of CsSr is seen as about $1 \mathrm{~kW} / \mathrm{t}(\mathrm{iHM})$ at 10 years ex-reactor. Alternatively the $\mathrm{CsSr}$ decay power can be expressed as $\sim 1 / 4 \mathrm{~kW} / \mathrm{kg}(\mathrm{CsSr})$ at 10 -years cooling. In engineering terms, the specific decay power of $\mathrm{CsSrOx}$ is about 10 times that of overall FPOx at 3-20 years cooling.

Recently, interest in separation of Cs and/or Sr during remediation of long-stored HLWs and for advanced fuel cycles has stimulated developments in waste forms tailored to $\mathrm{CsSr}$ immobilization. These include variants of waste forms for HLW and several new matrices (see Table A-4), which is representative rather than complete. The various minerals formed have differing capacities for Cs and Sr.

Table A-4. Representative CsSr-Rb-Ba waste forms.

\begin{tabular}{|c|c|c|c|c|}
\hline Matrix & CsSr Composition & $\begin{array}{c}\text { Composition Matrix, }(\% \\
\text { wt })\end{array}$ & Process & Reference \\
\hline Cement & $\begin{array}{l}4 \% \text { Zeolite A, } \\
5 \mathrm{mEq} / \mathrm{g}\end{array}$ & $\begin{array}{l}\text { PFA, Ordinary Portland } \\
\text { Cement }\end{array}$ & $\begin{array}{l}\text { Grouting ambient } \mathrm{T} \\
\text { Maintain } \leq 95^{\circ} \mathrm{C}\end{array}$ & $\begin{array}{l}\text { El-Kamash et al. } \\
2006\end{array}$ \\
\hline $\begin{array}{l}\text { Alumino- } \\
\text { silicate } \\
\text { (Steam reform) }\end{array}$ & $\begin{array}{l}27 \% \mathrm{Cs} / 8 \% \mathrm{Sr} \\
\mathrm{SrCO}_{3} \\
\mathrm{CsAlSi}_{2} \mathrm{O}_{4} \\
\end{array}$ & $\begin{array}{l}\text { Pollucite/hydroceramic } \\
\text { Slawsonite }\end{array}$ & $\begin{array}{l}\text { Steam reform } \mathrm{CsSr}-\mathrm{Ba} \\
\text { with carbon } \& \text { alumino- } \\
\text { silicate clay at } \sim 700^{\circ} \mathrm{C}\end{array}$ & $\begin{array}{l}\text { Ortega and } \\
\text { McDeavitt, } 2007 \\
\text { Law et al. } 2007\end{array}$ \\
\hline $\begin{array}{l}\text { Bentonite } \\
\text { (alumino- } \\
\text { silicate) } \\
\text { Dry sinter } \\
\end{array}$ & $\leq 40 \%$ Cs loading & $\begin{array}{l}\text { Celsian } \\
\text { Pollucite } \\
\text { Hydroxyl-apophyllite }\end{array}$ & $\begin{array}{l}\text { Dry sintering bentonite } \\
\text { clay containing } \mathrm{Cs}, \mathrm{Sr} \\
\mathrm{Rb}, \mathrm{Ba} \text { to } 600-1,000^{\circ} \mathrm{C}\end{array}$ & $\begin{array}{l}\text { Kaminski and Merz, } \\
\text { ANL. }\end{array}$ \\
\hline $\begin{array}{l}\text { Crystalline } \\
\text { Silicotitan-ate, } \\
\text { CST and } \\
\text { niobate IX }\end{array}$ & $\mathrm{Cs}_{2} \mathrm{O} \sim 20 \% \mathrm{wt}$ & $\begin{array}{l}\text { Cs2TiSi6O15 } \\
\text { Cs3TiSi3O9.5 } \\
\text { and Ti analogue of } \\
\text { Pollucite CsTiSi2O6 }\end{array}$ & $\begin{array}{l}\text { Calcining CsSr soaked } \\
\text { UOP CST IE-911 in air } \\
\text { at } 900-1,000^{\circ} \mathrm{C}\end{array}$ & $\begin{array}{l}\text { Balmer et al. } 2000 . \\
\text { Luca et al. } 2006\end{array}$ \\
\hline $\begin{array}{l}\text { Borosilicate } \\
\text { Glass } \\
\text { High mp glass }\end{array}$ & $\begin{array}{l}\mathrm{Cs}_{2} \mathrm{O} 13 \% \mathrm{wt} \\
\text { and } \\
\text { SrO } 7 \% \mathrm{wt} \\
\text { PNNL } \sim 40 \% \mathrm{wt}\end{array}$ & $\begin{array}{l}\mathrm{Na}_{2} \mathrm{O} 10-20 \\
\mathrm{~B}_{2} \mathrm{O}_{3} 10-17 \\
\mathrm{SiO}_{2} 45-50 \\
\mathrm{Al}_{2} \mathrm{O}_{3} 2-5 \\
\mathrm{Ba}, \mathrm{Pb}, \mathrm{TiOx} 4-6\end{array}$ & $\begin{array}{l}\text { Calcination and } \\
\text { Melting } \\
\text { High-melting glass }\end{array}$ & Aloy et al. 2007 \\
\hline $\begin{array}{l}\text { Hexagonal } \\
\text { Tunsten oxide } \\
\text { Bronze, HTB }\end{array}$ & $\begin{array}{l}\mathrm{Cs}_{2} \mathrm{O} \sim 12 \% \mathrm{wt} \\
\text { or } \\
\mathrm{SrO} \sim 5 \% \mathrm{wt}\end{array}$ & $\begin{array}{l}\mathrm{Cs}_{0.13} \mathrm{Mo}_{0.03} \mathrm{~W}_{0.97} \mathrm{O}_{3} \\
\mathrm{Sr}_{0.05} \mathrm{Mo}_{0.03} \mathrm{~W}_{0.97} \mathrm{O}_{3}\end{array}$ & $\begin{array}{l}\text { CsSr adsorbed } \\
\text { hexagonal tungsten } \\
\text { oxide bronze, Calcine } \\
500-1,000^{\circ} \mathrm{C} \text { in air. }\end{array}$ & Luca et al. 2006 \\
\hline $\begin{array}{l}\text { Synroc-C } \\
\text { Hydrous } \\
\text { titanium oxide } \\
\text { (HTO) }\end{array}$ & $\begin{array}{l}\text { CsSr-Rb-Ba } 12 \\
\text { \%wt }\end{array}$ & $\begin{array}{l}\text { Hollandite } \\
\text { Rutile } \\
\text { Titanates }\end{array}$ & $\begin{array}{l}\text { Calcination } 750^{\circ} \mathrm{C} \& \\
\mathrm{HIP} 1,275^{\circ} \mathrm{C}, 30 \mathrm{MPa} \text {, } \\
1 \mathrm{~h}\end{array}$ & Carter et al. 2007 \\
\hline $\mathrm{Cs} / \mathrm{Sr}$ Oxides & $\mathrm{Cs}_{2} \mathrm{O} / \mathrm{SrO}$ & Pure & Calcination & - \\
\hline $\mathrm{CsCl}$ & $\begin{array}{l}100 \% \text { Cs salt in } \\
\text { capsule in pool }\end{array}$ & $\begin{array}{l}\mathrm{CsCl}, 35 \mathrm{kCi} \\
190 \mathrm{~W}\end{array}$ & IX separation and Evap & Nat Acad Sci, 2003 \\
\hline $\mathrm{SrF}_{2}$ & $\begin{array}{l}\text { Sr salt in capsule in } \\
\text { pool }\end{array}$ & $\begin{array}{l}\mathrm{CsF}_{2}, 33 \mathrm{kCi} \\
260 \mathrm{~W}\end{array}$ & IX separation and Evap & Nat Acad Sci, 2003 \\
\hline
\end{tabular}


These potential CsSr waste storage forms evolved from upstream processing needs. Bentonite (including commercial UOP IE-911), and hydrous titanium oxide (HTO) are examples of IX materials used to selectively adsorb Cs, Sr, etc., from stored, complex chemistry salt HLWs to provide partitioning of waste for optimized waste management. These IX materials bearing low-medium Cs, Sr concentrations are heat treated by sintering, generally in the temperature range $500-1,000^{\circ} \mathrm{C}$. This causes removal of water, recrystallization, denitration and additional phases, and ultimately removal of hydroxyl groups. By contrast, the advanced fuel cycle processes (e.g., UREX+ [Vandegrift et al. 2004]) create salt free product streams of CsSr (e.g., nitrate and carboxylic acid based). These are not constrained by feed of mineral IX materials and can be used to form the complete range of waste forms from pure CsSr oxides/chlorides to glasses to freely tailored ceramics. The uses of zeolites (i.e., micro-porous crystalline solids with well-defined structures) generally contain silicon, aluminum, and oxygen in their framework and cations, and/or other molecules within their pores. For CsSr recovery in molten salt, electrochemical waste forms are more likely to be aluminosilicate ceramics or aluminosilicate glass ceramics.

High-level waste vitrification is well known as a complex technology with significant cost impact on existing PUREX commercial and defense recycling waste management. Advanced fuel processes generally partition FP and actinide species into more streams (e.g., seven for UREX+4). Some of these, (CsSr), have medium radioactive lifetimes and means have been sought to optimize the waste immobilization process to the waste lifetime, including storage requirements. For substantial masses, CsSr wastes need cooling for periods of 100-200 years. AFCI has examined use of a steam reforming process to fabricate alumino-silicate waste forms for CsSr storage (Law et al. 2006).

The UREX+ suite of processes has a separation segment, CCD-PEG in UREX+1a (Law et al. 2004) or FPEX in UREX+1b (Law et al. 2007), for recovery of CsSr-Rb-Ba from the raffinate of the UREX segment. Both of these technologies provide simultaneous solvent extraction of Cs and Sr together with the majority of $\mathrm{Rb}$ and $\mathrm{Ba}$. With CCD-PEG, the CsSr by-product is stripped using an organic amine and carboxylic acid mixture whilst FPEX uses dilute nitric acid as strip. Steam reforming has been developed for stabilization of streams because it can produce a solid waste form while retaining the Cs and $\mathrm{Sr}$ in the solid, destroy the nitrates and organics present in these aqueous solutions, and convert the Cs and Sr into leach resistant aluminosilicate minerals. The waste form is intended to meet a 300 year, 10 half-life period of storage prior to projected LLW disposal complying with Class C waste criteria.

A bench-scale steam reforming pilot plant has been operated at INL with several potential CsSr feed compositions and steam reformed product has been generated and analyzed (Law et al. 2006). A small, but representative fluidized-bed was used to conduct steam-reforming tests to produce mineralized granular product. Operating conditions of $700^{\circ} \mathrm{C}, \sim 3 \% \mathrm{H}_{2}, \sim 4 \% \mathrm{CO}, 70 \% \mathrm{CO}_{2}$, and $20 \%$ steam were used to decompose nitrates and organics. A starting bed of 100-300 micron aluminum oxide particles was used and Sagger clay slurried with the feed to produce pollucite and other alumino-silicate minerals. Excess clay was used to mineralize the cationic feed constituents. The clay particles are less than $10 \mu \mathrm{m}$ to achieve a high-surface area for reaction. The final bed material in each run was generally a granular material much like the initial aluminum oxide starting bed with some additional smaller diameter solids. The bench-scale steam reformer tests successfully converted cesium/strontium strip products to a solid form without volatilizing the Cs. Results also indicate that with optimization of the steam reforming operating parameters, $100 \%$ mineralization is possible (Law et al. 2006). The bed waste product material may be compacted, for example, within canisters to form pucks, which may be loaded into an over-pack.

A collection of scoping studies, entitled Engineering Alternative Studies (EAS), related to a commercial scale UREX+ separations plant were commissioned by DOE and carried out by a multinational laboratory team in 2006-2008. EAS investigated features of a canyon approach for a commercial plant, with expected throughput of $\sim 3,000 \mathrm{t}(\mathrm{HM})$ /year, with three solvent extraction lines. The Follow-on EAS (FOEAS) evaluation assumed a smaller plant throughput ( $>00 \mathrm{t}(\mathrm{HM}) /$ year UREX+) with re-examination of facility layout options, requirements, alternate flowsheets, etc. (Hebditch et al. 2007). 
In the EAS, an engineering proposal and costing of the proposed storage of the UREX + cesiumstrontium (CsSr) waste stream was presented. The study was based on the UREX+1a process, throughput of 3,000 $\mathrm{t}(\mathrm{iHM}) /$ year mixed LWR fuel of $60 \mathrm{GW}(\mathrm{t}) . \mathrm{d} / \mathrm{t}$ and formed a variety of products and wastes, including an aluminosilicate mineral powder $\mathrm{CsSr}$ waste intended for a 300-year period of storage prior to projected LLW disposal. This study demonstrated reasonable feasibility, but was not an economic optimization and further studies were performed.

The FOEAS was based on the UREX+1b process, throughput of $800 \mathrm{t}(\mathrm{iHM}) / \mathrm{year}$ mixed LWR fuel of $60 \mathrm{GW}(\mathrm{t}) \cdot \mathrm{d} / \mathrm{t}$ and formed a variety of products and wastes. For CsSr, three waste forms were examined conceptually (i.e., a sintered bentonite and two vitrified CsSr options with differing CsSr loadings). As for EAS, these would need nominal 300-years storage for compliance with Class $\mathrm{C}$ waste disposal. Other geological disposal scenarios may be feasible, but are not well defined yet and so are not considered here. This was a top-down assessment based on the above 3,000 $\mathrm{t}(\mathrm{HM}) / y e a r$ study with some variations to account for process changes and scale, etc. The use of sintered bentonite or vitrified CsSr wastes may possibly increase waste immobilization costs, but is expected to decrease overall waste storage costs life cycle costs (LCC) by increasing CsSr loading and canister diameter and by reducing waste volumes, total canisters, and required storage capacity. However, depending on design these may need periods of forced convection cooling and delayed potential for using passively cooled storage. If AFCI does call for CsSr separation, a vitrified CsSr waste form option is presently favored with a range of increased loading values being examined and this makes good use of state-of-the-art vitrification and waste storage technologies.

There was a third conceptual design, which was based on a high temperature, molten salt electrochemical process. This design was based on oxide fuel electrochemical reduction, uranium electrorefining, and transuranic product recovery by electrowinning. The design throughput is 300 $\mathrm{t}(\mathrm{HM}) /$ year mixed LWR fuel of $60 \mathrm{GW}(\mathrm{t}) . \mathrm{d} / \mathrm{t}$ and the process formed a variety of products and wastes including a glass-ceramic CsSr waste formed with zeolite used as an ion exchange material to recover CsSr from salt. The specific activity of this waste form is expected to be similar to or lower than the aluminosilicate waste and so costs for this case can be considered equivalent to EAS costs using a per $\mathrm{kg}(\mathrm{CsSr})$ basis. However, the electrometallurgical $\mathrm{CsSr}$ waste may have higher radioactive impurity levels (e.g., TRU), and require longer decay storage to comply with Class $\mathrm{C}$ limits or need disposal as GTCC.

In summary, the CsSr-Rb-Ba separated waste stream from UREX+ aqueous separations is salt free and can be decomposed thermally to the oxides and converted into a wide variety of waste forms and chemistries including particulate ceramics, cements, sintered ceramics, glass ceramic composites, and cast vitrified waste. The waste stream has few process additives so the CsSr waste form may be made as concentrated in $\mathrm{CsSr}$ as desired consistent with chemical, physical, and thermal waste-form properties. The Integrated Waste Management Strategy (IWMS) presently favors the CsSr vitrified waste option using existing waste storage and state-of-the-art vitrification technologies. Incorporation of $20 \% \mathrm{wt}$ $\mathrm{CsSrOx}$ in borosilicate glass has been reported and fully active samples made (Aloy 2007). Pacific Northwest National Laboratory is understood to be investigating higher incorporations, $\geq 40 \% \mathrm{wt}$. $\mathrm{CsSr}-\mathrm{Rb}-\mathrm{BaOx}$, which equates to $\geq 27 \%$ wt $\mathrm{CsSrOx}$. CsSr concentrations in commercial LWR vitrified waste are around an order of magnitude lower than the latter number so that borosilicate glass (BSG) waste container diameters may be need to be reduced from $0.4 \mathrm{~m}$ to $0.13 \mathrm{~m}$ (i.e., by factor $\sqrt{10}$ ), or $\mathrm{SNF}$ decayed stored for $>3$ CsSr half-lives (i.e., $~ 100$ years). Additionally, increasing the glass transition temperature by several hundred degrees centigrade may allow the canister diameter to be increased back to around $0.2 \mathrm{~m}$ with the same high $\mathrm{CsSr}$ incorporation. Glass formulations with higher devitrification temperatures generally require formulations with higher melting point as may be achieved by a cold crucible melter. 


\section{VITRIFICATION AND STORAGE OF LWR OXIDE HLW}

For LWR fuels, the main operating commercial separations plants in the world are UP2-800 and UP-3 at Cap La Hague and THORP at Sellafield (Rokkasho is believed to start full operation in 2008 and uses Japanese JCM vitrification technology). These French and UK plants have used French AVH vitrification technology for nearly 2 decades. Calcined fission product waste is mixed with glass frit in the ratio of around 1:3 by weight. The PUREX raffinate has low processing inerts and after calcination is mainly FP and minor actinide (MA) oxides with very low $\mathrm{U}, \mathrm{Pu}$ content, and moderate corrosion product concentrations.

Vitrification of commercial and/or defense HLW has taken place at Cap La Hague and Marcoule in France, Sellafield in the UK, Tokai in Japan, Karlsruhe in Germany, Savannah River and West Valley in U.S., Tarapur in India, Russian Federation, etc. Almost all of these facilities use air-cooled vault storage systems where waste canisters are stored in cooled thimble tubes. Most use forced air convection, at least initially, whereas one uses natural convection with forced convection as standby during early operation (IAEA 1992).

France first performed vitrification operations in the 1970s in the Ateliers Vitrification Marcoule facility (known as the AVM) and then in the late 1980s in the R7 and T7 facilities of the La Hague plant (referred to as the AVH). French vitrification technology uses a rotary calciner feeding a metallic inductively heated melter vessel, which siphons batches of vitrified waste into HLW canisters. The Marcoule vitrified waste store used HLW canisters of dimensions, $0.5 \mathrm{~m}$ diameter and $1 \mathrm{~m}$ height, for lower burnup, lower decay power gas-cooled reactor wastes. For AVM, three casts of glass (120kg each) totaling about $140 \mathrm{~L}$ are made into a single stainless steel canister. The vitrified waste store used thimble tubes (steel sleeves with base set into concrete) and stacked canisters, 10 high. The sleeves are $0.6 \mathrm{~m}$ diameter and $10 \mathrm{~m}$ height. The maximum output of the ten canisters in a sleeve is $8 \mathrm{~kW}$ (i.e., $0.8 \mathrm{~kW} /$ canister) on average, but $1 \mathrm{~kW}$ peak value. Forced convection cooling air normally flows between sleeves and canisters at velocities of $10-15 \mathrm{~m} / \mathrm{s}$ with filtration at outlet but can revert to natural convection without filtration for power failure conditions or after long storage, etc. Two vaults were built initially at Marcoule, one with 80 storage sleeves and the other with 60 storage sleeves. The maximum heat load of the whole store is 1MW. One AVM single line plant was constructed in the 1970s at Marcoule, to provide vitrification of low burnup gas reactor fuel with vitrified waste power densities of $\leq 8 \mathrm{~W} / \mathrm{L}$.

The AVH stainless steel canisters are cylindrical with overall dimensions of around $0.42 \mathrm{~m}$ diameter (17 inches) and height $1.3 \mathrm{~m}$ (52 inches). The canisters have a top flange of reduced diameter with welded closure following filling with two pours from the melter. After pouring, the canisters contain about $400 \mathrm{~kg}(150 \mathrm{~L})$ vitrified HLW and are around three-fourths filled. (In France, the residual space is filled with pucks of compacted leached fuel hulls.) Two AVH plants, designated R7 and T7 and each of three vitrification lines, were constructed in the late 1980s at La Hague, to provide vitrification of standard LWR fuel $(33 \mathrm{GW}(\mathrm{t}) . \mathrm{d} / \mathrm{t}) \mathrm{HLW}$ after 4 years of cooling. Preliminary evaluations foresaw glass center line temperatures $\leq 650^{\circ} \mathrm{C}$ and power densities of $\leq 60 \mathrm{~W} / \mathrm{L}$, which implied a maximum canister heat load of $9 \mathrm{~kW}$. Eventual design values were specified as $20 \mathrm{~W} / \mathrm{L}$ and $3 \mathrm{~kW}$, respectively.

The Sellafield waste vitrification plant was constructed with two AVH process lines, and first operated in the early 1990s. Its vitrified product store (VPS) accommodates up to 8,000 AVH canisters stacked 10 high (about $13 \mathrm{~m}$ ). Each canister (400 kg waste) typically contains vitrified waste from the recycling of $8 \mathrm{t}$ Magnox fuel or $2 \mathrm{t}$ oxide fuel (Dobson and Phillips 2006). There are 800 stainless steel storage thimble tubes into which the canisters are stacked through top plugs and seals. Each storage tube is within a rectangular compartment to guide cooling air. Decay heat is removed by natural convection cooling (Figure A-2) of the exterior of the sealed storage tubes, and due to multiple barriers and compliance with glass centerline limits and civil structural limits, no filtration of the cooling air is 
required. The Sellafield borosilicate glass formulations have waste oxide incorporations in the range 20 30 weight $\%$ with glass transformation temperature of around $550^{\circ} \mathrm{C} ; 500^{\circ} \mathrm{C}$ is taken as the glass center line temperature limit. VPS has capacity for vitrified HLW from two decades of THORP design throughput of $800 \mathrm{t}$ (oxide SNF)/year, that is 16,000 toxide SNF) equivalent.

British Nuclear Fuels plc (BNFL) commenced active commissioning of the third line at its Waste Vitrification Plant (WVP) at Sellafield in January 2002. The start of operation of the 320M UK pound $(\sim 2000$ m.v. $) \equiv$ U.S. \$485M (2000 dollars) line enables BNFL to meet its commitment to speed up the conversion of liquid HLW to borosilicate glass blocks for longer term storage. The UK regulator requires year-on-year reductions in highly active liquid waste down to buffer stocks of $200 \mathrm{~m}^{3}$ by 2015 .

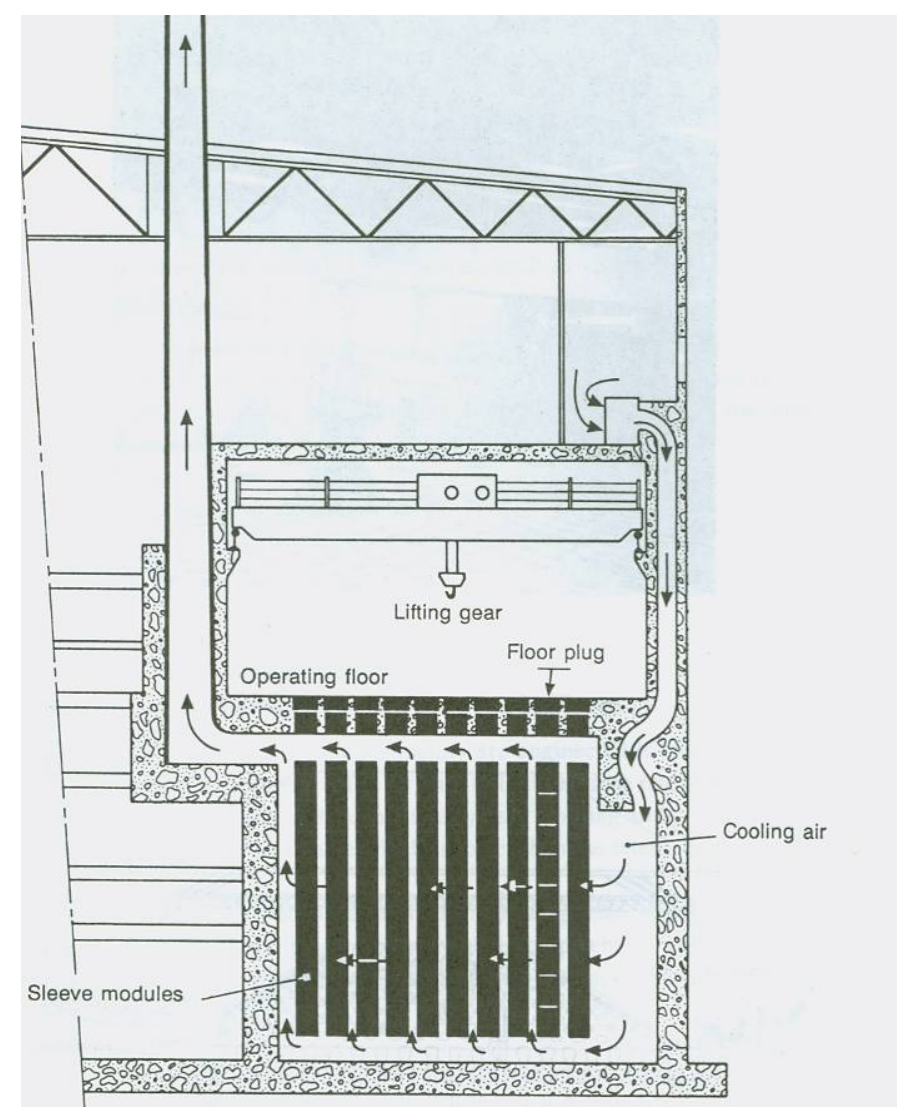

Figure A-2. WVP VPS natural convection air-cooled storage system.

The operation of WVP has led to the production of over 4,000 containers of vitrified waste to-date, which are currently stored within the VPS at Sellafield. The VPS is deemed suitable for this interim storage requirement, subject to regular maintenance and refurbishment, for at least 100 years. A proportion of the vitrified HLW will be returned to overseas customers at the appropriate time as set out in the recycling contracts. The canisters of vitrified HLW are kept in a purpose-built store (VPS) as shown in Figure A-3, which has passive cooling and a back-up forced cooling system. 


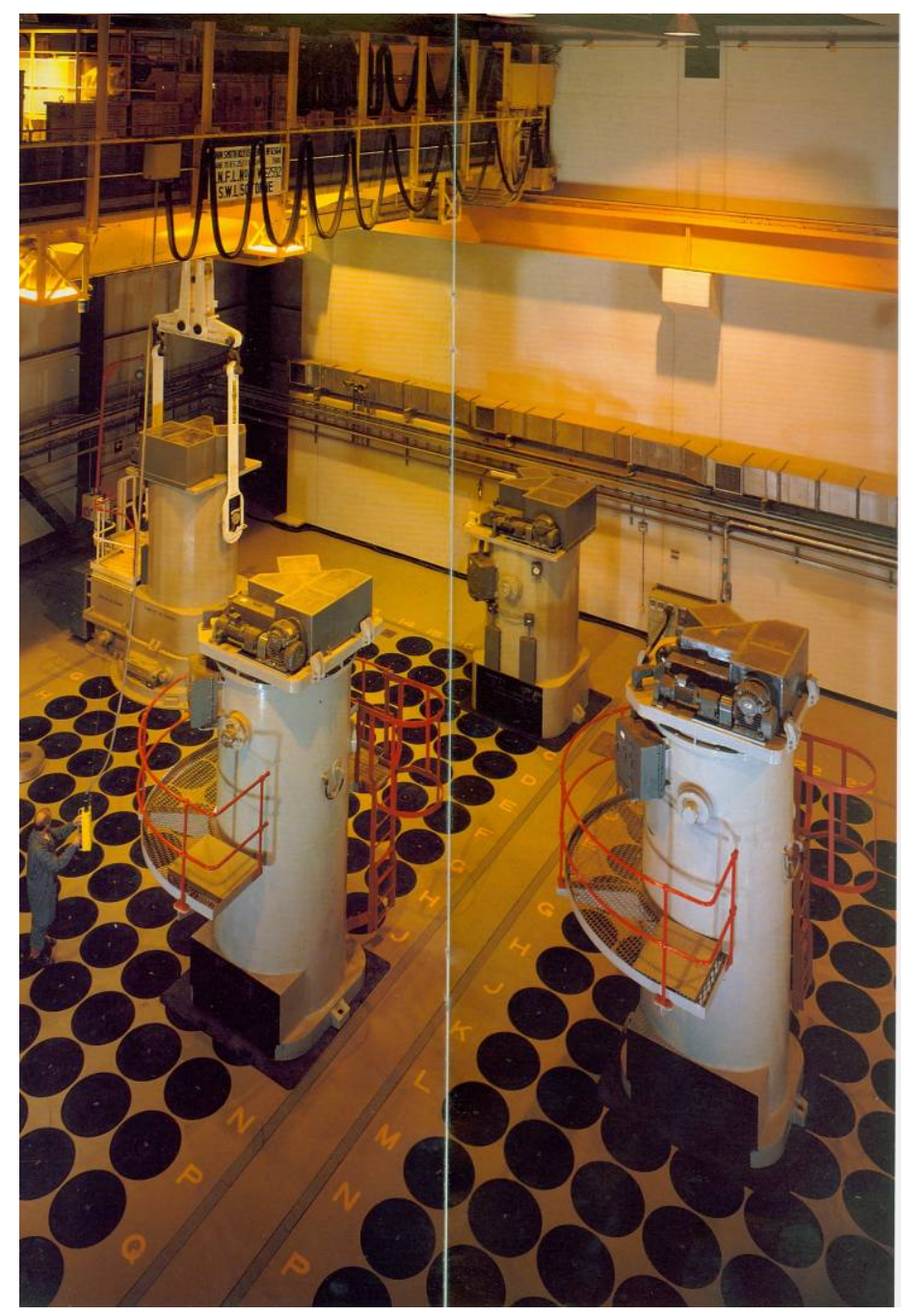

Figure A-3. WVP VPS charge face showing waste product flasks over storage channels (BNFL 1991).

The design and operation of HLW vitrification facilities has been well described for the major national nuclear programs (IAEA 1992). Following filling of stainless steel, cylindrical waste canisters with vitrified waste, various operations are used to prepare canisters for storage and ultimate disposal. Thermal conditioning of canisters to reduce heat shock and decrease glass cooling rate and fracture may be used. Tungsten Inert Gas (TIG), plasma torch or upset-resistance welding is used to seal the canisters with lids. Canister welds are normally inspected optically or by helium leak testing. Canister dimensions, weight temperature and dose rate may be determined. The exteriors of canisters are often decontaminated using high-pressure water, sand slurries, dry blasting, or electrochemical decontamination. Waste canisters must be cooled in storage to minimize devitrification and maintain store integrity.

Currently, operating and planned interim stores use air cooling of canisters. Air cooling can be achieved by conduction, or natural or forced convection. For some high-specific decay power glasses, forced cooling is combined with natural convection cooling. Canister, waste, and store characteristics for various national facilities are given in Table A-5. 
Table A-5. Canister and waste parameters for operating vitrified HLW stores.

\begin{tabular}{|c|c|c|c|c|c|}
\hline $\begin{array}{l}\text { Facility } \\
\text { Cooling }\end{array}$ & $\begin{array}{c}\text { Canister } \\
\text { I.D./height } \\
\mathrm{m} / \mathrm{m}\end{array}$ & $\begin{array}{c}\text { Glass Mass/ } \\
\text { volume, } \\
\text { kg/L }\end{array}$ & $\begin{array}{c}\text { Max activity } \\
\text { GBq } \\
\alpha / \beta\end{array}$ & $\begin{array}{c}\text { Maximum } \\
\text { canister power, } \\
\text { W }\end{array}$ & $\begin{array}{c}\text { Maximum } \\
\text { power } \\
\mathrm{W} / \mathrm{kg} \\
\end{array}$ \\
\hline AVM France & 0.49 & 360 & $3.0 \times 10^{7}$ & & \\
\hline Forced/Natural & 1.0 & 135 & $1.4 \times 10^{7}$ & 1,000 & 2.8 \\
\hline R7/T7 France & 0.42 & 400 & $1.4 \times 10^{5}$ & 2,980 peak & \\
\hline Forced/Natural & 1.34 & 150 & $2.8 \times 10^{7}$ & 2,100 average & 7.5 \\
\hline WVP-VPS UK & 0.42 & 400 & & Estimated & \\
\hline Natural & 1.34 & 150 & - & 2,000 & - \\
\hline DWPF-U.S. & 0.59 & & & & \\
\hline Forced & 3.0 & 670 & - & - & - \\
\hline TVF-Japan & 0.42 & 300 & $1.5 \times 10^{7}$ & & \\
\hline Forced & 1.0 & 110 & combined & 1,400 & 4.7 \\
\hline
\end{tabular}

BNFL WVP with lines 1 and 2 and VPS has dimensions $64 \mathrm{~m}$ long $\times 38 \mathrm{~m}$ wide $\times 40 \mathrm{~m}$ high, which gives footprint of $2,430 \mathrm{~m}^{2}$ (IAEA 1992). The capital cost is estimated as $250 \mathrm{M}$ GBP (1990 dollars) $\equiv \$ 446 \mathrm{M}$ (U.S. 1990 dollars $\left.^{\mathrm{u}}\right) \equiv 730 \mathrm{M}$ (U.S. 2008 dollars $^{\mathrm{v}}$ ). The two stores and access corridor have a footprint of around $25 \mathrm{~m} \times 40 \mathrm{~m}=1,000 \mathrm{~m}^{2}\left(10^{4} \mathrm{ft}^{2}\right)$ or $40 \%$ of WVP footprint. A pro rata capital cost for the VPS is then $\$ 292 \mathrm{M}$ (U.S. 2008 dollars) $\equiv 146 \mathrm{M}$ GBP with a capacity corresponding to $16,000 \mathrm{t}$ (LWR SNF). This corresponds to a facility square foot capital cost of $\$ 29 \mathrm{k}$. Commonly process areas have costs that are several times greater than waste storage areas. So, a value of $\$ 150 \mathrm{M}\left(\$ 15 \mathrm{k} / \mathrm{ft}^{2}\right)$ for the store may be appropriate here, and this is regarded as high although passive cooling favors lower long-term operational costs.

\section{COMPARISON WITH U.S. DOE HLW VITRIFICATION AND INTERIM STORAGE}

A valuable review and evaluation of interim storage facilities for application to Hanford Tank Waste Remediation System (TWRS) vitrified waste and Cs dry IX waste canisters was performed by Calmus (1996). He examined the four options of Standalone casks, Housing arrays, Vault storage, and Modification of existing major facilities to provide vault storage. The canister dimensions were vitrified waste: $0.61 \mathrm{~m}$ diameter $\times 3.0 \mathrm{~m}$ height $(\max 1 \mathrm{~kW})$ or $0.61 \mathrm{~m}$ diameter $\times 4.57 \mathrm{~m}$ height $(\max 1 \mathrm{~kW})$; and Cs IX waste: 0.33 diameter $\times 1.37 \mathrm{~m}$ height $(\max 1.5 \mathrm{kw})$. The design requirements included air cooling. It was concluded that forced convection with high-efficiency particulate air (HEPA) filtration would generally be needed for reuse of existing facilities but natural convection with use of indirect cooling by means of loading waste canisters into storage sleeves (thimbles) was preferred for new facilities. Standalone casks and Housing arrays were found to be substantially more expensive.

Using passive above-ground air-cooled MVDS for vitrified waste canisters, Hanford TWRS estimated overall facility capital costs based on $\$ 37,000(1996 \mathrm{mv})$ per storage tube of $\sim 5 \mathrm{~m}$ tube height (Calmus 1996). A total of 2,000 tubes of $\sim 5 \mathrm{~m}$ height then are required for 8,000 AVH type HLW

u. Measuring Worth - Exchange Rates Between the United States Dollar and Forty-one Currencies, http://www.measuringworth.com/datasets/exchangeglobal/result.php

v. Money values derived using: U.S. Army Corps of Engineers, Civil Works Construction Cost Index System (CWCCIS), Using CWBS Feature Code - 07 Power plant, Appendix A, EM 1110-2-1304, Appendix Revised September 30, 2007. 
canisters. This gives a capital cost of \$74M (1996 dollars), which equates to around \$106M (2008 dollars).

Idaho National Environmental and Engineering Laboratory (INEEL) reported a study of the Idaho Waste Vitrification Facilities Project-Vitrified Waste Interim Storage Facility (VWISF) (Aitken et al. 2001). Two scenarios were evaluated during this study. The first scenario includes individual storage tubes for the vitrified waste canisters (two canisters per tube) and a passive ventilation system. This option is called the "Hanford Option," because it is modeled after the Hanford vitrified waste storage design. The second scenario includes racks for holding the vitrified waste canisters and a mechanical ventilation system. The second option is labeled the "Savannah River Option," since it is modeled after the Savannah River Site's vitrified waste storage facility. The second option has lower total project cost, but higher life cycle cost. The costs interpolated here are for the first option. The major waste product resulting from the treatment process will be a vitrified waste glass. The glass will be placed in canisters approximately $15 \mathrm{ft}(4.5 \mathrm{~m})$ long $\times 2 \mathrm{ft}(0.6 \mathrm{~m})$ in diameter referred to as "Hanford Canisters." Under a "high" waste loading scenario in the IWVF melter, this will result in approximately 436 canisters of the treated SBW and 4,600 canisters of the treated calcine. Two canisters are stored in each Hanford thimble tube, which is equivalent to around $10 \mathrm{AVH}$ type HLW canisters. This gives a capital cost of $\sim \$ 95 \mathrm{M}$ (2001 dollars), which equates to around \$126M (2008 dollars).

\section{STORAGE OF SPENT OXIDE FUEL}

Further cost confirmation can be derived from data on storage of spent fuel. Storage of vitrified HLW is expected to not be dissimilar in cost to that of SNF since the thermal power and radiation level is similar though the mass of vitrified waste is about $80 \%$ less. Of the main alternatives of vault storage, casks, and housing arrays, the preferred option for long-term storage of large quantities of spent fuel and other heat generating wastes is dry vault storage. This concept for spent fuel was originated by Alstec and deployed in the U.S. by Foster Wheeler (Alstec 2008). There is considerable similarity between vault storage of vitrified waste and spent fuel.

Costs of dry cask interim storage of fuel are reported as about $\$ 250 \mathrm{M}$ for storage of $1,000 \mathrm{t}$ (LWR SNF) during 40 years generation and for 40 years following this (Bunn et al. 2001). Casks are licensed by the Nuclear Regulatory Commission (NRC) for 20 year operational periods. Research and development (R\&D) is underway to assess NRC's judgment is that storing fuel in dry casks would be safe for 100 years. This averages to $\$ 250 \mathrm{k} / \mathrm{t}(\mathrm{iHM})$. Costs for dry vault storage were said to be similar. For shutdown reactors with all spent fuel in dry storage the annual costs were estimated as $\$ 3-4 \mathrm{M}$, while for pool storage after reactor shutdown the annual costs were estimated as $\$ 9 \mathrm{M}$ (Bunn et al. 2001). Japanese and European SNF storage costs are in general higher than these costs.

The Modular Vault Dry Store system is used at the PAKS, Hungary (Figure A-4) and at Fort St. Vrain (Figure A-5) in the U.S. It is a passive system employing natural convection cooling. The Paks MVDS handles thermal power of up to $17 \mathrm{~kW} / \mathrm{t}$ of fuel when fully loaded compared with about 1.4 to $2 \mathrm{~kW} / \mathrm{t}$ for concrete casks. The PAKS MVDS uses a natural convection cooling system, as shown in Figure A-6. Vaults have sufficient heat removal capacity to keep fuel-cladding temperatures of 5-year-cooled PWR assemblies below $200^{\circ} \mathrm{C}$. The Fort St. Vrain MVDS was designed for a 40-year lifetime. 


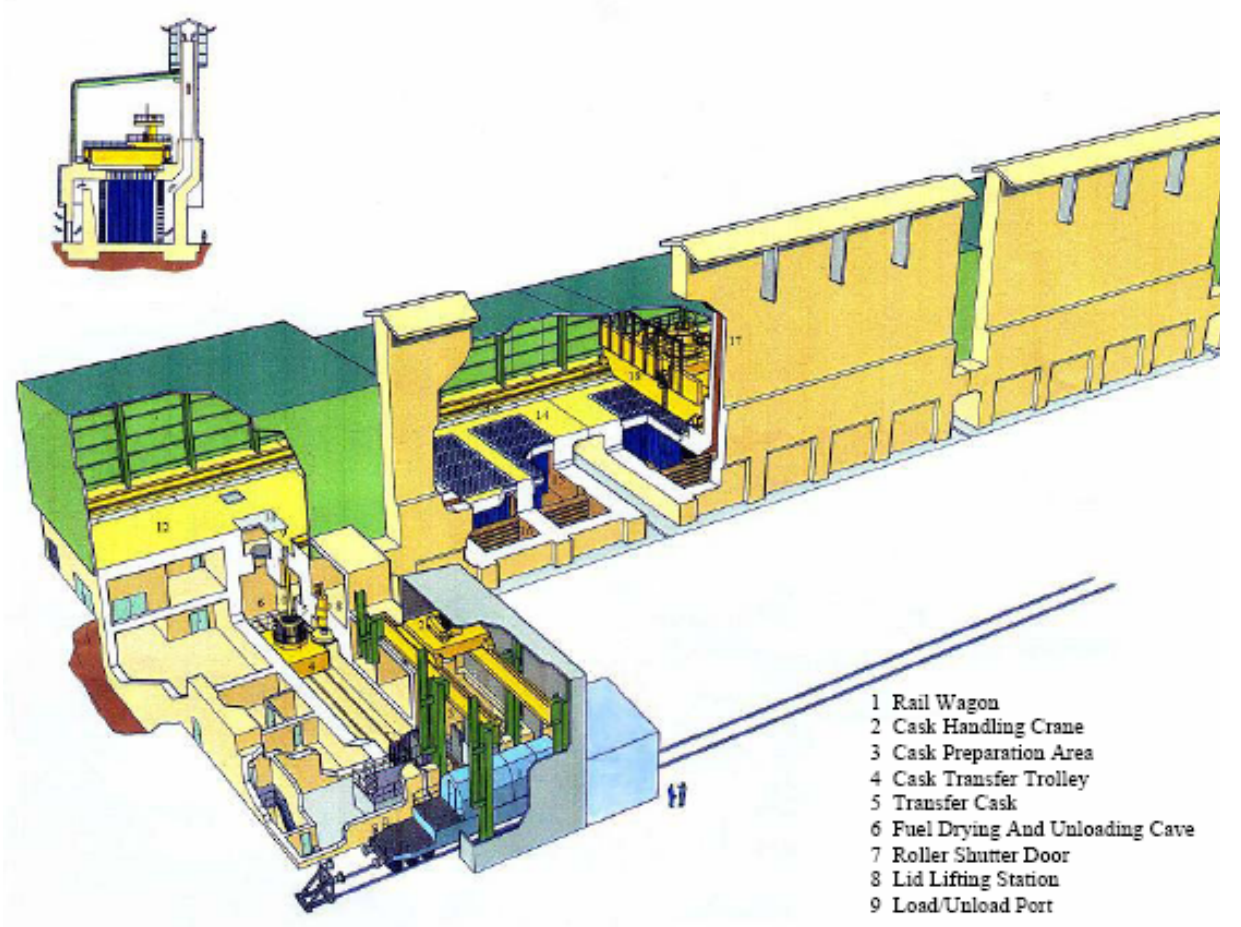

Figure A-4. Schematic of modular vault dry storage of spent fuel at PAKS, Hungary (Ordogh et al. 2004).

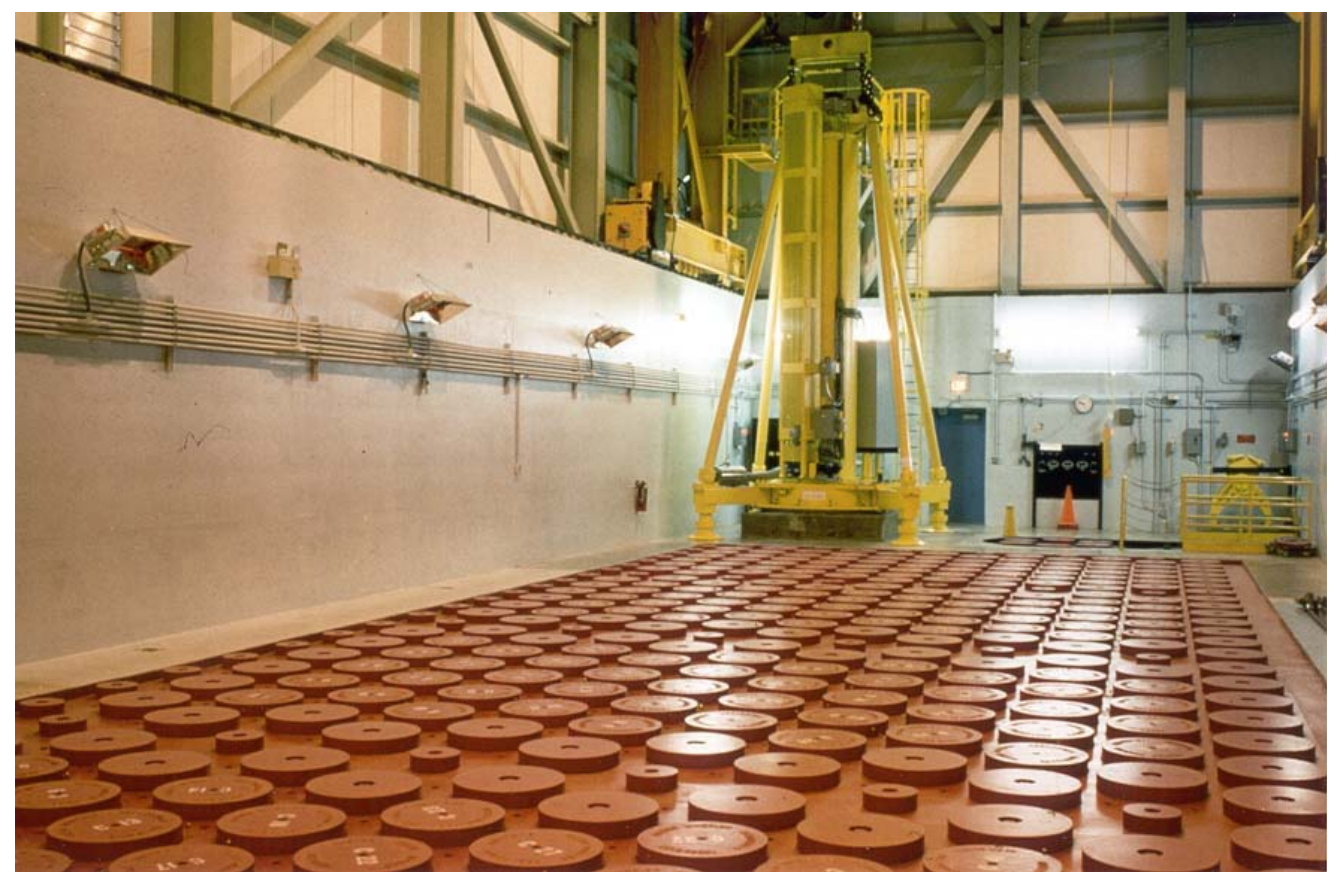

Figure A-5. Fort St. Vrain modular vault dry store (MVDS) showing charge face.

The status of MVDS facilities designed by Foster Wheeler and ALSTEC are shown in Table A-6, below: 
Table A-6. Status of MVDS facilities by Foster Wheeler and ALSTEC (Roberts et al. 2003).

\begin{tabular}{|c|c|c|c|c|}
\hline Facility & Type of Reactor/Fuel & $\begin{array}{l}\text { Dry Storage } \\
\text { Method }\end{array}$ & $\begin{array}{c}\text { Licensing Authority } \\
\text { and Date of License } \\
\text { Approval }\end{array}$ & $\begin{array}{c}\text { Date of } \\
\text { Operation }\end{array}$ \\
\hline MVDS Topical SAR & $\begin{array}{l}\text { PWR and BWR, } \\
\text { anywhere in USA }\end{array}$ & $\begin{array}{l}\text { Concrete vault - } \\
\text { MVDS }\end{array}$ & $\begin{array}{l}\text { USA NRC } \\
1988\end{array}$ & $\mathrm{n} / \mathrm{a}$ \\
\hline $\begin{array}{l}\text { Wylfa dry fuel cells } 1 \\
\text { to } 3 \\
\text { Anglesey, UK }\end{array}$ & $\begin{array}{l}\text { Gas Cooled Reactor } \\
\text { Magnox fuel }\end{array}$ & $\begin{array}{l}\text { Concrete vault, } \\
\text { tube storage }\end{array}$ & $\begin{array}{l}\text { UK NII } \\
1969\end{array}$ & 1969 \\
\hline $\begin{array}{l}\text { Wylfa dry fuel cells } 4 \\
\text { to } 5 \\
\text { Anglesey, UK }\end{array}$ & $\begin{array}{l}\text { Gas Cooled Reactor } \\
\text { Magnox fuel }\end{array}$ & $\begin{array}{l}\text { Concrete vault, } \\
\text { tube storage }\end{array}$ & $\begin{array}{l}\text { UK NII } \\
1979 \text { and } 1980\end{array}$ & $\begin{array}{l}\text { Cell 4: } 1979 \\
\text { Cell 5: } 1980\end{array}$ \\
\hline $\begin{array}{l}\text { Fort St Vrain MVDS } \\
\text { Colorado, USA }\end{array}$ & $\begin{array}{l}\text { High temperature gas } \\
\text { reactor } \\
\text { HTGR fuel blocks }\end{array}$ & $\begin{array}{l}\text { Concrete vault - } \\
\text { MVDS }\end{array}$ & $\begin{array}{l}\text { USA NRC } \\
1991\end{array}$ & 1991 \\
\hline $\begin{array}{l}\text { Paks MVDS } \\
\text { Paks, Hungary }\end{array}$ & $\begin{array}{l}\text { VVER } 440 \\
\text { VVER } 440 \text { fuel }\end{array}$ & $\begin{array}{l}\text { Concrete vault - } \\
\text { MVDS }\end{array}$ & $\begin{array}{l}\text { Hungary OAH } \\
\text { Feb } 1997\end{array}$ & $\begin{array}{l}\text { December } \\
1997\end{array}$ \\
\hline $\begin{array}{l}\text { Idaho Spent Fuel } \\
\text { Facility } \\
\text { Idaho, USA }\end{array}$ & $\begin{array}{l}\text { DOE owned fuels: } \\
\text { Peach Bottom Core } 1 \\
\text { Peach Bottom Core } 2 \\
\text { TRIGA aluminum clad } \\
\text { TRIGA stainless clad } \\
\text { Shippingport modules }\end{array}$ & $\begin{array}{l}\text { Concrete vault - } \\
\text { MVDS }\end{array}$ & $\begin{array}{l}\text { USA NRC } \\
\text { Planned } 2003\end{array}$ & Planned 2005 \\
\hline
\end{tabular}

Estimated dry storage costs from a variety of sources are given, see Table A-7 (Fairlie 2000), that provide discussion of wide differences observed. A reasonable value of $\$ 200 \mathrm{k} / \mathrm{t}(\mathrm{iHM})$ is adopted with 2008 money value.

Table A-7. Estimated dry storage costs for oxide spent fuels (Fairlie 2000).

\begin{tabular}{|c|c|}
\hline STUDY & LWR FUEL, \$k/t(iHM) \\
\hline Kf K-PAE NuclearFuel (1993b) ${ }^{a}$ & 220 \\
\hline OECD-NEA $(1994)^{\mathrm{b}}$ & 225 \\
\hline IAEA (1990) & $82-165$ \\
\hline Supko $(1995)^{\mathrm{c}}$ & $50-100$ \\
\hline Wisconsin PS C (1994) ${ }^{\mathrm{d}}$ & $35-68$ \\
\hline Ontario Hydro, (Stevens-Guille, 1994; Nash, 1997) & $15-20$ \\
\hline $\begin{array}{ll}\text { a. } & \text { Undiscounted } \\
\text { b. } & \text { Levelised fuel cycle costs } \\
\text { c. } & \text { Representative life cycle costs } \\
\text { d. } & \text { Constant } \$ \text { analysis } \\
\text { e. } & \text { Low burnup fuel. References given in Fairlie } 2000 .\end{array}$ & \\
\hline
\end{tabular}




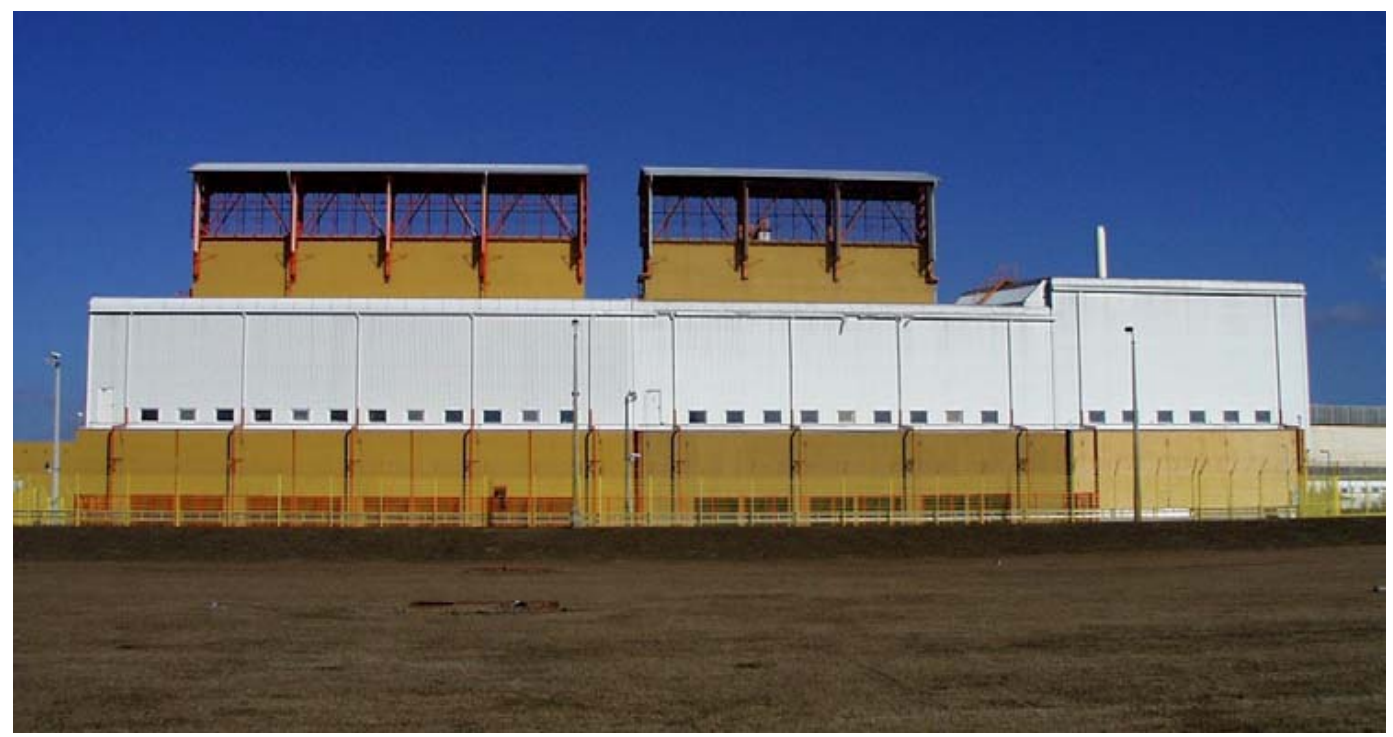

Figure A-6. Paks MVDS in 2000 showing natural convection cooling exhaust outlets (Ordogh et al. 2004).

German institutions give undiscounted estimates of about $\$ 225,000$ per tonne of LWR fuel over indeterminate periods. Relatively expensive CASTOR spent fuel casks were used in these calculations. Estimated costs of U.S. and Canadian dry storage systems are significantly lower than European systems.

\section{COST COMPARISON AND STORAGE ESTIMATE}

Sound cost estimates require the examination of relevant design parameters, existing industrial practice, and the definition of a reference design concept for immobilization and storage of CsSr waste. The reference process need not be fully optimal, but should be broadly representative to provide a conservative basis for costing. The reference process adopted here for CsSr immobilization and storage is that of vitrification to form borosilicate glass of CsSr loading in the range $2-10 \%$ by weight depending on decay time before separation and immobilization. The shortest decay period is likely to be around 4 years and this requires use of canisters of diameter $\sim 0.4 \mathrm{~m}$, (e.g., AVH type), and low CsSr loadings. A modular vault dry storage system is selected consistent with worldwide practice for long storage of heat generating wastes. CsSr waste storage costs are then taken to be the same as HLW vitrified waste storage costs on a capital cost and yearly operational expense basis. Vitrified waste is planned to be stored 50-100 years before disposal whereas CsSr waste is planned to be stored 300 years prior disposal.

The current use of commercial MVDS facilities for storage of vitrified HLW and spent fuels for planned periods of $\leq 100$ years gives confidence in this approach. However, the project and operational costs of these are often commercially protected. Estimates have been gathered where possible and values also taken from cost studies for planned facilities, which may not be constructed. Table A-8 summarizes cost data given above. 
Table A-8. Summary cost data for managed decay storage.

\begin{tabular}{|c|c|c|c|}
\hline Facility & $\begin{array}{l}\text { Est. Capital Cost, } \\
\text { \$M }\end{array}$ & $\begin{array}{l}\text { Est. Operating } \\
\text { Cost },{ }^{1} \$ \mathrm{M} / \mathrm{yr}\end{array}$ & Comments \\
\hline \multicolumn{4}{|l|}{ Vitrified Waste, MVDS } \\
\hline WVP-VPS (1990) & 150 & $4^{b}$ & $\begin{array}{l}8,000 \text { AVH canisters } \equiv \\
16,000 \mathrm{t}\left(\mathrm{SNF}^{\mathrm{c}}\right)\end{array}$ \\
\hline Hanford TWRS (1996) & 106 & $4^{b}$ & $\begin{array}{l}8,000 \text { AVH canisters } \equiv \\
16,000 \mathrm{t}\left(\mathrm{SNF}^{\mathrm{c}}\right)\end{array}$ \\
\hline INEEL VWISF (2001) & 126 & $4^{b}$ & $\begin{array}{l}8,000 \text { AVH canisters } \equiv \\
16,000 \mathrm{t}\left(\mathrm{SNF}^{\mathrm{c}}\right)\end{array}$ \\
\hline Oxide SNF, MVDS & Est. Life Cycle Cost & & \\
\hline Bunn et al. (2001) & $\$ 250 \mathrm{M}$ & $3-4$ & $\begin{array}{l}1,000 \mathrm{t}\left(\mathrm{SNF}^{\mathrm{c}}\right) \text { stored, } \mathrm{LWR} \\
\text { operation }+40 \text { years }\end{array}$ \\
\hline Fairlie (2000) & $\sim \$ 200 \mathrm{k} / \mathrm{t}\left(\mathrm{SNF}^{\mathrm{c}}\right)$ & - & \\
\hline \multicolumn{4}{|c|}{$\begin{array}{l}\text { a. } 2008 \text { money values - Derived using US Army Corps of Engineers, Civil Works Construction Cost Index System } \\
\text { (CWCCIS), Using CWBS Feature Code - } 07 \text { Power plant, Appendix A, EM 1110-2-1304, Appendix Revised September } \\
30,2007 \text {. } \\
\text { b. Operating cost based on team of } 20 \text { for } 24-\text { hour coverage at fully loaded cost of } \$ 200 \mathrm{k} / \mathrm{staff} \text {. } \\
\text { c. LWR oxide spent nuclear fuel. }\end{array}$} \\
\hline
\end{tabular}

The estimated capital costs of the vitrified waste stores are seen to be similar with possibly U.S. practice lower cost than UK.

Two sources provide similar values for annual operating costs for dry storage of vitrified waste and spent fuel. Due to the unusually long period of storage (300 years), the undiscounted total operating costs outweigh capital cost by around a factor of $\sim 10$.

Taking the operational expenses of a single module of vitrified $\mathrm{CsSr}$ waste air-cooled store, capacity of $64,000 \mathrm{~kg}(\mathrm{CsSr}$ elemental) $\equiv 16,000 \mathrm{t}$ (oxide $\mathrm{SNF}$ ), as $\$ 4 \mathrm{M} /$ year and the operational period as 300 years gives an operational expense of $\$ 1.2 \mathrm{~B}$. The capital cost of the store is estimated as $\$ 150 \mathrm{M}$. The cost of decommissioning of the store is taken as $10 \%$ of capital cost plus 5 years operational cost.

CsSr Waste Storage Life Cycle Cost

$=\$ 4 \mathrm{M} \times 300+\$ 150 \mathrm{M}+(5 \times \$ 4 \mathrm{M}+\$ 15 \mathrm{M})=\$ 1,385 \mathrm{M}$

CsSr Storage Unit Cost $=1,385 \mathrm{M} / 64,000=\$ 21,600 / \mathrm{kg}(\mathrm{CsSr}$ elemental $)$

The nominal cost of storage of $\mathrm{CsSr}$ is judged to be $\sim \$ 22.5 \mathrm{~K} / \mathrm{kg}(\mathrm{CsSr}$ elemental).

The low range cost is judged by halving the capital cost, discounting (starting at 100 years) the 2 nd and 3rd centuries of operation to about $30 \%$ of their nominal value and halving decommissioning costs. This gives an unit value of around $\$ 10 \mathrm{~K} / \mathrm{kg}(\mathrm{CsSr}$ elemental). Other opportunities include alternative glass formulations with high-temperature limits that enable increased $\mathrm{CsSr}$ concentrations and decreased numbers of waste canisters. Cold crucible induction melters are under development for HLW vitrification, which may supersede the two main industrial types, joule ceramic and hot wall induction, and provide higher temperature operation giving access to higher melting glass formulations with higher glass transition temperatures.

Risks for higher unit storage costs include requirements to increase operational team size, decrease the module storage capacity for each team, and/or increase of the required storage period to account for minor radionuclide decay. The high range cost is estimated as $60 \%$ higher than nominal (i.e., $\$ 35 \mathrm{k} / \mathrm{kg}([\mathrm{s}-\mathrm{Sr}$ elemental] $)$. 


\section{CONCLUSIONS}

- This module determined a cost range for 300-year decay storage of immobilized, heat-generating, mixed cesium-strontium ( $\mathrm{CsSr}-\mathrm{BaRb}$ ) waste such as generated by the AFCI UREX+ based fuel cycle and a reference waste immobilization and storage process defined here. The period of storage may allow subsequent shallow disposal as LLW.

- The life cycle cost estimates for storage of $\mathrm{CsSr}$ waste are $\$ 22.5 \mathrm{~K} / \mathrm{kg}(\mathrm{CsSr})$ nominal, $\$ 10 \mathrm{~K} / \mathrm{kg}(\mathrm{CsSr})$ low range, and $\$ 35 \mathrm{~K} / \mathrm{kg}(\mathrm{CsSr})$ high range. The estimates use 2008 money values and are based on mass of cesium and strontium in elemental form (i.e., do not include $\mathrm{Rb}, \mathrm{Ba}$, oxide, or other glass or ceramic forming elements).

- Due to the uniquely long lifecycle period, 2350 years for construction through decommissioning, of the $\mathrm{CsSr}$ storage facility (normally nuclear facilities have a lifecycle of $<100$ ) the summed operational costs significantly exceed the initial capital cost (e.g., by one order of magnitude). Under these circumstances, discounting of all costs following 100 years of operation may be more appropriate for decision analysis and the low range value includes this effect to some degree.

- The operational expenditure cost estimates are based on facility design that is operationally conservative (i.e., assumes full 24 hour, 365 day per year) manning for the full period of storage even when decay power has reduced substantially, and are technically conservative, appropriate to the long period of storage, through adoption of modular vault dry stores, passive cooling, multiple containment barriers, and refractory waste form (borosilicate glass) of relatively low specific decay power. New melter types and higher transition temperature glasses may enable higher CsSr-Rb-Ba loadings, which can reduce container numbers and storage requirements.

- Due to the unusual cost structure, optimization of facility design in relation to progressive reduction of staffing consistent with decreasing hazards with eventual remote monitoring and control and periodic inspection may yield significant reduction in undiscounted cost. This is analogous to "Safestor" designs for radioactive decay of nuclear reactor structures prior to decommissioning. Further cost and design studies are needed to substantiate potential cost reductions.

\section{References for Appendix A}

Aitken, S. et al., 2001, "Idaho Waste Vitrification Facilities Project Vitrified Waste Interim Storage Facility,” INEEL/EXT-01-01217, September 2001.

Aloy, A.S. et al., 2007, "Development of Glass Matrix for Immobilization of CsSr Fraction after HLW Partitioning," Proc. Global 2007 International Conference, Boise, ID, U.S., pp. 1017-1021, 2007.

Alstec, 2008, Fort St. Vrain Modular Vault Dry Storage (MVDS), USA and PAKS MVDS, http://www.alstec.com/Main/ALSTECGroup/Nuclear/Casestudies/FortStVrainModularVaultDryStore USA/tabid/149/Default.aspx http://www.alstec.com/Main/ALSTECGroup/Nuclear/Casestudies/PaksModularVaultDryStore/tabid/ 147/Default.aspx, 2008.

Alvarez, R., 2007, Radioactive Wastes and the Global Nuclear Energy Partnership, pp. 1-23, Institute for Policy Studies, Washington D.C., 2007.

Balmer, M. L. et al., 2000, New Silicotitanate Waste Forms: Development and Characterization, Environmental Management Science Program, Project Number: 60345, pp. 1.19-1.28, 2000.

Benedict, M. et al., 1981, Nuclear Chemical Engineering, $2^{\text {nd }}$ Ed, pp. 580-590, McGraw-Hill, 1981.

Bergelson, B. R. et al., 2005, Radiation Protection Dosimetry, Vol. 115, No. 1-4, pp. 445-447, 2005.

BNFL, 1991, The Vitrification Plant, British Nuclear Fuels plc, 1991. 
Bresee, J., et al., 2007, “Advanced Reactor, Fuel Cycle, and Energy Products," DOE Workshop for Universities, Hilton Hotel, Gaithersburg, MD, March 20, 2007.

Bunn, M., et al., 2001, "Interim Storage of Spent Nuclear Fuel, A Safe, Flexible and Cost-Effective Near-Term Approach to Spent Fuel Management," Harvard University and University of Tokyo, June 2001 .

Calmus, R. B., 1996, TWRS HLW Interim Storage Facility Search and Evaluation, WHC-SD-WM-ES-374, Rev. 0, May 1996.

Carter, M. L., et al., 2007, "HIPed Tailored Ceramic Waste Forms for the Immobilization of Cs, Sr and Tc,” Proc. Global 2007 International Conference, Boise, Idaho, U.S., pp. 1022-1028, September 2007.

Dobson, A. J. and C. Phillips, 2006, "High Level Waste Processing in the UK-Hard Won Experience that can benefit U.S. Nuclear Cleanup Work," WM'06 Conference, Tucson, Arizona, February-26-March 2, 2006.

El-Kamash, A. M., et al., 2006, Immobilization of Cesium and Strontium radionuclides in zeolite-cement blends, J. Hazardous Materials, Vol. 136, Issue 2, August 2006.

Fairlie, I., 2000, "Dry Storage of Nuclear Fuel: The Safer Alternative to Reprocessing," Response to Cogema Dossiers to the La Hague Public Inquiry, London, UK, May 2000.

Gombert, D., et al., 2007, "Global Nuclear Energy Partnership Integrated Waste Management Strategy Technical and Policy Issues for Implementation,” INL/EXT-07-12620, May 2007.

Hebditch, D., et al., 2007, "Issues for Conceptual Design of AFCF and CFTC LWR Spent Fuel Separations influencing Next-Generation Aqueous Fuel Reprocessing," Proc. Global 2007 International Conference, Boise, Idaho, USA, September 2007.

IAEA, 1992, Design and Operation of High Level Waste Vitrification and Storage Facilities, Technical Report Series, No. 339, Vienna, 1992.

Japan Nuclear Fuel Limited, 2008, http://www.jnfl.co.jp/english/wastestorage.html, Vitrified Waste Store, 2008.

Kaminski M. D. and C. J. Merz, 2007, Personal communication, Argonne National Laboratory, Illinois, 2007.

Law, J. D., et al., 2004, "Development of a Cobalt Dicarbollide/Polyethylene Glycol Solvent Extraction Process for Separation of Cesium and Strontium to Support Advanced Aqueous Reprocessing," Nuclear Technology, 147(2), pp. 284-290, 2004.

Law, J. D., et al., 2006, "Development of Cesium and Strontium Separation and Immobilization Technologies in Support of an Advanced Nuclear Fuel Cycle," WM'06 Conference, Tucson, Arizona, February 26-March 2, 2006.

Law, J. D., et al., 2007, "Flowsheet Testing of the Fission Product Extraction Process as Part of Advanced Aqueous Reprocessing," INL/CON-07-12827, PREPRINT 2007ANS National Meeting, June 2007.

Luca, V., et al., 2006, "Tungsten bronze-based nuclear waste form ceramics. Part 1 Conversion of microporous tungstates to leach resistant ceramics," J. Nuclear Materials, Vol. 358, pp. 139-150, 2006.

National Academy of Sciences, 2003, "Improving the Scientific Basis for Managing DOE's Excess Nuclear Materials and Spent Nuclear Fuel," Chapter 5, p. 52, 2003.

OECD-NEA, 2006, "The Roles of Storage in the Management of Long-lived Radioactive Waste, Practices and Potentialities in OECD Countries," pp. 1-64, NEA Report No. 6043, 2006. 
Ordogh, M., et al., 2004, "Operational Experiences and Future Plans for Hungarian Spent Fuel Storage," WM'04 Conference, Paper WM-4232, Tucson, Arizona, USA, March 2004.

Ortega, L. H. and S. M. McDeavitt, 2007, "Precursors for the Immobilization of Radioactive Cesium and Strontium from Spent Nuclear Fuel,” Global 2007, pp. 1385-88, Boise, Idaho, U.S., 2007.

Roberts, R., et al., 2003, “The Idaho Spent Fuel Project Update-January 2003,” WM'03 Conference, Tucson, Arizona, February 23-27, 2003.

Streatfield, R. E. et al., 2006, "Gas Generation in Radioactive Wastes - MAGGAS Predictive Life Cycle Model," Waste Management WM'06 Conference, Tucson, Arizona, Paper \#6241, February 26-March 2, 2006.

Vandegrift, G. F. et al., 2004, "Designing and Demonstration of the UREX+ Process Using Spent Nuclear Fuel," ATALANTE 2004, International Conference on Advances for Future Nuclear Fuel Cycles, Nimes, France, June 21-24, 2004.

Wigeland, R. A. et al., 2006, "Separations and Transmutation Criteria to Improve Utilization of a Geologic Repository,” Nuclear Technology, Vol. 154, pp. 95-106, April 2006. 
Appendix B

Recycled Product Storage Costs 


\section{Appendix B}

\section{Recycled Product Storage Costs}

\section{BASIC INFORMATION}

Recycled actinide products, actual and proposed, for potential storage include:

1. Recycled uranium oxide

2. Plutonium oxide

3. Blended uranium and plutonium oxides

4. Blended uranium, plutonium, and minor actinide $(\mathrm{Np}, \mathrm{Am}$, and $\mathrm{Cm})$ oxides

5. Selected blends of $\mathrm{U}, \mathrm{Pu}, \mathrm{Np}, \mathrm{Am}$, and $\mathrm{Cm}$ oxides as delivered as by-products from the various proposed UREX+ flow-sheets

6. Curium oxide.

From recycling of thermal oxide fuel, the first two, $\mathrm{UO}_{3}$ and $\mathrm{PuO}_{2}$ storage, are routinely performed by commercial industry and the third is starting at the Rokkasho recycling plant. The first five options are generally for buffer storage purposes while the sixth is more likely for decay purposes. Plant capital and operating costs are known in principle, but may be commercially restricted information.

The recycled uranium from LWR fuels is often low enriched, $\sim 1 \% \mathrm{U}-235$, and has higher U-232 concentration than natural uranium. The $\beta-\gamma$ dose in LWR recycled uranium, due to daughters of Th- 228 by $\alpha$ decay of U-232, increases by several orders of magnitude in several years' storage. Commercial recycling plants use mainly automated remote equipment for uranium finishing (formation of dry solid oxide product), sampling, metering to drums, closure, decontamination, transport, and storage. This equipment is normally maintained, following inventory removal, using contact maintenance. This modern approach is consistent with conventional industrial automation, and dose and toxic inhalation/ingestion minimization. The heat generation rate of a filled drum is low.

Plutonium separated from LWR fuels in commercial oxide reprocessing plants is purified by solvent extraction in pulse columns in cells and finished to solids using remotely operated plant in gloveboxes with contact maintenance. It is sampled, metered $(\sim 4 \mathrm{~kg} \mathrm{Pu})$ to canisters, and the canister seals are remotely welded and contaminated ends cut off (by laser for BNFL). Again, gloveboxes are used with contact maintenance. The higher burnups of LWR fuel give higher decay heats and penetrating dose from $\mathrm{Pu}$ products with isotopic spectrum of increased higher radionuclide atomic masses. These necessitate an all metal storage canister and an automated loading system for the Pu store. Criticality safety and package cooling require engineered features. At BNFL Sellafield, the loading/unloading system uses a trolley propelled by chain along a rail with lifting action for package deposition or retrieval, see Figure B-1. A second trolley provides equipment for in situ inspections of packages. The degree of automation appears quite basic, but is part of the criticality safety case. A cable is used as necessary for retrieval of a failed trolley. 


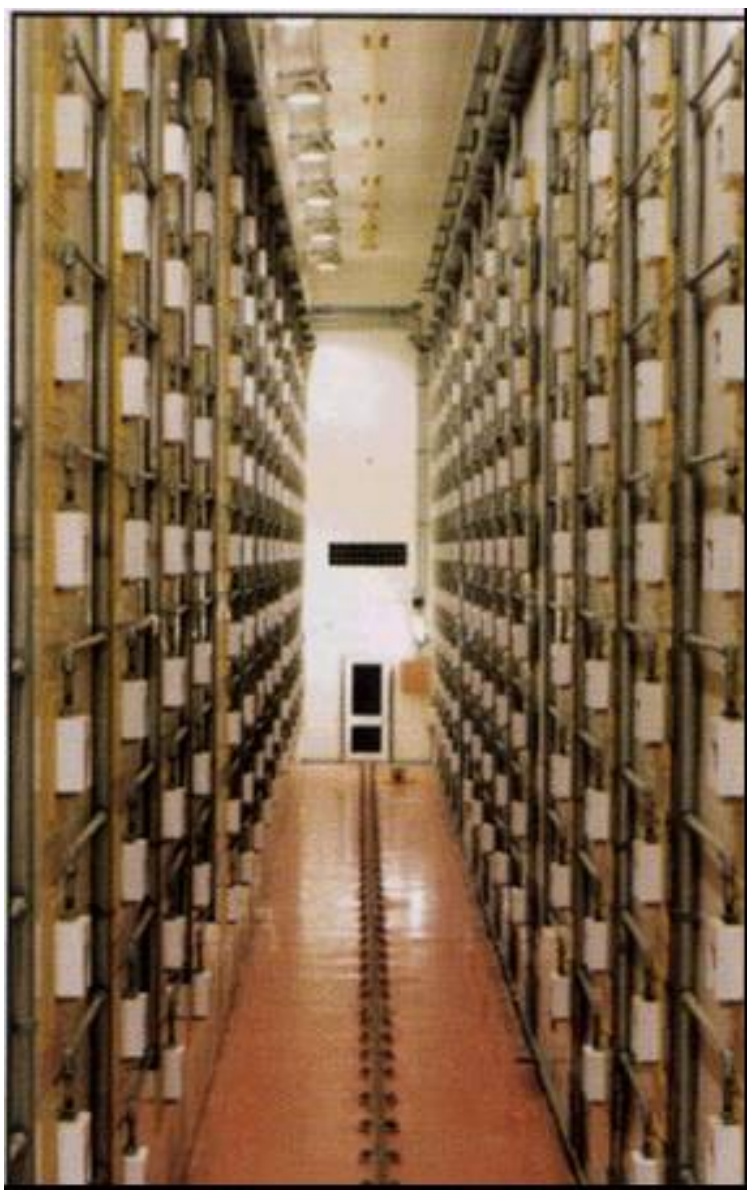

Figure B-1. Remotely operated store for Pure $\mathrm{PuO}_{2}$ in welded stainless steel canisters at BNFL Sellafield.

Mixed U and Pu product (MOx blend) (i.e., Category III) as used by Rokkasho, can be stored under similar conditions to $\mathrm{Pu}$ oxide storage. Costs per $\mathrm{kg}(\mathrm{Pu})$ may be greater due to the $\mathrm{U}$ diluent essentially increasing the number of canisters and storage positions needed. A conservative approach to design would be to retain the same size canisters for U-Pu oxide product as for $\mathrm{PuO}_{2}$ product $(4.4 \mathrm{~kg} / \mathrm{can})$ as a form of geometric limitation (take no safety credit for $U$ dilution). It seems possible that a middle course would be taken by the Japanese to increase the canister volume equivalent to $6.6 \mathrm{~kg}$ product and combine with administrative control. This would give a $30 \%$ storage capacity increase relative to pure $\mathrm{PuO}_{2}$ product.

The last three categories, IV-VI, are proposed for industrial deployment under AFCI and have much higher penetrating dose rates and decay heats than $\mathrm{Pu}$. The whole of the UREX+ flowsheet operation is likely to be deployed within fully remotely maintained hot-cells. Finishing of the actinide product, sampling, metering, canister welding, checking, and swabbing will be performed remotely with full remote maintenance of equipment. The actinide product (e.g., blended $\mathrm{U}, \mathrm{Pu}, \mathrm{Np}, \mathrm{Am}$, and $\mathrm{Cm}$ oxides) has higher decay heat, penetrating radiation, and still with high-fissile content. The FO-EAS proposed U content is $67 \mathrm{wt} . \%$ and the higher actinide content $33 \%$ by weight. The quantity of TRU is specified as $4.4 \mathrm{~kg}$ giving a product mass per canister of $13.2 \mathrm{~kg}$. Criticality safety would then depend on administrative control. Assuming a more conservative approach of $9 \mathrm{~kg}$ per canister would give a $50 \%$ increase of storage capacity relative to pure $\mathrm{PuO}_{2}$ product. 
Table B-1. Judged cost increase factor.

\begin{tabular}{|l|c|c|c|}
\hline \multicolumn{1}{|c|}{ Stored Material } & $\begin{array}{c}\text { Relative Factor- } \\
\text { Capacity Needed }\end{array}$ & $\begin{array}{c}\text { Relative Factor-Heat, } \\
\text { Activity, Dose }\end{array}$ & $\begin{array}{c}\text { Store Cost Factor- } \\
\text { c.f. } \mathrm{kg}(\mathrm{Pu})\end{array}$ \\
\hline $\mathrm{UO}_{3}$ drums & $1(100 \mathrm{~kg})$ & 1 & 1 \\
\hline $\mathrm{PuO}_{2}$ canisters & $1(4.4 \mathrm{~kg})$ & 1 & 1 \\
\hline $\mathrm{UO}_{3}-\mathrm{PuO}_{2}(50 \%)$ & 1.3 & 1 & 1.3 \\
\hline $\mathrm{UO}_{3}-\mathrm{TRUO}_{2}(33 \%)$ & 1.5 & 1.3 & 2 \\
\hline
\end{tabular}

The cost factor evaluation shown in Table B-1 is based on the store receiving intact and sealed canisters, returning defective ones, and eventually exporting intact cans to downstream plants.

\section{WORLDWIDE COMMERCIAL PU STORAGE CHARGES COMPARED TO U.S. COST DATA}

\section{Converting OECD-NEA Unit Storage Charge to Capital and Operational Costs}

The OECD-NEA report, "The Economics of the Nuclear Fuel Cycle," Section 4.3.2.6, Plutonium Storage, p. 40, 1994, estimates that the costs for Pu storage varies widely between countries and are usually taken to be in the region of $\$ 1$ to $\$ 2$ per gram (1994 dollars) of total Pu per year. This forms an essentially linear capacity versus cost correlation. OECD-NEA also states, "Both BNFL and COGEMA include the cost of short-term storage as a minor component of the overall recycling price, but some countries requiring longer-term storage are incurring additional prices of this order." As a price, this does include the reprocessor's profit element for the service element.

As seen in Module E3 and as expected, operational and maintenance costs for loading/unloading may differ considerably from those for long-term storage. Estimates of operational costs of $10 \%$ of capital per year during buffer storage with steady filling/emptying and 5\% of capital per year during long-term storage, where $\mathrm{Pu}$ inventories are retained in full stores pending potential future recycling, seem reasonable. A commercial PUREX plant with LWR oxide SNF throughput of $800 \mathrm{t}(\mathrm{HM}) / \mathrm{year}$ has a plutonium arising rate of about $1 \%$ of initial heavy metal input to reactor, which equates to 8,000 $\mathrm{kg}(\mathrm{Pu}) /$ year or around $1,800 \mathrm{PuO}_{2}$ canisters/year ( $\sim 6$ canisters/day). For a steady state buffer store of $50 \mathrm{t}(\mathrm{Pu})$ capacity servicing a typical PUREX oxide recycling plant, this then gives a mean residence time of 6 years, which would not be unreasonable for a developed nuclear fuel cycle.

For example, taking the upper range OECD-NEA overall estimate (1994 dollars) of $\$ 2,000 / \mathrm{kg}(\mathrm{Pu})$ year based on a $50 \mathrm{t}(\mathrm{Pu})$ store operated for 50 years.

Overall charge of $2,000 \$ \times 50,000 \mathrm{~kg}(\mathrm{Pu}) \times 50 \mathrm{year}=\$ 5.0 \mathrm{~B}(1994$ dollars $)$

Where $50,000 \mathrm{~kg}(\mathrm{Pu})$ at $4.5 \mathrm{~kg}(\mathrm{Pu}) /$ container $=11,110$ containers.

The period of steady loading/unloading and buffer storage is taken as 50 years at $10 \%$ of capital/year $\equiv$ $500 \%$ total

This gives total operating and capital costs of $600 \%$ of capital only (i.e., $6.0 \times$ Capital cost $=\$ 5.0 \mathrm{~B}$ $\rightarrow$ Capital cost $=\$ 5.0 \mathrm{~B} / 6.0=\$ 833 \mathrm{M}[1994$ dollars $] \equiv \mathrm{S} 1.2 \mathrm{~B}[2007$ dollars $]$ ).

This equates to an operational expenditure cost of about $\$ 83 \mathrm{M} /$ year (1994 dollars) $\equiv \$ 121 \mathrm{M}$ (2007 dollars) $\equiv \sim 600$ overall staffing (U.S. unit cost), which appears rather high even for continuous staffing including physical security. 


\section{Cost Estimates derived from CSF as a Representative US Pu Store Design}

A linear capital cost correlation for two U.S. plutonium oxide storage facilities with capacities of 9 and $45 \mathrm{t}(\mathrm{Pu})$ is provided in the "AFC Cost Basis" report, March 2008, page E3-4, as follows:

Cost $\$ \mathrm{M}(2007)=240.6+8.7417 \times[$ storage capacity, $\mathrm{t}(\mathrm{Pu})]$

This indicates high fixed cost and moderate consistency of costing data, but neither of the designs are considered to be state-of-the-art.

The larger of the two U.S. plutonium stores was the Consolidated Storage Facility (CSF) with capacity of $45 \mathrm{t}(\mathrm{Pu})$ and for this study capital and operating costs were linearly up-rated to $50 \mathrm{t}(\mathrm{Pu})$ to provide a good comparison with the OECD-NEA store costs, as a reasonable scale example. The CSF design was completed by SRS (it is believed to conceptual stage only) in 2001 and the cost value considered rough order of magnitude (ROM). This store was not constructed. The capital cost of the U.S. CSF was given as $\$ 600 \mathrm{M}$ with annual operating cost of $\$ 75 \mathrm{M}$ (13\% of capital/year) with store capacity of $45 \mathrm{t}(\mathrm{Pu})$, which gives a capital and operational cost of $\$ 4.35 \mathrm{~B}$ over 50 years. These costs are in 2005 dollars and are taken from the "AFC Cost Basis" report, INL/EXT-07-12107, March 2008, page E3-5/6.

The up-rated capital cost is $\$ 749 \mathrm{M}$ (2007 dollars) and the up-rated operational cost is $\$ 93.6 \mathrm{M}(2007$ dollars) $\equiv \sim 465$ overall staffing (U.S. unit cost), which appears more reasonable for continuous staffing including physical security. This gives an overall project and operational cost of \$5.43B (2007 dollars).

\section{Comparing OECD-NEA and CSF Cost Estimates}

The cost comparisons for the two $50 \mathrm{t}(\mathrm{Pu})$ stores are shown in Table $\mathrm{B}-2$.

Table B-2. Capital, total, and unit charges (50 t capacity store).

\begin{tabular}{|c|c|c|c|}
\hline $\mathrm{Pu}$ Store Cost Basis & $\begin{array}{c}\text { Capital Cost, } \\
\text { \$B }\end{array}$ & $\begin{array}{c}\text { Overall Cost, }{ }^{\mathrm{a}} \\
\left.\text { \$B (2007 dollars }{ }^{\mathrm{b}}\right)\end{array}$ & $\begin{array}{l}\text { Unit Charge, } \\
\$ / \mathrm{g}(\mathrm{Pu}) \text {.Year }\end{array}$ \\
\hline $\begin{array}{c}\text { OECD-NEA, } 1994^{\mathrm{c}} \\
50 \text { t capacity }\end{array}$ & $\begin{array}{l}0.83(1994 \text { dollars }) \\
\equiv 1.2(2007 \text { dollars })\end{array}$ & 7.4 & $\begin{array}{c}2.0(1994 \text { dollars }) \\
\equiv 2.9(2007 \text { dollars })\end{array}$ \\
\hline $\begin{array}{c}\text { AFC Cost Basis, } 2008^{\mathrm{d}} \\
\text { CSF uprated to } 50 \mathrm{t}\end{array}$ & $\begin{array}{c}0.67 \text { (2005 dollars }) \\
\equiv 0.75 \text { (2007 dollars) }\end{array}$ & 5.4 & 2.1 (2007 dollars) \\
\hline
\end{tabular}

a. Capital and operational expenditure (decommissioning not explicitly included)

b. Money values derived using: U.S. Army Corps of Engineers, Civil Works Construction Cost Index System (CWCCIS), Using CWBS Feature Code - 07 Power plant, Appendix A, EM 1110-2-1304, Appendix Revised September 30, 2007. \$1 (1994 dollars) $\equiv \$ 1.31$ (2005 dollars) $\equiv \$ 1.47$ (2007 dollars).

c. OECD-NEA, "The Economics of the Nuclear Fuel Cycle," Section 4.3.2.6, Plutonium storage, 1994, using overall cost value of \$2 (1994 dollars)/g(Pu).year.

d. AFC Cost Basis, March 2008, page E3-5/6. Capital cost of U.S. Consolidated Storage Facility (CSF) of \$600M with annual operating cost of $\$ 75 \mathrm{M}$ with store capacity of $45 \mathrm{t}(\mathrm{Pu})$. Costs in 2005 dollars.

For a $50 \mathrm{t}(\mathrm{Pu})$ capacity store, the unit storage charge range given by OECD-NEA of $\$ 1-2 \mathrm{~g}(\mathrm{Pu}) /$ year (1994 dollars) $\equiv \$ 1.5-2.9 \mathrm{~g}(\mathrm{Pu}) /$ year (2007 dollars) implies a store capital and operating cost range of \$3.7-7.4B (2007 m.v) with reasonable assumptions for operating costs over 50 years. At the same capacity, the SRS design for the US consolidated storage facility (CSF) for plutonium oxide has a store capital and operating cost estimate of \$5.4B (2007 dollars) over the same period. The CSF cost estimate is seen be to well within the estimated OECD-NEA price range. OECD-NEA is using commercial industry data, which may be more broadly based from worldwide nuclear operators. Also, OECD-NEA does 
comment on the wide spread of costs. However, it is considered that design requirements, particularly relating to safeguards, physical security, and non-proliferation aspects for storage of fissile material, have increased since the early 2000s and are likely do so further. The nominal cost value for storage of $\mathrm{PuO}_{2}$ is therefore judged to lie above values established by OECD-NEA in 1994 for stores constructed in the 1980-1990s period.

\section{CONCLUSIONS}

The following cost values are proposed:

- Nominal costs are estimated at $\$ 5,000 / \mathrm{kg} \mathrm{Pu}$ (costs based on the elemental composition of $\mathrm{Pu}$ ) for buffer storage of a combined recycled uranium/plutonium oxide (RUPu) product based on U.S. security requirements in 2008 dollars. No pure $\mathrm{Pu}$ is planned to be stored. The charge is incurred as a one time cost when the RUPu material is transferred into buffer storage (no annual storage charges). The assumed nominal mean storage period is 4.5 years. This estimate is about $30-50 \%$ higher in real terms than the upper value used by the OECD-NEA in 1994 . The high range estimate of $\$ 6,500 / \mathrm{kg} P u$ reflects a greater numbers of canisters and higher decay heat and radiation levels, over the nominal cost estimate. A low range cost value of $\$ 3,500 / \mathrm{kg} \mathrm{Pu}$, which corresponds to the lower range OECD-NEA estimate of 1994. It may also correspond to the cost value for long (e.g., 50-100 years storage of $\mathrm{PuO}_{2}$ inventories) where operating costs are reduced and loading/unloading operations become a much less significant part of overall costs.

- A nominal range cost value of $\$ 10,000 / \mathrm{kg}$ TRU corresponding to buffer storage of proposed $\mathrm{UO}_{3^{-}}$ TRUOx (33\%) AFCI material. The low range cost estimate of $\$ 7,000 / \mathrm{kg}$ TRU and the high range cost value of $\$ 13,000 / \mathrm{kg}$ TRU are estimated at twice the cost of the RUPu storage. These costs are charged as a one-time cost when the TRU material is transferred into recycled product storage (no annual storage charges).

\section{References for Appendix B}

OECD-NEA, 1994, "The Economics of the Nuclear Fuel Cycle," Section 4.3.2.6, Plutonium storage, 1994.

Shropshire, D. et al., 2008, “Advanced Fuel Cycle Cost Basis," INL/EXT-07-12107, pp. E3-4 to E3-8, March 2008.

U.S. Army Corps of Engineers, 2007, Civil Works Construction Cost Index System (CWCCIS), Using CWBS Feature Code - 07 Power Plant, Appendix A, EM 1110-2-1304, Appendix Revised September 30, 2007. 


\section{Attachment A}

\section{Static Equilibrium Analysis (G4 ECONS)}

This attachment includes the detailed G4 ECONS Excel spreadsheets that describe the costs for the AFCI once-through, 1-Tier, and 2-Tier cases. These static analyses are based on $100 \mathrm{GWe}$-yr of generation with an equilibrium balance of LWR and FRs in the closed fuel cycle cases. The three total system comprehensive flow sheets provide a breakdown of costs by module when the costs are set at low, nominal, and high. The results from this analysis provide the basis for the uncertainty analysis in Section 6 and the comparisons to the VISION.ECON results provided in Section 9. 
A-1. Total System Cost Comprehensive Spreadsheet (Low, Nominal, High) 


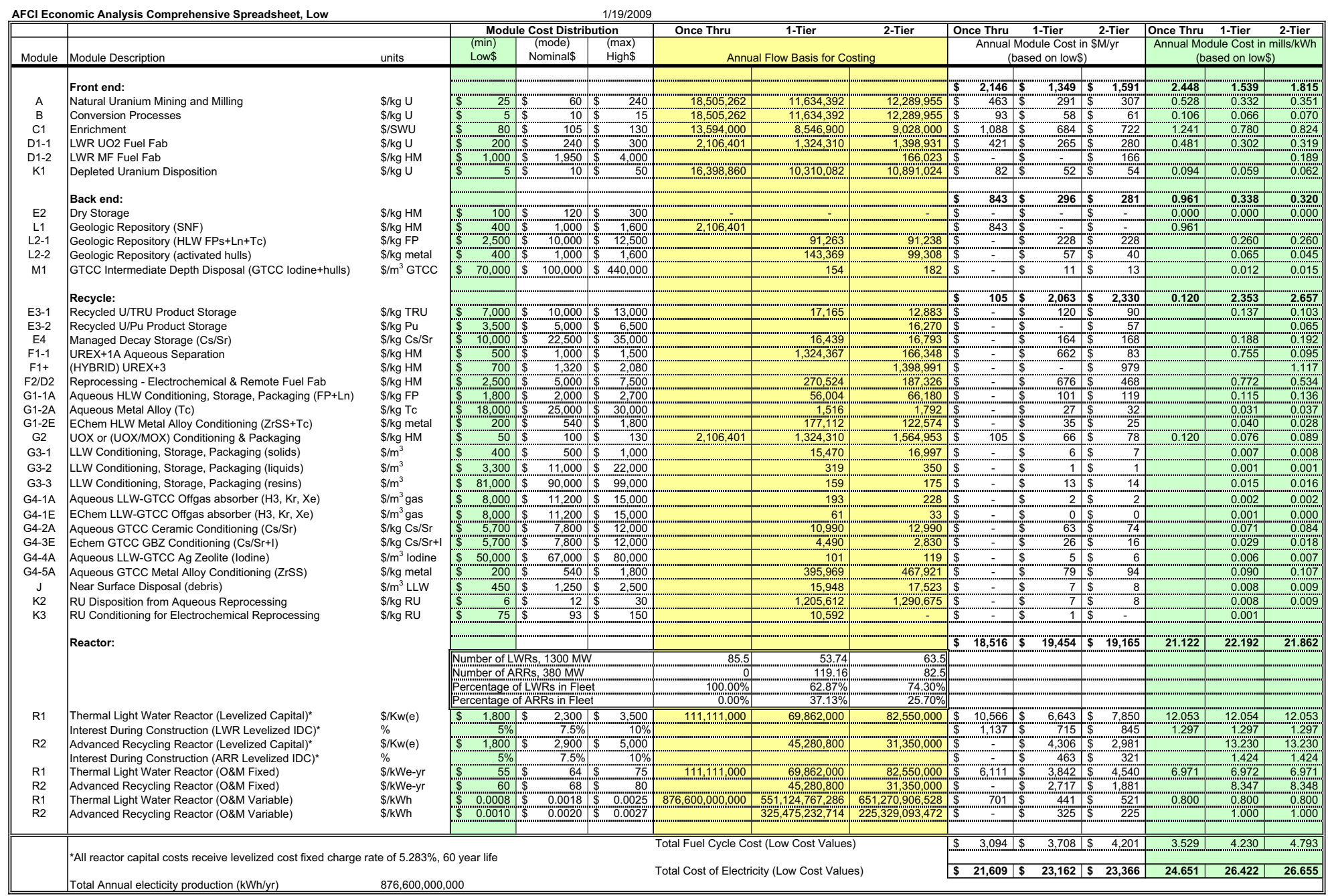




\begin{tabular}{|c|c|c|c|c|c|c|c|c|c|c|c|c|c|c|c|}
\hline \multirow{2}{*}{ Module } & \multirow{2}{*}{ Module Description } & \multirow[b]{2}{*}{ units } & \multicolumn{3}{|c|}{ Module Cost Distribution } & \multicolumn{2}{|l|}{ Once Thru } & \multirow{2}{*}{ 2-Tier } & \multirow{2}{*}{\multicolumn{4}{|c|}{$\begin{array}{|cc|}\text { Once Thru } & \text { 1-Tier } \\
\text { Annual Module Cost in } \$ \mathrm{M} / \mathrm{yr} \\
\text { (based on nominal\$) }\end{array}$}} & \multirow{2}{*}{\multicolumn{3}{|c|}{ 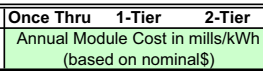 }} \\
\hline & & & $\begin{array}{l}(\mathrm{min}) \\
\text { Low\$ }\end{array}$ & \begin{tabular}{|c|} 
(mode) \\
Nominal\$
\end{tabular} & $\begin{array}{l}\text { (max) } \\
\text { High\$ }\end{array}$ & \multicolumn{2}{|c|}{ Annual Flow Basis for Costing } & & & & & & & & \\
\hline & Front end: & & & & & & & & 3,392 & $\$$ & 2,133 & $\$ \quad 2,577$ & 3.870 & 2.433 & 2.939 \\
\hline A & Natural Uranium Mining and Milling & $\$ / \mathrm{kg} \mathrm{U}$ & 25 & 60 & 240 & $18,505,262$ & $11,634,392$ & $12,289,955$ & 1,110 & $\$$ & 698 & 737 & 1.267 & 0.796 & 0.841 \\
\hline B & Conversion Processes & $\$ / \mathrm{kg} \mathrm{U}$ & 5 & 10 & 15 & $\begin{array}{l}18,505,262 \\
182\end{array}$ & $\begin{array}{l}111,634,392 \\
\end{array}$ & $\begin{array}{l}12,289,955 \\
-12\end{array}$ & 185 & $\$$ & - & 1233 & 0.211 & 0.0 & 0.140 \\
\hline C1 & Enrichment & \$/SWU & 80 & 105 & 130 & $\begin{array}{l}13,594,000 \\
\end{array}$ & $8,546,9000$ & $9,028,000$ & $(1,427$ & $s$ & 897 & 948 & - & 1.024 & 1.081 \\
\hline D1-1 & LWR UO2 Fuel Fab & $\$ / \mathrm{kg} U$ & 200 & 240 & 300 & $2,106,4011$ & $1,324,310$ & $1,398,931$ & 506 & $s$ & 318 & 336 & 0.577 & 0.363 & 0.383 \\
\hline D1-2 & LWR MF Fuel Fab & $\$ / \mathrm{kg} \mathrm{HM}$ & 1,000 & 1,950 & 4,000 & & & 166,023 & $\$$ & $\$$ & & 324 & & & 0.369 \\
\hline \multirow[t]{2}{*}{$\mathrm{K} 1$} & \multirow[t]{2}{*}{ Depleted Uranium Disposition } & $\$ / \mathrm{kg} U$ & 5 & 10 & 50 & $16,398,860$ & $10,310,082$ & $\begin{array}{l}10,891,024 \\
10,34\end{array}$ & 164 & $\$$ & 103 & 109 & 0.187 & 0.118 & 0.124 \\
\hline & & & & & & & & & 2,106 & $\$$ & 1,071 & 1,030 & 2.403 & 1.222 & 1.175 \\
\hline E2 & Dry Storage & $\$ / \mathrm{kg} \mathrm{HM}$ & 100 & 120 & 300 & & & & $\$$ & s' & & $\$$ & 0.000 & 0.000 & 0.000 \\
\hline L1 & Geologic Repository (SNF) & $\$ / k g ~ H M$ & 4000 & 1,000 & 1,600 & $2,106,401$ & & & 2,106 & $\$$ & "- & $\$$ & 2.403 & & \\
\hline L2-1 & Geologic Repository (HLW FPs+Ln+TC) & $\$ / k g$ FP & $\$ \quad 2,500$ & 10,000 & $\$ 12,500$ & & 91,263 & 91,238 & $\$$ & $\$$ & 913 & 912 & & 1.041 & 1.041 \\
\hline L2-2 & Geologic Repository (activated hulls) & $\$ / \mathrm{kg}$ metal & $s=400$ & 1,000 & $\$=1,600$ & & 143,369 & 99,308 & $\$$ & $\$$ & 143 & 99 & & 0.164 & 0.113 \\
\hline \multirow[t]{2}{*}{ M1 } & GTCC Intermediate Depth Disposal (GTCC Iodine+hulls) & $\$ / \mathrm{m}^{3}$ GTCC & $\$ \quad 70,000$ & $\$ 100,000$ & $\$ 440,000$ & & 154 & 182 & $\$$ & $\$$ & 15 & 18 & & 0.018 & 0.021 \\
\hline & Recycle: & & & & & & & & 2111 & 5 & 4.002 & 4.390 & 0.240 & 4.565 & 5.008 \\
\hline E3-1 & Recycled U/TRU Product Storage & $\$ / K g$ TRU & $\$ \quad 7,000$ & 10,000 & $\$ 13,000$ & & 17,165 & 12,883 & ㄴ.? & $\$$ & 172 & 129 & & 0.196 & 0.147 \\
\hline E3-2 & Recycled U/Pu Product Storage & $\$ / K g$ Pu & $\$-3,500$ & 5,000 & $\$$ & & & 16,270 & $\$$ & $\$$ & & 81 & & & 0.093 \\
\hline E4 & Managed Decay Storage $(\mathrm{Cs} / \mathrm{Sr})$ & $\$ / \mathrm{Kg} \mathrm{Cs} / \mathrm{Sr}$ & $\begin{array}{ll} & 10,000 \\
\end{array}$ & 22,500 & $\$ 35,000$ & & 16,439 & 16,793 & $\$$ & $\$$ & 370 & 378 & & 0.422 & 0.431 \\
\hline F1-1 & UREX+1A Aqueous Separation & $\$ / \mathrm{Kg} \mathrm{HM}$ & $\$ \quad 500$ & 1,000 & $\$ 1,500$ & & $\begin{array}{l}1,324,367 \\
1,3,3\end{array}$ & 166,348 & $\$$ & $\$$ & $\begin{array}{l}1,324 \\
1,324\end{array}$ & 166 & & 1.511 & 0.190 \\
\hline $\mathrm{F} 1+$ & (HYBRID) UREX+3 & $\$ / \mathrm{Kg} \mathrm{HM}$ & 700 & 1,320 & $\$-2,080$ & & & $1,398,991$ & $\$$ & $\$$ & & 1,847 & & & 2.107 \\
\hline F2/D2 & Reprocessing - Electrochemical \& Remote Fuel Fab & $\$ / K g H M$ & $\$ 2,500$ & 5,000 & 7,500 & & 270,524 & 187,326 & $\$$ & $\$$ & 1,353 & 937 & & 1.543 & 1.068 \\
\hline $\mathrm{G} 1-1 \mathrm{~A}$ & Aqueous HLW Conditioning, Storage, Packaging (FP+Ln) & $\$ / K g$ FP & $\$ 1,800$ & 2,000 & 2,700 & & 56,004 & 66,180 & $\$$ & $\$$ & 112 & 132 & & 0.128 & 0.151 \\
\hline $\mathrm{G} 1-2 \mathrm{~A}$ & Aqueous Metal Alloy (TC) & $\$ / K g$ Tc & $\$ 18,000$ & 25,000 & $\$ 30,000$ & & 1,516 & 1,792 & $\$$ & $\$$ & 38 & 45 & & 0.043 & 0.051 \\
\hline $\mathrm{G} 1-2 \mathrm{E}$ & EChem HLW Metal Alloy Conditioning (ZrSS+Tc) & $\$ / K g$ metal & $\$=200$ & 540 & $\$ \quad 1,800$ & & 177,112 & 122,574 & $\$$ & $\$$ & 96 & 66 & & 0.109 & 0.076 \\
\hline G2 & UOX or (UOX/MOX) Conditioning \& Packaging & $\$ / \mathrm{Kg} \mathrm{HM}$ & 50 & 100 & 130 & $2,106,401$ & $1,324,310$ & $1,564,953$ & 211 & $\$$ & 132 & 156 & 0.240 & 0.151 & 0.179 \\
\hline G3-1 & LLW Conditioning, Storage, Packaging (solids) & $\$ / m^{3}$ & 400 & 500 & $\$ 1,000$ & & 15,470 & 16,997 & $\$$ & $\$$ & 8 & 8 & & 0.009 & 0.010 \\
\hline G3-2 & LLW Conditioning, Storage, Packaging (liquids) & $\$ / m^{3}$ & $\$ 3,300$ & 11,000 & $\$ 22,000$ & & 319 & 350 & $\$$ & $\$$ & 4 & 4 & & 0.004 & 0.004 \\
\hline G3-3 & LLW Conditioning, Storage, Packaging (resins) & $\$ / m^{3}$ & $\$ 81,000$ & 90,000 & $\$ 99,000$ & & 159 & 175 & $\$$ & $\$$ & 14 & 16 & & 0.016 & 0.018 \\
\hline G4-1A & Aqueous LLW-GTCC Offgas absorber ( $\mathrm{H} 3, \mathrm{Kr}, \mathrm{Xe}$ ) & $\$ / m^{3}$ gas & $\$ \quad 8,000$ & 11,200 & $\$ 15,000$ & & 193 & 228 & $\$$ & $\$$ & 2 & 3 & & 0.002 & 0.003 \\
\hline G4-1E & EChem LLW-GTCC Offgas absorber $(\mathrm{H} 3, \mathrm{Kr}, \mathrm{Xe})$ & $\$ / m^{3}$ gas & $\$ 8,000$ & 11,200 & $\$ 15,000$ & & 61 & 33 & $\$$ & $\$$ & 1 & $\$$ & & 0.001 & 0.000 \\
\hline G4-2A & Aqueous GTCC Ceramic Conditioning (Cs/Sr) & $\$ / \mathrm{Kg}$ Cs/Sr & $\$ \quad 5,700$ & 7,800 & $\$ 12,000$ & & 10,990 & 12,990 & $\$$ & $\$$ & 86 & 101 & & 0.098 & 0.116 \\
\hline G4-3E & Echem GTCC GBZ Conditioning (Cs/Sr+l) & $\$ / K g \mathrm{Cs} / \mathrm{Sr}+1$ & $\$ \quad 5,700$ & 7,800 & $\$ 12,000$ & & 4,490 & 2,830 & $\$$ & $\$$ & 35 & 22 & & 0.040 & 0.025 \\
\hline G4-4A & Aqueous LLW-GTCC Ag Zeolite (lodine) & $\$ / \mathrm{m}^{3}$ Iodine & $\$ \quad 50,000$ & 67,000 & $\$ 80,000$ & & 101 & 119 & $\$$ & $\$$ & 7 & 8 & & 0.008 & 0.009 \\
\hline G4-5A & Aqueous GTCC Metal Alloy Conditioning (ZrSS) & $\$ / \mathrm{Kg}$ metal & $s-200$ & 540 & $\$-1,800$ & & 395,969 & 467,921 & $\$$ & $\$$ & 214 & 253 & & 0.244 & 0.288 \\
\hline $\mathrm{J}$ & Near Surface Disposal (debris) & $\$ / \mathrm{m}^{3} \mathrm{LLW}$ & 450 & 1,250 & 2,500 & & 15,948 & 17,523 & $\$$ & $\$$ & 20 & 22 & & 0.023 & 0.025 \\
\hline K2 & RU Disposition from Aqueous Reprocessing & $\$ / K g$ RU & 6 & 12 & 30 & & $1,205,612$ & $1,290,675$ & $\$$ & $\$$ & 14 & 15 & & 0.017 & 0.018 \\
\hline \multirow[t]{6}{*}{ K3 } & RU Conditioning for Electrochemical Reprocessing & $\$ / K g R U$ & 75 & 93 & 150 & & 10,592 & & $\$$ & $\$$ & 1 & $\$$ & & 0.001 & \\
\hline & Reactor: & & & & & & & & 531,366 & 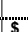 & 35,103 & $\$ 33,952$ & 35782 & 40.045 & 38.731 \\
\hline & & & Number of LI & WRs, $1300 \mathrm{MW}$ & & 85.5 & 2 & 263.5 & & & & & & & \\
\hline & & & Number of $A$ & RRs, $380 \mathrm{MW}$ & & - & 119.16 & 82.5 & & & & & & & \\
\hline & & & Percentage o & of LWRs in Flee & & $100.00 \%$ & $62.87 \%$ & $74.30 \%$ & & & & & & & \\
\hline & & & Percentage 0 & ff ARRs in Flee & & $0.00 \%$ & $37.13 \%$ & $25.70 \%$ & & & & & & & \\
\hline R1 & Thermal Light Water Reactor (Levelized Capital)* & $\$ / K w(e)$ & $\$ 1,800$ & 2,300 & $\$ \quad 3,500$ & $1111,111,000$ & $699,862,000$ & $82,550,000$ & $\$ 19,420$ & $\$$ & 12,210 & $\$ 14,428$ & 22.153 & 22.155 & 22.153 \\
\hline & Interest During Construction (LWR Levelized IDC) & & & $7.5 \%$ & $10 \%$ & & & & $\$$ & $\$$ & 2,048 & 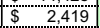 & 3.716 & 3.715 & 3.715 \\
\hline R2 & Advanced Recycling Reactor (Levelized Capital)* & $\$ / K w(e)$ & $\$ \quad 1,800$ & 2,900 & $\$ \quad 5,000$ & & $45,280,800$ & $31,350,000$ & $\$$ & $\$$ & 9,979 & $\begin{array}{l}9 \\
\$\end{array} 6,909$ & & 30.658 & 30.660 \\
\hline & Interest During Construction (ARR Levelized IDC) & & & $7.5 \%$ & $10 \%$ & & & & $\$$ & $\$$ & 1,673 & 1,159 & & 5.141 & 5.141 \\
\hline R1 & Thermal Light Water Reactor (O\&M Fixed) & $\$ / k W e-y r$ & & 64 & 75 & $111,111,000$ & $69,862,000$ & $82,550,000$ & 7,1111 & $\$$ & 4,471 & 5,283 & 8.112 & 8.113 & 8.112 \\
\hline R2 & Advanced Recycling Reactor (O\&M Fixed) & $\$ / k W e-y r$ & & 68 & (3) & & $45,280,800$ & $31,350,000$ & 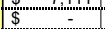 & \$ & 3,079 & 2,132 & & 9.460 & 9.461 \\
\hline R1 & Thermal Light Water Reactor (O\&M Variable) & $\$ / k W h$ & $\$ 0.0008$ & 0.0018 & $\$ 0.0025$ & $876,600,000,000$ & $551,124,767,286$ & $651,270,906,528$ & 1,578 & $\$$ & 992 & 1,172 & 1.800 & 1.800 & 1.800 \\
\hline R2 & Advanced Recycling Reactor (O\&M Variable) & $\$ / k W h$ & $\$$ & $\$=0.020$ & $\begin{array}{l}9 \\
\$\end{array} 0.0027$ & & $\begin{array}{l}325,475,232,714 \\
325,4\end{array}$ & $\begin{array}{l}225,329,093,472 \\
225,32,093 \\
\end{array}$ & $\$$ & $\$$ & 651 & 451 & & 2000 & 2.000 \\
\hline & & & & & & Total Fuel Cycle Co & st (Nominal Cost Va & lues) & \begin{tabular}{|l|l|}
$\$$ & 5,709 \\
\end{tabular} & $\$$ & 77,206 & \begin{tabular}{ll|}
$\$$ & 7,997 \\
\end{tabular} & 26.513 & 8.220 & 9.122 \\
\hline & *All reactor capital costs receive levelized cost fixed cha & te of $7.599^{\circ}$ & year life & & & & & & & & & & & & \\
\hline & & & & & & Total Cost of Electric & city (Nominal Cost $\mathrm{V}$ & /alues) & \begin{tabular}{|l|l|}
$\$ 37,076$ \\
\end{tabular} & $\$$ & 42,309 & $\$ 41,949$ & 42.295 & 48.265 & 47.854 \\
\hline
\end{tabular}




\begin{tabular}{|c|c|c|c|c|c|c|c|c|c|c|c|c|c|c|c|}
\hline \multirow[b]{2}{*}{ Module } & \multirow[b]{2}{*}{ Module Description } & \multirow[b]{2}{*}{ units } & \multicolumn{3}{|c|}{ Module Cost Distribution } & \multirow{2}{*}{\multicolumn{3}{|c|}{ Annual Flow Basis for Costing }} & \multirow{2}{*}{\multicolumn{4}{|c|}{ 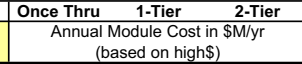 }} & \multirow{2}{*}{\multicolumn{3}{|c|}{ 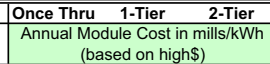 }} \\
\hline & & & $\begin{array}{l}(\min ) \\
\text { Low\$ }\end{array}$ & \begin{tabular}{c|} 
(mode) \\
Nominal\$
\end{tabular} & $\begin{array}{l}\text { (max) } \\
\text { High\$ }\end{array}$ & & & & & & & & & & \\
\hline & Front end: & & & & & & & & 7,938 & $\$$ & 4,991 & 5,936 & 9.055 & 5.693 & 6.772 \\
\hline A & Natural Uranium Mining and Milling & $\$ / \mathrm{kg} \mathrm{U}$ & 25 & 60 & 240 & $18,505,262$ & $11,634,392$ & $12,289,955$ & 4,441 & $\$$ & 2,792 & 2,950 & 5.066 & 3.185 & 3.365 \\
\hline B & Conversion Processes & $\$ / \mathrm{kg} \mathrm{U}$ & 5 & 10 & 15 & $18,505,262$ & $111,634,392$ & $12,289,955$ & 278 & $\$$ & 175 & 184 & 0.317 & 0.199 & 0.210 \\
\hline C1 & Enrichment & \$/SWU & "80 & 105 & 130 & $13,594,000$ & $8,546,900$ & $9,028,000$ & 1,767 & "\$ & 1,111 & "1,174" & 2.016 & 1.268 & 1.339 \\
\hline D1-1 & LWR UO2 Fuel Fab & $\$ / k g$ U & 200 & 240 & 300 & $2,106,401$ & $1,324,310$ & $1,398,931$ & 632 & $\$$ & 397 & 420 & 0.721 & 0.453 & 0.479 \\
\hline $\mathrm{D} 1-2$ & LWR MF Fuel Fab & $\$ / \mathrm{kg} \mathrm{HM}$ & $s-1,000$ & 1,950 & 4,000 & & & 166,023 & $s$ & $\$$ & & 664 & & & 0.758 \\
\hline \multirow[t]{2}{*}{$\mathrm{K} 1$} & Depleted Uranium Disposition & $\$ / \mathrm{kg} \mathrm{U}$ & 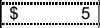 & -10 & 50 & $16,398,860$ & $10,310,082$ & $10,891,024$ & 820 & $\$$ & 516 & 545 & - 0.9355 & 0.588 & 0.621 \\
\hline & Back end: & & & & & & & & 3,370 & $\$$ & 1,438 & 1,379 & 3.845 & 1.640 & 1.574 \\
\hline E2 & Dry Storage & $\$ / \mathrm{kg} \mathrm{HM}$ & 100 & 120 & 300 & & $\cdots$ & $=$ & - n & $\$$ & - n & $=$ & 0.000 & 0.000 & 0.000 \\
\hline L1 & Geologic Repository (SNF) & $\$ / \mathrm{kg} \mathrm{HM}$ & 400 & 1,000 & 1,600 & $2,106,401$ & & & 3,370 & $s$ & & $\$$ & 3.845 & & \\
\hline L2-1 & Geologic Repository (HLW FPs+Ln+TC) & $\$ / k g ~ F P$ & $s-2,500$ & 10,000 & $\$ 12,500$ & & 91,263 & 91,238 & $s$ & $s$ & $-1,141$ & 1,140 & & 1.301 & 1.301 \\
\hline L2-2 & Geologic Repository (activated hulls) & $\$ / \mathrm{kg}$ metal & \begin{tabular}{|l}
$\$$ \\
\end{tabular} & 1,000 & $\$ \quad 1,600$ & & 143,369 & 99,308 & $\$$ & $\$$ & 229 & 159 & & 0.262 & 0.181 \\
\hline \multirow[t]{2}{*}{ M1 } & GTCC Intermediate Depth Disposal (GTCC lodine+hulls) & $\$ / \mathrm{m}^{3}$ GTCC & $\$ \quad 70,000$ & $\$ 100,000$ & $\$ 440,000$ & & 154 & 182 & $\$$ & $\$$ & 68 & 80 & & 0.077 & 0.091 \\
\hline & Recycle: & & & & & & & & & $\$$ & 6,528 & 7,252 & 0.312 & 7.447 & 8.873 \\
\hline E3-1 & Recycled U/TRU Product Storage & $\$ / K g$ TRU & $\$ \quad 7,000$ & 10,000 & $\$ 13,000$ & & 17,165 & 12,883 & $\$$ & \$ & 等, 223 & (167 & & $\begin{array}{l}1.441 \\
0.255\end{array}$ & (3.2191 \\
\hline E3-2 & Recycled U/Pu Product Storage & $\$ / K g$ Pu & $\$-3,500$ & 5,000 & $\$ 6,500$ & & & 16,270 & $\$$ & $\$$ & & 106 & & & 0.121 \\
\hline E4 & Managed Decay Storage (Cs/Sr) & $\$ / \mathrm{Kg} \mathrm{Cs} / \mathrm{Sr}$ & $\$ 10,000$ & 22,500 & $\$ 35,000$ & & 16,439 & 16,793 & $\$$ & \$ & 575 & 588 & & 0.656 & 0.670 \\
\hline F1-1 & UREX+1A Aqueous Separation & $\$ / \mathrm{Kg} \mathrm{HM}$ & $\$ \quad 500$ & 1,000 & $\$ \quad 1,500$ & & $1,324,367$ & 166,348 & $\$$ & $\$$ & 1,987 & 250 & & 2.266 & 0.285 \\
\hline $\mathrm{F} 1+$ & (HYBRID) UREX+3 & $\$ / \mathrm{Kg} \mathrm{HM}$ & 700 & 1,320 & $\$ \quad 2,080$ & & & $1,398,991$ & $\$$ & $\$$ & & $\$ \quad 2,910$ & & & 3.320 \\
\hline F2/D2 & Reprocessing - Electrochemical \& Remote Fuel Fab & $\$ / K g ~ H M$ & $\$ 2,500$ & 5,000 & 7,500 & & 270,524 & 187,326 & $\$$ & $\$$ & 2,029 & "'1,405 & & 2.315 & 1.603 \\
\hline G1-1A & Aqueous HLW Conditioning, Storage, Packaging (FP+Ln) & $\$ / K g$ FP & $\$=1,800$ & $\begin{array}{ll}2 \\
2,000 \\
2\end{array}$ & $\$=2,700$ & & 56,004 & - & $\$$ & $\$$ & 151 & (179 & & 0.172 & 0.204 \\
\hline G1-2A & Aqueous Metal Alloy (Tc) & $\$ / K g$ Tc & $\$ 18,000$ & 25,000 & $\$ 30,000$ & & 1,516 & 1,792 & $\$$ & "s.' & 45 & 54 & & 0.052 & 0.061 \\
\hline G1-2E & EChem HLW Metal Alloy Conditioning (ZrSS+Tc) & $\$ / K g$ metal & $s-200$ & 540 & $\$-1,800$ & & 177,112 & 122,574 & $\$$ & $s$ & 319 & 221 & & 0.364 & 0.252 \\
\hline G2 & UOX or (UOX/MOX) Conditioning \& Packaging & $\$ / \mathrm{Kg} \mathrm{HM}$ & 50 & 100 & 130 & $2,106,401$ & $1,324,310$ & $1,564,953$ & 274 & $\$$ & 172 & 203 & 0.312 & 0.196 & 0.232 \\
\hline G3-1 & LLW Conditioning, Storage, Packaging (solids) & $\$ / \mathrm{m}^{3}$ & 400 & 500 & 1,000 & & 15,470 & 16,997 & $\$$ & $\$$ & 15 & 17 & & 0.018 & 0.019 \\
\hline G3-2 & LLW Conditioning, Storage, Packaging (liquids) & $\$ / m^{3}$ & $\$ \quad 3,300$ & $\$ \quad 11,000$ & $\$ 22,000$ & & 319 & 350 & $\$$ & $\$$ & 7 & $\$$ & & 0.008 & 0.009 \\
\hline G3-3 & LLW Conditioning, Storage, Packaging (resins) & $\$ / m^{3}$ & $\$ 81,000$ & 90,000 & $\$ 99,000$ & & 159 & 175 & $\$$ & $\$$ & 16 & 17 & & 0.018 & 0.020 \\
\hline G4-1A & Aqueous LLW-GTCC Offgas absorber $(\mathrm{H} 3, \mathrm{Kr}, \mathrm{Xe})$ & $\$ / m^{3}$ gas & $\$ \quad 8,000$ & 11,200 & $\$ 15,000$ & & 193 & 228 & $\$$ & $\$$ & 3 & $\$$ & & 0.003 & 0.004 \\
\hline G4-1E & EChem LLW-GTCC Offgas absorber $(\mathrm{H} 3, \mathrm{Kr}, \mathrm{Xe})$ & $\$ / m^{3}$ gas & $\$ \quad 8,000$ & 11,200 & $\$ 15,000$ & & 61 & 33 & $\$$ & $\$$ & 1 & $\$$ & & 0.001 & 0.001 \\
\hline G4-2A & Aqueous GTCC Ceramic Conditioning (Cs/Sr) & $\$ / \mathrm{Kg} \mathrm{Cs} / \mathrm{Sr}$ & $s-5,700$ & 7,800 & $\$ 12,000$ & & 10,990 & $-12,990$ & $\$$ & $s$ & 132 & 156 & & 0.150 & 0.178 \\
\hline G4-3E & Echem GTCC GBZ Conditioning (Cs/Sr+l) & $\$ / K g \mathrm{Cs} / \mathrm{Sr}+1$ & $\$ \quad 5,700$ & 7,800 & $\$ 12,000$ & & 4,490 & 2,830 & $\$$ & $\$$ & 54 & 34 & & 0.061 & 0.039 \\
\hline G4-4A & Aqueous LLW-GTCC Ag Zeolite (lodine) & $\$ / \mathrm{m}^{3}$ lodine & $\$ 50,000$ & 67,000 & $\$ 80,000$ & & 101 & 119 & $\$$ & $\$$ & 8 & 10 & & 0.009 & 0.011 \\
\hline G4-5A & Aqueous GTCC Metal Alloy Conditioning (ZrSS) & $\$ / K g$ metal & & 540 & $\$ \quad 1,800$ & & 395,969 & 467,921 & $\$$ & $\$$ & 713 & 842 & & 0.813 & 0.961 \\
\hline $\mathrm{J}$ & Near Surface Disposal (debris) & $\$ / \mathrm{m}^{3} \mathrm{LLW}$ & 450 & 1,250 & $\$ 2,500$ & & 15,948 & 17,523 & $\$$ & $\$$ & 40 & 44 & & 0.045 & 0.050 \\
\hline K2 & RU Disposition from Aqueous Reprocessing & $\$ / \mathrm{Kg} \mathrm{RU}$ & & 12 & $=30$ & & $1,205,612$ & $1,290,675$ & $\$$ & $\$$ & 36 & 39 & & 0.041 & 0.044 \\
\hline K3 & RU Conditioning for Electrochemical Reprocessing & $\$ / K g R U$ & 75 & 93 & 150 & & 10,592 & & $\$$ & $\$$ & 2 & $\$$ & & 0.002 & \\
\hline & Reactor: & & & & & & & & 58,614 & $\$$ & 69,348 & $\$ 66,043$ & 66.865 & 79.110 & 75 \\
\hline & & & Number of $L$ & WRs, $1300 \mathrm{MW}$ & & 85.5 & $\overline{53.74}$ & "63.5 & & & & & & & \\
\hline & & & Number of $A$ & RRs, $380 \mathrm{MW}$ & & & 119.16 & 82.5 & & & & & & & \\
\hline & & & Percentage 0 & of LWRs in Flee & & $300.00 \%$ & $62.87 \%$ & $74.30 \%$ & & & & & & & \\
\hline & & & Percentage o & of ARRs in Flee & & - & $37.13 \%$ & $25.70 \%$ & & & & & & & \\
\hline R1 & Thermal Light Water Reactor (Levelized Capital)* & $\$ / K w(e)$ & $\$-1,800$ & 2,300 & 3,500 & $\begin{array}{ll}111,111,000 \\
\end{array}$ & $699,862,000$ & $82,550,000$ & $\$ 39,017$ & $\$$ & 24,532 & \$ 28,988 & 44.510 & 44.513 & 44.510 \\
\hline & Interest During Construction (LWR Levelized IDC) & & & $7.5 \%$ & $10 \%$ & & & & 9,072 & \$ & 5,702 & \$ 6,737 & 10.349 & 10.346 & 10.345 \\
\hline R2 & Advanced Recycling Reactor (Levelized Capital)" & $\$ / K w(e)$ & $\$ \quad 1,800$ & 2,900 & 5,000 & & $45,280,800$ & $31,350,000$ & $\$$ & $\$$ & 22,715 & $\$ \quad 15,727$ & & 69.791 & 69.794 \\
\hline & Interest During Construction (ARR Levelized IDC) & & & $7.5 \%$ & $10 \%$ & & & & $\$$ & $\$$ & 5,280 & 3,655 & & 16.221 & 16.222 \\
\hline R1 & Thermal Light Water Reactor (O\&M Fixed) & $\$ / k W e-y r$ & & 64 & 75 & $111,111,000$ & $69,862,000$ & $82,550,000 "$ & 8,333 & $\$$ & 5,240 & $\$ 6,191$ & 9.506 & 9.507 & 9.506 \\
\hline R2 & Advanced Recycling Reactor (O\&M Fixed) & $\$ / k W e-y r$ & 60 & 68 & 80 & & $45,280,800$ & $31,350,000$ & $\$$ & $\$$ & 3,622 & 2,508 & & "11.130' & 11.130 \\
\hline R1 & Thermal Light Water Reactor (O\&M Variable) & $\$ / k W h$ & $\$ 0.0008$ & 0.0018 & $\$ 0.0025$ & $876,600,000,000$ & $551,124,767,286$ & $651,270,906,528$ & 2,192 & $\$$ & 1,378 & 1,628 & 2.500 & 2.500 & 2.500 \\
\hline R2 & Advanced Recycling Reactor (O\&M Variable) & $\$ / \mathrm{kWh}$ & $\$=0.0010$ & $\$-0.0020$ & $\$ \quad 0.0027$ & & $325,475,232,714$ & $225,329,093,472$ & s & $\$$ & 879 & 608 & & 2.700 & 2.700 \\
\hline & & & & & & Total Fuel Cycle Cos & st (High Cost Values & & \begin{tabular}{|l|l|}
$\$$ & 11,582 \\
\end{tabular} & & $\overline{12,957}$ & & $\begin{array}{ll}13.212 \\
\end{array}$ & 14.780 & 16.618 \\
\hline & *All reactor capital costs receive levelized cost fixed charge & te of $10.033 \%$ & 60 year life & & & & & & & & & & & & \\
\hline & & & & & & Total Cost of Electr & city (High Cost Valu & & \begin{tabular}{|l|l|}
$\$ 70,196$ \\
\end{tabular} & $\$$ & 82,304 & $\$ 80,610$ & 80.077 & 93.890 & 91.956 \\
\hline
\end{tabular}




\section{A-2. Once-through Costs and Flows Worksheet}




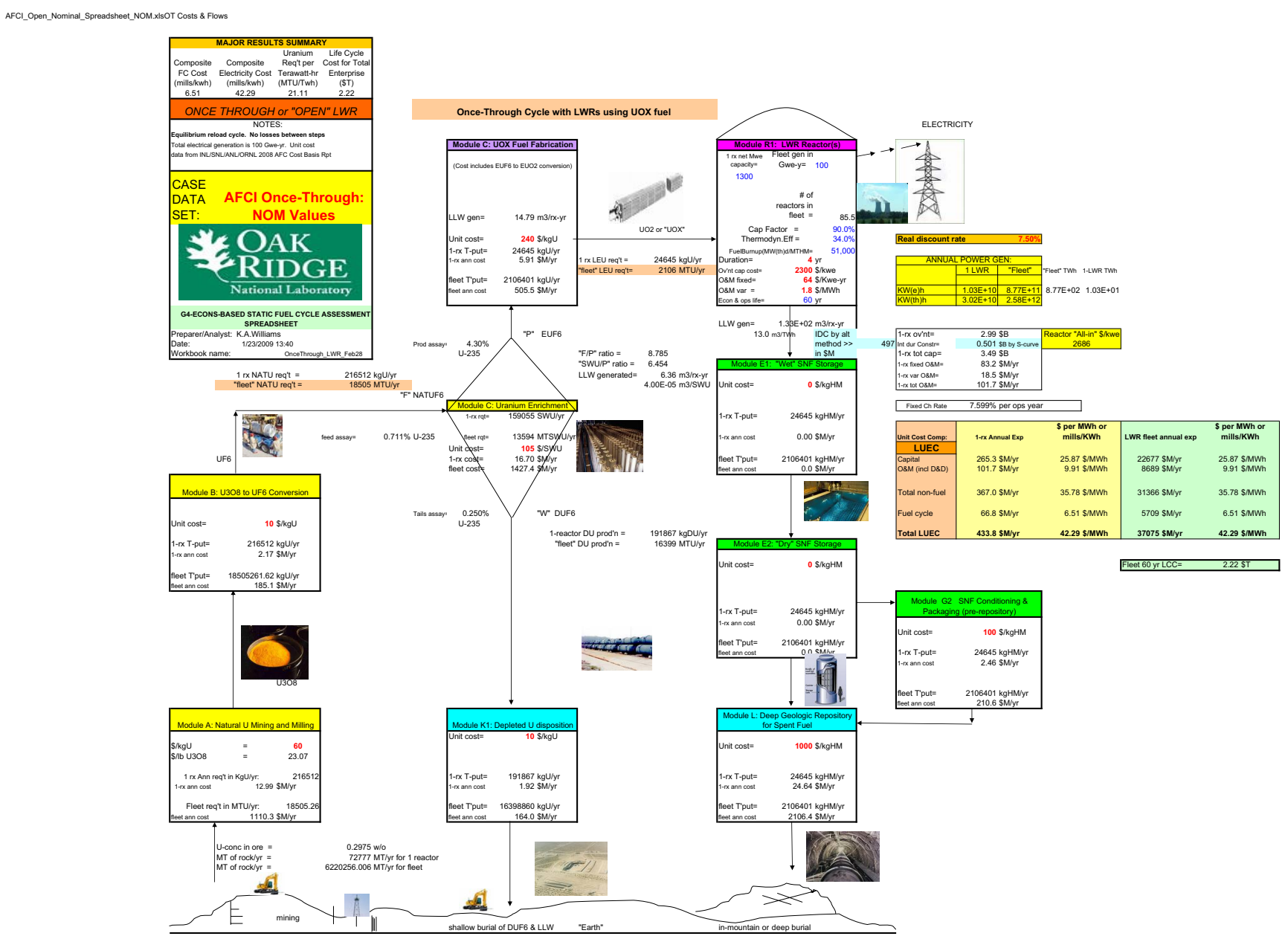




\section{A-3. 1-Tier Costs and Flows Worksheets}




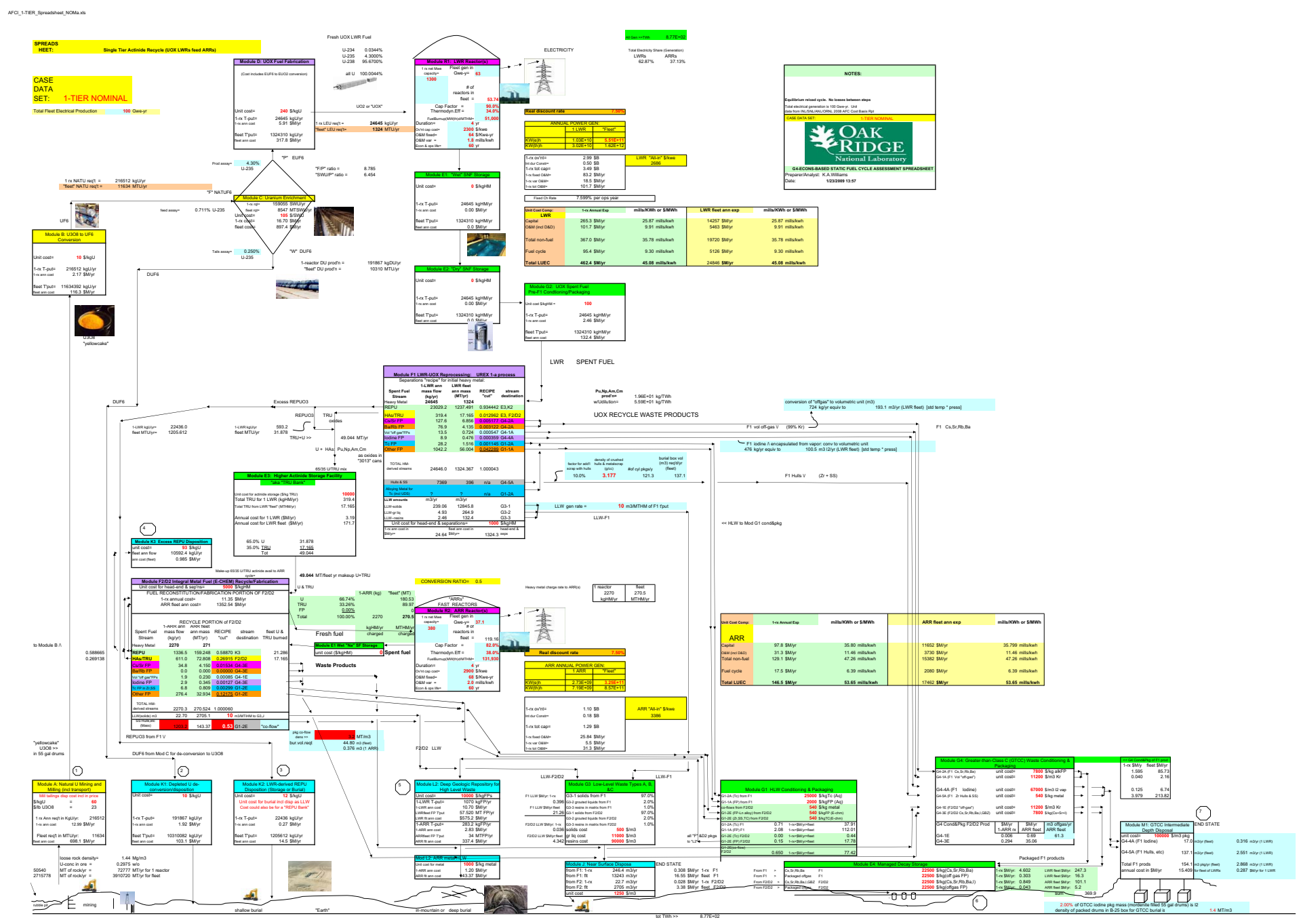


AFCI_1-TIER_Spreadsheet_NOMa.xls

\begin{tabular}{|c|c|c|c|c|c|c|}
\hline \begin{tabular}{|} 
Unit Cost Comp: \\
LWR
\end{tabular} & 1-rx Annual Exp & per kwh & LWR fleet ann exp & per kwh & Generation \% & \\
\hline Capital & $265.3 \$ M / y r$ & 25.87 mills/kwh & $14257 \$ M / y r$ & 25.87 mills/kwh & & \\
\hline O\&M (incl D\&D) & $101.7 \$ \mathrm{M} / \mathrm{yr}$ & 9.91 mills/kwh & $5463 \$ M / y r$ & 9.91 mills/kwh & & \\
\hline Total non-fuel & $367.0 \$ \mathrm{M} / \mathrm{yr}$ & 35.78 mills/kwh & 19720 \$M/yr & 35.78 mills/kwh & & \\
\hline Fuel cycle & $95.4 \$ \mathrm{M} / \mathrm{yr}$ & 9.30 mills/kwh & $5126 \$ M / y r$ & 9.30 mills/kwh & & \\
\hline Total LUEC & $462.4 \$ \mathrm{M} / \mathrm{yr}$ & $45.08 \mathrm{mills} / \mathrm{kwh}$ & $24846 \$ \mathrm{M} / \mathrm{yr}$ & 45.08 mills $/ \mathrm{kwh}$ & $62.9 \%$ & of power \\
\hline
\end{tabular}

\begin{tabular}{|c|c|c|c|c|c|c|}
\hline $\begin{array}{r}\text { Unit Cost Comp: } \\
\text { ARR }\end{array}$ & 1-rx Annual Exp & per kwh & ARR fleet ann exp & per kwh & Generation $\%$ & \\
\hline Capital & $97.8 \$ \mathrm{M} / \mathrm{yr}$ & 35.80 mills/kwh & $11652 \$ M / y r$ & 35.80 mills/kwh & & \\
\hline O\&M (incl D\&D) & $31.3 \$ \mathrm{M} / \mathrm{yr}$ & 11.46 mills/kwh & $3730 \$ M / y r$ & 11.46 mills/kwh & & \\
\hline Total non-fuel & $129.1 \$ \mathrm{M} / \mathrm{yr}$ & 47.26 mills/kwh & $15382 \$ M / y r$ & 47.26 mills/kwh & & \\
\hline Fuel cycle & $17.5 \$ \mathrm{M} / \mathrm{yr}$ & 6.39 mills/kwh & 2080 \$M/yr & 6.39 mills/kwh & & \\
\hline Total LUEC & $146.5 \$ \mathrm{M} / \mathrm{yr}$ & 53.65 mills $/ \mathrm{kwh}$ & $17462 \$ M / y r$ & 53.65 mills $/ k w h$ & $37.1 \%$ & of power \\
\hline
\end{tabular}

\begin{tabular}{|lr|}
\hline Fleet Average & mills/kwh \\
Cap & 29.56 \\
O\&M (Incl D\&D) & $\frac{10.49}{\text { Total Non-fuel }}$ \\
Fuel Cycle & 8.04 \\
Total & $\mathbf{4 8 . 2 6}$ \\
\hline Once -Through FC & 6.61 \\
\hline
\end{tabular}

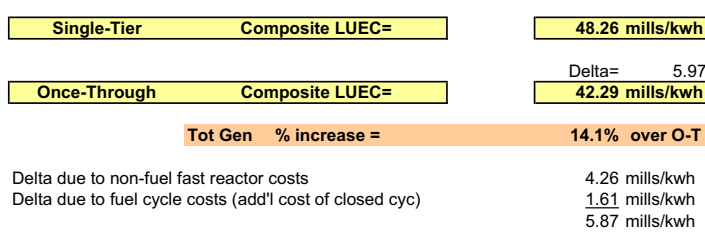

FC comp \% increase=

$24.4 \%$ over O-T 


\begin{tabular}{|c|c|c|c|c|}
\hline Module & $\begin{array}{l}\text { FUEL } \\
\text { CYCLE }\end{array}$ & $\mid \begin{array}{c}1-L W R \text { rx annual } \\
\text { in SM/yr }\end{array}$ & Skg EUU tuel & mills/kwh \\
\hline 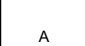 & \begin{tabular}{|l} 
LWR Front-end \\
Mine/mill
\end{tabular} & & & \\
\hline $\begin{array}{l}\text { A } \\
B\end{array}$ & 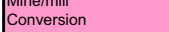 & 2.165 & & 0.21 \\
\hline$K-1$ & Enrichment & 16.701 & 678 & 1.63 \\
\hline $\begin{array}{l}\text { K-1 } \\
\text { D1-1 }\end{array}$ & $\begin{array}{l}\text { DUF6 Disp } \\
\text { Fabrication }\end{array}$ & $\begin{array}{l}1.9919 \\
5.915\end{array}$ & $\begin{array}{r}78 \\
240\end{array}$ & $\begin{array}{l}0.19 \\
0.58 \\
-0.5\end{array}$ \\
\hline & Front End Subtotal & 39.690 & 1610 & \\
\hline & LWR Back-end & & S/kgitHM & \\
\hline & SF Handling & & $t$ & \\
\hline $\begin{array}{l}\begin{array}{l}\text { E1 } \\
\text { E2 }\end{array} \\
\text { S }\end{array}$ & $\begin{array}{l}\text { Wet S S storage } \\
\text { Dry SF Storage }\end{array}$ & $\begin{array}{l}0.000 \\
0.000\end{array}$ & 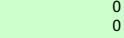 & $\begin{array}{l}0.00 \\
0.00\end{array}$ \\
\hline G2 & Jox SF Cond/Pkg & $\frac{2.464}{2.464}$ & $\frac{100}{100}$ & $\frac{0.24}{0.24}$ \\
\hline F1 & $\begin{array}{l}\text { Aq Repr Heprocossing } \\
\text { Aq Rep }\end{array}$ & 24.645 & 1000 & 2.40 \\
\hline K2 & Repuranium Prod Dis & 0.269 & 11 & 0.03 \\
\hline 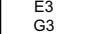 & $\mid \begin{array}{l}\text { TRU Un in AA storage } \\
\text { LW cond pokg Fi }\end{array}$ & $\begin{array}{l}3.194 \\
0.396\end{array}$ & $\begin{array}{r}130 \\
16\end{array}$ & $\begin{array}{l}0.04 \\
0.04\end{array}$ \\
\hline & LLW disposal: F1 & 0.308 & 13 & 0.03 \\
\hline G4-2A & Cs/Sr/Rb/Ba C\&P:F1 & 1.595 & 65 & 0.16 \\
\hline $\begin{array}{l}\text { G4-AA } \\
\text { G4-4A }\end{array}$ & vol "offgas" C\&P: F1 & 0.040 & ${ }_{5}^{2}$ & 0.00 \\
\hline $\begin{array}{l}\text { G4-4A } \\
\text { G4-5A }\end{array}$ & $\begin{array}{l}\text { lodine C\&P: F1 } \\
\text { Hulls, etc C\&Pkg:F1 }\end{array}$ & $\begin{array}{l}0.125 \\
3.979\end{array}$ & 161 & $\begin{array}{l}0.01 \\
0.39\end{array}$ \\
\hline E4 & Mng'd Prod Dy Stg:F1 & 4.905 & 199 & \\
\hline G1 & Other FPs C\&P: F1 & 2.084 & 85 & 0.20 \\
\hline G1 & To C8P: F1 & & & \\
\hline & Subtotal & 42.248 & 1714 & 4.12 \\
\hline M1 & $\begin{array}{l}\text { WR Recy Geoo Disp } \\
\text { GTCC Int Dep Disp:F1 }\end{array}$ & 0.287 & 12 & 0.03 \\
\hline L2 & HLW Deep Geo Disp & $\frac{10.704}{10.991}$ & $\frac{434}{446}$ & $\frac{1.04}{1.07}$ \\
\hline fleet kwh/y & LWR Back-end Subt & 55.703 & 2260 & 5.43 \\
\hline $5.51 \mathrm{E}+11$ & \begin{tabular}{|l|} 
Total LWR Fuel Cycle \\
\end{tabular} & 95.393 & 3871 & 9.30 \\
\hline & ARR Back-end & 1-ARR rx SM/yr & $\begin{array}{l}\text { S/kgiHM (ARR) } \\
\text { S/kgiHM }\end{array}$ & mills/kwh (ARR) \\
\hline & SF Handling & & $\downarrow$ & \\
\hline E1 & $\begin{array}{l}\text { Wet (Na) SF storage } \\
\text { Subtotal } \\
\text { ARR Repro/Refab }\end{array}$ & $\frac{0.000}{0.000}$ & $\frac{0}{0}$ & $\frac{0.00}{0.00}$ \\
\hline $\mathrm{F} 2 / \mathrm{D} 2$ & $\begin{array}{l}\text { E-Chem Reproce: Hd End, } \\
\text { Sep'n, \& Fuel Refaba }\end{array}$ & 11.351 & 5000 & 4.16 \\
\hline K3 & Repuranium Prod Dis & & 0 & \\
\hline G3 & $L W_{\text {cond }} \mathrm{pkg}: \mathrm{F} 2 / 1 \mathrm{D} 2$ & 0.036 & 16 & 0.01 \\
\hline G4-3E & \begin{tabular}{|l} 
LuW disposal: F2/D2 \\
Cs/SrrRb/Ra//2 C\&P:F2?DD2
\end{tabular} & $\begin{array}{l}0.028 \\
0.294\end{array}$ & 130 & $\begin{array}{l}0.01 \\
0.11\end{array}$ \\
\hline & Vol "offgas" C\&P: F2/D2 & 6 & 3 & 0.00 \\
\hline G1-2E & Hulls, etc C8PKg:F2/102 & 0.650 & 286 & 0.24 \\
\hline $\begin{array}{l}\text { E4 } \\
\text { G1 }\end{array}$ & |Mng'd Prod Dy Stg:F2:1D2 & 0.892 & 393 & $\begin{array}{l}0.33 \\
0.05\end{array}$ \\
\hline $\begin{array}{l}\text { G1 } \\
\text { G1 }\end{array}$ & 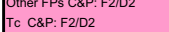 & $\begin{array}{l}0.149 \\
0.004\end{array}$ & & $\begin{array}{l}0.05 \\
0.00\end{array}$ \\
\hline & & 13.410 & $590 \overline{7}$ & $\frac{5.91}{4.91}$ \\
\hline L2/FP & $\begin{array}{l}\text { HNR Recyc ceo Ulsp } \\
\text { HLW Deep Geo Disp }\end{array}$ & 2.832 & 1247 & 1.04 \\
\hline L2/metal & HLW Metals (nuls, etc) & 1.203 & 530 & \\
\hline fleet kwh/y & & & & 1.48 \\
\hline $\begin{array}{l}3.25 \mathrm{E}+11 \\
\end{array}$ & \begin{tabular}{|l} 
Total ARR Fuel Cycle \\
\end{tabular} & 17.445 & 7685 & 6.39 \\
\hline \multicolumn{5}{|l|}{$8.77 \mathrm{E}+11$} \\
\hline osite & Fuel Cycle Cost for & & 8.22 & nills/kwh \\
\hline osi & Cost of Electricity & & $\frac{48.26}{2}$ & $\mathrm{~kW}$ \\
\hline
\end{tabular}




\section{A-4. 2-Tier Costs and Flows Worksheets}




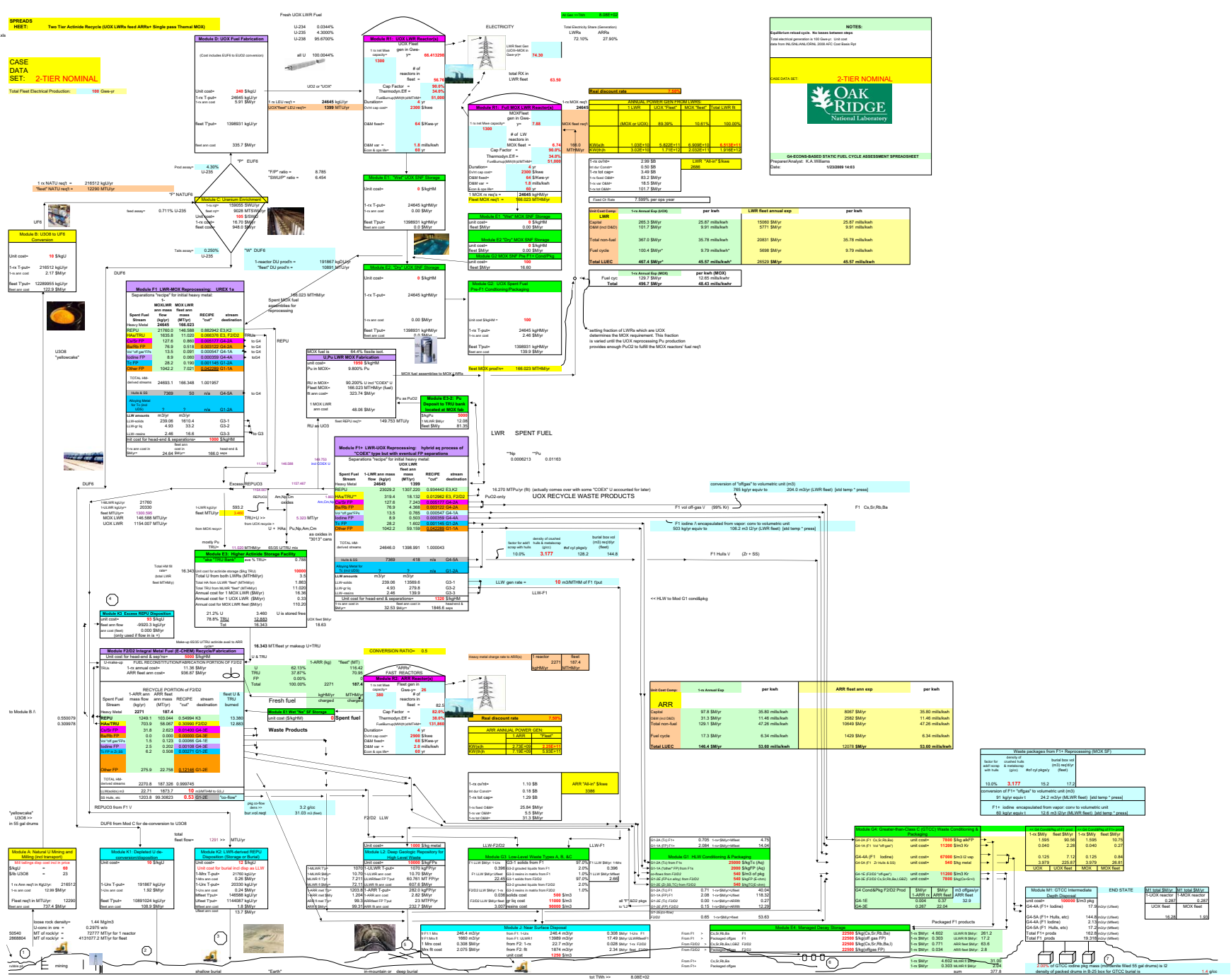


AFCI_2-TIER_Spreadsheet_NOM.xls

Power

Gen split Unit Cost Comp:

$66.41 \%$ UOX-LWRs

Capital

O\&M (incl D\&D)
Total non-fuel

1-rx Annual Exp

\begin{tabular}{l|l}
\hline per kwh & L \\
& \\
& \\
& \\
$9.87 \mathrm{mills} / \mathrm{kwh}$ &
\end{tabular}

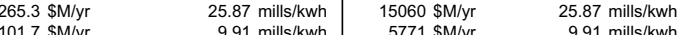

\begin{tabular}{lr|rr}
$101.7 \mathrm{\$ M} / \mathrm{yr}$ & $9.91 \mathrm{mills} / \mathrm{kwh}$ & $5771 \mathrm{\$ M} / \mathrm{yr}$ & $9.91 \mathrm{mills} / \mathrm{kwh}$ \\
$367.0 \mathrm{\$ M} / \mathrm{yr}$ & $35.78 \mathrm{mills} / \mathrm{kwh}$ & $20831 \mathrm{\$ M} / \mathrm{yr}$ & $35.78 \mathrm{mills} / \mathrm{kwh}$
\end{tabular}

Fuel cycle

$100.4 \mathrm{SM} / \mathrm{yr}$

35.78

35.78 mills/kwh

Total LUEC

$467.4 \$ \mathrm{M} / \mathrm{yr}$

$45.57 \mathrm{mills} / \mathrm{kwh}$

$\begin{array}{lr}5698 \$ \mathrm{M} / \mathrm{yr} & 9.79 \mathrm{mills} / \mathrm{kwh} \\ 26529 \mathrm{\$ M} / \mathrm{yr} & \mathbf{4 5 . 5 7} \mathrm{mills} / \mathrm{kwh}\end{array}$

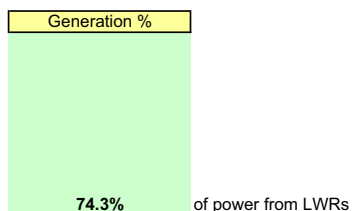

\subsection{8\% MOX-LWRs}

$\begin{array}{ll}\text { MOX-LWRS } & \\ \text { Capital } & 265.3 \$ \mathrm{M} / \mathrm{yr}\end{array}$ $129.7 \$ M / y r$

Fuel cycle

$129.7 \$ M / y r$

per kwh

LWR fleet ann exp

per kwh

Total LUEC

$259.4 \$ M / y r$

$25.87 \mathrm{mills} / \mathrm{kwh}$ 9.91 mills/kwh $35.78 \mathrm{mills} / \mathrm{kwh}$

$1787 \$ \mathrm{\$ M} / \mathrm{yr} \quad 25.87 \mathrm{mills} / \mathrm{kwh}$

$2472 \mathrm{SM} / \mathrm{yr} \quad \quad 9.91 \mathrm{mills} / \mathrm{kwh}$

$12.65 \mathrm{mills} / \mathrm{kwh}$

874 SM/yr

$35.78 \mathrm{mills} / \mathrm{kwh}$

$48.43 \mathrm{mills} / \mathrm{kwh}$

$3346 \$$ M/yr

$48.43 \mathrm{mills} / \mathrm{kwh}$

$74.3 \%$

of power from LWRs

\begin{tabular}{|c|c|c|}
\hline \multicolumn{2}{|r|}{ Unit Cost Comp: } & \multirow{2}{*}{ 1-rx Annua } \\
\hline $25.70 \%$ & ARRs & \\
\hline & $\begin{array}{l}\text { Capital } \\
\text { O\&M (incl D\&D) }\end{array}$ & $\begin{array}{l}97.8 \text { \$I } \\
31.3 \$ 1\end{array}$ \\
\hline & Total non-fuel & 129.1 \$ \\
\hline & Fuel cycle & $17.3 \$ 1$ \\
\hline & Total LUEC & $146.4 \$ 1$ \\
\hline \multicolumn{3}{|l|}{$100.00 \%$} \\
\hline & Fleet Average & mills/kwh \\
\hline & Cap & 28.42 \\
\hline & O\&M (Incl D\&D) & 10.31 \\
\hline & Total Non-fuel & 38.73 \\
\hline & Fuel Cycle & 9.13 \\
\hline & Total & 47.86 \\
\hline & Once -Through FC & 6.51 \\
\hline
\end{tabular}

\begin{tabular}{|l|rr|}
\hline per kwh & ARR fleet ann exp & per kwh \\
& & \\
$35.80 \mathrm{mills} / \mathrm{kwh}$ & $8067 \$ \mathrm{M} / \mathrm{yr}$ & $35.80 \mathrm{mills} / \mathrm{kwh}$ \\
$11.46 \mathrm{mils} / \mathrm{kwh}$ & $2582 \$ \mathrm{M} / \mathrm{yr}$ & $11.46 \mathrm{mills} / \mathrm{kwh}$ \\
$47.26 \mathrm{mills} / \mathrm{kwh}$ & $10649 \$ \mathrm{M} / \mathrm{yr}$ & $47.26 \mathrm{mills} / \mathrm{kwh}$ \\
& & \\
$6.34 \mathrm{mills} / \mathrm{kwh}$ & $1429 \$ \mathrm{M} / \mathrm{yr}$ & $6.34 \mathrm{mills} / \mathrm{kwh}$ \\
$\mathbf{5 3 . 6 0} \mathrm{mills} / \mathbf{k w h}$ & $12078 \$ \mathrm{M} / \mathrm{yr}$ & $\mathbf{5 3 . 6 0} \mathrm{mills} / \mathbf{k w h}$
\end{tabular}
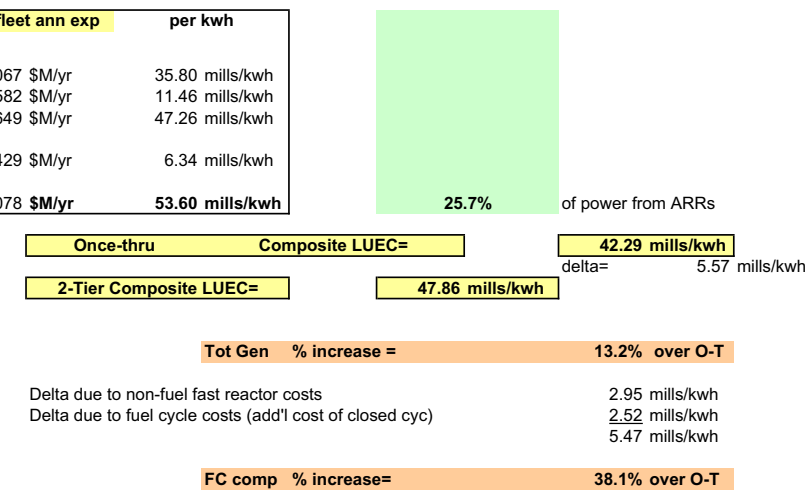


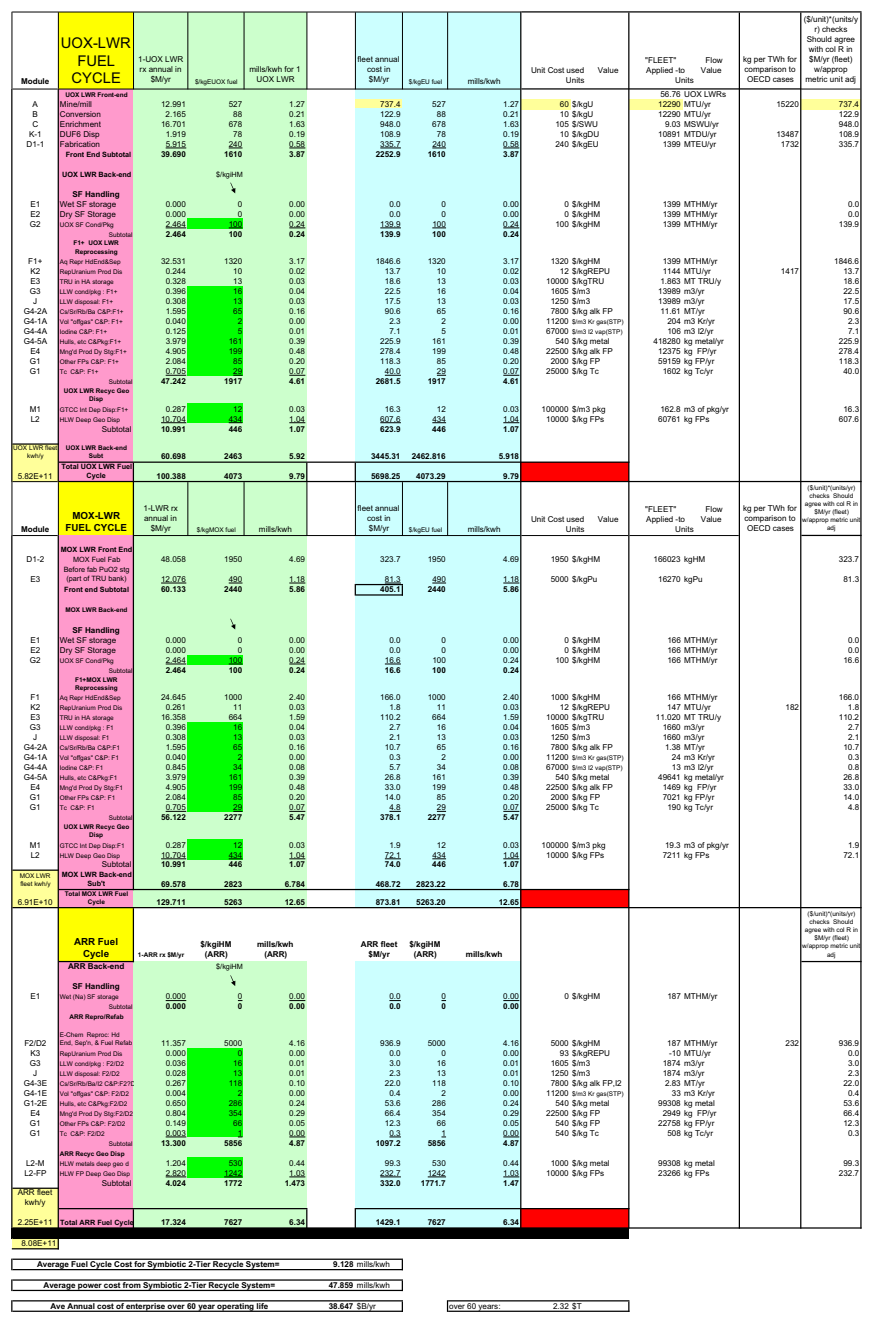




\section{Attachment B}

\section{Dynamic Analysis of AFCI Nominal Growth Cases}

This attachment provides an example of nominal cost analyses using VISION and VISION.ECON under dynamic growth scenarios. These analyses are complementary to the analysis being performed under the AFCI Analysis and Integration task. Each analysis compares the once-through, 1-Tier, and 2-Tier fuel cycles. A typical growth case scenario is at $1.2 \%$ growth. For the 1-Tier case, the initial reactors are light-water reactors (LWRs) using uranium oxide fuel (UOX) then switching to Fast Reactors at a 0.50 conversion ratio. The 2Tier case is LWRs moving to LWRs using a mixed oxide fuel (MOx) and then switching to fast reactors at a 0.50 conversion ratio. The difference in fuel cycle costs and total cost of electricity between the once-through, 1-Tier, and 2-Tier can be compared annually. These modeling capabilities are used to produce the results shown in Section 6.6 and the comparisons to the G4 ECONS static results provided in Section 9. 


\section{B-1. VISION.ECON Graphical Outputs}



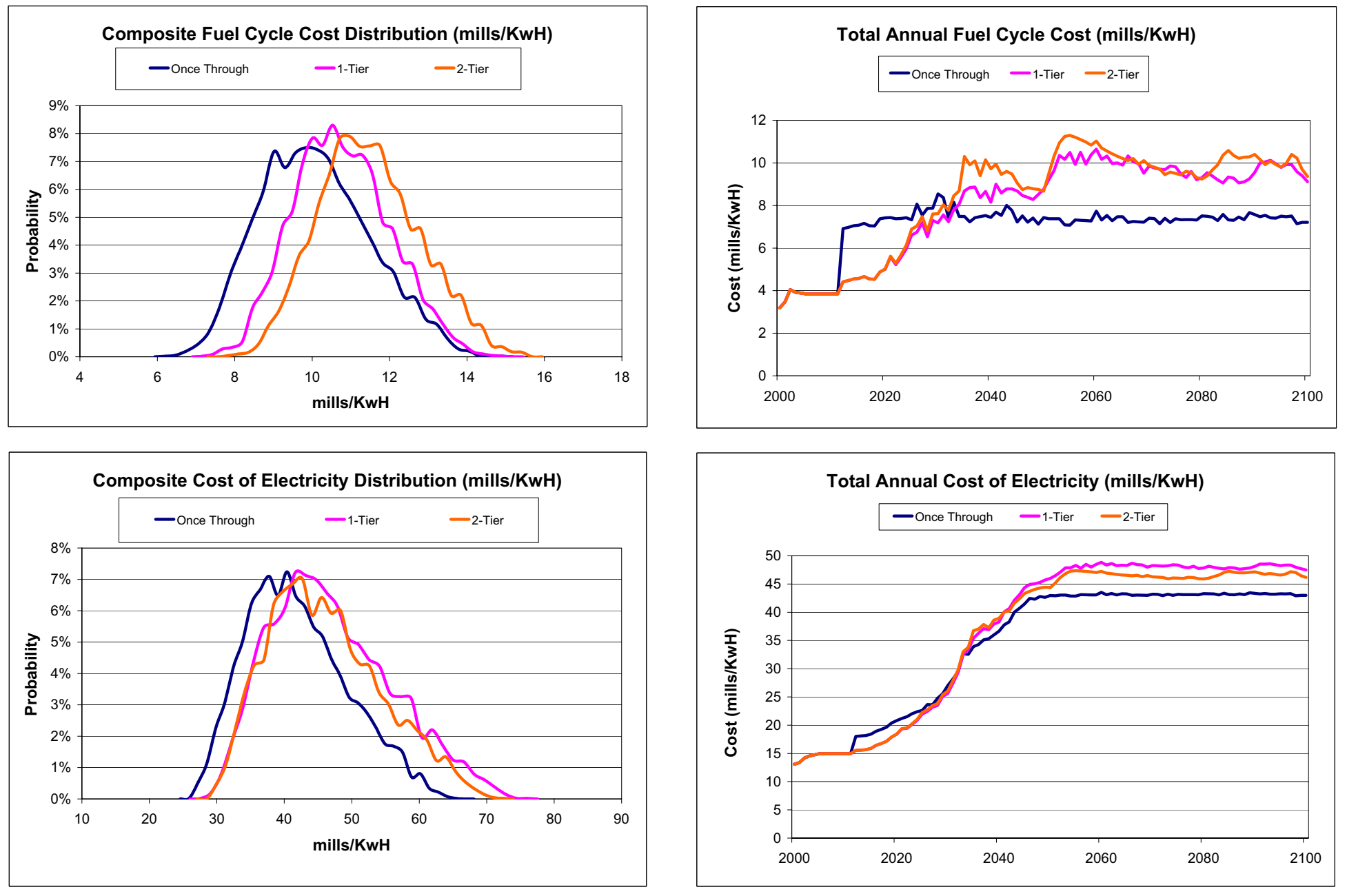

5 Run Comparative Graphs,econ data Output ver2-NominalEcon FORMATED2.xls 

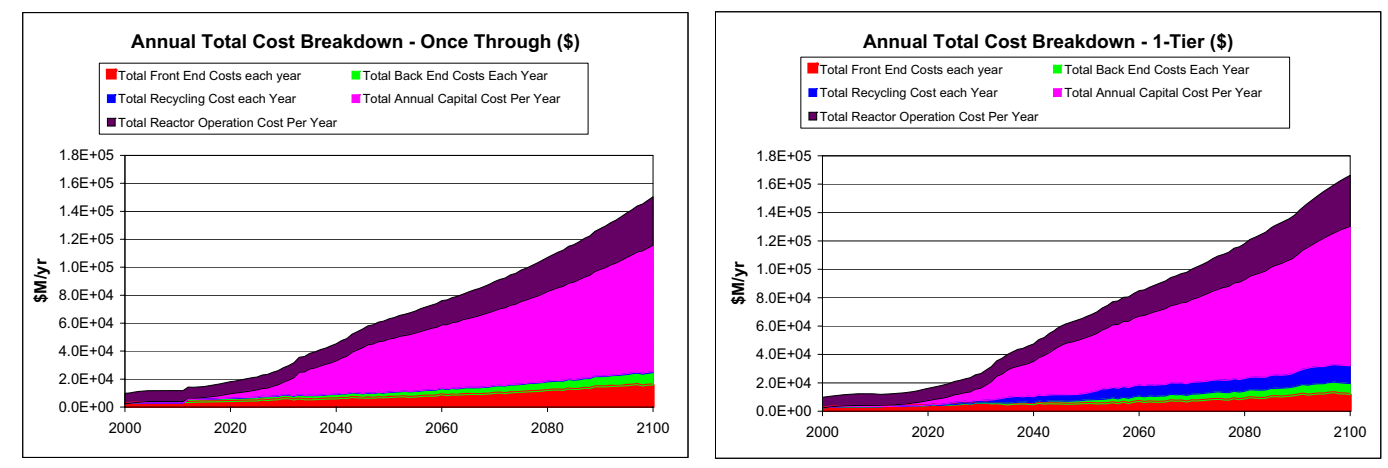

Annual Unit Cost Breakdown - Once Through (mills/KwH)

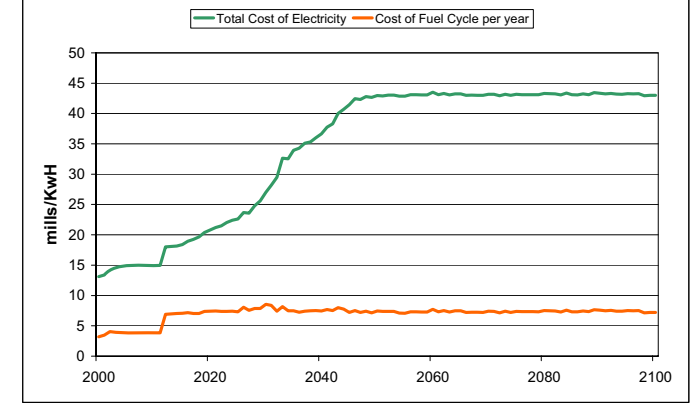

Annual Unit Cost Breakdown - 1-Tier (mills/KwH) -Total Cost of Electricity —Cost of Fuel Cycle per year

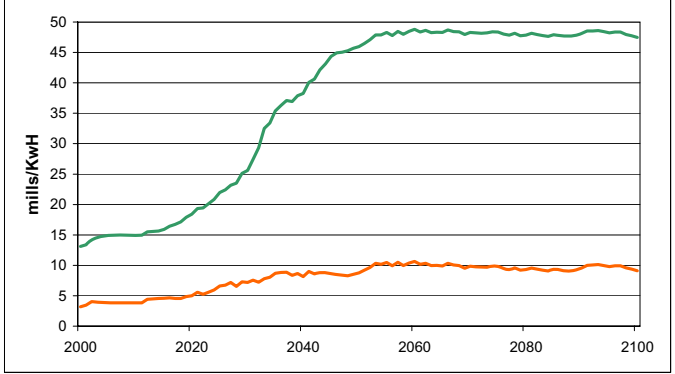

Annual Total Cost Breakdown - 2-Tier (\$) - Total Front End Costs each year $\quad$ Total Back End Costs Each Year
- Total Recycling Cost each Year - Total Recycling Cost each Year - Total Reactor Operation Cost Per Year

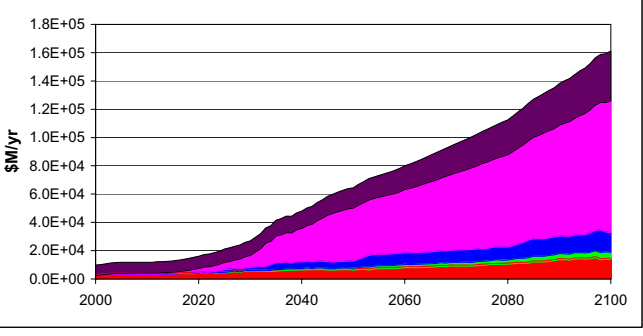

Annual Unit Cost Breakdown - 2-Tier (mills/KwH) -Total Cost of Electricity - Cost of Fuel Cycle per year

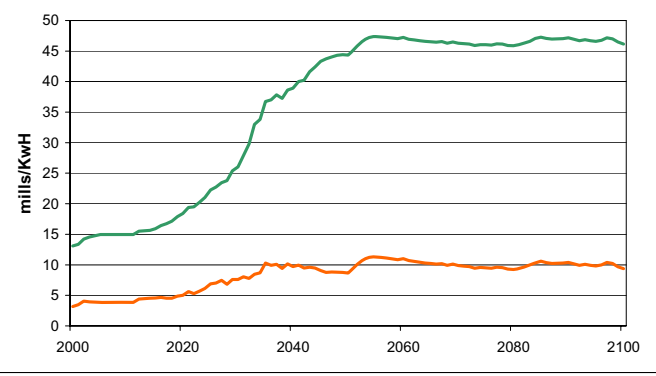



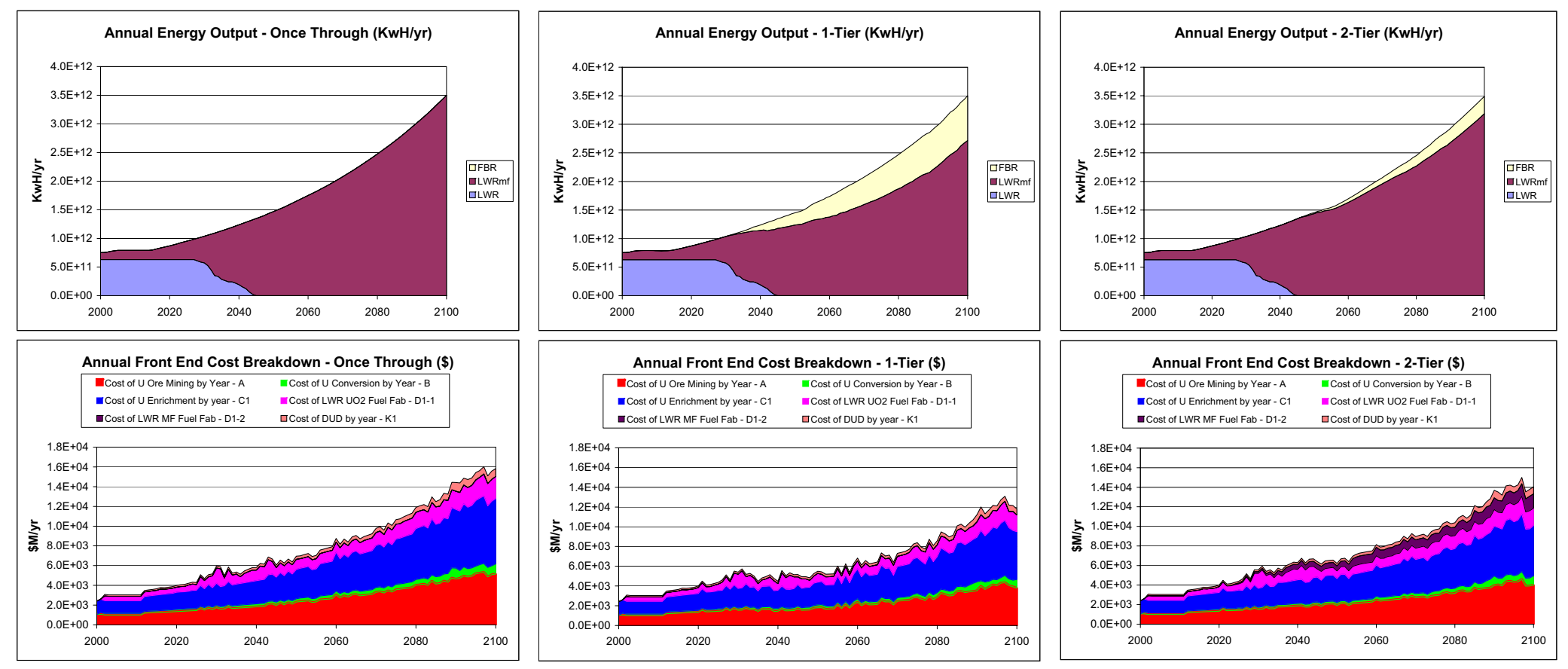


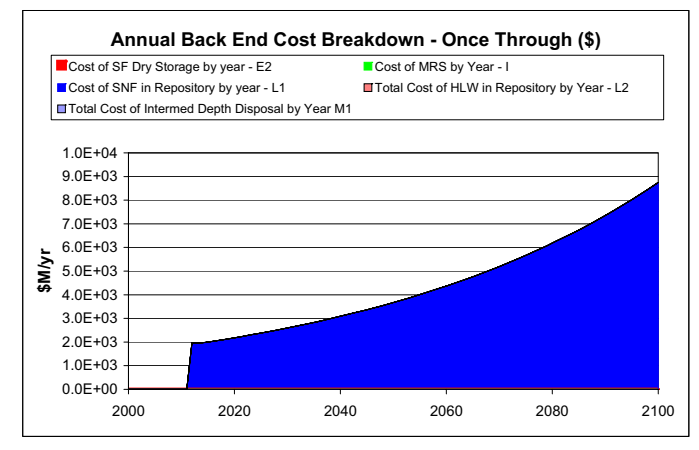

Annual Recycle Cost Breakdown - Once Through (\$) \begin{tabular}{|ll|}
\hline MAqueous UOX Separations Cost - F1 & पAqueous MOX Separations Cost - F1 \\
$\begin{array}{l}\text { EEchem Separations Cost F2D2 } \\
\text { ETotal Cost of Conditioning - G }\end{array}$ & व Total Cost Recycled Product Storage E3 \\
\hline
\end{tabular}

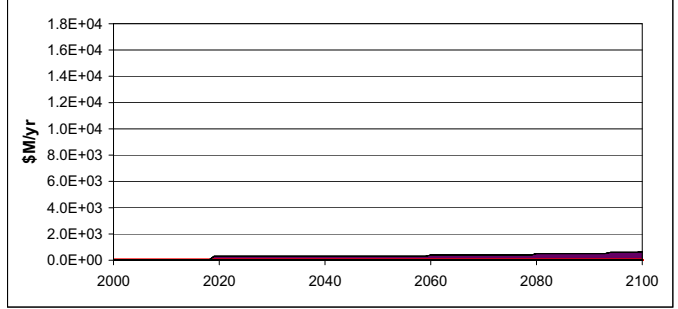

Annual Back End Cost Breakdown - 1-Tier (\$) Cost of SF Dry Storage by year - E2 2 - Cost of SNF in Repository by year - L1 पT Total Cost of HLW in Repository by Year - L2 $\square$ Total Cost of Intermed Depth Disposal by Year $\mathrm{M}$
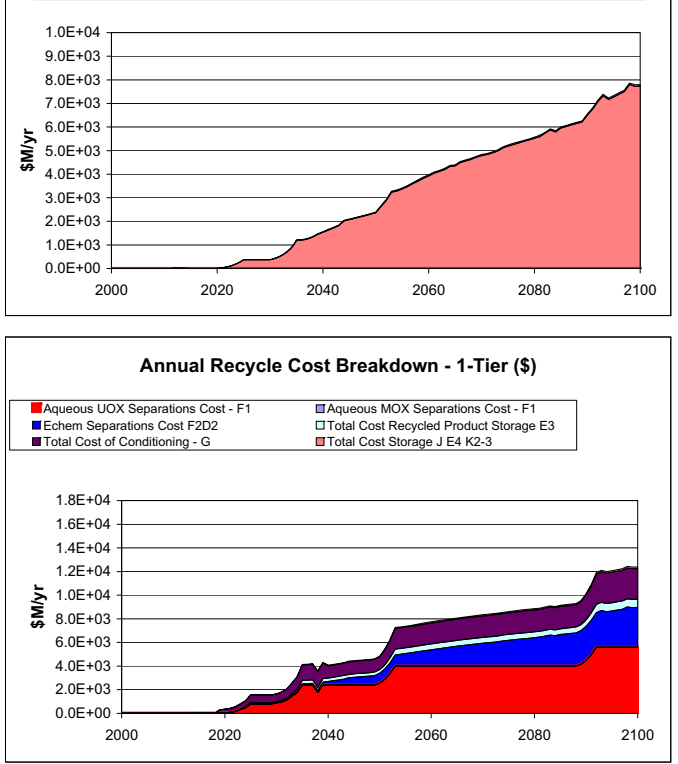

Annual Back End Cost Breakdown - 2-Tier (\$)

\begin{tabular}{ll}
\hline Cost of SF Dry Storage by year - E2 & Cost of MRS by Year - I \\
- Cost of SNF in Repository by year - L1 & प Total Cost of HLW in Repository by Year - L2
\end{tabular} पTotal Cost of Intermed Depth Disposal by Year $M$
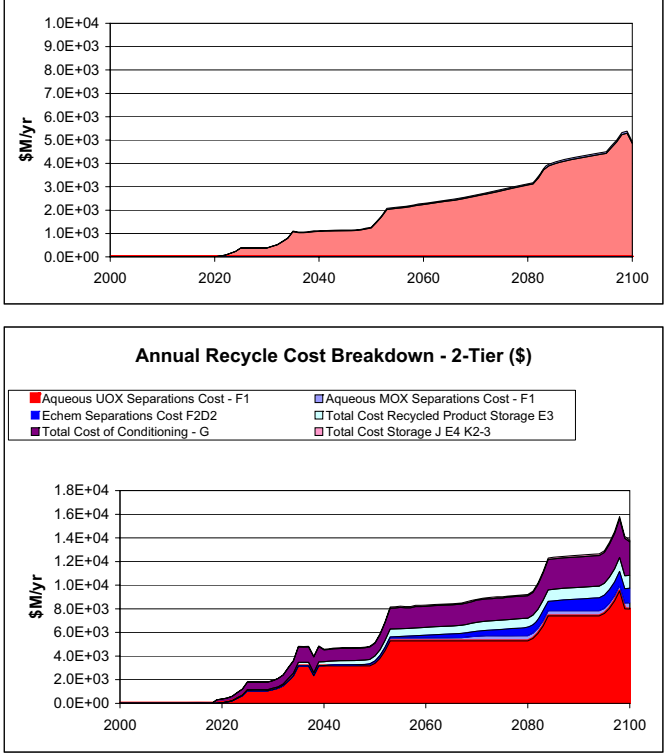


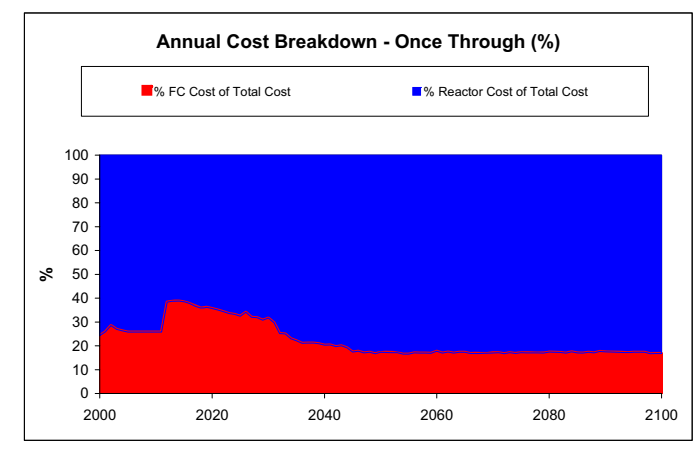

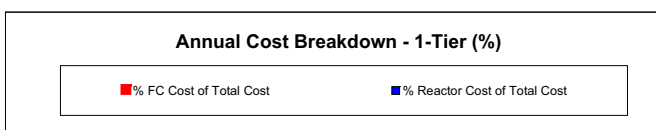
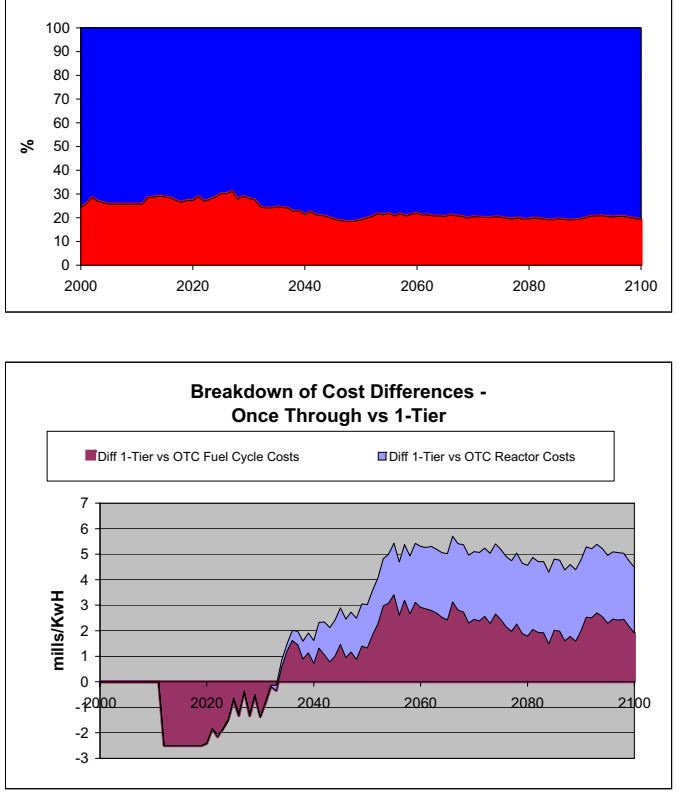
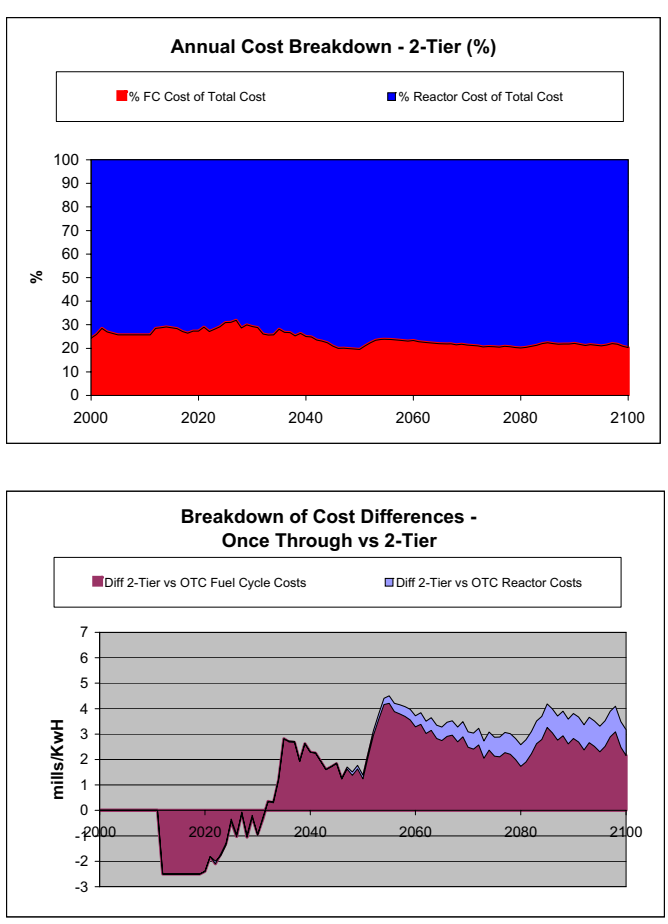


\section{B-2. VISION.ECON Module Outputs}



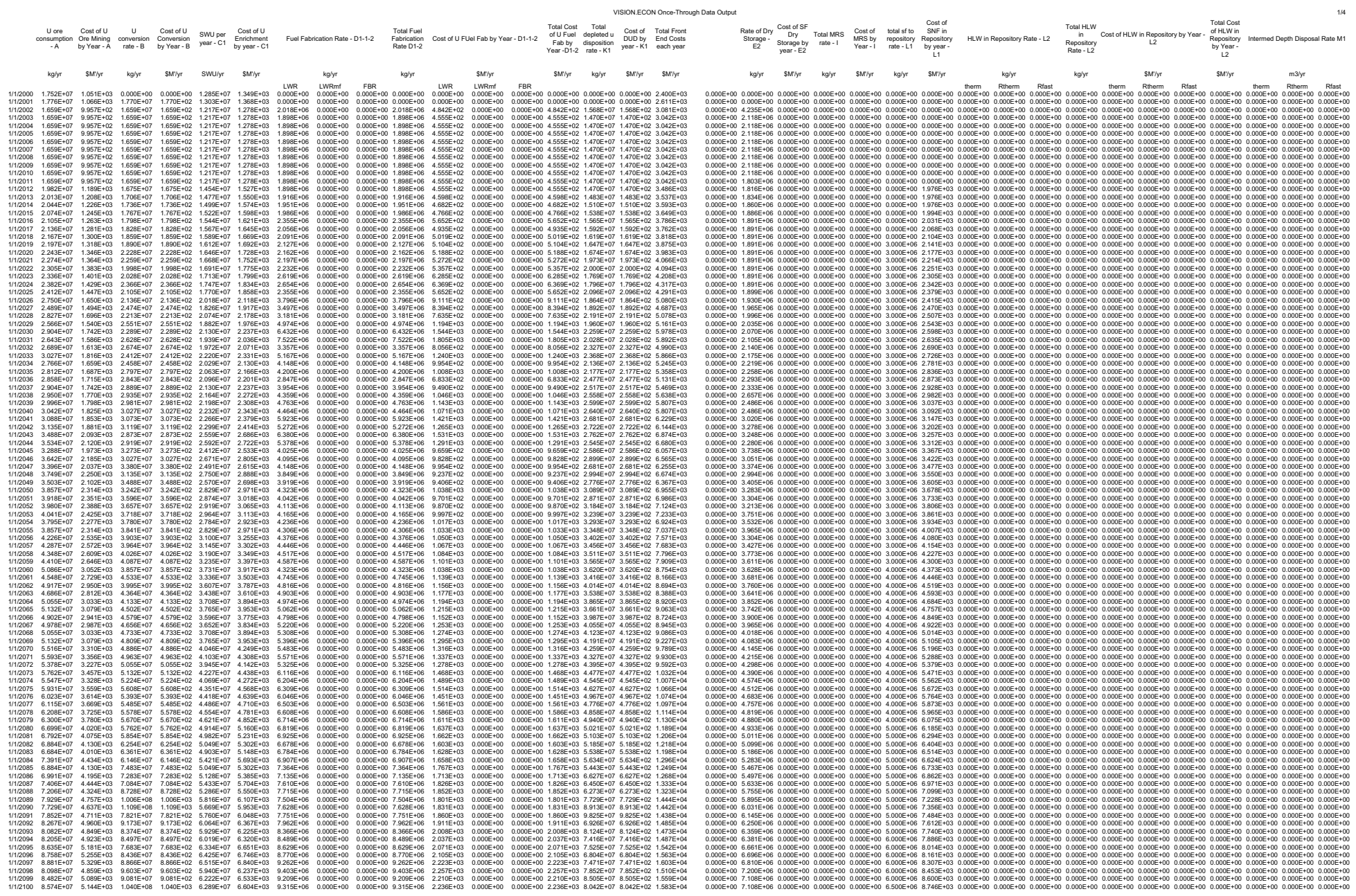


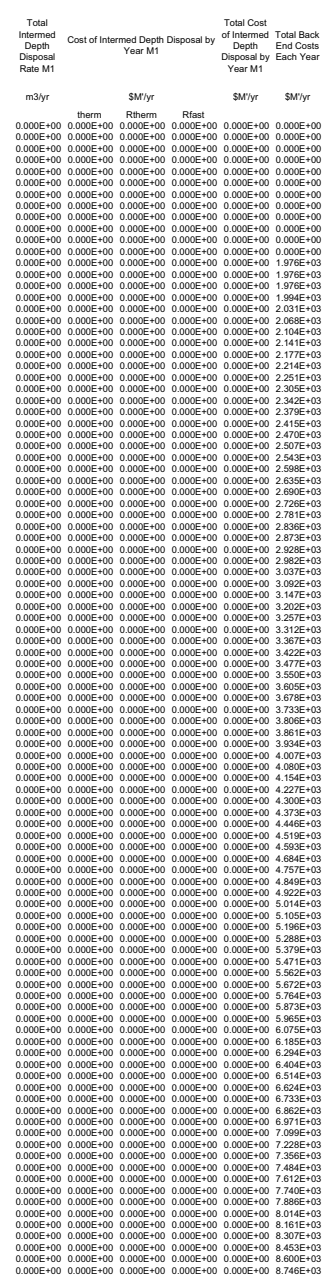

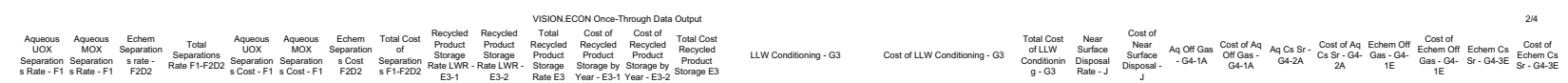

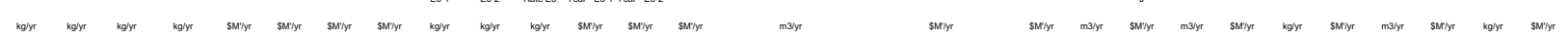

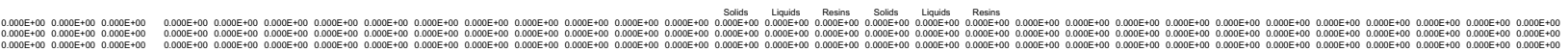

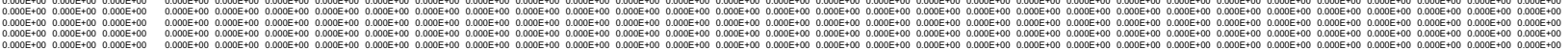

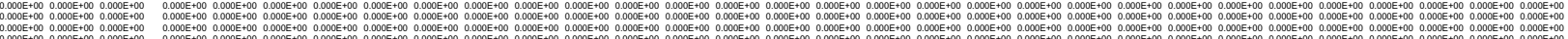

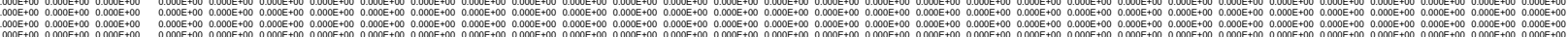

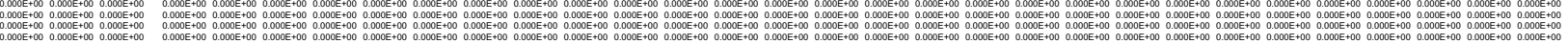

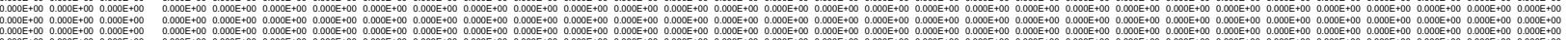

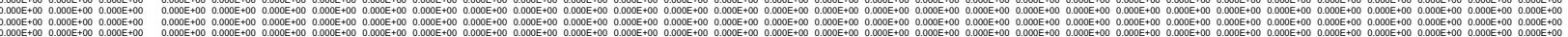

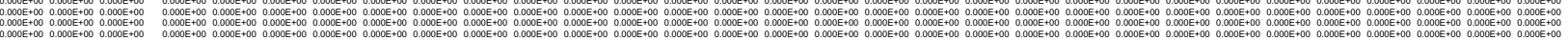

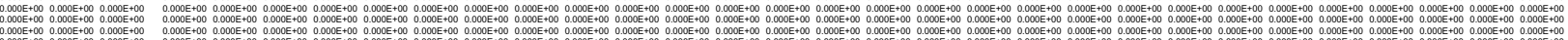

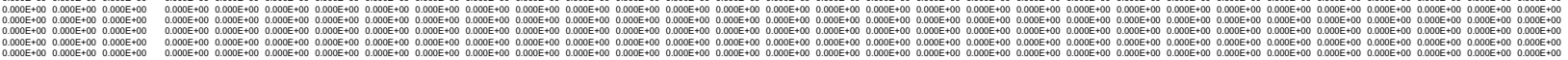

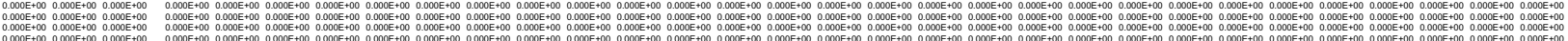

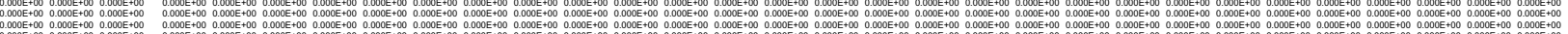

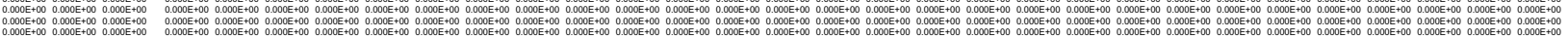

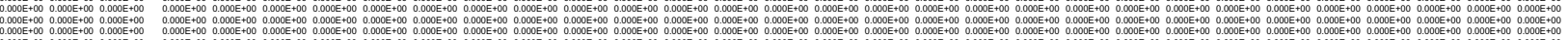

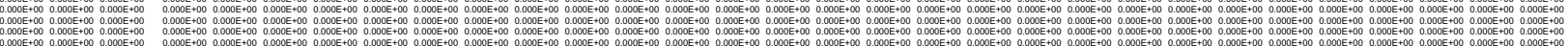

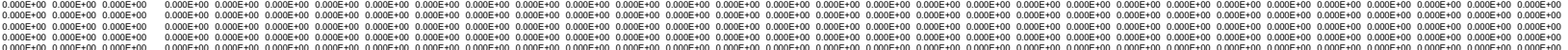

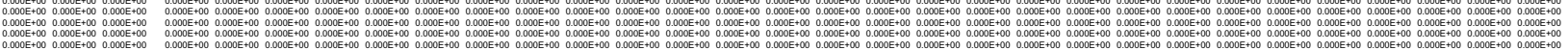

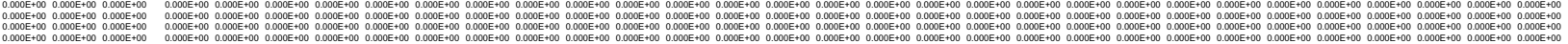

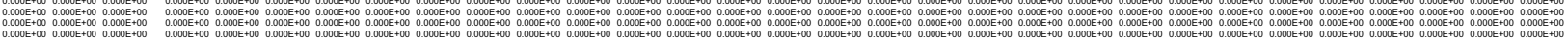

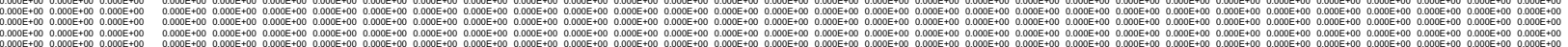

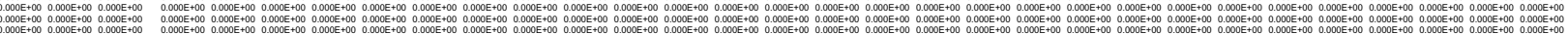

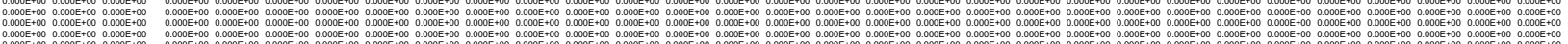

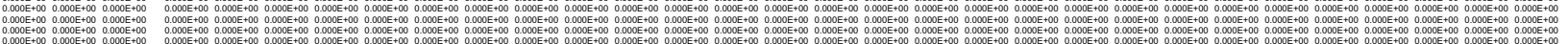

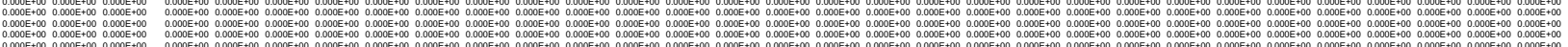

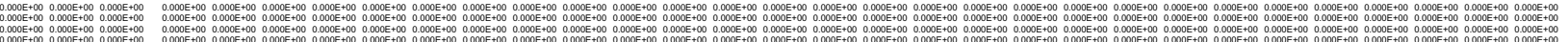

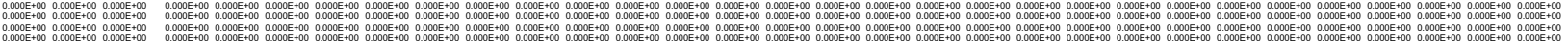
1
and

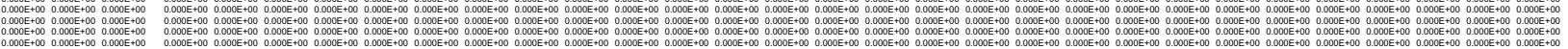

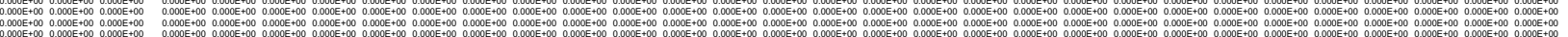

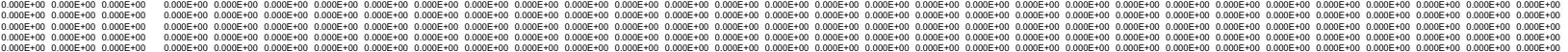

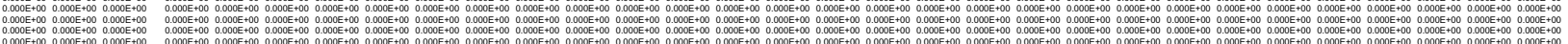

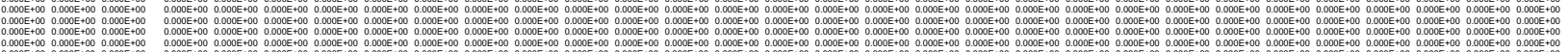

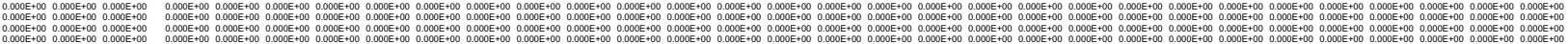

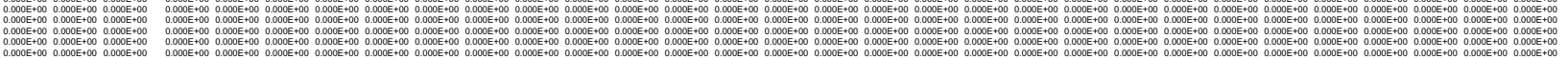



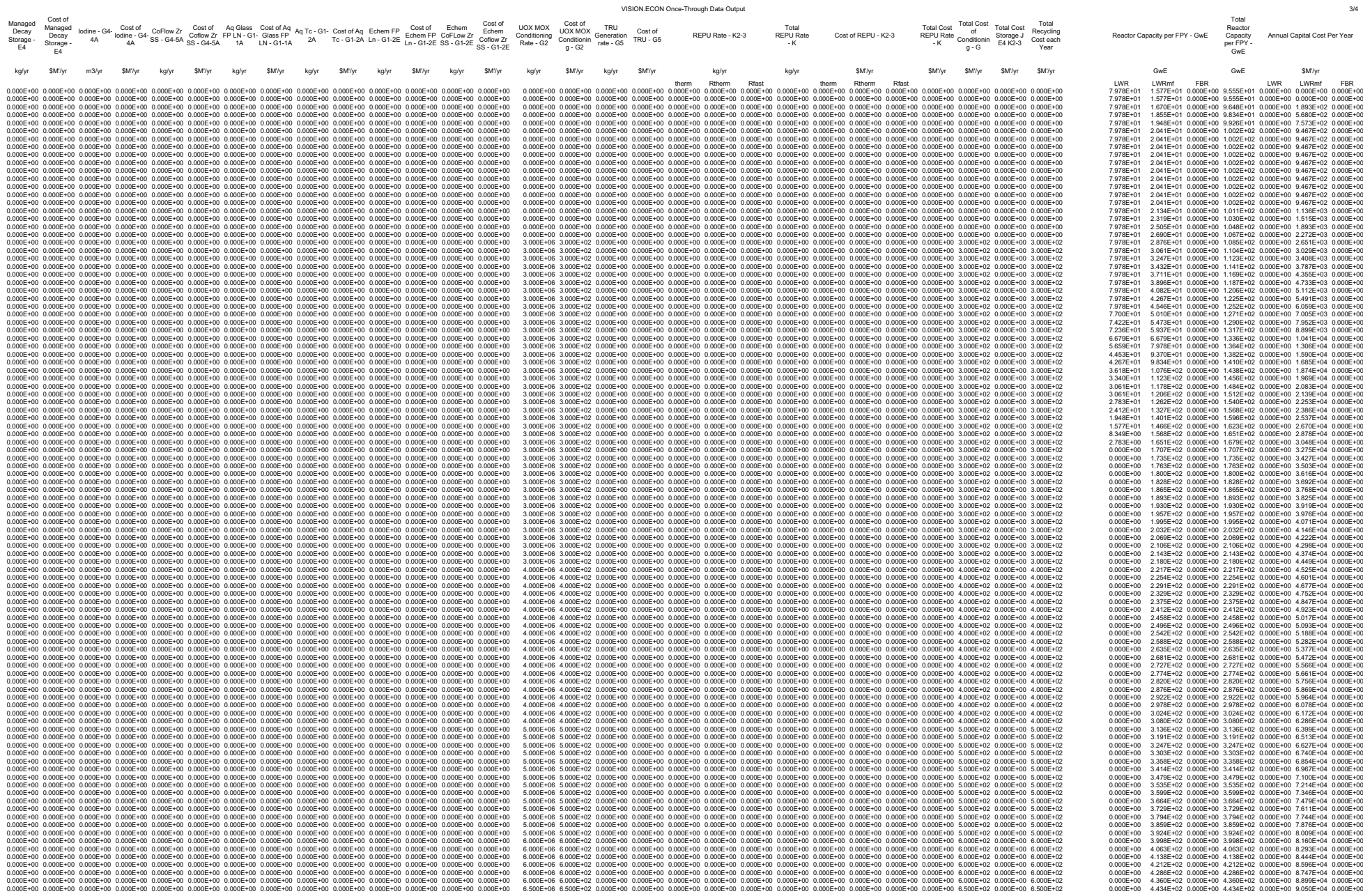

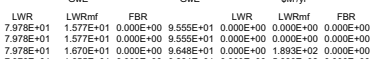

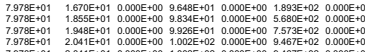

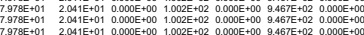

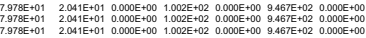

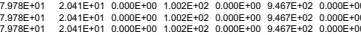

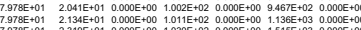

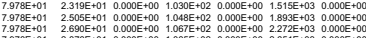

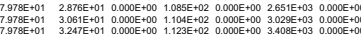

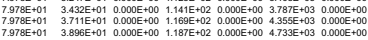

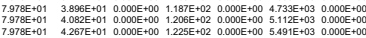

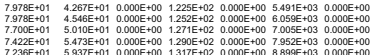

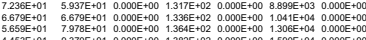

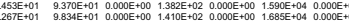

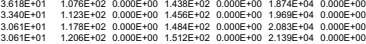

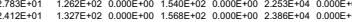

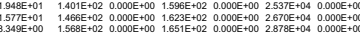

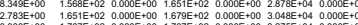

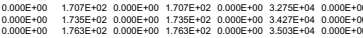

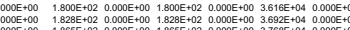
C.

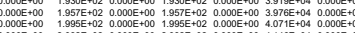

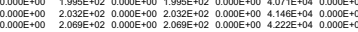

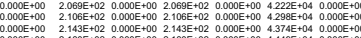

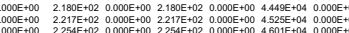

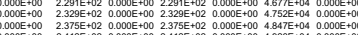

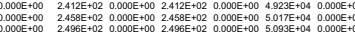

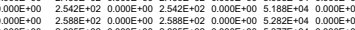

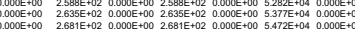

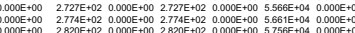

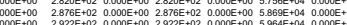

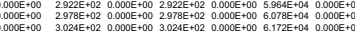

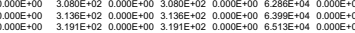

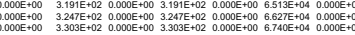

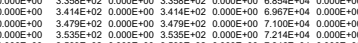

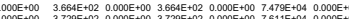

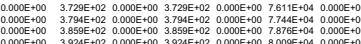

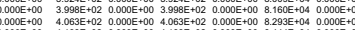

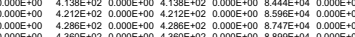

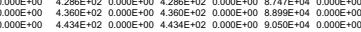



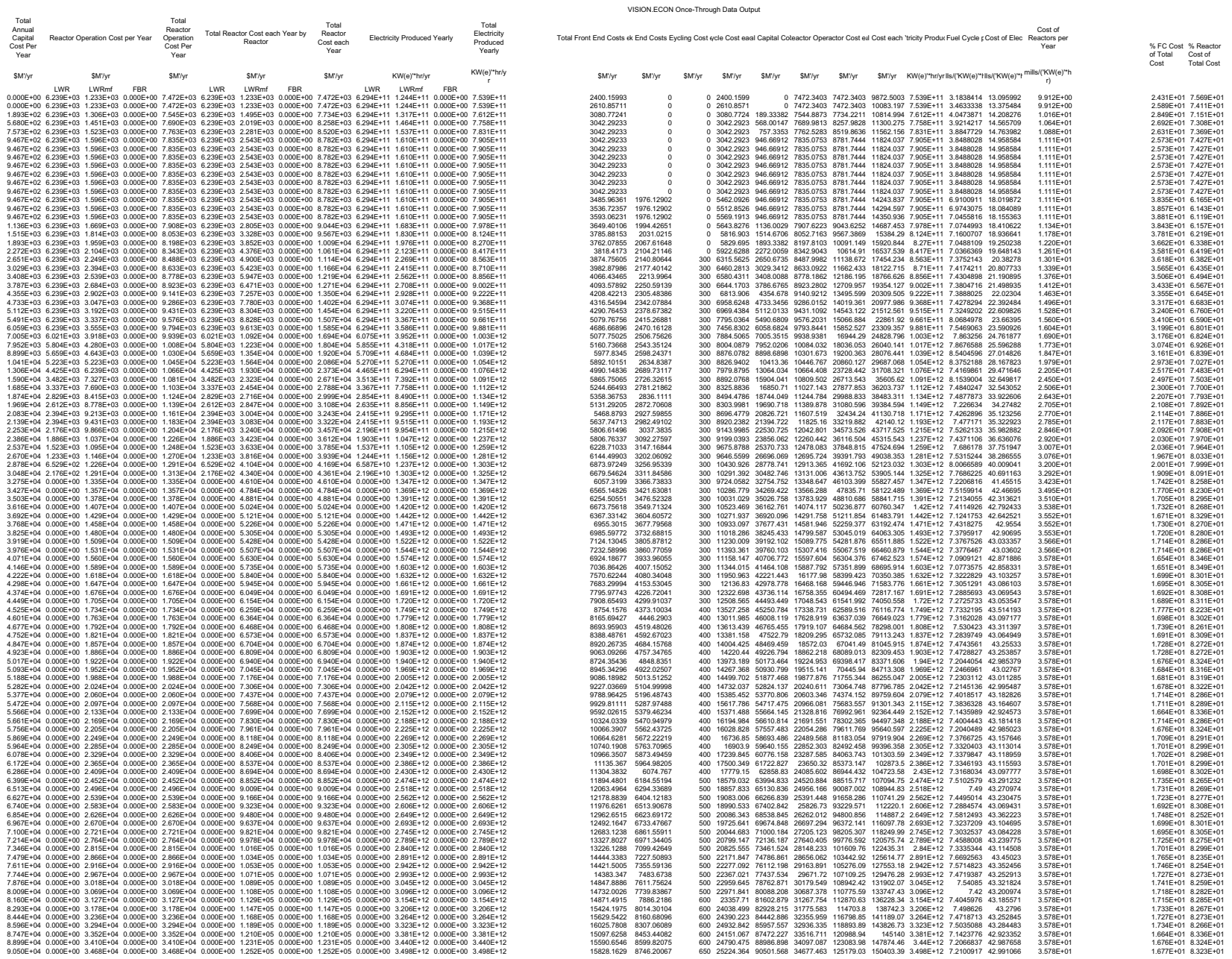

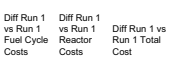

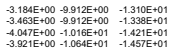

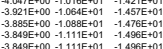

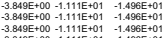

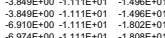

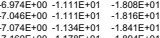

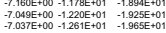

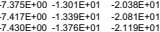

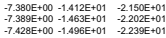

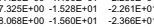

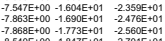

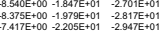

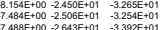

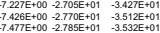

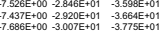

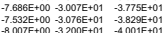

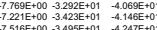

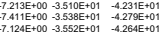

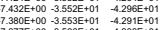

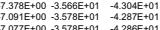

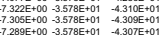

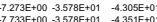

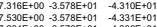

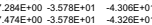

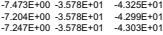

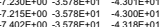

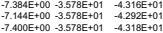

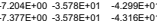

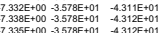

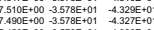

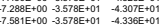

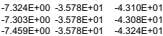

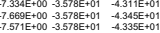

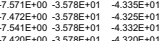

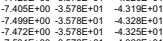

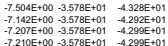




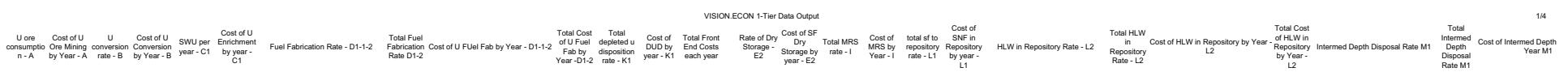

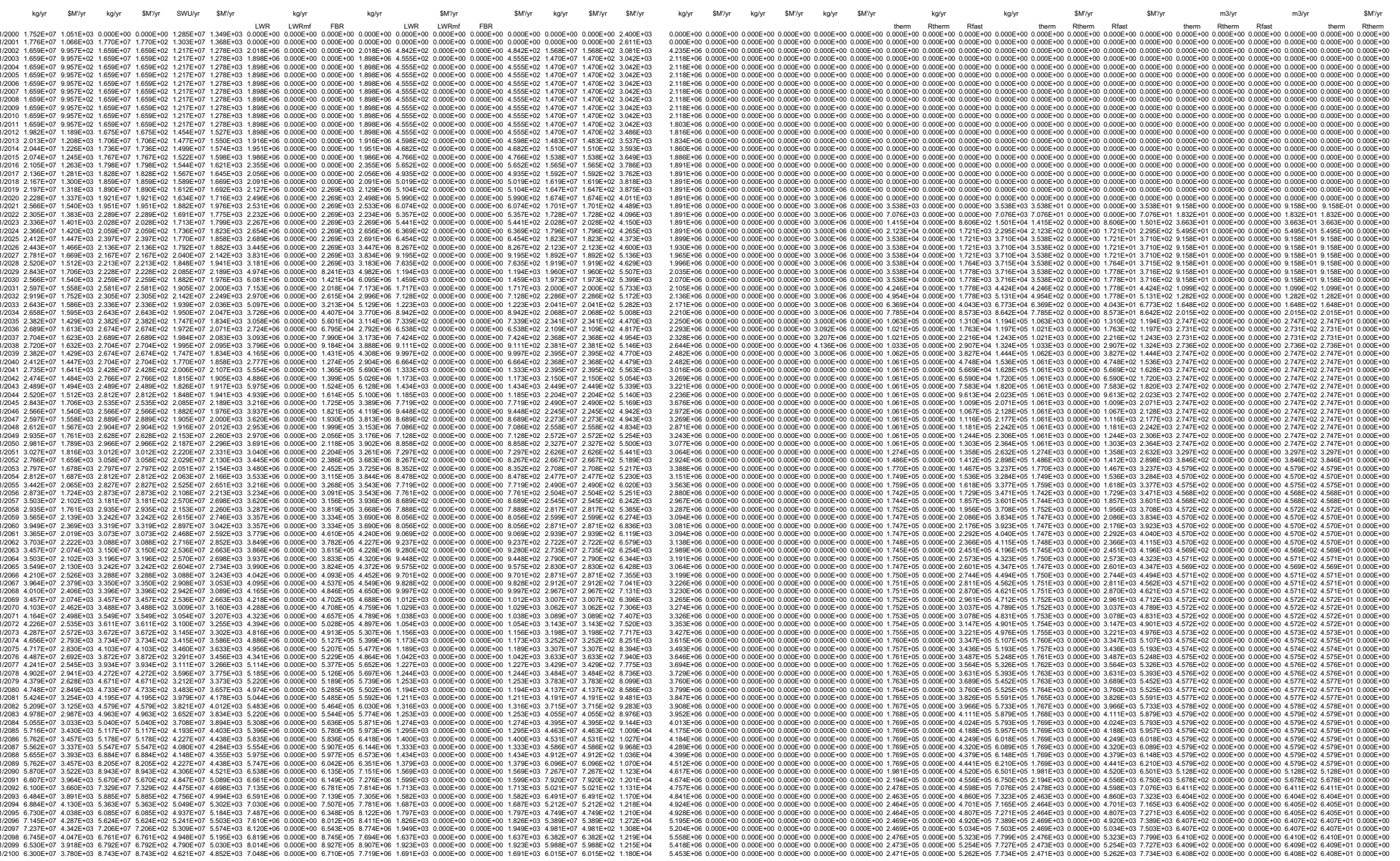




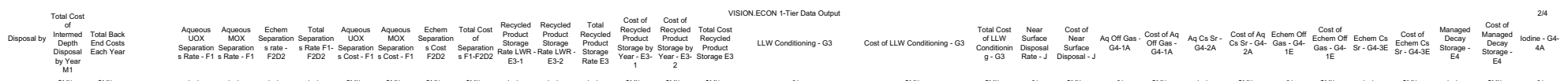

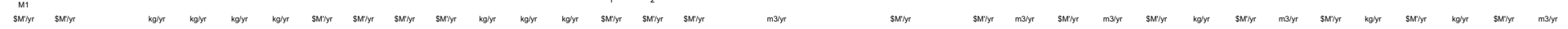

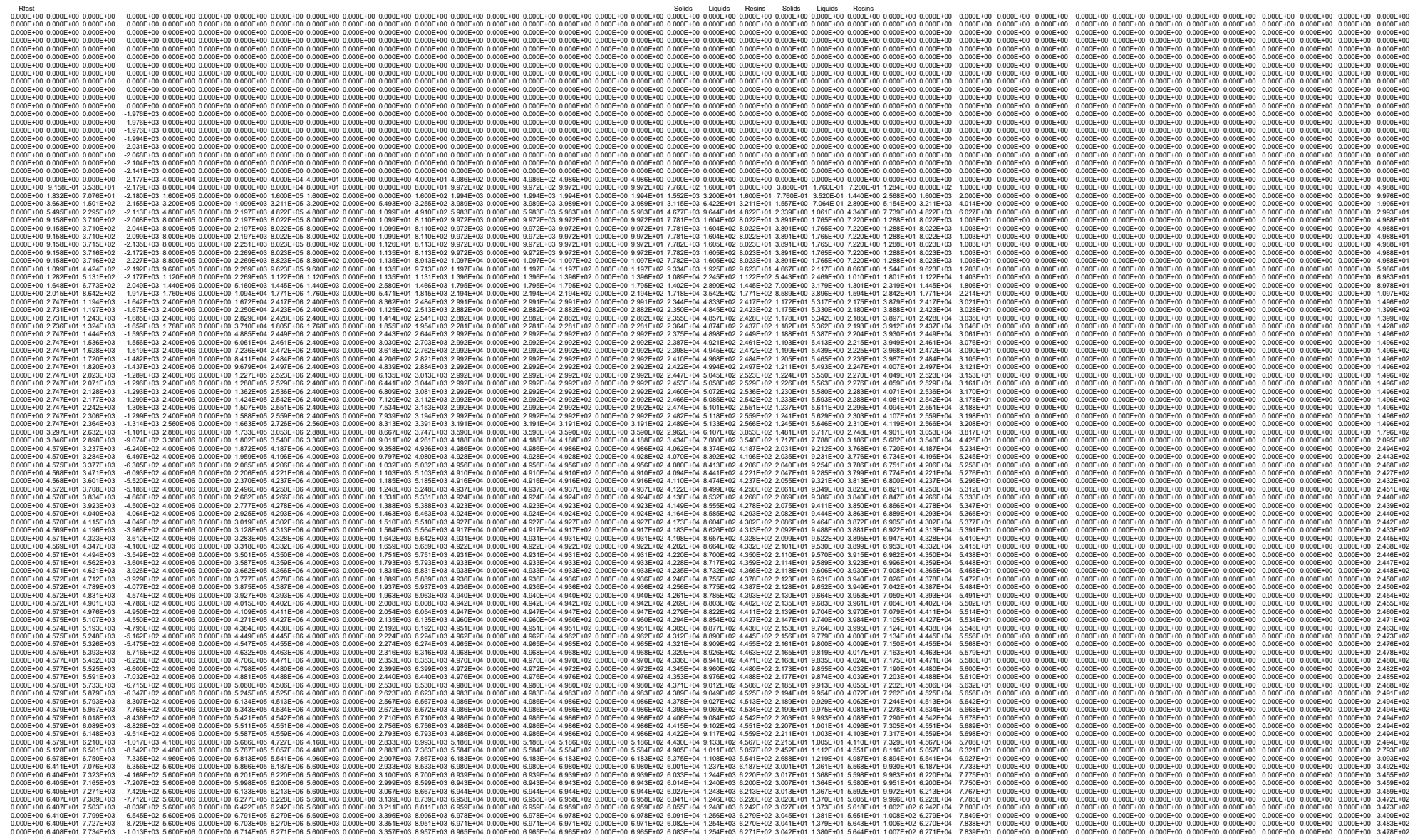




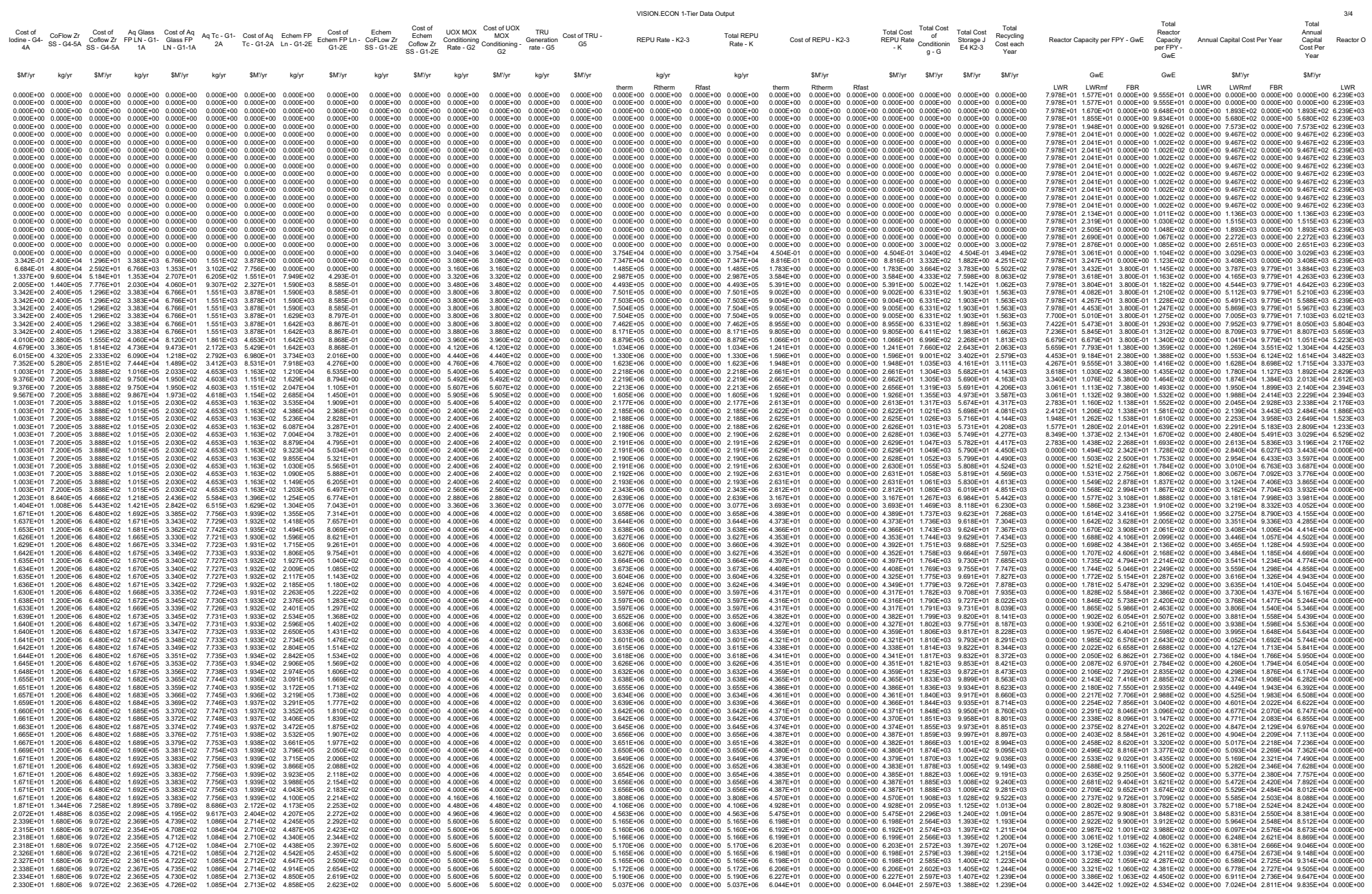




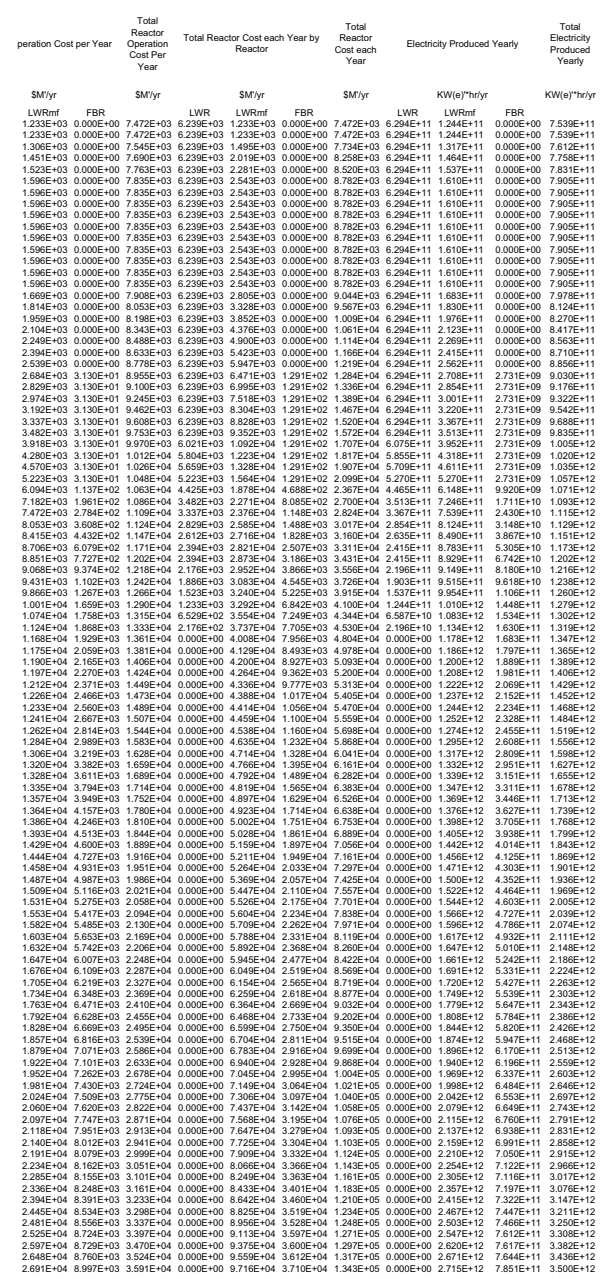

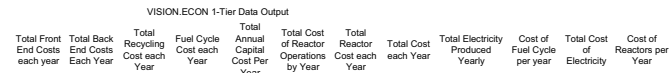

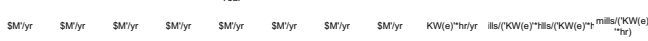

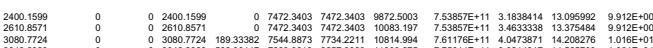

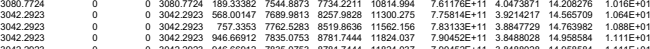

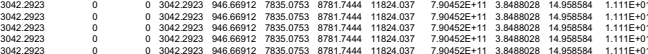

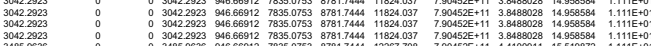

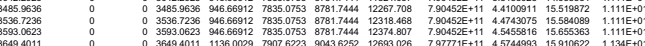

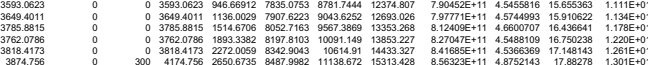

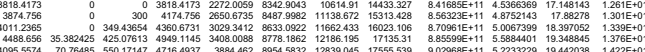

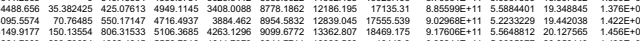

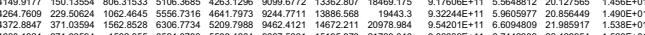

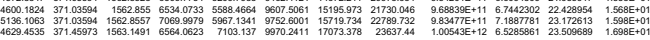

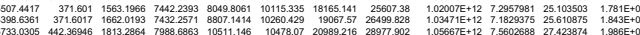

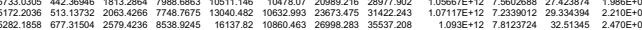

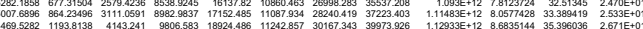

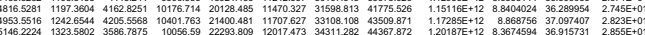

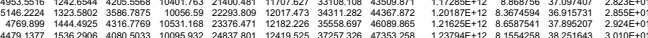

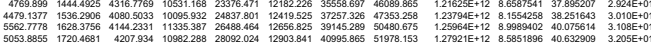

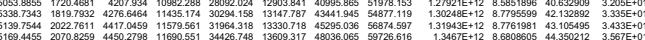

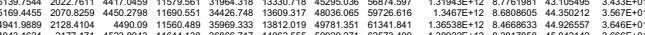

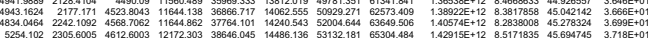

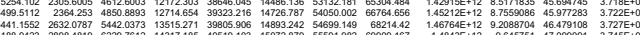

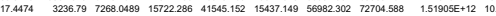

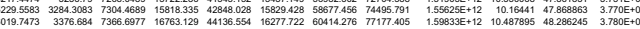

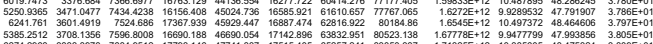

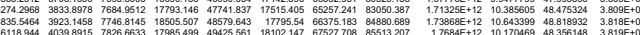

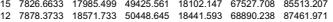

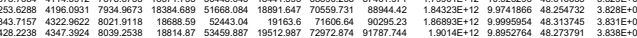

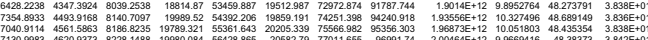

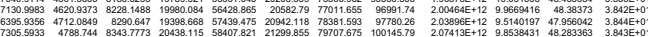

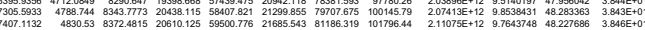

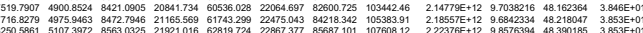

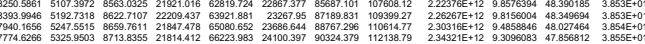

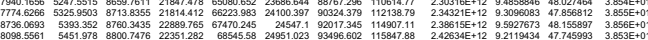

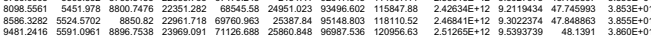

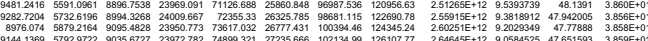

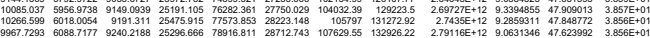

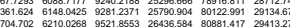

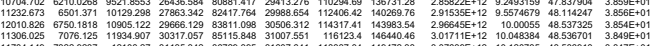

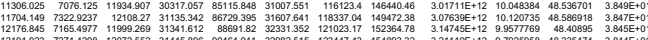

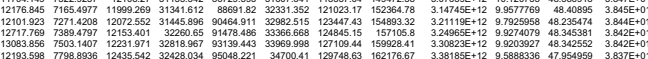

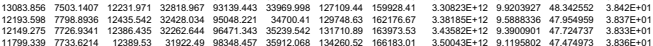

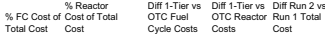

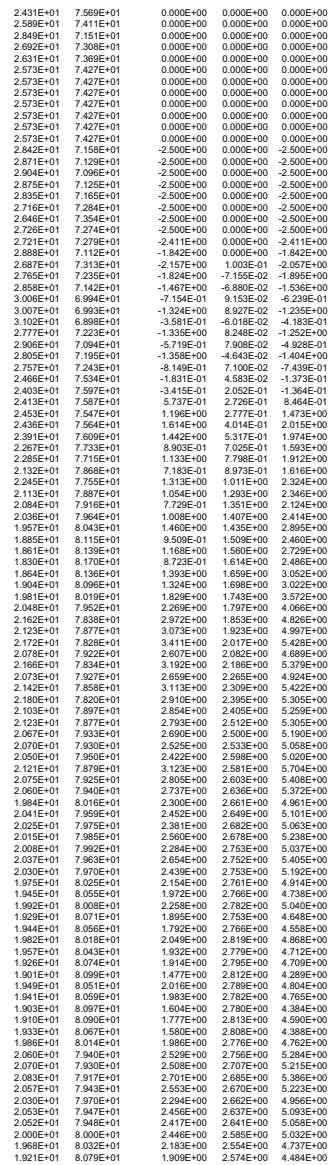




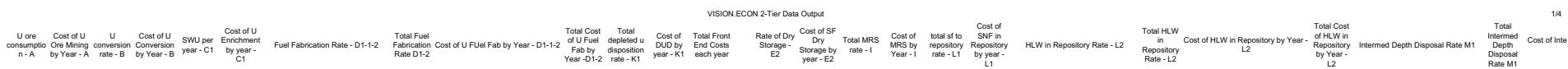

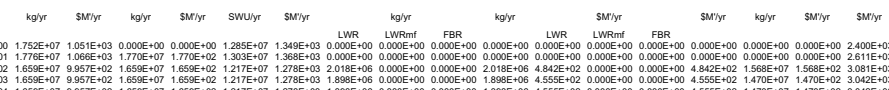

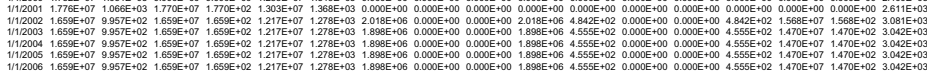

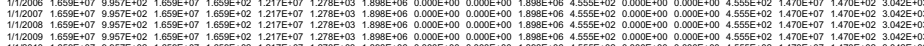

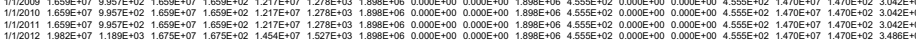

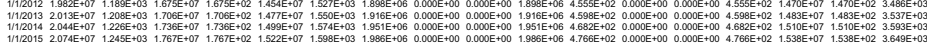

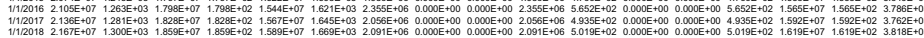

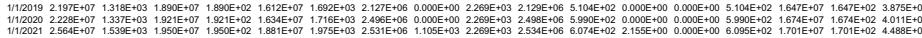

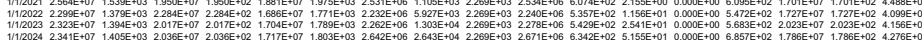

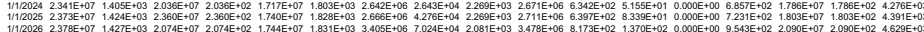

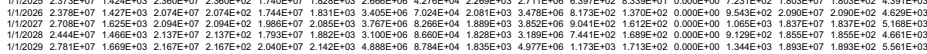

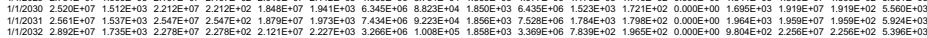

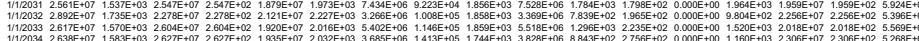

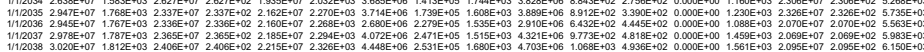

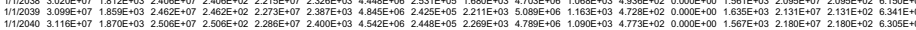

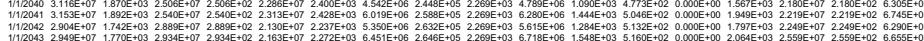

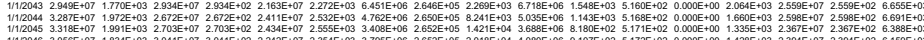

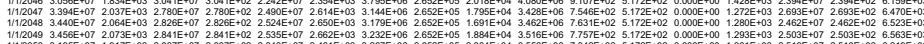

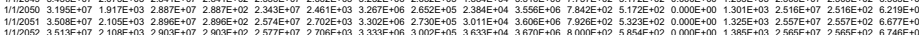

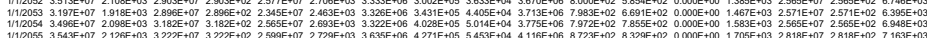

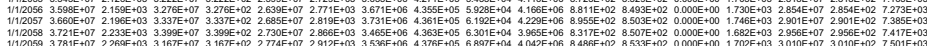

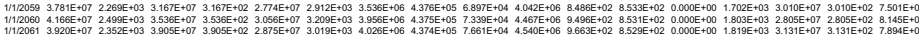

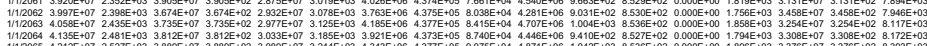

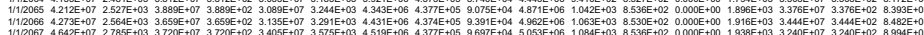

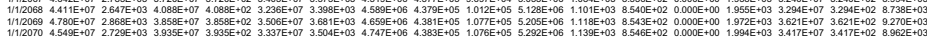

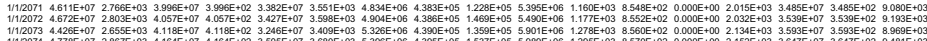

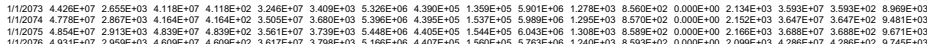

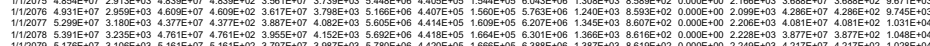

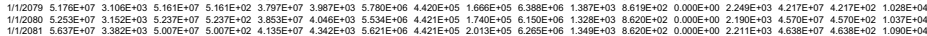

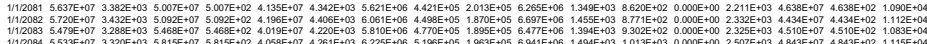

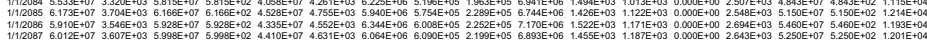

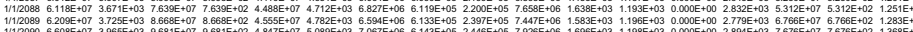

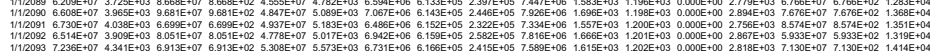

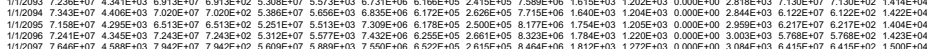

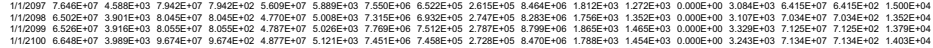




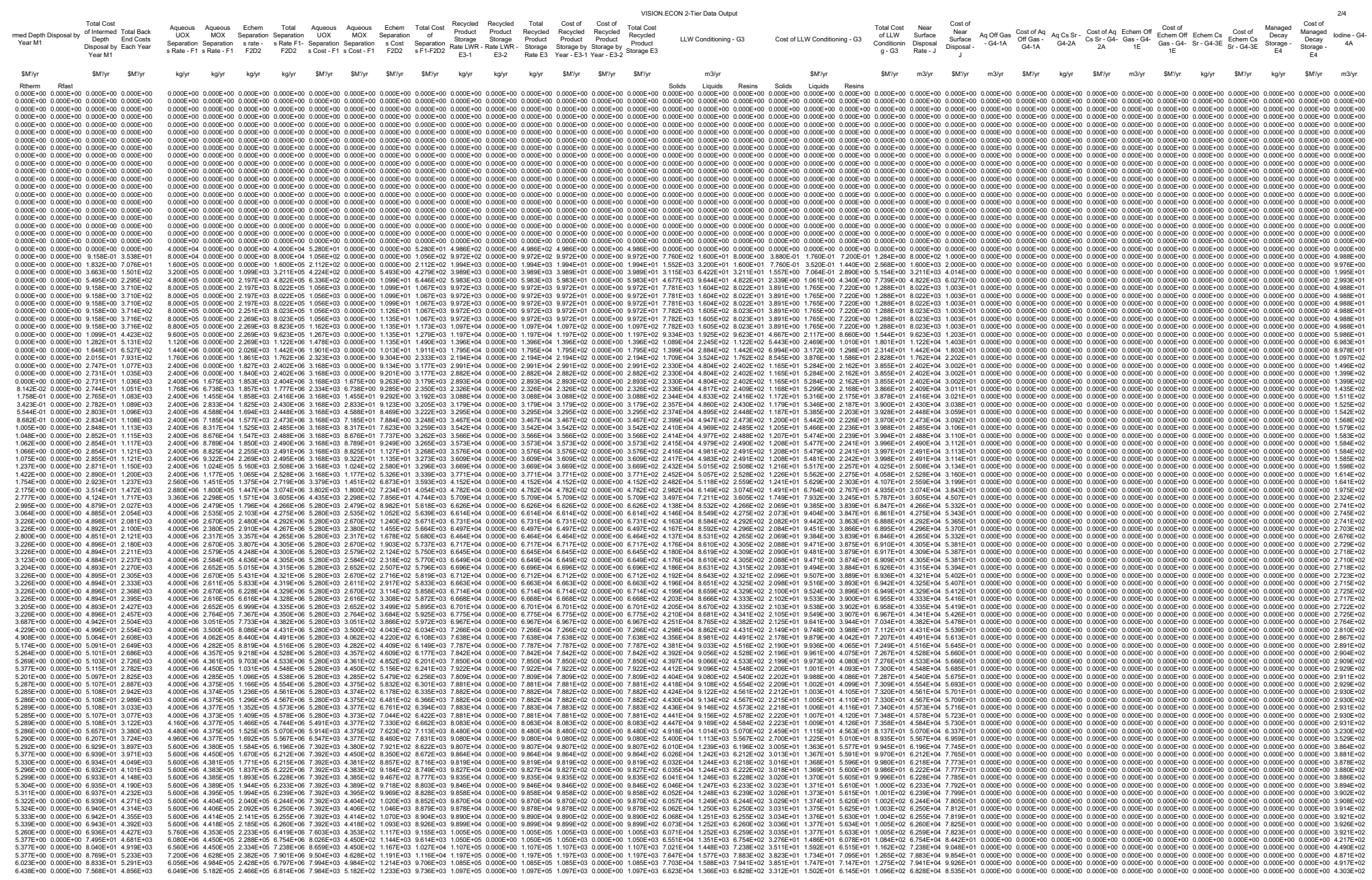



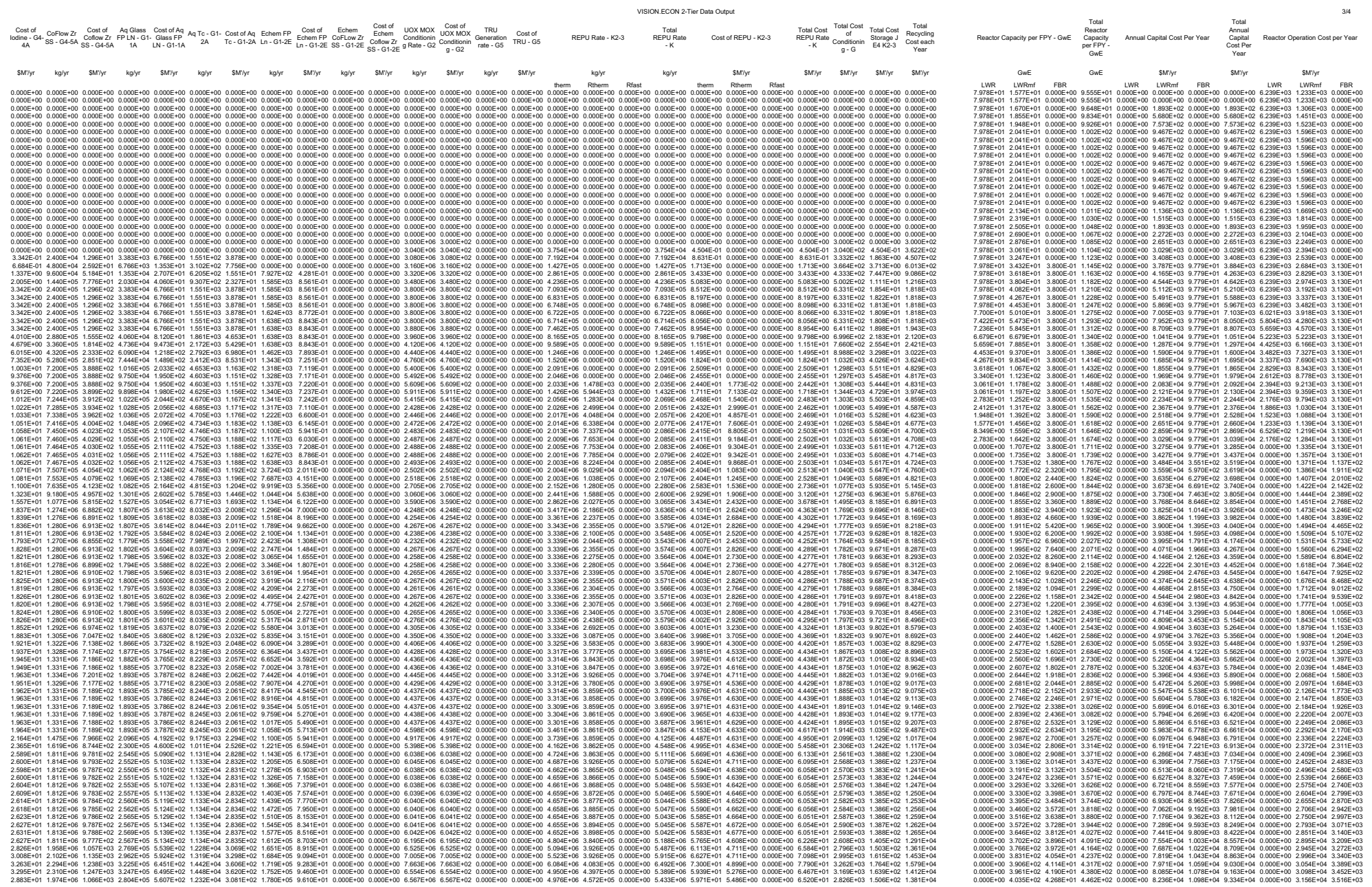

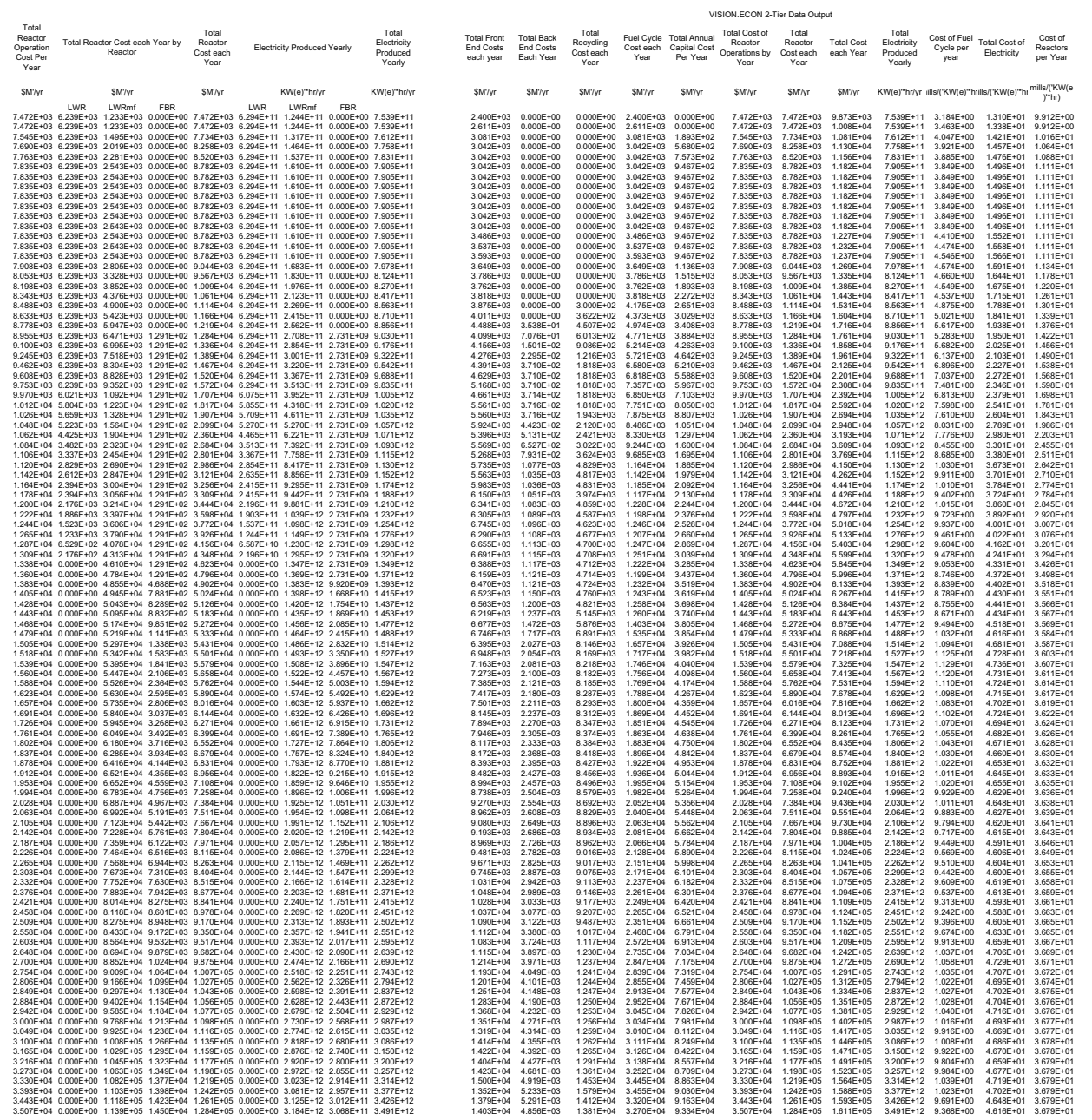

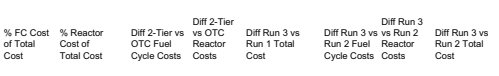

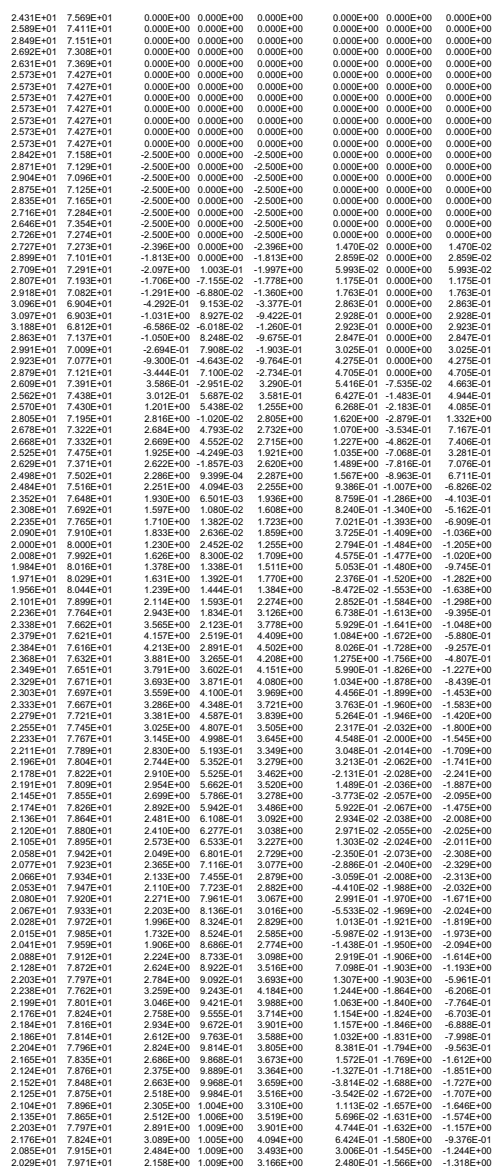

